*ak RMIS View/Frint Document Cover Sheet tow

This document was retrieved from the Documentation and Records Manaqement (DRM) ISEARCH System. It is intended for Information only and may not be the most recent or updated version. Contact a Document Service Center (see Hanford Info for locations) if you need additional retrieval information.

Accession \#: D196030799

Document \#: SD-WM-VI-031

Title/Desc:

VECTRA GSI INC LLW MELTER TESTING PHASE 1 TEST REPORT

Pages: 291 


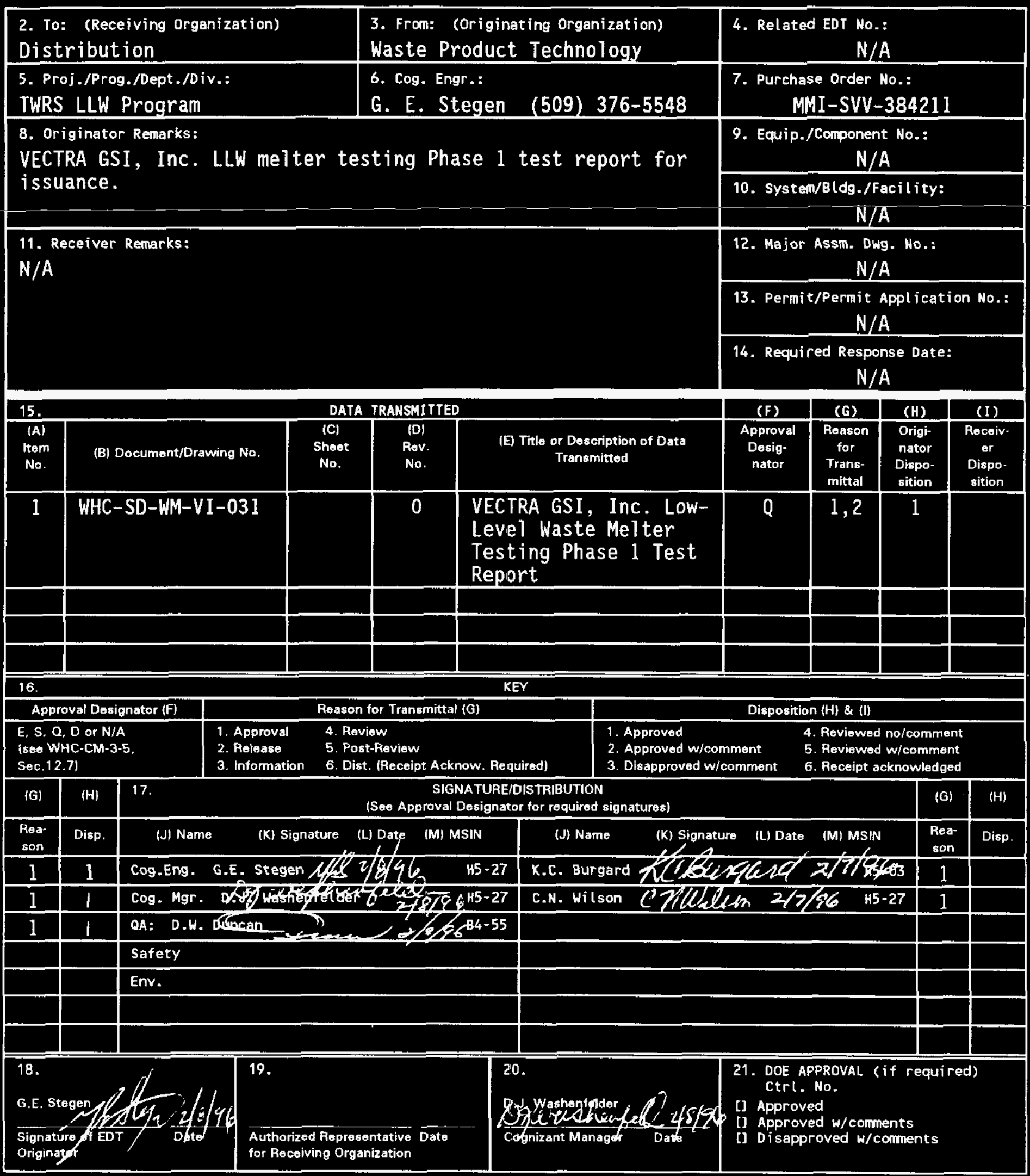

BD-7400-172-2 (04/94) GEF097 


\title{
Vectra GSI, Inc. Low-Level Waste Melter Testing Phase 1 Test Report
}

\author{
G. E. Stegen, C. H. Wilson
}

Westinghouse Hanford Company, Richland, WA 99352

U.S. Department of Energy Contract DE-ACO6-87RL10930
EDT/ECN: 611890
UC: 2020
Org Code: 73510
Charge Code: D4NCO
B\&R Code: EW3130010
Total Pages: 287

Key Words: Low-level waste, vitrification, melter, Hanford Site tank waste, fluid bed calcination, rotary dryer, slurry feed

Abstract: A multiphase program was initiated in 1994 to test commercially available melter technologies for the vitrification of the low-level waste (LLW) stream from defense wastes stored in underground tanks at the Hanford Site in southeastern Washington State. Vectra GSI, Inc. was one of seven vendors selected for Phase 1 of the melter demonstration tests using simulated LLW that were completed during fiscal year 1995. The attached report prepared by Vectra GSI, Inc. describes results of melter testing using slurry feed and dried feeds. Results of feed drying and prereaction tests using a fluid bed calciner and rotary dryer also are described.

TRADEMARK DISCLAIMER. Reference herein to any specific commercial product, process, or service by trade neme, tredemark, manufacturer, or otherwise, does not necessarily constitute or inply its endorsement, recommendation, or favoring by the United states Government or any agency thereof or its contractors or subcontractors.

Printed in the United States of America. Ta obtain copies of this document, contact: UHC/Bcs Document Control Services, P.0. Box 1970, Mailstop H6-08, Richland WA 99352, Phone (509) 372-2420; Fax (509) 376-4989.

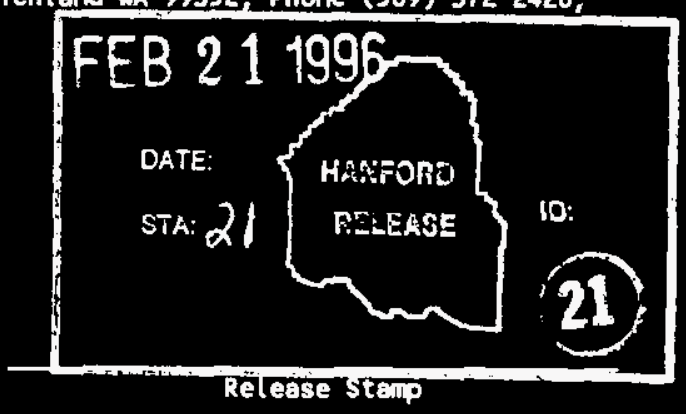

Approved for Public Release 
WHC-SD-WM-VI-031

Revision 0

\section{PART 1 - FLUID BED CALCINER TEST REPORT}


WHC-SD-WM-VI-031

Revision 0

FLUID BED CALCINER TEST REPORT - FINAL

For

WHC Contract No. MMI-SVV-384211

EVALUATION OF MELTER SYSTEM TECHNOLOGIES FOR VITRIFICATION OF HIGH SODIUM CONTENT LLRW

VECTRA GSI Report No. WHC-VIT-03

August 1995

by

VECTRA Government Services, Inc.

2939 Richardson Road

Richland, WA 99352

Prepared by:

John J. Koehr Rantecler
Approved by:

Brad Mason Mamadow 
WHC-SD-WM-VI-031

Revision 0

\section{CONTENTS}

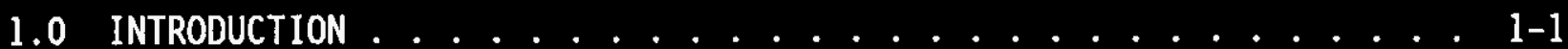

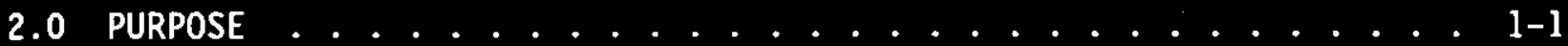

3.0 CHRONOLOGY OF EVENTS . . . . . . . . . . . . . . 1-2

4.0 EQUIPMENT OPERATING BEHAVIOR ............. 1-2

4.1 CALIBRATION . . . . . . . . . . . . . . 1-2

4.1.1 Waste Feed Pump . . . . . . . . . . . 1-2

4.1.2 Pressure Indicator (PI) Calibration ....... 1-2

4.1.3 Temperature Indicator (TI) Calibration....... 1-2

4.1.4 CEMS Calibration .............. . . 1-6

4.2 FLOW DIAGRAM ................... 1-6

4.3 CHEMICAL MIXING AND BATCHING ........... 1-7

4.3.1 Bench and Pilot Simulant Feed System ........ 1-7

4.3.2 Bench and Pilot Calciner Filter Blowback Gas . . . . . 1-7

4.3.3 Bench Scale Calciner Start-up and Shutdown Sequence + 1-8

4.3.4 Sampling .............. . . 1-8

4.3.5 Bench Scale Scrubber Solution ........... . 1-9

4.3.6 Bench Scale Dry Glass Former Feed System . . . . . . 1-9

5.0 BENCH SCALE TESTS AND OBSERVATIONS ............... 1-9

5.1 GLASS FORMER FLUIDIZATION TESTS . . . . . .... 1-9

5.2 FBC-SO: GLASS FORMERS W/ WATER/SIMULANT, NO REDUCTANTS . . 1-10

5.3 FBC-S1: GLASS FORMERS AND SIMULANT W/ 600\% SUCROSE AT

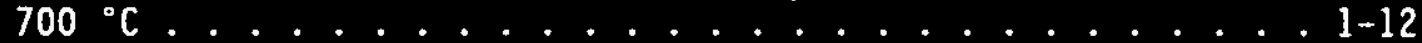

5.4 FBC-S2: GLASS FORMERS AND SIMULANT W/ 200\% AMMONIA AT $700{ }^{\circ} \mathrm{C}$. . . . . . . . . . . . . . . . . . . . . .

5.5 FBC-S3: GLASS FORMERS AND SIMULANT W/ $100 \%$ SUCROSE AT $700{ }^{\circ} \mathrm{C} . . . . .1-14$

5.6 FBC-S4: GLASS FORMERS AND SIMULANT W/ 125\% SUCROSE AT $500{ }^{\circ} \mathrm{C} \ldots$. 1-15

5.7 FBC-S5: GLASS FORMERS AND SIMULANT W/ 100\% SUCROSE AT

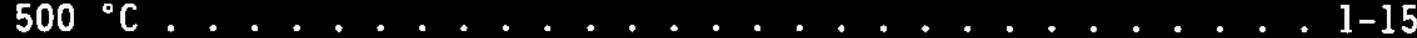

5.8 FBC-S6: GLASS FORMERS AND SIMULANT W/ 200\% AMMONIA AT

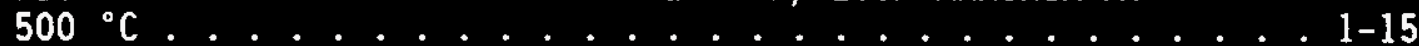

5.9 FBC-S7: GLASS FORMERS AND SIMULANT W/ 200\% HYDROGEN AT $300{ }^{\circ} \mathrm{C}$. . . . . . . . . . . . . . . . . . . . . . . .

5.10 FBC-S8: GLASS FORMERS AND SIMULANT W/ 200\% METHANE AT

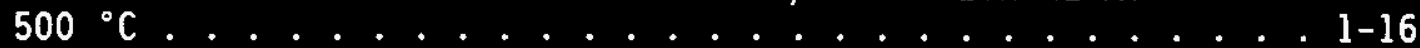

5.11 FBC-S9: GLASS FORMERS AND SIMULANT $W / 75 \%$ SUCROSE AT $500{ }^{\circ} \mathrm{C}$. . . . . . . . . . . . . . . . . . . . . .

5.12 FBC-S10: GLASS FORMERS W/ 100\% BORIC ACID FEED AT $500{ }^{\circ} \mathrm{C} \ldots 1-17$

5.13 FBC-S11: GLASS FORMERS (INCLUDING 9\% BORIC ACID) AND $16 \%$ BORIC ACID FEED AT $500^{\circ} \mathrm{C} \ldots \ldots$. . . . . . . . . . . . .

5.14 FBC-S12: GLASS FORMERS W/ BORIC OXIDE FEED AND SIMULANT W/ $100 \%$ SUCROSE AT $400{ }^{\circ} \mathrm{C} \ldots \ldots$. . . . . . . . . . . . . .

5.15 FBC-S13: GLASS FORMERS W/ BORIC OXIDE FEED AND SIMULANT W/ $100 \%$ SUCROSE AT $425{ }^{\circ} \mathrm{C} \ldots \ldots$. . . . . . . . . . . . . 19

5.16 BENCH SCALE TEST EVALUATION SUMMARY .......... . . 1-19 
WHC-SD-WM-VI-031

Revision 0

CONTENTS (cont)

6.0 PILOT SCALE TESTS AND OBSERVATIONS . . . . . . . . . . 1-21

6.1 FBC-P0: GLASS FORMERS WITH WATER ........... . 1-21

6.2 PRODUCTION RUN: GLASS FORMERS AND SIMULANT WITH

100\% SUCROSE . . . . . . . . . . . . . 1-22

7.0 CONCLUSIONS . . . . . . . . . . . . 1-23

8.0 RECOMMENDATIONS FOR FUTURE TESTING . . . . . . . . . 1-24

APPENDIXES

IA LAB ANALYSES .................. 1-25

1B MASS BALANCE CALCULATIONS ............... 1-71

IC DATA SHEETS ................... 1-91

\section{LIST OF TABLES}

1-1 Fluid Bed Calciner Test Log Summary . . . . . . . . . . 1-3

1-2 Fluid Bed Composition, Glass Former Mix A . . . . . . . 1-11

1-3 Fluid Bed Composition, Glass Former Mix B . . . . . . . . 1-12

1-4 Fluid Bed Composition, Glass Former Mix C . . . . . . . 1-14

1-5 Fluid Bed Composition, Glass Former Mix D . . . . . . 1-18

1-6 Fluid Bed Composition, Glass Former Mix E . . . . . . . . 1-19

1-7 Bench Scale fluid Bed Calciner Test Run Summary . . . . . . . . 1-20

1-8 Pilot Scale Glass Former Mix C . . . . . . . . . 1-21 
WHC-SD-WM-VI-031

Revision 0

\section{ACRONYHS}

GSI VECTRA Government Services, Inc.

LLRW low-level radioactive waste

MCW melter cooling water

RBEA Roger B. Ek Associates

RVR radwaste volume reduction

TPD tons per day

WHC Westinghouse Hanford Company 
WHC-SD-WM-VI-03I

Revision 0

This page intentionally left blank. 
WHC-SD-KM-VI-031

Revision 0

PART 1 - FLUID BED CALCINER TEST REPORT

\subsection{INTRODUCTION}

The Westinghouse Hanford Company (WHC) is conducting an evaluation of glass melter system technologies for vitrification of liquid low-level radioactive waste (LLRW) streams consisting mostly of sodium nitrate and nitrite salts in alkaline liquid slurry form. As part of this technology evaluation, VECTRA will demonstrate the performance of the EnviroGlass Vitrification System feed and VE-SKULL melter subsystems. The Fluid Bed Calciner system is part of the melter feed system and is intended to dry and react the liquid waste simulant and mix the simulant with glass formers prior to vitrification.

\subsection{PURPOSE}

This test report documents accomplishment of the Fluid Bed Calciner test objectives. Test objectives included the following:

2.1 Demonstrate that a fluidized bed can be operated successfully to produce a dry, prereacted material that is suitable as a melter feed using at least one reductant.

2.2 Demonstrate ability of fluid bed calciner to do the following:

- Evaporate water

- Volatize $\mathrm{CO}_{3}, \mathrm{NO}_{3}$, and $\mathrm{NO}_{2}$

- Reduce $\mathrm{NO}_{3}$ and $\mathrm{NO}_{2}$ with good yield of $\mathrm{N}_{2}$ gas

- Calcine inorganics (Na, K, Al, Ca, etc.)

- Pyrolyze/0xidize organics.

2.3 Determine throughput of calciner when operating within parameters for achieving objectives 2.1 and 2.2 .

2.4 Determine efficiency of $\mathrm{NO}_{3} / \mathrm{NO}_{2}$ reductions to $\mathrm{N}_{2}$ using the following additives: methane $\left(\mathrm{CH}_{4}\right)$ gas, hydrogen $\left(\mathrm{H}_{2}\right)$ gas, sol id sucrose $\left(\mathrm{C}_{12} \mathrm{H}_{22} \mathrm{O}_{11}\right)$, and ammonia $\left(\mathrm{NH}_{3}\right)$ gas. Investigate affects of reductant to $n$ itrate ratio over a wide temperature range.

TMVE-SKULL and EnviroGlass are trademarks of VECTRA Technologies, Inc. 
WHC-SD-WM-VI-031

Revision 0

\subsection{CHRONOLOGY OF EVENTS}

A detailed chronology of fluid bed calciner bench scale testing has been recorded in the Test Log. A brief summary is provided in Table 1-1. Numbers shown in parentheses following the VECTRA GSI test number are corresponding Procedyne test numbers.

\subsection{EQUIPNENT OPERATING BEHAYIOR}

\subsection{CALIBRATION}

Generally, equipment calibration was performed and documented as required by the Test Procedure. Any deviations from the test procedure or otherwise noteworthy equipment calibration related events are summarized below.

\subsubsection{Waste Feed Pump.}

1. Initial Waste feed pump (pump no. 1) was a laboratory, positive displacement, piston type, simplex pump. Pump calibration was performed at various piston stroke settings (0-10 range) to develop a curve of pump setting vs. flowrate. Pump no.l flowrates became inconsistent during $\mathrm{FBC}-\mathrm{SO}(\mathrm{I}-2)$ testing and the pump was unable to overcome vessel pressure, indicating pump failure.

2. A back-up feed pump (pump no. 2) was calibrated and installed. Pump no. 2 was a 20 GPD peristaltic tubing pump with variable speed and a discharge pressure rating of $25 \mathrm{psig}$. The pump was calibrated using water and various pump speeds. A curve of pump speed (rpm) vs. flow rate $(\mathrm{ml} / \mathrm{min})$ was generated over the range of the pump. Calibrated settings were verified at multiple discharge pressure settings and with simulant and sucrose mixtures. Calibration data are included in Reference 3.2. Pump no. 2 tubing $k$ it was-replaced on 2/15/95 prior to $\mathrm{FBC}-\mathrm{S3}$ ( $\mathrm{I}-7$ ) testing. A single point calibration check was performed and found to be consistent with the original calibration.

\subsubsection{Pressure Indicator (PI) Calibration}

Original pressure gauge PI-FG, for the fluidizing gas supply pressure to the vesse1, had a low range (0-100" water). Over the course of testing, this gauge was replace with a 0-10 psig gauge. Gauges were cal ibrated.

\subsubsection{Temperature Indicator (TI) Calibration}

Thermocouples Tl through T6 were calibrated using a manufacturer's calibrated thermocouple (TC) with 2-point temperature check. 
Table 1-1. Fluid Bed Calciner Test Log Summary. (4 sheets)

\begin{tabular}{|c|c|}
\hline Date & Activities/events \\
\hline $\begin{array}{l}2 / 8 / 95 \\
\text { FBC-SO }(I-1)\end{array}$ & $\begin{array}{l}\text { - Glass formers mix } A \text {, water injection only, no } \\
\text { reductants, at } 750 \text { 'C. Mix A included boric acid at } \\
\text { approximately } 9.2 \% \text { wt } \\
\text { - Good dry bed fluidization } \\
\text { - Obtained/confirmed fluid bed heat transfer data } \\
\text { ( } 20 \mathrm{ml} / \mathrm{min} \text { max feed flow) } \\
\text { - Feed pump no. I calibration point verification } \\
\text { failed/ recalibrated pump }\end{array}$ \\
\hline $\begin{array}{l}2 / 9 / 95 \\
\text { FBC-SO (I-2) }\end{array}$ & $\begin{array}{l}\text { - Glass formers mix A with simulant, no reductants, at } \\
750{ }^{\circ} \mathrm{C} \\
\text { - Feed pump no. } 1 \text { failed, test terminated early }\end{array}$ \\
\hline $\begin{array}{l}2 / 10 / 95 \\
\text { FBC-SO (I-3) }\end{array}$ & $\begin{array}{l}\text { - Glass formers mix } A \text { with simulant only, no } \\
\text { reductants, at } 700{ }^{\circ} \mathrm{C} \text {. } \\
\text { - Feed pump no. } 2 \text { calibrated and installed } \\
\text { - High NOx, rapid bed caking }\end{array}$ \\
\hline $\begin{array}{l}2 / 13 / 95 \\
\text { FBC-S1 (I-4) }\end{array}$ & $\begin{array}{l}\text { - Glass formers mix B, at } 700{ }^{\circ} \mathrm{C} \text {. Mix B included boric } \\
\text { acid at } 16.2 \% \mathrm{wt} \\
\text { - Modified glass former bed proportions based on } \\
\text { revised calculations. } \\
\text { - Lost fluidization prior to simulant injection due to } \\
\text { melting Boric Acid at higher relative weight percent. } \\
\text { - Glass former mix } \mathrm{C}, \mathrm{w} / \mathrm{simulant} \text { and } 600 \% \text { sucrose, at } \\
700{ }^{\circ} \mathrm{C} \text {. Mix } \mathrm{C} \text { had no boric acid. } \\
\text { - Good initial fluidization, no Nox } \\
\text { - Lost fluidization at } 3 \text { hours, continued for full run } \\
\text { - Vessel completely caked with carbon } \\
\text { - Vess). }\end{array}$ \\
\hline $\begin{array}{l}2 / 14 / 95 \\
F B C-S 2(I-6)\end{array}$ & $\begin{array}{l}\text { - Glass former mix } \mathrm{C}, \mathrm{W} / \text { simulant and } 200 \% \mathrm{NH}_{3} \\
\text { injection, at } 700{ }^{\circ} \mathrm{C} . \\
\text { - Feed injection nozzle plugged from previous sucrose } \\
\text { test, repaired } \\
\text { - Low Nox } \\
\text { - Lost fluidization rapidly, but continued full run } \\
\text { (4 hours) } \\
\text { - Severe caking in bed }\end{array}$ \\
\hline
\end{tabular}


Table 1-1. Fluid Bed Calciner Test Log Summary. (4 sheets)

\begin{tabular}{|c|c|}
\hline Date & Activities/events \\
\hline $\begin{array}{l}2 / 15 / 95 \\
\text { FBC-S3 (I-7) }\end{array}$ & $\begin{array}{l}\text { - Glass formers mix } C, W / \text { simulant and } 100 \% \text { sucrose, at } \\
700{ }^{\circ} \mathrm{C} \text {. } \\
\text { - Shifted to open testing with modified sucrose } \\
\text { percentages } \\
\text { - Replaced pump no. } 2 \text { hose. Performed single point } \\
\text { cal ibration check with simulant. } \\
\text { - Added water flush funne to feed suction } 1 \text { ine. } \\
\text { - Lost fluidization quickly. Terminated test early } \\
\text { - Bed inspection revealed caking } \\
\text { - Glass formers mix } C, W / \text { simulant and } 125 \% \text { sucrose, at } \\
\text { - Foo }{ }^{\circ} \mathrm{C} \\
\text { - Low Nox } \\
\text { - Post test bed inspection revealed successful run with } \\
\text { minimal caking }\end{array}$ \\
\hline $\begin{array}{l}2 / 16 / 95 \\
\text { FBC-S5 (I-9) }\end{array}$ & $\begin{array}{l}\text { - Glass formers mix } \mathrm{C}, \mathrm{W} / \mathrm{simulant} \text { and } 100 \% \text { sucrose, at } \\
500{ }^{\circ} \mathrm{C} \\
\text { - Ful } 4 \text {-hour run } \\
\text { - Low NOx } \\
\text { - Inspection revealed successful run } \\
\text { - Glass formers mix } \mathrm{C}, \mathrm{W} / \mathrm{simulant} \text { and } 200 \% \mathrm{NH}_{3} \\
\text { addition, at } 500{ }^{\circ} \mathrm{C} \\
\text { - Rapid loss of fluidization } \\
\text { - Low NOx } \\
\text { - Inspection revealed bed caking }\end{array}$ \\
\hline $\begin{array}{l}2 / 17 / 95 \\
\text { FBC-S7 (I-11) }\end{array}$ & $\begin{array}{l}\text { - Glass formers mix } \mathrm{C}, \mathrm{w} / \mathrm{simulant} \text { and } 200 \% \mathrm{H}_{2} \\
\text { addition, at } 300{ }^{\circ} \mathrm{C} \\
\text { - Rapid loss of fluidization } \\
\text { - Low NOx } \\
\text { - Inspection showed bed caking }\end{array}$ \\
\hline$F B C-S 8(I-12)$ & $\begin{array}{l}\text { - Glass formers mix } \mathrm{C}, \mathrm{w} / \mathrm{simulant} \text { and } 200 \% \mathrm{CH}_{4} \text {, at } \\
500{ }^{\circ} \mathrm{C} \\
\text { - Rapid loss of fluidization } \\
\text { - High NOx } \\
\text { - Terminated test early } \\
\text { - Inspection showed caking }\end{array}$ \\
\hline FBC-S9 (I-13) & $\begin{array}{l}\text { - Glass formers mix } C, W / \text { simulant and } 75 \% \text { sucrose at } \\
500{ }^{\circ} \mathrm{C} \\
\text { - Ful7 } 4 \text {-hour test run } \\
\text { - Low NOx } \\
\text { - Post test inspection revealed successful test with } \\
\text { minimal caking }\end{array}$ \\
\hline
\end{tabular}


Table 1-1. Fluid Bed Calciner Test Log Summary. (4 sheets)

\begin{tabular}{|c|c|}
\hline Date & Activities/events \\
\hline $\begin{array}{l}2 / 22 / 95 \\
F B C-S 10(I-14)\end{array}$ & $\begin{array}{l}\text { - Glass formers mix } \mathrm{C}, \mathrm{W} / 100 \% \text { solid boric acid } \\
\text { injected at } 500^{\circ} \mathrm{C} \text {. No simulant injected. } \\
\text { - } 75 \text { minute test run } \\
\text { - Feed line plugged with melted boric acid } \\
\text { - Post test inspection revealed bed agglomerations } \\
\text { - Glass former mix } \mathrm{D}, \mathrm{W} / 16 \mathrm{wt} \% \text { solid boric acid } \\
\text { injected at } 500^{\circ} \mathrm{C} \text {. Mix } \mathrm{D} \text { included boric acid at } \\
9.2 \mathrm{wt} \% \\
\text { - Good fluidization of initial bed } \\
\text { - Feed line plugged rapidly with melted boric acid } \\
\text { - Post test inspection revealed large hard lump at top } \\
\text { of bed }\end{array}$ \\
\hline $\begin{array}{l}2 / 28 / 95 \\
F B C-S 12(I-16)\end{array}$ & $\begin{array}{l}\text { - Glass formers mix E, w/ simulant and } 100 \% \text { sucrose at } \\
400{ }^{\circ} \mathrm{C} \text {. Mix E included boric oxide at } 8 \% \text { wt which } \\
\text { was fed separately into fluidizing bed. } \\
\text { - Glass former mix fluidized well throughout most of } \\
\text { test } \\
\text { - } 3-3 / 4 \text { four test run } \\
\text { - Fluctuating Nox levels } \\
\text { - Post test inspection revealed a free flowing reddish } \\
\text { bed below a soft black cake (from unreacted sucrose). }\end{array}$ \\
\hline $\begin{array}{l}3 / 6 / 95 \\
\text { FBC-S13 (I-17) }\end{array}$ & $\begin{array}{l}\text { - Glass formers mix E, w/ simulant and } 100 \% \text { sucrose at } \\
425{ }^{\circ} \mathrm{C} \text {. Mix E included boric oxide at } 8 \% \text { which } \\
\text { was fed separately into fluidizing bed. } \\
\text { - Glass former mix fluidized well throughout most of } \\
\text { test } \\
\text { - Fluctuating Nox levels } \\
\text { - Post test inspection revealed a free flowing reddish } \\
\text { bed below a soft black cake (from unreacted sucrose). }\end{array}$ \\
\hline $\begin{array}{l}3 / 8 / 95 \\
\text { FBC-PO (I-1) }\end{array}$ & $\begin{array}{l}\text { - Initial bed loading of } 2051 \mathrm{~b} \text { of glass former mix } \mathrm{C} \text {. } \\
\text { Mix } \mathrm{C} \text { included no boric acid } \\
\text { - Fluidization with } \mathrm{N}_{2} \text { at } 30 \mathrm{fpm} \text { and bed temperature at } \\
500{ }^{\circ} \mathrm{C} \text {. } \\
\text { - No simulant/glass former feed or product discharge } \\
\text { - Water injected at various flowrates to establish } \\
\text { thermodynamic performance. Bed fluidized well at a11 } \\
\text { flowrates. Bed temperatures were steady and the wall } \\
\text { temperatures stayed below } 540{ }^{\circ} \mathrm{C} \text {. }\end{array}$ \\
\hline FBC-PI (I-1) & $\begin{array}{l}\text { - Bed fluidization continued at } 500{ }^{\circ} \mathrm{C} \text {. } \\
\text { - Simulant with } 100 \% \text { stoichiometric sucrose injected to } \\
\text { establish initial simulant solid loading in bed. } \\
\text { - Batch fluidization with simulant injection continued } \\
\text { for } 4 \text { hours at } 500{ }^{\circ} \mathrm{C} \text {. } \\
\text { - Calciner cooled down and shutdown overnight }\end{array}$ \\
\hline
\end{tabular}


Table 1-1. Fluid Bed Calciner Test Log Summary. (4 sheets)

\begin{tabular}{|c|c|}
\hline Date & Activities/events \\
\hline $\begin{array}{l}3 / 9 / 95 \\
F B C-P 1(I-1)\end{array}$ & $\begin{array}{l}\text { - Batch fluidization resumed at } 500{ }^{\circ} \mathrm{C} \\
\text { - Simulant } w / 100 \% \text { stoichiometric sucrose injected to } \\
\text { achieve initial dry simulant bed loading. } \\
\text { - Balanced simulant injection, dry glass former feed, } \\
\text { and product discharge initiated. } \\
\text { - Steady state production continued for } 7 \text { hours } \\
\text { - } 181 \mathrm{Kg}(4001 \mathrm{~b}) \text { dry product processed } \\
\text { - Calciner cooled down and shutdown overnight }\end{array}$ \\
\hline $\begin{array}{l}3 / 9 / 95 \\
F B C-P 1\end{array}$ & $\begin{array}{l}\text { - Bed fluidization resumed at } 500{ }^{\circ} \mathrm{C} \text {. } \\
\text { - Balanced simulant/100\% sucrose injection, dry glass } \\
\text { formers, and product discharge resumed. } \\
\text { - Feed pump leak forced shutdown and cooldown. } \\
\text { Reinitiation of feed at } 464{ }^{\circ} \mathrm{C} \text { resulted in bed cakes, } \\
\text { loss of fluidization, and product discharge plugging. } \\
\text { - Steady state fluidization at } 500{ }^{\circ} \mathrm{C} \text { with balanced } \\
\text { feed and discharge was reinitiated after repairs. } \\
\text { - } 4 \text { hour mass balance performed } \\
\text { - Production complete after } 5 \text { hours continuous } \\
\text { operation, } 272 \mathrm{Kg}(6001 \mathrm{~b}) \text { product generated for } \\
\text { total of } 454 \mathrm{Kg} \mathrm{(1,000} 1 \mathrm{~b}) \text {. } \\
\text { - Calciner cooled down and shutdown. } \\
\text { - Inspection revealed free flowing product, some caking } \\
\text { on simulant injection } 1 \text { ine, and light freeboard } \\
\text { caking. }\end{array}$ \\
\hline
\end{tabular}

\subsubsection{CEMS Calibration}

A continuous emissions monitoring system (CEMS) combustion gas and pollution gas monitor was installed and calibrated by an EPA qualified vendor for $\mathrm{NO}, \mathrm{NO}_{2}$, and $\mathrm{NOx}$ only.

\subsection{FLON DIAGRAM}

The following changes to the Bench Scale Calciner Flow Diagram were incorporated during testing.

- Pressure gauge (PI-FEED) was added downstream of the feed pump. Although positive displacement peristaltic pump flowrates are independent of discharge pressure within the rating of the pump, PI-FEED was necessary to quickly give an indication of feed system problems (such as plugged injection line).

- A branch line with a funnel and isolation valve was added at the feed pump suction to allow water flush following tests. The injection line was found to plug rapidly on tests immediately following sucrose injection. 
WHC-SD-WM-VI-031

Revision 0

- PI-OUT was not part of the existing equipment, was considered unnecessary for operations or testing, and was deleted from the drawing. Filter differential pressure (DP-FF) taps into the calciner top freeboard and the off-gas line, downstream of the filters. In many cases during testing, a high vessel pressure ( $>3$ psig) required isolation of DP-FF to prevent over-ranging.

- TI-WALL, inside wall temperature, was deleted since installation was not practical. This temperature indication was not critical to bench scale fluid bed calciner operation or test results. A wall temperature indication was available on the pilot scale test bed.

- TI-Annulus (T6), external electric heater temperature, was added and recorded. Although not critical for testing results, this temperature readout is useful in verifying proper fluid bed calciner operation.

\subsection{CHEMICAL MIXING AND BATCHING}

\subsubsection{Bench and Pilot Simulant Feed System}

Two (2) DOT 17H 55-gallon drums were provided with DSSF simulant for Fluid Bed Calciner testing. These drums were lined with plastic bags prior to simulant fill and shipment to Procedyne. To avoid damaging the plastic bags which could result in plastic contamination of feed, these drums were allowed to settle. Liquid simulant was pumped from the simulant shipping drum to an intermediate 55-gallon poly mix drum. Residual solids within the plastic shipping bags were manually transferred into the intermediate mix drum.

The intermediate mix drum was equipped with an electric mixer, drum pump, and splash lid. DSSF simulant was thoroughly mixed within the intermediate mix drums and transferred via the installed drum pump into a portable container for manual filling of the $26 \mathrm{~L}$ (7 gal) stainless steel waste hold-up tank (feed tank). Sucrose $\left(\mathrm{C}_{12} \mathrm{H}_{22} \mathrm{O}_{11}\right)$ was manually added to the waste hold-up tank in a batch quantity necessary for the original sugar test (FBC-S1). Open sucrose testing (FBC-S3/4/5/9) utiljzed the original concentrated $600 \%$ stoichiometric simulant/sugar solution remaining from FBC-SI which was mixed with new simulant in the waste hold-up tank in the proportions necessary to achieve the proper stoichiometric ratios and test volumes.

The waste hold-up tank mixer was continuously operated during simulant feeding into the fluid bed calciner. The feed suction flush funnel allowed water flush through the feed line into the fluid bed prior to cooldown for all test after FBC-S3.

\subsubsection{Bench and Pilot Calciner Filter Blowback Cas}

Separate $\mathrm{N}_{2}$ bottles were not utilized for filter blowback gas. Filter blowback operated continuously with a 5 second blowback every 40 seconds during bed fluidization. Although filter blowback operation introduced $\mathrm{N}_{2}$ gas into the bed and ultimately in the off-gas, $N_{2}$ mass balance accountability was 
WHC-SD-WM-VI-031

Revision 0

not required for bench scale testing. Additionally, only $\mathrm{NO}, \mathrm{NO}_{2}$, and $\mathrm{NO}_{3}$ were monitored in the off-gas. Filter blowback $\mathrm{N}_{2}$ bottles were weighted during Pilot scale mass balance run (FBC-PI).

\subsubsection{Bench Scale Calciner Start-up and Shutdown Sequence}

The bench scale calciner start-up procedure varied slightly from that outlined in the Test Procedure (Reference 3.2). The fluidized alumina bath external heating furnace was preheated to expected operating temperature prior to each test.

Before insertion into the alumina bath furnace, the bench scale test vessel was loaded with a $5.5 \mathrm{~kg}$ (12 1b) premixed glass former bed. The test vessel was then mounted to the top assembly (freeboard) suspended from overhead rigging equipment. Fluidizing gas, $\mathrm{N}_{2}$ filter blowback, and off-gas hoses were attached. Fluidizing $\mathrm{N}_{2}$ gas flow and the $\mathrm{N}_{2}$ blowback auto sequence were then initiated. While continuously monitoring temperatures, the bench scale fluid bed test vessel was then lowered into the preheated fluidized external heating furnace bath to commence test bed heat-up. The feed line was then connected.

Once steady state temperatures were established in the test fluid bed, gaseous reductant flow was initiated for applicable tests. Feed injection was then initiated to start the test. Instead of varying feed flow up to the heat transfer 1 imits of the bed, a nominal $20 \mathrm{ml} / \mathrm{min}$ flowrate was maintained throughout all testing, based on the conservative heat transfer results with water injection.

The feed metering quantity was calculated using calibrated pump flowrates and resulting time to achieve the quantity of simulant necessary for proper glass ratios. Feed injection was stopped after the appropriate amount of simulant had been injected. For FBC-S3 and later tests, a water flush funnel was available at the feed pump suction. Potable water was added to the funnel, the waste hold-up tank was isolated, and water was injected to clear the feed line of any residual simulant. This was particularly important for tests with sucrose.

Following test completion, the feedline was isolated and disconnected from the test vessel. External cooling water flow and fluidizing gas flow were then initiated to the alumina cooldown bath, located next to the heating furnace. The test vessel was then lifted from the furnace and inserted in the alumina cooldown bath using overhead rigging equipment. Test bed fluidization continued until the bed was cooled down to a point where it could be safely handled.

\subsubsection{Sampling}

During testing, small test fluid bed samples were drawn from the lower bed region using $N_{2}$ motive gas and a sample line venturi eductor. In many cases, the inability to draw a large volume sample with the eductor indicated that the bed had lost fluidization. 
WHC-SD-WM-VI-031

Revision 0

\subsubsection{Bench Scale Scrubber Solution}

Soda ash $\left(\mathrm{Na}_{2} \mathrm{CO}_{3}\right)$ was added to the scrubber solution to maintain $\mathrm{pH}$. Scrubber solution replacement was required when temperature reached $120^{\circ} \mathrm{F}$ due to PVC components in the solution piping.

\subsubsection{Bench Scale Dry Glass Former Feed System}

For bench scale test FBC-S10 (I-14) through FBC-S13 (I-17), dry glass formers were fed into the fluidizing bed during testing in order to gradually add boric acid and boric oxide. The feed line utilized an existing un-used 3/4" penetration with a "feedlock" spool attached. The feedlock spool consisted of two ball valves with a pipe spool between equipped with a connection for $N_{2}$ pressurization. One end of the spool was connected to the top of the calciner freeboard. The other end was open for addition of dry feed material.

During dry feeding the bottom ball valve was closed and the top valve open to load the feed material. After loading, the top valve was closed and the feedlock spool between the valves was pressurized with $N_{2}$ to approximately $80 \mathrm{Kpag}$ (12 psig). With the fluid bed operating at approximately $20 \mathrm{Kpag}$ ( 3 psig), the bottom ball valve was opened and the feedlock contents were injected into the fluid bed.

\subsection{BENCH SCALE TESTS AND OBSERYATIONS}

\subsection{GLASS FORIER FLUIDIZATION TESTS}

Although not specifically required by the Test Procedure, fluidization tests were performed with bulk glass former materials and the premixed glass former bed in order to qualitatively evaluate the adequate fluidization of the glass formers and determine the optimum fluidization gas velocity for subsequent testing.

Fluidization tests were performed in transparent fluid bed test tubes 14 and $7.6 \mathrm{~cm}\left(5.5^{\prime \prime}\right.$ and $3^{\prime \prime}$ diameter). Compressed air was used as the fluidization gas. An initial $15 \mathrm{~cm}\left(6^{\prime \prime}\right)$ bed height was loaded. Fluidization gas flow was slowly initiated. The "incipient" velocity was recorded when the bed started to fluidize. The "operating" velocity was recorded when the bed stabilized at approximately 1.5 times the initial bed height. The "maximum" fluidizing velocity was noted when the fluidizing action became unstable and excessive dusting started to occur.

A nominal glass former bed flowrate was determined to be $6 \mathrm{~m} / \mathrm{min}$ (20 fpm) for glass former mix $A$ and $12 \mathrm{~m} / \mathrm{min}(40 \mathrm{fpm})$ for mix B. Mixed glass former fluid bed performance was determined to be acceptable. Fluidization velocity was maintained fairly high $(12 \mathrm{~m} / \mathrm{min})$ for most tests to minimize the potential loss of fluidization due to liquid feed injection. 
The original Alumina tested was hydrated and exhibited poor fluidizing performance during bulk testing. A larger particle size, calcined alumina was also tested and exhibited superior fluidizing characteristics. The larger particle size alumina was used in bench and pilot scale testing.

The bulk magnesium carbonate exhibited adequate fluidization characteristics but excessive dusting, which can cause elutriated fines in the off-gas. Since some of the magnesium carbonate required for the glass formulation was to be derived from the dolomite, the weight of bulk magnesium carbonate necessary in the fluid bed was relatively small. However, since magnesium carbonate has a small bulk density compared to other glass formers (See Table 6.1-1) the volume percent of magnesium carbonate was relatively large (approx. 30\%) for glass former mix $A$. The mixed bed dusting was attributed to the bulk magnesium carbonate. It is anticipated that a bulk magnesium carbonate with less water content will fluidize more efficiently.

Glass former mix B was generated based on revised calculations which accounted for impurities in various bulk glass formers. Mix B had considerable lower weight percentage of magnesium carbonate which resulted in a larger weight percentage of boric acid. Mix $B$ was tested in the fluidization test tube and found to be a superior fluidizing mixture. This is largely attributed to the lower magnesium carbonate percentage.

Glass former mix $C$ differed from mix $B$ only by the removal of boric acid. Glass former mix $C$ was not tested in the fluidizing test tube since the removal of boric acid was not expected to significantly affect fluidization characteristics. Actual testing showed that mix C fluidized easily at 6-12 $\mathrm{m} / \mathrm{min}(20-40 \mathrm{fpm})$.

Glass former Mix $D$ differed from Mix $C$ due to the addition of $9 \% \mathrm{wt}_{2} \mathrm{H}_{2} \mathrm{BO}_{3}$. Glass former mix $E$ differed from Mix $C$ due to the addition of $9 \%$ wt Boric Oxide. Neither Mix D nor Mix E was tested for fluidization but both performed well in the bench scale calciner at $9 \mathrm{~m} / \mathrm{min}(30 \mathrm{fpm})$ fluidization velocity.

\subsection{FBC-SO: GLASS FORNERS W/ WATER/SINULANT, NO REDUCTANTS}

The purpose of this test was to determine the fluidization performance of the mixed glass former bed, determine limiting feed flowrate based on heat transfer relationships with water, prove that a high temperature fluid bed could volatize NOX, and to verify that feed line caking would not result due to simulant melting. Portions of this test were performed between $2 / 8 / 95$ and $2 / 10 / 95$. Test data corresponds to Procedyne test numbers I-1 through I-3.

The glass former fluid bed composition used for this test is given in Table 1-2. 
Table 1-2. Fluid Bed Composition, Glass Former Mix A.

\begin{tabular}{|l|c|c|}
\hline \multicolumn{1}{|c|}{ Glass former } & $\begin{array}{c}\text { Weight } \\
\text { percent }\end{array}$ & $\begin{array}{c}\text { Weight, } \mathrm{kg}(\mathrm{lb}) \text { [rounded to } \\
\text { nearest .05 1b] }\end{array}$ \\
\hline Silica sand & 60.0 & $2.45(5.40)$ \\
\hline Boric acid & 9.2 & $0.39(0.85)$ \\
\hline Dolomite & 10.9 & $0.45(1.00)$ \\
\hline Alumina & 11.4 & $0.48(1.05)$ \\
\hline Iron oxide & 1.0 & $0.05(0.10)$ \\
\hline Magnesium carbonate & 7.5 & $0.29(0.65)$ \\
\hline Total & 100.0 & $4.10(9.05)$ \\
\hline
\end{tabular}

Initial concerns with bed fluidization at $700{ }^{\circ} \mathrm{C}$ included possibility that boric acid would decompose to boric oxide and me1t $\left(450^{\circ} \mathrm{C}\right)$ causing the bed to agglomerate and lose fluidization. Results of Procedyne test $I-1$ through $1-3$ revealed that in the above concentrations (mix $A$ ), the mixed glass former bed could be successfully fluidized at temperatures up to $750{ }^{\circ} \mathrm{C}$ without agglomeration.

An initial concern with injection of liquid slurry into the fluidizing bed was that excess 1iquid/moisture would result in bed caking causing a loss of fluidization. For conservatism, water was injected into the fluid bed to establish a maximum feed rate. Since the DSSF Simulant is approximately $60 \%$ by weight water, the heat transfer performance of the fluid bed is bounded by maximum feed rates of $100 \%$ water. Procedyne test I-1 demonstrated that a feed flowrate of up to $20 \mathrm{ml} / \mathrm{min}$ of water could be processed effectively with in the heat transfer capacity of the bench scale fluid bed without loss of fluidization.

Initial concerns with the injection of DSSF Simulant without reductants was that non-reacted salts would melt and/or decompose, resulting in bed caking, agglomeration, and loss of fluidization. Additionally, processing the simulant without reductants in the fluid bed was expected to release large volumes of NOx off-gas which is an atmospheric pollutant and should be minimized.

Procedyne test no. I-2 attempted the injection of simulant into a fluidizing bed of glass formers (mix A) at $750{ }^{\circ} \mathrm{C}$. Mechanical failure of feed pump no. 1 forced termination of test I-2. Feed pump no. 2, a peristaltic tubing pump, was installed and utilized for injecting simulant at $20 \mathrm{ml} / \mathrm{min}$ during Procedyne test no. I-3 and later tests. As expected, simulant injection without reductants resulted in rapidly diverging upper and lower fluid bed temperatures, indicating a loss of fluidization caused from caking. Additionally, NOx levels in the off-gas increased rapidly to over $12,000 \mathrm{ppm}$, indicating that the fluid bed was effectively volatizing NOx. 
WHC-SD-WM-VI-031

Revision 0

Although loss of fluidization was evident within 15 minutes of starting the test, the test was continued for a total of 1 hour to evaluate bed and feed line conditions. Inspection of the bed following testing revealed a very hard cake; however, the simulant feed line inside was not obstructed. Additionally, liberation of water from the bulk glass formers caused bed shrinkage. In order to guarantee that both the upper and lower thermocouples were covered during fluidization, $5.5 \mathrm{~kg}$ (12 1b) of glass former mixture was used on subsequent testing instead of $4.1 \mathrm{~kg}$ (9 $\mathrm{lb}$ ) for FBC-SO.

\subsection{FBC-S1: GLASS FORNERS AND SINULANT W/ 600\% SUCROSE AT $700^{\circ} \mathrm{C}$}

Glass former mix B was generated based on slightly revised calculations which accounted for impurities in various bulk glass formers. Mix $B$ had considerably lower weight percentage of magnesium carbonate which resulted in a larger weight percentage of boric acid. Glass former mix B composition is given in Table 1-3 and was used for FBC-S1 (I-4) conducted on 2/13/95.

The bulk sucrose reductant was added directly to the feed tank. Calculation of the initial weight of sucrose additive was based on the $100 \%$ stoichiometric reaction with $\mathrm{NaNO}_{3}$ in the simulant. This same concentration of sucrose was utilized for both Procedyne tests I-4 and I-5.

As the fluidizing bed was heating up to $700{ }^{\circ} \mathrm{C}$ prior to injection of the simulant/sucrose feed for test FBC-S1 (I-4), the upper and lower bed temperatures diverged rapidly, indicating loss of fluidization and caking. The test was terminated prior to feed injection. Inspection of the bed showed severe caking. This caking was attributed to the larger weight percent (16\%) of boric acid which melts at $169{ }^{\circ} \mathrm{C}$ and decomposes to $\mathrm{B}_{2} \mathrm{O}_{3}$ which melts at $450{ }^{\circ} \mathrm{C}$. Note that in test FBC-SO glass former mix $A$ boric acid weight percent was $9 \%$ and bed fluidization was acceptable.

Table 1-3. Fluid Bed Composition, Glass Former Mix B.

\begin{tabular}{|l|c|c|}
\hline \multicolumn{1}{|c|}{ Glass former } & $\begin{array}{c}\text { Weight } \\
\text { percent }\end{array}$ & $\begin{array}{c}\text { Weight, kg (1b) [rounded to } \\
\text { nearest .05 lb] }\end{array}$ \\
\hline Silica sand & 59.69 & $3.24(7.15)$ \\
\hline Boric acid & 16.19 & $0.88(1.95)$ \\
\hline Dolomite & 10.89 & $0.59(1.30)$ \\
\hline Alumina & 11.48 & $0.63(1.40)$ \\
\hline Iron oxide & 1.04 & $0.05(0.10)$ \\
\hline Magnesium carbonate & 0.71 & $0.05(0.10)$ \\
\hline Total & 100.0 & $5.44(12.00)$ \\
\hline
\end{tabular}


WHC-SD-WM-VI-031

Revision 0

The glass former mixture was reformulated to remove the boric acid and readjust remaining constituent weight percentages. The resulting mixture was glass former mix $C$, which was used for test FBC-S1 (I-5) also conducted on 2/13/94. Glass former mix $C$ composition is given in Table 1-4.

Test FBC-S1 (I-5) was reinitiated with glass former mix C. Adequate fluidization was observed during bed heat-up to $700{ }^{\circ} \mathrm{C}$ indicating that the boric acid had indeed been the cause of previous caking in glass former mix B.

Simulant with sucrose additive was injected at $20 \mathrm{ml} / \mathrm{min}$. Good fluidization characteristics were observed for the first 3 hours of the test. NOx levels in the off-gas were negligible, indicating a successful reaction between the sucrose and the simulant. NOx levels peaked at approximately $30 \mathrm{ppm}$ and dropped to $0 \mathrm{ppm}$ after the first half hour.

Fluid bed upper and lower temperatures began to diverge after 3 hours indicating bed agglomeration or caking. Efforts to bring the temperatures back together by adjusting fluidizing gas flowrate were unsuccessful. Additionally, Nox levels were detectable after the bed lost fluidization. The test was terminated approximately 45 minutes early.

Inspection of the fluid bed test vessel revealed a significant excess of carbon material and severe bed caking. The entire bed and freeboard region were packed with carbon material which resembled a soft black powder in the upper freeboard, a soft black cake in the lower freeboard, and a hard black cake in the bed region. Results of the inspection indicated that greater than $100 \%$ stoichiometric sucrose proportions had been added to the simulant in the feed tank.

Sucrose calculational assumptions were revised to account for actual concentrations of sodium nitrate $\left(\mathrm{NaNO}_{3}\right)$, and sodium nitrite $\left(\mathrm{NaNO}_{2}\right)$ and EDTA in the simulant. The results of the revised calculations required significantly less sucrose to achieve $100 \%$ stoichiometric concentrations. These results indicated that test $\mathrm{FBC}-\mathrm{S} 1$ (I-5) had been performed with approximately $600 \%$ stoichiometric sucrose. This number seemed reasonable compared to observations of bed caking.

\subsection{FBC-S2: GLASS FORMERS AND SIMULANT W/ $200 \%$ ANMONIA AT $700{ }^{\circ} \mathrm{C}$}

Test FBC-S2 (I-6) was conducted on $2 / 14 / 95$. Glass former mix $\mathrm{C}$ was loaded into the bench scale fluid bed calciner and preheated to $500{ }^{\circ} \mathrm{C}$. Ammonia $\left(\mathrm{NH}_{3}\right)$ reductant gas was added to the fluidization gas $\left(\mathrm{N}_{2}\right)$ and simulant was injected at $20 \mathrm{ml} / \mathrm{min}$. Ammonia addition was calculated based on $200 \%$ stoichiometric necessary to convert sodium nitrites/nitrates $\left(\mathrm{NaNO}_{2} / \mathrm{NaNO}_{3}\right)$ to sodium oxide $\left(\mathrm{Na}_{2} \mathrm{O}\right)$.

Initial testing was delayed due to feed injection tube plugging, believed to be caused by the previous sucrose testing. The feed 7 ine was cleared and testing resumed. 
Table 1-4. Fluid Bed Composition, Glass Former Mix C.

\begin{tabular}{|l|c|c|}
\hline \multicolumn{1}{|c|}{ Glass former } & $\begin{array}{c}\text { Weight } \\
\text { percent }\end{array}$ & $\begin{array}{c}\text { Weight, } \mathrm{kg}(\mathrm{lb}) \text { [rounded to } \\
\text { nearest .05 lb] }\end{array}$ \\
\hline Silica sand & 71.3 & $3.88(8.55)$ \\
\hline Boric acid & 0.0 & 0.00 \\
\hline Dolomite & 13.0 & $0.70(1.55)$ \\
\hline Alumina & 13.6 & $0.75(1.65)$ \\
\hline Iron oxide & 1.3 & $0.07(0.15)$ \\
\hline Magnesium carbonate & 0.8 & $0.05(0.10)$ \\
\hline Total & 100.0 & $5.44(12.00)$ \\
\hline
\end{tabular}

Fluid bed upper and lower temperatures began to diverge rapidly within the first 15 minutes of the test indicating loss of fluidization, caking, or agglomeration. Nox levels were negligible for the first hour of testing, but then increased to approximately $350 \mathrm{ppm}$. Although loss of fluidization was evident by the divergent temperatures, the test was continued for the full duration since NOx levels were low, indicating that the desired reaction was continuing.

Inspection of the bed following test completion showed severe hard caking which was difficult to clean from the inside surfaces of the fluid bed vessel.

\subsection{FBC-S3: GLASS FORNERS AND SINULANT W/ $100 \%$ SUCROSE AT $700{ }^{\circ} \mathrm{C}$}

Test FBC-S3 (I-7) was performed on 2/15/95. Although the Test Procedure (Reference 3.2) called for scoping tests with methane $\left(\mathrm{CH}_{4}\right)$ and hydrogen $\left(\mathrm{H}_{2}\right)$ first, previous successful sucrose results and limited schedule called for open testing of sucrose as a priority over continuing with the scoping tests with other reductants.

A $100 \%$ stoichiometric sucrose and simulant solution was mixed in the feed tank. The fluid bed vessel was preheated to $700{ }^{\circ} \mathrm{C}$ and injection began. NOx levels in the off-gas rose rapidly and stabilized at approximately $600 \mathrm{ppm}$. After approximately 20 minutes, fluid bed upper and lower temperatures began to diverge indicating loss of fluidization. After 30 minutes, Nox levels began to increase to approximately $1800 \mathrm{ppm}$. On line sampling yielded only small amounts of product from the bed. Since loss of fluidization was obvious, the test was terminated early after 50 minutes total run time.

Bed inspection revealed a soft cake with agglomerated chunks. It appeared that the reaction at $700{ }^{\circ} \mathrm{C}$ resulted in some melting salts. 
WHC-SD-WM-VI-031

Revision 0

\subsection{FBC-S4: GLASS FORNERS AND SINULANT W/ $125 \%$ SUCROSE AT $500^{\circ} \mathrm{C}$}

Based on the results of test $\mathrm{FBC}-\mathrm{S3}$, sucrose concentration in the simulant feed was increased to $125 \%$ stoichiometric and fluid bed temperature was lowered to $500^{\circ} \mathrm{C}$. Test FBC-S4 was conducted on $2 / 15 / 95$.

The feed line became blocked upon initiation of feed. Following on-line removal, the lower $15 \mathrm{~cm}\left(6^{\prime \prime}\right)$ of the feed line was discovered to be blocked with carbon cake and required cutting off that section of tubing. Following repairs, simulant and $125 \%$ sucrose were injected at $20 \mathrm{ml} / \mathrm{min}$. The fluid bed (mix C) exhibited good fluidization characteristics. NOx levels initially rose to approximately $1200 \mathrm{ppm}$ and then gradually lowered to approximately $200 \mathrm{ppm}$ after 2 hours where levels remained reasonably stable for the duration of the test. The test was continued to completion with a total duration of 4 hours.

Post test inspection of the bed revealed a free flowing dry product with a thin, soft cake on the filters and vessels walls. This product could easily be handled for melter feed. Final bed weight of $7.6 \mathrm{Kg}$ (16.75 lb) resulted from the addition of simulant. Solids from simulant made up $28 \%$ of final bed weight.

\subsection{FBC-S5: GLASS FORMERS AND SIMULANT W/ $100 \%$ SUCROSE AT 500 'C}

Following the success and experience of test FBC-S4, 100\% stoichiometric sucrose was again attempted, but FBC-S4 (I-9) was conducted with the fluid bed at $500{ }^{\circ} \mathrm{C}$, on $2 / 16 / 95$.

The $100 \%$ stoichiometric sucrose and simulant was injected at $20 \mathrm{ml} / \mathrm{min}$. NOx levels in the off-gas rose rapidly to over $2500 \mathrm{ppm}$ and gradually lowered over the 4-hour test period to approximately $1500 \mathrm{ppm}$. The bed exhibited excellent fluidizing characteristics. The test was continued until completion.

Post test inspection of the fluid bed revealed a dry free flowing product with minor lumps/agglomeration. This product could also be easily handled for melter feed. Final bed weight of $7.0 \mathrm{~kg}(15.5 \mathrm{lb})$ resulted from the addition of simulant. Solids from simulant accounted for $23 \%$ of final bed weight.

\subsection{FBC-S6: GLASS FORHERS AND SIMULANT W/ $200 \%$ ANMONIA AT $500^{\circ} \mathrm{C}$}

Based on success with sucrose at a lower temperature and previous success converting nitrites/nitrates with $\mathrm{NH}_{3}$ addition, open testing with ammonia as the reductant at $500{ }^{\circ} \mathrm{C}$ was performed during test FBC-S6 (I-10) on $2 / 16 / 95$.

The glass former bed ( $\operatorname{mix} C$ ) was preheated with nitrogen fluidization to $500{ }^{\circ} \mathrm{C}$. Ammonia was added to the fluidizing gas flow based on $200 \%$ stoichiometric necessary to convert sodium nitrites/nitrates $\left(\mathrm{NaNO}_{2} / \mathrm{NaNO}_{3}\right)$ to sodium oxide $\left(\mathrm{Na}_{2} \mathrm{O}\right)$. NOx levels in the off-gas rose initially to over $5,600 \mathrm{ppm}$ but dropped rapidly over a 2 hour period to approximately $300 \mathrm{ppm}$ indicating that nitrite/nitrate conversion was taking place. 
WHC-SD-WM-VI-031

Revision 0

The bed upper and lower temperatures began to diverge after approximately 30 minutes indicating loss of fluidization. The test was continued for a total of 2 hours and 15 minutes.

Bed inspection revealed caking and channeling with some free flowing dry product within.

\subsection{FBC-S7: GLASS FORNERS AND SIMULANT W/ 200\% HYDROGEN AT $300^{\circ} \mathrm{C}$}

Scoping tests were resumed to evaluate the affect of hydrogen as a reductant. The calculation for hydrogen addition was based on $200 \%$ stoichiometric hydrogen necessary for conversion of sodium nitrite $\left(\mathrm{NaNO}_{2}\right)$ and nitrate $\left(\mathrm{NaNO}_{3}\right)$ to sodium oxide $\left(\mathrm{Na}_{2} \mathrm{O}\right)$. Test $\mathrm{FBC}-\mathrm{S7}(\mathrm{I}-11)$ was performed $2 / 17 / 95$. Since ammonia was unsuccessful at $500{ }^{\circ} \mathrm{C}$ and the hydrogen reaction was expected to be similar, a lower temperature $\left(300^{\circ} \mathrm{C}\right)$ was investigated.

The glass former bed (mix C) was preheated to $300{ }^{\circ} \mathrm{C}$. Hydrogen reactant gas was added to the fluidizing gas flow to achieve expected $200 \%$ stoichiometry. Simulant was then injected at $20 \mathrm{ml} / \mathrm{min}$. NOx levels in the off-gas stream remained less than approximately $300 \mathrm{ppm}$ throughout the test. After approximately 20 minutes, upper and lower fluid bed temperatures began to diverge. The test was terminated early after 30 minutes due to 10 ss of fluidization.

Post test inspection of the fluid bed revealed bed caking.

\subsection{FBC-S8: GLASS FORNERS AND SINULANT W/ 200\% METHANE AT $500{ }^{\circ} \mathrm{C}$}

Scoping tests continued with investigation of the affects of methane $\left(\mathrm{CH}_{4}\right)$ as a reductant. The calculation for methane addition was based on $200 \%$ stoichiometric methane necessary for conversion of sodium nitrite $\left(\mathrm{NaNO}_{2}\right)$ and nitrate $\left(\mathrm{NaNO}_{3}\right)$ to sodium carbonate $\left(\mathrm{Na}_{2} \mathrm{CO}_{3}\right)$. Test $\mathrm{FBC}-\mathrm{S8}(\mathrm{I}-12)$ was performed $2 / 17 / 95$.

The glass former bed $\left(\operatorname{mix} \mathrm{C}\right.$ ) was preheated to $500{ }^{\circ} \mathrm{C}$. Methane reductant gas was added to the fluidizing gas flow to achieve expected $200 \%$ stoichiometry. Simulant was then injected at $20 \mathrm{ml} / \mathrm{min}$. NOx levels in the off-gas stream rose rapidly to over $6,000 \mathrm{ppm}$ and then lowered to less than $1200 \mathrm{ppm}$ over a 30 minute period. After approximately 15 minutes, upper and lower fluid bed temperatures began to diverge. The test was terminated early (after 45 minutes) due to loss of fluidization.

Post test inspection of the fluid bed revealed hard caking on top of the bed with lumps in the lower bed region.

\subsection{FBC-S9: GLASS FORHERS AND SINULANT W/ $75 \%$ SUCROSE AT $500^{\circ} \mathrm{C}$}

Since testing has already proven that a fluid bed without reductants would agglomerate and lose fluidization, and that a bed with $100 \%$ stoichiometric sucrose could process simulant at $500{ }^{\circ} \mathrm{C}$ and maintain 
WHC-SD-WM-VI-031

Revision 0

fluidization, the affects of a smaller stoichiometric sucrose concentration (75\%) were investigated. Test $\mathrm{FBC}-\mathrm{S9}$ was performed on $2 / 17 / 95$.

The glass former bed $(\operatorname{mix} C)$ was preheated to $500{ }^{\circ} \mathrm{C}$. $75 \%$ stoichiometric sucrose and simulant were added to the fluid bed at $20 \mathrm{ml} / \mathrm{min}$. NOx levels in the off-gas rapidly stabilized at approximately 7,000 $\mathrm{ppm}$ and remained relatively stable for the duration of the run. Excellent fluidization characteristics were exhibited for the full duration of the test, 4 hours.

Post test inspection revealed a reddish colored free-flowing bed with some agglomerates. Freeboard region was coated with fines. Final bed product weight of $6.8 \mathrm{Kg}(15 \mathrm{lb})$ resulted from addition of simulant. Solids added to bed from simulant accounted for $20 \%$ of final weight.

\subsection{FBC-S10: GLASS FORMERS W/ 100\% BORIC ACID FEED AT $500^{\circ} \mathrm{C}$}

Since the presence of boric acid (oxide) in the final product bed is desirable and premixed boric acid attempts at $700{ }^{\circ} \mathrm{C}$ had failed, the possibility of slowly added boric acid to a fluidizing bed was investigated.

The bench scale calciner was modified to allow addition of a second feed stream through the feed lock in the freeboard region. Solid 100\% boric acid was selected as the second feed stream. Test FBC-S10 was performed on $2 / 22 / 95$.

Glass former Mix $C$ was preheated to $500{ }^{\circ} \mathrm{C}$. Once stable at temperature, solid $100 \%$ boric acid feed was slowly initiated through the feedlock. Feed rate was limited to very small amounts. No simulant or reductants were injected. After 75 minutes approximately 0.45 pounds of boric acid had been fed into the fluid bed. The bed still exhibited excellent fluidization characteristics; however, the test was terminated due to feed 1 ine plugging.

Post test inspection revealed that the boric acid had melted in the feed pipe which was completely plugged at the $45^{\circ}$ elbow closest to the freeboard. The product bed was free-flowing with some hard agglomerates mixed throughout. The bed agglomerates were believed to be melted boric acid.

\subsection{FBC-S11: GLASS FORMERS (INCLUDIMG 9\% BORIC ACID) AND $16 \%$ BORIC ACID FEED AT $500{ }^{\circ} \mathrm{C}$}

Since test $\mathrm{FBC}-\mathrm{S} 10$ proved that $100 \%$ boric acid would melt in and plug the feed 1 ine, it was decided to test the use of a $16 \mathrm{wt} \%$ boric acid/glass former mix as a feed material.

Additionally, since test $\mathrm{FBC}-\mathrm{SO}(1-1,2,3)$ demonstrated that a boric acid hydrate weight percent of $9 \%$ would fluidize well at $700^{\circ} \mathrm{C}$, a new bed of premixed glass formers was prepared with $9 \%$ wt boric acid and loaded into the reactor. The $16 \%$ wt boric acid/glass former mix was to be fed into the $9 \%$ bed while some of the bed material would be extracted through the eductor. This would slowly raise the boric acid level in the reactor to the desired $16 \% \mathrm{wt}$. The initial glass former mixture (Mix D) is shown in Table 1-5. 
Table 1-5. Fluid Bed Composition, Glass Former Mix D.

\begin{tabular}{|l|c|c|}
\hline \multicolumn{1}{|c|}{ Glass former } & $\begin{array}{c}\text { Weight } \\
\text { percent }\end{array}$ & $\begin{array}{c}\text { Weight, kg (1b) [rounded to } \\
\text { nearest .05 lb] }\end{array}$ \\
\hline Silica sand & 64.88 & $3.54(7.80)$ \\
\hline Boric acid & 9.0 & $\begin{array}{c}0.50(1.10) \text { [initial load, } \\
16 \text { wt\% added during test] }\end{array}$ \\
\hline Dolomite & 11.83 & $0.63(1.40)$ \\
\hline Alumina & 12.38 & $0.68(1.50)$ \\
\hline Iron oxide & 1.18 & $0.68(0.15)$ \\
\hline Magnesium carbonate & .73 & $0.05(0.10)$ \\
\hline Total & 100.0 & $5.46(12.05)$ \\
\hline
\end{tabular}

Glass former mix $D$ was preheated to $500{ }^{\circ} \mathrm{C}$. Good fluidization characteristics were observed as the bed reached steady state. The $16 \%$ solid boric acid mixture feed was then initiated. After approximately 10 minutes, $2 \mathrm{lb}$ of mixture had been injected, and the feed line plugged.

Post test inspection revealed a completely plugged feed 1 ine and a large hard lump at the top of the bed. Once again, this caking was attributed to melting boric acid.

\subsection{FBC-S12: GLASS FORMERS W/ BORIC OXIDE FEED AND SINULANT W/ $100 \%$ SUCROSE AT $400^{\circ} \mathrm{C}$}

Previous tests proved that boric acid mixed with the other glass formers in the proper proportions (Mix B) caused bed agglomeration at temperatures above $500{ }^{\circ} \mathrm{C}$. Since the melting point of boric oxide $\left(\mathrm{B}_{2} \mathrm{O}_{3}\right)$ is $450{ }^{\circ} \mathrm{C}$, it was hoped that operation of the fluid bed calciner at temperatures below $450{ }^{\circ} \mathrm{C}$ could successfully process the complete glass former mixture, simulant, and sucrose reductant.

Addition of boric oxide $\left(\mathrm{B}_{2} \mathrm{O}_{3}\right)$ to the glass former mixture instead of boric acid $\left(\mathrm{H}_{2} \mathrm{BO}_{3}\right)$ was investigated in order to minimize boric acid melting and transition to boric oxide, which occurs at $169^{\circ} \mathrm{C}$. Additionally, the boric oxide was not mixed in with the initial fluidizing bed but was added throughout test period. The revised glass former mixture (Mix E) is shown in Table 1-6. Test FBC-S12 was performed on 2/28/95.

Glass former mix $E$ was preheated to $400{ }^{\circ} \mathrm{C}$. $100 \%$ stoichiometric sucrose $\left(\mathrm{C}_{12} \mathrm{H}_{22} \mathrm{O}_{11}\right)$ was added to the fluidizing bed at $20 \mathrm{ml} / \mathrm{min}$. Boric oxide was slowty added to the fluidizing bed over a 15 minute period, within the first 35 minutes of testing. NOx levels fluctuated between 400 and 7,000 ppm throughout the test. Good glass former bed fluidizing characteristics were initially observed. The test was terminated early (after 3-3/4 hours) when bed temperatures began to diverge, and a sample could not be drawn via the sample eductor. 
Table 1-6. Fluid Bed Composition, Glass Former Mix E.

\begin{tabular}{|l|c|c|}
\hline \multicolumn{1}{|c|}{ Glass former } & $\begin{array}{c}\text { Weight } \\
\text { percent }\end{array}$ & $\begin{array}{c}\text { Weight, } \mathrm{kg} \text { (1b) [rounded to } \\
\text { nearest } 0.05 \mathrm{lb} \text { ] }\end{array}$ \\
\hline Silica sand & 64.8 & $3.54(7.80)$ \\
\hline Boric oxide & 9.1 & $0.50(1.10)$ [added during test] \\
\hline Dolomite & 11.8 & $0.63(1.40)$ \\
\hline Alumina & 12.4 & $0.68(1.50)$ \\
\hline Iron oxide & 1.2 & $0.07(0.15)$ \\
\hline Magnesium carbonate & 0.7 & $0.05(0.10)$ \\
\hline Total & 100.0 & $5.46(12.05)$ \\
\hline
\end{tabular}

Post test inspection revealed a reddish colored, free-flowing bed of glass formers. A soft, black, airy cake filled the freeboard region above the glass former bed. Final bed product weight of $6.01 \mathrm{Kg}(13.25 \mathrm{lb})$ resulted from addition of simulant. Solids added to bed from simulant accounted for $9 \%$ of final weight.

\subsection{FBC-S13: GLASS FORNERS W/ BORIC OXIDE FEED AND SIMULANT W/ $100 \%$ SUCROSE AT $425{ }^{\circ} \mathrm{C}$}

Results of previous testing indicated that sucrose would completely react with the simulant above $500{ }^{\circ} \mathrm{C}(\mathrm{FBC}-\mathrm{S3}, 4,9)$ but not at temperatures below $400{ }^{\circ} \mathrm{C}$ (FBC-S12). Since the melting point of boric oxide $\left(\mathrm{B}_{2} \mathrm{O}_{3}\right)$ is $450{ }^{\circ} \mathrm{C}$, a temperature of $425{ }^{\circ} \mathrm{C}$ was selected in order to determine the minimum temperature for the sucrose/simulant reaction, and prevent bed agglomeration resulting from boric oxide melting. FBC-S12 (I-16) was repeated with the temperature at $425^{\circ} \mathrm{C}$. Test $\mathrm{FBC}-\mathrm{S} 13$ was performed on $3 / 6 / 95$.

Glass former mix $E$ was preheated to $425{ }^{\circ} \mathrm{C}$. 100\% -stoichiometric sucrose $\left(\mathrm{C}_{12} \mathrm{H}_{22} \mathrm{O}_{11}\right)$ was added to the fluidizing bed at $20 \mathrm{ml} / \mathrm{min}$. Boric oxide was slowly added to the fluidizing bed over a 15 minute period. As observed in test FBC-S12, NOx levels fluctuated throughout the test. Good initial glass former bed fluidizing characteristics were observed. The test was terminated early when bed temperatures began to diverge, and a sample could not be drawn via the sample eductor.

Post test inspection revealed a reddish colored free-flowing bed of glass formers. A soft, black, airy cake filled the freeboard region above the glass former bed.

\subsection{BENCH SCALE TEST EVALUATION SUMHARY}

A summary of test data and conditions for the bench scale fluid bed calciner test runs is given in Table 1-7. 
Table 1-7. Bench Scale Fluid Bed Calciner Test Run Summary.

\begin{tabular}{|c|c|c|c|c|c|c|c|c|c|c|c|c|c|c|}
\hline VECTRA tost & FBC-So & FBC-S1 & FBC-S2 & FBC-S3 & FBC.S4 & FBC.:S5 & FBC-S6 & FBC-S7 & FBC-SB & FBC-S9 & FBC-S10 & FBC-S11 & FBC-S12 & FBC-S13 \\
\hline Procedyne Test & $\{-1,2,3$ & $1-4,5$ & $1-6$ & $1-7$ & I-B & $1-9$ & $1 \cdot 10$ & $\mid-11$ & +12 & +13 & $1-14$ & $1-15$ & $1-16$ & $1-17$ \\
\hline Reductant & none & sucrose & $\mathrm{NH}_{3}$ & sucrose & sucrose & eucrose & $\mathrm{NH}_{3}$ & $\mathrm{H}_{2}$ & $\mathrm{CH}_{4}$ & eucrose & nono & nono & sucroses & sucroses \\
\hline Stoichiometry (\%) & $N / A$ & 600 & 200 & 100 & 125 & 100 & 200 & 200 & 200 & 75 & N/A & N/A & 100 & 100 \\
\hline Tomp $\left({ }^{\circ} \mathrm{C}\right)$ & 700 & 700 & 700 & 700 & 500 & 500 & 500 & 300 & 500 & 500 & 500 & 500 & 400 & 425 \\
\hline Glase Former Mix & A & B.C & c & c & C & c & c & c & c & c & c & D & $\mathbf{E}$ & $\mathbf{E}$ \\
\hline $\begin{array}{l}\text { Simulant Foed } \\
\text { Rate }(\mathrm{m} / \mathrm{min})\end{array}$ & 20 & 20 & 20 & 20 & 20 & 20 & 20 & 20 & 20 & 20 & $\begin{array}{l}0.45 \mathrm{lb} / \\
\mathrm{H}_{2} \mathrm{BO}_{3}\end{array}$ & $\begin{array}{l}21 \mathrm{~b} / 16 \% \\
\mathrm{H}_{2} \mathrm{BO}_{3}\end{array}$ & 20 & 20 \\
\hline $\begin{array}{l}\text { Fluidizing Velocity } \\
\mathrm{m} / \mathrm{min} \text { (fpm) }\end{array}$ & $\begin{array}{l}9-12 \\
(30-40)\end{array}$ & $\begin{array}{l}9-14 \\
(30-45)\end{array}$ & $\begin{array}{l}12 \\
(40)\end{array}$ & $\begin{array}{l}12 \\
(40)\end{array}$ & $\begin{array}{l}12 \\
(40)\end{array}$ & $\begin{array}{l}12 \\
(40)\end{array}$ & $\begin{array}{l}12 \\
(40)\end{array}$ & $\begin{array}{l}11 \\
\text { (35) }\end{array}$ & $\begin{array}{l}11 \\
(35)\end{array}$ & $\begin{array}{l}11 \\
|35|\end{array}$ & $\begin{array}{l}9 \\
(30)\end{array}$ & $\begin{array}{l}9 \\
(30)\end{array}$ & $\begin{array}{l}9 \\
(30)\end{array}$ & $\begin{array}{l}9 \\
\text { (30) }\end{array}$ \\
\hline $\begin{array}{l}\text { Gaseous } \\
\text { Reductant Flow } \\
\mathrm{Nm}^{3} / \mathrm{hr} \text { (SCFM) }\end{array}$ & N/A & $N / A$ & $\begin{array}{l}0.406 \\
(0.239)\end{array}$ & N/A & N/A & N/A & $\begin{array}{l}0.406 \\
(0.239)\end{array}$ & $\begin{array}{l}0.612 \\
(0.360)\end{array}$ & $\begin{array}{l}0.153 \\
10.090 \mid\end{array}$ & $N / A$ & N/A & N/A & N/A & N/A \\
\hline Test Date\{'95\} & $2 / 8-10$ & $2 / 13$ & $2 / 14$ & $2 / 15$ & $2 / 15$ & $2 / 16$ & $2 / 16$ & $2 / 17$ & $2 / 17$ & $2 / 17$ & $2 / 22$ & $2 / 22$ & $2 / 28$ & $3 / 6$ \\
\hline $\begin{array}{l}\text { Tost Duration } \\
\text { (min.) }\end{array}$ & 60 & 195 & $\begin{array}{l}240 \\
\text { end }\end{array}$ & 50 & $\begin{array}{l}240 \\
\text { end }\end{array}$ & $\begin{array}{l}240 \\
\text { and }\end{array}$ & 135 & 30 & 45 & $\begin{array}{l}240 \\
\text { ond }\end{array}$ & 75 & 78 & 225 & 240 \\
\hline $\begin{array}{l}\text { WHC-VIT-02A } \\
\text { soction }\end{array}$ & 4.3 .2 & 4.3 .3 & 4.3 .4 & 4.3 .9 & 4.3 .9 & 4.3 .9 & 4.3 .9 & 4.3 .6 & 4.3 .5 & 4.3 .9 & 4.3 .9 & 4.3 .9 & 4.3 .9 & 4.3 .9 \\
\hline $\begin{array}{l}\text { Final Bed } \\
\text { Characteriatics }\end{array}$ & $\begin{array}{l}\text { hard } \\
\text { cake } \\
\text {-rod }\end{array}$ & $\begin{array}{l}\text {-oxcoss } \\
\text { carbon } \\
\text {-bed } \\
\text { soglom. } \\
\text {-black }\end{array}$ & $\begin{array}{l}\text { hard caks } \\
\text {-rod }\end{array}$ & $\begin{array}{l}\text {-hard cake } \\
\text {-red }\end{array}$ & $\begin{array}{l}\text {-free } \\
\text { flowing } \\
\text {-black }\end{array}$ & $\begin{array}{l}\text {-free } \\
\text { flowing } \\
\text { orey }\end{array}$ & $\begin{array}{l}\text { - hard cake } \\
\text {-channels } \\
\text {-rod }\end{array}$ & $\begin{array}{l}\text {-hard cake } \\
\text {-rod }\end{array}$ & $\begin{array}{l}\text {-hard cako } \\
\text {-rod }\end{array}$ & $\begin{array}{l}\text {-froe } \\
\text { flowing } \\
\text {-red }\end{array}$ & $\begin{array}{l}\text {-feed plus } \\
\text {-free flow } \\
\text {-hard } \\
\text { eaglom. }\end{array}$ & $\begin{array}{l}\text {-feed plus } \\
\text {-hard cake } \\
\text { e top } \\
\text {-bed froe } \\
\text { flow }\end{array}$ & $\begin{array}{l}\text {-not } \\
\text { uniform } \\
\text {-upper: } \\
\text { bleck cake } \\
\text {-bed; rod/ } \\
\text { free } \\
\text { flowing }\end{array}$ & $\begin{array}{l}\text {-not } \\
\text { uniform } \\
\text {-uppor: } \\
\text { black cake } \\
\text {-bod: redl } \\
\text { free } \\
\text { flowing }\end{array}$ \\
\hline $\begin{array}{l}\text { NOx:-high } \\
\text { (ppm)-low } \\
\text {-avg }\end{array}$ & $\begin{array}{l}12000 \\
1380 \\
3700\end{array}$ & $\begin{array}{l}75 \\
0 \\
3\end{array}$ & $\begin{array}{l}390 \\
0 \\
144\end{array}$ & $\begin{array}{l}1815 \\
150 \\
795\end{array}$ & $\begin{array}{l}1275 \\
120 \\
424\end{array}$ & $\begin{array}{l}2760 \\
660 \\
1821\end{array}$ & $\begin{array}{l}6750 \\
300 \\
1732\end{array}$ & $\begin{array}{l}300 \\
120 \\
190\end{array}$ & $\begin{array}{l}6990 \\
195 \\
2863\end{array}$ & $\begin{array}{l}9750 \\
465 \\
6422\end{array}$ & $N / A$ & N/A & $\begin{array}{l}30720 \\
15 \\
3415\end{array}$ & $\begin{array}{l}30720 \\
16 \\
3415\end{array}$ \\
\hline $\begin{array}{l}\text { Molt } \\
\text { Characteristics }\end{array}$ & N/A & $\begin{array}{l}\text {-no foam } \\
\text {-cryatals }\end{array}$ & $N / A$ & N/A & $\begin{array}{l}\text {-no foam } \\
\text {-crystals }\end{array}$ & $\begin{array}{l}\text {-no foam } \\
\text {-cryetale }\end{array}$ & N/A & N/A & N/A & $\begin{array}{l}\text {-no foam } \\
\text {-cryatals }\end{array}$ & $N / A$ & N/A & N/A & $\mathbf{N} / \mathbf{A}$ \\
\hline Overall Evaluation & Poor & Poor & Poor & Poor & Good & Good & Poor & Poor & Poor & Good & Poor & Poor & Poor & Poor \\
\hline
\end{tabular}


WHC-SD-WM-VI-031

Revision 0

\subsection{PILOT SCALE TESTS AND OBSERVATIONS}

Based on the results of the bench scale testing, the optimum process was determined to be $100 \%$ stoichiometric sucrose at $500{ }^{\circ} \mathrm{C}$ with initial Mix $\mathrm{C}$ bed composition. The glass former mix $C$ fluid bed composition used for the pilot scale production run is given in Table 1-8.

Nitrogen was utilized as the fluidizing gas. No other gasses were added.

Based on observations of the bench scale tests, the following process parameters were initially selected for pilot scale testing:

Reductant:

Reductant to $\mathrm{NO}_{3} / \mathrm{NO}_{2}$ Ratio:

Waste Feed Injection Rate:

Glass Former Charge:

Fluidizing Gas Velocity:

Gas Preheat Temperature:

Calciner Bed Operating Temperature:

Freeboard Temperature:
Sucrose, Powdered

$100 \%$ stoichiometric

$100 \mathrm{ml} / \mathrm{min}$

$93 \mathrm{~kg}(205 \mathrm{lb})$ (Mix C)

$9.1 \mathrm{~m} / \mathrm{min}$ (30 feet $/ \mathrm{min}$ )

$240{ }^{\circ} \mathrm{C}$

$500{ }^{\circ} \mathrm{C}$

$500{ }^{\circ} \mathrm{C}$

\subsection{FBC-P0: GLASS FORMERS YITH WATER}

The initial bed was charge with $92.97 \mathrm{~kg}$ (205 $\mathrm{lb})$ of mix $\mathrm{C} \mathrm{glass}$ formers. On the morning of March 8, 1995, bed fluidization was initiated with $\mathrm{N}_{2}$ only and the bed was preheated to $500^{\circ} \mathrm{C}$. Fluidizing gas was initially preheated to $240{ }^{\circ} \mathrm{C}$ and the calciner freeboard was preheated to $520^{\circ} \mathrm{C}$. The off-gas scrubber was started up with $\mathrm{pH}$ of 12.

Table 1-8. Pilot Scale Glass Former Mix C.

\begin{tabular}{|l|r|r|}
\hline \multicolumn{1}{|c|}{ Glass former } & $\begin{array}{c}\text { Weight } \\
\text { percent }\end{array}$ & $\begin{array}{c}\text { Weight, kg (1b) [rounded to } \\
\text { nearest } 0.05 \mathrm{lb})\end{array}$ \\
\hline Silica sand & 74.66 & $69.42(153.05)$ \\
\hline Boric acid & 0.00 & 0.00 \\
\hline Dolomite & 13.62 & $12.66(27.90)$ \\
\hline Alumina & 9.54 & $8.87(19.55)$ \\
\hline Iron oxide & 1.30 & $1.21 \quad(2.65)$ \\
\hline Magnesium carbonate & 0.88 & $0.82(1.80)$ \\
\hline Total & 100.0 & $92.98(205.00)$ \\
\hline
\end{tabular}


WHC-SD-WM-VI-031

Revision 0

Once the bed was fluidizing at steady state, potable water feed injection was initiated at $100 \mathrm{ml} / \mathrm{min}$ in order to establish thermodynamic performance characteristics. The pilot scale calciner was operated for approximately 4 hours at steady state in the batch mode with water injection into the fluidizing bed of glass formers. The pump speed was gradually increased throughout the batch test in order to evaluate thermodynamic performance. Pump speed was adjusted between $100 \mathrm{ml} / \mathrm{min}$ and $400 \mathrm{ml} / \mathrm{min}$. Good bed fluidization characteristics were observed for all feed rates. The critical data point was the inside wall temperature of the calciner. If temperatures were too high, caking could start on the walls. A feed rate of about $350 \mathrm{ml} / \mathrm{min}$ showed good performance.

\subsection{PRODUCTION RUN: GLASS FORMERS AND SIMULANT WITH 100\% SUCROSE}

The bed remained fluidizing at $500{ }^{\circ} \mathrm{C}$ while simulant with $100 \%$ sucrose reductant was mixed in the feed tank. Simulant injection began at $225 \mathrm{ml} / \mathrm{min}$. Fluidization gas preheated temperature was $250{ }^{\circ} \mathrm{C}$ and freeboard temperature was $550{ }^{\circ} \mathrm{C}$. After approximately 50 minutes with simulant injection at $225 \mathrm{ml} / \mathrm{min}$ and stable fluidization, feed rate was raised to $275 \mathrm{ml} / \mathrm{min}$. After another 50 minutes of stable fluidization, feed pump speed was raised to $350 \mathrm{ml} / \mathrm{min}$. High off-gas flow was observed $1-1 / 2$ hours into the test. Injection of simulant and batch fluidization at $500{ }^{\circ} \mathrm{C}$ were continued for a total duration of 4 hours. The pilot scale calciner was then cooled down and shutdown on the evening of March 8, 1995. The initial simulant loading ( $20 \mathrm{wt} \% \mathrm{Na}_{2} \mathrm{O}$ equivalent) had not yet been reached so the fluid bed was not changed out.

On the morning of March 9, 1995, the pilot scale calciner fluidization was reinitiated at. $9.1 \mathrm{M} / \mathrm{min}(30 \mathrm{fpm})$ and the bed was preheated to $500{ }^{\circ} \mathrm{C}$ with the fluidization gas and freeboard at $270{ }^{\circ} \mathrm{C}$ and $570{ }^{\circ} \mathrm{C}$, respectively. After the fluid bed stabilized, simulant injection with $100 \%$ stoichiometric sucrose was initiated at $110 \mathrm{ml} / \mathrm{min}$. Feed injection was gradually increased from $110 \mathrm{ml} / \mathrm{min}$ to $350 \mathrm{ml} / \mathrm{min}$ over a 10 minute period to maintain good fluidization and minimize wall temperature transients. Batch operation was continued for 1 hour until the calculated bed simulant loading had been achieved.

Fluidization gas preheated temperature was stabilized at approximately $337{ }^{\circ} \mathrm{C}$, and freeboard temperature was stabilized at approximately $550{ }^{\circ} \mathrm{C}$. Continuous glass former feed was initiated at $20.4 \mathrm{Kg} / \mathrm{hr}(45 \mathrm{lb} / \mathrm{hr})$, and continuous product discharge was initiated at $25.4 \mathrm{Kg}(56 \mathrm{lb} / \mathrm{hr})$. Simulant feed was continued at $350 \mathrm{ml} / \mathrm{min}$. Continuous processing with balanced feed and discharge rates was continued for over 7 hours. The pilot scale calciner was cooled down and shutdown on the evening of March 9, 1995. Up to this point a cumulative product total weight of $181 \mathrm{~kg}(400 \mathrm{lb})$ had been generated. The observed throughputs exceeded expected levels by a factor of 3 .

On the morning of March 10, 1995, pilot scale fluidization was again initiated and stabilized at $9.1 \mathrm{~m} / \mathrm{min}$ fluidization gas velocity, $500{ }^{\circ} \mathrm{C}$ bed temperature, $300{ }^{\circ} \mathrm{C}$ fluidization gas preheat temperature, and $500{ }^{\circ} \mathrm{C}$ freeboard temperature. After the fluid bed was preheated and stable, simulant feed with $100 \%$ stoichiometric sucrose was initiated. Additionally, continuous dry glass former feed and product discharge were initiated to balance feed and discharge. 
A feed pump leak developed early in the test run and required securing all feed and discharge. Additionally, calciner heaters were turned off during feed pump repair. When repairs were complete, the fluidizing bed temperature had cooled to $464{ }^{\circ} \mathrm{C}$. Heaters were turned on to begin heat-up to $500^{\circ} \mathrm{C}$. Balanced simulant injection, dry glass former feed, and product discharge were all reinitiated while the bed was heating up from $464^{\circ} \mathrm{C}$. Initial feed pump setting was $350 \mathrm{ml} / \mathrm{min}$. Within 45 minutes, fluid bed temperatures were above $480{ }^{\circ} \mathrm{C}$ and began to diverge. Additionally, product discharge flow was restricted and ultimately became plugged with small chunks. All feed and discharges were secured and the discharge line was cleared. Some bed material was removed during discharge line corrective maintenance.

After repairs were complete, the fluid bed was allowed to preheat and stabilize at $500{ }^{\circ} \mathrm{C}$. Balanced simulant injection, dry glass former feed, and product discharge were initiated and gradually increased to match a feed rate of $350 \mathrm{ml} / \mathrm{min}$. Good bed fluidization characteristics were observed. Steady state operations were continued for over 5 hours. The corresponding calculations are presented in Appendix 1B. A 4 hour mass balance was performed during this period. In the afternoon of March 10, 1995 the required quantity of dry product had been produced, feed and discharge were secured, the pilot scale calciner was cooled down, the bed was unloaded through the screw cooler discharge line, and then the calciner was opened and inspected. A total of $454 \mathrm{Kg}(1,000 \mathrm{lb})$ of dry product was generated by pilot scale testing.

Post test inspection revealed free flowing product, minor caking in the freeboard region, and a large lump at the product exit nozzle. Loss of fluidization at $464{ }^{\circ} \mathrm{C}$ was attributed to incomplete reaction of sucrose and melting of unreacted salts. This is believed to also be the source of lumps in the bed.

\subsection{CONCLUSIONS}

- Mixed glass formers fluidized well at ambient temperature in the proportions of mix $A, B, C, D$ and $E$.

- Operation of bed at less than $460{ }^{\circ} \mathrm{C}$ or greater than $700{ }^{\circ} \mathrm{C}$ results in bed caking and agglomeration.

- Sucrose $\left(\mathrm{C}_{12} \mathrm{H}_{22} \mathrm{O}_{11}\right)$ proved to be the optimal reductant by efficiently converting nitrates and nitrites while maintaining stable bed operating conditions.

- Addition of less than $100 \%$ stoichiometric sucrose in the simulant resulted in lower conversion of nitrites/nitrates to $\mathrm{N}_{2}$ and resulted in higher NOx off-gas levels. Higher levels of sucrose (up to 125\%) resulted in lower NOx levels.

- Operation of fluidized bed with addition of simulant and hydrogen, methane or ammonia reductants was unsuccessful, even at operating 
WHC-SD-WM-VI-031

Revision 0

temperatures as $10 \mathrm{w}$ as $300{ }^{\circ} \mathrm{C}$ as bed caking and loss of fluidization resulted.

- Operation of bed at $480-525{ }^{\circ} \mathrm{C}$ produced well fluidized, uniform bed with reasonable conversion efficiencies when sucrose is used as reductant.

- Boric acid addition of greater than 9 wt\% in glass former mix causes early bed agglomeration and loss of fluidization, even at temperatures as $10 \mathrm{~W}$ as $500{ }^{\circ} \mathrm{C}$.

- Boric oxide in the glass former bed fluidizes well at temperatures below $425{ }^{\circ} \mathrm{C}$.

- Sucrose does not completely react with simulant in the fluid bed at temperatures below $480^{\circ} \mathrm{C}$. Incomplete reaction results in caking and loss of bed fluidization.

- Feed addition of glass formers with boric acid (at greater than $16 w t \%$ ) into a fluidizing bed at $500^{\circ} \mathrm{C}$ causes feed line plugging and bed agglomeration.

\subsection{RECONHENDATIONS FOR FUTURE TESTINE}

Additional testing is recommended to bound fluidized bed calciner operating temperature range. Suggested test range would be $480{ }^{\circ} \mathrm{C}$ to $650{ }^{\circ} \mathrm{C}$, with $60 \%$ stoichiometric sucrose addition.

Testing is also recommended to investigate glass former constituents that can be used to incorporate boron into initial bed mix by use of high melting point minerals, such as use of calcium borate, in lieu of boric acid. 
WHC-SD-WM-VI-031

Revision 0

\section{APPENDIX 1A}

\section{LAB ANALYSES}

1-25 


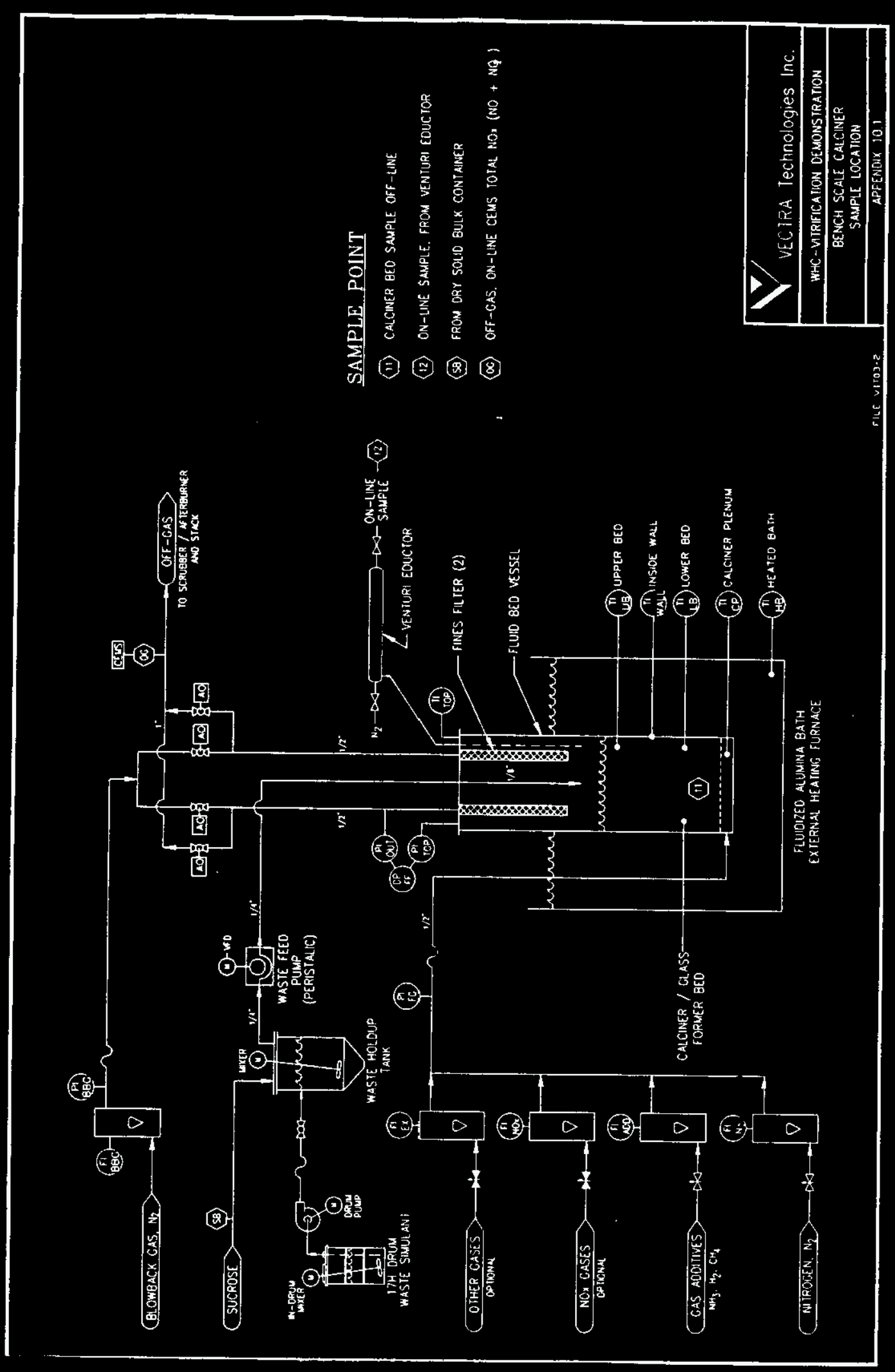




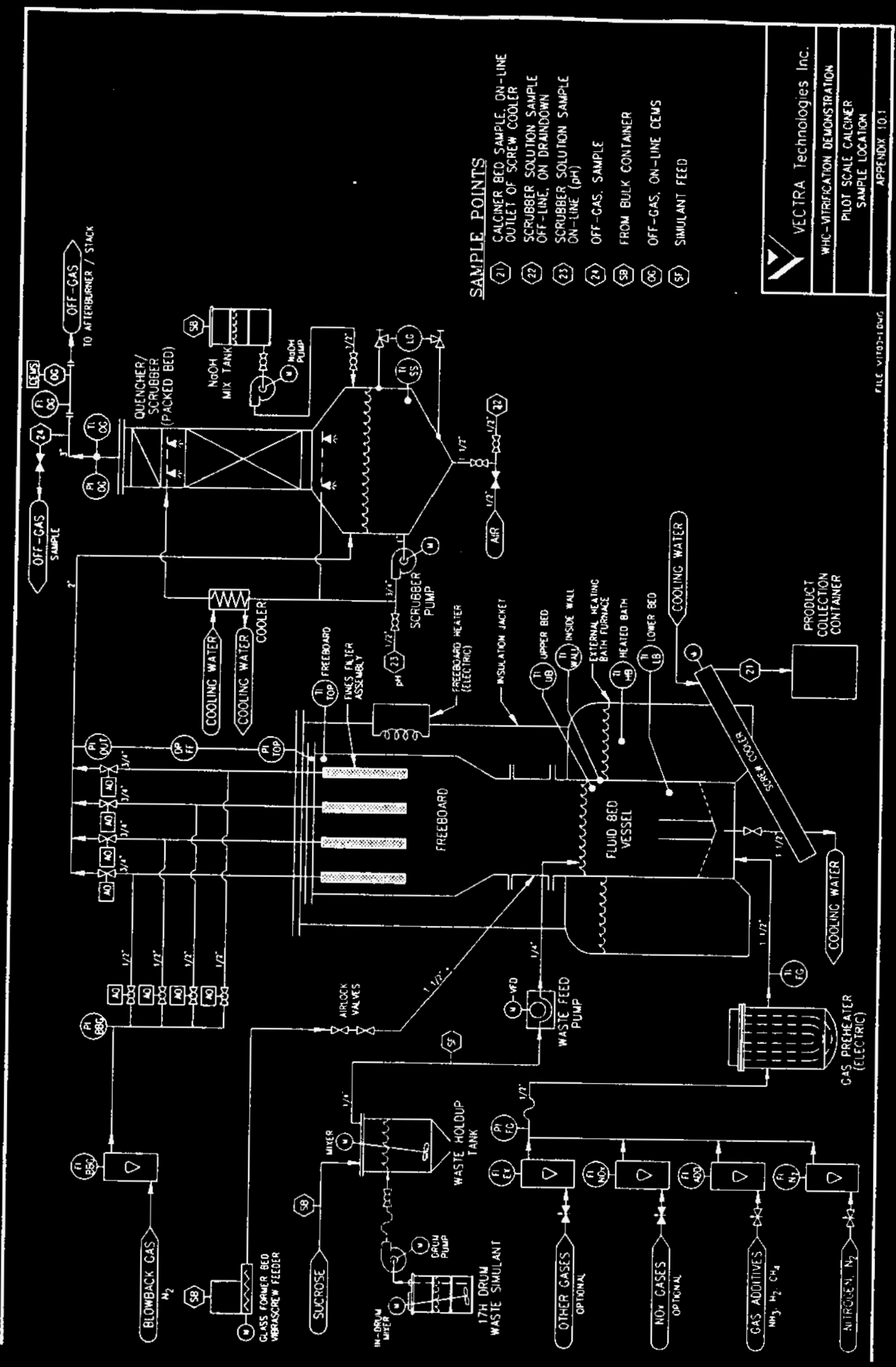




\section{SAMPLE LOG}

\begin{tabular}{|c|c|c|c|c|c|c|}
\hline SAMPLE NUMBER & DATE & TMIE & SAMIPLE TYPE & DESTINATION LAB & SHIPMENT DATE & LAB SAMPLE \# \\
\hline $\mathrm{V} \perp \mathrm{FSO} / \mathrm{FSB}-001$ & $8 / 2$ & 1155 & Initial Bedmix (F) & & & \\
\hline $\mathrm{V} 1 \mathrm{FSO} / 112-002$ & $8 / 2$ & 1210 & Bed sample during (I) & & & \\
\hline $\mathrm{V} 1 \mathrm{FSO} / \mathrm{I} 12-003$ & $8 / 2$ & 1315 & Bed sample during (I) & & & \\
\hline $\mathrm{V} 1 \mathrm{FSO} / 111-004$ & $8 / 2$ & 1630 & Clump sample (I) & & & \\
\hline $\mathrm{V} 1 \mathrm{FSO} / \mathrm{I} 12-005$ & $8 / 2$ & 1203 & Bed sample (I) & & & \\
\hline V 1 FSO /I $12-006$ & $9 / 2$ & 1330 & Bed sample (I) & & & \\
\hline V 1 FSO/I $12-007$ & $10 / 2$ & 1725 & Bed sample (I) & & & \\
\hline$V 1$ FSO/I $11-008$ & $13 / 2$ & 900 & Bed sample & & & \\
\hline$V \perp F S 0 / 111-009$ & $13 / 2$ & 900 & Bed sample & & & \\
\hline V 1 FSO/111-010 & $13 / 2$ & 900 & Bed santiple & & & \\
\hline VIFS0/111-011 & $13 / 2$ & 900 & Clump sample & & & \\
\hline $\mathrm{V} 1 \mathrm{FSO} / \mathrm{I} 11-012$ & 132 & 900 & Bed sample & & 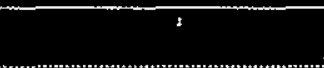 & \\
\hline $\mathrm{V} 1 \mathrm{FSI} / \mathrm{I} 12-013$ & $13 / 2$ & 1630 & Bed sample (1) & & & \\
\hline V 1 FS1 / I $11-014$ & $14 / 2$ & 0840 & Upper freeboard & & & \\
\hline VIFS1/I $11-015 \mathrm{H}$ & $14 / 2$ & 0840 & Lower freeboard & & & \\
\hline $\mathrm{V} 1 \mathrm{FSI} / \mathrm{I} 11-016 \mathrm{H}$ & $14 / 2$ & 0845 & $\mathrm{Bed}$ & & & \\
\hline V1FS1/111-017H & $14 / 2$ & 0845 & Bed & & & \\
\hline V 1 FS1/I $11-018 \mathrm{H}$ & $14 / 2$ & 0845 & Bed & & & \\
\hline $\mathrm{V} 1 \mathrm{FS} 1 / 111-019 \mathrm{H}$ & $14 / 2$ & 0845 & Bed & & & \\
\hline
\end{tabular}

SAMPLE LOG 


\begin{tabular}{|c|c|c|c|c|c|c|}
\hline SAMPLE NUMBER & DATE & TIME & SAMPLE TYPE & DESTINATION LAB & SHIPMENT DATE & LAB SAMPLE \# \\
\hline $\mathrm{V} 1 \mathrm{FSI} / \mathrm{I} 11-020 \mathrm{H}$ & $14 / 2$ & 0845 & Bed Cake & & & \\
\hline $\mathrm{V} 1 \mathrm{FS} 1 / \mathrm{I} 11-021 \mathrm{H}$ & $14 / 2$ & 0845 & Bed & Melt Test & & \\
\hline$V 1 F S 2 / 112-022$ & $14 / 2$ & 1305 & Bed & & & \\
\hline $\mathrm{V} 1 \mathrm{FS} 2 / \mathrm{I} 11-023 \mathrm{H}$ & $15 / 2$ & 0800 & Bed Cake & & & \\
\hline $\mathrm{V}_{1} \mathrm{FS} 2 / \mathrm{I} 11-024 \mathrm{H}$ & 1512 & 0800 & Bed & & & \\
\hline $\mathrm{V}_{1} \mathrm{FS} 2 / \mathrm{I} 11-025 \mathrm{H}$ & $15 / 2$ & 0800 & Bed. & & & \\
\hline $\mathrm{V} 1 \mathrm{FS} 2 / \mathrm{I} 11-026 \mathrm{H}$ & $15 / 2$ & 0800 & Bed & & & \\
\hline $\mathrm{V} 1 \mathrm{FS} 2 / \mathrm{I} 11-027 \mathrm{H}$ & $15 / 2$ & 0800 & Bed & & & \\
\hline $\mathrm{V} 1 \mathrm{FS} 3 / \mathrm{I} 11-028 \mathrm{H}$ & $15 / 2$ & 1400 & Bed Cake & & & \\
\hline $\mathrm{V} 1 \mathrm{FS} 3 / \mathrm{I11}-029 \mathrm{H}$ & $15 n$ & 1400 & $\mathrm{Bed}$ & & & \\
\hline $\mathrm{V} 1 \mathrm{FS} 3 / \mathrm{I} 11-030 \mathrm{H}$ & $15 / 2$ & 1400 & Bed & & & \\
\hline $\mathrm{V} 1 \mathrm{FS} 3 / 111-031$ & $15 / 2$ & 1400 & Bed & & & \\
\hline $\mathrm{V} 1 \mathrm{FS} 3 / \mathrm{I} 11-032$ & $15 / 2$ & 1400 & Bed & & & \\
\hline V1 FS4/I11-033 & $16 / 2$ & 1030 & $\mathrm{Bed}$ & WHC Archive & $3 / 1 / 95$ & \\
\hline $\mathrm{V} 1 \mathrm{FS} 4 / \mathrm{I} 11 \mathrm{ll}-034$ & $16 / 2$ & 1030 & $\mathrm{Bed}$ & PED & $2 / 24 / 95$ & \\
\hline V 1 FS4/I $11-035$ & $16 / 2$ & 1030 & Bed & & & \\
\hline V 1 FS4/I $11-036$ & $16 / 2$ & 1030 & Bed & & & \\
\hline V 1 FS4 $/ I 11-037$ & $16 / 2$ & 1030 & Freeboard & & & \\
\hline V 1 FS4/I $11-038$ & $16 / 2$ & 1030 & Crust/Cake & & & \\
\hline$V 1$ FS5/I $12-039$ & $16 / 2$ & 1200 & Bed & & & \\
\hline V 1 FSS/I $11-040$ & $16 / 2$ & 1800 & Bed & & & \\
\hline V1FS5/I $11-041$ & $16 / 2$ & 1800 & Bed & WHC Archive & $3 / 1 / 95$ & \\
\hline
\end{tabular}




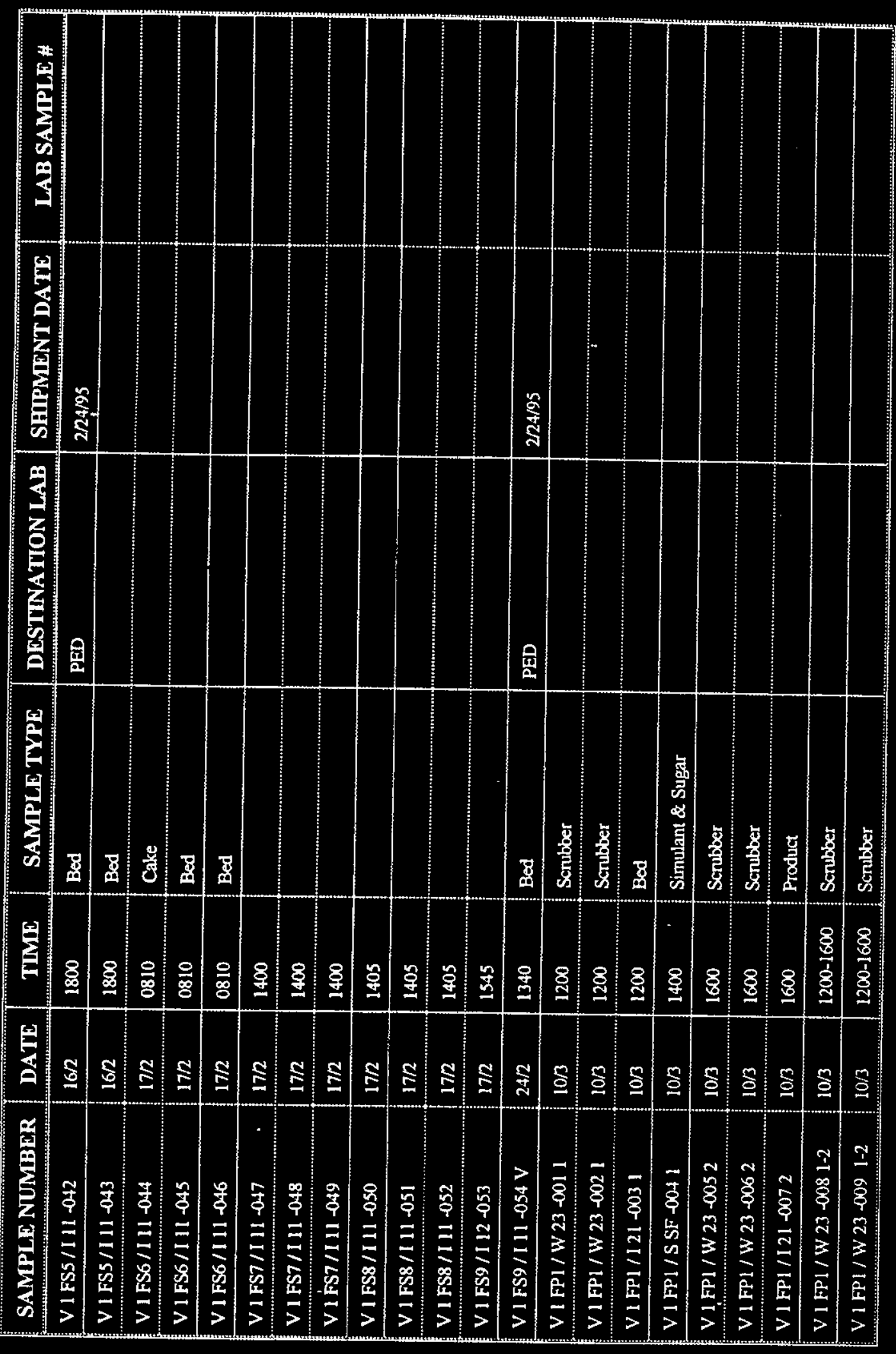




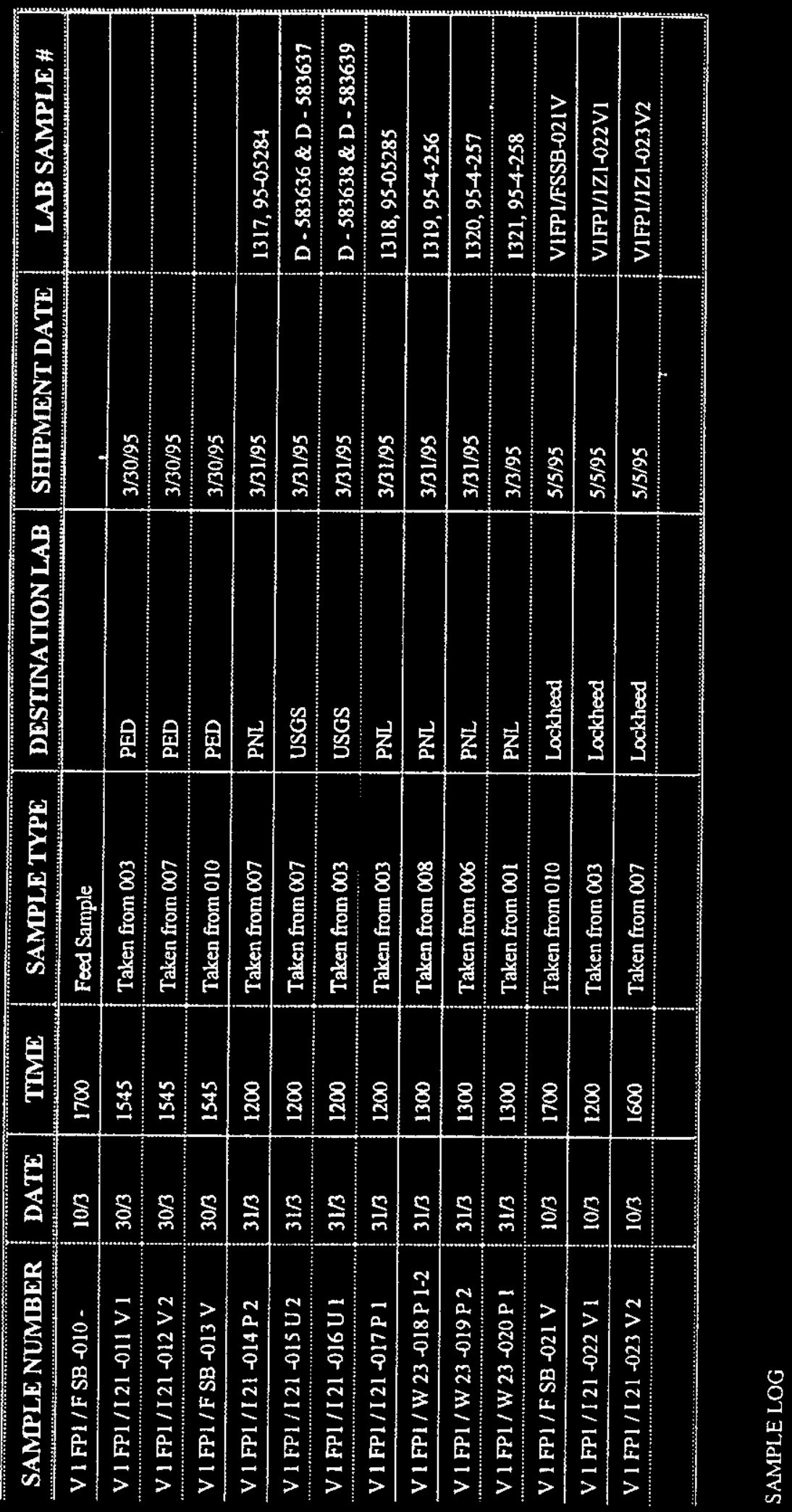


WHC-SD-WM-VI-031

Revision 0

Lockheed Analytical Services

METALS RESULTS 


\section{METALS RESULTS}

\begin{tabular}{|l|l|l|}
\hline Client Sample 1D: V1FP1/FS8-021V & Date Collected: 03-10-95 & Matrix: solid waste \\
\hline LAL Batch ID(s): 509 vc & Date Received: 05-09-95 & \\
\hline
\end{tabular}

\begin{tabular}{|c|c|c|c|c|c|c|}
\hline Constituents & Method & $\begin{array}{c}\text { Concentration } \\
(\mathrm{mg} / \mathrm{kg})\end{array}$ & $\begin{array}{c}\text { Peporting } \\
\text { Detection Limit } \\
\text { (mg/kg) }\end{array}$ & $\begin{array}{c}\text { Data } \\
\text { Qualifier(s) }\end{array}$ & $\begin{array}{c}\text { Date } \\
\text { Analyzed }\end{array}$ & $\begin{array}{l}\text { LAL } \\
\text { Sample ID }\end{array}$ \\
\hline Aluminum & 6010 & 3000 & 40 & & $05-26-95$ & L4472-1 \\
\hline Boron & 6010 & 2000 & 40 & $\bullet$ & $05 \cdot 26-95$ & L4472-1 \\
\hline Calcium & 6010 & 31,000 & 1000 & & $05-26-95$ & L4472-1 \\
\hline Chromium & 6010 & 4.2 & 2 & C & $05-26-95$ & L4472-1 \\
\hline Cesium & 3500 & $<800$ & 800 & & 05.18 .95 & 14472.1 \\
\hline Iron & 6010 & 6,400 & 20 & & $05-26-95$ & L4472-1 \\
\hline Magnesium & 6010 & 20,000 & 1000 & & $05-26-95$ & L4472.1 \\
\hline Manganese & 6010 & 36 & 3 & & $05-26-95$ & L4472-1 \\
\hline Molybdenum & 6010 & 40 & 40 & & 05.26 .95 & L4472-1 \\
\hline Nickel & 6010 & $<8$ & 8 & & $05-26-95$ & L4472-1 \\
\hline Potassium & 6010 & $<1000$ & 1000 & C & $05-26-95$ & $L 4472-1$ \\
\hline Silicon & 6010 & 85,000 & 400 & $D(1: 5)$ & $05-17-95$ & L4472-1 \\
\hline Sodium & 6010 & $<1000$ & 1000 & & $05-26-95$ & $L 4472.1$ \\
\hline Strontium & 6010 & $<20$ & 20 & & $05-26-95$ & L4472-1 \\
\hline Zinc & 6010 & 6.9 & 4 & & 05.26 .95 & $L 4472.1$ \\
\hline
\end{tabular}

\section{Comments:}


WHC-SD-WM-VI-031

Revision 0

\section{METALS RESULTS}

\begin{tabular}{|l|l|l|}
\hline Client Sample ID: V1FP1/Z1-022V1 & Date Collected: 03-10-95 & Matrix: solid waste \\
\hline LAL Batch ID(s): 509 vc & Date Received: 05-09-95 & \\
\hline
\end{tabular}

\begin{tabular}{|c|c|c|c|c|c|c|}
\hline Constituents & Method & $\begin{array}{c}\text { Concentration } \\
(\mathrm{mg} / \mathrm{kg})\end{array}$ & $\begin{array}{c}\text { Reporting } \\
\text { Detection Limit } \\
(\mathbf{m g} / \mathbf{k g})\end{array}$ & $\begin{array}{c}\text { Data } \\
\text { Qualifier(s) } \\
\end{array}$ & $\begin{array}{l}\text { Date } \\
\text { Analyzed }\end{array}$ & $\begin{array}{c}\text { LAL } \\
\text { Sample ID }\end{array}$ \\
\hline Aluminum & 6010 & 12000 & 40 & & $05-26-95$ & $14472-2$ \\
\hline Boron & 6010 & 960 & 40 & $\sim$ & 05.26 .95 & $\mathrm{~L} 4472.2$ \\
\hline Calcium & 6010 & 21.000 & 1000 & & $05-26-95$ & L4472-2 \\
\hline Chromium & 6010 & 180 & 2 & C & $05-26-95$ & $\llcorner 4472-2$ \\
\hline Cesium & 3500 & $<800$ & 800 & & $05-18-95$ & L4472-2 \\
\hline Iron & 6010 & 400 & 20 & & $05-26-95$ & L4472-2 \\
\hline Magnesium & 6010 & 13,000 & 1000 & & $05-26-95$ & $\lcm{4} 4472-2$ \\
\hline Manganese & 6010 & 35 & 3 & & $05-26-95$ & L4472-2 \\
\hline Molybdenum & 6010 & 610 & 40 & & $05-26-95$ & 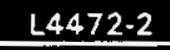 \\
\hline Nickel & 6010 & 15 & 8 & & $05-26.95$ & $\llcorner 4472-2$ \\
\hline Potassium & 6010 & 7,500 & 1000 & C & $05-26-95$ & L4472-2 \\
\hline Silicon & 6010 & 69,000 & 400 & $D(1: 5)$ & $05-17.95$ & $\mathbf{L} 4472.2$ \\
\hline Sodium & 6010 & 91,000 & 1000 & & $05.26-95$ & $-\lfloor 4472.2$ \\
\hline Strontium & 6010 & 570 & 20 & & $05-26-95$ & $\llcorner 4472.2$ \\
\hline Zinc & 6010 & 16 & 4 & & $05-26-95$ & L4472-2 \\
\hline
\end{tabular}

\section{Comments:}


:sมขอแu०0

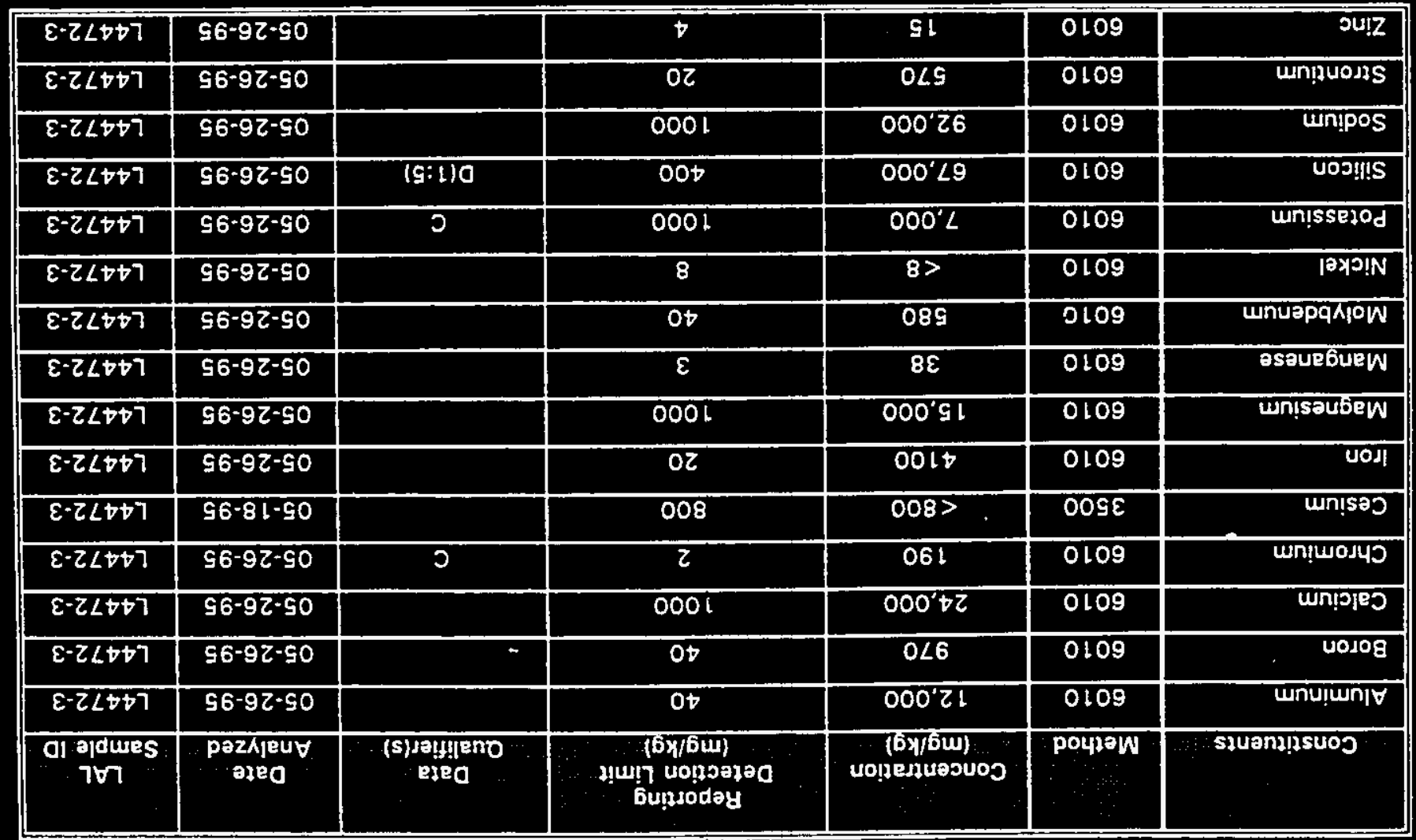

\begin{tabular}{|c|c|c|}
\hline & 96-60-90 :pan!องวy әдеg & 5^ 609:(s)al 4ग18g 787 \\
\hline 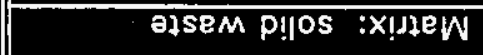 & 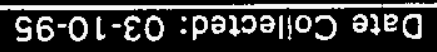 & 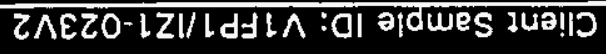 \\
\hline
\end{tabular}

\section{SI7nS $3 y$ S $7 \forall \perp \exists W$}


WHC-SD-WM-VI-031

Revision 0

METALS QC DATA SUMMARY

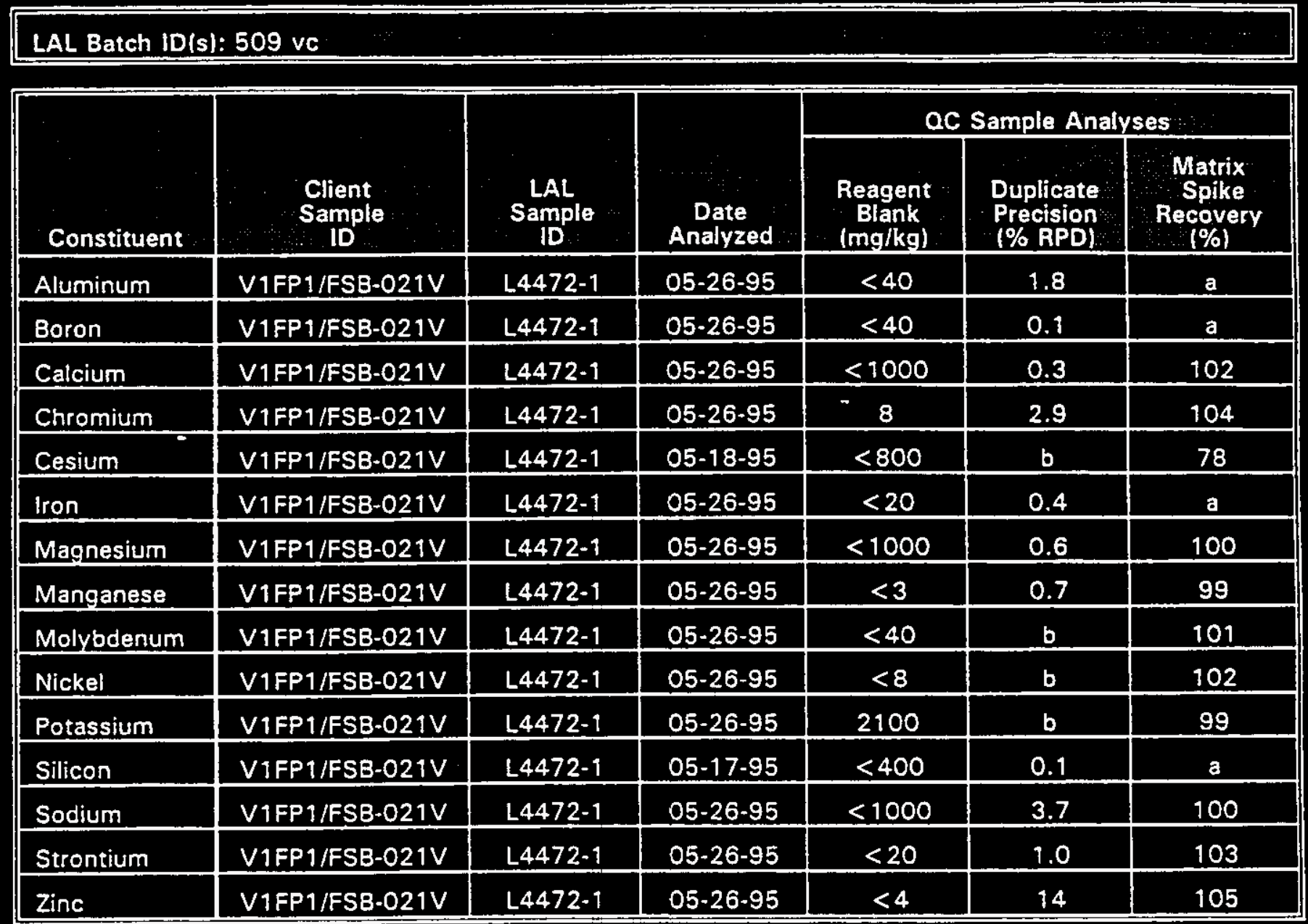

"b" - The RPD cannot be computed, because the sample or the duplicate concentration was below the Reporting Detection Limit.

Comments: 
$H=-5 z-4 M-1 I-031$

Revisioll 0

Lockheed Analytical Se "ivices

NON-METALS RESULTS 


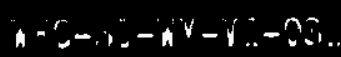

Rovision 0

LOCKHEEY, AUILITTICA:- SERVICES

COMMON IONS INTI AODITIONAL ANALYTES

Gamp:.e! Restilt:s

\begin{tabular}{|l|l|}
\hline Client Sample ID: V1FP1/FSB-021V & Date Collected: 10-MAR-95 \\
\hline Matrix: SolidWaste & Date Received: 09-MAY-95 \\
\hline
\end{tabular}

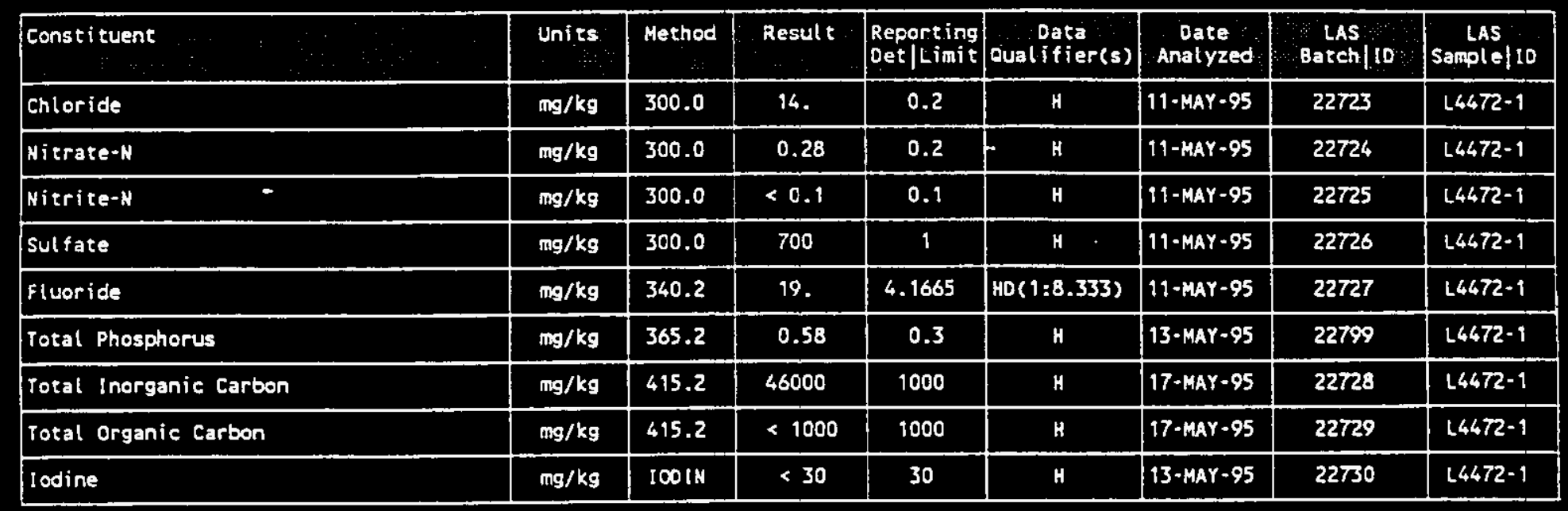




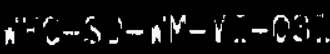

Rervision 0

LOCKFEET HNWHSICAI, SIERVICES

COMMON IONS AND WIDIMIONEL ANALYTES

Sitinpl $\Leftrightarrow$ Resultsi

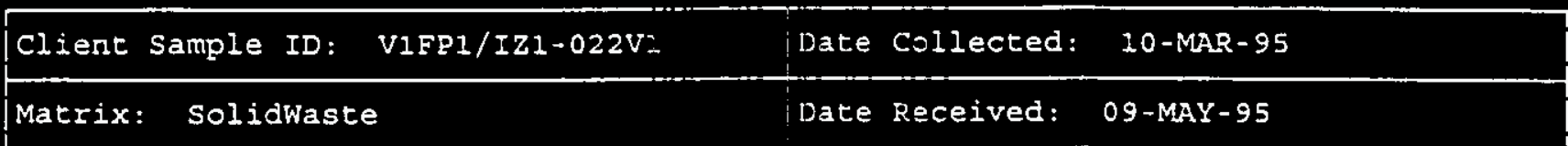

\begin{tabular}{|c|c|c|c|c|c|c|c|c|}
\hline Const i tuent & Units & Method & Result & $\begin{array}{l}\text { Reporting } \\
\text { Det|Limit }\end{array}$ & Qualifier(s) & $\begin{array}{l}\text { Date } \\
\text { Analyzed }\end{array}$ & $\begin{array}{l}\text { LAS } \\
\text { Batch /10 }\end{array}$ & $\begin{array}{c}\text { LAS } \\
\text { Sample } \$ 10\end{array}$ \\
\hline Chloride & $\mathrm{mg} / \mathrm{kg}$ & 300.0 & 2200 & 2 & $H D(1: 10)$ & 11 -MAY -95 & 22723 & $44472 \cdot 2$ \\
\hline Nitrate-N & $\mathrm{mg} / \mathrm{kg}$ & 300.0 & 71. & 2 & $H O(1: 10)$ & $11-$ MAY-95 & 22724 & $16472 \cdot 2$ \\
\hline Nitrite-N & $\mathrm{mg} / \mathrm{kg}$ & 300.0 & 19. & $i$ & $\mathrm{HO}(1: 10)$ & 11 - MAY-95 & 22725 & $L 4472-2$ \\
\hline Sulfate & $\mathrm{mg} / \mathrm{kg}$ & 300.0 & 1900 & 10 & $H D(1: 10)$ & 11-MAY-95 & 22726 & $44472 \cdot 2$ \\
\hline Fluoride & $\mathrm{mg} / \mathrm{kg}$ & 340.2 & 49. & 4.1665 & KD ( $1: 8.333)$ & $11-$ MAY-95 & 22727 & $14472-2$ \\
\hline Total Phosphorus & $\mathrm{mg} / \mathrm{kg}$ & 365.2 & $<0.3$ & 0.3 & H & 13-MAY-95 & 22799 & $14472 \cdot 2$ \\
\hline Total Inorganic Carbon & $\mathbf{m g} / \mathbf{k g}$ & 415.2 & 22000 & 1000 & H & 17-MAY-95 & 22728 & $L 4472-2$ \\
\hline Total Organic Carbon & $\mathbf{m g} / \mathbf{k g}$ & 415.2 & 12000 & 1000 & $H$ & 17-MAY-95 & 22729 & $44472-2$ \\
\hline lodine & $\mathrm{mg} / \mathrm{kg}$ & $1001 \mathrm{~N}$ & 590 & 30 & H & 13-MAY-95 & 22730 & $14472-2$ \\
\hline
\end{tabular}




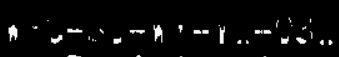

Revision 0

LOCKHEE) ANALY'TICAI, SERVICES

COMMON IONS ANI AI:IIIIIONAL ANALYTES

Gample Resiuts

\begin{tabular}{|l|l|l|}
\hline Client Sample ID: VIFPI/IZl-023V2 & Date Collected: 10-MAR-95 \\
\hline Matrix: SolidWaste & | Date Received: 09-MAY-95 \\
\hline
\end{tabular}

\begin{tabular}{|c|c|c|c|c|c|c|c|c|}
\hline Const ituent 1 & Units & Method & Result & $\begin{array}{l}\text { Reparting } \\
\text { Det ltimit }\end{array}$ & $\begin{array}{l}\text { Data } \\
\text { Oual ifier(s) }\end{array}$ & $\begin{array}{l}\text { Date } \\
\text { Analyzed }\end{array}$ & LAS & $\begin{array}{l}\text { LAS } \\
\text { Sample | } 10\end{array}$ \\
\hline Chloride & $\mathrm{mg} / \mathrm{kg}$ & 300.0 & 1600 & 2 & HD $(1: 10)$ & $11-$ MAY-95 & 22723 & $L 4472 \cdot 3$ \\
\hline Nitrate-N & $\mathrm{mg} / \mathrm{kg}$ & 300.0 & $<2$ & 2 & $H D(1: 10)$ & 11 - MAY-95 & 22724 & $24472-3$ \\
\hline Nitrite-N & $\mathrm{mg} / \mathrm{kg}$ & 300.0 & 20. & 1 & $H O(1: 10)$ & $11-$ MAY-95 & 22725 & $L 4472 \cdot 3$ \\
\hline Sulfate & $\mathrm{mg} / \mathrm{kg}$ & 300.0 & 1700 & 10 & $H D(1: 10)$ & $11-$ MAY-95 & 22726 & $14472-3$ \\
\hline Fluoride & $\mathrm{mg} / \mathrm{kg}$ & 340.2 & 400 & 2.5 & $\mathrm{HD}(1: 5)$ & 11 - MAY-95 & 22727 & $16472-3$ \\
\hline Total Phosphorus & $\mathrm{mg} / \mathrm{kg}$ & 365.2 & $<0.3$ & 0.3 & H & $13-$ MAY -95 & 22799 & $L 4472-3$ \\
\hline Total Inorganic Carbon & $\mathrm{mg} / \mathrm{kg}$ & 415.2 & 32000 & 1000 & $H$ & 17-MAY-95 & 22728 & $14472-3$ \\
\hline Total Organic Carbon & $m g / k g$ & 415.2 & 11000 & 1000 & H & $17 \cdot$ MAY-95 & 22729 & $L 4472 \cdot 3$ \\
\hline lodine & $\mathrm{mg} / \mathrm{kg}$ & $100 \mathrm{IN}$ & 710 & 30 & H & $13-$ MAY- 95 & 22730 & $44472-3$ \\
\hline
\end{tabular}


WHC-SD-WH-VI-031

Revision 0

\section{USGS Results}

1-41 
$0:$ Gary $\leftleftarrows$ Stegen at - WHC50 Subject: VECTRA feeds
WHC-S[D-WH- $\mathrm{VI}-0: 3]$

Revision 3

Text item 1: Text Item

R (ved: by ccmail from fepl.rl.gov

Frow plamotheehel ios.cr.usgs.govefepo

$X$-Envelope-From: plamothe@hel jos.cr.usgs.govefepo

Received: by fepl.rl.gov (5.51/5.17.r1-1)

id AA14587; Tue, 16 May 95 09:53:20 PDT

Received: from helios.cr.usgs.gov (helios.cr.usgs.gov [136.177.21.1]) by isdsun.c

r.usgs.gov (8.6.9/8.6.9) with SMTP id KAA11185; Tue, 16 May 1995 10:48:01 -0600

Received: by helios.cr.usgs.gov (4.1/SMI-4.1)

id AA04202; Tue, 16 May 95 10:48:56 MDT

Date: Tue, 16 May 1995 10:48:56 -0600 (MDT)

From: Paul Lamothe <plamothechelios.cr.usgs.govefepos

To: Joy Smith <Joy Y Smitherl.gov>

Cc: Eric Mast 〈Eric $\$$ Mastori.gov>

Subject: VECTRA feeds

Message-Id: 〈Pine.SUN.3.91.950516104444.4151A-100000@hel ios>

Mime-Version: 1.0

Content-Type: TEXT/PLAIN; charset=US-ASCII

Joy,

Here is all the data for the VECTRA feed samples. The previous email transmission contained only the VECTRA glass samples. The only thing pending on these feeds is the total nitrogen analysis. We'll have to send the samples to our Virginia lab because we don't have the necessary equipment here in Denver. We should have the total nitrogen data in ab 2 weeks. Hope you can read this file. Let me know if you need me to mik this data. Thanks.

IECTRA Feed Samples

\begin{tabular}{|c|c|c|c|c|c|c|c|}
\hline ZieldNo & Lab No & $\underset{\text { XRF }}{\mathrm{SiO}_{0}} \%$ & $\underset{\text { XRF }}{A 1203} \%$ & $\underset{\text { XRF }}{\text { FeT03 }} \%$ & $\begin{array}{l}\mathrm{MgO} \% \\
\text { XRF }\end{array}$ & $\begin{array}{l}\mathrm{CaO} \% \\
\text { XRF }\end{array}$ & $\underset{\text { XRF }}{\mathrm{Na20}} \%$ \\
\hline $\begin{array}{l}\text { VIFPII21-015U } \\
\text { VIFP1I21-015U } \\
\text { VIFP1I21-016U } \\
\text { VIFPII21-016U }\end{array}$ & $\begin{array}{l}D-583636 \\
D-583637 \\
D-583638 \\
D-583639\end{array}$ & $\begin{array}{l}58.0 \\
57.9 \\
57.9 \\
57.9\end{array}$ & $\begin{array}{l}8.48 \\
8.51 \\
9.08 \\
8.94\end{array}$ & $\begin{array}{l}0.65 \\
0.65 \\
0.65 \\
0.66\end{array}$ & $\begin{array}{l}2.44 \\
2.41 \\
2.32 \\
2.30\end{array}$ & $\begin{array}{l}3.16 \\
3.16 \\
3.01 \\
3.01\end{array}$ & $\begin{array}{l}13.2 \\
13.2 \\
13.3 \\
13.4\end{array}$ \\
\hline
\end{tabular}

ECTRA Feed Samples

\begin{tabular}{|c|c|c|c|c|c|c|c|}
\hline ieldNo : & Lab No & $\begin{array}{l}\text { K20 \% } \\
\text { XRF }\end{array}$ & $\begin{array}{l}\text { TiO2 } \% \\
\text { XRF }\end{array}$ & $\begin{array}{c}\text { P205 \% } \\
\text { XRF }\end{array}$ & $\begin{array}{l}\text { MnO \% } \\
\text { XRF }\end{array}$ & LOI $\underset{X R F}{925 C}$ & $\underset{\text { ISE }}{\text { CI } \%}$ \\
\hline $\begin{array}{l}1 F P 1121-015 U \\
1 F P 1121-015 U \\
\text { 'IFPII21-016U } \\
\text { 'IFPIIII-016U }\end{array}$ & $\begin{array}{l}D-583636 \\
D-583637 \\
D-583638 \\
D-583639\end{array}$ & $\begin{array}{l}0.93 \\
0.94 \\
0.97 \\
0.97\end{array}$ & $\begin{array}{l}0.02 \\
0.02 \\
0.02 \\
0.02\end{array}$ & $\begin{array}{l}0.17 \\
0.19 \\
0.16 \\
0.17\end{array}$ & $\begin{array}{l}<0.01 \\
<0.01 \\
<0.01 \\
<0.01\end{array}$ & $\begin{array}{l}11.8 \\
11.8 \\
11.2 \\
11.2\end{array}$ & $\begin{array}{l}0.24 \\
0.18 \\
0.26 \\
0.27\end{array}$ \\
\hline
\end{tabular}

ECTRA Feed Samples

\begin{tabular}{|c|c|c|c|c|c|c|c|}
\hline jeldNo & Lab No & $\begin{array}{l}\text { F } \% \\
\text { ISE }\end{array}$ & $\begin{array}{l}\text { FeO \% } \\
\text { TITRN }\end{array}$ & $\begin{array}{l}\text { TOTAL S\% } \\
\text { S-ANALZR }\end{array}$ & $\begin{array}{l}\text { B } P \text { PPM } \\
\text { ICP }\end{array}$ & $\begin{array}{l}\mathrm{Ba} \text { ICPM } \\
I C P\end{array}$ & $\begin{array}{l}\mathrm{Cr} P P M \\
\text { ICP }\end{array}$ \\
\hline $\begin{array}{l}1 F P 1121-015 U \\
1 F P 1121-015 U \\
1 F P 1121-016 U \\
1 F P 1121-016 U\end{array}$ & $\begin{array}{l}D-583636 \\
D-583637 \\
D-583638 \\
D-583639\end{array}$ & $\begin{array}{l}0.14 \\
0.15 \\
0.13 \\
0.14\end{array}$ & -- & $\begin{array}{l}0.06 \\
0.06 \\
0.06 \\
0.06\end{array}$ & $\begin{array}{l}1300 \\
1300 \\
1300 \\
1300\end{array}$ & $\begin{array}{l}8 \\
8 \\
9 \\
9\end{array}$ & $\begin{array}{l}210 \\
210 \\
250 \\
230\end{array}$ \\
\hline
\end{tabular}


-ab vo Cs PPM Mo PP' Ni PPM Sr PPM $\operatorname{lr}$ PPM TOTAL C INAA ICP ICP ICP ICP C-ANALZ

\begin{tabular}{|c|c|c|c|c|c|c|c|}
\hline $\begin{array}{l}\text { V]FP' }[2]-015 \mathrm{U} \\
\text { V]FF1 } 121-015 \mathrm{U} \\
\text { VIFP1 } 21-016 \mathrm{U} \\
\text { IFP1 } 121-016 \mathrm{U}\end{array}$ & $\begin{array}{l}\text { D) }-58: 3636 \\
\text { [) }-58: 3637 \\
\text { [) }-.58: 3638 \\
{[]-58: 3639}\end{array}$ & $\begin{array}{l}7513 \\
763 \\
8015 \\
8015\end{array}$ & $\begin{array}{l}66) \\
671) \\
7019 \\
7010\end{array}$ & $\begin{array}{r}15 \\
<8 \\
44 \\
29\end{array}$ & $\begin{array}{l}620 \\
630 \\
620 \\
620\end{array}$ & $\begin{array}{l}160 \\
210 \\
220 \\
190\end{array}$ & $\begin{array}{l}3.1: \\
3.07 \\
2.93 \\
2.9\end{array}$ \\
\hline
\end{tabular}

VECTRA Feed Samples

FieldNo Lab No ORGNC C\% CRBNT C\% Calc Co3-anal

VIFPII2]-015U

VIFPII2]-015U

VIFPII21-016U

VIFP1I21-016U

I ppm Nitrite \%Nitrate \%Total N \% INAA Ion Chromion ChromCHN-Analz

\begin{tabular}{|c|c|c|c|c|c|}
\hline $\begin{array}{l}536 \\
537\end{array}$ & $\begin{array}{r}0.02 \\
0.07 \\
<0.01 \\
0.03\end{array}$ & $\begin{array}{l}3.08 \\
3.02 \\
2.94 \\
2.92\end{array}$ & $\begin{array}{c}797 \\
-- \\
-866\end{array}$ & $\begin{array}{l}<0.001 \\
<0.001 \\
<0.001 \\
<0.001\end{array}$ & $\begin{array}{l}<0 \\
<0 \\
<0 \\
<0\end{array}$ \\
\hline
\end{tabular}

Pau1 J. Lamothe

U.S. Geological Survey

plamothechel ios.cr.usgs.gov

. (303) 236-1923 
WHC-SD-WM-VI-031

Revision 0

\section{PNL Results}




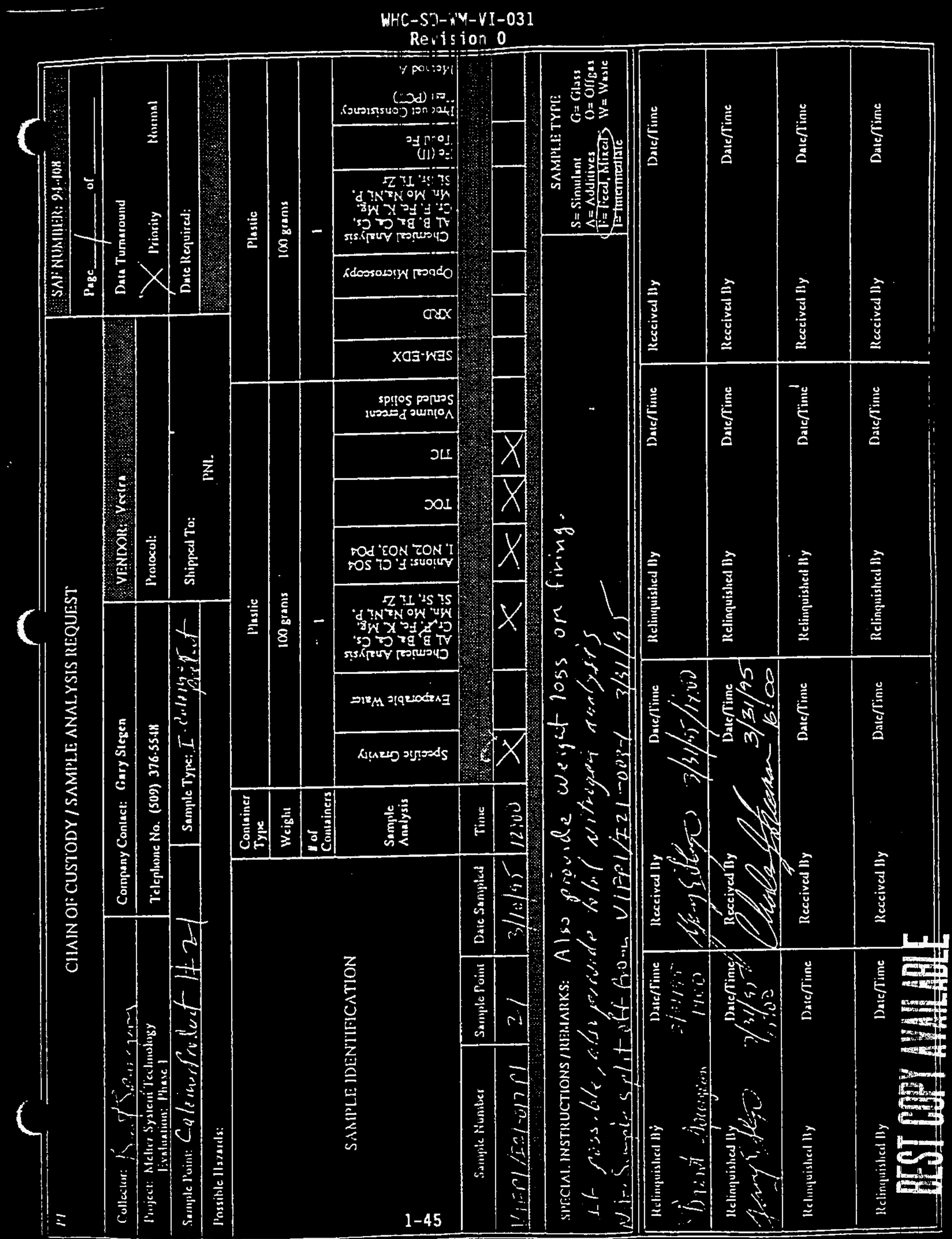




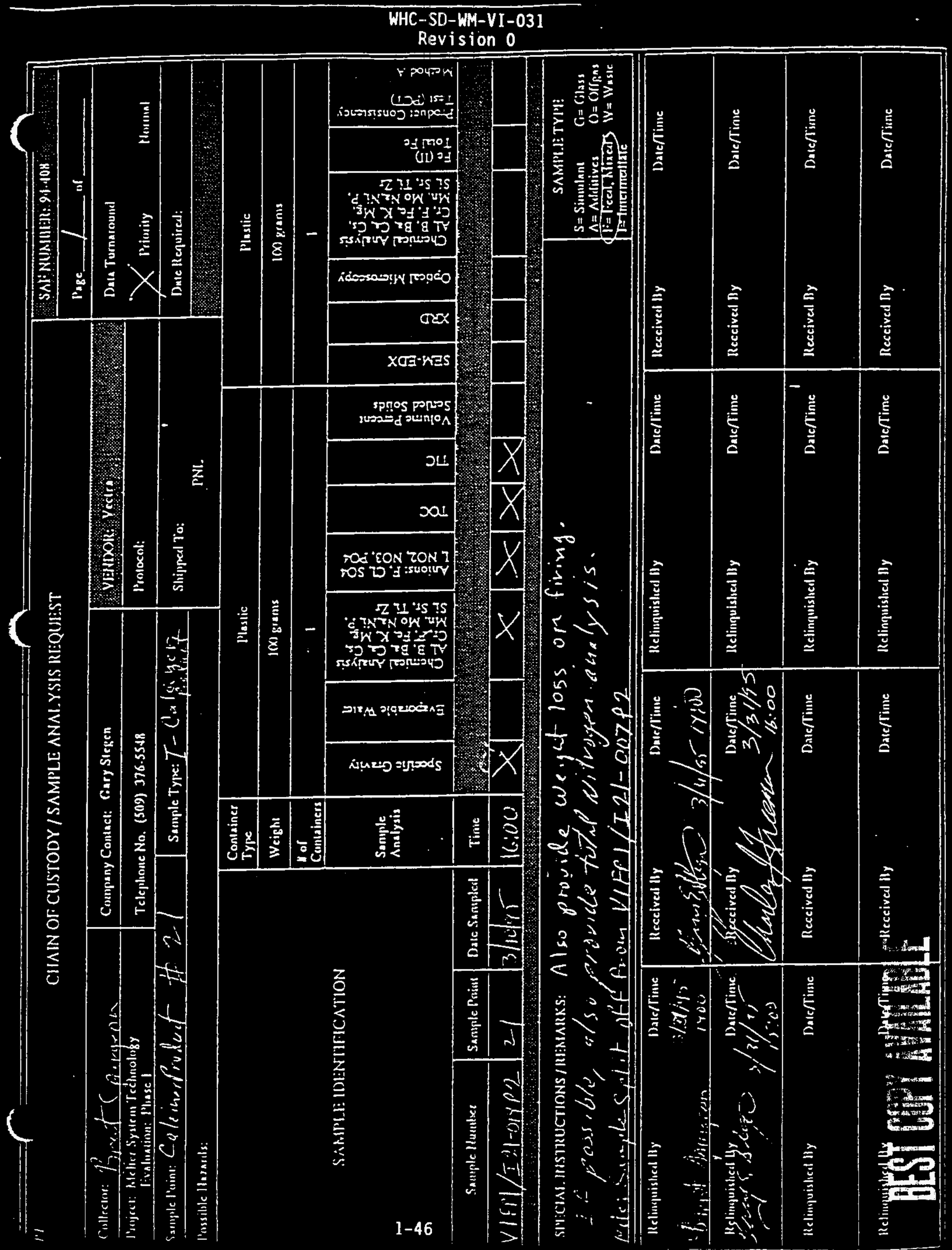




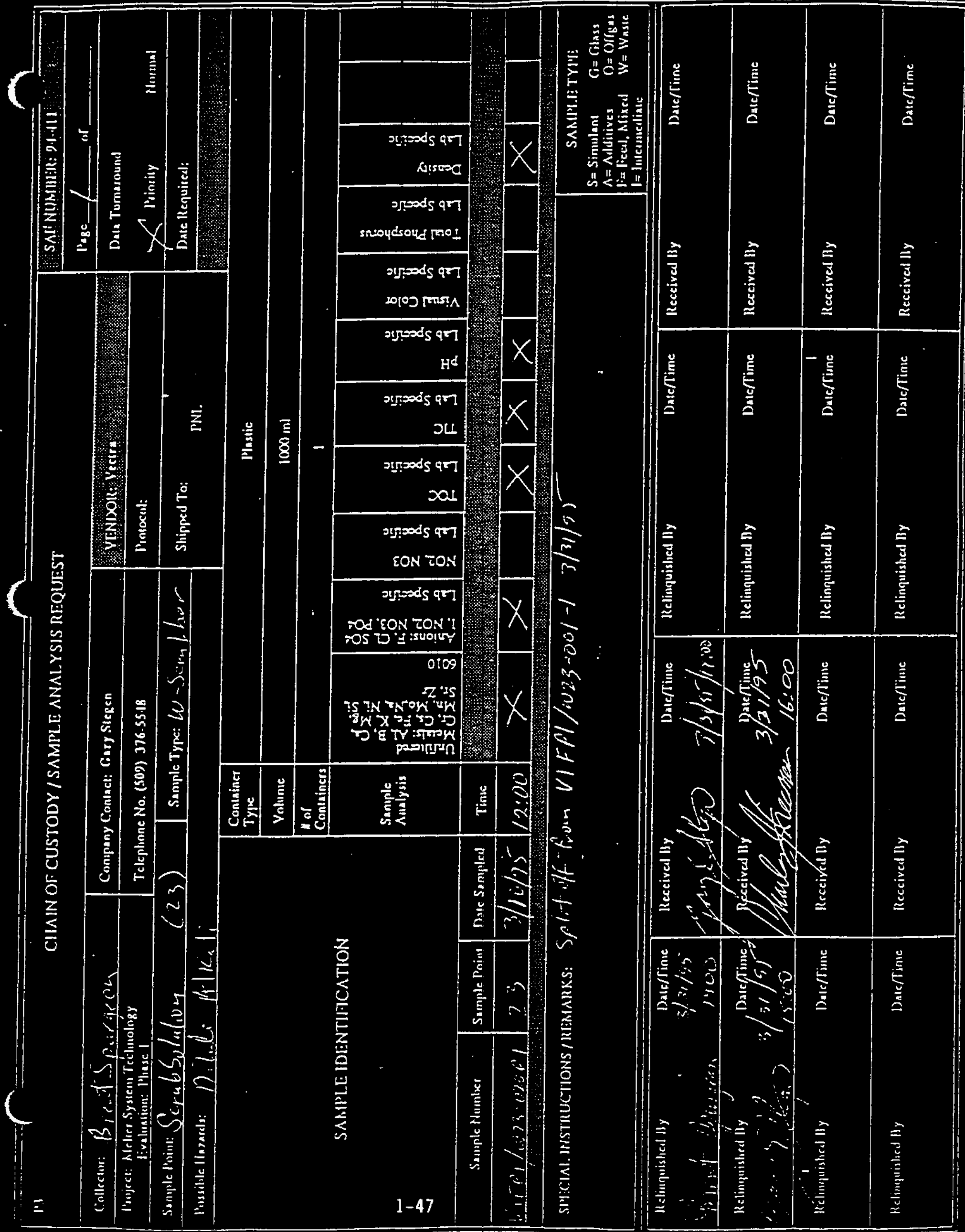




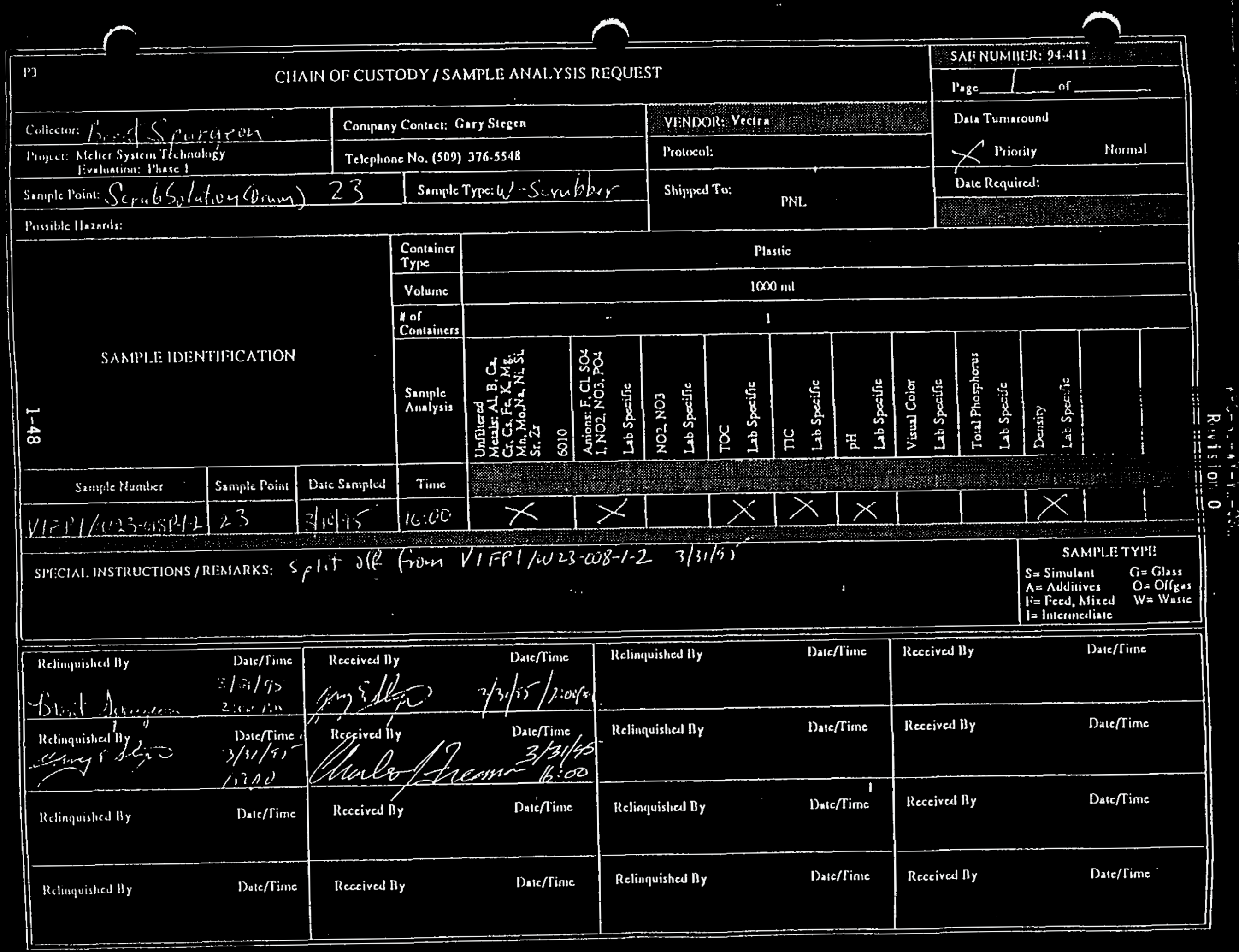




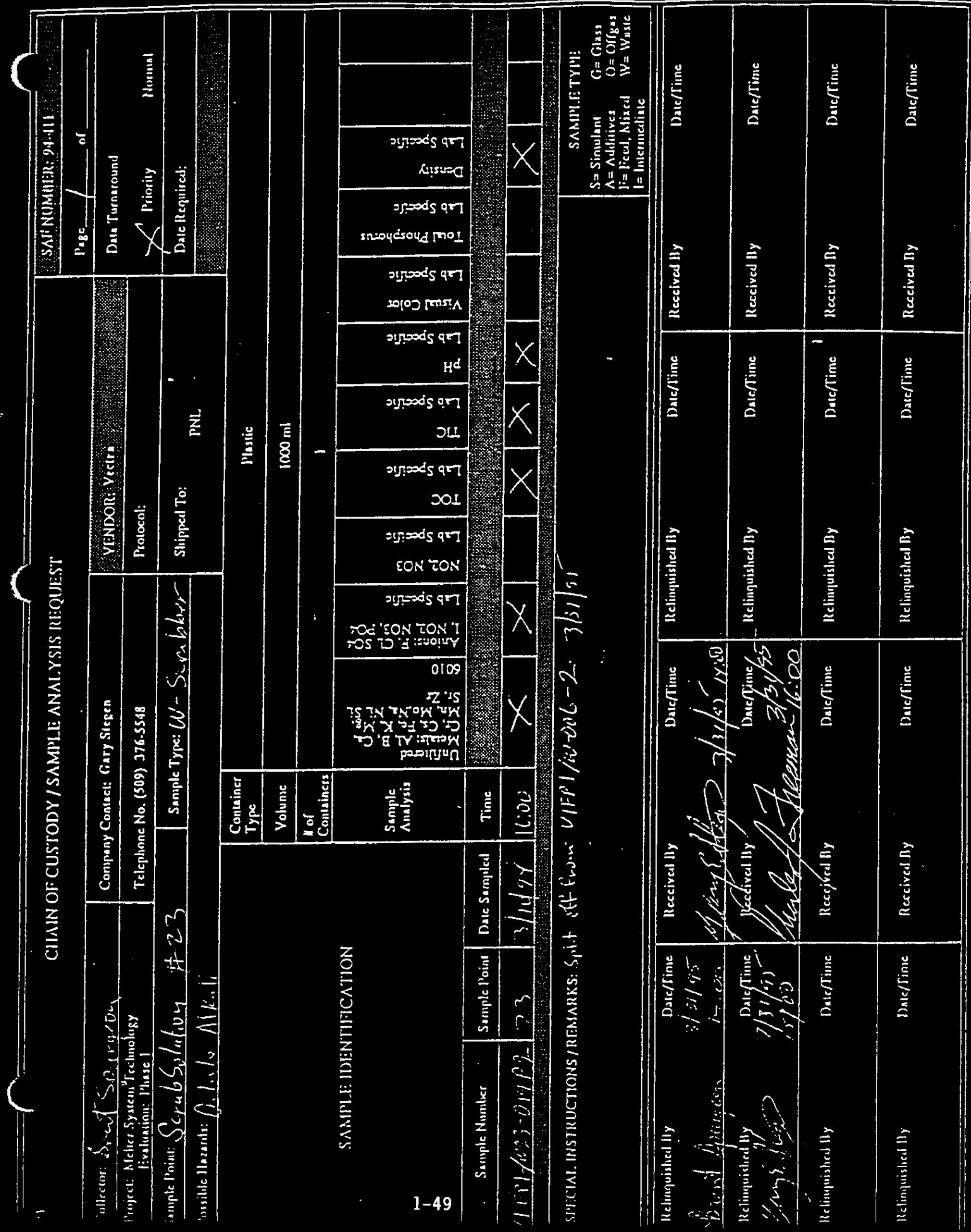


WHC-SD-WM-VI-031

Revision 0

C

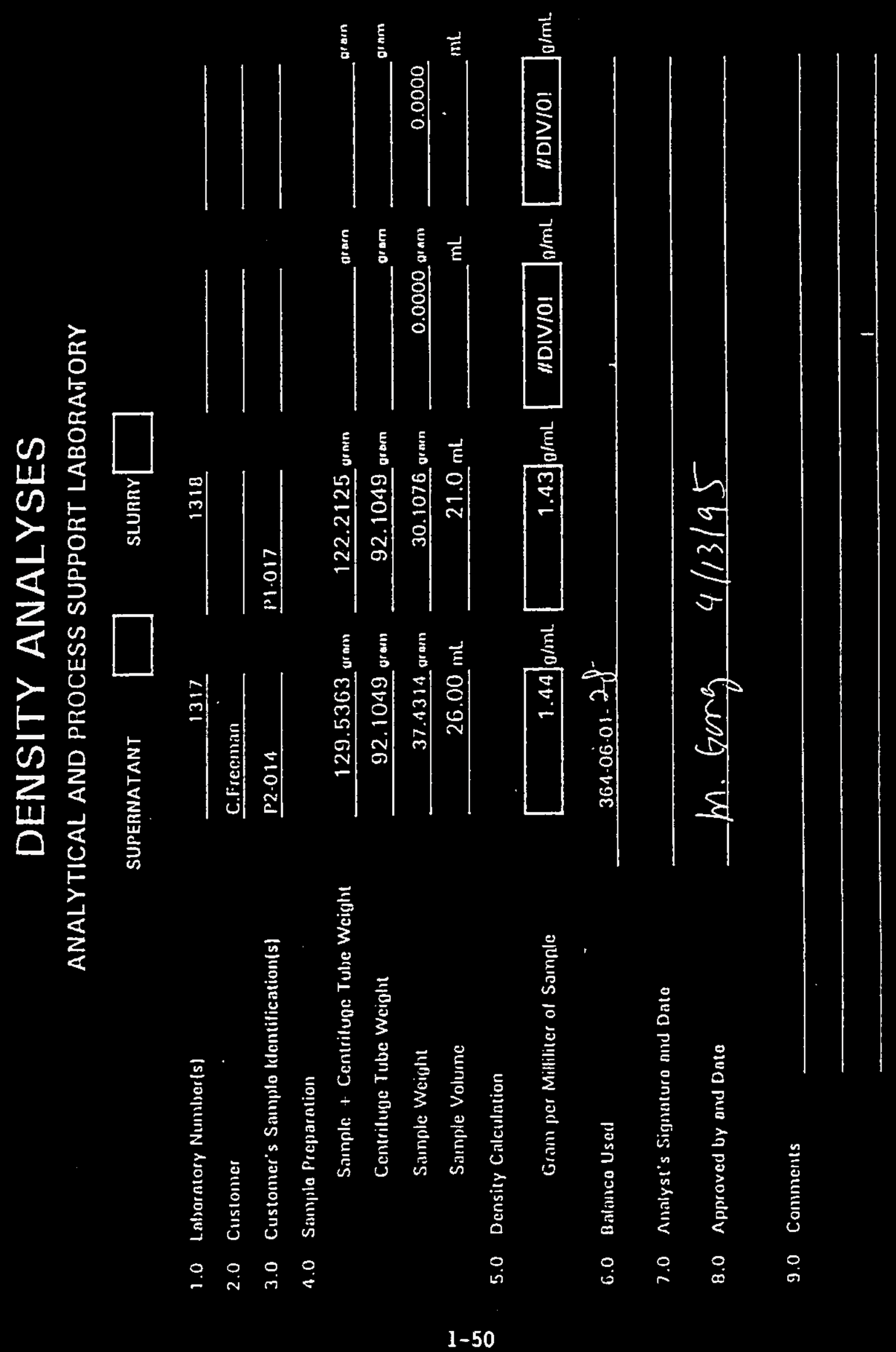




\section{WHC-SD-WH-VI-031 \\ Revision 0}

\section{ICP ANALYSIS ON FUSED SAMPLES}

Analytical and Procese Support Laboratory

1EP Anainie

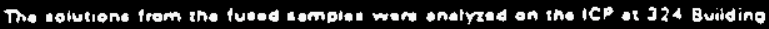

2.0 Lat No

3.0 Curtamer

4.0 Customert: 10

5.0 Dera File 10

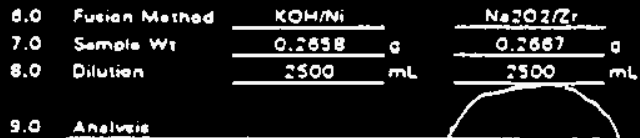

\begin{tabular}{|c|c|c|c|}
\hline Pont & $\begin{array}{c}w_{1} x \\
-0.005 \%\end{array}$ & & $\begin{array}{c}m x \\
-0.005 \%\end{array}$ \\
\hline At & $1.959 \%$ & . & $3.758 \%$ \\
\hline$B$ & $0.162 \%$ & & $0.166 \%$ \\
\hline 5. & $-0.003 \%$ & : & $-0.003 \%$ \\
\hline Go & $.0 .003 \%$ & & $-0.003 \%$ \\
\hline Bi & $.0 .056 \%$ & & $-0.056 \%$ \\
\hline e. & $2.734 \%$ & & $2.825 \%$ \\
\hline $\mathrm{cd}$ & $-0.006 \%$ & & $-0.006 \%$ \\
\hline s. & $-0.038 \%$ & 1 & $-0.037 \%$ \\
\hline $\mathrm{Ca}$ & .0 .0098 & & $-0.009 \%$ \\
\hline $\mathrm{Cr}_{\mathrm{r}}$ & $0.030 \%$ & & $0.028 \%$ \\
\hline $\mathrm{Cu}$ & $-0.006 \%$ & & $-0.006 \%$ \\
\hline Or & $-0.006 \%$ & & $-0.006 \%$ \\
\hline$\varepsilon_{4}$ & -0.0049 & & $-0.004 \%$ \\
\hline E. & $0.547 \%$ & & $0.553 \%$ \\
\hline$x$ & & ' & $0.418 \%$ \\
\hline Le & $-0.009 \%$ & $\vdots$ & $-0.009 \%$ \\
\hline Li & $-0.005 \%$ & 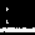 & $-0.005 \%$ \\
\hline $\mathrm{Mo}$ & $1.658 \%$ & : & $1.612 \%$ \\
\hline $\mathrm{Mn}$ & $0.005 \%$ & ; & $0.005 \%$ \\
\hline Mo & $0.091 \%$ & i & $0.087 \%$ \\
\hline No & $13.905 \%$ & ' & \\
\hline No & $-0.019 \%$ & & $0.023 \%$ \\
\hline $\mathrm{Ni}$ & & & $-0.019 \%$ \\
\hline$p$ & $0.290 \%$ & & $0.216 \%$ \\
\hline Po & $-0.075 \%$ & & $-0.075 \%$ \\
\hline 5 & $0.161 \%$ & & $0.126 \%$ \\
\hline si & $5.826 \%$ & & $20.501 \%$ \\
\hline sn & $-0.075 \%$ & & $.0 .075 \%$ \\
\hline 5 & $0.083 \%$ & & $0.090 \%$ \\
\hline T. & $-0.056 \%$ & & $-0.058 \%$ \\
\hline$\pi$ & $0.006 \%$ & & $0.005 \%$ \\
\hline$v$ & $.0 .009 \%$ & & -0.0098 \\
\hline$y$ & $-0.003 \%$ & & $.0 .003 \%$ \\
\hline $2 n$ & $0.186 \%$ & & $0.184 \%$ \\
\hline 24 & $.0 .009 \%$ & & \\
\hline
\end{tabular}

100 Sub-tolete $27.64 x+39.60 x$

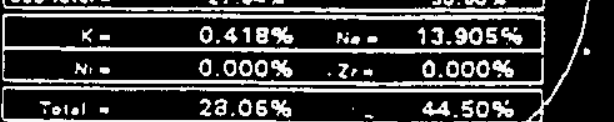
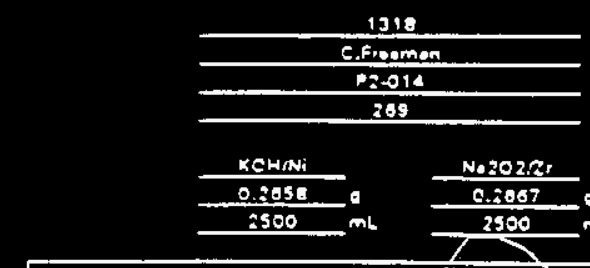

$\frac{0.2050}{2500} \mathrm{~mL} \frac{0.2067}{2500} \mathrm{~mL}$

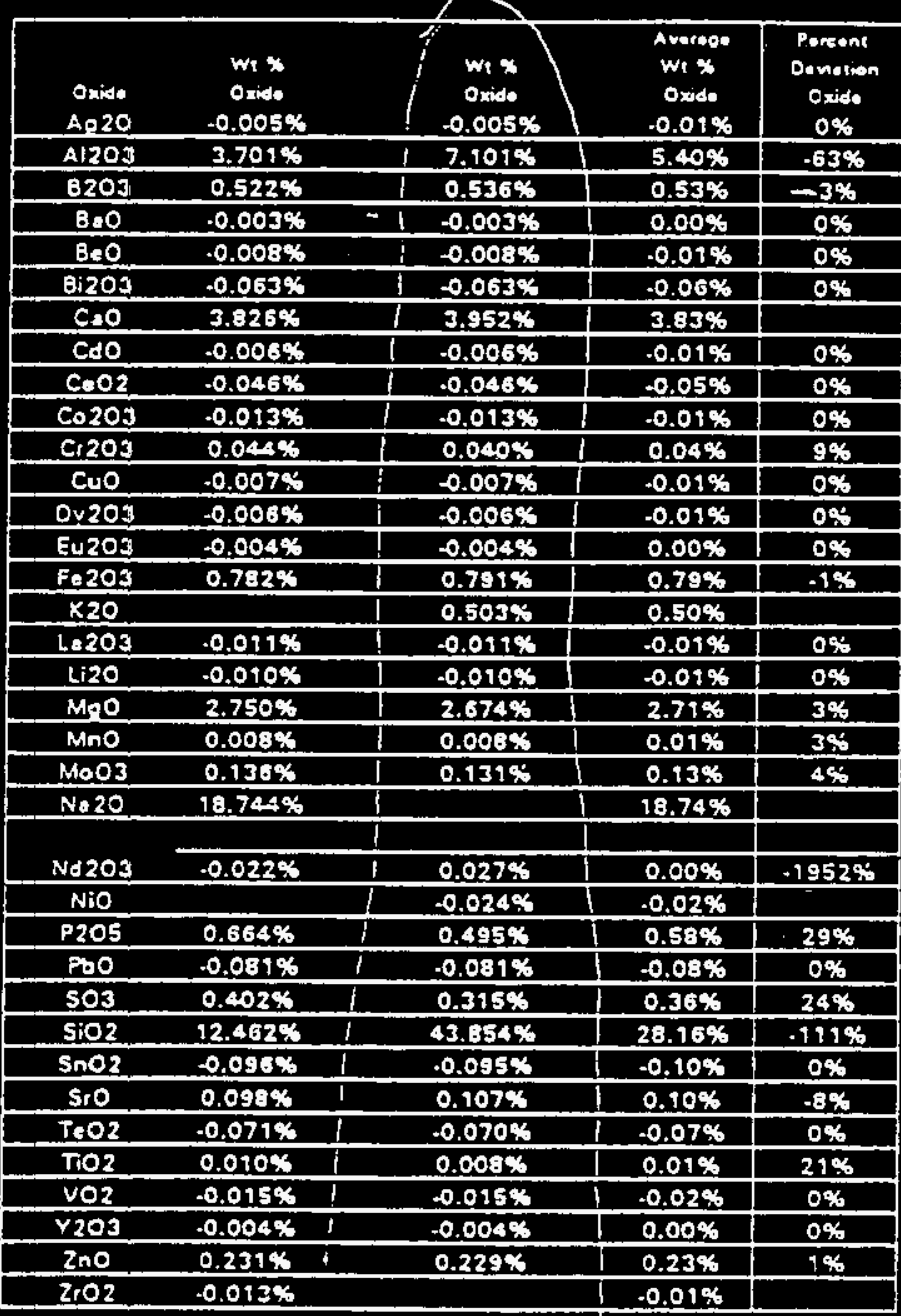

\begin{tabular}{|c|c|c|}
\hline Sub-10inel - & $4.3102 x$ & $60.772 x$ \\
\hline$k 20=$ & $0.503 \%$ & $18.744 \%$ \\
\hline
\end{tabular}

Nio $=0.000 \% \quad 2.02=0.000 \%$

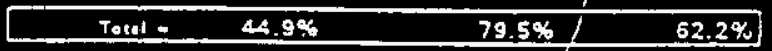

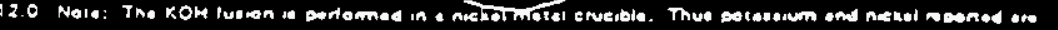

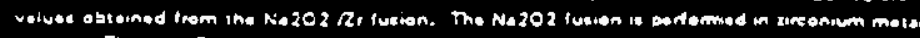

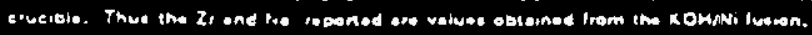

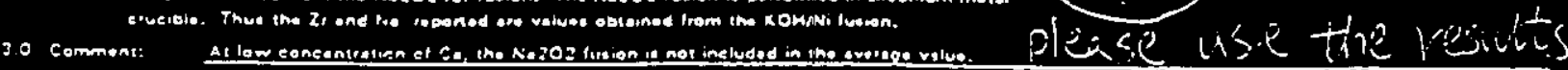

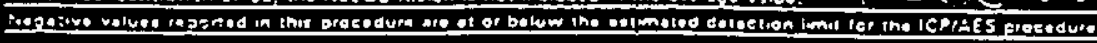

14.0 Celeviatec by end de:-

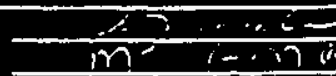

is o Aporave sy and date

$4 / 8: 1.1$

j) $1-7-i, 1$ 
WHC-SD-WM-VI-031

Revision 0

ICP ANALYSIS ON FUSED SAMPLES

Analytical and Process Support Laboratory

1.0 ICP Anolves

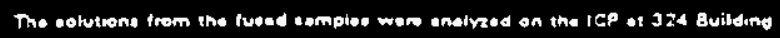

2.0 t.0 No

3.0 Customer

4.0 Cuctomatis ID

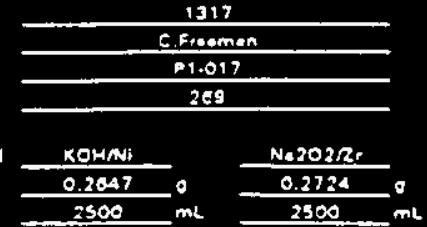

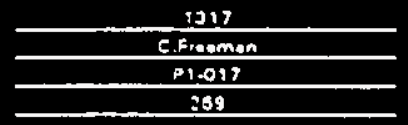

6.0 Fusion Mothod
7.0 Semple We

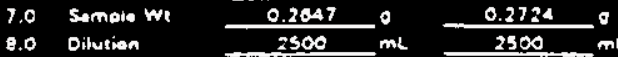

9.0
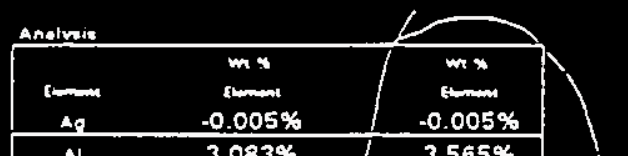

\begin{tabular}{|llll|}
\hline $\mathrm{Al}$ & $3.083 \%$ & $/$ & $3.565 \%$ \\
\hline $\mathrm{B}$ & $0.215 \%$ & $/$ & $0.240 \%$ \\
\hline
\end{tabular}

日 $\quad 0.215 \%$ / $0.240 \%$

\begin{tabular}{ll|l|}
\hline en & $-0.003 \%$ & $0.003 \%$ \\
\hline s. & $0.007 \%$ & $-0.003 \%$ \\
\hline
\end{tabular}

\begin{tabular}{llll}
\hline 0 & $0.057 \%$ & $-0.055 \%$ \\
\hline
\end{tabular}

\begin{tabular}{llll} 
C. & $4.197 \%$ & $4.374 \%$ \\
\hline C. & $0.009 \%$ & 1 & $-0.006 \%$ \\
\hline
\end{tabular}

\begin{tabular}{llll} 
co & $-0.038 \%$ & 1 & $-0.037 \%$ \\
\hline co & $-0.009 \%$ & 1 & $-0.009 \%$
\end{tabular}

c. $0.058 \%$ । $0.040 \%$

\begin{tabular}{llll}
$c_{\mu}$ & $-0.006 \%$ & $/$ & $-0.006 \%$ \\
\hline
\end{tabular}

\begin{tabular}{lll|l} 
or & $-0.006 \%$ & $0.006 \%$
\end{tabular}

\begin{tabular}{llll} 
Eu & $-0.004 \%$ & $!$ & $-0.004 \%$ \\
\hline Fo & $0.546 \%$ & $!$ & $0.603 \%$
\end{tabular}

\begin{tabular}{|c|c|c|}
\hline R. & $0.546 \%$ & $0.603 \%$ \\
\hline$K$ & & $0.659 \%$ \\
\hline L. & $-0.009 \%$ & $-0.009 \%$ \\
\hline
\end{tabular}

\begin{tabular}{ll|l} 
L & $-0.009 \%$ & $-0.009 \%$ \\
\hline$L_{i}$ & $0.012 \%$ & $0.005 \%$ \\
\hline$M_{q}$ & $2.421 \%$ & $2.439 \%$
\end{tabular}

\begin{tabular}{|c|c|c|}
\hline$M_{p}$ & $2.421 \%$ & $2.439 \%$ \\
\hline$M_{n}$ & $0.009 \%$ & $0.008 \%$ \\
\hline$M_{0}$ & $0.099 \%$ & $0.101 \%$ \\
\hline
\end{tabular}

No $19.108 \%$

(2)

\begin{tabular}{|c|c|c|}
\hline \multirow[b]{2}{*}{ Nd } & \multicolumn{2}{|c|}{1} \\
\hline & $-0.019 \%$ & $0.045 \%$ \\
\hline $\mathrm{Ni}$ & & $-0.018 \%$ \\
\hline
\end{tabular}

\begin{tabular}{|c|c|c|}
\hline$?$ & $0.325 \%$ & $0.519 \%$ \\
\hline$P_{b}$ & $-0.076 \%$ & $.0 .073 \%$ \\
\hline$s$ & $0.195 \%$ & $0.193 \%$ \\
\hline si & $10.994 \%$ & $11.742 \%$ \\
\hline Sn & $0.076 \%$ & $-0.073 \%$ \\
\hline St & $0.116 \%$ & $0.127 \%$ \\
\hline$T_{0}$ & $-0.057 \%$ & $.0 .055 \%$ \\
\hline $\mathrm{T}$ & $0.005 \%$ & $0.006 \%$ \\
\hline$v$ & $-0.009 \%$ & $.0 .009 \%$ \\
\hline$y$ & $0.008 \%$ & $-0.003 \%$ \\
\hline $2 n$ & $0.162 \%$ & $0.263 \%$ \\
\hline 2 & $0.019 \%$ & \\
\hline
\end{tabular}

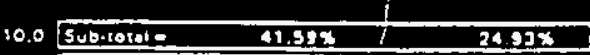

\begin{tabular}{|cc|cc|}
\hline$K_{0}$ & $0.659 \%$ & $N_{0}=$ & $19.108 \%$ \\
\hline $\mathrm{Ni}=$ & $0.000 \%$ & $z_{r}=$ & $0.019 \%$ \\
\hline
\end{tabular}

11,0 Tolol - $42.25 \%$ i $44.06 \%$

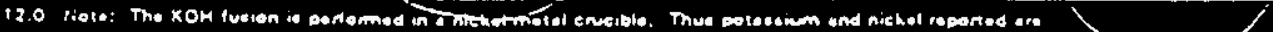

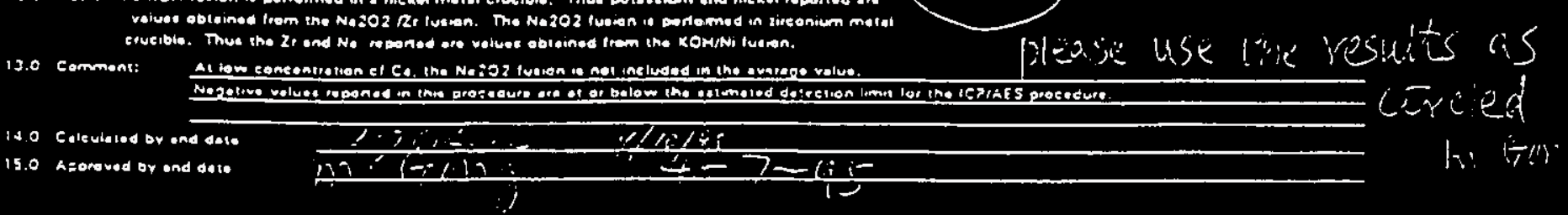

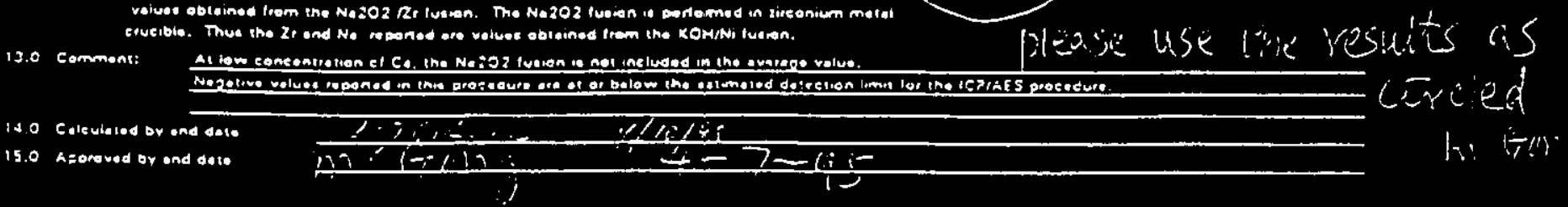

$\frac{K_{0 H} / N_{i}}{0.2547} \cdot \frac{N 4202 R 6}{\frac{0.2724}{2500}} \cdot \mathrm{mL}$

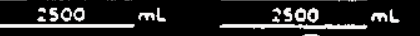

\begin{tabular}{|c|c|c|c|c|}
\hline $\begin{array}{l}\text { Oxido } \\
\text { Ar } 20\end{array}$ & $\begin{array}{c}\text { Wi } \% \\
\text { oxids } \\
-0.005 \%\end{array}$ & $\begin{array}{c}W \% \\
\text { Oride } \\
.0 .005 \%\end{array}$ & $\begin{array}{c}\text { Avorose } \\
\text { We } \% \\
\text { Oxide } \\
.0 .01 \%\end{array}$ & $\begin{array}{l}\text { Porcent } \\
\text { Doviation } \\
\text { Oxide } \\
3 \%\end{array}$ \\
\hline $\mathrm{A} 1203$ & $5.826 \%$ & $6.737 \%$ & $6.28 \%$ & $-15 \%$ \\
\hline 8203 & $0.692 \%$ & $0.773 \%$ & $0.73 \%$ & $-11 \%$ \\
\hline 800 & $-0.003 \%$ & $0.003 \%$ & $0.00 \%$ & $-12221 \%$ \\
\hline $\mathrm{BeO}$ & $0.020 \%$ & $-0.008 \%$ & $0.01 \%$ & $438 \%$ \\
\hline
\end{tabular}

\begin{tabular}{ll|lll|l}
$\mathrm{Bi} 203$ & $-0.063 \%$ & $-0.061 \%$ & $-0.06 \%$ & $3 \%$
\end{tabular}

\begin{tabular}{ll|l|l|}
$\mathrm{CaO}$ & $5.872 \%$ & $5.119 \%$ & $5.87 \%$ \\
\hline
\end{tabular}

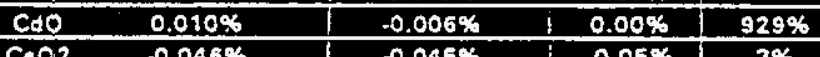

\begin{tabular}{ll|l|l|l|}
$\mathrm{Ce} 02$ & $-0.046 \%$ & $-0.045 \%$ & $-0.05 \%$ & $3 \%$ \\
\hline
\end{tabular}

\begin{tabular}{ll|l|l|l|}
0.0203 & $0.013 \%$ & $-0.013 \%$ & $-0.01 \%$ & $3 \%$
\end{tabular}

\begin{tabular}{ll|l|l|l|}
$\mathrm{Cr} 203$ & $0.085 \%$ & $0.059 \%$ & $0.07 \%$ & $37 \%$ \\
\hline $\mathrm{CuO}$ & $-0.007 \%$ & $0.007 \%$ & $0.01 \%$ & $3 \%$
\end{tabular}

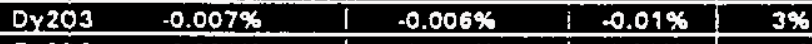

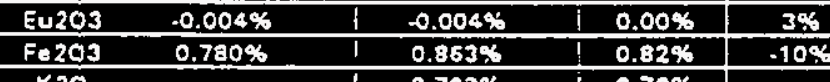

\begin{tabular}{ll|l|l|l|}
$\operatorname{lo} 203$ & $-0.011 \%$ & $0.0 .011 \%$ & $0.01 \%$ & $3 \%$
\end{tabular}

\begin{tabular}{ll|l|l|l} 
Li20 & $0.025 \%$ & $0.010 \%$ & $0.02 \%$ & $85 \%$
\end{tabular}

\begin{tabular}{l|l|l|l|l} 
MoO & $4.016 \%$ & $4.045 \%$ & $4.03 \%$ & $.1 \%$ \\
\hline MnO & $0.015 \%$ & $0.013 \%$ & $0.01 \%$ & $12 \%$ \\
\hline
\end{tabular}

\begin{tabular}{ll|l|l|l}
$\mathrm{MOO}$ & $0.148 \%$ & $0.152 \%$ & $0.15 \%$ & $.3 \%$ \\
\hline
\end{tabular}

$\mathrm{No20} \quad 25.758 \%$

\begin{tabular}{|c|c|c|c|c|}
\hline $\mathrm{Nd} 203$ & $.0 .022 \%$ & $0.052 \%$ & $0.02 \%$ & $.492 \%$ \\
\hline
\end{tabular}

\begin{tabular}{ll|l|l|l|} 
NiO & & $0.023 \%$ & $\mid 0.02 \%$ & \\
\hline P205 & $0.745 \%$ & $1.189 \%$ & $0.97 \%$ & $46 \%$
\end{tabular}

\begin{tabular}{ll|l|l|l|}
$\mathrm{Pag}$ & $-0.081 \%$ & $-0.079 \%$ & $-0.08 \%$ & $3 \%$ \\
\hline
\end{tabular}

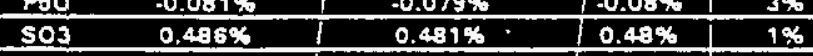

\begin{tabular}{ll|l|l|l} 
sio2 $23.518 \%$ & $25.117 \%$ & $24.32 \%$ & $.7 \%$ \\
\hline
\end{tabular}

\begin{tabular}{ll|l|l|l}
$\sin 02-0.096 \%$ & $\mid$ & $0.093 \%$ & $-0.09 \%$ & $3 \%$
\end{tabular}

\begin{tabular}{ll|l|l|l|}
$5 r 0$ & $0.138 \%$ & $0.150 \%$ & $0.14 \%$ & $.9 \%$ \\
\hline
\end{tabular}

\begin{tabular}{|c|c|c|c|c|c|}
\hline ToOl & $-0.071 \%$ & $-0.069 \%$ & $i$ & $-0.07 \%$ & $3 \%$ \\
\hline
\end{tabular}

\begin{tabular}{lll|l|l|l} 
TIO2 & $0.009 \%$ & $\mid$ & $0.010 \%$ & $0.01 \%$ & $-8 \%$ \\
\hline
\end{tabular}

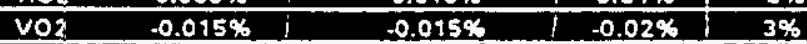

\begin{tabular}{lll|l|l|l|l|}
\hline 203 & $0.010 \%$ & $0.003 \%$ & $0.00 \%$ & $432 \%$
\end{tabular}

\begin{tabular}{lll|l|l|}
\hline $\mathrm{nO}$ & $0.202 \%$ & $0.00 \%$ & $432 \%$ \\
\hline & $0.025 \%$ & $0.328 \%$ & $0.26 \%$ & $.48 \%$ \\
\hline
\end{tabular}

$\begin{array}{lll}2102 & 0.025 \%: 0.03 \%\end{array}$

\begin{tabular}{|c|c|c|c|}
\hline Subriatel. & $68.379 x$ & 46.3965 & \\
\hline $\mathbf{1} 20=$ & $0.793 \%$ & $1 N+20=25.758 \%$ & $!$ \\
\hline NiO - & $0.000 \%$ & $0.025 \%$ & \\
\hline
\end{tabular}

NiO - $0.000 \% \frac{12102=0.025 \%}{2}$

$72.7 \% / 70.9 \%$ 
$\varepsilon \mathcal{E}-\mathrm{I}$

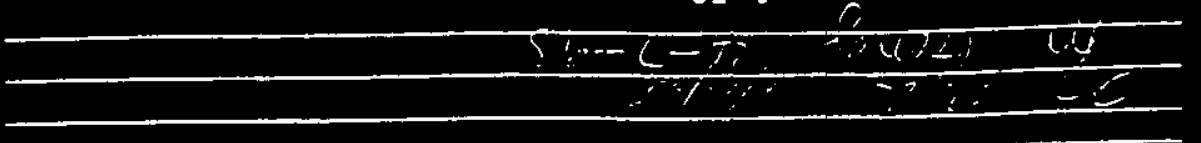

JAP DU AQ POAOICEY 021

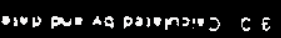

$$
\text { nowwos } c \text {; }
$$

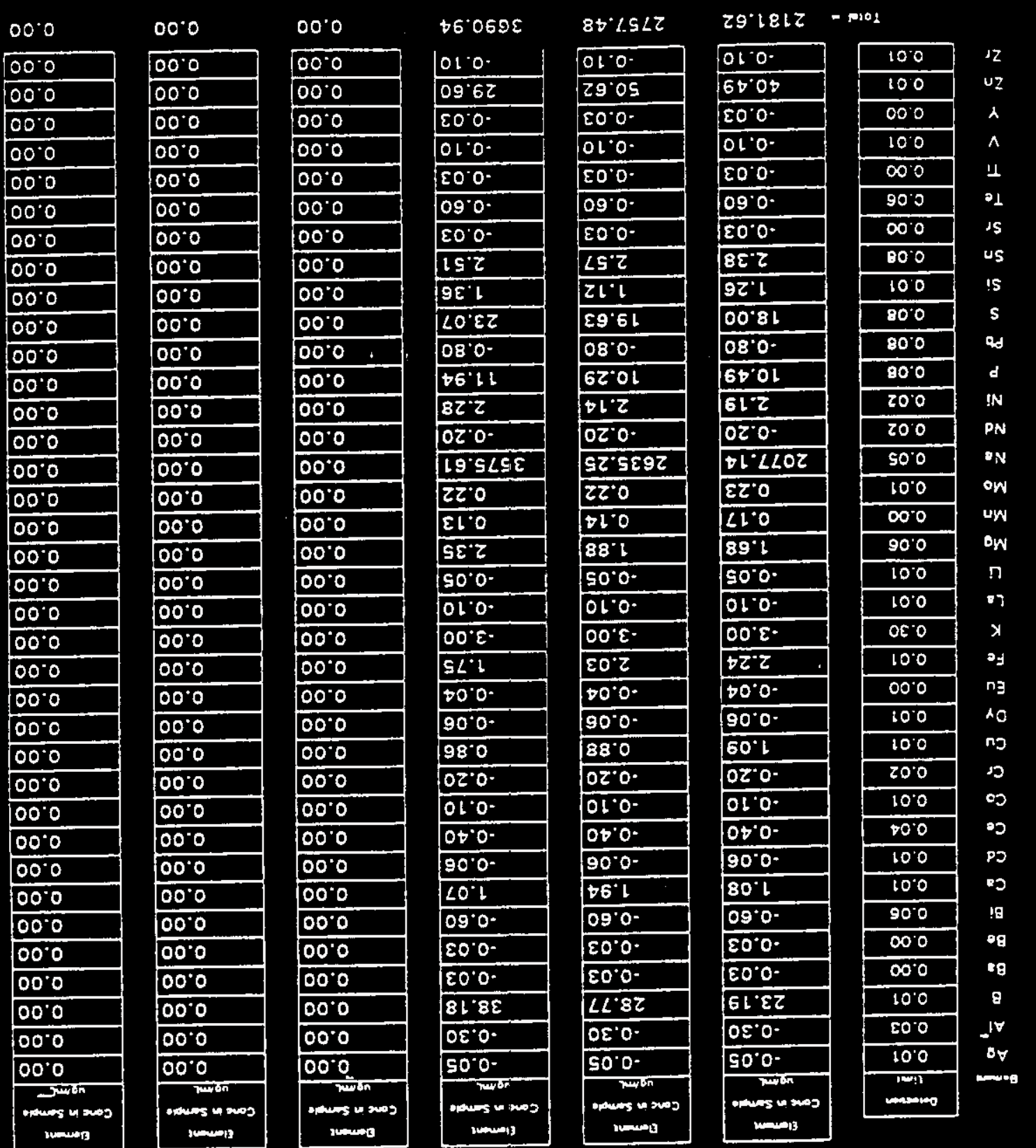

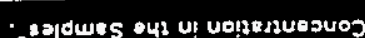

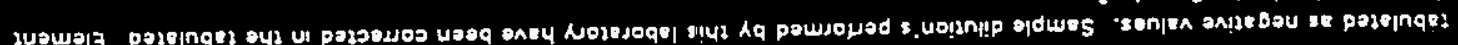
(1)

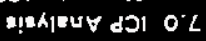

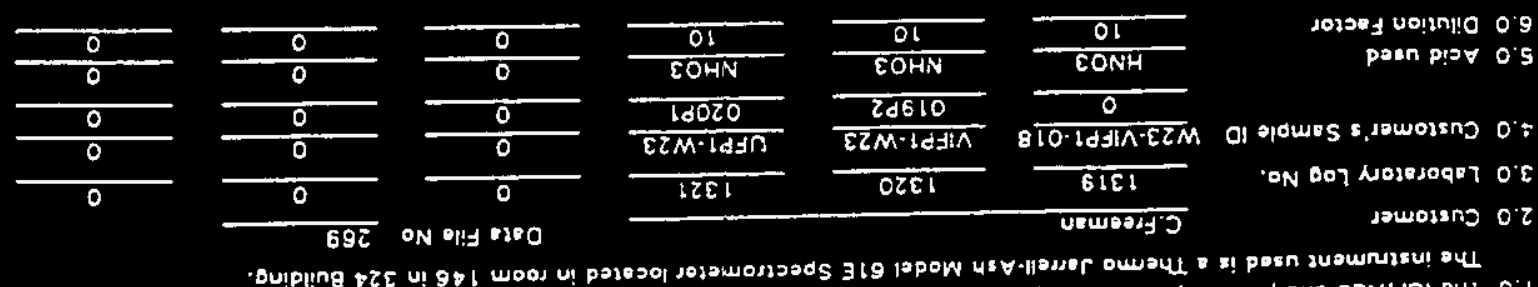

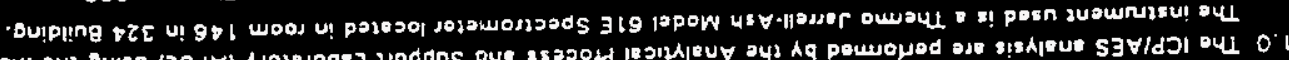

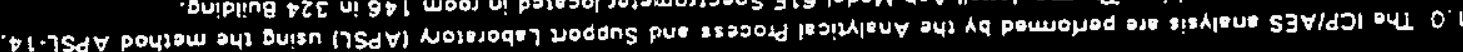

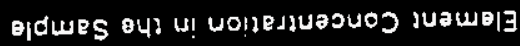

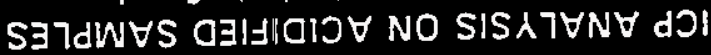

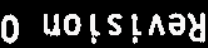

IEO-IA-WH-OS-JHM 


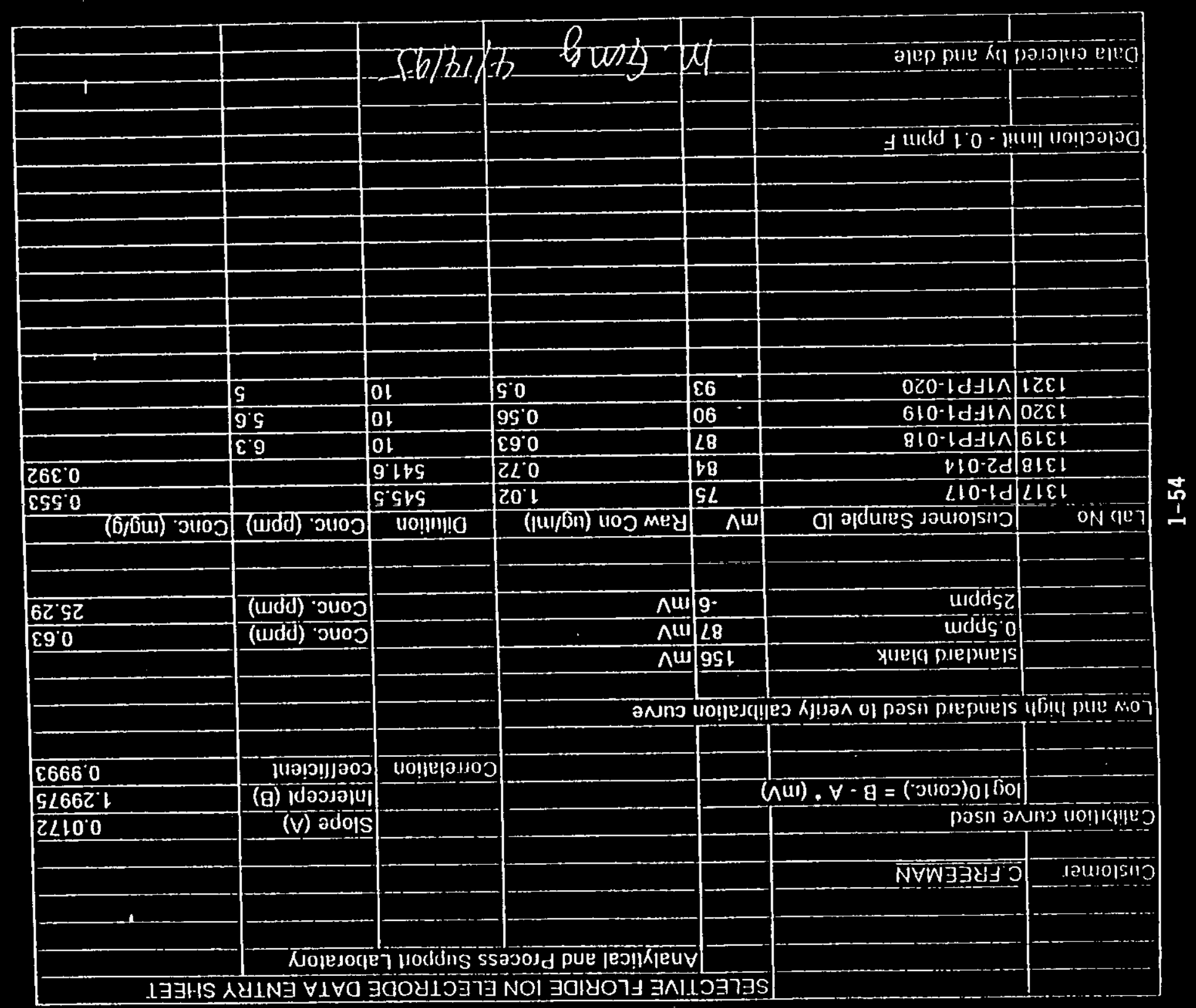



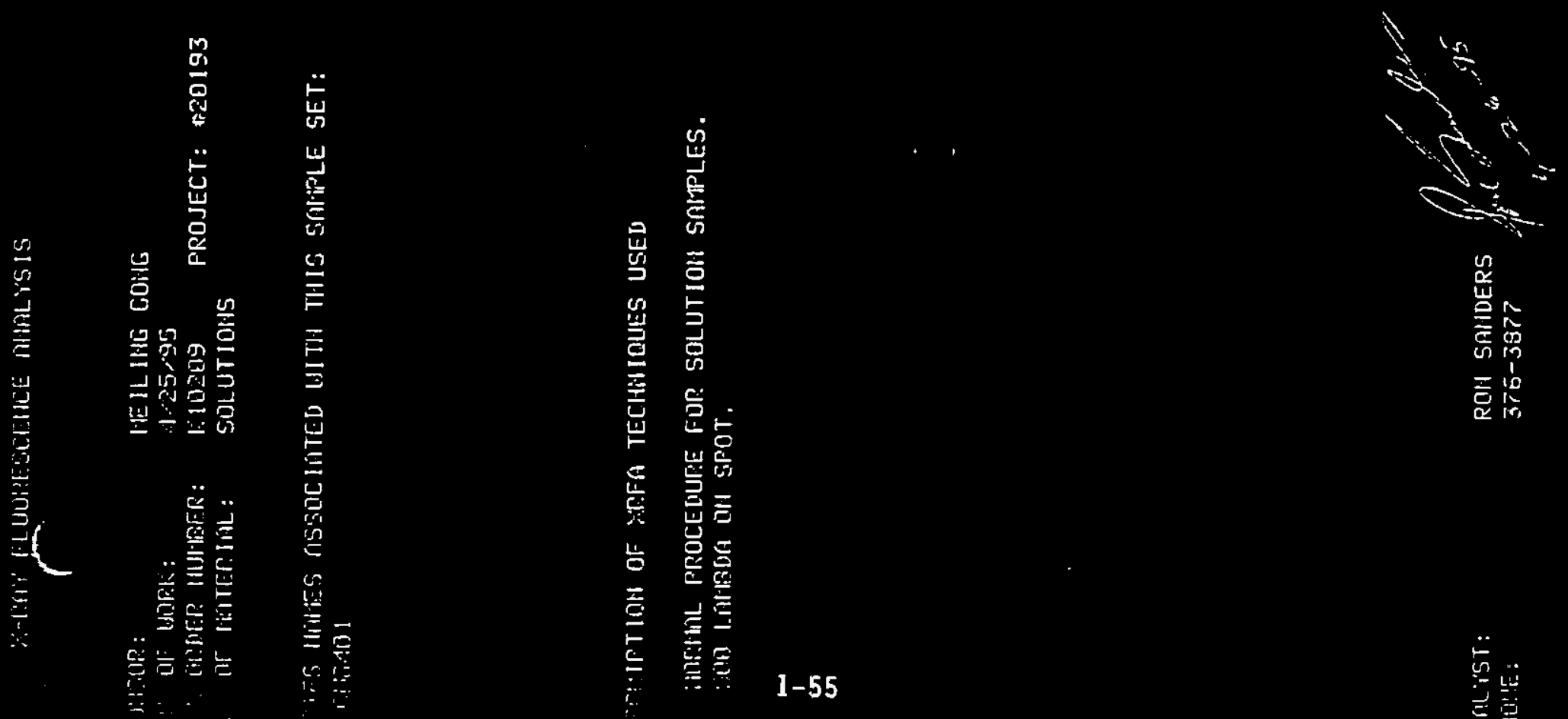


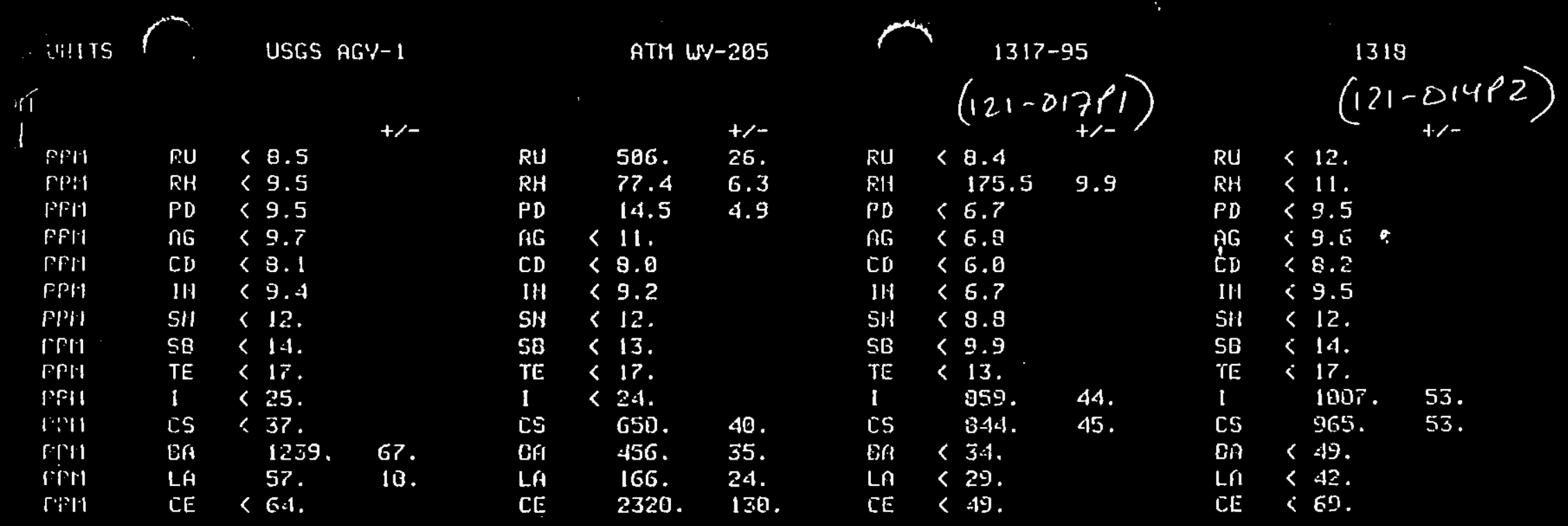




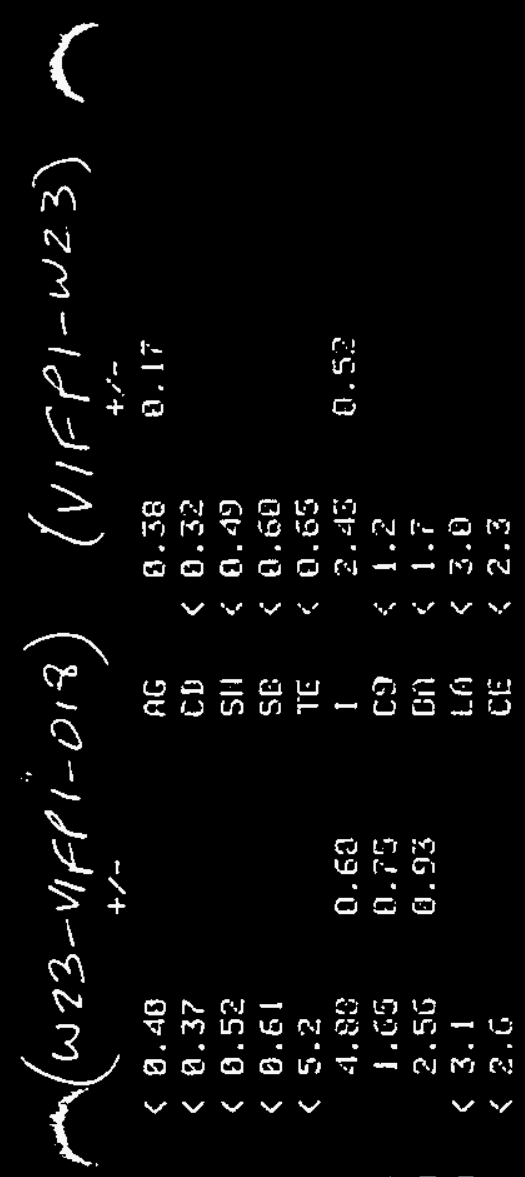

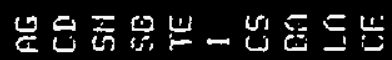

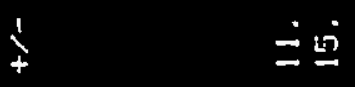

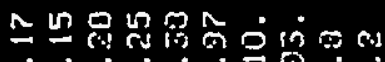

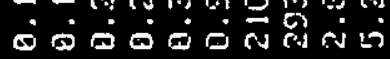
レレレレレン $レ \vee$

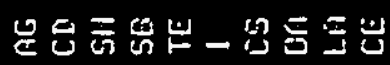

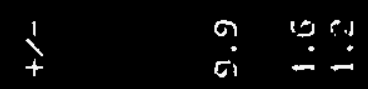

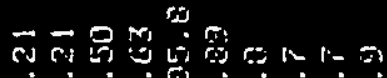

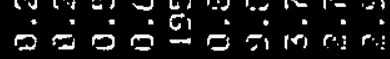
レレレレ v $\because \mho$

(

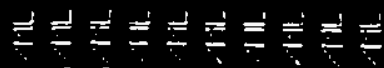

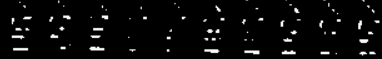


(

$+\quad$ is

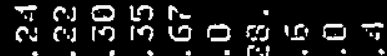

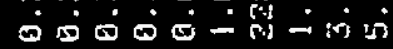

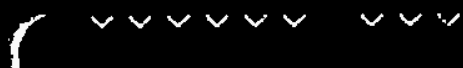

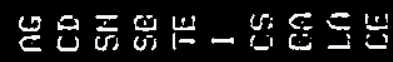

1
+

ง ف बه レレレレ レ レレV

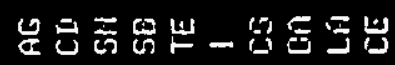

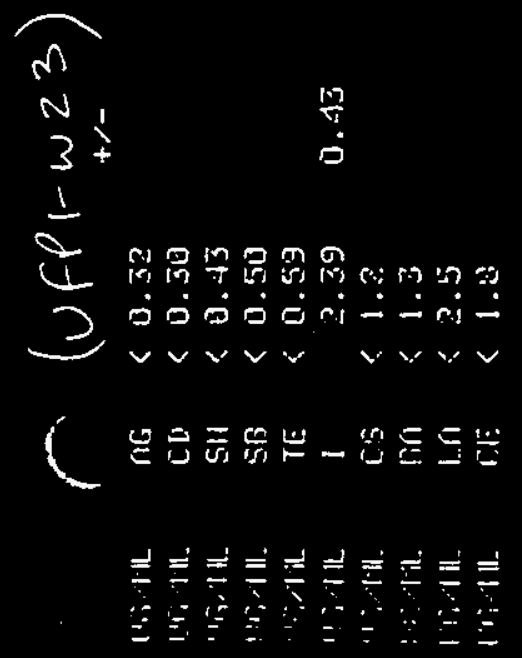




\section{WHC-SD-WM-VI-031}

Revision 0

ARVANCED INORGANIC AVALYSIS GROUP

ATONHC AND MCLECULAR CHEMISTRY SECTION, CHEMICAL SCIENCES DEPAATMENT BATEILLE, FACIFIC NORTHWEST LASORATORIES, RICHLAND, WA

pH. Conductivity. Titration, Ion Chromatograph, and Carbon Analyzer RESULTS

Name: Long for Chavie Frecman

Work Package \# K10209

Report Date: $x-1+95$

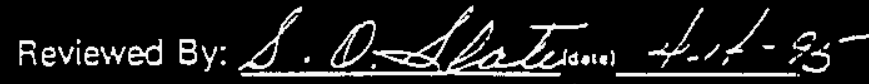
Analysis Performed By: Karen Achellu.

\section{Report Date:}

Concentration in $\mathrm{mg} / \mathrm{L}$

or as specified

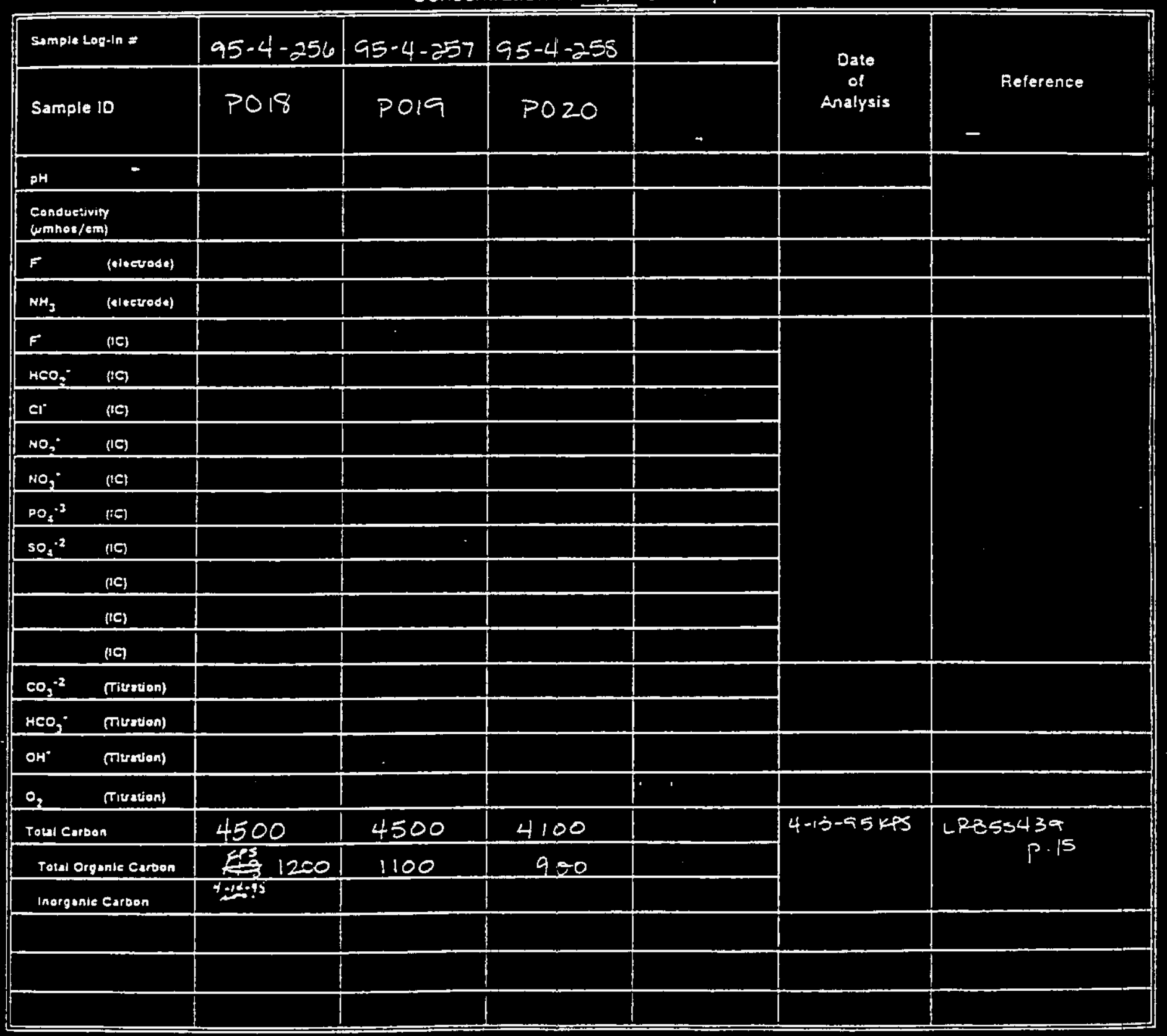

Anal; tical Equipment:
口 pit Meter, Orion Hodel 501 (S/N 51137)
C Conductivicy Bridge, Yeliow Spring, inc. Model 32 (S/N 2404)
口 Ion Chromaiegraph (iC). Dionex Series 4000i (Vv337 427$)$

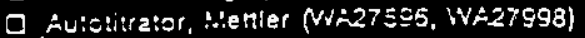

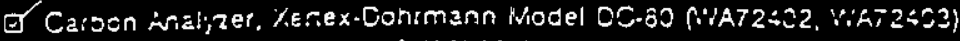

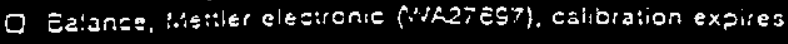




\section{ION CHROMATOGRAPHY \\ Analytical and Process Support Laboratory}

Lab No

Customer

Customers's ID

Woter Leach OF

Sample OF

IC values

(ug/mL)

Fluaride

Chloride

Nitrite

Nitrate

Phosphate $\quad 2.435$

Sulfats $\quad 8.663$

Oxalote

Formate

INO

9.347

$-$

Lab No

Customer

Customers's i0

Watar Leach dilution

Second dilution

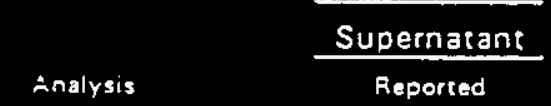

$\begin{array}{lc}\text { Analysis } & \begin{array}{c}\text { IC values } \\ \text { (ug/mL) } \\ \text { Fivoride }\end{array} \\ \text { Chloride } & \\ \text { Nitrite } & \\ \text { Nitrate } & \\ \text { Phosphate } & 1.568 \\ \text { Sulfate } & 10.056 \\ \text { Oxolate } & \\ \text { Formote } & \end{array}$

volues

ugiml

\begin{tabular}{|r|}
\hline \multicolumn{1}{l}{ ug/mL } \\
\hline \#VALUE! \\
\hline-2.50 \\
\hline-5.00 \\
\hline-5.00 \\
\hline 7.84 \\
\hline 50.28 \\
\hline .5 .00 \\
\hline-2.50 \\
\hline
\end{tabular}

ug/g

\begin{tabular}{|r|}
\hline \#VALUE! \\
\hline 2039.33 \\
\hline-218.18 \\
\hline-218.18 \\
\hline 531.27 \\
\hline 1890.09 \\
\hline-218.18 \\
\hline-109.09 \\
\hline
\end{tabular}

$$
\begin{gathered}
\frac{1318.95}{\text { Freemen }} \\
\hline \frac{121.014 P 2}{108.33} \\
\hline 2 \\
\hline \text { Slurry } \\
\text { Reported }
\end{gathered}
$$

IC values

$\frac{\frac{1320.95}{\text { Freeman }}}{\frac{\text { W23.019P2 }}{1}}$

Dionex Model 3000X (ug $/ \mathrm{mb}$

IND

9.771

cl

No3

PO4

504

$(\mathrm{COOH}) 2$

(HCOO)
$\mathrm{NO} 2$$$
\begin{array}{|}
\hline \\
\hline \\
\hline
\end{array}
$$

values

\begin{tabular}{|r|}
\hline \multicolumn{1}{|l}{ Ug/9 } \\
\hline \#VALUE! \\
\hline 2116.98 \\
\hline .216 .66 \\
\hline .216 .66 \\
\hline 466.04 \\
\hline 2349.46 \\
\hline .216 .66 \\
\hline-108.33 \\
\hline
\end{tabular}

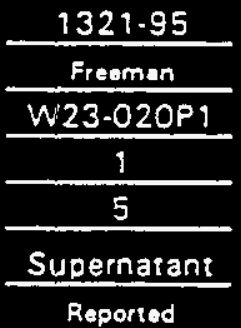

iC values

values

$(u g / m L) \quad u g / m L$

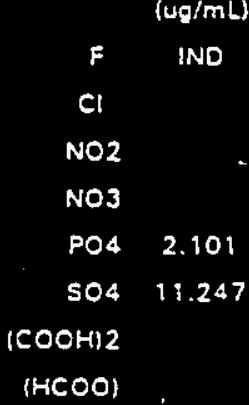

\#VALUEI

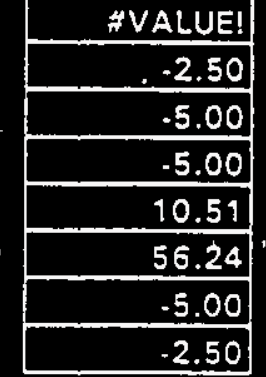

$\frac{\frac{1319.95}{\text { Froeman }}}{\frac{\text { W23-018P-1-2 }}{1}}$

Supernatant

Reported

IC volues

values

(ug/mL)

ug/mL

IND

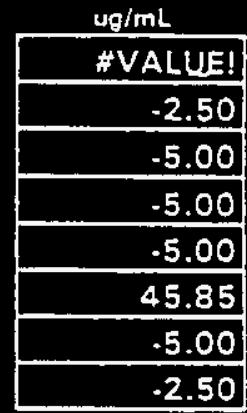

(HCOO)

9.109

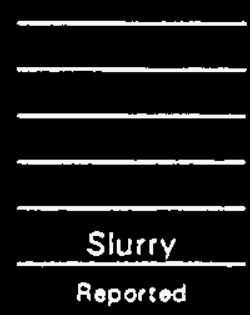

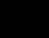

cl

IC values

values

\begin{tabular}{|c|c|}
\hline Fivoride $=0.5 \mathrm{ug} / \mathrm{mL}$ & Phosphare $=1.0 \mathrm{ug} / \mathrm{mL}$ \\
\hline Chlcride $=0.5 \mathrm{ug} / \mathrm{mL}$ & Sulfate $=1.0$ ugi $/ \mathrm{mL}$ \\
\hline Formate $=0.5 \mathrm{ug} / \mathrm{mL}$ & Oxalace $=1.0 \mathrm{ug} / \mathrm{mL}$ \\
\hline Nitrite $=1.0 \mathrm{ug} / \mathrm{mL}$ & \\
\hline Nitrate $=1.0$ ugiml & \\
\hline
\end{tabular}

(ug/mL)

ug/mL

\begin{tabular}{|r|}
\hline 0.00 \\
\hline 0.00 \\
\hline 0.00 \\
\hline 0.00 \\
\hline 0.00 \\
\hline 0.00 \\
\hline 0.00 \\
\hline 0.00 \\
\hline
\end{tabular}

Analyst Signature and Date

Reviewed by and Date

Comments Estimated detection limit of the anions analvsed in the diluted solution. 
WHC-SD-WM-VI-031

Revision 0

Off Gas Results 


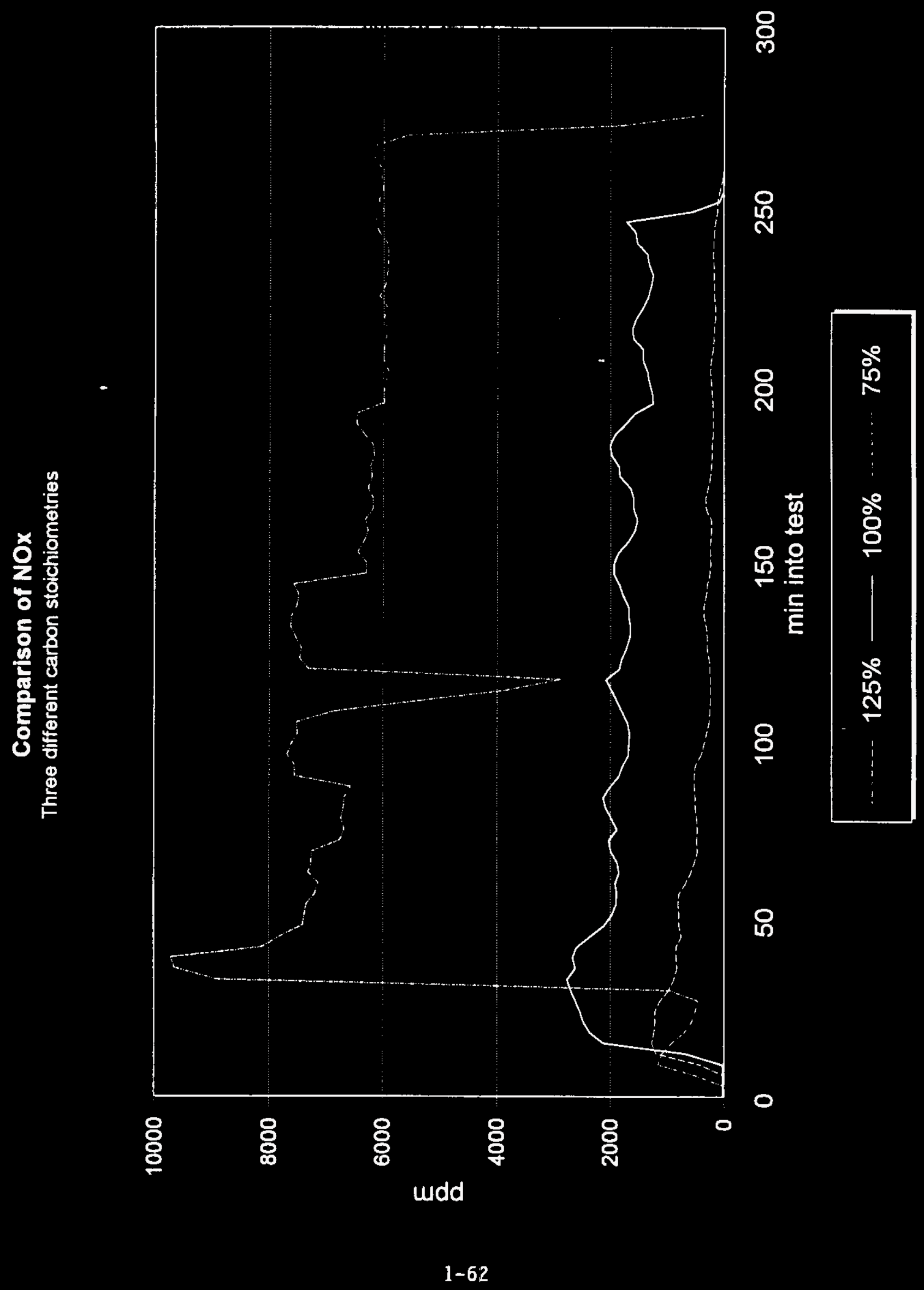


Revision 0

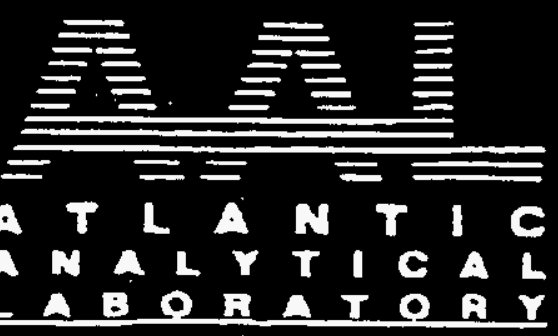

\section{Gas Analysis Report}

First Environment. 90 Riverdale Road Riverdale, N 07457 201-616-9700 (Fax 201-616-1930) Aln.: Mr I arry.Bnit Sample ID 1: Chemical Process Gas Sample ID 2: Project \# VEG001, Procedyne Comments: 5, 15L Tedlar bags containing brownish gas.
AAL Number: 4607 Received On: 03/10/95

Report Date: 03/14/95

PO Number: VEG001

Sampled On: $3 / 8-3 / 10 / 95$

Sample Point: see COC

\section{Date Taken/Samnle Identification}

3/8/95, 1500h, Bag \#1

$3 / 9 / 95,1135 \mathrm{~h}, \mathrm{Bag} \# 2$

$3 / 9 / 95,1600 \mathrm{~h}, \mathrm{Bag} \# 3$

$3 / 10 / 95,1305 h$, Bag $\$ 4$

$3 / 10 / 95,1600 \mathrm{~h}, \mathrm{Bag}$ if
Nitrogen 1 Nitrous Oride

\% $\mathrm{F} / \mathrm{V}$ Domp/r

$\begin{array}{ll}94.6 & 890 \\ 93.8 & 750 \\ 92.2 & 1,680 \\ 96.1 & 1,980 \\ 95.5 & 2,170\end{array}$

Note 1: Nitrogen $\left(N_{2}\right)$ Detection Limit $=0.0015$

Note 2: Nitcous Oxide $\left(\mathrm{N}_{2} \mathrm{O}\right)$ Detection Limit $=5 \mathrm{gpm}$ v/v

nd = indientes the concentration is less than the report deccetion limic. - - test got perfomed. $\%$ = parts per bundred

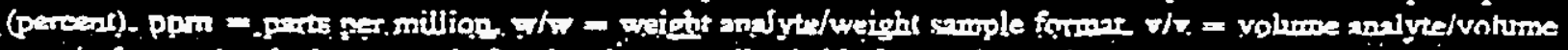
somplo form (equicylent to mole frection for nowmilized, ideal gas uixtares).

\section{Comments:}

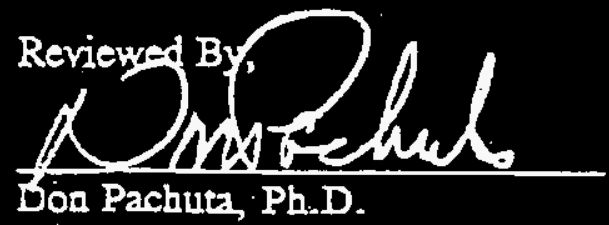

Atrachments:

-Chain of Cuscody

Addendom:

Chromatograrns on-file

-Notebook data on-tile 


\section{( ENVHRSTMENT}

May 8, 1995

Mr. Jim Care

Vectra Government Services, Inc.

2939 Richardson Road

Richland, Washington 99352

Ro: Emission Tests - Particulate Sampling Results

Procedyne Facility

Dear Jim,

The particulate analyses have been completed for the samples collected at the Procedyne facility. The results are summarized below:

$\begin{array}{llc}\text { Run No. } & \text { Sample I.D. } & \text { Particulate Concentration, mg/ } \\ 1 & \text { P-1 } & 0.146 \\ 2 & \text { P-2 } & 0.141 \\ 3 & \text { P-3 } & 0.143 \\ 4 & \text { P-4 } & 0.125 \\ 5 & \text { P-5 } & 0.113\end{array}$

These results should be the final data required from the emission testing completed at the Procedyne facility. Please check your records to ensure that you have received all the necessary data.

If you have any questions, please do not hesitate to call، .

Very truly yours,

FIRST ENVIRONMENT, INC.

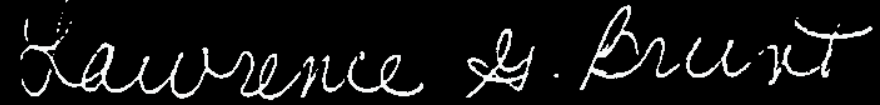

Lawrence G. Brunt, P.E.

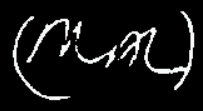

Manager - Riverdale Office

cc: $\quad$ R. Biasca 
ZENON

Enviranmental

Laboratories

Hegronas

Laboralorics:

Brition Columbias

Onisiro

Ouobee

\begin{abstract}
5555 North Service Foad Tel 905 332978
\end{abstract}
Burlington Ontario

Fax 9053329163

Canada LIL SH7

\title{
Certificate of Analysis
}

\section{CLIENT INFORMATION}

Attention: L.Brunt

Client Name: First Environnent Inc.

Project: VEG001

Project Desc: Vectri Govemment Corp.

Address: $\quad 90$ Riverdale Road

Riverdale, New Jersey

NJ 07457

Fax Number: 201-616-1930

Phone Number: 201-616-9700
ZENON

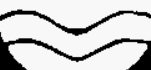

\section{LABORATORY WNORMATION}

Contact:

Yvonne Bond/Ron MeLeod

Project: AN950257

Date Received: $\quad 95 / 03 / 15$

Date Reported: 95/04/24

Submission Na.: $\quad$ \$C0317

Sample No.:

008169-008174

\section{NOTES:}

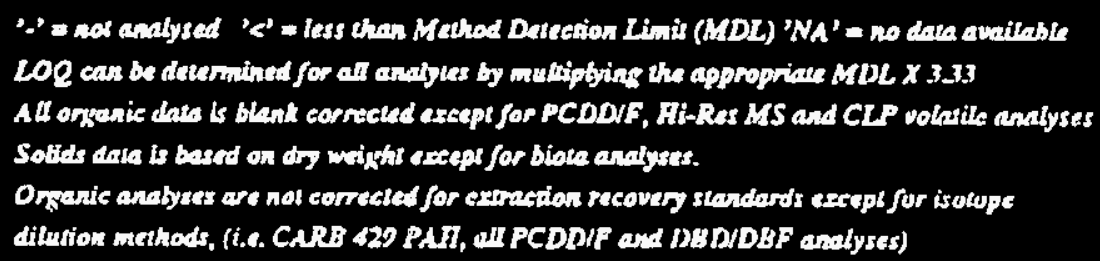

Methods used by Zenon are based upon those found in 'Stundard Mechods for the Eramination of Water and Wastewater", Seventeenth Elition. Other methods are based on the principles of MISA or EPA methodologics.

All wot recorded herein hus heen dune in decordanee wish normal professional standards using accepted resting methodologics, quality assurance and quality contol proxedures except where otherwise agreed to by the clienl and resting exmpany in writing. Any and alt use of these test resules shall be limitod to the actual cost of the pertinent analysis done. There is no olher warranty expressed or implied. Your samples will be retained it Zenon for a period of three weeks from recsipe of data or as per contraet

\section{COMMENTS:}




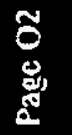

WHC-SD-WM-VI-031

Revision 0

erc

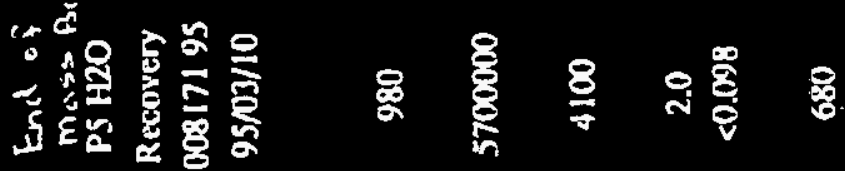

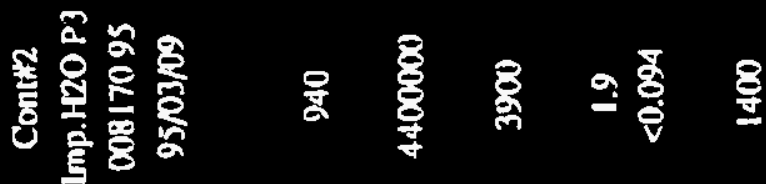

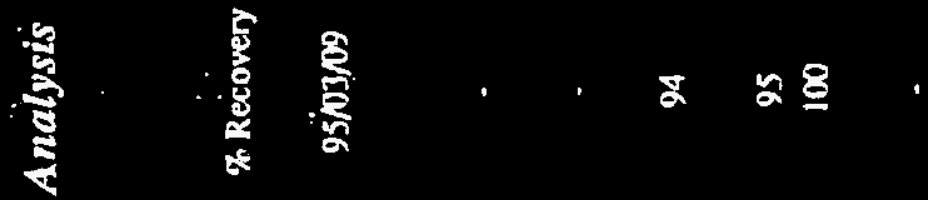

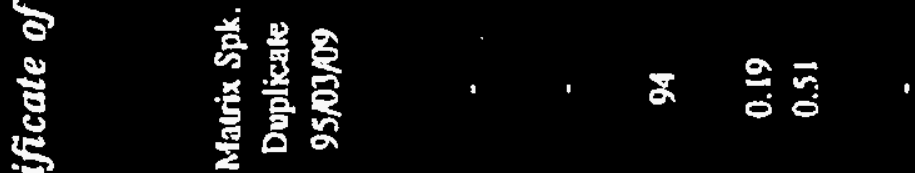

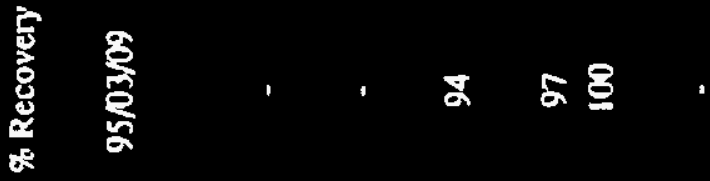

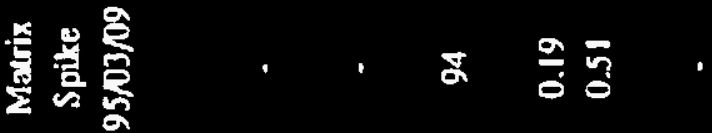

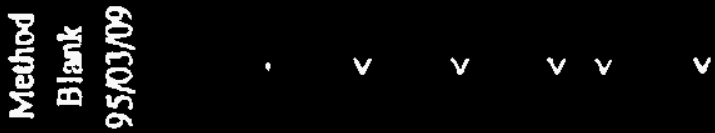

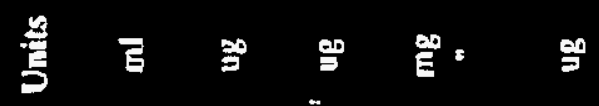

客害害官

총

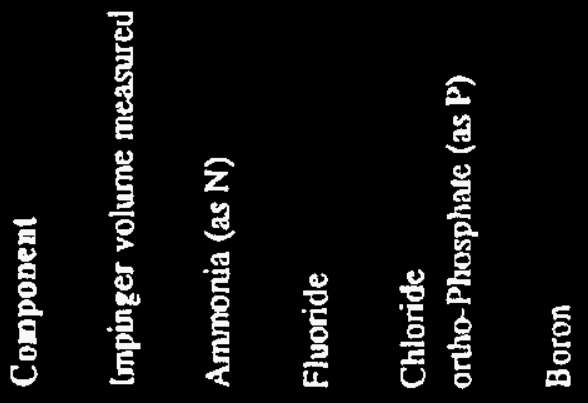




\begin{tabular}{|c|c|c|c|c|c|c|c|}
\hline & & p5 Han & DF HOO & & $\begin{array}{l}\text { Besgining of } \\
\text { Mass Balance }\end{array}$ & & \\
\hline Client ID: & & Recovery & Recovery & Recovery & Recovery & Inp.H2O & Imp.H2O \\
\hline Zenon ID: & & 00817195 & 00817195 & 00817195 & 00817295 & 00817395 & 00817495 \\
\hline Dale Sompled: & & $95 / 03 / 10$ & $95,03 / 10$ & $95,03 / 10$ & $95 / 03 / 10$ & $95 / 03 / 08$ & $95 / 03 / 09$ \\
\hline MDL & Units & Duplicate & M. Spike & $\%$ Recovery & & & \\
\hline 0 & $\mathrm{ml}$ & - & - & - & 990 & 490 & 870 \\
\hline 3.0 & บg & 5700000 & 11000000 & 82 & 6300000 & 1800000 & 5700000 \\
\hline 3.0 & ' ug & - & - & - & 4900 & 540 & 4800 \\
\hline 0.005 & $m g$ & - & - & - & 2.0 & 0.19 & 0.69 \\
\hline 0.010 & $"$ & - & - & - & $<0.099$ & $<0.049$ & $<0.087$ \\
\hline 1.0 & 鸧 & - & - & - & 860 & 5600 & 4100 \\
\hline
\end{tabular}

穴 Boron

Impinger volume neasured

Aminonia (as $\mathrm{N}$ )

Furoride

Chkoride

ortho-Phosphaze (as P) 
Revision 0

Batch Code:

Impinger volume measured etc.

Analysis Date:

Preparation Date:

Batch Code:

Ammonia (as N) ets:

Preparation Date:

Bateh Code:

Fluoride ek:

\section{PSA 1}

008169

008170

008171

008172

008173

008174

$95 / 04 / 10$

95/04/10

0407FSA1 0407FSA2

$008169 \quad 008171$

008170

008171

008172

008173

008174

95/04/07 95/04/07

95/04/07 95/04/07

\section{O412MNA1}

008169

008170

008171

008172

008173

008174

$95 / 04 / 12$

$95 / 04 / 12$

Prepauation Dalte:

Batch Code:

Chlaride etc.

\section{OSL2MNA1}

008169

008170

008171

008172

008173

008174

$95 / 04 / 12$

95/04/12

\section{M412MNA1}

008169

008170

008171

008172

008173 
008174

Analysis Dare:

Preparation Date:

Batch Code:

Boron etc.

Analysis Date:

Preparation Date:
95/04/12

$95 / 04 / 12$

0421BMB1

008169

008170

008171

008172

008173

008174

$95 / 04 / 21$

$95 / 04 / 21$ 
WHC-SD-WM-VI-031

Revision 0

This page intentionally left blank. 
WHC-SD-WM-VI-031

Revision 0

APPENDIX 1B

wass balance calculations 
$A--\therefore-A \cdot-{ }^{\prime}, .-\cdots$

Revision 0

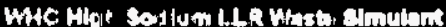

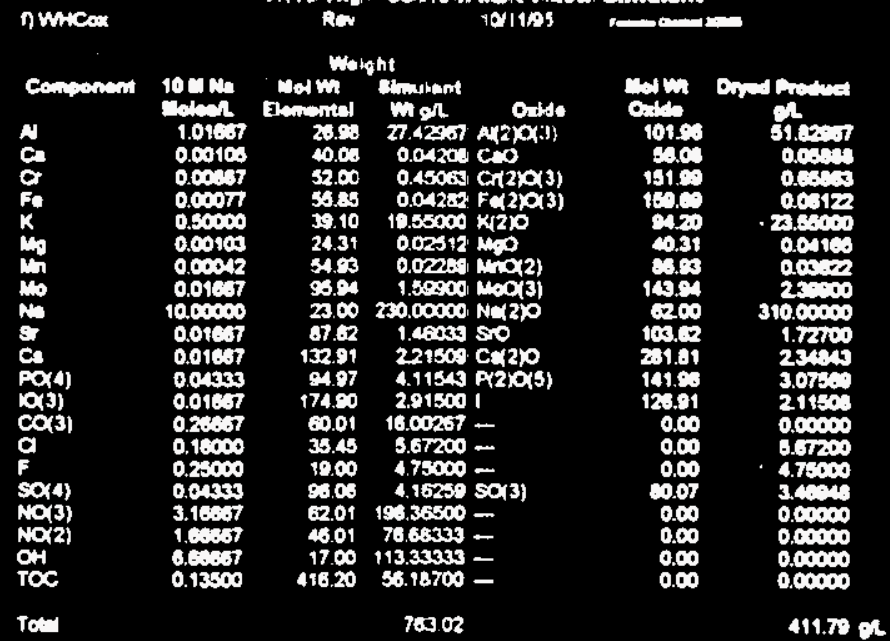
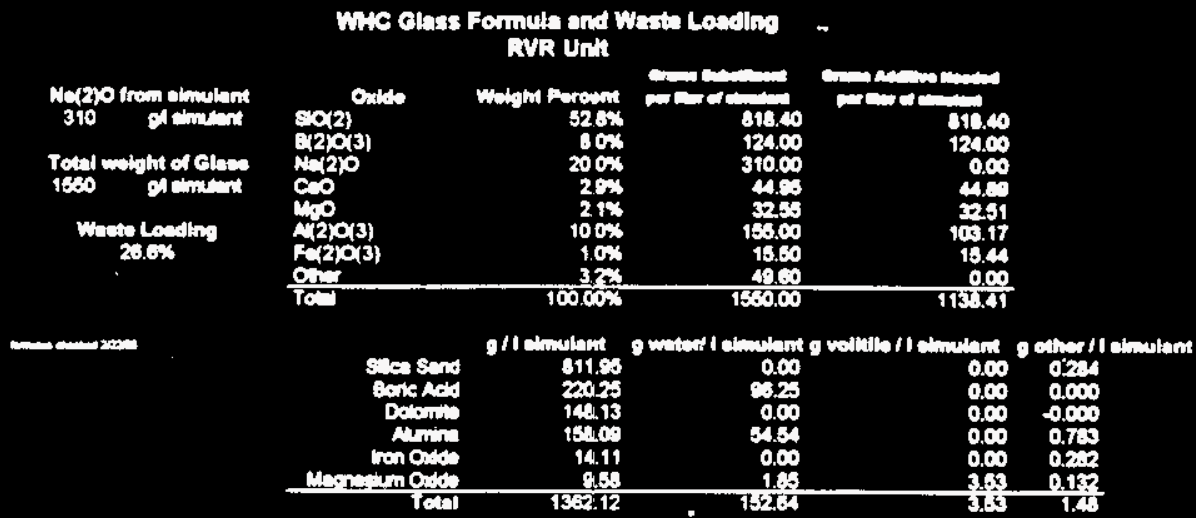

Wire olese Formula and What Leath' Cakiner Fluld Ead
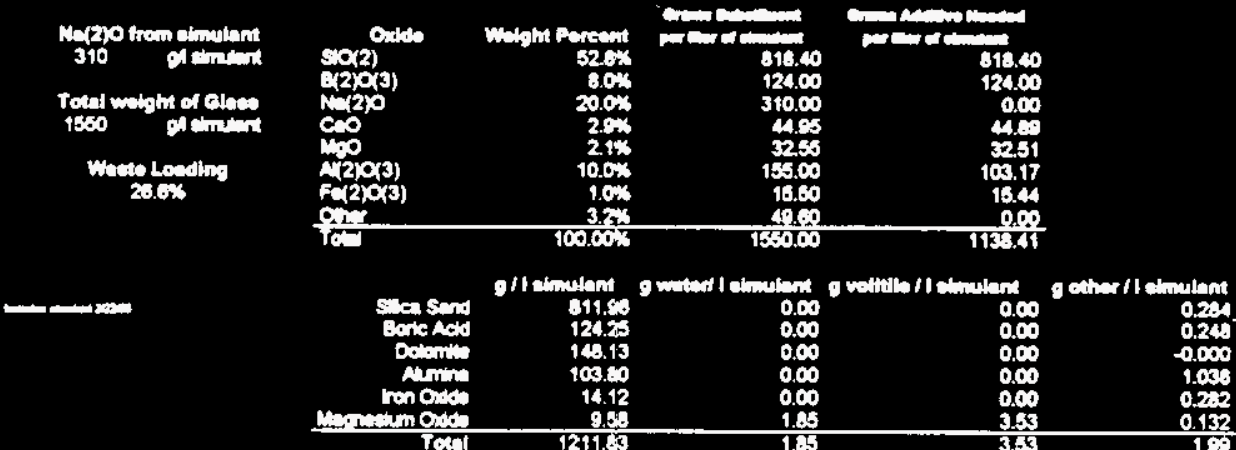

RVR Glase Fomers

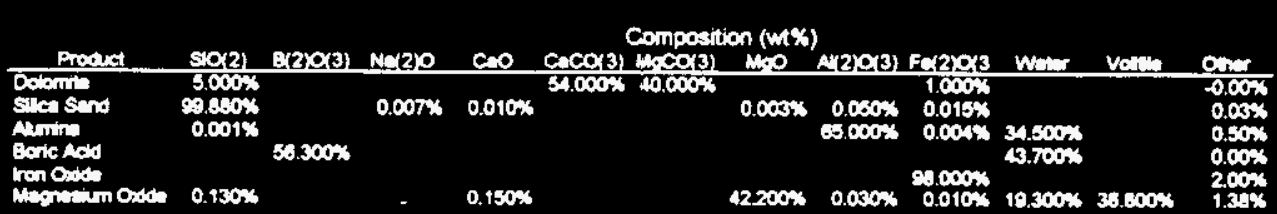

Caleiner Glass Formors

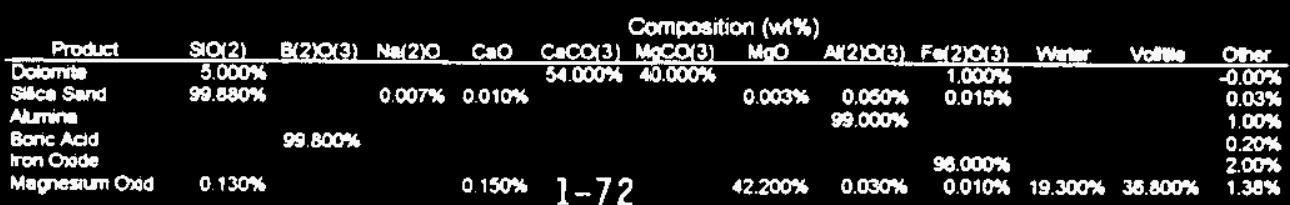


Mass Balance, Procedyne Pilot Scale

\begin{tabular}{ll} 
Prepared by: Brent Spurgeon $4 / 2$ & Date: $08 / 17 / 95$ \\
Checked by: John Koehr AK & Date: $08 / 17 / 95$ \\
\hline
\end{tabular}

Total Simulant injection

\begin{tabular}{cccc} 
& High & Low & Difference \\
Total Inches & 12.625 & 5.250 & 7.375 \\
& 17.125 & 6.125 & 11.000 \\
& 17.375 & 5.000 & 12.375 \\
& 17.000 & 5.750 & 11.250 \\
- & 18.500 & 5.500 & 13.000 \\
& 16.500 & 7.000 & 9.500 \\
\hline & & & 64.500
\end{tabular}

Density of Simulant $\quad 1.49 \mathrm{~kg} / \mathrm{it}$

Assume $\quad 0.61\left(\mathrm{~cm}^{\wedge} 3 / \mathrm{g}\right.$ Sugar) volume increase manured during tont

$15.1 \mathrm{lb}$ Sugar = $\quad 6849.244 \mathrm{~g}$ Sugar

$180 \mathrm{lb}$ Simulant $=\quad 81.64662 \mathrm{~kg}$ Simulant

$=\quad 54.79639$ liters of Simulant

Sugar concentration $\quad 125 \mathrm{~g}$ sugar / 1 simulant

125 g sugar / / simulant *

1 I simulant $=$

1.08 sugar simulant

$116.14 \mathrm{~g}$ sugar

I sim w/ sugar

(C) $100 \%$ Stoich C

64.5 in. $x$

1.25 liter/in. $=80.625$ liters with sugar

per Procodyno Col po. 30

80.625 liters w/sugar $x \quad 116.14 \frac{\mathrm{g} \mathrm{sugar} x}{\mathrm{l} / \mathrm{w} \text { sugar }} \quad 0.61 \frac{\mathrm{ml}=}{\mathrm{g} \text { sugar }} \quad 5712 \mathrm{ml}$ sugar

$\mathbf{8 0 . 6 2 5}$ liters w/sugar - $\quad \mathbf{5 . 7 1 2}$ liter sugar $=\quad \mathbf{7 4 . 9 1}$ liter simulant

Total Glass Fomers In: $\quad 170.00 \mathrm{lb}=\quad 77.11 \mathrm{~kg}$

Glass Former Composition:

Silica Sand

Dolomite

Alumina

Iron Oxide

Magnesium Carbonate
$74.66 \%$

$13.62 \%$

$9.54 \%$

$1.30 \%$

$0.88 \%$ 
Total Product Out:

$$
223.85 \mathrm{lb}=101.54 \mathrm{~kg}
$$

Off-Gas:

Average Reading = Flow Rate

Assume Gas Temp:

$V_{2}=T_{2} T_{1} * V_{1}$

Standard Flow (V2) @ 298.15 K (T2):

14.82 SCFM

25. $19 \mathrm{~N} \mathrm{m3/hr}$

Scrubber:

Start Volume: $\quad 22.00 \mathrm{gal}=$

End Volume: $\quad 36.02 \mathrm{gal}=$
83.30 liter 136.37 liter 


\section{Water Balance}

Water in:

Total Simulant In: $\quad \mathbf{7 4 . 9 1}$ liters

Water In Simulant:

\begin{tabular}{lrl} 
Density of Simulant: & $1490.00 \mathrm{~g} n$ \\
Other chemicals in simulant: & 763.02 \\
\hline Weight of Water in simulant: & $726.98 \mathrm{~g} \Lambda$
\end{tabular}

This equals: $\quad 0.727$ I water / $\quad$ simulant
Total water in:
54.46 liters

Water Out:

$117 \mathrm{lb}=\quad \mathbf{5 3 . 0 7 0 3}$ liters of water plus contaminant 


\section{Nitrogen Balance}

NITROGEN IN:

$\mathrm{NO}(3)$

$$
\begin{gathered}
\times \begin{array}{r}
196.37 \\
\times \begin{array}{r}
74.91 \\
14710
\end{array} \text { g liter of so(3) }
\end{array} \\
\% \mathrm{~N}=\frac{14.01}{62.01} \mathrm{~g} \mathrm{~N}=22.59 \% \mathrm{NO}(3)
\end{gathered}
$$

$$
\mathbf{N} \text { in } N O(3)=
$$

$3323.52 \mathrm{~g} N$

NO(2)

$$
\begin{gathered}
\times \quad \begin{array}{l}
76.68 \mathrm{~g} \text { diter of simulant } \\
74.91 \\
5745 \mathrm{~g} \mathrm{NO}(2)
\end{array} \\
\% \mathrm{~N}=\frac{14.01}{46.01} \mathrm{~g} \mathrm{~N}=30.45 \% \mathrm{~N}(2)
\end{gathered}
$$

$$
\mathrm{N} \text { in } \mathrm{NO}(2)=\quad 1749.22 \mathrm{~g} \mathrm{~N}
$$

EDTA:

concentration: $\quad 0.135$ mol EDTAliter Simulant

Simulant Used: $\quad \mathbf{7 4 . 9 1}$ liter

EDTA: $\quad 10.11 \mathrm{~mol}$ EDTA

$\times 416.20 \mathrm{~g} / \mathrm{mol}=\quad 4209 \mathrm{~g}$ EDTA

$\frac{28 \mathrm{gN}=}{416.2 \mathrm{gNa}(4) E D T A} \quad 6.73 \%$

$$
\mathrm{N} \text { in } \mathrm{Na}(4) E D T A=283.17 \mathrm{~g} \mathrm{~N}
$$

Fluidizing Gas:

$\begin{array}{cc}\text { \%flow of flow meter } & 30.00 \% \\ \text { Actual Flow }= & 12.00 \mathrm{SCFM} N(2) \\ \text { or } & 20.39 \mathrm{~N} \mathrm{m3Mhr}\end{array}$

$n=P$ VIRT

$\frac{101.0 \mathrm{kPa}+}{8.314 \mathrm{kPa} 3 \mathrm{k}_{\mathrm{kmark}}} \frac{20.39 \mathrm{~N} \mathrm{~m} 3 \mathrm{Mr}=}{298.15 \mathrm{k}} \quad 0.831 \mathrm{kmol} / \mathrm{hr}$

\begin{tabular}{lc} 
Total Time $=$ & $4.0 \mathrm{hr}$ \\
Total $\mathrm{kmol}=$ & $3.32 \mathrm{kmol}$ \\
mw of $\mathrm{N}(2)$ & $28.01 \mathrm{~kg} / \mathrm{kmol}$ \\
\multicolumn{1}{c}{ Nitrogen from fluidizing gas: }
\end{tabular}

$93.07 \mathrm{~kg} \mathrm{~N}$

Filter Blowback Nitrogen: 
flow rate $=$

$$
\frac{14.7}{1.75} \mathrm{lb}=\quad 8.4 \mathrm{lb} / \mathrm{hr}
$$

test time:

$4 \mathrm{hr}$

Total N(2):

Total Nitrogen In: $\quad 113.67 \mathrm{~kg} \mathrm{~N}$ 
NITROGEN OUT:

Product: $\quad$ Total Product $=\quad 101.54 \mathrm{~kg}$

Nitrogen in Product is Negligible

Off-Gas:

Flow Rate: $\quad 25.19 \mathrm{~N} \mathrm{m3/hr}$

\section{Elemental Nitrogen:}

Average N(2) level in off-gas:

Nitrogen flow.

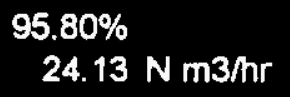

$95.80 \%$

$24.13 \mathrm{~N} \mathrm{~m} 3 \mathrm{hr}$

per mentyis

$-$

$n=P V / R T$

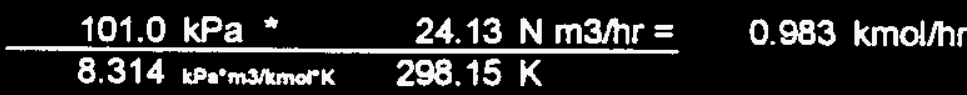

$=\quad 27.54 \mathrm{~kg} / \mathrm{hr}$

Test Time:

$4.00 \mathrm{hr}$

total

$110.15 \mathrm{~kg} \mathrm{~N}$

N(2)O:

Average N(2)O Reading:

2075 ppm

per enthers

$N(2) O$ flow.

$0.0523 \mathrm{~N} \mathrm{m3/hr}$

$n=P V / R T$

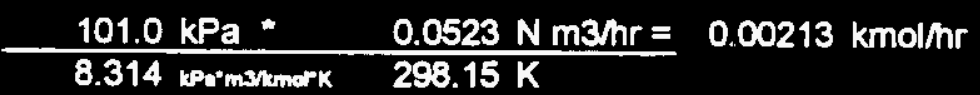

total $\mathrm{kmol}$ for $4 \mathrm{hr}$.

$0.008518 \mathrm{kmol}$

$\frac{28.01 \mathrm{~kg} N(\mathrm{mnol} N(2) \mathrm{O}=}{44.01 \mathrm{~kg} N(2) O \operatorname{Non} \mathrm{N}(2) \mathrm{O}}$

$0.636 \mathrm{~kg} \mathrm{~N} / \mathrm{kg} \mathrm{N}(2) \mathrm{O}$

Total N(2)O:

$0.375 \mathrm{~kg}$

Total Nitrogen from N(2)O: 
NO:

Average NO Reading:

$$
362 \mathrm{ppm}
$$

per intore tat

NO flow.

$0.0771 \mathrm{~N} \mathrm{m3} / \mathrm{hr}$

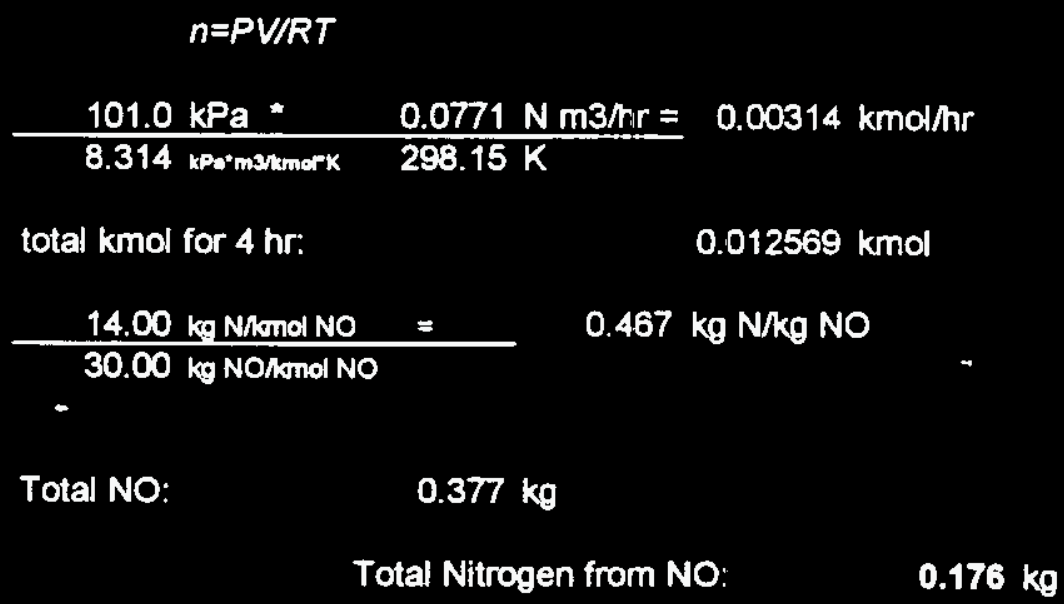

NO(2):

Average NO(2) Reading:

$133.5 \mathrm{ppm}$

per ofloges dite

NO(2) flow.

$3.36 E-03 \mathrm{~N} \mathrm{m3} / \mathrm{hr}$

$n=P$ VRT

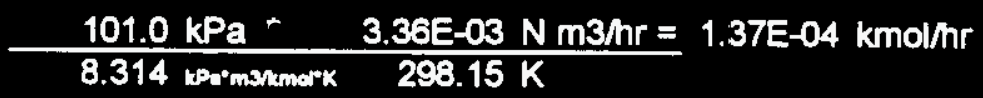

total $\mathrm{kmol}$ for $4 \mathrm{hr}$ :

$0.000548 \mathrm{kmol}$

$\frac{14.00 \mathrm{kn} N \text { mmal } N(2) \mathrm{O}}{46.00 \mathrm{~m} N(2) 0 \mathrm{knnol} N(2) \mathrm{O}}=$

$0.304 \mathrm{~kg} \mathrm{~N} / \mathrm{kg} \mathrm{N}(2) \mathrm{O}$

Total NO(2):

$0.025 \mathrm{~kg}$

Total Nitrogen from NO(2): $\quad \quad 7.67 E-03 \mathrm{~kg} \mathrm{~N}$ 


\section{NH(3):}

Average NH(3) Reading:

$6.00 \mathrm{~g} / \mathrm{hr}$

per Zomon Arabyels nuts ext and:

total $\mathrm{kmol}$ for $4 \mathrm{hr}$ :

$$
24.00 \mathrm{~g}
$$

$\frac{14.00 \mathrm{~kg} \mathrm{~N}}{17.00 \mathrm{~kg} \mathrm{NH}(3)}=$

$0.824 \mathrm{~kg} \mathrm{~N} / \mathrm{kg} \mathrm{NH}(3)$

Total Nitrogen from $\mathrm{NH}(3)$ :

Total Nitrogen Out:

Total Nitrogen In:

Difference

\% Missing
$19.765 \mathrm{~g} \mathrm{~N}$

ERR $\mathrm{kg} \mathrm{N}$

$113.67 \mathrm{~kg} \mathrm{~N}$

ERR $\mathrm{kg}$

ERR 


\section{Carbon Balance}

Carbon In:

sucrose:

for $100 \%$ Stoichiometry

116. $14 \mathrm{~g}$ sucrose/ sim whh sucrose

Simulant/sucrose Solution used: $\quad \mathbf{8 0 . 6 2 5}$ liter

Total sucrose in:

9364 g sucrose

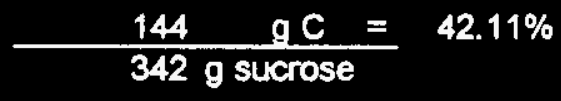

Total C from sucrose:

$-3.94 \mathrm{~kg} \mathrm{C}$

EDTA:

concentration: $\quad 0.135$ mol EDTAMiter Simulant

Simulant Used: $\quad \mathbf{7 4 . 9 1}$ liter

EDTA: $\quad 10.11 \mathrm{~mol}$ EDTA

$\times 416.20 \mathrm{~g} / \mathrm{mol}=\quad 4209 \mathrm{~g}$ EDTA

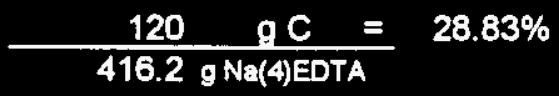

Total C from EDTA: $\quad 1.21 \mathrm{~kg} \mathrm{C}$

glass formers:

Dolomite In:

$10.50 \mathrm{~kg}$

$\mathrm{CaCO}(3)$ from Dolomite $\quad 5.67 \mathrm{~kg}$

$\mathrm{MgCO}(3)$ from Dolomit $\quad 4.20 \mathrm{~kg}$

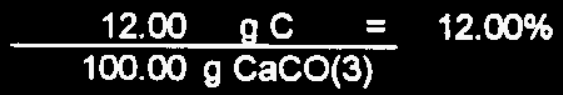

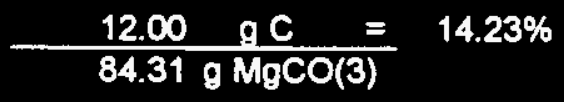

Total C from Dolomite: $\quad 1.28 \mathrm{~kg} \mathrm{C}$

Mag. Carb. In:

$\% \mathrm{C}$ in MagCarb:
$0.68 \mathrm{~kg}$
$10.09 \%$
Total C from Mag Carb:
per mat cut shent
Total C in:
$0.0685 \mathrm{~kg} \mathrm{C}$
$6.503 \mathrm{~kg} \mathrm{C}$ 
Carbon Out:

Product:

Total Product Out:

$101.54 \mathrm{~kg}$

Average TIC in Product:

$2.78 \%$

$2.82 \mathrm{~kg}$

ov. of enalyis

Average TOC in Product:

$0.18 \%$

$0.18 \mathrm{~kg}$

Total C in Product:

$3.01 \mathrm{~kg}$

Off-Gas:

Flow Rate:

$25.19 \mathrm{~N} \mathrm{~m} 3 / \mathrm{hr}$

Total Volume out:

$100.75 \mathrm{~m}^{\wedge} 3$

CO:

Avg Reading:

3485 ppm

Volume of $\mathrm{CO}$ :

$0.351 \mathrm{~m}^{\wedge} 3$

$n=P V / R T$

$\frac{101.0 \mathrm{kPa} *}{8.314 \text { kPatmshmork }} \quad \frac{0.35 \mathrm{~N} \mathrm{m3}=}{298.15 \mathrm{k}} \quad 0.014 \mathrm{kmol}$

Total C from CO: $\quad 0.172 \mathrm{~kg} \mathrm{C}$

CO(2):

Avg Reading:

$3.31 \%$

Volume of $\mathrm{CO}(2)$ :

$3.33 \mathrm{~m}^{\wedge} 3$

$n=P V / R T$

$101.0 \mathrm{kPa}$ *

$3.33 \mathrm{Nm3}=$

8.314 kpamatmmerk $298.15 \mathrm{~K}$

$0.136 \mathrm{kmol}$

Total C from $\mathrm{Co}(2)$ :

$1.63 \mathrm{~kg} \mathrm{C}$

TOC:

Avg Reading:

Volume of TOC:

$8.87 \mathrm{ppm}$

$0.000894 \mathrm{~m}^{\wedge} 3$

$n=P V / R T$

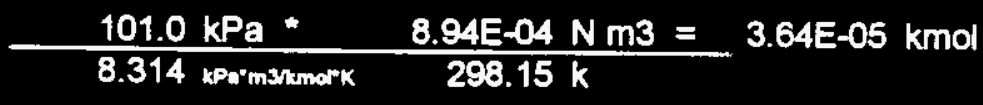

Total C from TOC:

4.37E-0.4 $\mathrm{kg} \mathrm{C}$ 
Scrubber Solution:

Start C concentration:

Total C at Start:

End $\mathrm{C}$ concentration:

Total $\mathrm{C}$ at End:

$$
\begin{aligned}
& 4.100 \mathrm{mg} / \mathrm{L} \\
& 0.342 \mathrm{~kg} \\
& 4500 \mathrm{mg} / \\
& 0.614 \mathrm{~kg}
\end{aligned}
$$

carbon accumulation:

Total Carbon Out:

$5.080 \mathrm{~kg} \mathrm{C}$

Total Carbon In:

$6.503 \mathrm{~kg} \mathrm{C}$

percent missing:

$21.88 \%$ 


\section{Sodium Balance}

\begin{tabular}{|c|c|}
\hline $\mathrm{Na}(2) \mathrm{O}$ in Simulant: & $\begin{array}{l}310.00 \mathrm{~g} / \text { iter } \\
23.22 \mathrm{~kg}\end{array}$ \\
\hline Silica Sand contains: & $0.0040 \mathrm{~kg}$ \\
\hline Total Na(2)O in: & $23.227 \mathrm{~kg}$ \\
\hline $\mathrm{Na}(2) \mathrm{O}$ in product: & $\begin{array}{l}16.27 \% \\
16.52 \mathrm{~kg}\end{array}$ \\
\hline $\mathrm{Na}$ in Scrubber @ start: & $\begin{array}{l}3576 \mathrm{mg} / \mathrm{Alter} \\
0.298 \mathrm{~kg}\end{array}$ \\
\hline Na in Scrubber (9) end: & $\begin{array}{l}2356 \mathrm{mg} / \mathrm{hiter} \\
0.321 \mathrm{~kg}\end{array}$ \\
\hline $\begin{array}{l}\text { Na accumulation: } \\
\text { corresponding } \mathrm{Na}(2) \mathrm{O} \text { weight: }\end{array}$ & $\begin{array}{l}0.023 \mathrm{~kg} \\
0.031 \mathrm{~kg}\end{array}$ \\
\hline Total Na(2)O out: & $16.547 \mathrm{~kg}$ \\
\hline
\end{tabular}

$\%$ missing:

$28.76 \%$ 


\section{Halide Balances}

Total Simulant In:

Total Off-Gas out:

Total Product Out:
74.91 liter

$100.75 \mathrm{~N} \mathrm{~m} 3 / \mathrm{hr}$

$101.54 \mathrm{~kg}$

Fluoride Balance:

$F$ in Simulant:

Total $F$ in:

Avg $F$ in Product:

Total $F$ in product:

Avg $F$ in Off-Gas:

Total F in Off-Gas:

Fluoride in scrubber (1) Start:

Total F at Start:

Fluoride in scrubber $(9)$ End:

Total $F$ at End:

Accumulation of $F$ :

Total F out:

$\%$ missing:
$4.75 \mathrm{~g} / \mathrm{iter}$

$0.356 \mathrm{~kg}$

$0.473 \mathrm{~g} / \mathrm{kg}$

$48.03 \mathrm{~g}$

$0.0045 \mathrm{~g} / \mathrm{hr}$ $0.018 \mathrm{~g}$

$5.00 \mathrm{mg} / \mathrm{iter}$ $0.416 \mathrm{~g}$

$6.00 \mathrm{mg} / \mathrm{diter}$

$0.818 \mathrm{~g}$

$0.402 \mathrm{~g}$

$0.0484 \mathrm{~kg}$

$86.39 \%$ per WHCox.mb1

PEN PNL

DER ZENON

per PNL

$$
0.425 \mathrm{~kg}
$$

$0.24 \%$

$0.24 \mathrm{~kg}$

Total $\mathrm{Cl}$ in product:

Avg Cl in Off-Gas:

Total $\mathrm{Cl}$ in Off-Gas:

$2.00 \mathrm{mg} / \mathrm{hr}$

$8.005-06 \mathrm{~kg}$

Chloride in scrubber:

ND -

PM PNL

per uses

per ZaNON

Total Cl out:

$\%$ missing:

$0.244 \mathrm{~kg}$

$42.65 \%$

lodide Balance:

I in Simulant:

2.12 gniter

Total 1 in:

$0.158 \mathrm{~kg}$

Avg I in product:

832 ppm

Total I out:

$0.084 \mathrm{~kg}$

$\%$ missing

$46.68 \%$ 


\section{Cesium Balance:}

Cesium In:

$$
74.91 \text { liter * } \quad 2.21509 \text { Cesilum / liter simulant }=\quad 165.94 \mathrm{~g}
$$

Cesium Out:

$$
101.54 \mathrm{~kg} \text { product } \quad 781 \mathrm{ppm}=\quad 79.30 \mathrm{~g} \quad \text { per uses }
$$

\section{Strontium Balance:}

Strontium In:

$$
74.91 \text { liter } \quad 1.46 \mathrm{~g} \text { Strontium } / \text { liter Simulant }=\quad 109.40 \mathrm{~g}
$$

Strontium Out:

$101.5366 \mathrm{~kg}$ product *

$0.11 \% \mathrm{ppm}=\quad 111.69 \mathrm{~g}$

$$
\text { per PNL }
$$

\section{Chromium Balance:}

Chromium In:

74.91 liter

Chromium Out:

101.5366 kg product *

\section{Potassium Balance:}

Potassium In:

74.91 liter

$$
0.03 \%
$$$$
=
$$

$34.52 \mathrm{~g}$

$33.76 \mathrm{~g}$

$0.45 \mathrm{~g}$ Chrom / liter Simulant $=$

per PNL

$19.55 \mathrm{~g}$ potassium / liter Simulant $=1464.552 \mathrm{~g}$

Potassium Out:

$101.5366 \mathrm{~kg}$ product

$0.54 \%=$

$546.77 \mathrm{~g}$

per PNL 
$H(D-S]-W M-V I-03]$

08/17 PROCMASS.WB2

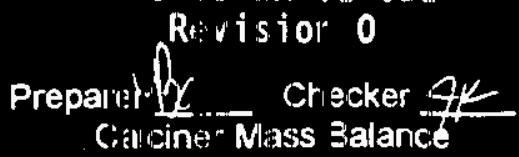

Manganese Balance:

Manganese in:

74.91 liter * $0.02289 \mathrm{~g}$ Manganese / liter simulant $=\quad 1.71 \mathrm{~g}$

Manganese Out:

$101.5366 \mathrm{~kg}$ product *

$0.01 \%=$

$6.60 \mathrm{~g}$

Par PNL

Mn in Scrubber @ Start:

$0.130 \mathrm{mg} / \mathrm{iter}$

Per PNL

Mn in Scrubber (1) End:

$0.011 \mathrm{~g}$

$0.155 \mathrm{mg} / \mathrm{hter}$

$0.021 \mathrm{~g}$

Mn Accumulation:

$0.010 \mathrm{~g}$

Total Manganese Out:

6.61

\section{Molybdenum Balance:}

Moly In:

74.91 liter * $\quad 1.599 \mathrm{~g}$ moly / liter simulant =

$119.79 \mathrm{~g}$

Moly Out:

$101.5366 \mathrm{~kg}$ product *

$0.09 \%=$

$95.44 \mathrm{~g}$

Per PNL

Mo in Scrubber (e) Start:

$0.220 \mathrm{mg} / \mathrm{hiter}$

Per PNE

Mo in Scrubber (e) End:

$0.018 \mathrm{~g}$

$0.225 \mathrm{mg} / \mathrm{iter}$

$0.031 \mathrm{~g}$

Mo Accumulation:

$0.012 \mathrm{~g}$

per PNL

Total Moly Out:

95.46

\section{Phosphorous Balance:}

Phosphorous in:

74.91 liter * $\quad 1.343333 \mathrm{~g}$ Phosphorus / liter simulant $=\quad 100.63 \mathrm{~g}$

Phosphorous out:

$101.5366 \mathrm{~kg}$ product *

$0.37 \%=$

$373.15 \mathrm{~g}$

Per PNL

$P$ in Scrubber @ Start:

11.940 mg/iter

per PNL

$P$ in Scrubber (e) End:

$0.995 \mathrm{~g}$

$10.390 \mathrm{mg} / \mathrm{iter}$

per PNL

P Accumulation:

$1.417 \mathrm{~g}$

$0.422 \mathrm{~g}$

Total Phosphorous Out:

373.57 


\section{Glass Former Balances}

Total Glass Formers In:

$$
170 \mathrm{\| b}=
$$

$77.11069 \mathrm{~kg}$

Glass Former Composition:

$\begin{array}{lr}\text { Silica Sand } & 74.66 \% \\ \text { Dolomite } & 13.62 \% \\ \text { Alumina } & 9.54 \% \\ \text { Iron Oxide } & 1.30 \% \\ \text { Magnesium Carbonate } & 0.88 \%\end{array}$

SiO(2):

\begin{tabular}{lr} 
Silica Sand & $57.50 \mathbf{~ k g}$ \\
Dolomite & $0.53 \mathbf{~ k g}$ \\
Alumina & $0.00 \mathbf{~ k g}$ \\
Iron Oxide & $0.00 \mathbf{~ k g}$ \\
Magnesium Carbonate & $0.00 \mathbf{~ k g}$ \\
\hline SiO(2) from G.F.: & $\mathbf{5 8 . 0 3} \mathbf{~ k g}$
\end{tabular}

Silica in product: $\quad \mathbf{5 5 . 0 0 \%}$ $55.85 \mathrm{~kg}$

Avo uses a PNt
$\%$ Missing:
$3.76 \%$

CaO:

\begin{tabular}{ll} 
Silica Sand & $0.006 \mathrm{~kg}$ \\
Dolomite & $2.949 \mathrm{~kg}$ \\
Alumina & $0.000 \mathrm{~kg}$ \\
Iron Oxide & $0.000 \mathrm{~kg}$ \\
Magnesium Carbonate & $0.001 \mathrm{~kg}$ \\
\hline CaO from G.F. & $2.956 \mathrm{~kg}$ \\
CaO in Simulant: & $0.059 \mathrm{~g}$ hiter \\
& $0.004 \mathrm{~kg}$ \\
Total CaO in: & $2.960 \mathrm{~kg}$ \\
& $3.09 \%$ \\
CaO in Product: & $3.13 \mathrm{~kg}$ \\
& \\
& $-5.81 \%$
\end{tabular}


$-.0-r^{2}-r^{2}-133^{\circ}$

Revisiori 0

08/17 PROCMASS.WB2

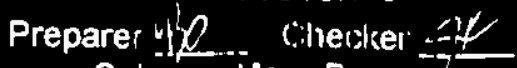

Cali: her Vasis B.a aris

Al(2)O(3):

\begin{tabular}{ll} 
Silica Sand & $0.0: 19 \mathrm{~kg}$ \\
Dolomite & $0.010 \mathrm{~kg}$ \\
Alumina & $7.266 \mathrm{~kg}$ \\
Iron Oxide & $0.000 \mathrm{~kg}$ \\
Magnesium Carbonate & $0.000 \mathrm{~kg}$ \\
\hline Al(2)O(3) from G.F. & $7.315 \mathrm{~kg}$
\end{tabular}

Al(2)O(3) in Simulant: $\quad \mathbf{5 1 . 8 3 0}$ gniter

$3.883 \mathrm{~kg}$

Total Al(2)O(3) In: $\quad 11.198 \mathrm{~kg}$

$\mathrm{Al}(2) \mathrm{O}(3)$ in product: $\quad \mathbf{8 . 1 4 \%} \quad$ Avo usas \& PNL

$8.27 \mathrm{~kg}$

$26.18 \%$

Fe(2)O(3):

Silica Sand

$0.009 \mathrm{~kg}$

Dolomite

$0.105 \mathrm{~kg}$

Alumina

$0.000 \mathrm{~kg}$

Iron Oxide

$0.981 \mathrm{~kg}$

Magnesium Carbonate $6.8 E-05 \mathrm{~kg}$

Fe(2)O(3) from G.F.: $\quad 1.095 \mathrm{~kg}$

$\mathrm{Fe}(2) \mathrm{O}(3)$ in Simulant: $\quad 0.061$ gliter

$0.005 \mathrm{~kg}$

Total Fe(2)O(3) in: $\quad 1.099 \mathrm{~kg}$

$\mathrm{Fe}(2) \mathrm{O}(3)$ in Product: $\quad 0.71 \%$

$0.72 \mathrm{~kg}$

Ave USCS \& PNL

\% Missing:

$34.51 \%$

MgO:

\begin{tabular}{ll} 
Silica Sand & $0.002 \mathrm{~kg}$ \\
Dolomite & $2.008 \mathrm{~kg}$ \\
Alumina & $0.000 \mathrm{~kg}$ \\
Iron Oxide & $0.000 \mathrm{~kg}$ \\
Megnesium Cartonate & $0.287 \mathrm{~kg}$ \\
\hline MgO from G.F: & $2.296 \mathrm{~kg}$ \\
& $0.042 \mathrm{~g} / i t e r$ \\
MgO in Simulant: & $0.0031 \mathrm{~kg}$ \\
& $2.300 \mathrm{~kg}$ \\
Total MgO in: & $2.37 \%$ \\
MgO in Product: & $2.40 \mathrm{~kg}$ \\
& \\
& $-4.54 \%$
\end{tabular}


WHC-SD-WM-VI-031

Revision 0

This page intentionally left blank. 
WHC-SD-WM-VI-031

Revision 0

APPENDIX IC

DATA SHEETS

1-91 
WHC-SD-WM-VI-031

Revision 0

Pilot-Scale Test - Datasheet

$\operatorname{Pg} \underline{2}$ of $\underline{2}$

\begin{tabular}{|c|c|c|c|c|c|c|c|}
\hline \multirow{2}{*}{$\frac{\text { Test No. FBC-S }}{\text { Parameter }}$} & \multirow{3}{*}{$\begin{array}{r}\text { Units } \\
{ }^{\circ} \mathrm{C}\end{array}$} & \multicolumn{3}{|c|}{ ADDITIVE $100 \%$ sucrase } & \multicolumn{3}{|c|}{ Sht 1 of 17} \\
\hline & & \multicolumn{6}{|c|}{ Readings } \\
\hline TI-Heated Bath & & & & & & & \\
\hline TI-Fluidizing Gas & ${ }^{\circ} \mathrm{C}$ & & 107 & 240 & 244 & 245 & 247 \\
\hline TI-Lower Bed & ${ }^{\circ} \mathrm{C}$ & & 299 & 504 & 502 & 502 & 502 \\
\hline TI-Upper Bed & ${ }^{\circ} \mathrm{C}$ & & 302 & soy & 502 & 502 & 502 \\
\hline TI-Freeboard & ${ }^{\circ} \mathrm{C}$ & & 337 & 521 & 515 & 51.3 & $5 / 6$ \\
\hline TI- off-gas & ${ }^{\circ} \mathrm{C}$ & & 56 & 86 & 92 & 97 & 102 \\
\hline TI- & ${ }^{\circ} \mathrm{C}$ & & & & & & \\
\hline & & & & & & & \\
\hline PI-Blowback Gas & $\mathrm{kPag}$ & & 138 & 138 & 138 & 138 & 138 \\
\hline PI-Fluidizing Gas & kPag & & 17 & 14 & 15 & 15 & 15 \\
\hline PI-Calciner Freeboard & $\mathrm{kPag}$ & & & & & & \\
\hline PI-Off-gas line $(\Delta P)$ & kPag & & 0.8 & 0,4 & 0.4 & 0.4 & 0.3 \\
\hline PI-Fines Filter $\quad \Delta /$ & $\mathrm{kPag}$ & & 1.5 & 1.2 & 1.5 & 1.7 & 2.0 \\
\hline $\mathrm{PI}$ & $\mathrm{kPag}$ & & & & & & \\
\hline & & & & & & & \\
\hline Simulant Injection Rate & $\mathrm{ml} / \mathrm{min}$ & $\mathrm{H}, \mathrm{O}$ & & & 50 & 77 & 77 \\
\hline Total Simulant Injected & & 0 & & & & & ש \\
\hline Scrubber Level & $\mathrm{cm}$ & & & & & & \\
\hline Blowback Gas & kPag & $138<10$ & $<<<10$ & $139<10$ & $138<10$ & $138 \div 10$ & $13 \%=10$ \\
\hline off-Gas in $H_{2} \mathrm{O}$ & Ninkpers & 3.2 & +2 & $1 \div 6$ & 16 & 12 & 1.26 \\
\hline fluding $\mathrm{N}_{z}$ & $\mathrm{Nm}^{3} / \mathrm{hr}$ & 50,52 & 26.93 & 20.14 & 20.14 & 20,14 & 20,14 \\
\hline & & & & & & & \\
\hline $3-8-95$ & & 0800 & 0845 & 0945 & 1000 & 1015 & 1030 \\
\hline Initials & & & & & & & \\
\hline
\end{tabular}


WHC-SD-WM-VI-031

Revision 0

Pilot-Scale Test - Datasheet

$\mathrm{Pg} 2$ of $\underline{2}$

\begin{tabular}{|c|c|c|c|c|c|c|c|}
\hline \multirow{3}{*}{$\frac{\text { Test No. FBC-S }}{\text { Parameter }}$} & \multirow{3}{*}{$\begin{array}{r}\text { Units } \\
{ }^{\circ} \mathrm{C}\end{array}$} & \multicolumn{3}{|c|}{ ADDITIVE $100 \%$ Sucress } & & \multicolumn{2}{|c|}{ Sht 2 of 17} \\
\hline & & \multicolumn{6}{|c|}{ Readings } \\
\hline & & & & & & & \\
\hline TI-Fluidizing Gas & ${ }^{\circ} \mathrm{C}$ & 249 & 254 & 254 & 256 & 258 & 256 \\
\hline TI-Lower Bed & ${ }^{\circ} \mathrm{C}$ & 502 & 502 & 502 & 502 & 501 & 502 \\
\hline TI-Upper Bed & ${ }^{\circ} \mathrm{C}$ & 501 & 502 & 502 & $502^{\circ}$ & 502 & 502 \\
\hline TI-Freeboard & ${ }^{\circ} \mathrm{C}$ & 519 & 518 & 522 & 522 & 524 & 532 \\
\hline TI- OffGAS & ${ }^{\circ} \mathrm{C}$ & 105 & 108 & 110 & 116 & 116 & 117 \\
\hline$T I-$ & ${ }^{\circ} \mathrm{C}$ & & & & & & \\
\hline & & & & & & & \\
\hline PI-Blowback Gas & $\mathrm{kPag}$ & 138 & 138 & 138 & 138 & 138 & 13 \\
\hline PI-Fluidizing Gas & $\mathrm{kPag}$ & 14 & 15 & 16 & 16 & 17 & 19 \\
\hline PI-Calciner Freeboard & $\mathrm{kPag}$ & & & & & & \\
\hline PI-Off-gas line $(\Delta \rho)$ & $\mathrm{kPag}$ & 0.25 & 37 & 47 & 0.40 & 0,55 & 0.65 \\
\hline PI-Fines Filter $(\Delta P)$ & $\mathrm{kPag}$ & 1,5 & 2.0 & 2.5 & 2.5 & 3.2 & 4.0 \\
\hline $\mathrm{PI}$ & $\mathrm{kPag}$ & & & & & & \\
\hline & & & & & & & \\
\hline Simulant Injection Rate & $\mathrm{ml} / \mathrm{min}$ & 110 & 16.5 & 223 & 223 & 275 & 340 \\
\hline Total Simulant Injected & & 0 & & & & & \\
\hline Scrubber Level & $\mathrm{cm}$ & & & & & & \\
\hline Blowback Gas & $\mathrm{kPag}$ & $139 \leqslant 10$ & $\sum_{<10}^{1.38}$ & $\frac{139}{<10}$ & $\int_{-10}^{138}$ & $\sum_{39}^{139}<10$ & $130^{2}=10$ \\
\hline Off-Gas & $\operatorname{sex}^{2} / \mathrm{hr}$ & 1.0 & 1,5 & 1.9 & 1.6 & 2.2 & 2. $c$ \\
\hline $\mathrm{N}_{2}$ fluarlizing y as & $\mathrm{Nm}^{3} / \mathrm{hr}$ & 19.52 & 19.52 & 19.52 & 19.52 & 18,84 & 20.19 \\
\hline & & & & & & & \\
\hline $3-8-95$ - Tine & & 1050 & 1100 & 1115 & 1130 & 120 & 1245 \\
\hline Initials & & & & & & & \\
\hline
\end{tabular}


WHC-SD-WM-VI-031

Revision 0

Pilot-scale Test - Datasheet

Pg $\underline{2}$ of $\underline{2}$

\begin{tabular}{|c|c|c|c|c|c|c|c|}
\hline \multicolumn{2}{|l|}{ Test No. FBC-S } & \multicolumn{3}{|c|}{ ADDITIVE $100 \%$ Sucase } & & \multicolumn{2}{|c|}{ Sht 3 of 17} \\
\hline Parameter & Units & \multicolumn{6}{|c|}{ Readings } \\
\hline TI-Heated Bath & ${ }^{\circ} \mathrm{C}$ & & & & & & \\
\hline TI-Fluidizing Gas & ${ }^{\circ} \mathrm{C}$ & 258 & 259 & 254 & 253 & 253 & 253 \\
\hline TI-Lower Bed & ${ }^{\circ} \mathrm{C}$ & 499 & 500 & 503 & 502 & 502 & 503 \\
\hline TI-Upper Bed & ${ }^{\circ} \mathrm{C}$ & 469 & 500 & 502 & 502 & 502 & 50.3 \\
\hline TI-Freeboard & ${ }^{\circ} \mathrm{C}$ & 531 & 532. & 554 & 540 & 540 & 541 \\
\hline TI- OffIAS & ${ }^{\circ} \mathrm{C}$ & off & & & & & - \\
\hline TI- & ${ }^{\circ} \mathrm{C}$ & & & & & & \\
\hline & & & & & & & \\
\hline PI-Blowback Gas & $\mathrm{kPag}$ & 138 & 138 & 138 & 138 & 138 & 138 \\
\hline PI-Fluidizing Gas & $\mathrm{kPag}$ & 19 & 20 & 17 & 17 & 17 & 17 \\
\hline PI-Calciner Freeboard & $\mathrm{kPag}$ & & & & & & \\
\hline PI-off-gas line $(\Delta P)$ & $\mathrm{kPag}$ & 1.29 & 1.29 & 1.42 & 1.42 & 1.42 & 1.47 \\
\hline PI-Fines Filter $(\Delta P)$ & $\mathrm{kPag}$ & 5.0 & 3,555 & Sro & $3-5$ & 3.7 & 37 \\
\hline PI & $\mathrm{kPag}$ & & & & & 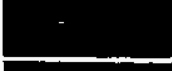 & \\
\hline & & & & & & & \\
\hline & & & Simulan & $E$ & & & \\
\hline Simulant Injection Rate & $\mathrm{ml} / \mathrm{min}$ & 395 & 395 & 223 & 223 & 223 & 223 \\
\hline Total Simulant Injected & $\ell$ & 0 & 0 & 0 & & & 12,19 \\
\hline Scrubber Level & $\mathrm{cm}$ & & & & & & \\
\hline Blowback Gas & $\mathrm{kPag}$ & $138 \quad<10$ & $\sum_{=10}^{133}$ & $138<10$ & $138<10$ & $\frac{133<10}{610}$ & $135<10$ \\
\hline Off-Gas & $2 x^{3}+2 x=$ & 5.2 & 5.2 & 5.7 & 5.7 & 5.7 & 5.9 \\
\hline $\mathrm{N}_{2}$ fludiziny & $\mathrm{Nm}^{3} / \mathrm{hr}$ & 20.19 & 19.52 & 20.14 & 20.19 & 20,19 & 20,19 \\
\hline & & & & & & & \\
\hline 3.8 .95 Tiuse & & 1300 & 1315 & 1330 & 1345 & 1400 & 1405 \\
\hline Initials & & & & & & & \\
\hline
\end{tabular}


S6-I

\begin{tabular}{|c|c|c|c|c|c|c|c|}
\hline & & & & & & & SโeTฺtuI \\
\hline$-\operatorname{sh}(1)$ & O\&SI & 5151 & oos/ & Shil & oहhl & & shs. \\
\hline & & & & & & & \\
\hline का'02 & & bin2 & b) & bा'ce & b1'C2 & ग्या० $\mathrm{N}$ & NYE TY' \\
\hline $0 !$ & & $\varepsilon 8$ & 28 & $9 \bar{S}$ & 75 & axthenttr- & $\operatorname{sed}-170$ \\
\hline 017551 & & $c^{\prime}>\sum_{\varepsilon=1}$ & $61>881$ & 0178 & 01735 & $6 \mathrm{~d} x$ & seg уреqMoIg \\
\hline & & & & & & wo & 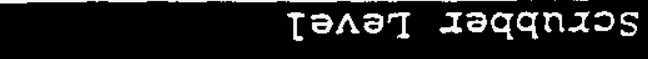 \\
\hline & & 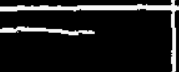 & 6092 & 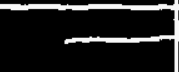 & $=$ & & 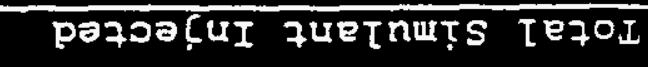 \\
\hline ORE & & OhE & $s t z$ & $s t \vec{c}$ & 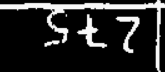 & UTTU/TH & azed Uot70a!UI quetnuts \\
\hline & & & & & & & \\
\hline & & & & & & bedX & Id \\
\hline-57 & & $5^{2}$ & $s \cdot b$ & $\operatorname{cis}$ & 53 & bedX & (dV) xa7tTa saut?a-Id \\
\hline $6+2$ & & 602 & hor & $b \varepsilon^{\prime}$ & $b \varepsilon \cdot$ & $6 e d x$ & 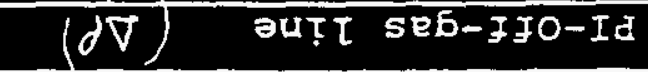 \\
\hline & & & & & & bedX & 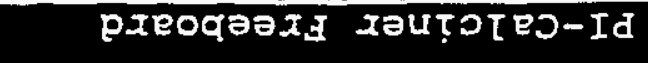 \\
\hline 12 & & b/ & $b 1$ & - & - & bedX & sed butzTpT?nTd-Id \\
\hline $8 \varepsilon^{\prime}$ & & 8हा & \&हा & 891 & $8 \& 1$ & bedx & ses xoeqMoTg-Id \\
\hline & & & & & & & \\
\hline & & & & & & & \\
\hline & & & & & & Jo & $-I L$ \\
\hline & & & & & & J. & -II \\
\hline Etis & & Ots & ots & $-b \varepsilon s$ & SES & 50 & pxеоqәәxI-II \\
\hline 205 & & 205 & 295 & 295 & 205 & Jo & pag Iədd $\Lambda$-II \\
\hline$\varepsilon 0 S$ & & 205 & $\sum \Phi$ & $\varepsilon 05$ & 205 & 5 & POg IOMOI-II \\
\hline$L+2$ & $f$ & 152 & 152 & 052 & 152 & Jo & seg buțTtpTกTd-IU \\
\hline & 1 & & & & & J。 & 47еg pa7еәH-IL \\
\hline \multicolumn{6}{|c|}{ s. } & s7? & xafourexed \\
\hline \multicolumn{3}{|c|}{ tा70 745} & \multicolumn{3}{|c|}{ 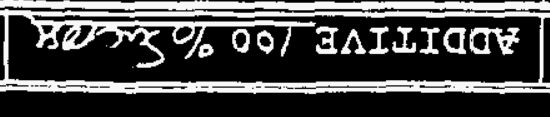 } & & $5-38 A \cdot 0 N 752 I$ \\
\hline
\end{tabular}

$\bar{z}$ รо० $\bar{z}$ 6d

$780458780-7501$ 0I80s-70ITa 
WHC-SD-WM-VI-031

Revision 0

Pilot-Scale Test - Datasheet

Pg $\underline{2}$ of $\underline{2}$

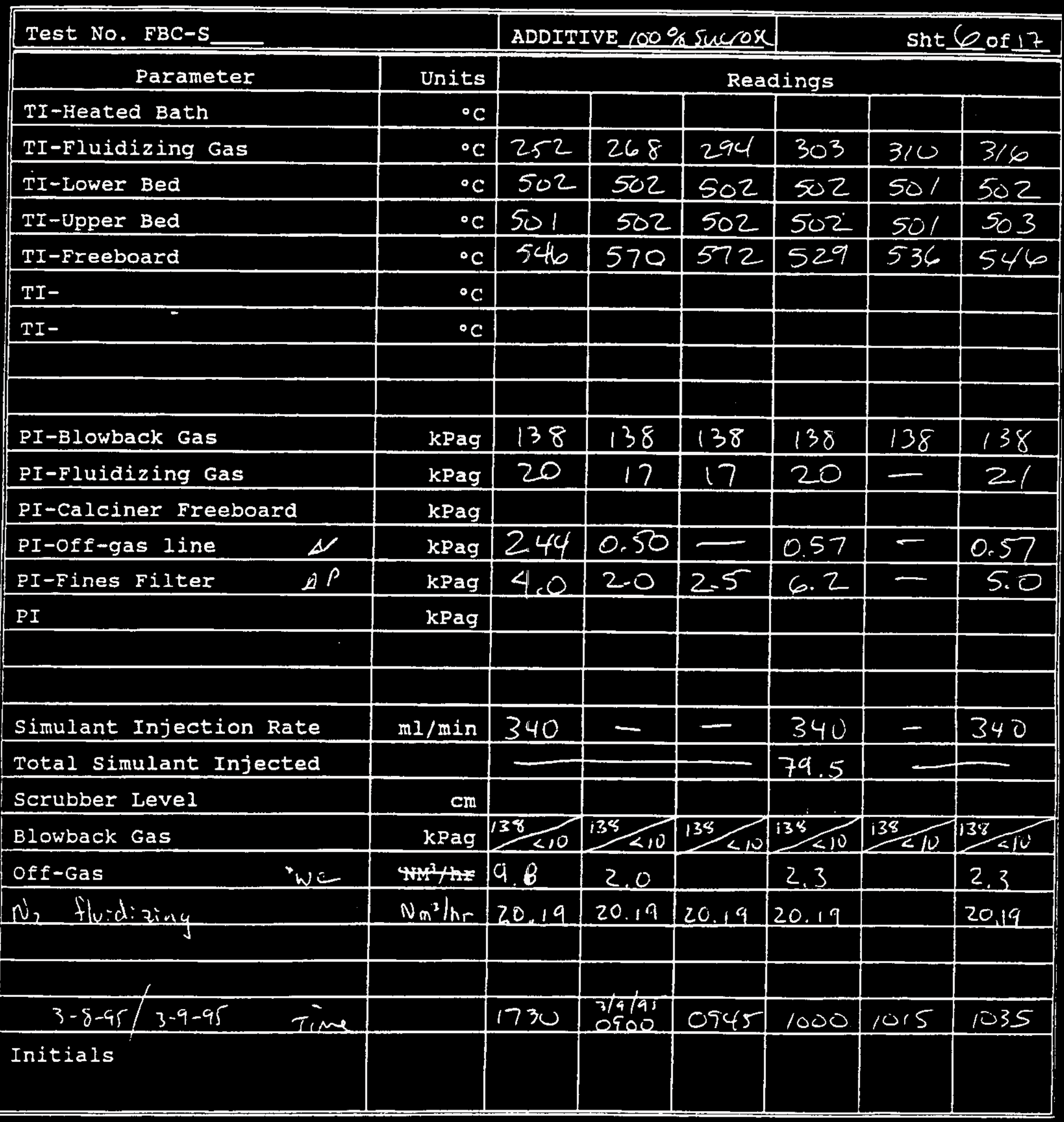


WHC-SD-WM-VI-031

Revision 0

Pilot-scale Test - Datasheet

Pg 2 of 2

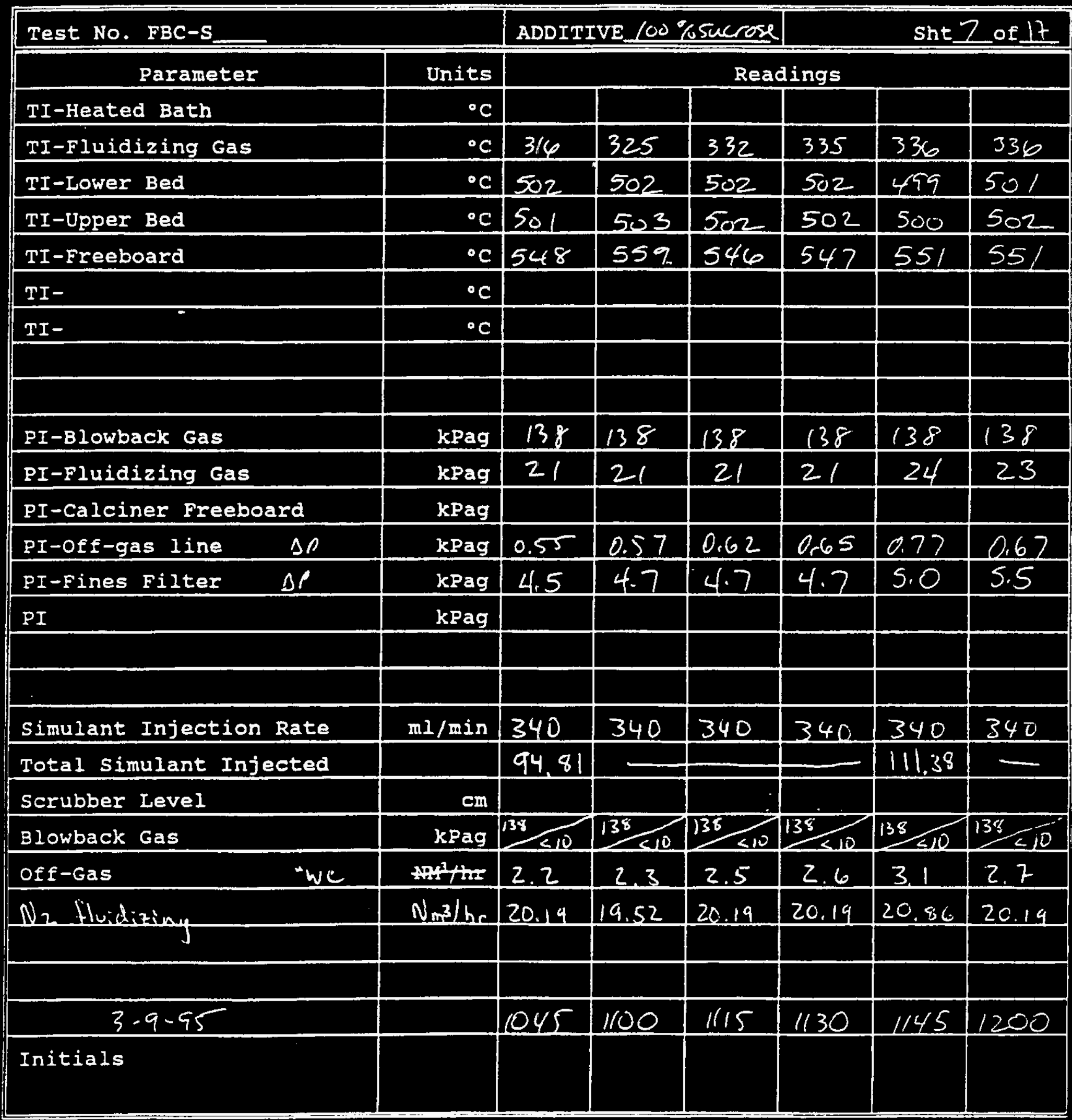


WHC-SD-WM-VI-031

Revision 0

Pilot-Scale Test - Datasheet

Pg 2 of 2

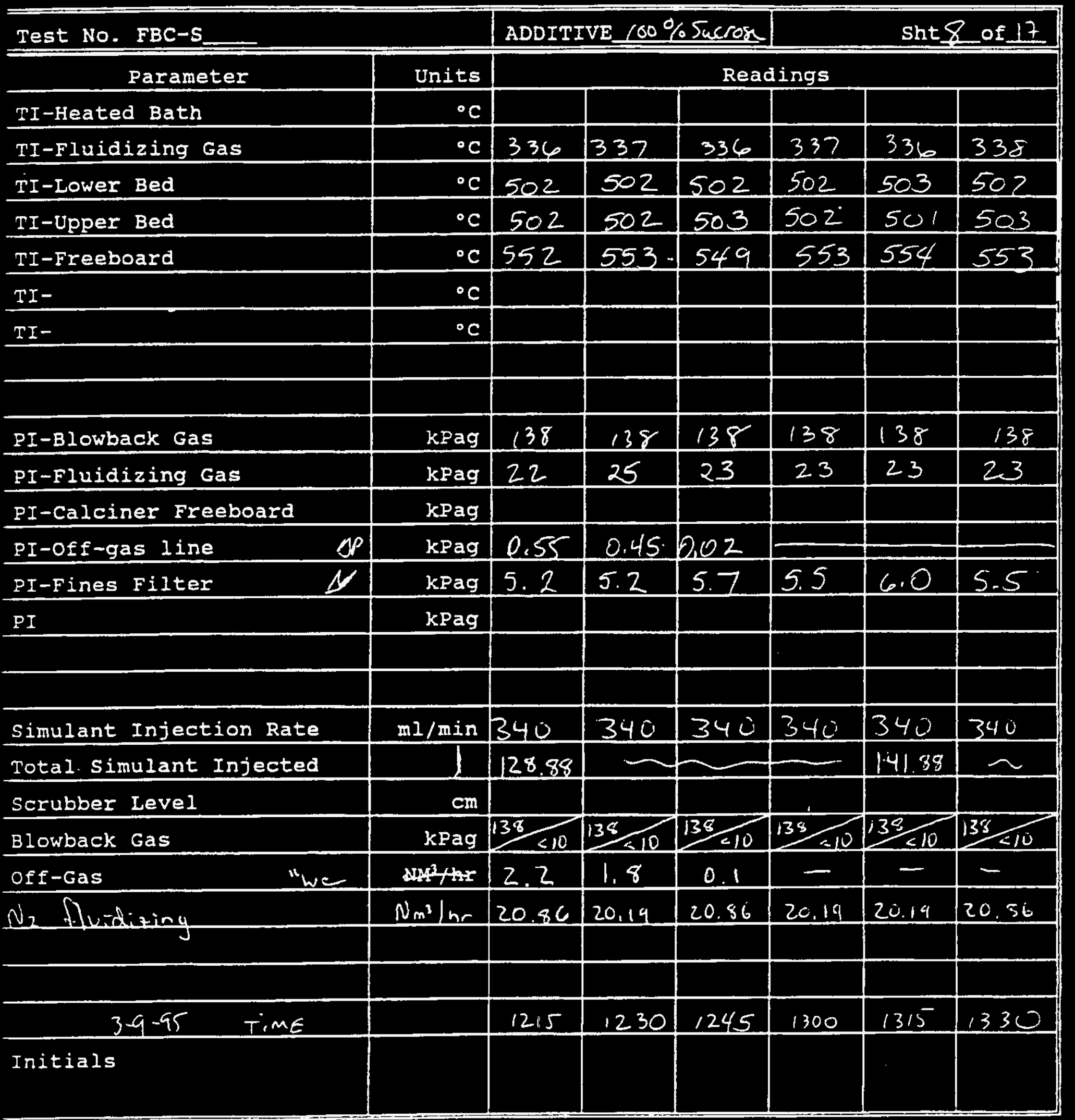


WHC-SD-WM-VI-031

Revision 0

Pilot-scale rest - Datasheet

$\mathrm{Pg} \underline{2}$ of $\underline{2}$

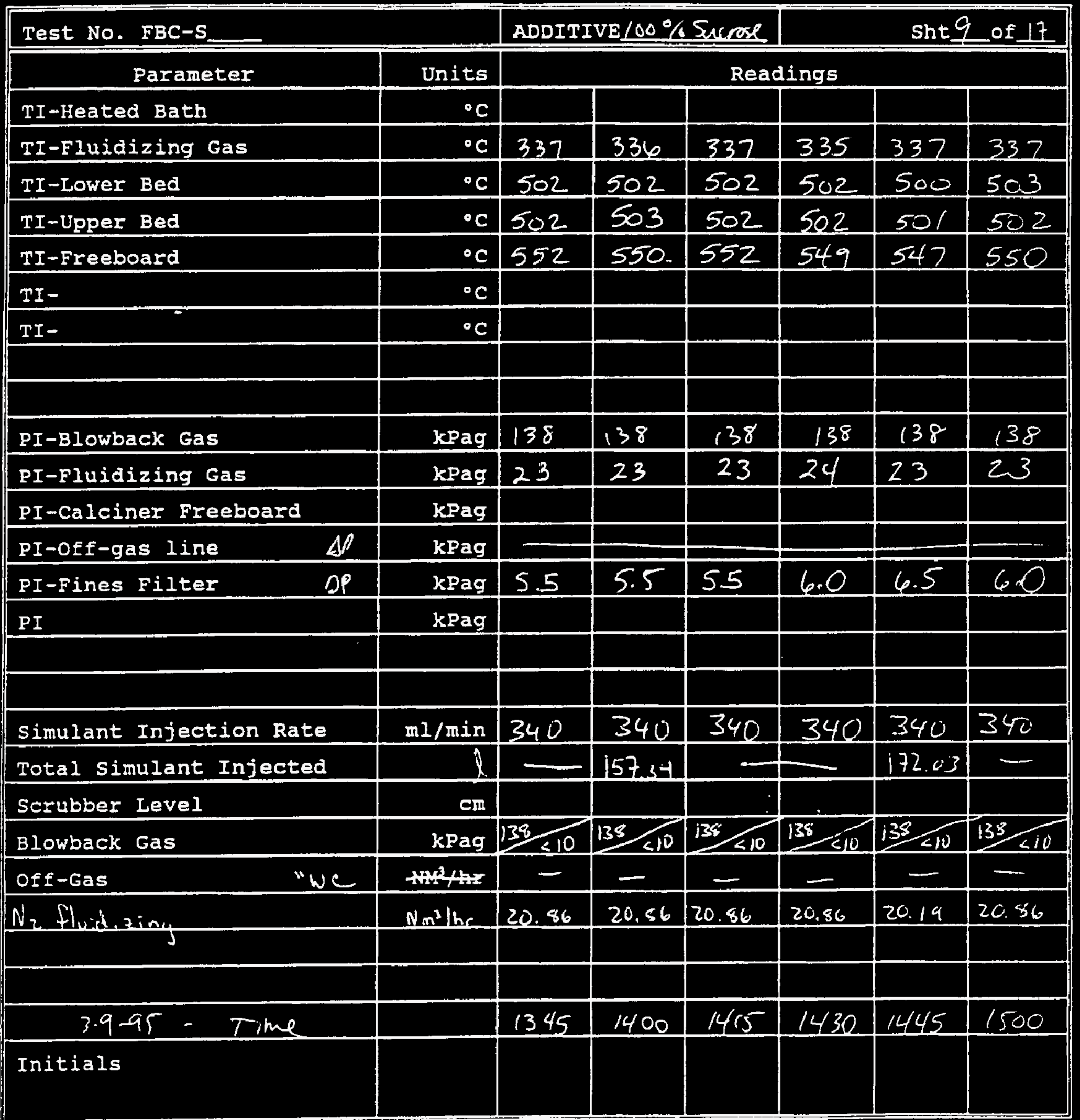


IOI-I

\begin{tabular}{|c|c|c|c|c|c|c|c|}
\hline & . & & & & & & sโeṬ 7 TuI \\
\hline$O \varepsilon 9$ & 319 & 0091 & Shsi & $O \varepsilon \Omega$ & sisl & & क्ता $S b-b^{-2}$ \\
\hline & & & & & & & \\
\hline 3502 & 902 & 98102 & ab' & 4502 & 18.02 & गप/20 N & किस:P:n LN \\
\hline - & - & - & - & -1 & 一 & $x+4+2 x$ & sed-IJ0 \\
\hline 015 & $\frac{017}{851}$ & 278 & 017881 & 978 8 & a) 8 81 & bedX & Ses Xoeqmota \\
\hline & & & & & & แอ & 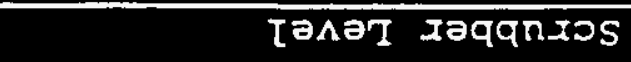 \\
\hline tb $28 !$ & & & & & & 8 & pa70อโuI 7uetnuțs Te70I \\
\hline abE & गTE & पhह & Oh. & Oमू & काह & UT⿱世-TW & 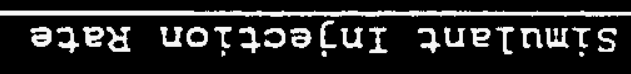 \\
\hline & & & & & & & \\
\hline & & & & & & 6edX & Id \\
\hline $2 \cdot 2$ & $O L$ & L.9 & $2 \cdot 9$ & $2 \cdot 5$ & $2 \cdot 7$ & GedX & Iə7โT! sauțg-Id \\
\hline 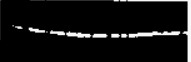 & 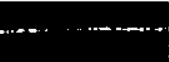 & $\cdots \cdots+\cdots$ & $-\cdots$ & -1 & $-\ldots$ & bedX & auț seb-IJO-Id \\
\hline & & & & & & bedX & pxeoqәaxs xaut?วtej-Id \\
\hline $\bar{E}$ & $\overline{\varepsilon z}$ & $\bar{\varepsilon}$ & $\overline{\varepsilon \tau}$ & $\overline{\varepsilon z}$ & $\overline{\varepsilon \tau}$ & bedx & ses buțzTpțnta-Id \\
\hline $8 \varepsilon l$ & $8 \varepsilon)$ & $8 \varepsilon 1$ & \&हा & $8 \varepsilon l$ & $8<1$ & 6edX & sed yрeqMoโg-Id \\
\hline & & & & & & & \\
\hline & & & & & & ग。 & -II \\
\hline & & & & & & Jo & $-I I$ \\
\hline 875 & LAS & ahs & shs & 2,5 & $8+5$ & 50 & pxeoqeaxa-IL \\
\hline$\overline{\varepsilon O S}$ & 105 & 205 & 105 & $\overline{\varepsilon S}$ & 205 & Jo & peg Ieddn-IL \\
\hline 205 & 205 & $\overline{\mathrm{EOS}}$ & 205 & 205 & 205 & 5. & Pəg $x ә M O I-I L$ \\
\hline $8 \varepsilon \varepsilon$ & $52 \varepsilon$ & 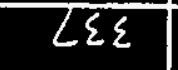 & LEs & 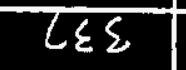 & बहट & So & ses buțzṬpṭโI-IU \\
\hline & & & & & & 50 & 478g pə7еәH-II \\
\hline \multicolumn{6}{|c|}{ sbuṭpead } & s7tun & xazaurexed \\
\hline \multicolumn{3}{|c|}{ चा $10 \overline{0 / 74 S}$} & \multicolumn{3}{|c|}{ fophs \% $\%$ cola $\triangle$ IIIda } & & S-JaS $\cdot 0 N$ 75EI \\
\hline
\end{tabular}

$\bar{z}$ Jo $\bar{\tau}$ 6d

700458780 - 780d 0T80s-70TTa 
WHC-SD-WM-VI-031

Revision 0

Pilot-scale Test - Datasheet

Pg $\underline{2}$ of $\underline{2}$

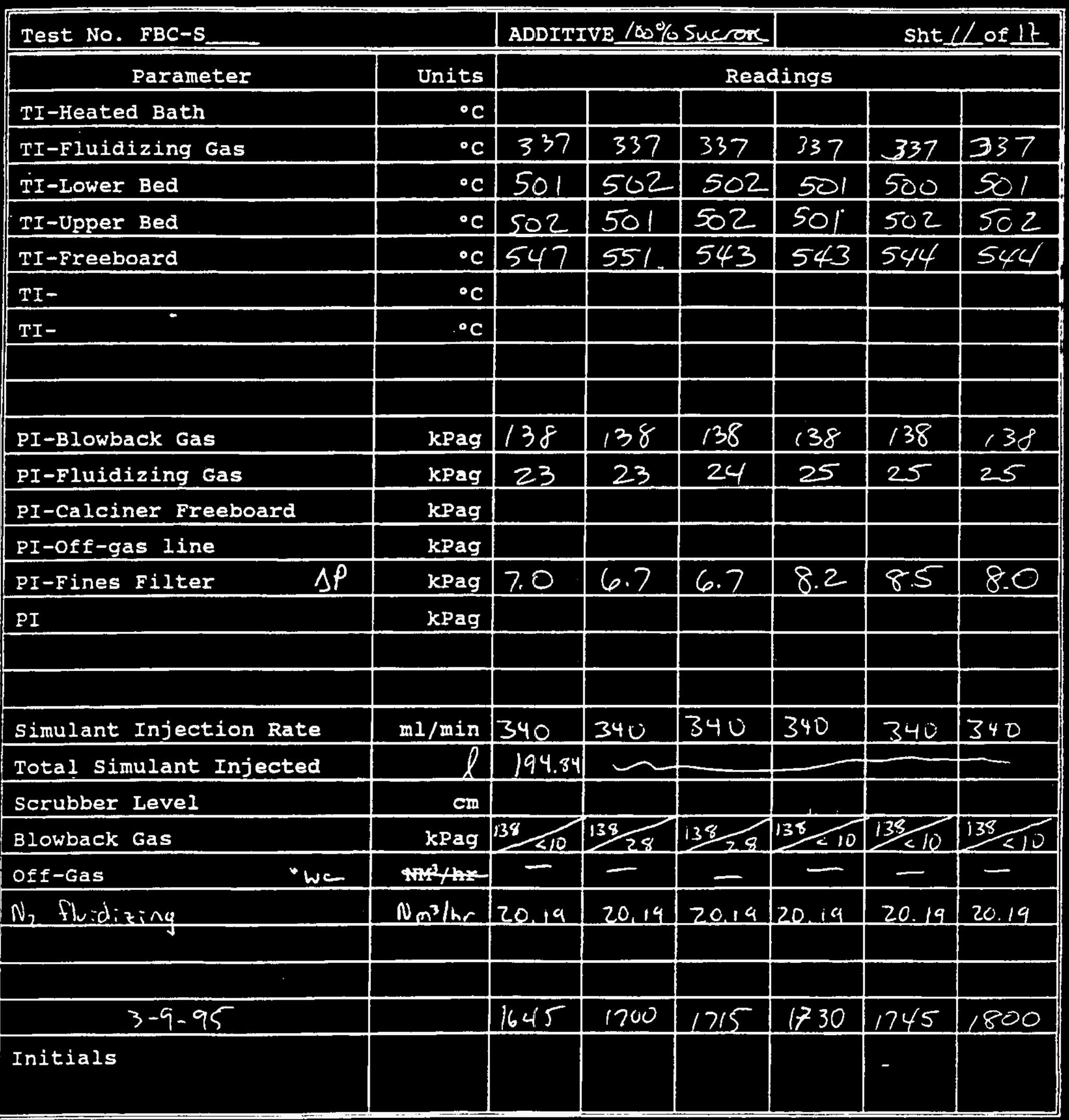


WHC-SD-WM-VI-031

Revision 0

Pilot-scale rest - Datasheet

$\mathrm{Pg} \underline{2}$ of $\underline{2}$

\begin{tabular}{|c|c|c|c|c|c|c|c|}
\hline Test No. FBC-S & & ADDITI & $\mathrm{VE} 100 \%$ & Sucrose & & Sht 12 & 2 of $L 7$ \\
\hline Parameter & Units & & & Reac & lings & & \\
\hline TI-Heated Bath & ${ }^{\circ} \mathrm{C}$ & & & & & & \\
\hline TI-Fiuidizing Gas & ${ }^{\circ} \mathrm{C}$ & 258 & 281 & 304 & 304 & 297 & $3, c 5$ \\
\hline TI-Lower Bed & ${ }^{\circ} \mathrm{C}$ & 501 & 504 & 496 & 504 & 464 & 480 \\
\hline TI-Upper Bed & ${ }^{\circ} \mathrm{C}$ & 501 & 504 & 502 & $503^{\circ}$ & 464 & 479 \\
\hline TI-Freeboard & ${ }^{\circ} \mathrm{C}$ & 492 & 512 & 514 & 547 & 511 & 513 \\
\hline TI- & ${ }^{\circ} \mathrm{C}$ & & & & & & \\
\hline TI- & ${ }^{\circ} \mathrm{C}$ & & & & & & \\
\hline & & & & & & & \\
\hline PI-Blowback Gas & kPag & $13 \delta$ & 138 & 138 & 138 & 38 & 159 \\
\hline PI-Fluidizing Gas & $\mathrm{kPag}$ & 20 & 17 & 19 & 18 & 23 & 23 \\
\hline PI-Calciner Freeboard & $\mathrm{kPag}$ & & & & & & \\
\hline PI-off-gas line & kPag & 0.75 & 0.85 & 0.75 & 0.67 & 0.70 & 0.82 \\
\hline PI-Fines Filter & $\mathrm{kPag}$ & 3.0 & 4.7 & 3.5 & 3.2 & 9.5 & 8.0 \\
\hline PI & $\mathrm{kPag}$ & & & & & & \\
\hline & & & & & & & \\
\hline Simulant Injection Rate & $\mathrm{mI} / \mathrm{min}$ & DCt & 223 & - & - & 350 & 250 \\
\hline Total Simulant Injected & $l$ & 4 & & & $-\infty$ & & - \\
\hline Scrubber Level & $\mathrm{cm}$ & & & & & & \\
\hline Blowback Gas & $\mathrm{kPag}$ & $\frac{138}{<10}$ & $\frac{138}{410}$ & $\frac{138}{410}$ & $139-10$ & $138<10$ & $159 / 14$ \\
\hline Off-Gas & $-\mathrm{Anx}^{3}+\mathrm{hx}$ & 3 & 3.4 & 3 & 2.7 & 2.8 & 3.3 \\
\hline$N_{2}$ Hvidizing & $\mathrm{N}_{n^{3}} / \mathrm{hr}$ & 20,19 & 18.84 & 15.84 & 18.17 & 18.17 & 20.19 \\
\hline & & & & & & & \\
\hline $3-10-95$ & & 0745 & 0800 & 0820 & 0845 & 0925 & 0945 \\
\hline Initials & & & & & & & \\
\hline
\end{tabular}


WHC-SD-WM-VI-031

Revision 0

Pilot-Scale Test - Datasheet

$\operatorname{Pg} \underline{2}$ of $\underline{2}$

\begin{tabular}{|c|c|c|c|c|c|c|c|}
\hline Test No. FBC-S & & ADDITI & VE $100 \%$ & sucrose & & $\sin \underline{L}$ & 3 of 17 \\
\hline Parameter & Units & & & Reac & ings & & \\
\hline TI-Heated Bath & ${ }^{\circ} \mathrm{C}$ & & & & & & \\
\hline TI-Fluidizing Gas & ${ }^{\circ} \mathrm{C}$ & 327 & 334 & 345 & 347 & 348 & 348 \\
\hline TI-Lower Bed & ${ }^{\circ} \mathrm{C}$ & 489 & 485 & 510 & 494 & 504 & 503 \\
\hline TI-Upper Bed & ${ }^{\circ} \mathrm{C}$ & 489 & 477 & 501 & 497 & 503 & $50 /$ \\
\hline TI-Freeboard & ${ }^{\circ} \mathrm{C}$ & 516 & 512 & 535 & 526 & 520 & 520 \\
\hline TI- & ${ }^{\circ} \mathrm{C}$ & & & & & & \\
\hline TI- & $\cdot \mathrm{C}$ & & & & & & \\
\hline & & & & & & & \\
\hline & & & & & & & \\
\hline PI-Blowback Gas & $\mathrm{kPag}$ & 165 & 159 & 159 & - & 172 & 172 \\
\hline PI-Fluidizing Gas & kPag & 23 & 21 & 21 & 22 & 25 & 24 \\
\hline PI-Calciner Freeboard & $\mathrm{kPag}$ & & & & & & \\
\hline PI-Off-gas line & $\mathrm{kPag}$ & 0.82 & 0.37 & 0.27 & 0.37 & 0.85 & 0.90 \\
\hline PI-Fines Filter & $\mathrm{kPag}$ & 9.2 & 9.5 & 10.0 & 8.5 & 8.5 & 7.5 \\
\hline $\mathrm{PI}$ & $\mathrm{kPag}$ & & & & & & \\
\hline & & & & & & & \\
\hline Simulant Injection Rate & $\mathrm{ml} / \mathrm{min}$ & 350 & $\longrightarrow$ & $36 \lambda$ & 320 & 350 & 350 \\
\hline Total Simulant Injected & $l$ & - & 206,22 & & 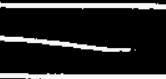 & 221.22 & - \\
\hline Scrubber Level & Cin & & & & & & \\
\hline Blowback Gas & kPag & $165 / 14$ & $\frac{154 / 14}{154}$ & $\frac{159}{14}$ & 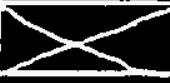 & $\frac{172}{21}$ & 172 \\
\hline Off-Gas & $2 x^{2}+4 x$ & 3.3 & 1.5 & 1.1 & 1.5 & 3.4 & 36 \\
\hline$N_{2}$ fluidiziny & $\mathrm{Nm}^{3} / \mathrm{he}$ & 20.19 & 20.19 & & 18.84 & 20.14 & 20.19 \\
\hline & & & & & & & \\
\hline $3-10-95$ & & 1000 & 1015 & 1030 & 1130 & 1145 & 1215 \\
\hline Initials & & & & & & & \\
\hline
\end{tabular}




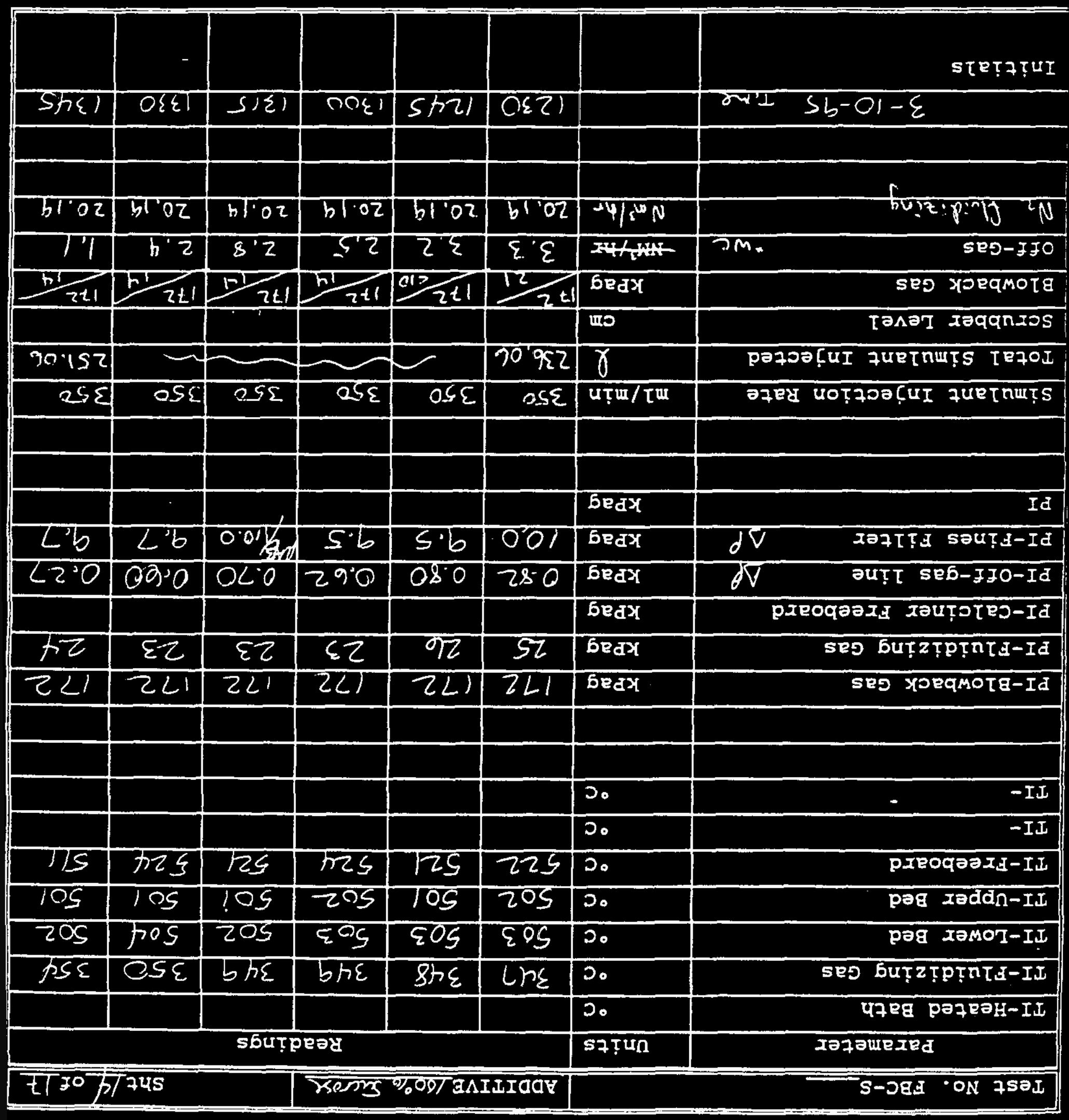




\section{$90 \llbracket-\mathrm{I}$}

\begin{tabular}{|c|c|c|c|c|c|c|c|}
\hline & & & & & & & sโeț 7 tuI \\
\hline S)ST & oesl & Sमी & och & Sinit & oohi & & \\
\hline & & & & & & & \\
\hline & & & & & & & \\
\hline 7.92 & 1,02 & $51 \cdot 02$ & bा.02 & b1.52 & b.वे & $-4 / 2^{4} n$ & 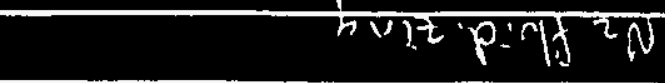 \\
\hline$h \cdot 2$ & $\varepsilon \cdot 2$ & 812 & $t \cdot 2$ & $8^{\prime 2}$ & $b 1$ & $x+4 x+4$ & seg-ija \\
\hline$\frac{\sqrt{2}}{2 t 1}$ & $\frac{12}{2+1}$ & $\frac{12}{221}$ & 27 & $\frac{12}{2+1}$ & $22+1$ & bedX & Sej xoeqmota \\
\hline & & & & & & mo & 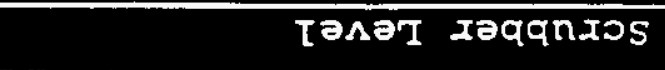 \\
\hline-1 & Fn & $\infty \pm 92$ & 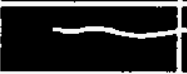 & $\infty$ & $\approx$ & $\gamma$ & pe700[̧uI zueTnuts te70I \\
\hline $0.5 \Sigma$ & $0 S \varepsilon$ & OSE & OSE & $a S \varepsilon$ & os & uṬu/โw & 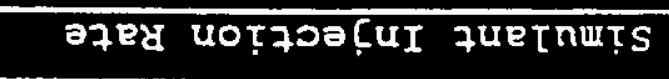 \\
\hline & & & & & & & \\
\hline & & & & & & bedx & $\overline{I d}$ \\
\hline $2 \cdot 8$ & $5 \cdot 8$ & 0.8 & $S \cdot L$ & 0.8 & $2 \cdot 01$ & bedX & 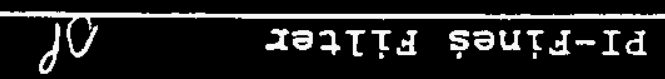 \\
\hline 090 & $\angle 5^{\circ} 0$ & $O L O$ & $\log$ & $O L \cdot \sigma$ & $\ln \theta$ & bedx & $\partial V$ aut s seb-JłO-Id \\
\hline & & & & & & bedx & 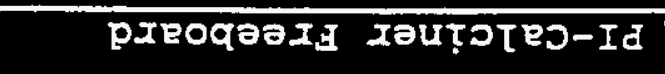 \\
\hline$p \zeta$ & $\varepsilon z$ & $\bar{z}$ & $\sigma$ & $\varepsilon \tau$ & rz & bedP & Seg buṬzṬpṬnTA-Ia \\
\hline 2LI & $2 t$ & $2 t 1$ & $2 t 1$ & $2 z 1$ & $7 t 1$ & bedX & Sed XoeqmoI8-Id \\
\hline & & & & & & & \\
\hline & & & & & & & \\
\hline & & & & & & 5 & $-\mathrm{IL}$ \\
\hline & & & & & & 20 & $-I \amalg$ \\
\hline Rिद्ध & क25 & 225 & 275 & 525 & 525 & 50 & pxeoqeax $x_{I}-I L$ \\
\hline हैछद्धि & 105 & EOS & 205 & 105 & 105 & 5 & pag xaddn-II \\
\hline EOS & $\sum O S$ & $\varepsilon O S$ & EQS & 295 & EOS & 5 & PEg xәMOI-IU \\
\hline उतह & bhe & 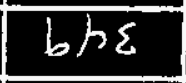 & $25 \varepsilon$ & $\varepsilon S \varepsilon$ & $\varepsilon S \varepsilon$ & 5 & seg GuțzTpțTI-Id \\
\hline & & & & & & 50 & 478g pə7eəH-II \\
\hline \multicolumn{6}{|c|}{ s6uṭpeay } & s7țun & xazaurexed \\
\hline \multicolumn{3}{|c|}{$t 17051^{745}$} & \multicolumn{3}{|c|}{ 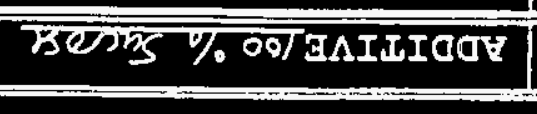 } & & $5-38 \mathrm{O} \cdot \mathrm{ON} 750 \mathrm{~L}$ \\
\hline
\end{tabular}

$\bar{\tau}$ 70 $\bar{\tau} 6 \mathrm{~d}$

78eqse7zd - 780T 8โE08-70TTd 
WHC-SD-WM-VI-031

Revision 0

Pilot-gcale rest - Datasheet

$\mathrm{Pg} \underline{2}$ of $\underline{2}$

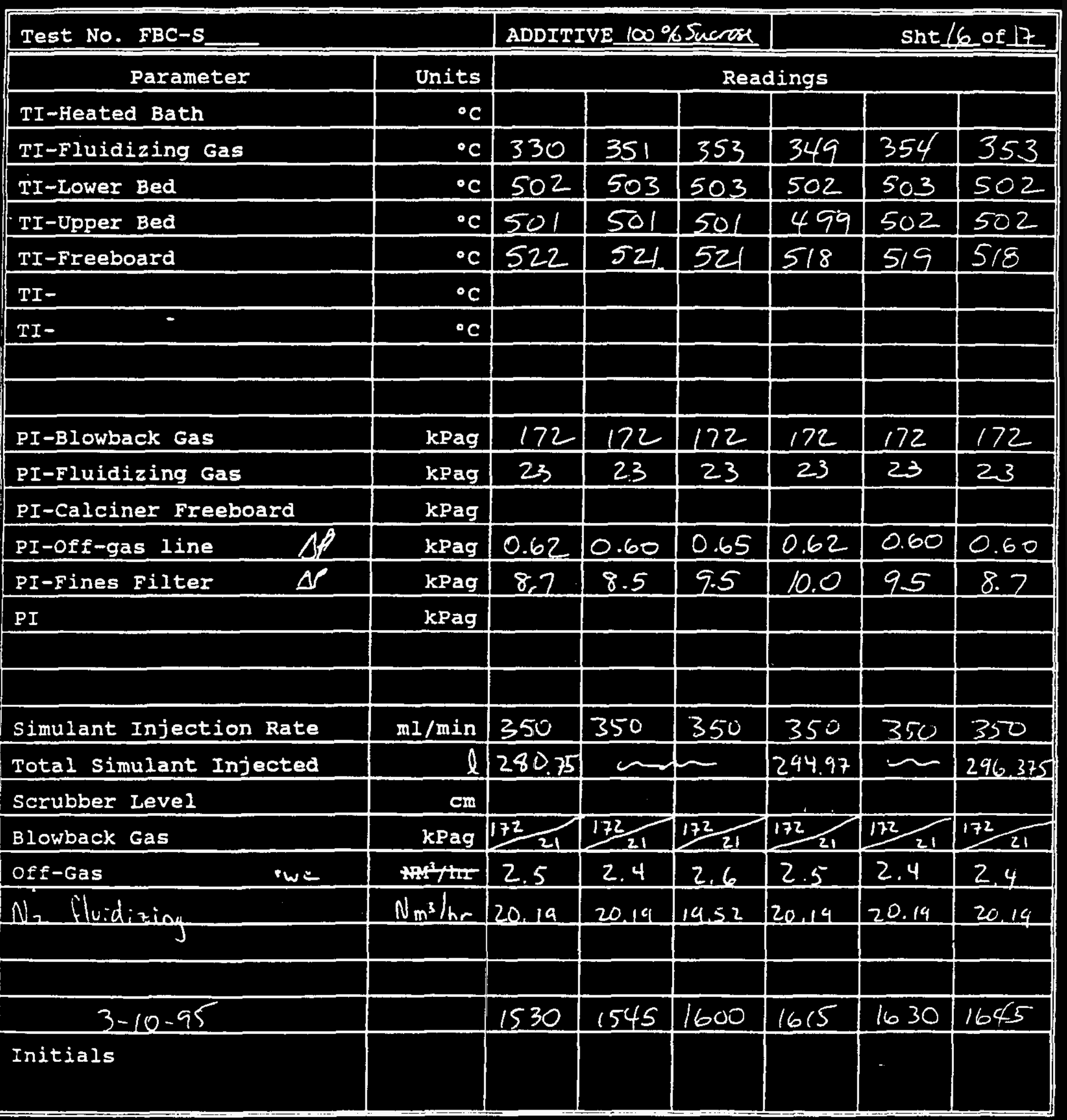


WHC-SD-WM-VI-031

Revision 0

Pilot-scale Test - Datasheet

$\mathrm{Pg} \underline{2}$ of 2

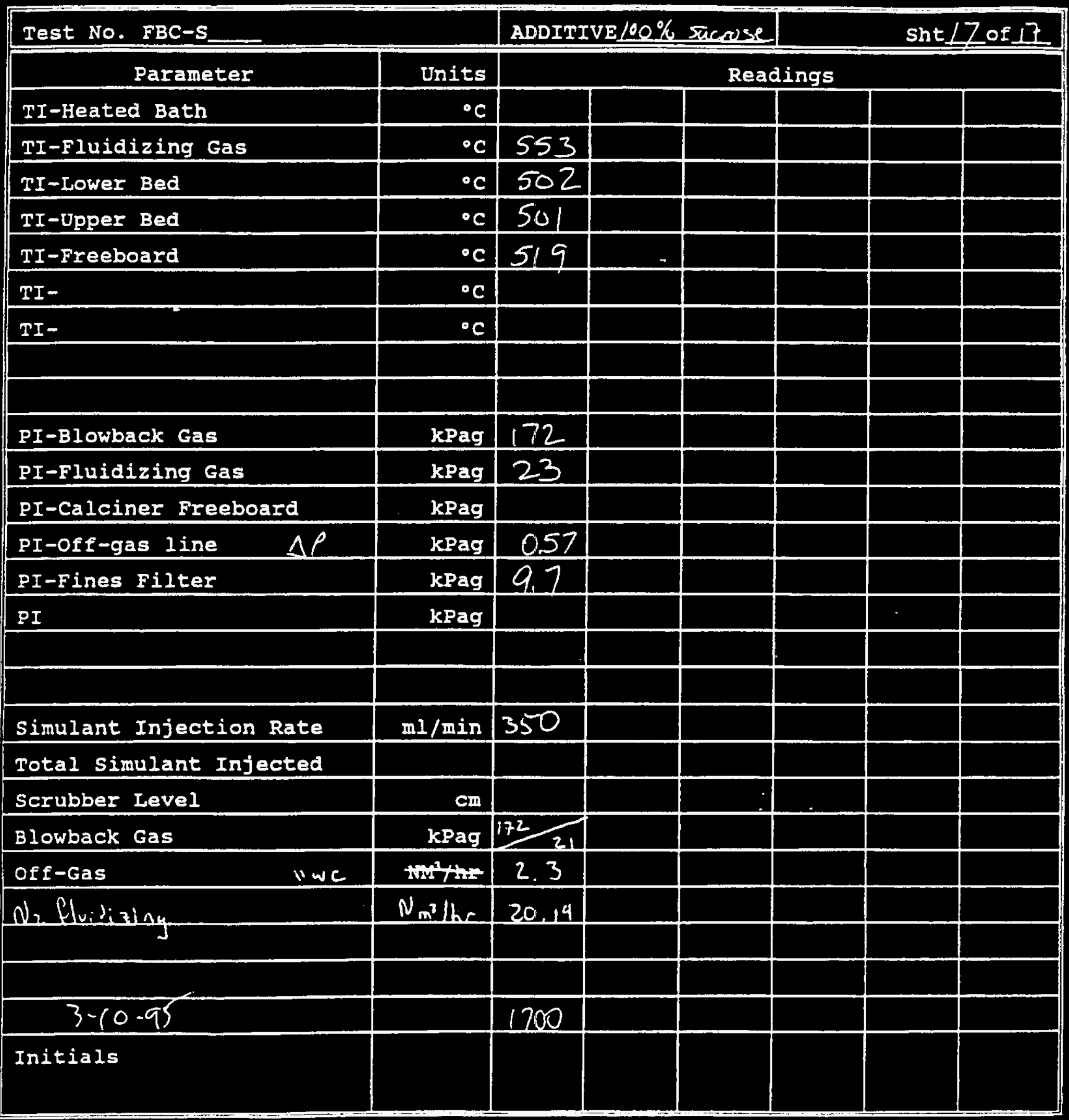


WHC-SD-WM-VI-031

Revision 0

\section{PART 2 - RVR DRYER/CALCINER TEST REPORT}


WHC-SD-WM-VI-031

Revision 0

RVR DRYER/CALCINER - TEST REPORT - FINAL

FOR

\section{EVALUATION OF MELTER SYSTEM TECHNOLOGIES FOR VITRIFICATION OF HIGH SODIUM CONTENT LLRW}

VECTRA GSI Report No. WHC-VIT-04

August 1995

for

WHC Contract No. MMI-SVV-384211

by

VECTRA Government Services, Inc.

2939 Richardson Road

Richland, WA 99352

(509) 375-3535

Prepared by:

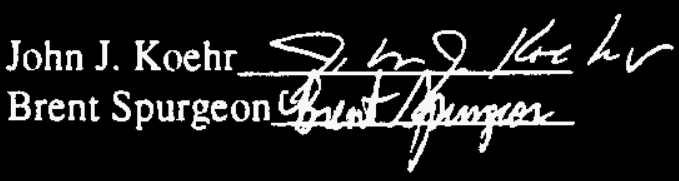

Approved by:

Brad Mason_f PMaton 
WHC-SD-WM-VI-031

Revision 0

\section{CONTENTS}

1.0 INTROOUCTION .................. 2-1

2.0 PURPOSE ....................... 2-1

3.0 CHRONOLOGY OF EVENTS ............... 2-1

4.0 EQUIPMENT OPERATING BEHAVIOR .......... 2-5

4.1 CHEMICAL AND GLASS FORMER MIXING, BATCHING, AND FEEDING ... 2-5

4.1.1 Simulant Feed System ............. 2-5

4.1 .2 Glass Former Feed System . . . . . . . . . . 2-6

4.2 OFF-GAS SYSTEM . . . . . . . . . . . 2-6

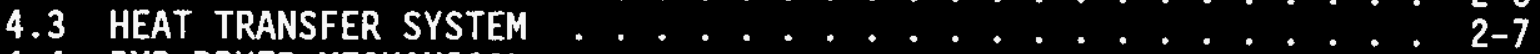

4.4 RVR DRYER MECHANICAL .............. 2- . . .

5.0 SCOPING TESTS AND OBSERVATIONS . . . . . . . . 2-8

5.1 TESTING WITH GLASS FORMERS AND WASTE FEED $. . . .2-8$

5.2 INITIAL RVR TESTING WITH LLRW SIMULANT AND GLASS FORMERS $: 2-10$

5.2.1 RVR-S1: Glass Former Mix C, Liquid Simulant, $100 \%$ Stoichiometric Sucrose Reductant .... . . 2-10

5.2.2 Glass Former Mix C, Liquid Simulant, 60\%

Stoichiometric Sucrose . . . . . . . 2-11

5.2.3 RVR-S3: Glass Former Mix C, Liquid Simulant, No Additives . . . . . 2-12

5.3 RVR LABORATORY SCALE OVEN TESTS W/ SUCROSE AND FORMIC ACID

REDUCTANTS . . . . . . . . . . 2-12

5.3.1 Oven Reaction Tests: Dried Glass Formers and

Simulant with $60 \%$ and $100 \%$ Dry Sucrose Reductant . . . . 2-13

5.3.2 Oven Reaction Tests: Glass Formers and Liquid

Simulant with $60 \%$ sucrose and $100 \%$ Formic Acid

Reductants ............ . 2- 2- . . . . .

5.4 RVR SIMULANT VACUUM DRYING PCP TESTS ........... 2-14

5.5 RVR TWO STAGE DRYING/REACTING .......... 2-15

5.5.1 RVR-S4: Pre-Dried Glass Formers and Simulant

W/ $60 \%$ Stoichiometric Sucrose . . . . . . . 2-16

5.5.2 RVR-S5: Pre-Dried Glass Formers and Simulant

5.5.3 RVR-S6: Pre-Dried GTass Formers and Simulant

W/ 100\% Stoichiometric Sucrose . . . . . . . 2-17

5.5.4 RVR-S7: Pre-Dried Glass Formers and Simulant

W/ 125\% Stoichiometric Sucrose ......... 2-18

5.6 SCOPING TEST EVALUATION SUMMARY ........... $2-19$

6.0 CONCLUSIONS ........................... 2- . . . . .

7.0 RECOMMENDATION FOR FUTURE TESTS ............. 2-19 
WHC-SD-WM-VI-031

Revision 0

CONTENTS (cont)

APPENDIXES

2A DATA SHEETS . . . . . . . . . . . . . . . . . . . 2-21

2B RVR TESTING SAMPLE LOG ....................... 2-51

\section{LIST OF TABLES}

2-1 RVR Dryer Testing Chronology Summary . . . . . . . . . . . . 2-2

2-2 Glass Former Mix B . . . . . . . . . . . . . . . . 2-8

2-3 Glass Former Mix C . . . . . . . . . . . . . . . . . 2-9

2-4 Oven Reaction Tests with Sugar and Formic Acid Reductants . . . . . 2-13

2-5 Product Generated During Two Stage RVR Drying Tests . . . . . . . 2-15

2-6 Average Steady State Bed Temperature Profiles Observed During

Second Stage RVR Drying Tests . . . . . . . . . . . . . 2-16

2-7 RVR Test Evaluation Summary .................... 2-20 
WHC-SD-WM-VI-031

Revision 0

PART 2 - REY DRYER/CALCINER TEST REPORT

\subsection{INTRODUCTION}

The Westinghouse Hanford Company (WHC) is conducting an evaluation of glass melter system technologies for vitrification of 1 iquid low-level radioactive waste (LLRW) streams consisting mostly of sodium nitrate and nitrite salts in alkaline liquid slurry form. As part of this technology evaluation, VECTRA will demonstrate the performance of the EnviroGlass Vitrification System feed and VE-SKULL melter subsystens. The RVR Bryer System is a potential Enviroglass ${ }^{\text {Th }}$ feed subsystem and is intended to dry, $m i x$, and react the 1 iquid waste simulant together with glass formers and reductant additives prior to vitrification.

\subsection{PURPOSE}

This test report documents accomplishment of the RVR Dryer/Calciner test objectives. Test objectives include the following:

2.1 Generate dry LLRW simulant for use in VE-SKULL" melter testing.

2.2 Demonstrate the ability of the VECTRA RVR Dryer to do the following:

Evaporate water to produce a dry, free flowing solid Volatize $\mathrm{NO}_{2}$ and $\mathrm{NO}_{3}$

Convert $\mathrm{NaNO}_{3}$ and $\mathrm{NaNO}_{3}$ salts to $\mathrm{Na}_{2} \mathrm{CO}_{3}$ salts

Reduce $\mathrm{NO}_{3}$ and $\mathrm{NO}_{2}$ to $\mathrm{N}_{2}$ gas

- Mix dry simulant with glass formers and reductant additive

2.3 Determine throughput of RVR Dryer/Calciner when operating within parameters for achieving objectives number one and two.

2.4 Determine efficiency of $\mathrm{NO}_{2} / \mathrm{NO}_{3}$ reductions using the following additives to dryer: sucrose $\left(\mathrm{C}_{12} \mathrm{H}_{22} \mathrm{O}_{11}\right)$, formic acid $\left(\mathrm{HCO}_{2} \mathrm{H}\right)$.

\subsection{CHRONOLOGY OF EVENTS}

A detailed chronology of RVR dryer scoping testing has been recorded in the Test $\log$ and data sheets. A brief summary is provided in Table 2-1. 
Table 2-1. RVR Dryer Testing Chronology Summary. (4 sheets)

\begin{tabular}{|c|c|}
\hline Dates & Activities/events \\
\hline $\begin{array}{l}3 / 14 / 95 \\
\text { (Water Feed) }\end{array}$ & $\begin{array}{l}\text { - Dry glass formers } \mathrm{W} / \text { boric acid (Mix } \mathrm{B} \text { ) } \\
\text { - Water feed, no simulant, no reductants } \\
\text { - Hot oil temp } 570^{\circ} \mathrm{F} \text {. } \\
\text { - Slurry leaks at rotor shaft packing seals } \\
\text { - Bed caking developed after approx. } 1 \text { hour } \\
\text { - Following cooldown, bed solidified due to presence of } \\
\mathrm{H}_{2} \mathrm{BO}_{3} \text { in bed }\end{array}$ \\
\hline $\begin{array}{l}3 / 16 / 95 \\
\text { (Water Feed) }\end{array}$ & $\begin{array}{l}\text { - Discharge weir removed to lower bed level for future } \\
\text { tests. } \\
\text { - Dry glass formers w/o boric acid (Mix } \mathrm{C} \text { ) } \\
\text { - Water feed, no simulant, no reductants } \\
\text { - Initial hot oil temp } 570^{\circ} \mathrm{F} \text {. Hot oil temp could not } \\
\text { be maintained above } 483^{\circ} \mathrm{F} \text {. } \\
\text { - Hard crust developed on wetted heat transfer surfaces } \\
\text { - Bed free-flowing, no solidification on cooldown. }\end{array}$ \\
\hline $\begin{array}{l}3 / 20 / 95 \\
\text { (Water Feed) }\end{array}$ & $\begin{array}{l}\text { - RVR dryer barrel external jacket insulated } \\
\text { - Dry glass formers w/o boric acid (Mix } \mathrm{C} \text { ) } \\
\text { - Water feed, no simulant, no reductants } \\
\text { - Hot oil temp maintained near } 570{ }^{\circ} \mathrm{F} \\
\text { - Minor caking observed, bed free flowing }\end{array}$ \\
\hline $\begin{array}{l}3 / 21 / 95 \\
\text { (RVR-S1) }\end{array}$ & $\begin{array}{l}\text { - Dry glass formers } w / 0 \text { boric acid (Mix } \mathrm{C} \text { ) } \\
\text { - Liquid LLW simulant injection } \mathrm{w} / \mathrm{100 \%} \text { sucrose } \\
\text { reductant } \\
\text { - Hot oil temp } 570{ }^{\circ} \mathrm{F} \\
\text { - } \text { Test duration approx. } 6 \text { hours } \\
\text { - Average NOx } 1353 \mathrm{ppm} \text {, average } \mathrm{CO} 446 \mathrm{ppm} \\
\text { - Off-gas carryover and leaks noted } \\
\text { - Periodic exothermic reactions raised bed } \\
\text { temperatures, hot oil system tripped on high } \\
\text { temperature. } \\
\text { - Peak NOx and CO observed during exothermic reaction } \\
\text { periods } \\
\text { - } 161.9 \mathrm{~kg}(357 \text { lbs) black product generated }\end{array}$ \\
\hline $\begin{array}{l}3 / 24 / 95 \\
(60 \% \text { sucrose) }\end{array}$ & $\begin{array}{l}\text { - Off-gas system modified } \\
\text { - More insulation and jacketing added to RVR dryer and } \\
\text { hot oil system piping } \\
\text {-dry glass formers } w / 0 \text { boric acid (Mix C) } \\
\text { - Liquid LLW simulant injection } \\
\text { - } 60 \% \text { sucrose reductant } \\
\text { - Hot oil temp } 570^{\circ} \mathrm{F} \\
\text { - Bag filter in new off-gas drum became plugged } \\
\text { - Bed developed cake, required clean-out } \\
\text { - No usable product generated }\end{array}$ \\
\hline
\end{tabular}


Table 2-1. RVR Dryer Testing Chronology Summary. (4 sheets)

\begin{tabular}{|c|c|}
\hline Dates & Activities/events \\
\hline $\begin{array}{l}3 / 25 / 95 \\
\text { ( } 60 \% \text { sucrose) }\end{array}$ & $\begin{array}{l}\text { - Dry glass formers w/o boric acid (Mix C) Repeat of } \\
\text { test from } 3 / 24 / 95 \text {. } \\
\text { - Liquid LLW simulant injection } \\
\text { - } 60 \% \text { sucrose reductant } \\
\text { - Hot oil temp } 570 \text { of } \\
\text { - Bed developed hard cake which required clean-out. } \\
\text { - No usable product generated } \\
\text { - Unable to duplicate results of RVR-Sl. }\end{array}$ \\
\hline $\begin{array}{l}3 / 26 / 95-3 / 27 / 95 \\
\text { (RVR-S3) }\end{array}$ & $\begin{array}{l}\text { - Dry glass formers w/o boric acid (Mix C) } \\
\text { - Liquid LLW simulant injection only, no reductants } \\
\text { - Hot oil temperature } 225-325{ }^{\circ} \mathrm{F} \\
\text { - Slow drying, wet zone migrated toward discharge end. } \\
\text { - Operated in drying mode for approx. } 5 \text { hours } \\
\text { - Severe bed caking resulted }>240{ }^{\circ} \mathrm{F} \\
\text { - } 81 \mathrm{~kg} \text { (179 lbs) dry glass formers and simulant } \\
\text { product generated }\end{array}$ \\
\hline $\begin{array}{l}3 / 27 / 95 \\
\text { (Oven Reaction } \\
\text { Testing) }\end{array}$ & $\begin{array}{l}\text { - Oven test: } 200 \mathrm{~g} \text { dry glass formers and simulant (RVR- } \\
\text { S1 product) } \\
\text { - Dry } 100 \% \text { stoich. sucrose reductant } \\
\text { - Oven temp } 530 \circ \mathrm{o} \text {. } \\
\text { - Dark black free-flowing product generated } \\
\text { - Oven test: } 200 \mathrm{~g} \text { dry glass formers and simulant } \\
\text { (RVR-S1 product) } \\
\text { - Ory } 60 \% \text { stoich. sucrose reductant } \\
\text { - Oven temperature } 530{ }^{\circ} \mathrm{F} \\
\text { - Lighter black free-flowing product generated }\end{array}$ \\
\hline $\begin{array}{l}3 / 29 / 95 \\
\text { (Oven Drying/ } \\
\text { Reaction Testing) }\end{array}$ & $\begin{array}{l}\text { - Oven test: dry glass formers } \\
\text { - Liquid LLRW simulant } \mathrm{W} / 60 \% \text { stoich. sucrose reductant } \\
\text { - Oven temperature } 530{ }^{\circ} \mathrm{F} \\
\text { - Dark black, hard cake formed } \\
\text { - Oven test: dry glass formers } \\
\text { - Liquid LLRW simulant } \\
\text { - Dry } 100 \% \text { stoich. formic acid reductant } \\
\text { - Formic reacted w/ } \mathrm{NaNO}_{2} \text { at room temperature, volatized } \\
\text { some NOx } \\
\text { - Oven temperature } 5300^{\circ} \mathrm{F} \\
\text { - Formic volatized prior to reacting with } \mathrm{NaNO}_{3} \\
\text { - Hard red cake formed upon cool ing indicating no } \\
\text { reaction and resulting melted salts }\end{array}$ \\
\hline
\end{tabular}


Table 2-1. RVR Dryer Testing Chronology Summary. (4 sheets)

\begin{tabular}{|c|c|}
\hline Dates & Activities/events \\
\hline $\begin{array}{l}3 / 29 / 95-3 / 31 / 95 \\
\text { (RVR Vacuum Dryer } \\
\text { PCP) }\end{array}$ & 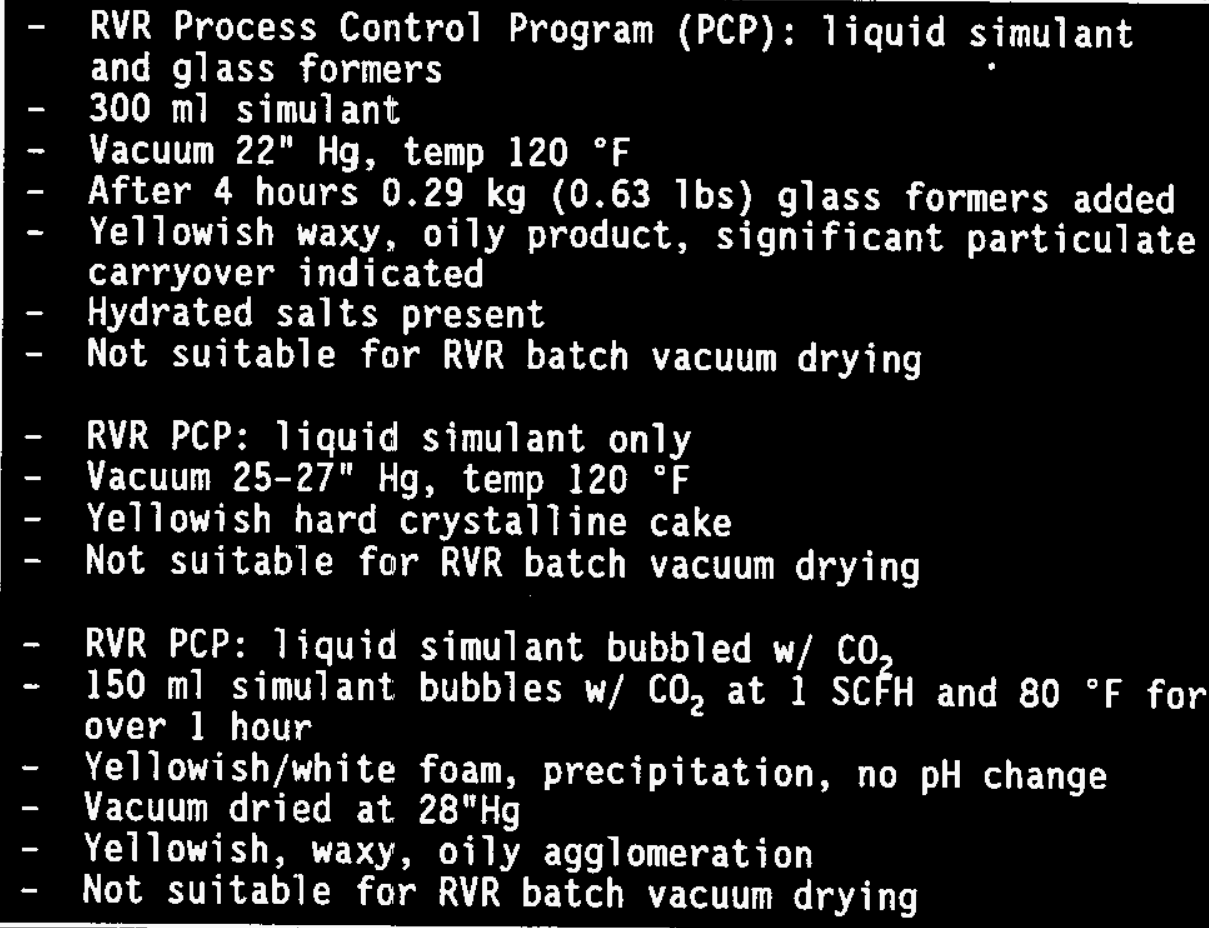 \\
\hline $\begin{array}{l}3 / 29 / 95-4 / 2 / 95 \\
\text { (RVR Drying) }\end{array}$ & $\begin{array}{l}\text { - RVR stage } 1 \text { drying: dry glass formers (Mix C), liquid } \\
\text { simulant, no reductants } \\
\text { - Hot oil } 230 \text { o } \mathrm{F} \\
\text { - } 195 \mathrm{~kg}(430 \mathrm{lbs} \text { ) additional dry reddish product } \\
\text { generated for use in stage } 2 \text { RVR reactions (RVR-S4 } \\
\text { through S7). Total of } 276 \mathrm{~kg}(609 \mathrm{lbs}) \text { dry RVR } \\
\text { product available. }\end{array}$ \\
\hline $\begin{array}{l}4 / 5 / 95 \\
\text { (RVR-S4) }\end{array}$ & $\begin{array}{l}\text { - RVR stage } 2 \text { reaction test: dry glass formers and } \\
\text { simulant (RVR 1st stage product) } \\
\text { - } 60 \% \text { stoich. sucrose } \\
\text { - Hot oil } 570{ }^{\circ} \mathrm{F} \\
\text { - Exothermic reactions ( } 574{ }^{\circ} \mathrm{F} \text { peak) } \\
\text { - Average N0x } 45.8 \mathrm{ppm} \text {, average C0 } 18.4 \mathrm{ppm} . \\
\text { - Minor caking } \\
\text { - Free flowing black product generated } \\
\text { - RVR stage } 2 \text { reaction test: dry glass formers and } \\
\text { - simulant (RVR lst stage product) } \\
\text { - } 80 \% \text { stoich. sucrose } \\
\text { - Hot oil } 5700^{\circ} \mathrm{F} \\
\text { - Exothermic reactions (653 }{ }^{\circ} \mathrm{F} \text { peak) } \\
\text { - Average NOx } 68.2 \text { ppm, average CO } 36.1 \mathrm{ppm} \\
\text { - Free flowing black product generated }\end{array}$ \\
\hline
\end{tabular}


WHC-SD-HM-VI-031

Revision 0

Table 2-1. RVR Dryer Testing Chronology Summary. (4 sheets)

\begin{tabular}{|c|c|}
\hline Dates & Activities/events \\
\hline $\begin{array}{l}\text { 4/11/95 } \\
\text { RVR-S6 }\end{array}$ & $\begin{array}{l}\text { - RVR stage } 2 \text { reaction test: dry glass formers and } \\
\text { simulant (RVR 1st stage product) } \\
\text { - } 100 \% \text { stoich. sucrose } \\
\text { - Hot oi } 570^{\circ} \mathrm{F} \\
\text { - Exothermic reactions ( } 573{ }^{\circ} \mathrm{F} \text { peak) } \\
\text { - Average NOx } 185.4 \mathrm{ppm} \text {, average CO } 52.0 \mathrm{ppm} \\
\text { - Free flowing black product generated }\end{array}$ \\
\hline RVR-S7 & $\begin{array}{l}\text { - RVR stage } 2 \text { reaction test: dry glass formers and } \\
\text { simulant (RVR lst stage product) } \\
\text { - } 125 \% \text { stoich. sucrose } \\
\text { - Hot oil } 570 \text { of } \\
\text { - Exothermic reactions ( } 587{ }^{\circ} \mathrm{F} \text { peak) } \\
\text { - Average NOx } 220.0 \mathrm{ppm} \text {, average CO } 60.5 \mathrm{ppm} \\
\text { - Free flowing black product generated }\end{array}$ \\
\hline
\end{tabular}

\subsection{EQUIPNENT OPERATING BEHAVIOR}

\subsection{CHEMICAL AND GLASS FORNER MIXING, BATCHING, AND FEEDING}

\subsubsection{Simulant Feed System}

DSSF simulant was provided in DOT 17H 55-gallon drums. Drums were staged adjacent to the RVR feed tank (Waste Hold-up Tank). The drum was opened and fitted with an electric mixer, drum pump, and splash lid. DSSF simulant was thoroughly mixed within the drum and transferred via the installed drum pump and hose directly into the RVR feed tank.

A 378 liter (100 gal) stainless steel RVR feed tank was equipped with a top mounted electric tank mixer. The tank was calibrated for liquid level by means of visual level indication in the sight glass. As needed, calculated simulant quantities were pumped from the simulant drums until the desired RVR feed tank level was achieved. For testing requiring addition of sucrose $\left(\mathrm{C}_{12} \mathrm{H}_{22} \mathrm{O}_{11}\right)$ reductant, dry sucrose was manually added to the RVR feed tank in weighed quantities necessary to achieve desired stoichiometric concentration. The RVR feed tank mixer was continuously operated during batching and subsequent simulant feeding to assure a homogeneous slurry.

Liquid slurry was pumped from the RVR mix tank into the RVR dryer via a stainless steel tubing feed line using a variable speed, positive displacement, peristaltic tubing pump. The simulant feed pump stainless steel suction tubing was provided with a connection for water. This connection was utilized for water injection testing and to flush the feed line following simulant injection. A separate stainless steel tubing feed line was also installed for liquid formic acid injection but never used. 
WHC-SD-WM-VI-031

Revision 0

\subsubsection{Glass Former Feed System}

A variable speed dry product feeder/mixer was utilized for dry glass former feed. The feeder hopper could hold over $45 \mathrm{~kg}$ (100 lbs) of dry material. During RVR dryer operation, the feeder was kept full above the top of the discharge pipe in order to prevent dryer off-gas from escaping through the dry feed line.

Dry glass formers were premixed by the supplier and shipped in $34 \mathrm{~kg}$ (75 1b) bags to VECTRA's Richland, Washington facility. Prior to each test, calculated dry glass former quantities were weighed and added to the dry feeder hopper. During stage two reaction scoping tests (RVR-S4, S5, S6, and S7) dry sucrose reductant was calculated for desired stoichiometric ratio, weighed, premixed with glass formers, and added to the feeder hopper.

In order to achieve proper weight percentages of constituents in the RVR discharge, the dry glass former feed rate and 1 iquid slurry feed rate were carefully balanced based on calculated ratios.

\subsection{OFF-GAS SYSTEN}

The off-gas system for RVR Dryer testing up to and including RVR-S1 $(3 / 21 / 95)$ consisted of a spray scrubber/condenser (T-SCB), a scrubber discharge vent blower (P-VS), a vent separator tank (T-VS), site vent stack and dilution ventilation blower (P-SB). Accumulated condensate and liquid carryover in the spray scrubber was pumped to a drain tank using an air driven diaphragm drain pump. Scrubber spray flow was provided by a centrifugal pump which took a suction on the drain tank and discharged into the scrubber spray header. The drain pump and spray pump were operated cont inuously to recirculate the scrubber. The small scrubber discharge blower took a suction on the scrubber and discharged off-gas into the vent separator tank. The spray scrubber and scrubber discharge blower were physically located within the test building, in close proximity to the RVR dryer. A short run of hose (less than 10') ran directly from the top of the RVR dryer to the spray scrubber.

During test RVR-S1 (100\% sucrose) significant carryover was observed in the off-gas system as evidenced by the accumulation of glass former material in the scrubber tank. Additionally, off-gas fumes were observed leaking out of the ductwork at the scrubber discharge blower into the test area atmosphere.

Following RVR-S1 testing, the off-gas system was modified. The scrubber and small vent fan were retired in place. Vent flow was directed from the RVR dryer lid into a modified 55 gallon drum which served as a dry product/liquid carryover collection reservoir. The drum outlet was fitted with a bag filter. off-gas flow traveled through the filter, off-gas hoses, and off-gas

instrumentation to the suction side of a vent blower (located outside the test building) which discharged into the vent separator. The vent separator interfaced with centrifugal pumps for continuous vent separator draindown to a large poly tank and continuous vent separator return spray flow from the poly tank. The vent separator was vented into the stack and joined dilution air flow at the discharge of a large vent blower. 
WHC-SD-WM-VI-031

Revision 0

During subsequent testing, the off-gas system was further modified by removal of the bag filter mounted within the 55-gallon collection reservoir.

\subsection{HEAT TRAWSFER SYSTEM}

During initial testing with dry glass former feed and water injection $(3 / 16 / 95)$, hot oil inlet temperature setpoint could not be maintained by heater $\mathrm{H}-\mathrm{HO}(40 \mathrm{kw})$. In order to avoid limiting liquid feed rates to below acceptable levels, additional thermal insulation was added to the RVR dryer barrel. The addition of insulation significantly reduced thermal losses to ambient and a more stable hot oil inlet temperature was maintained during subsequent testing with water injection.

Additional insulation and jacketing was added to the RVR barrel, top, and hot oil system piping following test RVR-S1 (3/21/95). No further difficulty was experienced in maintaining hot oil inlet temperature.

\subsection{RYR DRYER MECHANICAL}

During initial testing with dry glass former and water feed, liquid slurry was observed leaking from the RVR paddle shaft packing seals when bed level was raised above the paddles. In order to minimize leakage of slurry from the paddle shaft seals, the RVR bed discharge weir was removed to allow earlier discharge of dry product and limit the bed height. This effectively limited the total bed quantity to approximately $23 \mathrm{~kg}$ (50 1bs) of material, which is approximately half the maximum bed capacity.

Hot oil leaks were observed at the rotary joints connecting hot oil system hoses to the counter-rotating RVR dryer shafts. Repair required parts for rebuilding the rotary joint seals. Parts were not available, and the maintenance was not performed. Since leakage was minor, testing continued.

During the initial testing with glass formers (boric acid) and water injection (3/14/95), severe bed hardening restricted movement of the rotary paddles and caused the RVR dryer drive motor to trip on over-torque (overcurrent). No equipment damage was observed.

During test RVR-S3 $(3 / 27 / 95)$, the RVR drive motor chain had become loose and required tightening. No subsequent problems were experienced with the drive unit.

During many RVR dryer tests, hard caking developed within the dryer bed, on barrel wall surfaces, and on the paddles. Due to the configuration of the equipment and the composition of the dried material, this hard, water soluble cake was difficult to remove. 
WHC-SD-WM-VI-031

Revision 0

\subsection{SCOPING TESTS AND OBSERVATIOMS}

The continuous RVR dryer, hot oil system, off-gas system, simulant and glass former feed systems, and CEMS were installed at VECTRA's Richland, Washington facility. All RVR scoping tests were performed in Richland.

\subsection{TESTING WITH GLASS FORNERS AND WASTE FEED}

Following RVR Oryer initial check-out and calibration of equipment and instrumentation, initial testing was required to determine acceptability of the glass former mix, envelope heat transfer performance of the RVR dryer, and observe drying characteristics with injection of water only. This testing was performed from $3 / 14 / 95$ to $3 / 20 / 05$ and was in addition to the testing detailed in the Test Plan. Following initial testing, Scoping Test Procedure RVR-Sl was performed on $3 / 21 / 95$.

Glass formers were premixed to the composition of Mix B shown in Table 2-2. Note that this is the same weight percent as Mix B used in the Fluid Bed Calciner testing (WHC-VIT-03).

On 3/14/95, the RVR dryer was preheated to $570^{\circ} \mathrm{F}$ with the hot 0 il system operating. The RVR counter-rotating mixing paddles were energized at $30 \mathrm{rpm}$. The dry glass former feeder was filled with pre-mixed glass formers and energized for an initial dry feed rate of $68 \mathrm{~kg} / \mathrm{hr}(150 \mathrm{lb} / \mathrm{hr})$. Once the initial RVR bed was established, water injection began at $800 \mathrm{ml} / \mathrm{min}$.

Liquid slurry was observed leaking from the RVR paddle shaft seals when bed level was raised above the paddles. Additionally, rapid bed caking and coating of wetted heat transfer surfaces (paddles and barrel) were experienced. After approximately 1 hour, the RVR paddle motor tripped on over-torque resulting from excessive bed caking. The hot oil system was shutdown and the bed allowed to cooldown. Following cooldown, the bed solidified to an extremely hard cake throughout.

Table 2-2. Glass Former Mix B.

\begin{tabular}{|l|c|}
\hline \multicolumn{1}{|c|}{ Glass former } & Weight percent \\
\hline Silica sand & 59.69 \\
\hline Boric acid & 16.19 \\
\hline Dolomite & 10.89 \\
\hline Alumina & 11.48 \\
\hline Iron oxide & 1.04 \\
\hline Magnesium carbonate & 0.71 \\
\hline Total & 100.0 \\
\hline
\end{tabular}


It is believed that the initial caking developed due to melting boric acid which solidified upon cooling, forming an extremely hard cake.

Adjustments were made to minimize problems observed during the initial test run. The glass former mixture was reformulated to remove boric acid. The resulting RVR dryer test glass former Mix $\mathrm{C}$ is shown in Table 2-3. In order to minimize leakage of slurry from the paddle shaft seals, the RVR bed discharge weir was removed to allow earlier discharge of dry product and 1 imit the bed height. The RVR bed and heat transfer surfaces were cleaned out. The hard dry cake was discovered to be water soluble.

On 3/16/95 the RVR dryer was preheated to $570^{\circ} \mathrm{F}$ with the hot oil system. After heat-up, the paddles were energized and dry glass formers were fed into the bed. The dry glass former feeder was filled above the feed nozzle in order to seal off the potential vent path. The scrubber water spray flow was initiated and the vent fan was energized. Water injection was initiated at $500 \mathrm{ml} / \mathrm{min}$. After about 1 hour, hot oil system inlet temperature dropped to $483{ }^{\circ} \mathrm{F}$ with outlet at $477^{\circ} \mathrm{F}\left(6^{\circ} \mathrm{F}\right.$ differential temperature). The steady state bed temperature profile started with boiling $\left(212^{\circ} \mathrm{F}\right)$ in the first $25 \%$ of the bed, heat-up in the second $25 \%$ of the bed $\left(300^{\circ} \mathrm{F}\right)$, bed temperature peak in the bed center $\left(453^{\circ} \mathrm{F}\right)$, and gradual cooldown toward the discharge end of the bed $\left(416^{\circ} \mathrm{F}\right)$. Dry product was produced. Minor caking developed on wetted heat transfer surfaces, but the bed was free-flowing. No additional caking or hardening was observed following bed cooldown, confirming the previously observed results attributed to-boric acid.

The heat transfer capability of the hot oil system appeared to be limiting feed rates and temperatures. It was hoped that subsequent tests with simulant (lower percentage of water) would allow higher feed rates.

Additionally, insulation was added to the RVR dryer barrel to reduce heat losses to ambient.

Table 2-3. Glass Former Mix C.

\begin{tabular}{|l|c|}
\hline \multicolumn{1}{|c|}{ Glass former } & Weight percent \\
\hline Silica sand & 71.3 \\
\hline Boric acid & 0.0 \\
\hline Dolomite & 13.0 \\
\hline Alumina & 13.6 \\
\hline Iron oxide & 1.3 \\
\hline Magnesium carbonate & 0.8 \\
\hline Total & 100.0 \\
\hline
\end{tabular}


WHC-SD-WM-VI-031

Revision 0

On $3 / 20 / 95$, the RVR dryer was again tested with glass former mix $C$ and water injection to evaluate the effectiveness of the added insulation. Hot oil temperature dropped slowly to $566^{\circ} \mathrm{F}$ with a $9{ }^{\circ} \mathrm{F}$ differential temperature. Similar to previous testing, the resulting steady state bed temperature profile started with boiling $\left(210^{\circ} \mathrm{F}\right)$ in the first $25 \%$ of the bed, heat-up in the second $25 \%$ of the bed $\left(423^{\circ} \mathrm{F}\right)$, temperature peak in the bed center $\left(533^{\circ} \mathrm{F}\right)$, and gradual cooldown toward the discharge end of the bed $\left(514^{\circ} \mathrm{F}\right)$. Dry product was produced. Similar to previous test results, minor caking was observed on wetted heat transfer surfaces, but the bed was free-flowing. A dry product generation rate of $1001 \mathrm{~b} / \mathrm{hr}$ was projected.

\subsection{INITIAL RYR TESTING WITH LLRW SINULANT AND GLASS FORMERS}

Initial RVR drying runs were made feeding liquid LLRW simulant with different reductant additives and dry glass former mix C. A planned test (RVR-S2) using formic acid reductant was deleted based on oven reaction crucible test results.

\subsubsection{RVR-S1: Glass Former Mix C, Liquid Simulant, 100\% Stoichiometric Sucrose Reductant}

On $3 / 21 / 95$ the RVR dryer was preheated to $570{ }^{\circ} \mathrm{F}$ in preparation for test RVR-S1. Sucrose and liquid simulant were mixed in the feed tank to achieve $100 \%$ stoichiometric sucrose concentration (110 grams sucrose/1iter simulant). Dry glass former feed and liquid simulant/sugar feed were initiated into the RVR. Dry feed and liquid simulant/sugar feed were balanced to achieve a target $20 \% \mathrm{Na}_{2} \mathrm{O}$ concentration in the final glass form.

The RVR dryer was operated continuously for over 5 hours. During this time period, simulant injection rate was maintained at $425 \mathrm{ml} / \mathrm{min}$ and balanced dry glass former feed rate was maintained at $45.4 \mathrm{~kg} / \mathrm{hr}(100 \mathrm{lb} / \mathrm{hr})$. Plugging in the liquid simulant feed line temporarily forced feed shutdown and replacement of a feed line valve.

Throughout testing, hot $0 i 1$ system supply temperature averaged $566{ }^{\circ} \mathrm{F}$ with a $5.5^{\circ} \mathrm{F}$ differential temperature. "Steady state" was achieved after approximately 1 hour of operation. Average temperature profile was near boiling $\left(222^{\circ} \mathrm{F}\right)$ in the first $25 \%$ of the bed, heating up ( $\left.385^{\circ} \mathrm{F}\right)$ in the second $25 \%$ of the bed, peak temperature $\left(558^{\circ} \mathrm{F}\right)$ in the center of the bed, and gradual cooldown ( $507^{\circ} \mathrm{F}$ ) through the last half of the bed. Average NOx and c0 levels of $1353 \mathrm{ppm}$ and $446 \mathrm{ppm}$, respectively, were observed in the off-gas.

Sucrose reaction with the simulant was noted on several occasions due to exothermic reaction, as evidenced by NOX and CO spikes in the off-gas, and black colored product. The reaction initially began in the center of the bed. Temperatures in excess of $600^{\circ} \mathrm{F}$ were observed. As the reaction progressed, the temperature peak moved toward the bed inlet until slurry was encountered and temperature dropped. The temperature peak would then move back toward the center of the bed. The cycle repeated several times. At one point the exotherm was so extreme that the hot oil outlet temperature increased above the inlet temperature to the high temperature trip setpoint. NOx and CO spikes in the off-gas were observed simultaneously. 
WHC-SD-WM-VI-031

Revision 0

A total of $161.9 \mathrm{~kg}$ (357 lbs) of dried product was produced. Samples were drawn. Significant carryover was observed in the off-gas system as evident by the accumulation of glass former material in the scrubber tank. Additionaliy, off-gas fumes were observed leaking out of the ductwork at the vent fan connection into the test area atmosphere. Modification to the offgas system was necessary.

\subsubsection{Glass Former Mix C, Liquid Simulant, 60\% Stoichiometric Sucrose}

Following the results of the RVR-S1 testing, the off-gas system was modified. The scrubber and small vent fan were retired in place. Vent flow was directed from the RVR dryer 1 id into a modified 55 gallon drum which served as a dry product/liquid carryover collection reservoir. The drum outlet was fitted with a bag filter. Off-gas flow traveled through the filter, off-gas hoses, and off-gas instrumentation to the suction side of a vent fan (located outside the test building) which discharged into the vent separator. The vent separator flow loop contained centrifugal pumps for continuous vent separator draindown to a large poly tank and continuous vent separator return spray flow from the poly tank. The vent separator was vented into the stack and joined dilution air flow at the discharge of a large vent
blower.

In addition to the off-gas system modification, additional insulation and jacketing material was added to the RVR dryer barrel and hot oil system
piping.

On $3 / 24 / 95$, the impact of reducing the sucrose stoichiometric ratio to $60 \%$ was investigated. The RVR dryer was once again pre-heated to $570^{\circ} \mathrm{F}$. $60 \%$ stoichiometric sucrose was mixed in with the liquid LLRW simulant in the feed tank to achieve 51 grams sucrose per 1 iter of simulant. Balanced dry glass $825 \mathrm{ml} / \mathrm{min}$, respectively.

Excessive glass former carryover was again observed and resulted in rapid plugging of the off-gas system bag filter. Additionally, the RVR bed developed severe caking on wetted heat transfer surfaces. Testing was terminated early to clean-out the bed and remove the off-gas system bag
filter. No usable dry product was generated.

On $3 / 25 / 95$, following bed clean-out and removal of the off-gas filter, the $60 \%$ stoichiometric sucrose testing cont inued. The RVR dryer was once again heated to $570^{\circ} \mathrm{F}$. Balanced dry glass former feed and liquid simulant feed were initiated at $60 \mathrm{lb} / \mathrm{hr}$ and $400 \mathrm{ml} / \mathrm{min}$ respectively.

Rapid bed caking resulted and required shutdown after less than 1 hour operation. A hard cake had developed on wetted heat transfer surfaces requiring dryer clean-out. No usable product was generated. The reasonably steady state operating conditions and periodic sucrose reactions experience during the RVR-S1 testing could not be duplicated. 


\subsubsection{RVR-S3: Glass Former Mix C, Liquid Simulant, No Additives}

Due to the 1 imited success with $100 \%$ stoichiometric sucrose and no success with $60 \%$ stoichiometric sucrose, investigation of the impact of drying simulant with no reductants was necessary. Since high temperatures were causing bed caking, dryer performance was investigated at lower temperatures.

On $3 / 26 / 95$ the RVR dryer was heated up to $225^{\circ} \mathrm{F}$. Balanced dry glass former (mix C) feed and liquid simulant feed were initiated at $60 \mathrm{lb} / \mathrm{hr}$ and $400 \mathrm{ml} / \mathrm{min}$, respectively. A reasonable flat temperature profile was observed across the bed with gradual heat-up averaging from $140^{\circ} \mathrm{F}$ at the beginning of the bed to $188^{\circ} \mathrm{F}$ at the discharge end of the bed. Extremely slow drying rates were observed. The bed became wet throughout with moist product discharging. Dry and liquid feeds were secured to allow the bed to dry out. When paddle caking began to develop, testing was secured. The bed required clean-out prior to resumption of testing.

On the morning of $3 / 27 / 95$ the RVR dryer was preheated to $235^{\circ} \mathrm{F}$. Dry glass former feed was initiated at $601 \mathrm{~b} / \mathrm{hr}$. After $1-1 / 2$ hours cont inuous operation with dry feed only a dry product discharge rate of $54 \mathrm{lb} / \mathrm{hr}$ was observed. Balanced liquid simulant/sucrose injection was initiated at $300 \mathrm{ml} / \mathrm{min}$. As drying cont inued, the wet portion of the bed was observed steadily extending toward the discharge end of the bed. Hot oil inlet temperature was gradually increased to $340{ }^{\circ} \mathrm{F}$ over a 1 hour period before the wet zone was observed to trave? back toward the front half of the bed.

Bed caking was observed after hot oil temperature was raised above $240{ }^{\circ} \mathrm{F}$. Reasonably flat temperature profiles were observed across the bed with gradual heating throughout with an average heat-up of $53^{\circ} \mathrm{F}$ across the bed. After about 3-1/2 hours of continuous operation at $340^{\circ} \mathrm{F}$ a hard cake developed on the wetted heat transfer surfaces and the RVR dryer was secured. A total of $81 \mathrm{~kg}(179 \mathrm{lb})$ of dry product was produced.

In order to generate dry product for subsequent RVR scoping tests RVR-S4 through S7 (described later), additional RVR drying with glass formers (mix C), 1 iquid simulant, and no reductants was performed from $3 / 29 / 95$ to $4 / 2 / 95$. Hot oil temperature was 1 imited to $230^{\circ} \mathrm{F}$. A total of $195 \mathrm{~kg}$ (430 1b) of additional dried glass former/simulant (no additives) was produced.

\subsection{RVR LABORATORY SCALE OVEN TESTS W/ SUCROSE AND FORMIC ACID REDUCTANTS}

Based on the limited success with drying and reacting glass formers and simulant with one pass through the continuous RVR dryer, and the resulting excessive dryer clean-up requirements, laboratory scale tests were initiated to investigate drying and reacting options. 


\subsubsection{Oven Reaction Tests: Dried Glass formers and Simulant with $60 \%$ and $100 \%$ Dry Sucrose Reductant}

Oven reaction tests were performed to determine if a dried RVR product of glass formers and simulant (no reductants) would react at high temperatures in the presence of dry sucrose reductant at $60 \%$ and $100 \%$ stoichiometric ratios.

On $3 / 27 / 95$, two separate crucibles were prepared with approximately $200 \mathrm{~g}$ of dry glass formers and simulant (no reductants) produced with the RVR dryer during test RVR-S3 (3/27/95). This material was reddish in color. Dry sucrose was added to the two crucibles one with $100 \%$ and the other with $60 \%$ stoichiometric ratio. The dry sucrose was white in color.

Both crucibles were placed in a laboratory oven and slowly heated to $530^{\circ} \mathrm{F}$. After approximately 1 hour, both crucibles were observed to have reacted. The product in the $100 \%$ crucible was a homogeneous, dark black, free flowing, dry powder. The $60 \%$ crucible was similar in appearance, but a lighter color.

\subsubsection{Oven Reaction Tests: Glass Formers and Liquid Simulant with $60 \%$ sucrose and $100 \%$ Formic Acid Reductants}

Oven reaction tests were performed to determine if dry glass formers, liquid simulant, and liquid formic acid reductant could be successfully dried and reacted to produce a desired product in a single step. Additionaliy, ability to process dry glass formers and 1 iquid simulant with $60 \%$ sucrose reductant was also investigated in an effort to compare laboratory results with previous (poor) RVR dryer test results. On $3 / 29 / 95$, two crucibles were prepared as described in Table 2-4.

Brownish fumes (NOx) were observed when the formic acid was added to crucible 1.

Both crucibles were placed in a laboratory oven, slowly heated to $330{ }^{\circ} \mathrm{F}$ and held for 90 minutes. The crucibles were then heated to $530^{\circ} \mathrm{F}$ and held for 1 hour.

Table 2-4. Oven Reaction Tests with Sugar and Formic Acid Reductants.

\begin{tabular}{|l|c|c|}
\hline \multicolumn{1}{|c|}{ Constituent } & Crucible 1 & Crucible 2 \\
\hline Dry glass formers, mix C (grams) & 153.2 & 98 \\
\hline Liquid simulant (m1) & 134 & \\
\hline Liquid simulant with 60\% sucrose (m1) & & 88 \\
\hline Liquid formic acid (ml) & 60 & \\
\hline
\end{tabular}


Bubbles were observed in crucible 1 (formic). The reddish product appeared slightly molten and not completely dried. A film formed in crucible 2 (sucrose). The crucible 2 product was black, not completely dry, and slightly molten with evidence of foaming. The product in both crucibles solidified to a hard material following removal from the oven and cooldown.

The fumes observed after initial addition of formic acid to crucible 1 were believed to be from volatilization of $\mathrm{NO}_{2}$ from formic acid reaction with the $\mathrm{NaNO}_{2}$ in the simulant at room temperature. It was believed that residual formic acid then volatized at higher temperatures prior to reacting with the $\mathrm{NaNO}_{3}$ in the simulant and the resulting hard product in crucible 1 was due to melting of salts in the simulant. Based on the results of the formic acid laboratory scale testing, test RVR-S2 (100\% stoichiometric formic acid) was not performed.

\subsection{RVR SINULANT YACUUM DRYING PCP TESTS}

As discovered in test RVR-S3, a hard cake develops on the RVR dryer heat transfer surfaces when dry glass formers and liquid simulant are mixed with hot oil temperature greater than $240^{\circ} \mathrm{F}$. High temperatures $\left(>570^{\circ} \mathrm{F}\right)$ are required to produce the desired reaction between sucrose and simulant. Based on results of previous oven tests, pre-dried glass formers and simulant will react with dry sucrose reductant at high temperatures. In order to produce dry product at a low temperature and react at a high temperature, investigation of a two stage drying/reacting alternative was necessary.

Drying of simulant using the atmospheric RVR continuous dryer was observed to be extremely slow with hot oil temperatures 1 imited to $240{ }^{\circ} \mathrm{F}$. Since liquid saturation temperatures are lower and evaporation rates are higher with in a vacuum, VECTRA RVR vacuum dryer Process Control Program (PCP) tests were performed to determine if 1 iquid simulant could be successfully dried by the batch in VECTRA's RVR Vacuum Dryer.

On $3 / 29 / 95$, a $P C P$ test was performed to dry liquid simulant and glass formers. Liquid simulant $(300 \mathrm{ml})$ and a magnet ic stirring bar were added to a flask. The flask was placed on a magnetic stirring/hot plate and connected to the PCP vacuum and condensate system. A vacuum of 22 " $\mathrm{Hg}$ was drawn on the flask, stirring was initiated, and heat-up to $120^{\circ} \mathrm{F}$ commenced. Significant particulate carryover was noted. After 4 hours of boiling, $0.29 \mathrm{~kg}(0.63 \mathrm{lbs})$ of dry glass former mix (mix C) was added to the flask. The final dried product was a yellowish, oily, waxy agglomeration. The dried product was water soluble.

On $3 / 30 / 95$, a PCP was performed to dry liquid simulant at lower temperatures (higher vacuum). Liquid simulant $(150 \mathrm{ml})$ were added to a flask and 27" $\mathrm{Hg}$ vacuum was drawn in the flask. Stirring and heat-up were initiated. After 30 minutes, the flask was boiling at $93^{\circ} \mathrm{F}$. Forty minutes later, the flask was boiling at $97^{\circ} \mathrm{F}$ and a vacuum of $25^{\prime \prime} \mathrm{Hg}$. Solution internal temperature was measured at $119.2^{\circ} \mathrm{F}$. The final dried product was a hard crystalline substance caked to the bottom of the flask with a soft, oily material around the flask bottom radius. The dried product was water soluble. This product was determined to be unsuitable for RVR vacuum dryer processing. 
WHC-SD-WM-VI-031

Revision 0

Inability to vacuum dry liquid simulant was in part attributed to the presence of hydrated salts, which held on to unliberated water at low temperatures. Conversion of hydrated salts within the simulant to carbonates by exposure to $\mathrm{CO}_{2}$ was investigated.

On 3/31/95 $150 \mathrm{ml}$ of liquid simulant was added to a flask. Initial pH was measured at greater than 10.6. $\mathrm{CO}_{2}$ was bubbled through the solution in the flask at 1 SCFH while the flask was stirred and heated to $80^{\circ} \mathrm{F}$. After 30 minutes of $\mathrm{CO}_{2}$ exposure, a yellowish/white film formed on the top of the solution and precipitates began to form in the bottom of the flask. The $\mathrm{pH}$ was still greater than 10.6. After over 1 hour of $\mathrm{CO}_{2}$ exposure, the $\mathrm{CO}_{2}$
bubbling was stopped.

The flask with simulant was connected to the laboratory vacuum and condensate systems, 28" $\mathrm{Hg}$ vacuum was drawn and stirring and heat-up initiated. After 2-1/2 hours, vacuum drying was complete. The final dried product formed a waxy agglomeration on the bottom of the flask. This product was determined to be unsuitable for RVR vacuum dryer processing.

\subsection{RYR TWO STAGE DRYING/REACTING}

Since laboratory scale oven tests had shown that dry glass formers and simulant (RVR product) and dry sucrose would react at high temperatures $\left(530^{\circ} \mathrm{F}\right.$ ), this process was performed using the RVR continuous dryer. First stage RVR drying had produced $276 \mathrm{~kg}(609 \mathrm{1b})$ of dried glass formers and simulant (no reductants). This first stage dried product was combined with various stoichiometric ratios of sucrose reductant for the high temperature second stage reaction tests, RVR-S4 through RVR-S7. The quantities of product generated in these second stage RVR tests are given in Table 2-5. The RVR temperature profiles measured during these tests are given in Table 2-6.

Table 2-5. Product Generated During Two Stage RVR Drying Tests.

\begin{tabular}{|l|c|c|}
\hline \multicolumn{1}{|c|}{ Date } & Test & $\begin{array}{c}\text { Product generated, } \\
\mathrm{kg}(1 \mathrm{~b})\end{array}$ \\
\hline $03 / 27 / 95$ & RVR-S4 & $81.2(179)$ \\
\hline $03 / 29 / 95$ & RVR-S5 & $70.3(155)$ \\
\hline $03 / 31 / 95$ & RVR-S6 & $51.2(113)$ \\
\hline $04 / 02 / 95$ & RVR-S7 & $73.5(162)$ \\
\hline Tota1 & & $276.2(609)$ \\
\hline
\end{tabular}


Table 2-6. Average Steady State Bed Temperature Profiles Observed During Second Stage RVR Drying Tests.

\begin{tabular}{|l|c|c|c|c|c|}
\hline \multirow{2}{*}{ Test } & \multicolumn{5}{|c|}{ Temperature, ${ }^{\circ} \mathrm{C}\left({ }^{\circ} \mathrm{F}\right)$} \\
\cline { 2 - 6 } & Inlet & $25 \%$ point & $50 \%$ point & $75 \%$ point & Discharge \\
\hline RVR-S4 & 183.7 & 284.9 & 272.6 & 275.4 & 201.8 \\
$60 \%$ sucrose & $(362.6)$ & $(544.8)$ & $(522.6)$ & $(527.8)$ & $(395.2)$ \\
\hline RVR-S5 & 197.6 & 274.2 & 272.2 & 277.7 & 200.1 \\
80\% sucrose & $(387.7)$ & $(525.6)$ & $(521.9)$ & $(531.9)$ & $(392.1)$ \\
\hline RVR-S6 & 173.9 & 265.1 & 272.2 & 284.6 & 255.8 \\
100\% sucrose & $(345.0)$ & $(509.2)$ & $(522.0)$ & $(544.2)$ & $(492.4)$ \\
\hline RVR-S7 & 166.0 & 266.4 & 291.0 & 286.0 & 248.8 \\
125\% sucrose & $(330.8)$ & $(511.5)$ & $(555.8)$ & $(546.8)$ & $(479.8)$ \\
\hline
\end{tabular}

\subsubsection{RVR-S4: Pre-Dried Glass Formers and Simulant W/ 60\% Stoichiometric Sucrose}

On $4 / 5 / 95$, the impact of combining dried glass formers and simulant with $60 \%$ stoichiometric sucrose in the RVR dryer at high temperature $\left(570^{\circ} \mathrm{F}\right)$ was investigated. $56.7 \mathrm{~kg}$ (125 lbs) of dried glass formers/simulant was premixed with $1.57 \mathrm{~kg}$ (3.46 lbs) of dry sucrose reductant to achieve $60 \%$ stoichiometric ratio. The RVR dryer was preheated with a hot oil supply temperature of $566^{\circ} \mathrm{F}$. The off-gas system was started up. RVR dryer rotors were energized and set at $34 \mathrm{rpm}$. Dry feed was initiated at $54 \mathrm{~kg}(120 \mathrm{lb} / \mathrm{hr})$.

After approximately 15 minutes a reasonable steady bed temperature profile was observed. Lower temperatures were observed at the inlet and discharge ends of the bed. Peak temperatures were observed in the first half of the bed. The average temperature profile for run RVR-S4 is given in Table 2-6.

Reaction between the sucrose and dried simulant was observed. Average NOX and CO levels throughout the test were $45.8 \mathrm{ppm}$ and $27.3 \mathrm{ppm}$, respectively. A dry black product was generated. Additionally, exothermic reactions within the bed occasionally resulted in local bed temperatures slightly higher than hot oil supply temperature. Exothermic temperature peaks $\left[301{ }^{\circ} \mathrm{C}\left(574^{\circ} \mathrm{F}\right)\right]$ did not necessarily correspond to NOx peaks (130 $\left.\mathrm{ppm}\right)$ in the off-gas. CO peaks (43 ppm) did not occur with NOx peaks, as was observed in test RVR-S1. Average hot oil differential temperature was only $4^{\circ} \mathrm{F}$.

When all the premixed dry material had been fed into the RVR dryer, sand was added to the glass former feeder to help clear out the bed for the next test. Representative samples were drawn at the RVR discharge during the middle of each scoping test. The dry product was a black, free flowing granular powder. Minor caking was observed on the dryer heat transfer surfaces. 
WHC-SD-WM-VI-031

Revision 0

\subsubsection{RVR-S5: Pre-Dried Glass Formers and Simulant W/ 80\% Stoichiometric Sucrose}

RVR scoping tests continued on $4 / 5 / 95$ by investigating the impact of combining dried glass formers and simulant with $80 \%$ stoichiometric sucrose in the RVR dryer at high temperature $\left(570^{\circ} \mathrm{F}\right) .56 .7 \mathrm{~kg}$ (125 1bs) of dried glass formers/simulant was premixed with $2.49 \mathrm{~kg}(5.50 \mathrm{lbs})$ of dry sucrose reductant to achieve $80 \%$ stoichiometric ratio. The RVR dryer was preheated with a hot oil supply temperature of $565^{\circ} \mathrm{F}$. The off-gas system was started up. RVR dryer rotors were energized and set at $34 \mathrm{rpm}$. Dry feed was initiated at $54 \mathrm{~kg}(120 \mathrm{lb} / \mathrm{hr})$.

As in the $60 \%$ sucrose run (RVR-S4) a reasonable steady bed temperature profile was observed. Lower temperatures were again observed at the inlet and discharge ends of the bed. Peak temperatures were observed in the first half of the bed. The average temperature profile is given in Table 2-6.

Reaction between the sucrose and dried simulant was observed. Average NOX and CO levels throughout the test were $180 \mathrm{ppm}$ and $60 \mathrm{ppm}$, respectively. The average NOX and $\mathrm{CO}$ off-gas levels were higher than the $60 \%$ sucrose run. Dry black product was generated. Additionally, exothermic reactions within the bed resulted in local bed temperatures slightly higher than hot oil supply temperature. Exothermic temperature peaks appeared to correspond with NOx and $\mathrm{CO}$ peaks in the off-gas. Exothermic temperature peaks $\left[345^{\circ} \mathrm{C}\left(653{ }^{\circ} \mathrm{F}\right)\right]$ and NOx peaks (215 ppm) were higher than the 60\% run. Average hot oil differential temperature was only $2.3^{\circ} \mathrm{F}$.

When all the premixed dry material had been fed into the RVR dryer, sand was added to the glass former feeder to help clear out the bed for the next test. Representative samples were drawn at the RVR discharge during the middle of the scoping test. The dry product was a black, free flowing granular powder slightly lighter than the RVR-S4 (60\%) product.

\subsubsection{RVR-S6: Pre-Dried Glass Formers and simulant W/ $100 \%$ Stoichionetric Sucrose}

RVR scoping tests continued on $4 / 11 / 95$ by investigating the impact of combining dried glass formers and simulant with $100 \%$ stoichiometric sucrose in the RVR dryer at high temperature $\left(570^{\circ} \mathrm{F}\right) .56 .7 \mathrm{~kg}$ (125 1bs) of dried glass formers/simulant was premixed with $3.39 \mathrm{~kg}$ (7.47 1bs) of dry sucrose reductant to achieve $100 \%$ stoichiometric ratio. The RVR dryer was preheated with a hot oil supply temperature of $571^{\circ} \mathrm{F}$. The off-gas system was started up. RVR dryer rotors were energized and set at $34 \mathrm{rpm}$. Dry feed was initiated at $24 \mathrm{~kg} / \mathrm{hr}(60 \mathrm{lb} / \mathrm{hr}$ ) but later increased to approximately $40.8 \mathrm{~kg} / \mathrm{hr}$ (90 $\mathrm{ib} / \mathrm{hr}$ ).

Similar to the previous two second stage scoping runs (RVR-S4, S5) a reasonable steady bed temperature profile was observed. Lower temperatures were observed at the inlet ends of the bed. Peak temperatures were observed toward the second half of the bed. The average temperature profile is given in Table 2-6. 
Reaction between the sucrose and dried simulant was observed. Average NOX and CO levels throughout the test were $220 \mathrm{ppm}$ and $70 \mathrm{ppm}$, respectively. The average NOX and CO off-gas levels were higher than the $80 \%$ sucrose run. Dry black product was generated. Additionally, exothermic reactions with in the bed resulted in local bed temperatures sightly higher than hot oil supply temperature. Exothermic temperature peaks $\left[301^{\circ} \mathrm{C}\left(573^{\circ} \mathrm{F}\right)\right]$ and $\mathrm{NOx}$ peaks $(280 \mathrm{ppm})$ were higher than the $80 \%$ run. Average hot oil differential temperature was only $3.6^{\circ} \mathrm{F}$.

When all the premixed dry material had been fed into the RVR dryer, sand was added to the glass former feeder to help clear out the bed for the next test. Representative samples were drawn at the RVR discharge during the middle of scoping test. The dry product was a dark gray, free flowing granular powder slightly lighter in color than the RVR-S5 (80\%) product.

The results of RVR-S1 (1 iquid simulant $w / 100 \%$ sucrose) can be compared to the results of RVR-S6 (dry simulant $w / 100 \%$ sucrose). A much more stable and even temperature profile was observed during RVR-S6. Additionally, RVR-S6 reactions appeared less violent and more controlled. Bed caking was experienced during RVR-S1 and not during RVR-S6. Average NOx and CO leve1s in the RVR-Sl off-gas were nearly an order of magnitude higher, at 1352.8 and $445.6 \mathrm{ppm}$ respectively. However, this may be attributable to exhaust gas flow rates that are such lower in the $S 1$ testing.

\subsubsection{RVR-S7: Pre-Dried Glass Formers and simulant W/ 125\% Stoichiometric Sucrose}

RVR scoping tests continued on $4 / 11 / 95$ by investigating the impact of combining dried glass formers and simulant with $125 \%$ stoichiometric sucrose in the RVR dryer at high temperature $\left(570^{\circ} \mathrm{F}\right) .56 .7 \mathrm{~kg}$ (125 1bs) of dried glass formers/simulant was premixed with $4.56 \mathrm{~kg}$ (10.05 1bs) of dry sucrose reductant to achieve $100 \%$ stoichiometric ratio. The RVR dryer was preheated with a hot 0 il supply temperature of $568^{\circ} \mathrm{F}$. The off-gas system was started up. RVR dryer rotors were energized and set at $34 \mathrm{rpm}$. Dry feed was initiated at approximately $40.8 \mathrm{~kg} / \mathrm{hr}(90 \mathrm{lb} / \mathrm{hr})$.

Similar to the previous scoping run (RVR-S6) a reasonable steady bed temperature profile was observed. Lower temperatures were observed at the inlet ends of the bed. Peak temperatures were observed in the center of the bed. The average temperature profile is given in Table 2-6.

Reaction between the sucrose and dried simulant was observed. Average NOX and CO levels throughout the test were $220 \mathrm{ppm}$ and $85 \mathrm{ppm}$, respectively. The average NOX and $\mathrm{CO}$ off-gas levels, were about the same as the $100 \%$ run. Dry black product was generated. Additionally, exothermic reactions within the bed resulted in local bed temperatures slightly higher than hot oil supply temperature. Exothermic temperature peaks $\left[308{ }^{\circ} \mathrm{C}\left(587^{\circ} \mathrm{F}\right)\right]$ and $\mathrm{NOx}$ peaks (335 ppm) were higher than a11 previous second state scoping runs. NOx and co peaks in the off-gas were observed to correspond with exothermic temperature peaks. Average hot oil differential temperature was only $1.8^{\circ} \mathrm{F}$. 
When all the premixed dry material had been fed into the RVR dryer, representative samples were drawn at the RVR discharge. The dry product was a dark gray, free flowing granular powder very similar to the RVR-S6 (100\%) product.

\subsection{SCOPING TEST EVALUATION SUMHARY}

A summary of the RVR drying/reacting tests is given in Table 2-7.

\subsection{CONCLUSIONS} tests.

The following conclusions are drawn from the results of the RVR drying

- Boric acid in the glass former mixture melts in the RVR dryer at $570^{\circ} \mathrm{F}$ hot $0 i 1$ inlet temperatures causing hard caking.

- Bed caking results when glass formers and liquid simulant are dried by the RVR continuous dryer. Principal caking occurs in the transition zone where the final free water is evaporated and the product bed temperature begins to exceed the boiling point of water. Liquid simulant drying at $<115^{\circ} \mathrm{C}\left(<240{ }^{\circ} \mathrm{F}\right)$ and reacting at $300^{\circ} \mathrm{C}$ $\left(570^{\circ} \mathrm{F}\right.$ ) cannot be performed consistently with a single pass through the RVR dryer.

- Laboratory scale vacuum drying of liquid simulant results in a waxy product which is undesirable for RVR rotary vacuum dryer processing.

- Under laboratory conditions, Formic Acid volatizes before it can completely react with 1 iquid simulant at $530^{\circ} \mathrm{F}$ and is therefore, not suitable as a reductant for RVR dryer processing.

- Glass formers and predried simulant will react with Sucrose $\left(\mathrm{C}_{12} \mathrm{H}_{22} \mathrm{O}_{31}\right)$ at stoichiometric ratios of $60 \%, 80 \%, 100 \%$, and $125 \%$ in the RVR dryer with $570^{\circ} \mathrm{F}$ hot oil inlet temperature. The reaction is exothermic and non-uniform. NOx and $\mathrm{CO}$ off-gas concentrations increase as stoichiometric sucrose ratio increases. Two stage RVR drying/reacting can be successfully performed with the continuous RVR dryer. The first stage drying could be a pulse or spray dryer with the second stage high temperature reacting process performed in an RVR dryer.

\subsection{RECONHENDATION FOR FUTURE TESTS}

VECTRA does not recommend any additional testing be considered for any rotary dryer process. VECTRA recommends that future tests target nonmechanical dryers such as pulse or spray dryers. 


\begin{tabular}{|c|c|c|c|c|c|c|c|}
\hline 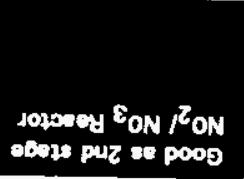 & $\begin{array}{l}\text { sojonct } \varepsilon_{\text {ON }} \tau_{\text {ON }} \\
\text { obsus puz se poog }\end{array}$ & 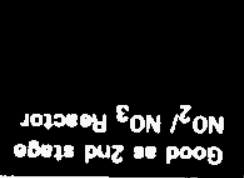 & 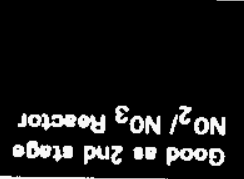 & $\begin{array}{r}\text { (Oupyeos) } \\
\text { 9quzdoson zoN }\end{array}$ & 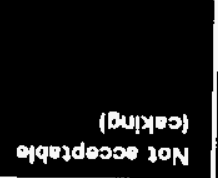 & 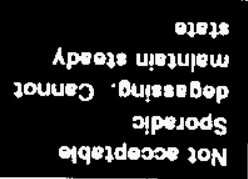 & บоㄱำрекз \\
\hline$\nabla / N$ & $\forall / N$ & 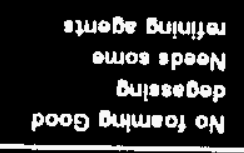 & $\begin{array}{r}\text { ojuobe Buyujes } \\
\text { oujoe spoon } \\
\text { Bulsse Bop } \\
\text { poos oujueof oN }\end{array}$ & $\mathbf{V} / \mathbf{N}$ & $\forall / N$ & $\mathbf{V} / \mathbf{N}$ & 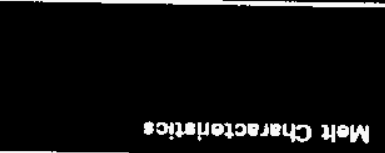 \\
\hline 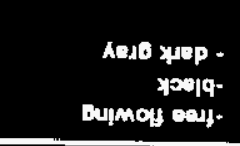 & $\begin{array}{l}\text { Aert wap- } \\
\text { BumMolf oaf- }\end{array}$ & $\begin{array}{l}\text { umara Asepe- } \\
\text { Bumaly caly. }\end{array}$ & 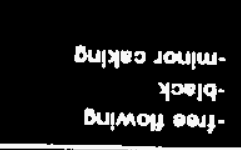 & $\begin{array}{l}\text { Dupyes ozesopour. } \\
\text { pes. } \\
\text { Bumaㄱ oos. }\end{array}$ & 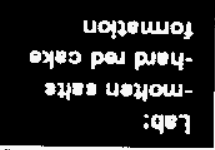 & 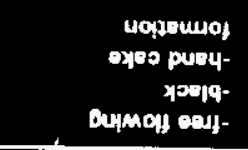 & $\begin{array}{l}\text { do100 - } \\
\text { Du!moly oaly - } \\
\text { osuereoddy }\end{array}$ \\
\hline $\begin{array}{c}908 \\
880 \\
07 z 22 \\
988-0\end{array}$ & $\begin{array}{l}0289 \\
78-0 \\
t-98 t \\
002-0\end{array}$ & $\begin{array}{l}1 \cdot 92 \\
z<-0 \\
2 \cdot 80 \\
912-0\end{array}$ & $\begin{array}{l}t \cdot 81 \\
8 t-0 \\
8 \cdot 9 t \\
0 \varepsilon 1 \cdot 0\end{array}$ & $\forall / N$ & $\forall / N$ & 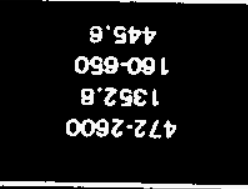 & 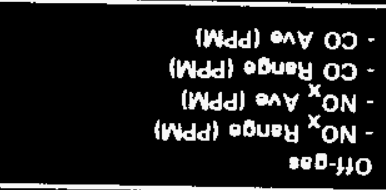 \\
\hline Kazt-orowons & Kool- - ssourns & \%or-esousns & \%09-oyouns & aUoN & $\begin{array}{c}\text { \%ool } \\
\text { pay syuroy }\end{array}$ & \%ool asourns & 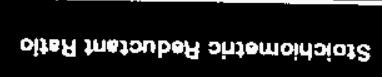 \\
\hline LS-HAY & 9s-tung & gs-yny & tS-tม & 8s-yny & . ZS-४४甘 & IS-女nย & \multirow{2}{*}{ sezowesesed } \\
\hline \multicolumn{7}{|c|}{ uny 2001} & \\
\hline
\end{tabular}

- Rueuums uotzenleaz 7501 yay $\angle-z$ olqe 
WHC-SD-WM-VI-031

Revision 0

APPENDIX 2A

DATA SHEETS

2-21 
WHC-SD-WM-VI-031

Revision 0

Scopteg Teat - Datashot

Page 1 of 1

\begin{tabular}{|c|c|c|c|c|c|c|}
\hline \multirow{2}{*}{\multicolumn{7}{|c|}{ Dace: $\quad 3 / 21 / 95$}} \\
\hline & & & & & & \\
\hline Time: & & 1130 & 1200 & 1230 & 1300 & 1330 \\
\hline \multicolumn{7}{|l|}{ Hoc oil syscem } \\
\hline Pump Inlet pressure PI-IN & $\mathrm{kPag}$ & 0 & C) & 2 & 0 & 0 \\
\hline Pump Outlet pressure PI-OUT & $\mathrm{kPag}$ & 124 & 124 & 124 & 124 & 124 \\
\hline Hot 011 supply, temperature & ${ }^{\circ} \mathrm{C}$ & 249 & 249 & 248 & 2.48 & 298 \\
\hline Hot Oi Return, temperature & ${ }^{\circ} \mathrm{C}$ & 297 & 20 & 295 & 217 & 295 \\
\hline Expansion Tank level 1 & $\mathrm{Cm}$ & & 20 & 20 & 20 & 20 \\
\hline \multicolumn{7}{|l|}{ RVR Dryer Bed Temoeracures } \\
\hline$T I-1$ & ${ }^{\circ} \mathrm{C}$ & 248 & 145 & 49 & 103 & 166 \\
\hline$T I-2$ & ${ }^{\circ} \mathrm{C}$ & 266 & 236 & 206 & 273 & 202 \\
\hline$T I-3$ & ${ }^{\circ} \mathrm{C}$ & 284 & 280 & 281 & 292 & 270 \\
\hline$T I-4$ & ${ }^{\circ} \mathrm{C}$ & 260 & 253 & 250 & 262 & 265 \\
\hline$T I-5$ & ${ }^{\circ} \mathrm{C}$ & -243 & 251 & 242 & 248 & 253 \\
\hline Rotor Speed Setting & $R P M$ & 30 & 30 & 30 & 30 & $30^{\circ}$ \\
\hline Glass Former Feed Rate & $\begin{array}{l}\text { Kg/hr } \\
\mathrm{Dial}\end{array}$ & 4570 & $45 \%$ & $45 \%$ & $45 / 10$ & $45 / 10$ \\
\hline Total glass fcrmers fed (this period) & $\mathrm{kg}$ & 0 & 46 & 45 & & \\
\hline Total dried product this period & $\mathrm{kg}$ & 0 & 21 & 13 & & 6,3 \\
\hline Total dried mixture output (accumulated) & $\mathrm{kg}$ & $\theta$ & & & & \\
\hline Tank Ievel reading & $\mathrm{L}$ & 120 & 116 & 114 & 108 & 98 \\
\hline Simulant/Additive Injection Rate & $m L / m i n$ & 425 & 425 & 425 & 425 & 425 \\
\hline Total Simulant/Additive Input & $L$ & $C$ & & & & \\
\hline \multicolumn{7}{|l|}{ off-Gas System } \\
\hline Off-gas Elow annemometer & $m / s+n^{2}+h=$ & 0.44 & (c) & $\underline{C}$ & & \\
\hline No Reading & pem. & 0 & (1) & 300 & & 500 \\
\hline $\mathrm{NO}_{2}$ Reading & ppm & 0 & Q & 172 & & \\
\hline Total No Reading & Dpm & 0 & (1) & 472 & & \\
\hline Co Reading & ppm & 0 & & 160 & & 500 \\
\hline Recisc Line Temp - TI-SCS & ${ }^{\circ} \mathrm{C}$ & 17 & 18 & 19 & 19 & 19 \\
\hline Scrubber Level - LG-SCB & $\mathrm{cm}$ & 0 & & & & \\
\hline Off-gas Pressure - PI-OFG & $\mathrm{kPag}$ & 0 & C) & 0 & 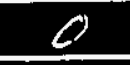 & 0 \\
\hline Vent Separacor Level - LG-VONT & $\mathrm{cm}$ & & & & & \\
\hline Vent separator Temp - TI-VENT & ${ }^{\circ} \mathrm{C}$ & & & & & \\
\hline & & & & & & \\
\hline & & & & & & \\
\hline
\end{tabular}

(1) INST. NOT REABING. 
WHC-SD-WM-VI-031

Revision 0

Scopteg Test - Daeabeot

Page 1 of 1

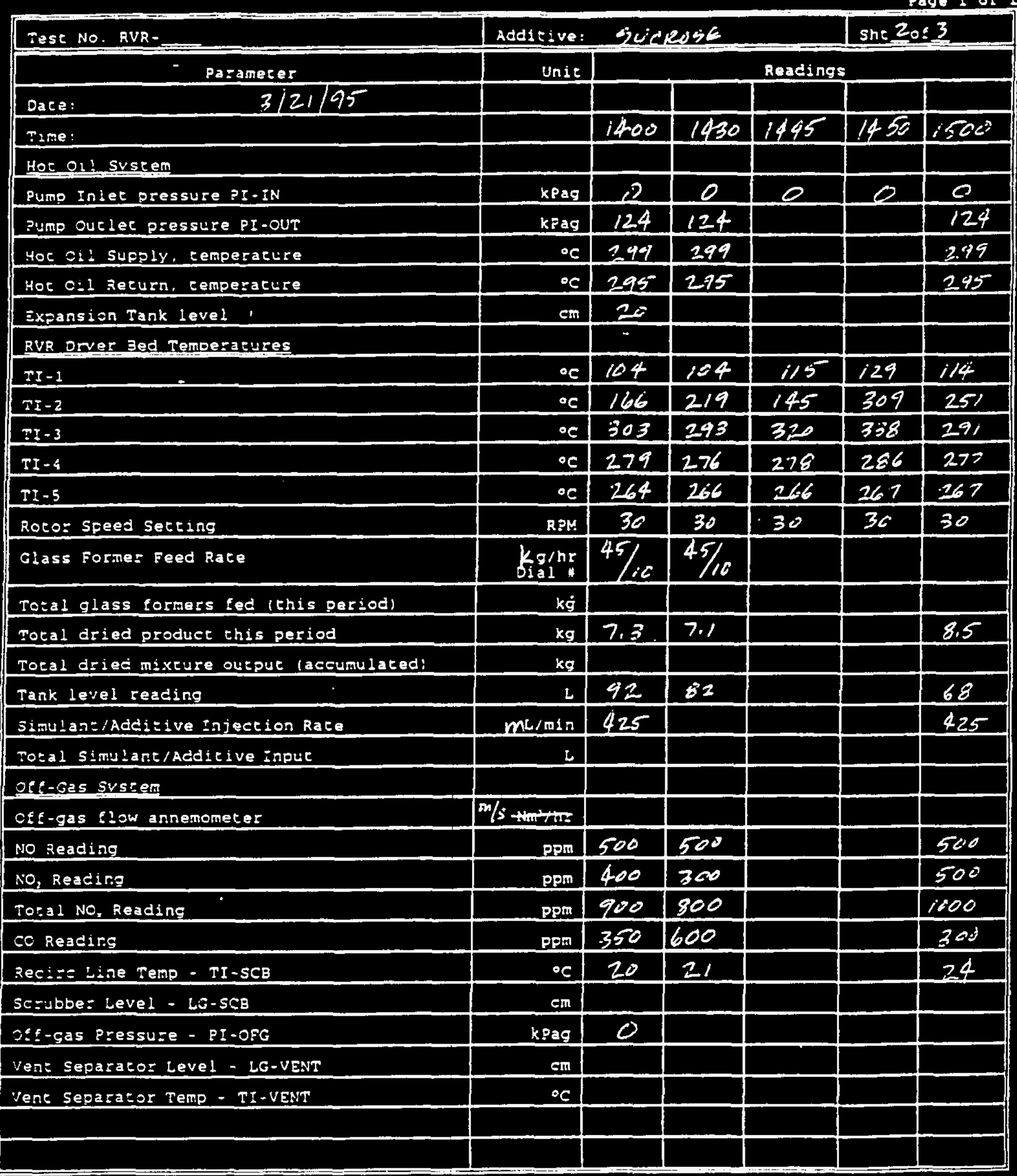


WHC-SD-WM-VI-031

Revision 0

Scoptar rest - Datashaet

Page : of :

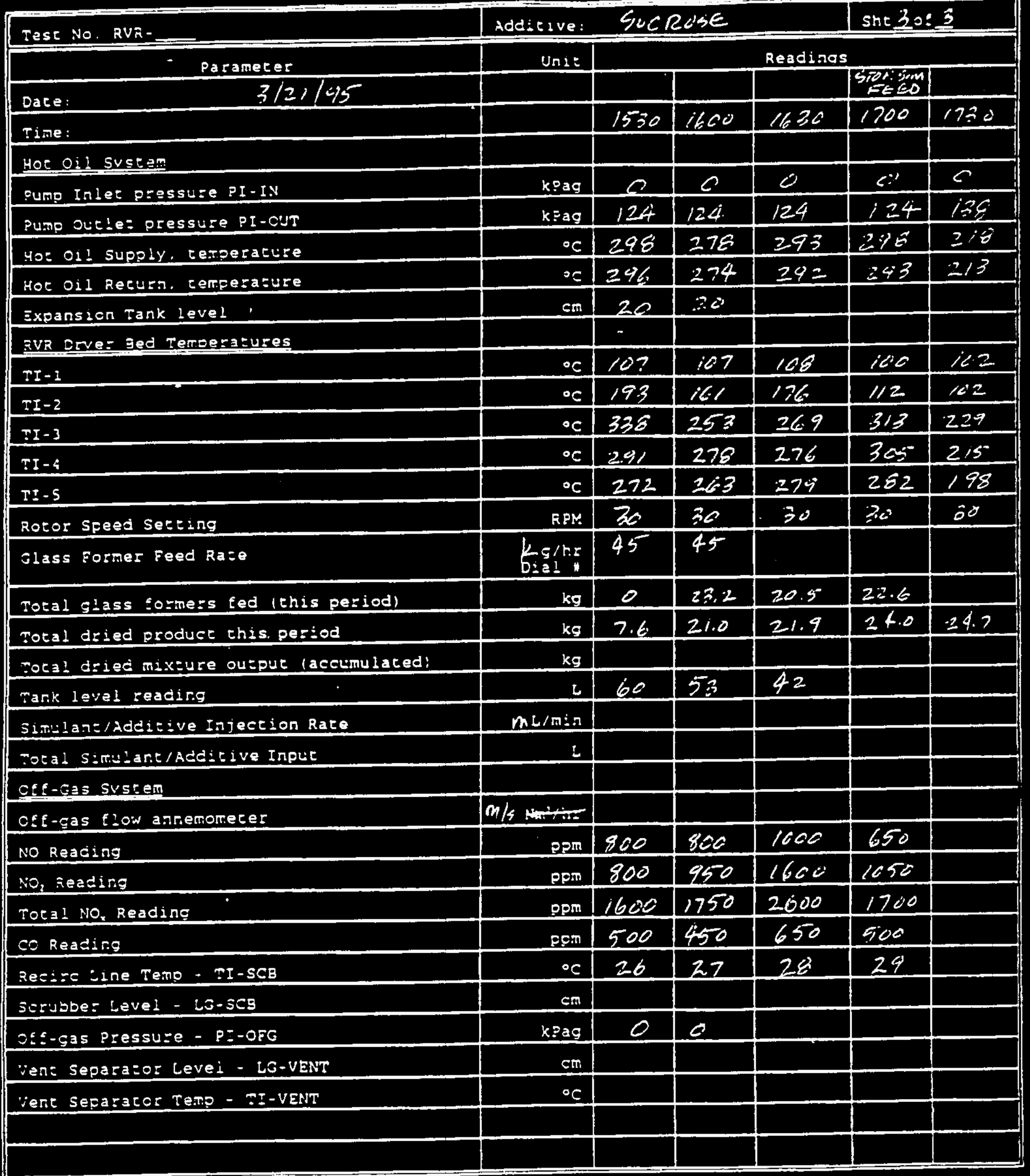


WHC-SD-WM-VI-031

Revision 0

Scoptag Tast - Dataghoet

Page 1 of 1

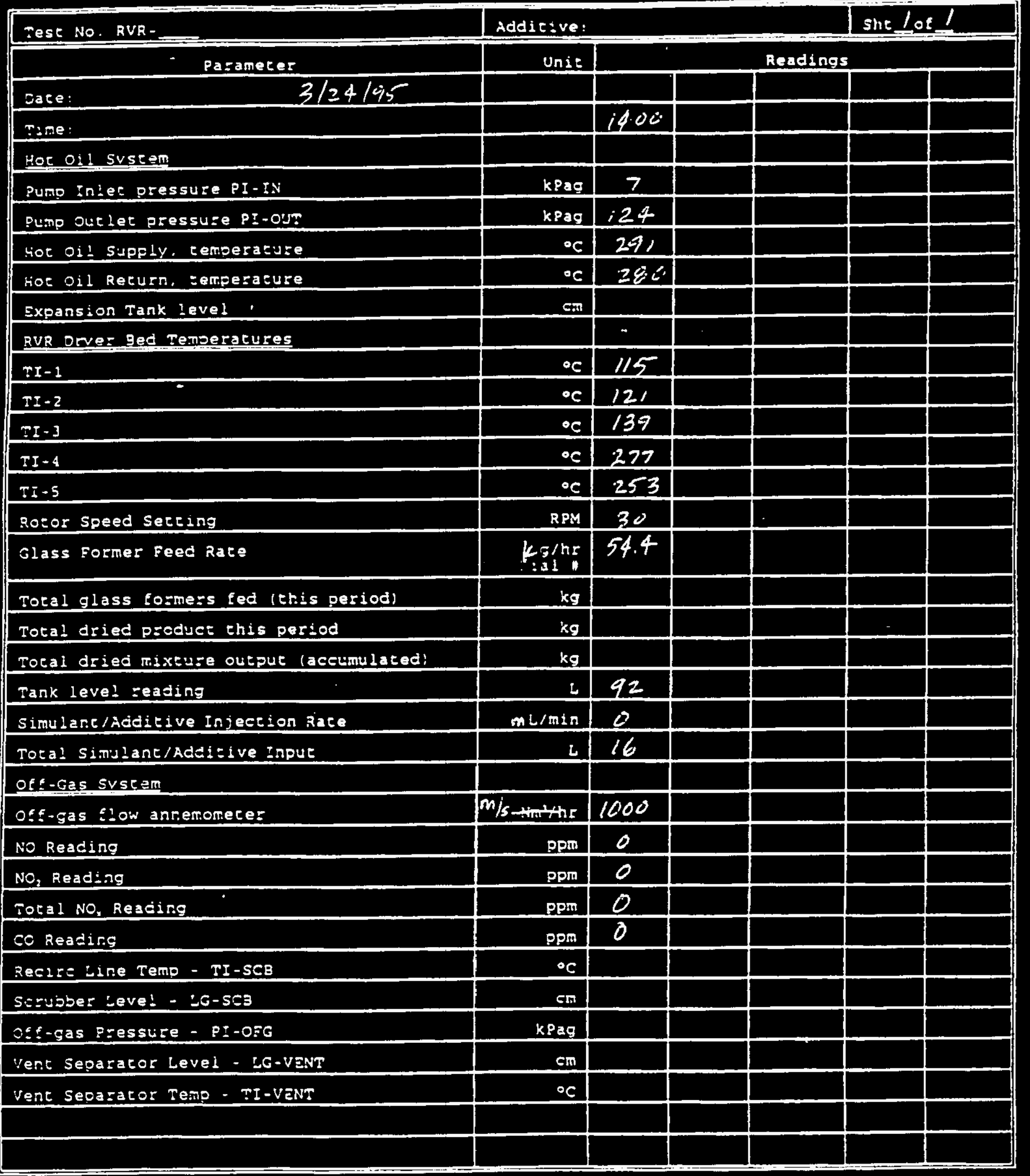


WHC-SD-WM-VI-031

Revision 0

scoptoo Tast - Datambet

Page 1.0: :

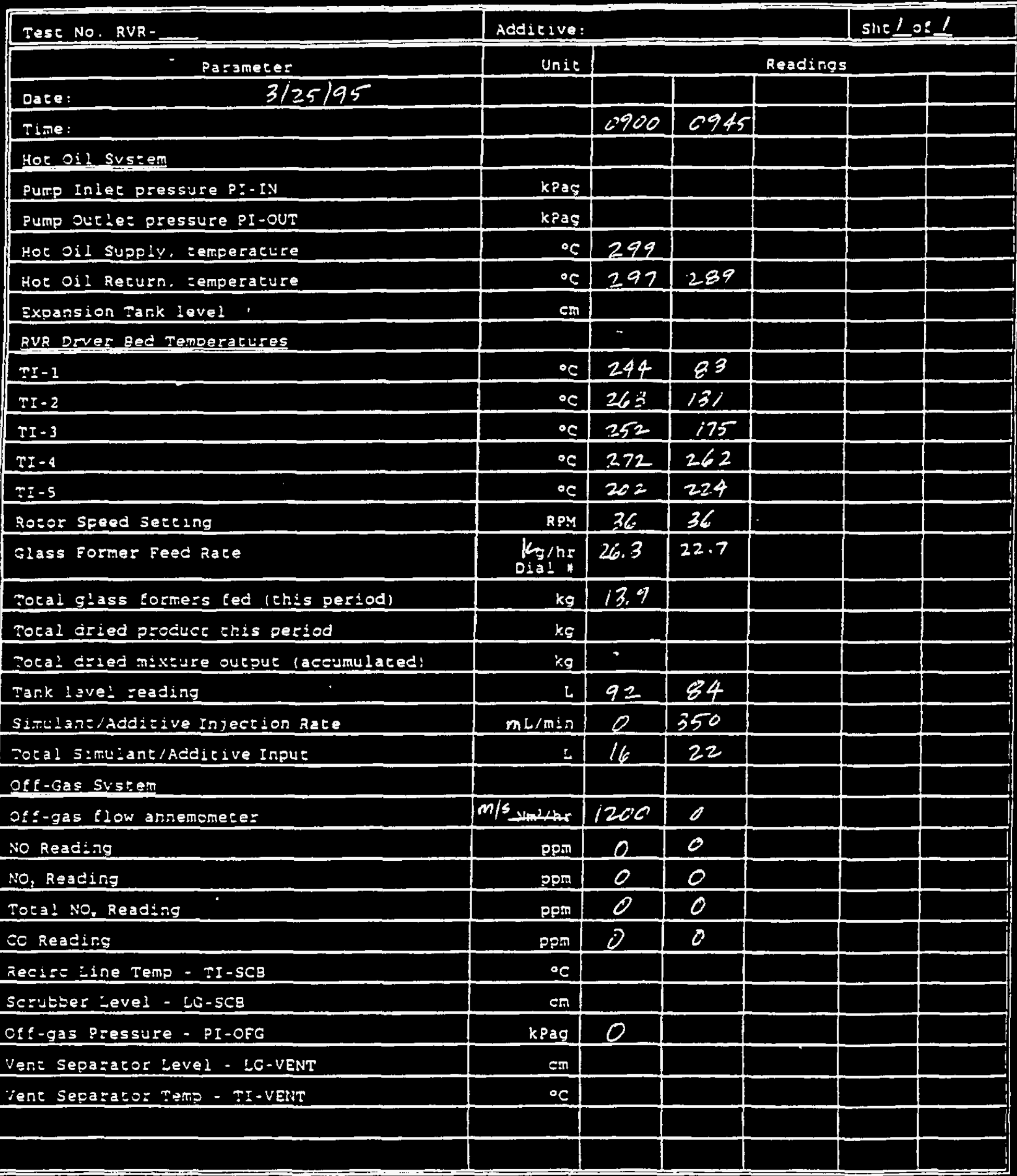


WHC-SD-WM-VI-031

Revision 0

Scoplag Tate - Dacadhat

Page : of 1

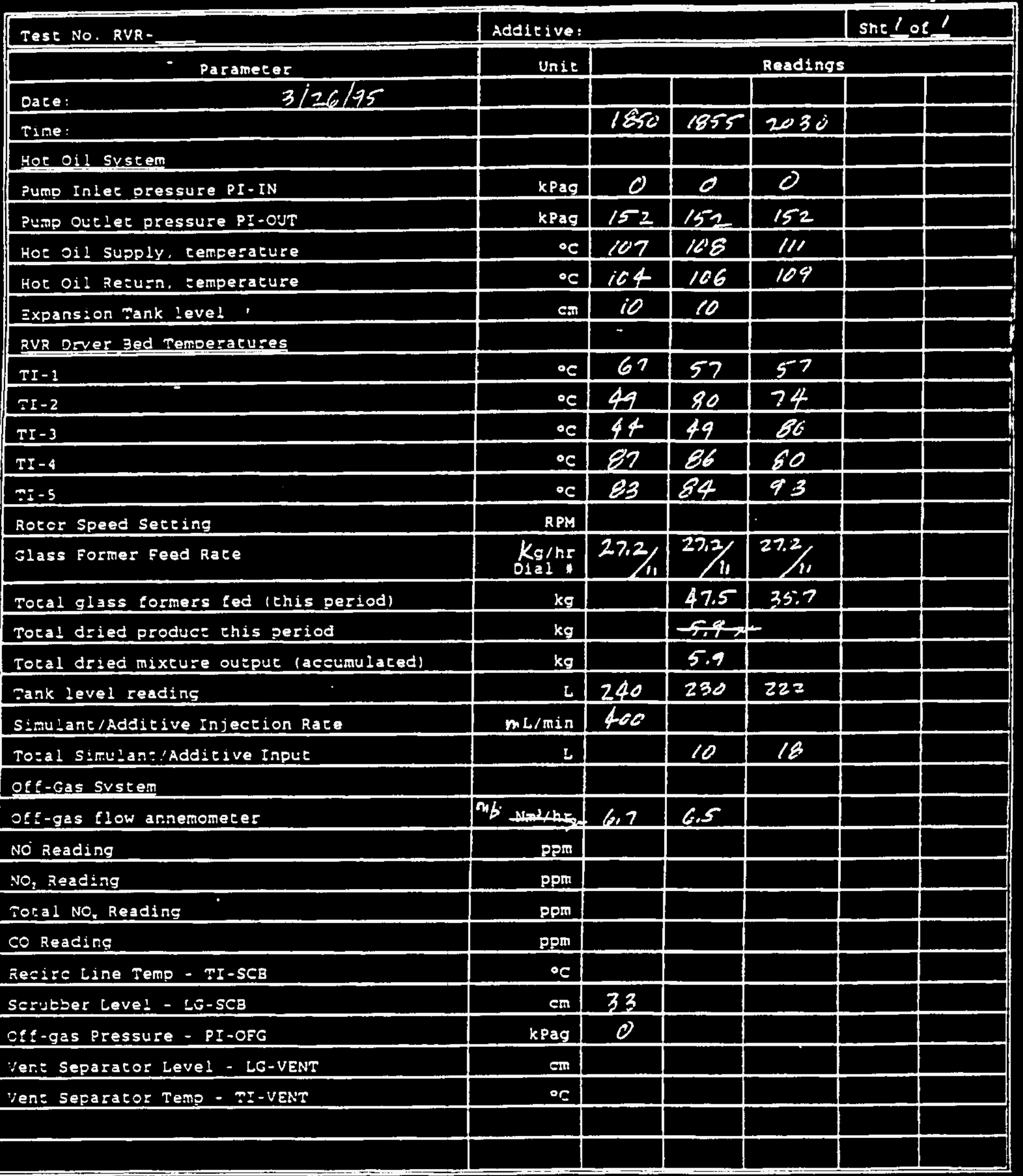


WHC-SD-WM-VI-031

Revision 0

Scopler Tate - Databhet

Page : of :

\begin{tabular}{|c|c|c|c|c|c|c|}
\hline \multicolumn{7}{|l|}{$3 / 27 / 95$} \\
\hline Time: & & oisc & $<400$ & $04.30^{\circ}$ & iseic & $c^{2} 530$ \\
\hline \multicolumn{7}{|l|}{ Hot o: system } \\
\hline Punc Inler oressure PI-IN & $\mathrm{kPag}$ & 6 & ¿? & ? & 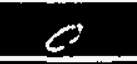 & 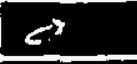 \\
\hline Puno Outies pressure PI-OUT & kpag & 152 & 152 & 152 & 152 & 152 \\
\hline Hot Cil supfly, semperature & ${ }^{\circ} \mathrm{C}$ & 112 & 129 & $i 6 /$ & $16=$ & 165 \\
\hline Hot OL! Recura, cemperacure & ${ }^{\circ} \mathrm{C}$ & $i / c^{\prime}$ & 117 & 157 & 161 & 161 \\
\hline Expansion Tank level. & Gr: & 10 & 10 & io & 10 & 16 \\
\hline \multicolumn{7}{|l|}{ RVR Dever Bed Temperacures } \\
\hline$T I-1$ & ${ }^{\circ} \mathrm{C}$ & 74 & 69 & 8.3 & 11 & 116 \\
\hline$T I-2$ & ${ }^{\circ} \mathrm{C}$ & 62 & 57 & 6.8 & $? 2$ & 125 \\
\hline$T I-3$ & $\circ \mathrm{C}$ & 80 & 87 & $11 S$ & $i 24$ & 128 \\
\hline$T I-4$ & ${ }^{\circ} \mathrm{C}$ & 77 & 69 & ic9 & 118 & 116 \\
\hline$I I .5$ & ${ }^{\circ} \mathrm{C}$ & 92 & 99 & 109 & 132 & 132 \\
\hline Rotor Speed Secting & RPM & 34 & 34 & 34 & 24 & 34 \\
\hline Glass Former Feed Rate & $\begin{array}{c}\mathrm{kg} / \mathrm{hr} \\
\mathrm{giaj}\end{array}$ & 27,2 & 27,2 & 27.2 & 27.2 & $2: 22$ \\
\hline Tota! glass formers fed (this period) & $\mathrm{kg}$ & & & & & 35.5 \\
\hline Total dried product this period & $\mathrm{kg}$ & & 8.7 & 7.6 & 8.5 & 2.4 \\
\hline Dotal dried mixture output (accumulaced) & $\mathrm{kg}$ & & 4,7 & 16.3 & 24.8 & 32.4 \\
\hline Tark level zeadirg & $\mathrm{L}$ & 220 & 210 & 201 & 192 & 164 \\
\hline Simulantidddicive Injection Race & $m i / m i r$ & 200 & 300 & 300 & 30 & 300 \\
\hline Tozal Simliant/Additive Inpue & $L$ & & 10 & 19 & 28 & 36 \\
\hline \multicolumn{7}{|l|}{ off-Gas Svstam } \\
\hline Cff-gas flow annemomecer & $\ln / \mathrm{s}+\mathrm{nn}^{2}+\mathrm{t}+\mathrm{x}=\mathrm{m}$ & 7,0 & $\infty$ & - & i. 1 & 5.5 \\
\hline No Reading & pen & 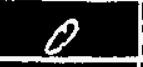 & 0 & 0 & 0 & 0 \\
\hline $\mathrm{NO}_{2}$ Reading & Ppm & $\theta$ & 0 & $\sigma$ & 0 & C \\
\hline Totai No, Reading & ppm & 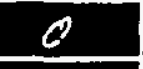 & 0 & $\infty$ & $C$ & C? \\
\hline Co Reading & pem & 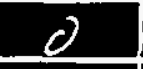 & C) & C) & C) & C) \\
\hline Recizs -ine Temo - TI-SCB & ${ }^{\circ} \mathrm{C}$ & 13 & 13 & 13 & 14 & 15 \\
\hline Scrubber jevel - LG-SCB & $\mathrm{cm}$ & 16 & $3 z$ & 33 & 66 & 22 \\
\hline Off-gas zzessure - PI-CFG & $\mathrm{kPag}$ & 0 & 0 & 2 & 0 & 0 \\
\hline Vert Sepazacor ievel - LG-VENT & $\sin$ & & & & & \\
\hline Jen: Separacor Teme - TI-VENT & ${ }^{\circ} \mathrm{C}$ & & & & & \\
\hline & & & & & & \\
\hline & & & & & & \\
\hline
\end{tabular}


WHC-SD-WM-VI-031

Revision 0

Scoptoo Tare - Dacaphat

Page 1 of 1

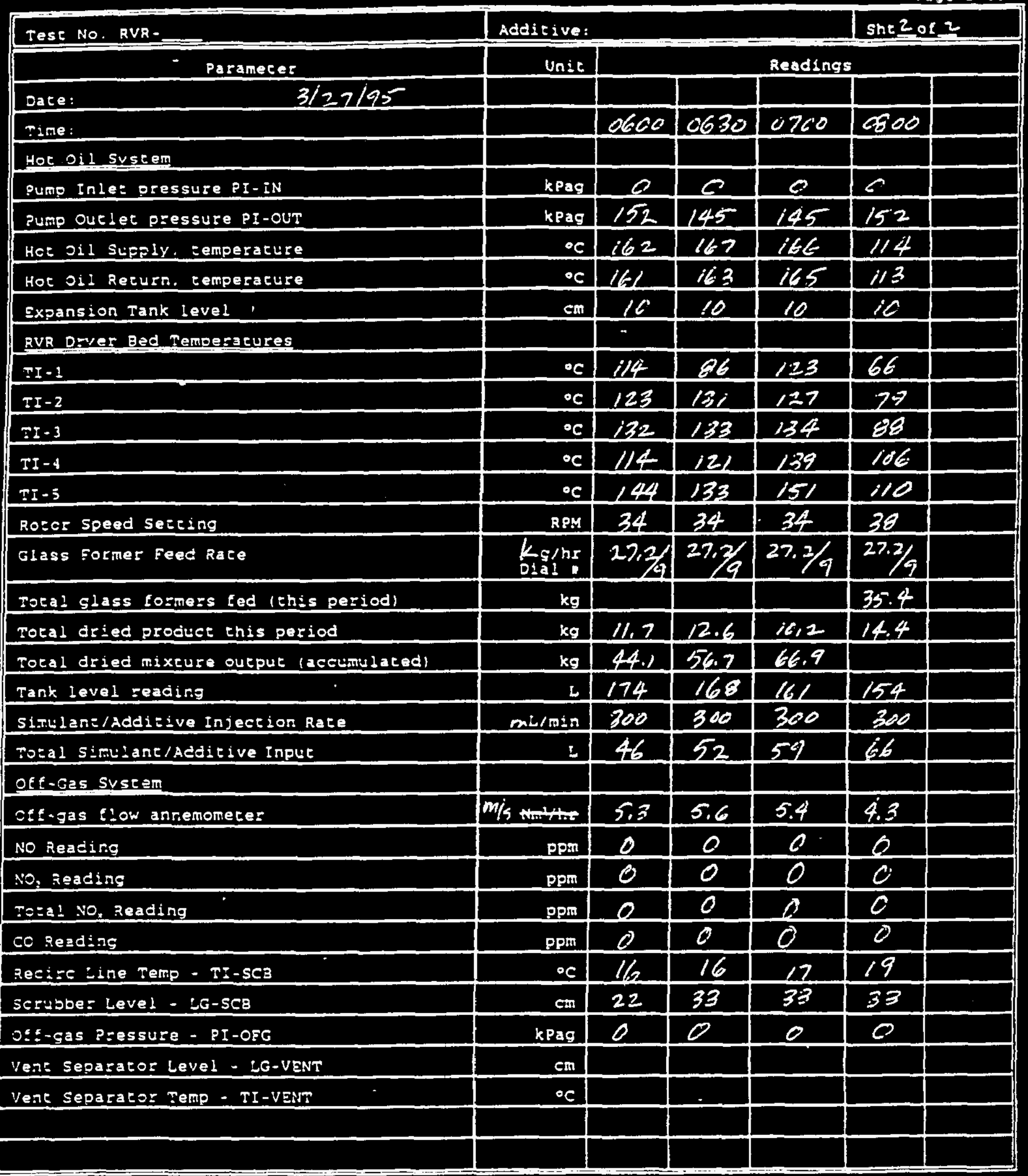


WHC-SD-WM-VI-031

Revision 0

Scoping rast - Datagheat

Page 1 of

\begin{tabular}{|c|c|c|c|c|c|c|}
\hline Tese No. RVR- & \multicolumn{4}{|c|}{ Additive: NGYe } & \multicolumn{2}{|c|}{ she/ of 4} \\
\hline Parameter & Unit & \multicolumn{5}{|c|}{ Readings } \\
\hline \multicolumn{7}{|l|}{ Dace: $\quad 3-29-55$} \\
\hline Time: & & 0932 & 1000 & 1030 & 1100 & 1130 \\
\hline \multicolumn{7}{|l|}{ Hot oil system } \\
\hline \multirow{2}{*}{$\begin{array}{l}\text { Eump Inlet pressure PI-IN } \\
\text { gump Outlet pressure RI-OUT }\end{array}$} & kPag & 0 & 0 & $C$ & C) & 0 \\
\hline & keag & 165 & 165 & 165 & 165 & 165 \\
\hline Hot oil supply, temperature & "c & 110 & 112 & 109 & 109 & 112 \\
\hline Hot oil Return, temperature & ${ }^{\bullet} \mathrm{C}$ & 109 & 109 & 108 & 109 & 111 \\
\hline Expangion Tank level & $\operatorname{em}$ & -10 & 10 & 10 & 10 & 10 \\
\hline \multicolumn{7}{|l|}{ RVR Dryer Bed Temperatures } \\
\hline$T I-1$ & ${ }^{\circ} \mathrm{C}$ & 59 & 68 & 66 & & 71 \\
\hline$T I-2$ & ${ }^{\circ} \mathrm{C}$ & 32 & 46 & 50 & 76 & 53 \\
\hline$T I-3$ & ${ }^{\circ} \mathrm{C}$ & 78 & 64 & 86 & 85 & 84 \\
\hline$T I-4$ & 'c & 52 & 66 & 62 & 62 & 65 \\
\hline$T I-5$ & 'c & 53 & 72 & 83 & 76 & 90 \\
\hline Rotor Speed setting & REM & 30 & 30 & 34 & 34 & 34 \\
\hline Glass Former Feed Rate & Kialar & $\begin{array}{c}27.21 \\
9\end{array}$ & & & & $\rightarrow$ \\
\hline Total glass formers fed (this pasiod) & kg & STimer All & & & & - \\
\hline Total dried produet this pertod & $\mathrm{kg}$ & - & 一 & 0.76 & 2.29 & 3.19 \\
\hline Total dried mixture output (accumulated) & $\mathrm{kg}$ & & & & 3.10 & 6.29 \\
\hline \multirow{2}{*}{$\begin{array}{l}\text { Tank level reading } \\
\text { Simulant/Additive Injection Rate }\end{array}$} & I & 150 & 144 & 140 & 136 & 134 \\
\hline & $m=/ m s a$ & 400 & & & & $=$ \\
\hline & $\mathbf{L}$ & & 6 & 10 & 14 & 16 \\
\hline \multicolumn{7}{|l|}{ Off-Gas system } \\
\hline Off-gas flow annemometer & $M / S$ siantares & 6.3 & 0.36 & 1.33 & 6.35 & 6.12 \\
\hline \multicolumn{7}{|l|}{ No Reading } \\
\hline \multicolumn{7}{|l|}{$\mathrm{NO}_{2}$ Reading } \\
\hline \multicolumn{7}{|l|}{ Total $\mathrm{NO}_{\mathrm{k}}$ Reading } \\
\hline co Reading & Ppin & & & & & \\
\hline Recirc Iine Temp - TI-SCB & 'c & 13 & 15 & 16 & 17 & 18 \\
\hline Scrubber Level - LG-SCB & $\mathrm{cm}$ & 33 & 33 & 33 & 22 & 22 \\
\hline Off-gas gressure - EI-OFG & kgag & & & & & \\
\hline Vent Separator Ievel $\rightarrow$ IG-VENI & $\mathrm{cs} /$ is & & & & & \\
\hline Vent separator Temp - TI-VaNT & $\bullet c$ & & & & & \\
\hline & & & & & & \\
\hline & & & & & & \\
\hline
\end{tabular}


WHC-SD-WM-VI-031

Revision 0

scoping reat - Datahoet

Rage 1 of

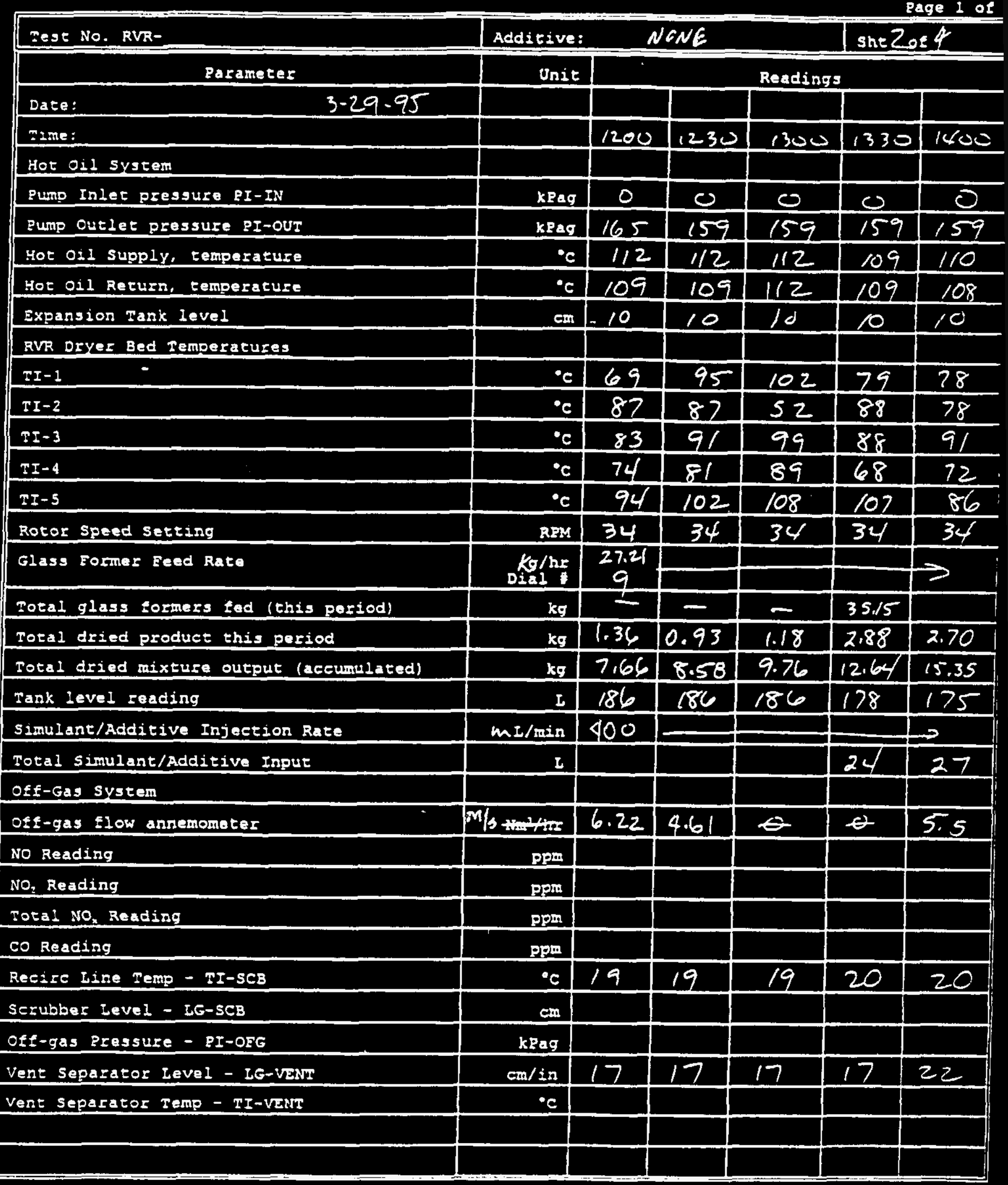


WHC-SD-WM-VI-031

Revision 0

Seoping Tort - Databeet

Page 1 of

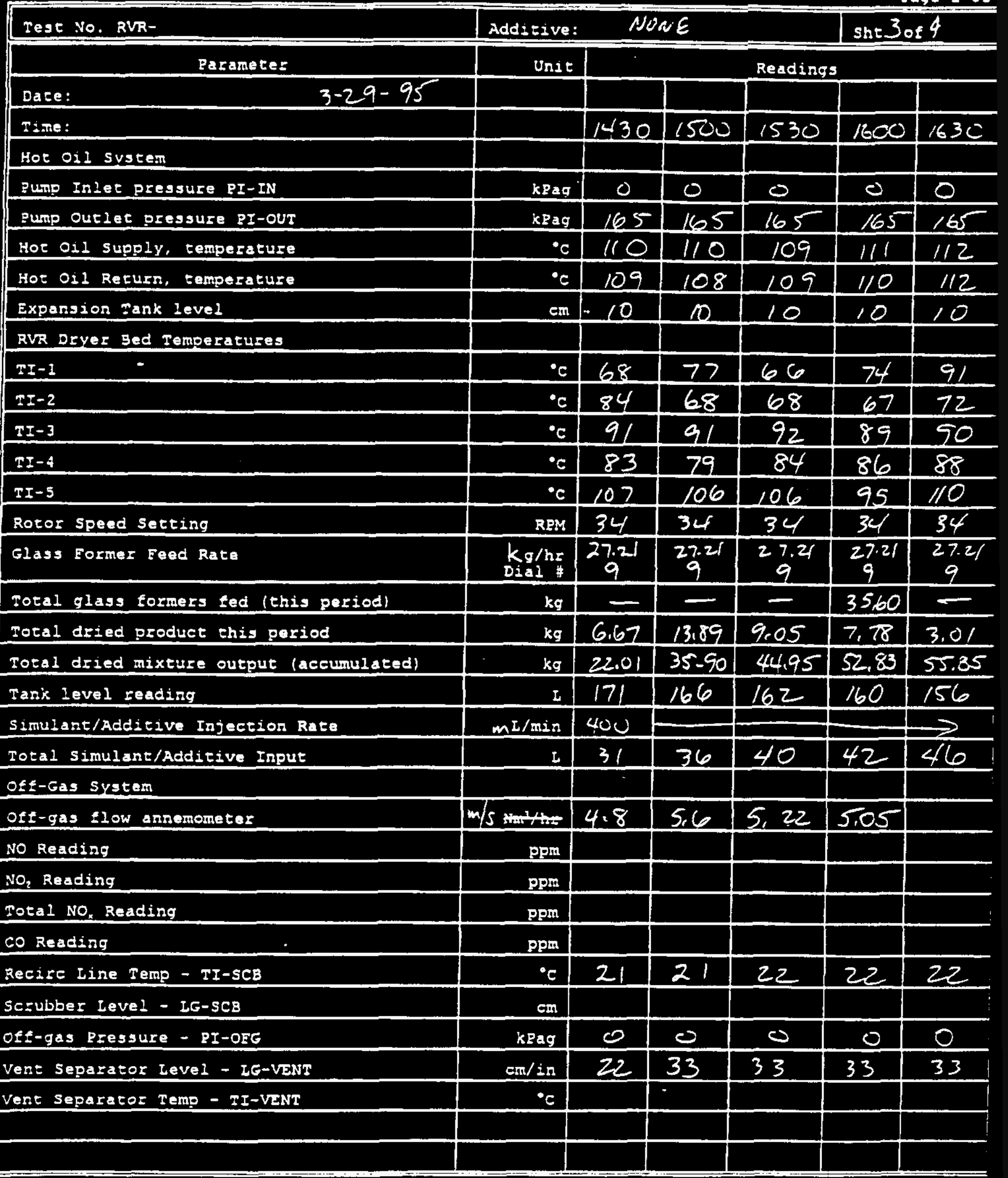


WHC-SD-WM-VI-03I

Revision 0

scoptag Teat - Datahoet

Page 1 of

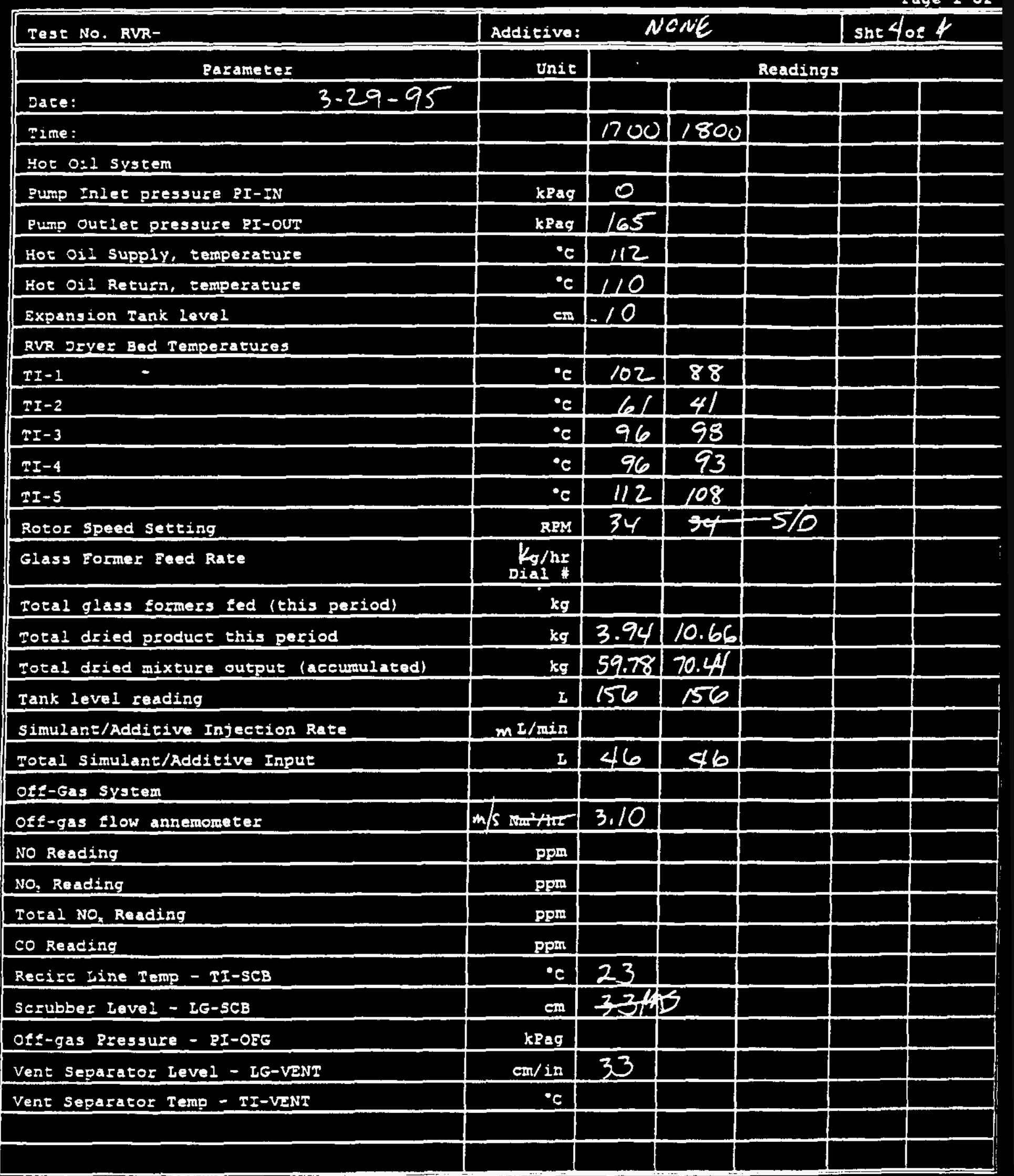


WHC-SD-WM-VI-031

Revision 0

Seoping rest - Datamhot

Page 1 of

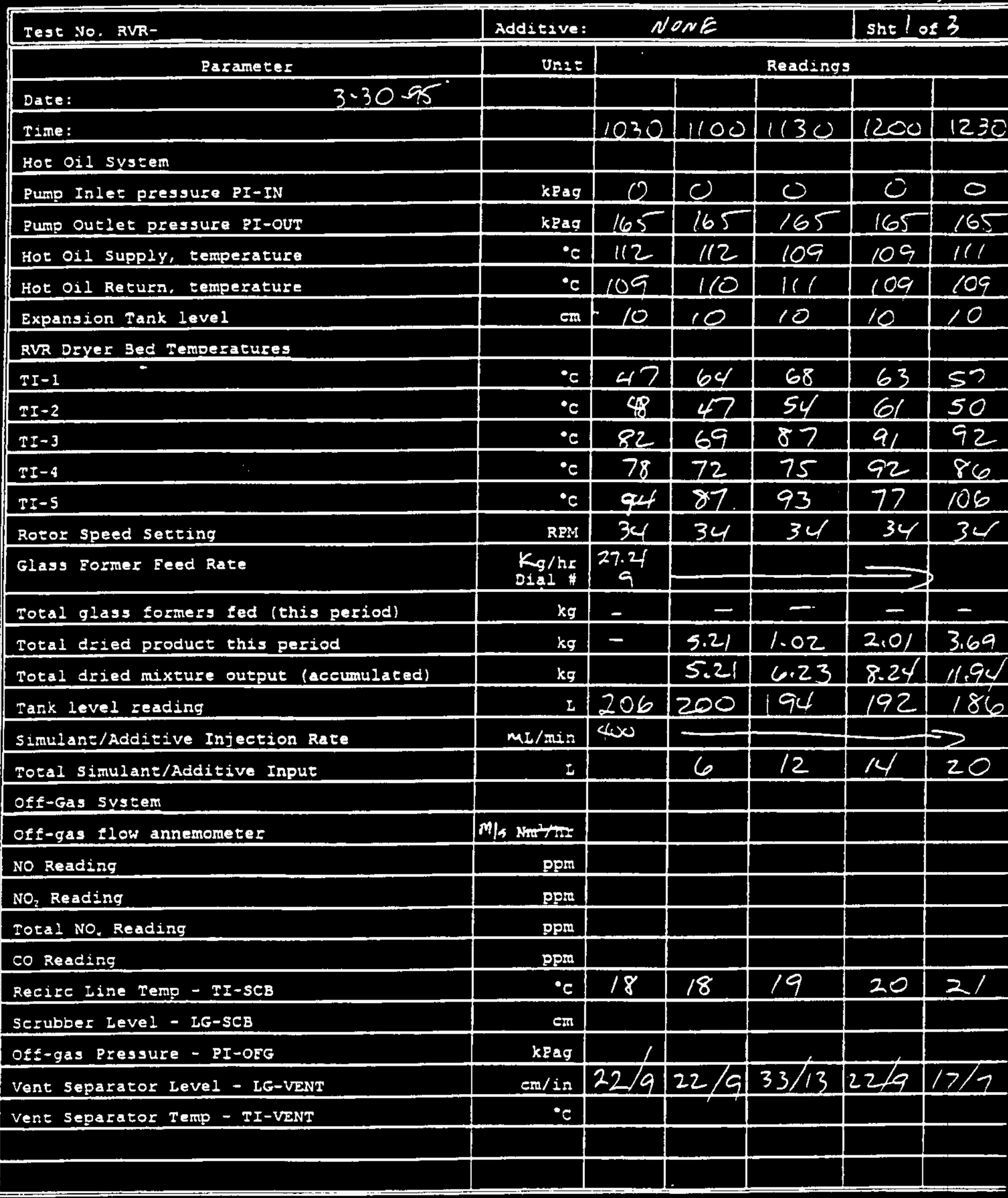


WHC-SD-WM-VI-031

Revision 0

Scoping reat - Datagheot

rage 1 of

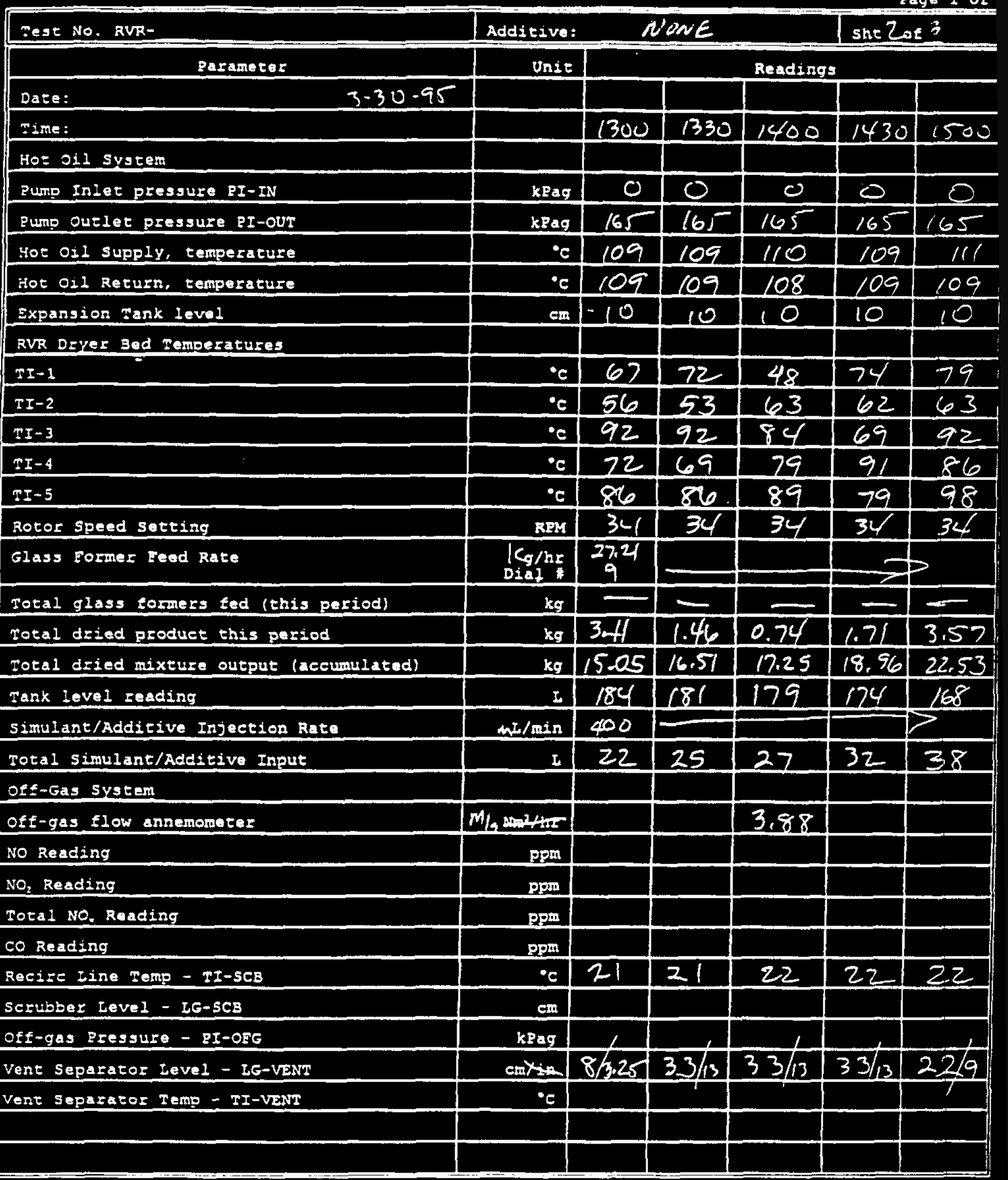




\section{Scoping Taet - Datraheot}

Page 1 of

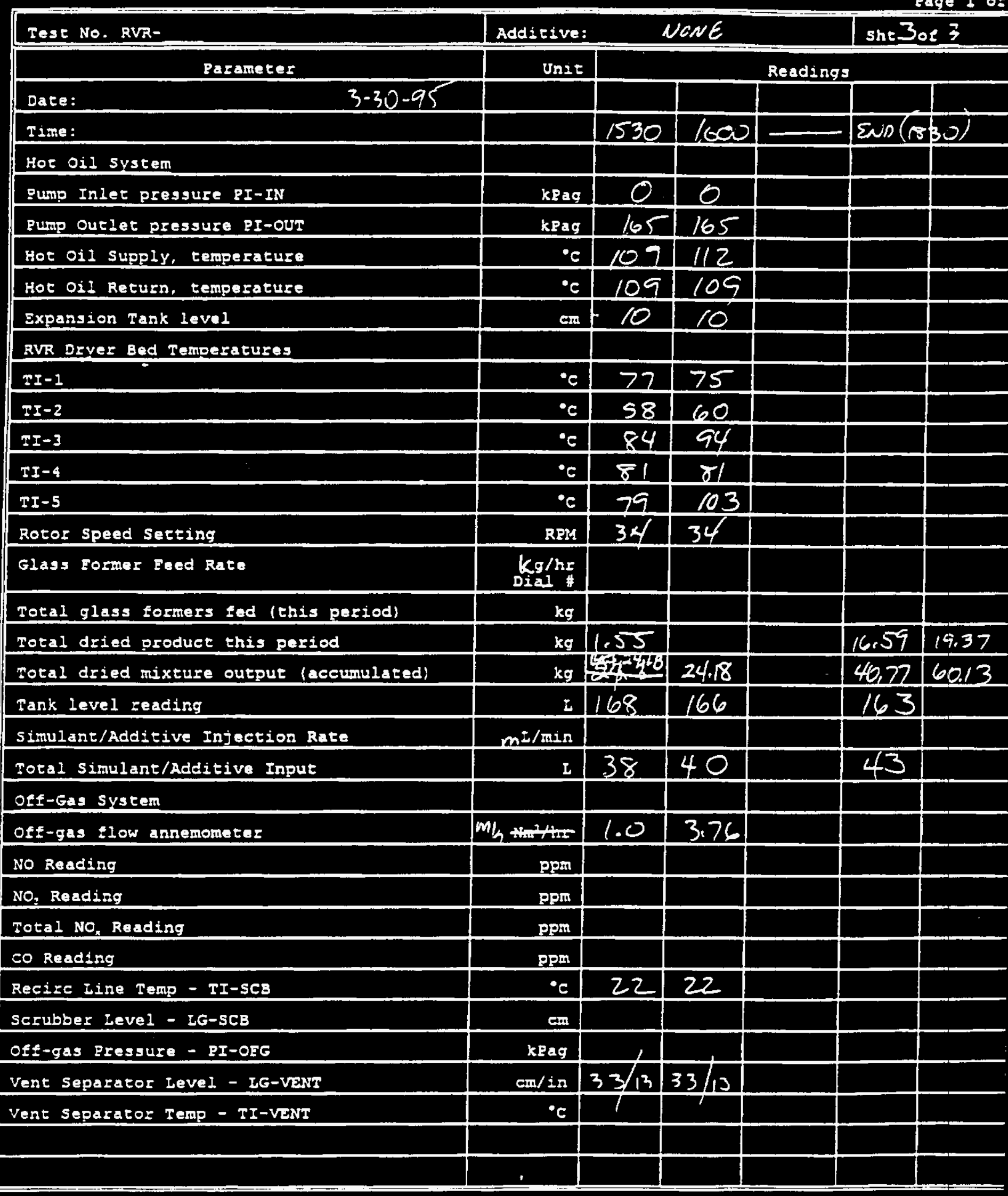


WHC-SD-WM-VI-031

Revision 0

Scoping rout - Datubhoet

Page 1 of

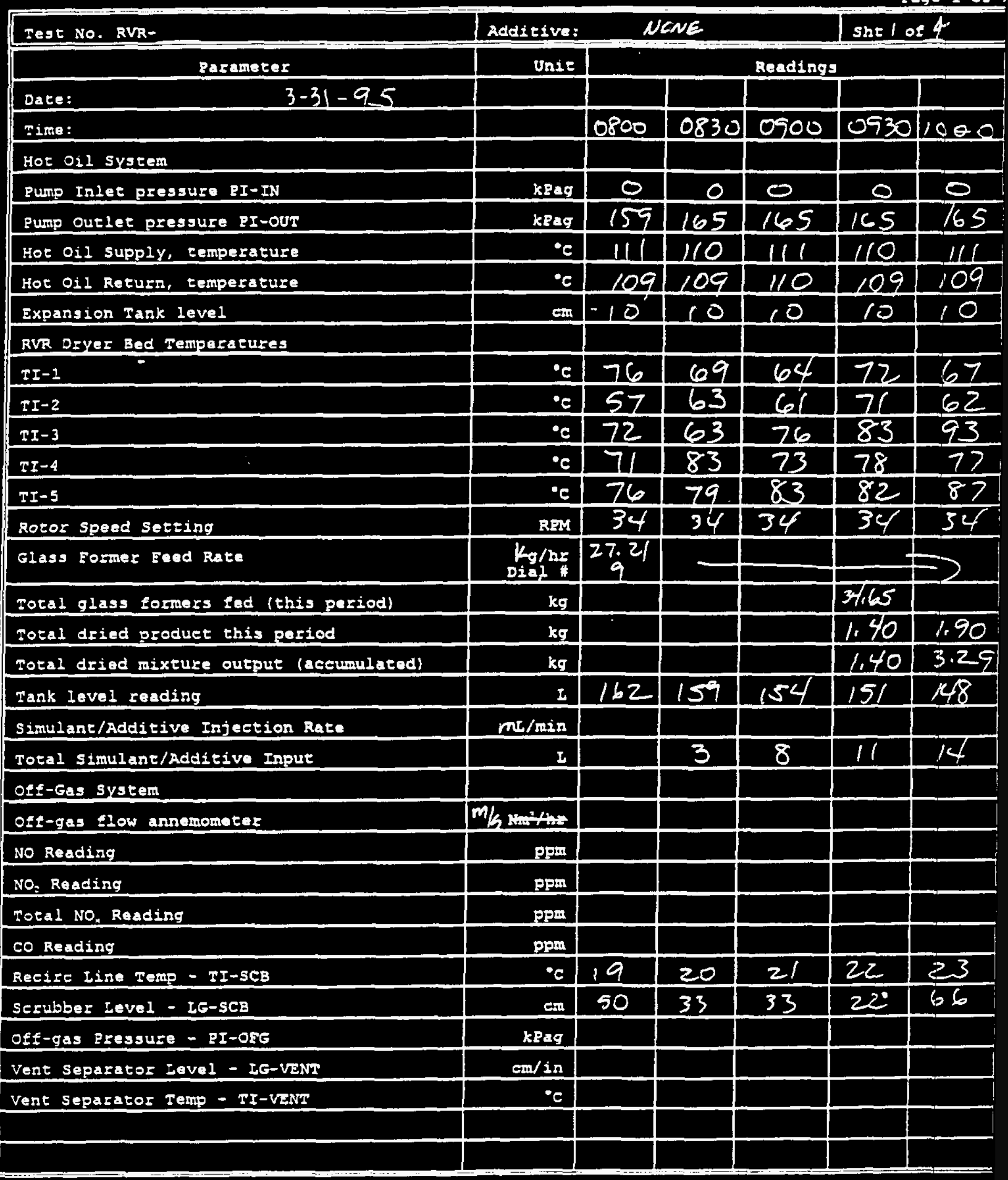




$$
\text { WHC-SD-WM-VI-031 }
$$

Revision 0

Seoping Jest - Datumbet

Rage: of

\begin{tabular}{|c|c|c|c|c|c|c|}
\hline Test No. RVR- & Additive: & $N$ & ME & & she Zo: & 4 \\
\hline \multirow{2}{*}{\multicolumn{7}{|c|}{$3-31-95$}} \\
\hline & & & & & & \\
\hline Time: & & 1030 & 1100 & 1130 & 1200 & 1230 \\
\hline \multicolumn{7}{|l|}{ Hot oil system } \\
\hline Punp Inler pressure EI-IN & $\mathrm{kPag}$ & 0 & () & 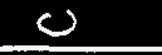 & 0 & 0 \\
\hline Eump Outlet pressure PI-OUT & $\mathrm{krag}$ & 165 & 165 & 165 & 165 & 165 \\
\hline Hot oil supply, temperature & $\bullet$ & 112 & 111 & 112 & 112 & 110 \\
\hline Hot oil Return, temperature & ${ }^{\circ} \mathrm{C}$ & 110 & 11 & 11 & 111 & 108 \\
\hline Expangion rank level & ear & $-/ 0$ & 10 & 10 & $\angle 0$ & \\
\hline \multicolumn{7}{|l|}{ RVR Dryez Bed Temperatures } \\
\hline$I-1$ & ${ }^{\circ} \mathrm{C}$ & 77 & 78 & 68 & 71 & 64 \\
\hline$T I-2$ & cc & 69 & 76 & 66 & 67 & 66 \\
\hline$I I-3$ & $\cdot c$ & 78 & 86 & 88 & 92 & 96 \\
\hline$I I-4$ & ${ }^{\circ} \mathrm{c}$ & 86 & 85 & & 68 & -78 \\
\hline $5 I-5$ & $\cdot c$ & 72 & 99 & 99 & 76 & 97 \\
\hline Rotor Speed Setting & REM & $3 c$ & 34 & 34 & 34 & 34 \\
\hline \multicolumn{7}{|l|}{ Glass Formex Eeed Rate } \\
\hline Total glass formers fod (this period) & $\mathrm{kg}$ & & & & & \\
\hline \multicolumn{7}{|l|}{ Total dried product this period } \\
\hline \multicolumn{7}{|l|}{ Total dried mixture output (accumulated) } \\
\hline \multicolumn{7}{|l|}{ Tank level reading } \\
\hline \multicolumn{7}{|l|}{ Simulant/Additive Injection Rate } \\
\hline Total simulant/Additive Input & L. & 18 & 20 & 22 & 26 & 32 \\
\hline \multicolumn{7}{|l|}{ off-Gas system } \\
\hline off-gas flow annemometer & $11 / 6 \sin ^{2}+4 x$ & & & & & \\
\hline \multicolumn{7}{|l|}{ No Reading } \\
\hline \multicolumn{7}{|l|}{$\mathrm{NO}_{2}$ Reading } \\
\hline \multicolumn{7}{|l|}{ Total NO, Reading } \\
\hline \multicolumn{7}{|l|}{ Co Reading } \\
\hline \multicolumn{7}{|l|}{ Recire Iine Temp - TI-SCB } \\
\hline \multicolumn{7}{|l|}{ Scrubbex Level - LG-SCB } \\
\hline \multicolumn{7}{|l|}{ Off-gas Pressure - PI-OFG } \\
\hline Vent Separator Level - IG-VaNr & $\cos /$ in & & & & & \\
\hline Vent Separator Temp - TI-VENT & 'c & & & & & \\
\hline & & & & & & \\
\hline & & & & & & \\
\hline
\end{tabular}


WHC-SD-WM-VI-031

Revision 0

scoping poat - Daterboot

Page 1 of

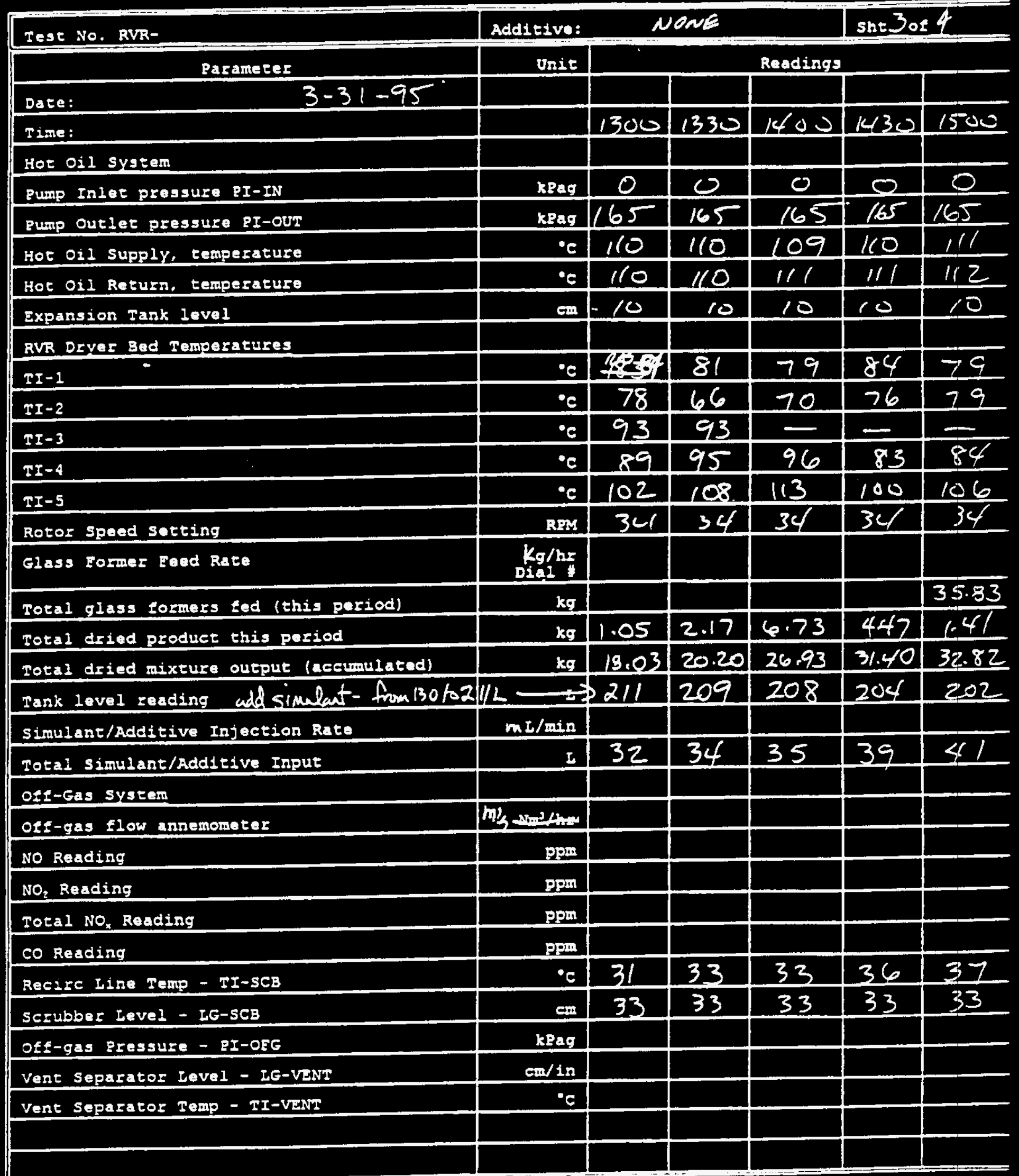


WHC-SD-WM-VI-031

Revision 0

scoping Tast - Dataghet

Eage 1 of

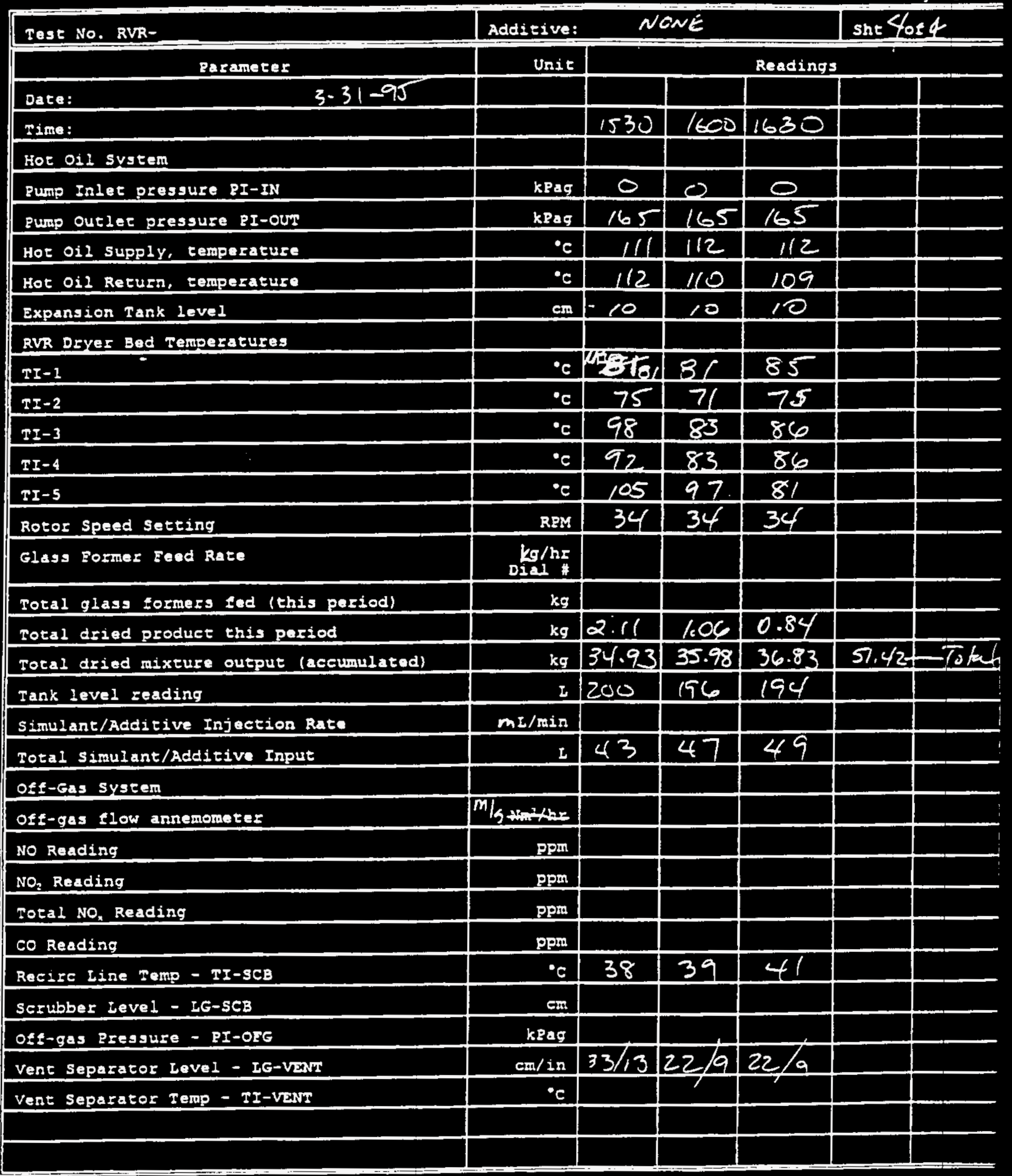


Scoping roet - Datanhoet

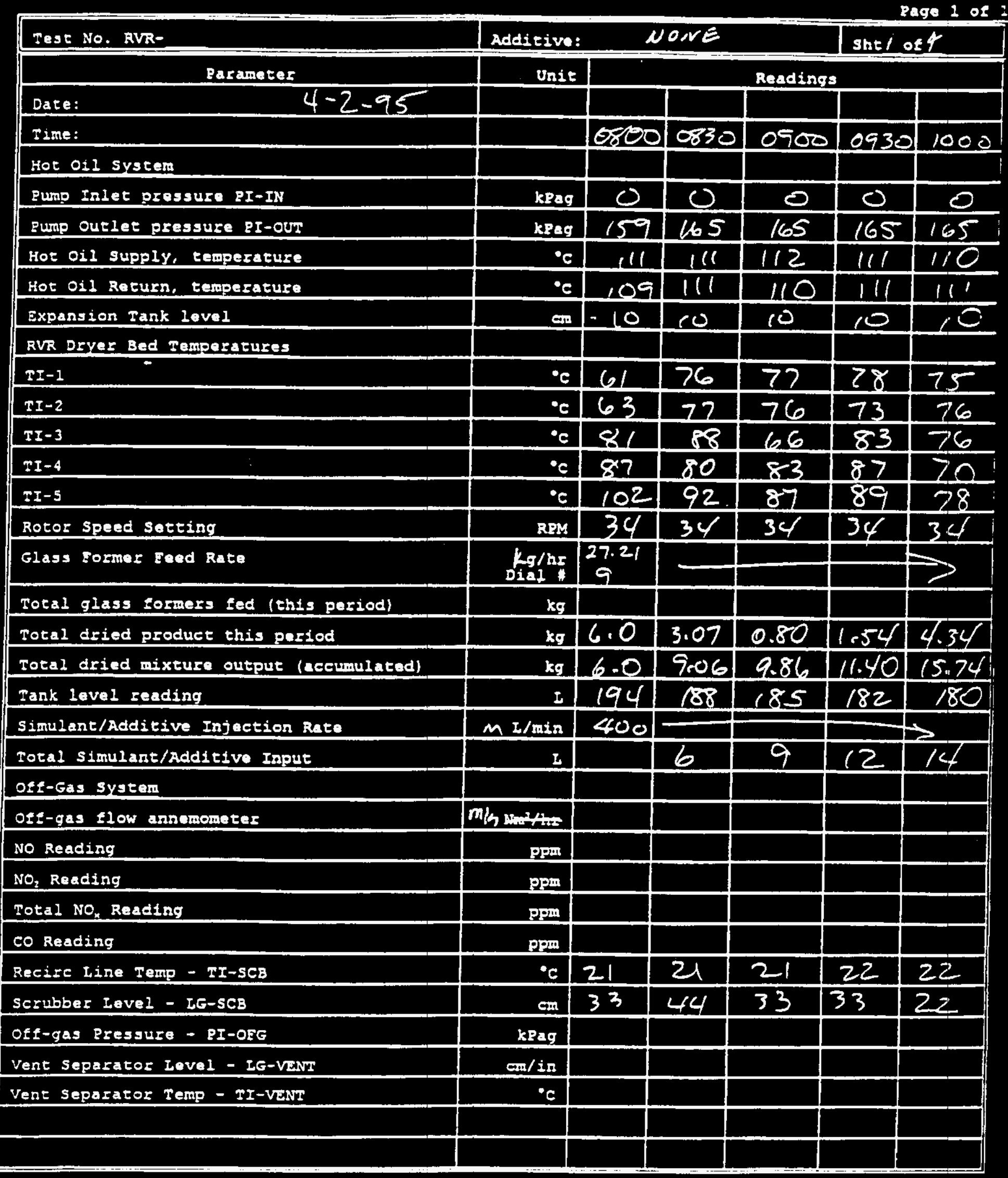


WHC-SD-WM-VI-031

-Revision 0

Sooping Port - Dataghedt

Page 1 of

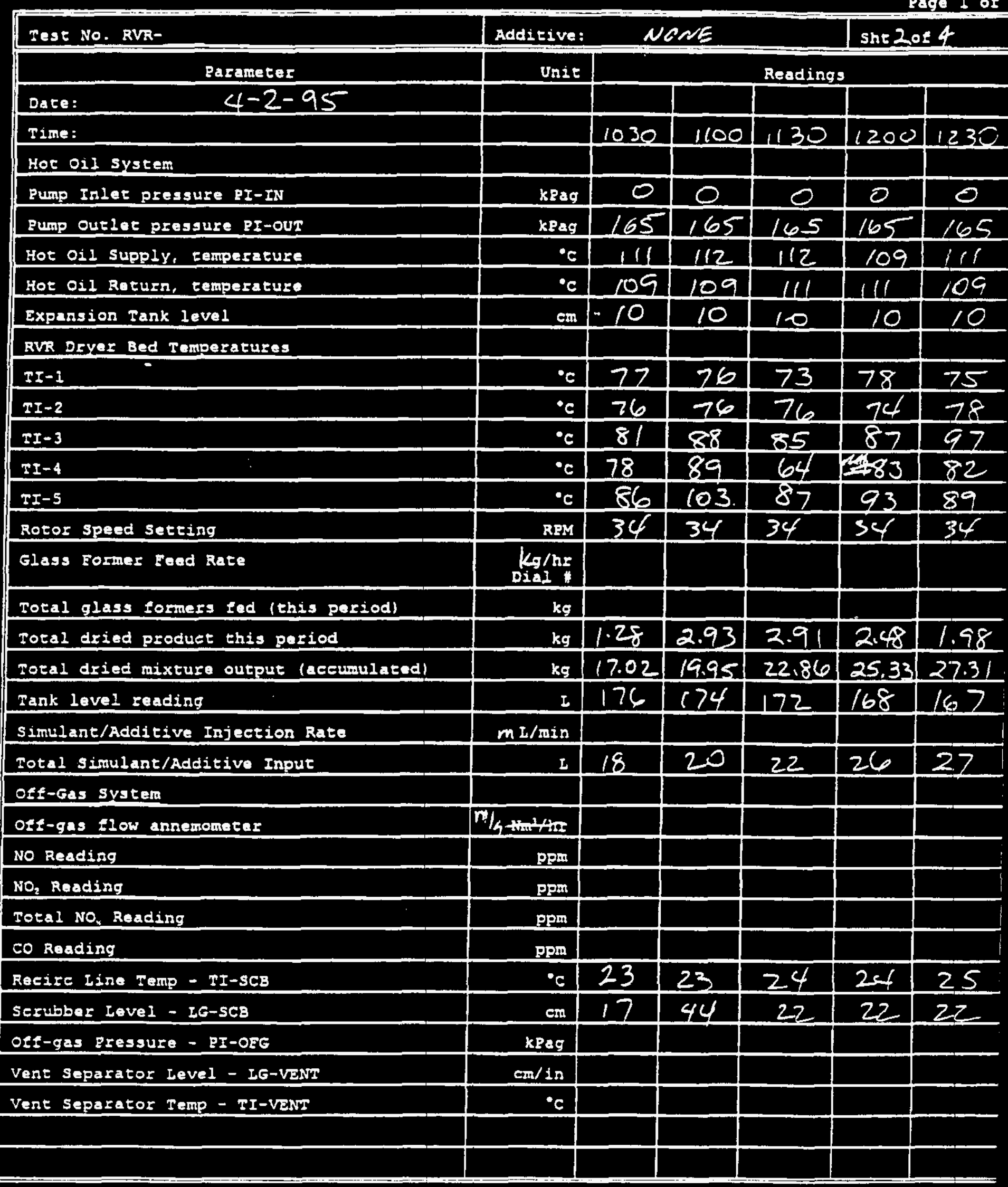




\section{WHC-SD-WM-VI-031 \\ Revision 0}

geoping reat - Datachor

Rage 1 of

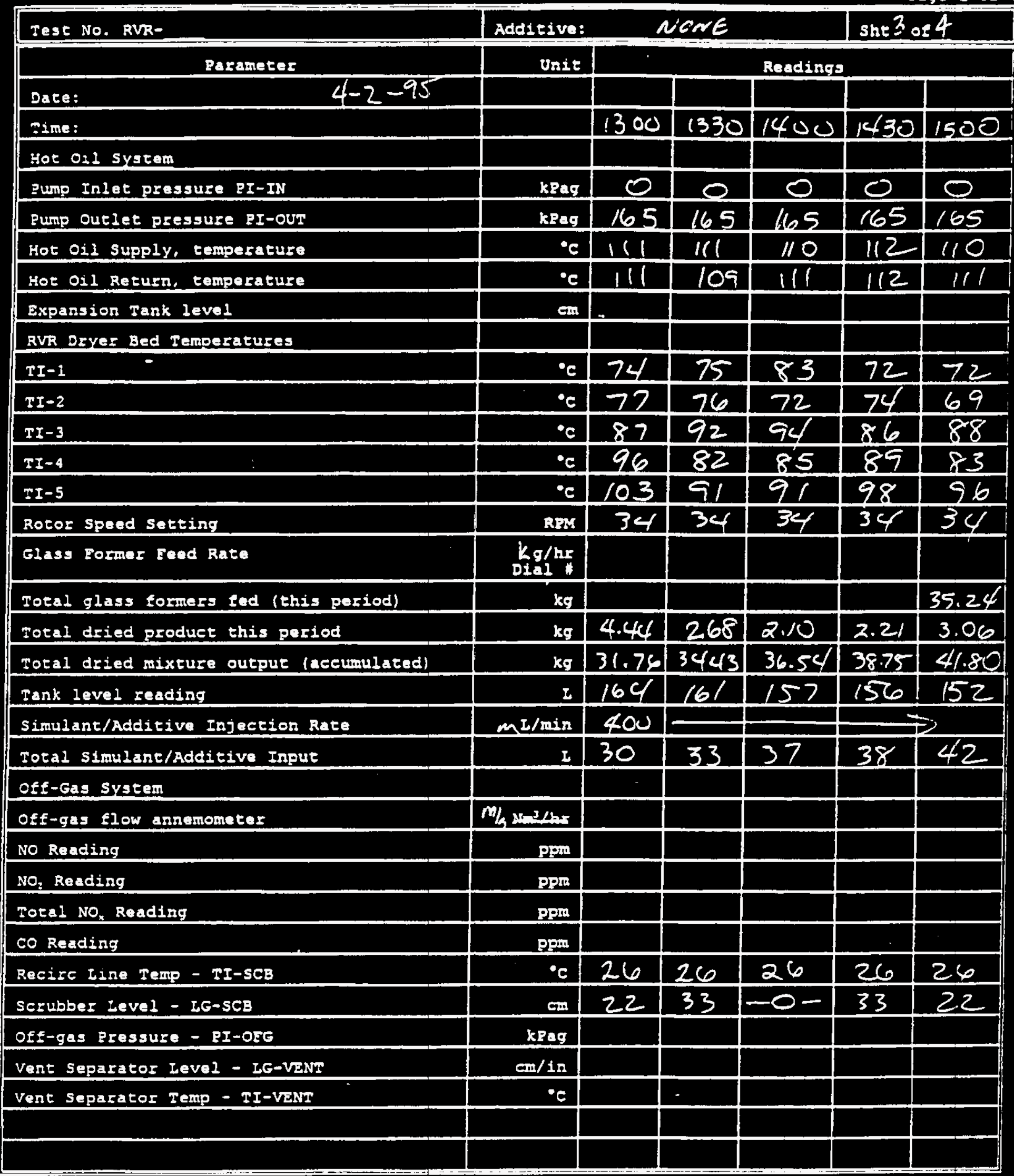


WHC-SD-WM-VI-031

Revision 0

Scopsing Toat - Datasheat

\begin{tabular}{|c|c|c|c|c|c|c|}
\hline Test No. RVR- & Additive: & \multicolumn{2}{|c|}{ NGNE } & & \multicolumn{2}{|c|}{$\sin 4$ of 4} \\
\hline Parameter & Unit & \multicolumn{5}{|c|}{ Readings } \\
\hline $4-2-95$ & & & & & & \\
\hline Time: & & 1530 & 1600 & 1630 & 1700 & \\
\hline \multicolumn{7}{|l|}{ Hor oil syarem } \\
\hline Pump Inlet presgure BI-IN & kPag & 0 & 0 & 0 & 0 & \\
\hline Pump outles pressure PI-ouT & krag & 165 & 165 & 165 & 165 & \\
\hline Hot oil supply, temperatuze & •c & 111 & 112 & 111 & 112 & \\
\hline Hot oil Return, temperature & ${ }^{\circ} \mathrm{C}$ & 111 & 111 & 112 & 111 & \\
\hline Expansion Tank level & $\mathrm{em}$ & 10 & co & 10 & 10 & \\
\hline \multicolumn{7}{|l|}{ RVR Dryer Bed Temperatures } \\
\hline$T I-1$ & •c & 84 & 84 & 88 & 82 & \\
\hline$T I-2$ & ${ }^{\circ} \mathrm{c}$ & 70 & 77 & 71 & 101 & \\
\hline$T I-3$ & ${ }^{\circ} \mathrm{C}$ & 27 & 96 & 96 & 92 & \\
\hline$T I-4$ & ${ }^{\circ} \mathrm{C}$ & 84 & 93 & 96 & 101 & \\
\hline$T I-5$ & $\cdot c$ & 68 & 90 & 105 & 112 & \\
\hline Roror speed Sotting & REM & 34 & 34 & 34 & 34 & \\
\hline \multicolumn{7}{|l|}{ Glags Former Feed Rate } \\
\hline potal glass tormers fed (this pariod) & $\mathrm{kg}$ & Q. & & & & \\
\hline Total dried product this period & $\mathrm{kg}$ & 0.44 & 0.99 & 4.15 & 5,96 & \\
\hline Total dried mixture output (aceurulated) & $\mathrm{kg}$ & 42,24 & 43.23 & 47.37 & 53,33 & 73,43 \\
\hline Tank level reading & 2 & 152 & 151 & 150 & 148 & \\
\hline Simulant/Additive Injection Rate & $m \pm / \min$ & & & & & \\
\hline Total Simulant/Additive Input & 2 & 42 & 43 & 44 & 46 & \\
\hline \multicolumn{7}{|l|}{ Off-Gas system } \\
\hline Off-gas flow annemometer & $M / 6 x^{2}+4=$ & & & & & \\
\hline No Reading & pem & & & & & \\
\hline $\mathrm{NO}_{2}$ Reading & ppm & & & & & \\
\hline Total NO, Reading & ppm & & & & & \\
\hline CO Reading & Pem & & & & & \\
\hline Recirc Line Temp - TI-SCB & •c & 26 & 26 & 26 & 27 & \\
\hline Serubber LeveI - LG-SCB & em & 33 & 33 & 33 & 33 & \\
\hline Off-gas Pressure - PI-OFG & $\mathrm{kgag}$ & & & & & \\
\hline Vent Suparator Level - IG-VENT & $\mathrm{cm} / \mathrm{in}_{\mathrm{n}}$ & & & & & \\
\hline Vent Separator Temp - TI-VENT & ${ }^{\circ} \mathrm{C}$ & & & & & \\
\hline & & & & & & \\
\hline & & & & & & \\
\hline
\end{tabular}


WHC-SD-WM-VI-031

Revision 0

Scoping Toat - Dataheot

Eage 1 of

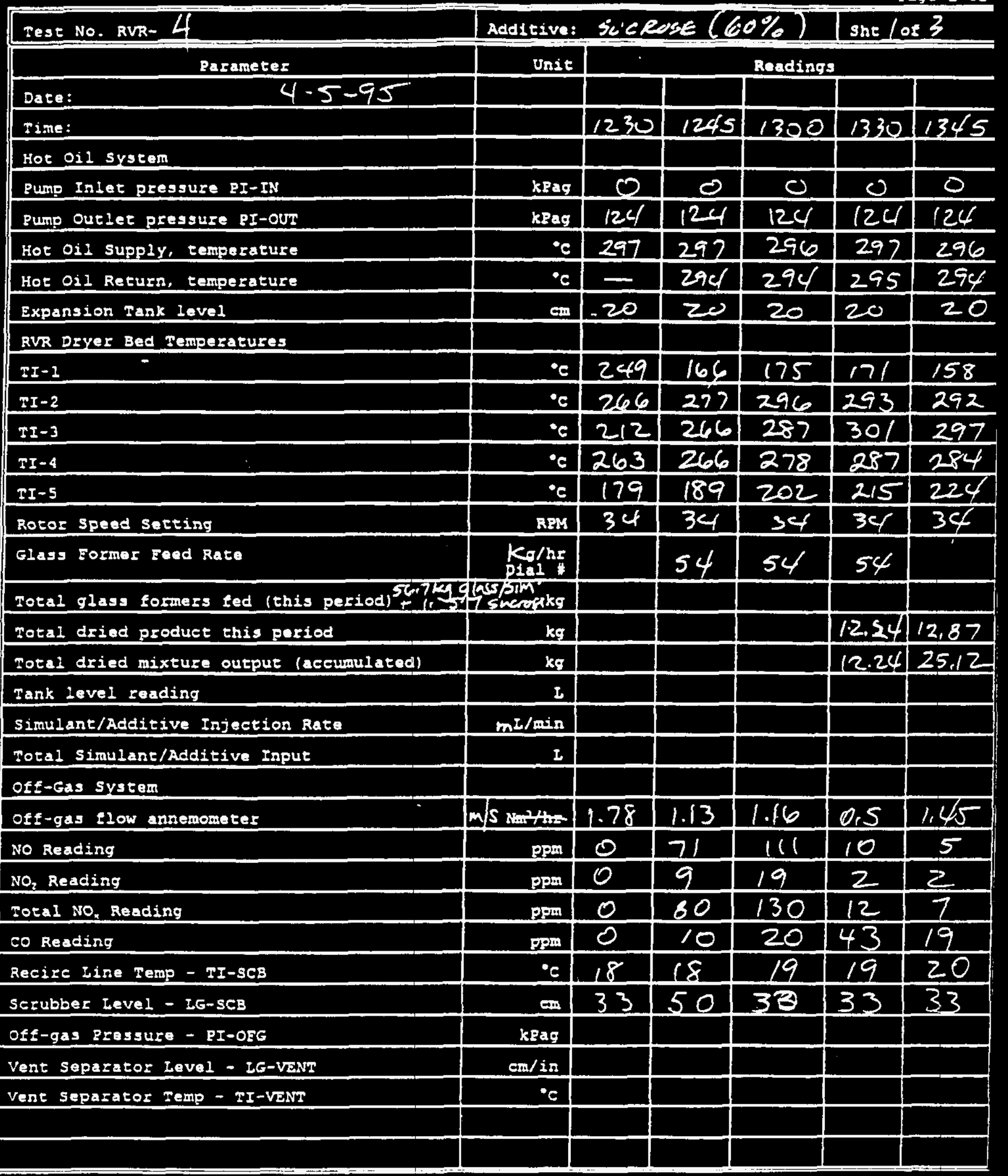


WHC-SD-WM-VI-03I

Revision 0

Scoping reat - Databhed

Rage 1 of

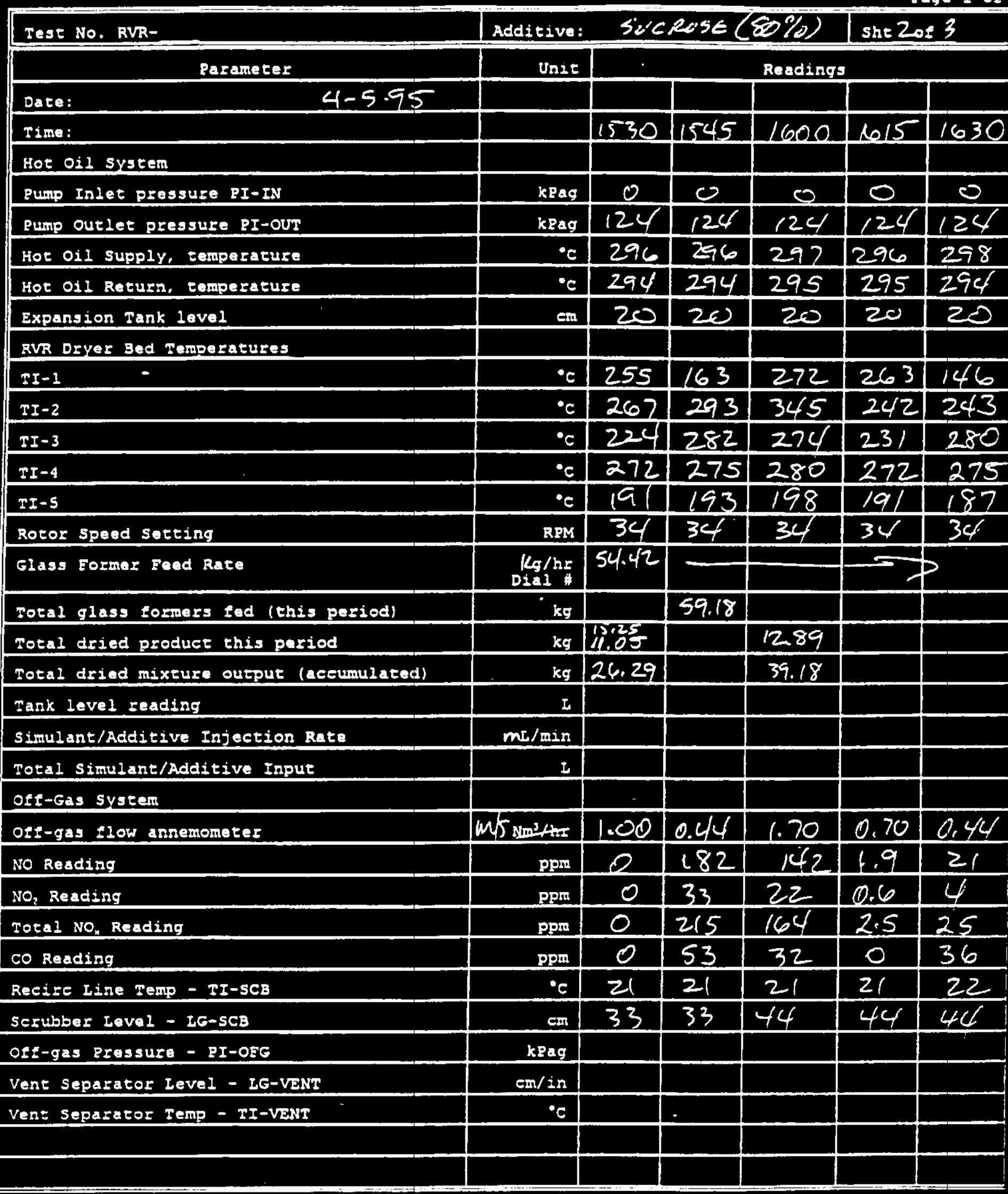


WHC-SD-WM-VI-03I

Revision 0

Scoptag poot - Dotaphoot

Rage 1 of

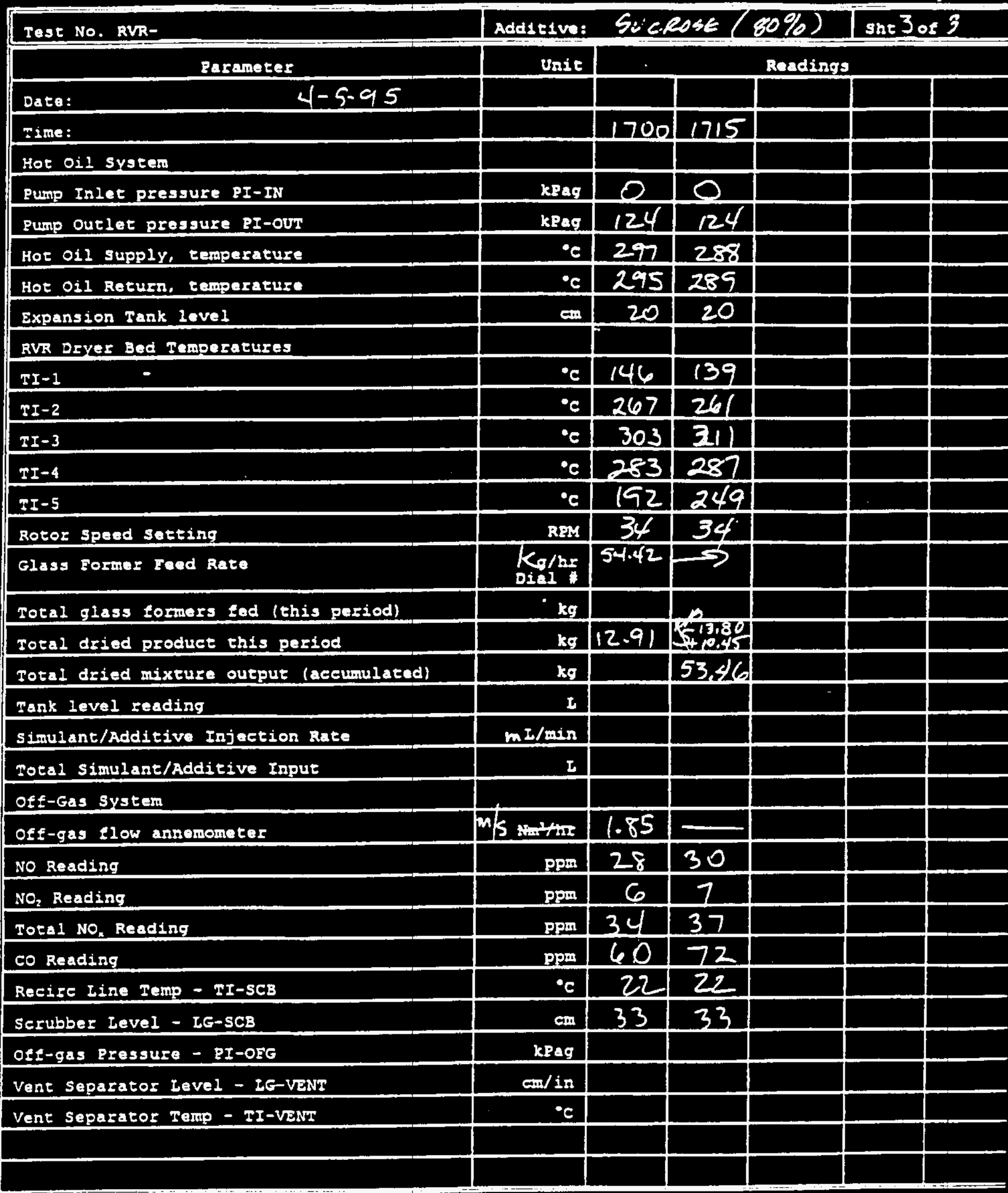


WHC-50-WH-VI-031

Revision 0

Scoping Pagt - Datuaheat

Eage 1 os

\begin{tabular}{|c|c|c|c|c|c|c|}
\hline Pacapeter & Unit & \multicolumn{5}{|c|}{ Readings } \\
\hline \multicolumn{7}{|l|}{ Date: } \\
\hline Time: & & 1200 & 1230 & 130 & 1330 & 2 \\
\hline \multicolumn{7}{|l|}{ Hot o11 system } \\
\hline \multicolumn{7}{|l|}{ Eung Inlet pressure EI-IN } \\
\hline Burp outlet presare PI-OUT & kgag & 124 & 12 & 124 & 124 & 124 \\
\hline Hot oil supgly, temperature & ${ }^{\circ} \mathrm{C}$ & & 298 & 299 & 299 & 299 \\
\hline Hot oil Return, temperature & ${ }^{\circ} \mathrm{C}$ & 258 & 297 & 298 & 298 & 298 \\
\hline Expansion Tank level & $\mathrm{cm}$ & & 10 & 10 & 10 & $\%$ \\
\hline \multicolumn{7}{|l|}{ RVR Dryer Bed Temperatures } \\
\hline$I I-1+-$ & ${ }^{\circ} \mathrm{C}$ & 253 & 156 & & 147 & 148 \\
\hline$I I-2$ & ${ }^{\circ} \mathrm{C}$ & 252 & 258 & 264 & 286 & 281 \\
\hline $2 I-3$ & ${ }^{\circ} \mathrm{C}$ & 212 & 274 & 2 & 296 & 301 \\
\hline$T I-4$ & ${ }^{\circ} \mathrm{C}$ & 282 & 282 & 285 & 287 & 282 \\
\hline $2 I-5$ & ${ }^{\circ} \mathrm{C}$ & 254 & 26.3 & 252 & 257 & 263 \\
\hline Rotor Speed Setting & REM & 34 & 34 & 34 & 34 & 34 \\
\hline \multicolumn{7}{|l|}{ Glass Former Feed Rate } \\
\hline \multirow{2}{*}{$\begin{array}{l}\text { Total glass formers fed (this period) } \\
\text { Total dried product this poriod }\end{array}$} & $\mathbf{k g}$ & & & & & - \\
\hline & $\mathrm{kg}$ & & & 15.66 & 18.03 & 10.93 \\
\hline Total dried mixture output (aceurulated) & kg. & & & 15.66 & 35.70 & 44.63 \\
\hline Tank level reading & L & & & & & \\
\hline simulant/Additive Injection Rate & $\mathrm{mI} / \mathrm{min}$ & & & & & \\
\hline Total simulant/Additive Input & 2 & & & & & \\
\hline \multicolumn{7}{|l|}{ Oft-Gas syaters } \\
\hline off-gas flow anmenoneter & Wam $1+4=$ & 1.55 & 3 & 1. & 1.20 & 1.30 \\
\hline No Reading & pom & 0 & 200 & & 183 & 182 \\
\hline $\mathrm{NO}_{2}$ Reading & Ppin & 2 & 58 & 27 & 39 & 98 \\
\hline Total NO Reading & Ppin & 0 & 258 & 167 & 222 & 280 \\
\hline Co Reading & PPsm & 0 & 82 & 43 & 72 & 63 \\
\hline Recirc Iine Temp - TI-sCB & ${ }^{\circ} \mathrm{C}$ & 17 & 17 & 18 & 19 & 20 \\
\hline Scrubber Level - IG-SCB & en & 22 & 22 & 33 & 33 & 50 \\
\hline Off-gas Rressure - gI-OFG & kPag & & & & & \\
\hline Vent Separator Level - LE-VONI & $\mathrm{cm} / \mathrm{in}$ & & & & & \\
\hline Vent separator Temp - TI-VSNP & ${ }^{\circ} \mathrm{C}$ & & & & & \\
\hline & & & & & & \\
\hline & & & & & & \\
\hline
\end{tabular}


WHC-SD-WM-VI-031

Revision 0

Seoping laot - Datorhoet

Rega 1 of

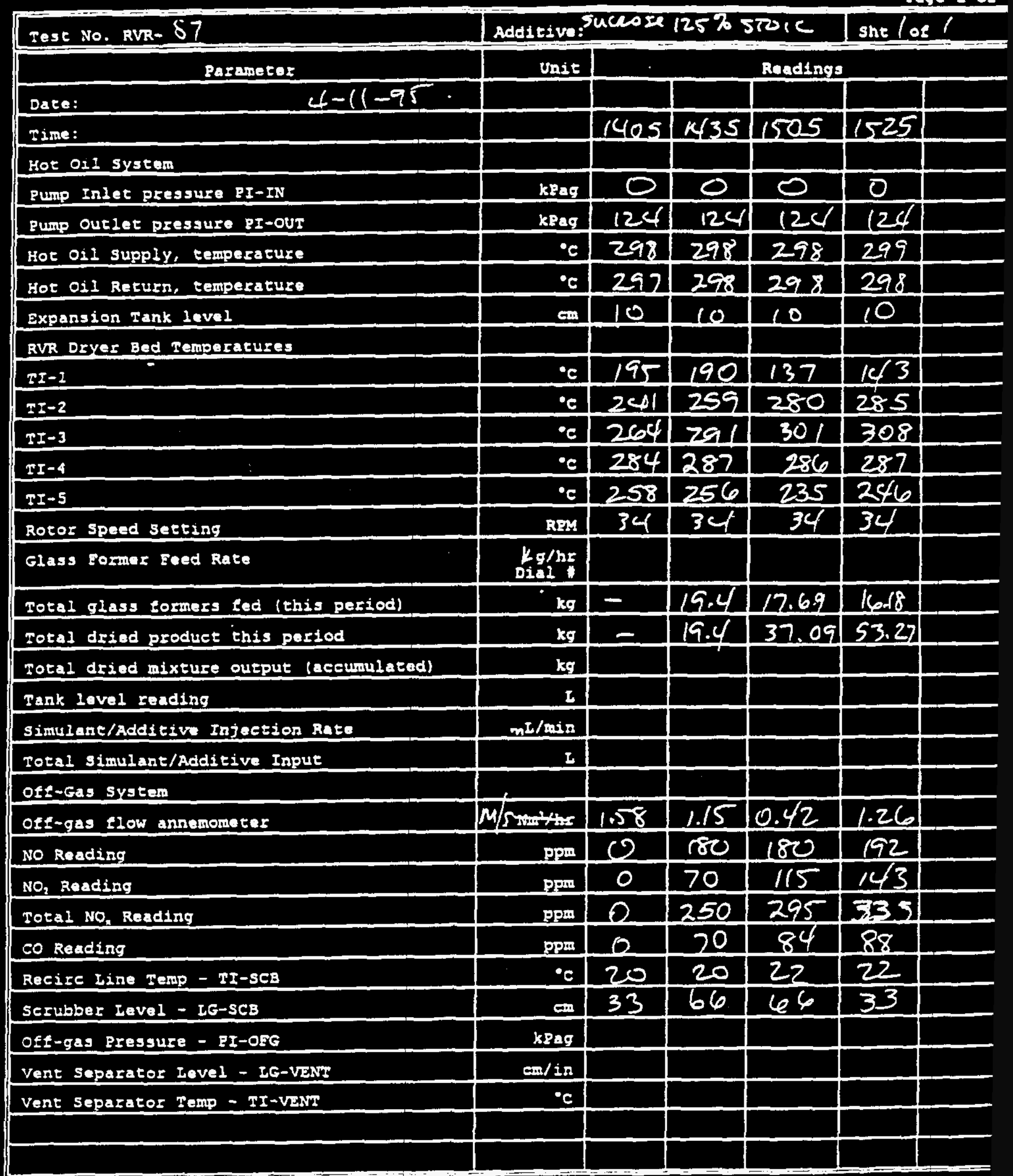


WHC-SD-WM-VI-031

Revision 0

This page intentionally left blank. 
WHC-SD-WM-VI-031

Revision 0

APPENDIX 2B

RVR TESTING SAMPLE LOG 


\section{SAMPLE LOG}

\begin{tabular}{|c|c|c|c|c|c|c|}
\hline SAMPLE NUMBER & DATE & TIME & SAMPLE TYPE & DESTINATION LAB & SHIPMINT DATE & LAB SAMPLE \# \\
\hline V 1 RS1 / 121-001 & $21 / 3$ & 1625 & Product & & & \\
\hline V 1 RS1 / $121-002$ & $21 / 3$ & 1745 & Product & & & \\
\hline V 1 RS3 / I $21-003$ & $5 / 4$ & 1245 & Dry SIM & & & \\
\hline V 1 RS1 / I $21-004$ & $5 / 4$ & 1345 & Reacted Product & & & \\
\hline V 1 RS4 / I $21-005$ & $5 / 4$ & 1740 & Reacted Product & & & \\
\hline V 1 RSS/I $21-006$ & $11 / 4$ & 1350 & Reacted Product & & & \\
\hline V 1 RS6/1 $21-007$ & $11 / 4$ & 1525 & Reacted Product & & & \\
\hline $\mathrm{V} 1 \mathrm{RS} 1-7 / \mathrm{W} 22-008$ & $11 / 5$ & 0910 & Scrubber Solution Final & & & \\
\hline
\end{tabular}


WHC-SD-WM-VI-031

Revision 0

\section{PART 3 - VE-SKULL MELTER PHASE 1 FINAL TEST REPORT}


WHC-SD-WM-VI-031

Revision 0

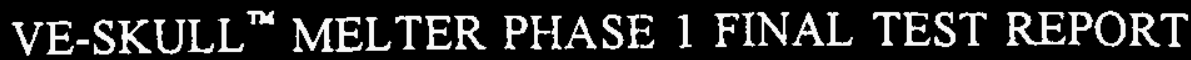

For

WHC Contract No. MMI-SVV-384211

EVALUATION OF MELTER SYSTEM TECHNOLOGIES FOR VITRIFICATION OF HIGH SODIUM CONTENT LLRW

VECTRA GSI Report No. WHC-VIT-05

August 1995

by

VECTRA Government Services, Inc. 2939 Richardson Road

Richland, WA 99352

Prepared by:

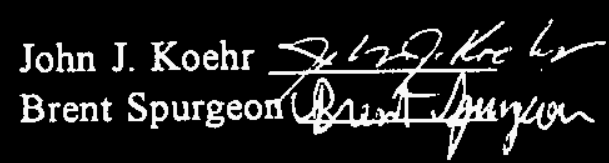

Approved by:

Brad Mason Zyplason 


\section{CONTENTS}

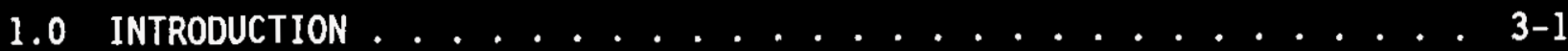

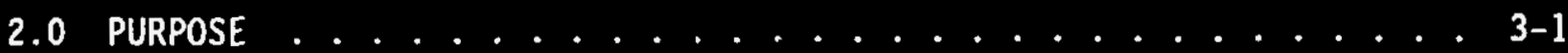

3.0 CHRONOLOGY OF EVENTS ................. 3-1

4.0 EQUIPMENT OPERATING BEHAVIOR .............. 3-4

4.1 START-UP . . . . . . . . . . . . . . 3-4

4.2 BOTTOM DRAIN ASSEMBLY ................. 3-5

4.3 GLASS FRIT SYSTEM ............. 3-6

4.4 SLURRY FEED SYSTEM . . . . . . . . . . . . 3-6

4.5 ELECTRODES ..................... 3-7

4.6 MELTER OPERATING BEHAVIOR ................. 3-8

4.7 MELTER SAMPLING ............... 3-8

4.8 OFF-GAS SYSTEM . . . . . . . . . . . . 3-9

4.9 REFRACTORY ................... 3- . . . . . .

4.10 SERVICE LOADS .................... 3- . . . . . . .

5.0 MELTER TESTS AND OBSERVATIONS ................ 3-10

5.1 MT-1: MELTER TEST PREPARATIONS . . . . . . . . 3-10

5.1.1 Melter Start-up .............. 3-10

5.1.2 VECTRA Glass Dry Feed Operations ....... 3-12

5.1.3 LLRW S1urry Feed Operations . . . . . . . . . 3-14

5.2 MT-2: REACT/VITRIFY GLASS FORMER, LLRW SIMULANT, AND

5.3 MT-3: VITRIFY SIMULATED DRY CALCINED PRODUCT $\therefore: \cdots 3-15$

5.4 MT-4: VITRIFY DRY CALCINED PRODUCT .......... 3-17

5.5 V-SIM HIGH THROUGHPUT TEST . ............ 3-19

5.6 SLURRY HIGH THROUGHPUT TEST ............. 3-19

5.7 HIGH SODIUM TESTING ................ 3-19

5.8 MELTER DRAINDOWN, SHUTDOWN, AND INSPECTION ........ 3-21

5.9 MELTER THROUGHPUT . . . . . . . . . . . . 3-22

5.10 MELTER MASS BaLANCE . . . . . . . . . . . . 3-22

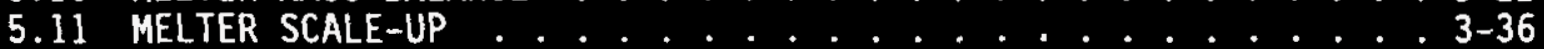

6.0 CONCLUSIONS $\ldots \ldots \ldots \ldots . \ldots \ldots . \ldots \ldots$

\section{APPENDIXES}

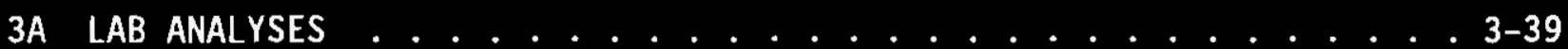

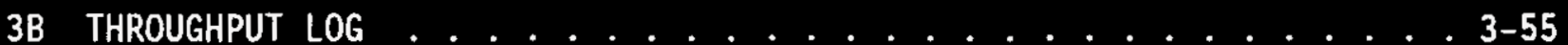

3C MASS BALANCE CALCULATIONS .................. 3-75 
WHC-SD-WM-VI-031

Revision 0

\section{LIST OF FIGURES}

3-1 MT-1 VECTRA Glass Throughput .............. 3-24

3-2 MT-1 VECTRA Glass Peak Throughput . . . . . . . . . 3-25

3-3 VECTRA Glass Idle Throughput ................... 3-26

3-4 MT-1 Slurry Throughput . . . . . . . . . . . . . 3-27

3-5 MT-1 Slurry Peak Throughput . . . . . . . . . . . . 3-28

3-6 MT-2 Slurry Throughput . . . . . . . . . . . . . 3-29

3-7 MT-3 V-SIM Throughput . . . . . . . . . . . 3-30

3-8 MT-4 Calcined Throughput .................. 3-31

3-9 V-SIM High Throughput (HT) Run ............ . 3-32

3-10 V-SIM High Throughput (HT) Run Peak Throughput . . . . . . . 3-33

3-11 Slurry High Throughput (HT) Run . . . . . . . . . . 3-34

3-12 High Sodium (Na) Run Throughput ............. 3-35 
WHC-SD-WM-VI-03I

Revision 0

\section{LIST OF TABLES}

3-1 VE-SKULL Melter Testing Chronology Summary . . . . . . . . . 3-2

3-2 Electrode Weight Losses . . . . . . . . . . . . . . . . 3-7

3-3 Melter System Phase 1 Testing Service Loads . . . . . . . . . . . 3-11

3-4 VECTRA G1ass Feed Composition . . . . . . . . . . . . . 3-13

3-5 RVR Glass Formers (Mix B) Composition . . . . . . . . . . . . 3-14

3-6 STurry Feed Batch Recipes . . . . . . . . . . . . . . . 3-15

3-7 Target Glass Composition . . . . . . . . . . . . . . . 3-16

3-8 MT-2 Slurry Feed Composition . . . . . . . . . . . . . 3-16

3-9 V-SIM Feed Composition ..................... 3-18

3-10 Boric Acid Addition to Dry Calcined Feed Product . . . . . . . 3-19

3-11 V-SIM High Throughput Summary . . . . . . . . . . . . . . . . 3-20

3-12 LLRW Slurry Feed High Throughput Summary . . . . . . . . . . . . . 3-20

3-13 High Sodium Run Batch Makeup . . . . . . . . . . . . . . . 3-20

3-14 High Sodium Run Throughput Summary . . . . . . . . . . . . . 3-21

3-15 Phase 1 Throughput Summary . . . . . . . . . . . . . . . 3-23

3-16 VE-SKULL Melter Sizing Specifications . . . . . . . . . . . 3-37 
WHC-SD-WM-VI-031

Revision 0

This page intentionally left blank. 
WHC-SD-WM-VI-031

Revision 0

PART 3 - VE-SKULL MELTER PHASE 1

FINAL. TEST REPORT

\subsection{INTRODUCTION}

The Westinghouse Hanford Company (WHC) is conducting an evaluation of glass melter system technologies for vitrification of liquid low-level radioactive waste (LLRW) streams consisting mostly of sodium nitrate and nitrite salts in alkal ine liquid slurry form. As part of this technology evaluation, VECTRA has demonstrated performance of the EnviroGlass ${ }^{\text {th }}$ Vitrification System feed and VE-SKULL" melter subsystems. The VE-SKULL" melter is the heart of the EnviroGlass ${ }^{\text {m }}$ Vitrification System. Performance testing for EnviroGlass th $^{\mathrm{H}}$ feed system components has been completed and documented in Part 1 (Fluid Bed Calciner Test) and Part 2 (RVR Dryer/Calciner Test) of this report.

\subsection{PURPOSE}

This final test report (Part 3 ) documents accomplishment of the VE-SKULL" melter test objectives. Test objectives include the following:

2.1 Demonstrate ability of VE-SKULL" melter to melt dry/calcined LLRW (from fluid bed Calciner and RVR dryer tests), simulated calcined LLRW, and simulant slurry feed combined with glass formers and reductants to create a product glass formulation which exceeds performance criteria including PCT tests.

2.2 Verify operating parameters while processing with high-sodium LLRW for test basis glass compositions, i.e., power supply voltage and amperage, throughput capability, cold-cap (batch blanket) depth, degree of phase separation, glass foaming, melt characteristics of dry/calcined feed materials, glass pour nozzle operation, and offgas rates and compositions.

2.3 Investigate throughput capability of melter.

\subsection{CHRONOLOGY OF EVENTS}

A detailed chronology of VE-SKULL" melter Phase 1 testing has been recorded in the Test Log. Detailed times for specific tests are recorded in the Throughput Log (Appendix $3 C$ ). A brief test chronology summary is provided in Table 3-1. 
Table 3-1. VE-SKULL Melter Testing Chronology Summary. (3 sheets)

\begin{tabular}{|c|c|}
\hline Date & Activities/events \\
\hline $\begin{array}{l}3 / 28 / 95 \\
\text { (MT-1, start-up, } \\
\text { boring bar) }\end{array}$ & $\begin{array}{l}\text { - } 976 \mathrm{~kg}(2,152 \mathrm{1b}) \mathrm{glass} \text { cullet in melter } \\
\text { - Attempted start-up with boring bars } \\
\text { - Poor success due to boring bar high iron/low } \\
\text { magnesium content } \\
\text { - } 11 \text { boring bars consumed, unable to establish molten } \\
\text { glass pool between electrodes } \\
\text { - Boring Bar start-up terminated due to melter head MCW } \\
\text { jacket erosion }\end{array}$ \\
\hline $\begin{array}{l}3 / 30 / 95-4 / 1 / 95 \\
\text { (MT-1, start-up, } \\
\text { gas burner) }\end{array}$ & $\begin{array}{l}\text { - Melter head MCW jacket repaired } \\
\text { - Performed start-up with gas burners } \\
\text { - Melter in auto temperature control } \\
\text { - } 408 \mathrm{~kg}(900 \text { 1b) cullet added as make-up. }\end{array}$ \\
\hline $\begin{array}{l}4 / 2 / 95-4 / 6 / 95 \\
(M T-1, \text { transition } \\
\text { batch) }\end{array}$ & $\begin{array}{l}\text { - } 179 \mathrm{~kg}(395 \mathrm{lb}) \text { of various transition batches } \\
\text { periodically added to melter } \\
\text { - Establish joule heat through drain ring and pin to } \\
\text { melt bottom glass } \\
\text { - On-line replacement of drain ring }\end{array}$ \\
\hline $\begin{array}{l}4 / 7 / 95-4 / 17 / 95 \\
\text { (MT-1, VECTRA } \\
\text { batch) }\end{array}$ & $\begin{array}{l}\text { - VECTRA batch } \\
\text { - Performed initial drain of } 233 \mathrm{~kg}(513 \mathrm{lb}) \mathrm{glass} \\
\text { - } 5401 \mathrm{~kg}(11,909 \mathrm{lb}) \text { VECTRA glass dry batch added } \\
\text { - }>3886 \mathrm{~kg}(8569 \mathrm{~b}) \text { VECTRA glass drained } \\
\text { - Idled melter over Easter Weekend }(4 / 14-4 / 16) ; 135 \mathrm{~kg} \\
\text { (297 1b) dry VECTRA glass batch feed; } 136 \mathrm{~kg}(300 \mathrm{lb}) \\
\text { glass drained } \\
\text { - Average feed throughput } 30.2 \mathrm{~kg} / \mathrm{hr}(66.6 \mathrm{lb} / \mathrm{hr}) \\
\text { - Peak feed throughput } 88.5 \mathrm{~kg} / \mathrm{hr}(195.2 \mathrm{lb} / \mathrm{hr}), \\
156 \mathrm{~kg} / \mathrm{hr}^{2} \mathrm{~m}^{2}\left(32.01 \mathrm{~b} / \mathrm{hr}-\mathrm{ft}^{2}\right)\end{array}$ \\
\hline $\begin{array}{l}4 / 17 / 95-4 / 21 / 95 \\
\text { (MT-1, s1urry } \\
\text { feed) }\end{array}$ & $\begin{array}{l}\text { - Initial slurry feed system arrangement resulted in } \\
\text { 1ine plugging } \\
\text { - Additional slurry feed tank, mixer, and recirc hoses } \\
\text { added on melter test stand. Injection pump moved to } \\
\text { test stand. } \\
\text { - Commenced slurry feeding } \\
\text { - } 3563 \mathrm{~kg}(7857 \mathrm{lb}) \text { slurry feed added } \\
\text { - }>1437 \mathrm{~kg}(3169 \mathrm{~b}) \mathrm{glass} \text { drained } \\
\text { - Average feed throughput: } 36.2 \mathrm{~kg} / \mathrm{hr}(79.8 \mathrm{lb} / \mathrm{hr}) \\
\text { - Peak feed throughput: } 77.3 \mathrm{~kg} / \mathrm{hr}(170.5 \mathrm{lb} / \mathrm{hr}) \\
136.4 \mathrm{~kg} / \mathrm{hr}-\mathrm{m}^{2}\left(28.01 \mathrm{~b} / \mathrm{hr}^{2} \mathrm{ft}^{2}\right)\end{array}$ \\
\hline $\begin{array}{l}4 / 21 / 95-4 / 22 / 95 \\
\text { (MT-2, slurry } \\
\text { feed mass } \\
\text { balance) }\end{array}$ & $\begin{array}{l}\text { - Started slurry feed mass balance at } 2300 \text { on } 4 / 21 / 95 \text {. } \\
\text { - CEMS monitoring } \\
\text { - } 14 \text { hour steady state mass balance run ended at } 1300 \\
\text { on } 4 / 22 / 95 \text {. } \\
\text { - } 865 \mathrm{~kg}(1907 \mathrm{lb}) \text { slurry feed added } \\
\text { - }>406 \mathrm{~kg}(8961 \mathrm{~b}) \text { glass drained } \\
\text { - Average feed throughput: } 59.9 \mathrm{~kg} / \mathrm{hr}(132.0 \mathrm{lb} / \mathrm{hr}) \\
105.6 \mathrm{~kg} / \mathrm{hr}-\mathrm{m}^{2}\left(21.6 \mathrm{~b} / \mathrm{hr}^{\left.-\mathrm{ft}^{2}\right)}\right.\end{array}$ \\
\hline
\end{tabular}


Table 3-1. VE-SKULL Melter Testing Chronology Summary. (3 sheets)

\begin{tabular}{|c|c|}
\hline Date & Activities/events \\
\hline $\begin{array}{l}4 / 22 / 95 \\
\text { (MT-3, simulated } \\
\text { dry calcined } \\
\text { feed) }\end{array}$ & $\begin{array}{l}\text { - Prebatched dry "V-SIM" feed to simulate dry calcined } \\
\text { fluid bed product. Started a mass balance at } 1900 \text { on } \\
4 / 22 / 95 \text {. } \\
\text { - CEMS monitoring } \\
\text { - } 3 \text { hour steady state mass balance run ended at } 2200 \text { on } \\
4 / 22 / 95 . \\
\text { - } 100 \mathrm{~kg}(220 \mathrm{lb}) \mathrm{V} \text {-SIM added } \\
\text { - }>69 \mathrm{~kg}(151 \mathrm{lb}) \mathrm{glass} \text { drained } \\
\text { - Average feed throughput: } 43.2 \mathrm{~kg} / \mathrm{hr}(95.2 \mathrm{lb} / \mathrm{hr}), \\
76.2 \mathrm{~kg} / \mathrm{hr}^{2} \mathrm{~m}^{2}\left(15.6 \mathrm{~b} / \mathrm{hr}-\mathrm{ft}^{2}\right)\end{array}$ \\
\hline $\begin{array}{l}4 / 23 / 95 \\
\text { (MT-4, dry } \\
\text { calcined feed) }\end{array}$ & 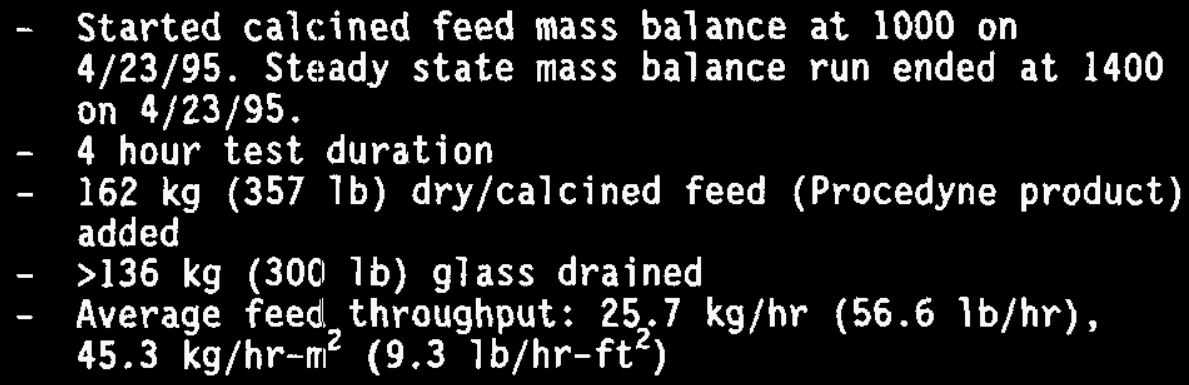 \\
\hline $\begin{array}{l}4 / 24 / 95-4 / 26 / 95 \\
\text { (V-SIM high } \\
\text { throughput) }\end{array}$ & $\begin{array}{l}\text { - Performed high throughput run with simulated } \\
\text { dry/calcined feed (V-SIM) } \\
\text { - } 2809 \mathrm{~kg}(6193 \mathrm{lb}) \mathrm{V} \text {-SIM feed added } \\
\text { - }>2101 \mathrm{~kg}(4632 \mathrm{lb}) \mathrm{glass} \text { drained } \\
\text { - Average feed throughput: } 47.4 \mathrm{~kg} / \mathrm{hr}(104.6 \mathrm{lb} / \mathrm{hr}) \\
\text { - Peak feed throughput: } 71.0 \mathrm{~kg} / \mathrm{hr}(156.5 \mathrm{lb} / \mathrm{hr}), \\
125.2 \mathrm{~kg} / \mathrm{hr}-\mathrm{m}^{2}\left(25.7 \mathrm{~b} / \mathrm{hr}-\mathrm{ft}^{2}\right) \\
\text { - Average Power: } 119.4 \mathrm{kw}\end{array}$ \\
\hline $\begin{array}{l}4 / 26 / 95 \\
\text { (Slurry high } \\
\text { throughput) }\end{array}$ & $\begin{array}{l}\text { - Performed high throughput run with liquid LLW } \\
\text { simulant, dry glass formers, and dry reductant slurry } \\
\text { feed } \\
\text { - } 375 \mathrm{~kg}(826 \mathrm{lb}) \text { slurry feed added } \\
\text { - }>310 \mathrm{~kg}(684 \mathrm{lb}) \text { glass drained } \\
\text { - Average feed throughput: } 67.5 \mathrm{~kg} / \mathrm{hr}(148.9 \mathrm{lb} / \mathrm{hr}) \text {, } \\
119.1 \mathrm{~kg} / \mathrm{hr}-\mathrm{m}^{2}\left(24.4 \mathrm{~b} / \mathrm{hr}^{2} \mathrm{ft}^{2}\right) \\
\text { - Average Power: } 121.3 \mathrm{kw}\end{array}$ \\
\hline $\begin{array}{l}4 / 26 / 95-4 / 27 / 95 \\
\text { (High Na high } \\
\text { throughput) } \\
4 / 27 / 95 \\
\text { (draindown) }\end{array}$ & 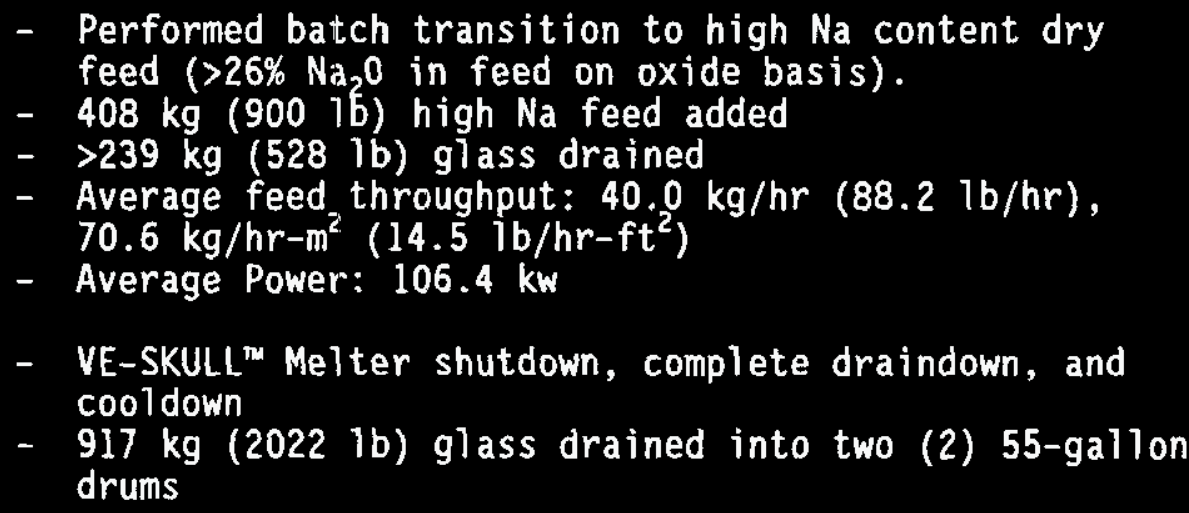 \\
\hline
\end{tabular}


WHC-SD-WM-VI-031

Revision 0

Table 3-1. VE-SKULL Melter Testing Chronology Summary. (3 sheets)

\begin{tabular}{|l|l|}
\hline \multicolumn{1}{|c|}{ Date } & \multicolumn{1}{c|}{ Activities/events } \\
\hline $\begin{array}{l}4 / 28 / 95 \\
\text { (inspection) }\end{array}$ & - Performed VE-SKULL" melter post test inspection \\
\hline
\end{tabular}

\subsection{EQUIPNENT OPERATING BEHAVIOR}

The Phase 1 Melter test equipment was installed within VECTRA's maintenance building in Richland, Washington. The Melter and dry feeder were mounted on a large structural steel test stand which contained space for the SCADA control system, CCTV, and melter watch standers. The Melter Cooling Water (MCW) tank, pump, heat exchanger, and ion exchanger were staged under the Melter Test Stand.

The slurry feed system was mounted on a test stand staged at ground leve] across from the melter test stand and consisted of a mix tank, glass former feeder, and two diaphram recirculation pumps. Slurry feed recirculation hoses ran from the diaphram recirculation pumps to the melter test stand batch drum and peristaltic feed pump. The glass quench trough and tank were staged at ground level between the slurry feed and melter stands.

The off-gas skid was mounted outside the maintenance building and contained the scrubber, vent fans and stack. The scrubber drain tank, drain pump, and spray pump were staged outside with hoses run to the scrubber. The quench water recirculation tanks, quench water supply pump, quench water return pump were staged outside the maintenance building with hoses run to the quench trough and tank inside the building. The cooling tower was staged outside the maintenance building with hoses run to the MCW heat exchanger under the melter test stand.

Other equipment stage outside included the diesel generator, service air compressor, nitrogen tanks, and propane tanks.

\subsection{START-UP}

Initial start-up was attempted using magnesium boring bars. Tempered glass cullet was loaded into the melter. A boring bar was attached to an oxygen supply hose, ignited, and manually inserted into the melter through an open 6" port between electrodes. The operator attempted to melt glass cullet between electrodes so that molten glass continuity could allow melter electrode operation for continued heat-up. Reddish smoke and a significant quantity of "slag" generated by the boring bars indicated a high iron content. A total of eleven (11) boring bars were expended attempting to start the melter. A flaw in the side of bar number 11 resulted in the erosion of the melter head MCW jacket. An MCW leak was created which required terminating boring bar start-up, isolating MCW, weld repair to the melter head jacket within the 6" nozzle, hydrostatic test to 45 psig, and inspection by an ASME certified inspector. 
WHC-SD-WM-VI-031

Revision 0

Following unsuccessful boring bar start-up, gas burners were positioned in two of the melter head 6" nozzles between electrode phases. The burners were ignited and glass cullet start-up was successfully accomplished.

\subsection{BOTTON DRATN ASSENBLY}

The bottom drain assembly consisted of a drain ring inserted inside melter refractory liner. The ring provided a small diameter orifice to control glass drain rate. The drain ring was held in place with four (4) lugs attached to a water cooled bottom support ring which was mounted to the melter bottom plate. The ring support lugs were electrically isolated from the ring by insulated pads. High drain ring temperatures melted the original isolation pads which required replacement with a material rated for higher temperature.

Sparge lines penetrated the bottom support ring through refractory into the melter to allow glass pool sparging with nitrogen. Nitrogen sparging was performed throughout Phase 1 testing.

An air cylinder operated, water cooled, stopper plug was utilized to plug the drain ring orifice when glass draining was not desired. The stopper plug performed very well as long as glass buildup broke away from the plug. Air and water tubes were directed at the bottom of the ring and could be utilized as a back-up method to cool the ring and help reduce ring temperature. Air cooling was periodically utilized to stop any leakage around ring perimeter following glass drain operations.

In order to allow glass draining following melter start-up, the glass on the bottom of the melter above the drain ring had to be fully melted. Heat generated by the three main electrodes was insufficient to melt the glass at the bottom of the melter. For the Test Program, the electrodes could not be fully extended due to fixed electrode holder design. A moveable electrode design will correct this limitation. A welding machine provided a single phase joule heating loop between one electrode phase (A) and the drain ring. Initially, a pin was inserted through the drain ring into the hot glass pool. The joule heating loop was established through this pin which was slowly withdrawn out the drain ring orifice as heat-up progressed. When the pin was fully withdrawn, the bottom joule heating loop was shifted back to the drain ring. Throughout the test program, drain ring temperature was maintained by the welding machine. Glass draining was initiated by opening the drain stopper plug and raising the drain ring to $>871^{\circ} \mathrm{C}\left(1600^{\circ} \mathrm{F}\right)$. Drain ring temperature was maintained below $1093^{\circ} \mathrm{C}\left(2000^{\circ} \mathrm{F}\right)$.

No drain ring damage or erosion was observed throughout phase 1 melter testing except minor damage resulting from metal deposits restricting orifice on the final day of testing.

The metal deposits originated from the boring bars used during the first attempted start up and also the erosion of the electrodes and the electrode holders. To remove this metal and restore the drain ring to an acceptable operating condition, the ring would have to be remachined to open the orifice back to its original diameter. 
WHC-SD-WM-VI-031

Revision 0

\subsection{GLASS FRIT SYSTEY}

The glass frit system functioned to cool the molten glass draining from the melter, and allow the glass to cool and shatter into granular pieces under controlled conditions. The glass product was collected in the glass frit tank. Quench water recirculated from two (2) $10,000 \mathrm{~L}$ poly tanks with a centrifugal supply pump, through the glass frit chute and into the glass frit tank. Molten glass was dumped from the melter into the quench water stream within the glass frit chute. Quench water was pumped from the frit tank by a variable speed centrifugal pump back into the poly tanks. The quench water was cooled by tank ambient losses.

The glass frit system produced a fine to medium granular product at moderate glass drain rates $[>4.5 \mathrm{~kg} / \mathrm{min}(10 \mathrm{lb} / \mathrm{min})]$. At drain rates less than approximately $2.3 \mathrm{~kg} / \mathrm{min}(5 \mathrm{lb} / \mathrm{min})$, or with excessive chute water flow, spaghetti-like glass strands were produced.

\subsection{SLURRY FEED SYSTEX}

The original slurry feed system consisted of a slurry mix tank with internal mixer, diaphragm recirculation pump, $25 \mathrm{ft}^{3}$ dry feeder, LLRW simulant drum mixer, and LLRW simulant drum transfer pump. The slurry mix tank was mounted on a scale which provided constant readout of mix tank weight. LLRW Simulant bulk drums were continuously mixed with installed drum mixers. LLRW simulant was transferred in calculated quantities from the drums to the slurry mix tank with a drum transfer pump. Calculated quantities of dry glass formers and sucrose were added to the slurry mix tank directly or with the dry feeder. The slurry mix tank was continuously agitated with the tank mixer and recirculated with the diaphragm pump. The melter feed line ran from the diaphragm pump discharge to the melter off-gas/feed plenum. The slurry mix tank weight was monitored to observe quantities of feed transferred from the recirculating slurry tank into the melter. During initial slurry feed operations feed line plugging resulted, and steady feed flow could not be maintained.

The slurry feed system was modified. A batch drum with mixer was placed on the melter test stand near the top of the Melter to serve as a slurry feed day tank. Larger diaphragm pumps were installed as recirculation pumps, and recirculation lines were run up to the melter stand. Intermediate quantities of slurry feed were transferred from the recirculation 1 ine to the batch drum as necessary. A positive displacement peristaltic tubing pump took a suction on the batch drum and injected slurry through tubing into the melter via the 16" off-gas/feed plenum. The feed tube extended down to about $15 \mathrm{~cm}$. (6 in) above the glass pool. The batch drum was calibrated for level which was periodicaliy monitored. Weight of simulant transferred from the slurry mix tank to the salvage drum was monitored on the slurry mix tank scale. This modified slurry feed system was utilized for melter test preparation slurry operations (MT-1), the slurry feed mass balance testing (MT-2), and the slurry feed high throughput testing. 
WHC-SD-WM-VI-031

Revision 0

\subsection{ELECTROOES}

The Phase 1 VE-SKULL" melter utilized three (3) 2-1/2" diameter vertical molybdenum electrodes. The electrodes were powered from a diesel generator through a 120V transformer and SCR. Electrode power levels ranged from 0 $145 \mathrm{kw}$. Idle power level was approximately $85 \mathrm{kw}$.

The electrodes penetrated the melter head and were held in position by stainless steel holders and water cooled stainless steel castings and stainless steel pipe sleeves. The castings were designed for water cooling to control electrode temperature and form a cold glass seal at the holder/ electrode interface, thus preventing corrosion from the molten glass. The cooling water was not initiated to the electrode holders throughout the test program due to presence of casting defects that caused minor water leakage into melter. The electrode thermocouples and electrode holder cooling water tubes failed as a result of no cooling water. Severe erosion of the stainless steel holders occurred below the upper glass melt line levels.

The electrodes were adjustable in height only during this test. The electrodes required very little adjustment during testing other than to extend two electrodes as they eroded. During testing, electrode phase B developed a phase imbalance due to electrode erosion. Electrode B was lowered further into the melter and the phase imbalance was corrected. Just prior to final melter drain down, electrode $A$ was also extended.

Inspection of the electrodes following final melter drain down indicated severe erosion of all three electrodes. Electrodes $A$ and B actually eroded to such an extent that a "bubble" erosion pocket in each electrode developed and a $12^{\prime \prime}-16^{\prime \prime}$ section of each electrode separated from the main electrode after 28 days of high temperature operation.

Erosion of electrodes was most severe in glass/gas interface zone as indicated by "necking down" of electrode just below electrode holder area. Pre- and post-testing electrode weight given in Table 3-2 indicate the extent of electrode wear.

Table 3-2. Electrode Weight Losses.

\begin{tabular}{|c|c|c|c|}
\hline \multirow{2}{*}{ Electrode } & \multicolumn{3}{|c|}{ Weight } \\
\cline { 2 - 4 } & Before & After & Loss \\
\hline$A^{*}$ & $195.47 \mathrm{~kg}$ & $150.22 \mathrm{~kg}$ & $45.25 \mathrm{~kg}$ \\
\hline $\mathrm{B}^{*}$ & $195.47 \mathrm{~kg}$ & $109.07 \mathrm{~kg}$ & $86.4 \mathrm{~kg}$ \\
\hline $\mathrm{C}$ & $195.47 \mathrm{~kg}$ & $186.90 \mathrm{~kg}$ & $8.57 \mathrm{~kg}$ \\
\hline
\end{tabular}

* Loss includes pieces that physically separated and

fell to the melter floor. 
WHC-SD-WM-VI-031

Revision 0

The electrodes were operated under high power operation for 28 days with glass melt temperature $>1400^{\circ} \mathrm{C}\left(2600^{\circ} \mathrm{F}\right)$. It is estimated that the melter was operated for at least 14 days at extreme high glass temperatures $>1480^{\circ} \mathrm{C}$ $\left(2700^{\circ} \mathrm{F}\right)$ with operation at $>1540^{\circ} \mathrm{C}\left(2800^{\circ} \mathrm{F}\right)$ for more than 7 days. The glass composition was at approximately 20 wt\% $\mathrm{Na}_{2} \mathrm{O}$ for 26 of the 28 days of operation. Electrode erosion is attributed primarily to lack of cooling water, glass pool level excursions below holder, and lack of sufficient inert gas purge during draindown.

\subsection{MELTER OPERATING BEHAVIOR}

The goal during dry feeding was to maintain glass pool level above the electrode holders, provide make-up for melter losses through volatization or draining, and maintain a stable "batch blanket" on top of the molten glass pool. Optimization of batch blanket thickness allowed control of off-gas particulate carry-over, minimized melter plenum temperature, and allowed operator observation of melter pool refinement via the CCTV system.

Glass pool "freeboard" level and batch blanket thickness were periodically checked by the operators by removing a $6^{\prime \prime}$ insulated plug from the melter head and inserting a calibrated dip stick into the molten glass pool. Glass draining was periodically performed throughout all phases of testing to control glass pool level. When operator inspection revealed sufficient glass pool level, glass draining was initiated by opening the remote drain stopper plug. Melter weight was transmitted from load cells and monitored by the operator at the control system. Drain rate was dependent upon glass and drain ring temperature. Draining was stopped by closing the drain plug when the desired weight of glass had been removed or when the desired freeboard level was achieved.

The melter electrodes were operated in automatic power control after start-up throughout all phases of testing. Glass temperature and refining rate were controlled by limiting electrode power level. One electrode phase supplied power to a welding machine connected to the drain ring lug. Power to the welding machine was manually adjusted to maintain drain ring temperature less than $1093^{\circ} \mathrm{C}\left(2000^{\circ} \mathrm{F}\right)$ and to keep the melter bottom glass molten. Following start-up, melter internal thermocouples failed and glass pool temperature indication was not available. Glass pool temperature was controlled by operator observation via the CCTV system, removal of insulated plugs for direct inspection through 6" melter head nozzles, and operator experience. Periodic dip thermocouple readings indicated glass temperature to be $1093-1560^{\circ} \mathrm{C}\left(2000-2850^{\circ} \mathrm{F}\right)$ following start-up throughout all phases of testing.

The nitrogen sparge system was operated continuously to facilitate molten glass pool mixing and refinement. Nitrogen sparge was maintained at a low level (5-20 scfh) throughout all testing.

\subsection{MELTER SAMPLING}

Product glass sampling was performed by inserting circular graphite forms into the molten glass drain stream. Alternatively, stainless steel ladles 
WHC-SD-WM-VI-031

Revision 0

were filled with molten glass and poured into forms. Resultant glass disks would shatter upon cooling unless annealed in an oven. Glass frit samples were withdrawn directly from the glass frit tank.

Product glass was originally green with glass cullet, transition batch, and VECTRA Glass feed. When LLRW simulant slurry feed testing was initiated the glass gradually turned darker with each pour. Final glass product was black.

\subsection{OFF-GAS SYSTEN}

The melter off gas was drawn out through the 16" combined off-gas/ feed plenum. Off-gas piping, metal hoses, and off-gas instrumentation were installed between the melter plenum and the suction side of a vent blower (located outside the test building) which discharged into the vent separator. The vent separator interfaced with centrifugal pumps for continuous vent separator draindown to a large poly tank and continuous vent separator return spray flow from the poly tank. The vent separator was vented into the stack and joined dilution air flow into stack at the discharge of a large vent blower.

The scrubber solution was not treated to control $\mathrm{pH}$. Original scrubber solution remained from previous RVR Dryer testing and contained noticeable amounts of particulates and glass formers from carryover. The vent separator tank and drain levels were monitored, during melter testing.

\subsection{REFRACTORY}

The melter is provided with a thin refractory liner inside the inner vessel she11. Following 26 days of high temperature operation with high (20 wt\%) $\mathrm{Na}_{2} \mathrm{O}$ glass the refractory appeared to be in original condition with no visible erosion except for:

- Several minor $(<1 \mathrm{~cm})$ concave indentations in refractory block in the glass/gas interface area. It is unknown if the concave indentations were initially present in refractory blocks.

- Mortar between blocks was partially eroded at interior surface leaving minor gaps $0-4 \mathrm{~mm}$ (0-0.16 inches) wide by $0-25 \mathrm{~mm}$ (0-1 inches deep) between blocks.

Refractory life is estimated to match 10 year design life of melter, if refractory seams-gaps are reduced to $<1 \mathrm{~mm}$ during initial refractory installations.

Mass balance calculations indicated an excess amount of chromium in the product glass. This excess chromium may be the result of minor refractory erosion. Approximately $1 \mathrm{~kg}$ of excess $\mathrm{Cr}_{2} \mathrm{O}_{3}$ resulted from $\mathrm{MT}-2$ testing, and approximately $180 \mathrm{~g}$ of excess $\mathrm{Cr}_{2} \mathrm{O}_{3}$ resulted from MT-3 testing. These values are consistent with observed refractory erosion. 
WHC-SD-WM-VI-031

Revision 0

\subsection{SERYICE LOADS}

The VE-SKULL" Melter was operated from 3/26/95 to 4/27/95 for the performance of Phase 1 testing. During this time period, various services were provided to sustain melter operation. These services include the following:

- Electrical power for melter electrodes, cooling tower, MCW pump, quench water recirculation pumps, off-gas blowers, scrubber drain and spray pumps, and slurry tank mixers (provided by diesel generator)

- Nitrogen gas for melter sparging and electrode holder cooling

- Propane gas for melter start-up burners

- Service water for cooling tower system, glass quench system, and off-gas system

- Service air for operation of slurry feed recirculation pumps and the melter drain plug valve.

A summary of service loads during the Phase 1 melter test period is given in Table 3-3.

\subsection{MELTER TESTS AND OBSERVATIONS}

\subsection{MT-1: MELTER TEST PREPARATIOHS}

VE-SKULL ${ }^{m}$ melter start-up and test preparations were performed from $3 / 28 / 95$ through $4 / 21 / 95$. During this period melter start-up was performed and the VE-SKULL" melter was operated cont inuously with glass cullet, dry VECTRA glass (20 wt $\% \mathrm{Na}_{2} \mathrm{O}$ ), and LLW simulant slurry feed.

\subsubsection{Melter Start-up}

VE-SKULL" melter initial start-up was performed from 3/28/95 through $4 / 2 / 95$. The cold melter was initially loaded with $976 \mathrm{~kg}(2152 \mathrm{lb})$ of tempered glass cullet. The off-gas, cooling water, and MCW systems were started up. "Magnesium" boring bars were initially utilized to attempt melter start-up. Eleven (11) boring bars were consumed attempting to start the melter, but generation of a molten glass pool between the electrodes was unsuccessful. Additionally, a boring bar flaw caused erosion of the melter head MCW jacket and resulted in an MCW leak which required weld repair. No further boring bar start-ups were attempted. 
WHC-SD-WM-VI-031

Revision 0

Table 3-3. Melter System Phase 1 Testing Service Loads.

\begin{tabular}{|c|c|c|}
\hline Service & Quantity & $\begin{array}{l}\text { Average daily } \\
\text { operating cost } \\
\text { (over } 32 \text {-day } \\
\text { test period) }\end{array}$ \\
\hline $\begin{array}{l}\text { Electrical Power (Electrodes } \\
\text { Only) }\end{array}$ & $55,736 \mathrm{KW}-\mathrm{HR}$ & \\
\hline Average Electrode Power Level & $96.9 \mathrm{KW}$ & \\
\hline Diese1 Fuel (for generator) & $\begin{array}{l}26,047 \text { liters }(6,881 \text { US } \\
\text { Gallons); } \$ .819 / g a 11 \text { on }\end{array}$ & $\$ 176.08$ \\
\hline Cumulative Diesel Run Hours & 647.79 hours & \\
\hline $\begin{array}{l}\text { Average Diesel Fuel } \\
\text { Consumption }\end{array}$ & $\begin{array}{l}40.2 \text { liters } / \mathrm{hr}(10.62 \\
\text { gal lons/hr) }\end{array}$ & \\
\hline Nitrogen & $\begin{array}{l}5148 \mathrm{~kg}(11,3521 \mathrm{~b}) ; 43 \\
\text { bottles, } 120 \mathrm{~kg}(264 \mathrm{lb}) \\
\mathrm{N}_{2} / \text { bottle, } \$ 117 / \text { bottle }\end{array}$ & $\$ 164.00$ \\
\hline Propane & $\begin{array}{l}1022 \text { liters }(270 \\
\text { gallons); } \$ 1.25 / \text { gallon }\end{array}$ & \\
\hline $\begin{array}{l}\text { Service Water (initial fill: } \\
\text { cooling water, glass quench, } \\
\text { scrubber) }\end{array}$ & $\begin{array}{l}\text { approx. } 26,500 \text { liters } \\
\text { (7000 US Gallons) }\end{array}$ & \\
\hline $\begin{array}{l}\text { Service Water (cooling tower } \\
\text { make-up) }\end{array}$ & $\begin{array}{l}\text { approx. } 3785 \text { liters (1000 } \\
\text { US Galions) }\end{array}$ & \\
\hline $\begin{array}{l}\text { Service Air (during feed } \\
\text { recirc pump operations only) }\end{array}$ & approx. 120 SCFM & \\
\hline $\begin{array}{l}\text { Consumable Molybdenum } \\
\text { Electrodes }\end{array}$ & $\begin{array}{l}121.3 \mathrm{~cm}(47.75 \text { inches }) \\
6.35 \mathrm{~cm}(2.5 \mathrm{in}) 0 \mathrm{0D} \\
0.323 \mathrm{~kg} / \mathrm{cm}(1.81 \mathrm{~b} / \mathrm{in}) \\
\$ 25 / 1 \mathrm{~b}\end{array}$ & $\$ 67.53$ \\
\hline TOTAL DAILY OPERATING COST & & $\$ 408.53$ \\
\hline
\end{tabular}

Following weld repair to the melter head MCW jacket, melter start-up strategy was shifted to gas burners instead of the boring bars which had proven ineffective. Melter start-up with burners was performed from 3/30/95 through 4/1/95. Gas burners were ignited and manually inserted into the melter through the two 6" openings in the top. The glass cullet was slowly melted to establish a conductive molten glass pool between the electrodes. Glass cullet temperature was monitored by thermocouples inserted into the melter through the head. Electrodes were periodically energized to monitor melt progress. After over 20 hours of slow burner heat-up, the glass cullet was molten between electrodes with glass pool temperature $760-982^{\circ} \mathrm{C}(1400-$ $1800^{\circ} \mathrm{F}$ ). The melter electrodes were shifted to automatic current control, and the burners were turned off. Glass pool heat-up to approximately $1540^{\circ} \mathrm{C}$ 
WHC-SD-WM-VI-031

Revision 0

$\left(2800^{\circ} \mathrm{F}\right)$ with the electrodes continued for approximately 2 days. During this period a total of $408 \mathrm{~kg}(900 \mathrm{1b})$ additional glass cullet were added to the melter.

In order to gradually adjust the melter glass pool chemistry toward the test chemistry expected for LLRW testing, transition batch high sodium feed was added to the melter. Transition batch consisted of various mixtures of soda ash, boric acid, and alumina. A total of $179 \mathrm{~kg}$ (395 1b) of transition batch feed was added to the melter over a 4-1/2 day period $(4 / 2 / 95-4 / 6 / 95)$.

During the transition batch period, operation of the melter electrodes alone would not melt the glass at the bottom of the melter above the bottom drain assembly due to the fixed electrode angle. Heating of the bottom glass was initiated by establishment of an additional single phase joule heating loop through the drain ring orifice and one electrode phase. A metal pin was inserted through the drain ring into the glass pool. The joule heating loop was established through this pin, the glass, and one electrode phase. As heat-up progressed, the pin was slowly withdrawn. At one point the pin could not be withdrawn due to deformation caused by excessive temperatures. This required on-1ine replacement of the drain ring and insertion of a new pin. When the pin was fully withdrawn, the bottom joule heating loop was shifted back to the drain ring.

To initiate glass draining, cooling water to the bottom drain assembly was stopped, and joule heating was again initiated to the drain ring. On $4 / 6 / 95$, the first VE-SKULL" melter glass drain was performed when $>233 \mathrm{~kg}$ (513 1b) of glass was drained from the melter. The glass product was green upon cooling.

\subsubsection{VECTRA Glass Dry Feed Operations}

From 4/7/95 through 4/17/95, the VE-SKULL" phase 1 melter was operated cont inuously with VECTRA Glass dry feed. The VECTRA Glass feed composition is presented in Table 3-4.

With the melter in steady state operation following start-up with the cullet and transition batch, the VECTRA Glass feed was initiated. Pre-mixed VECTRA Glass feed was supplied in pre-weighed bulk bags [approximately $36 \mathrm{~kg}$ $(80 \mathrm{1b})$ each $]$. The premixed bags were loaded into the $0.425 \mathrm{~m}^{3}\left(15 \mathrm{ft}^{3}\right)$ melter dry feeder hopper which was mounted on the melter test stand and connected directly to the 16" off-gas/feed plenum. Since the feeder was constant speed only, the motor was periodically operated for discrete time periods (usually 20 seconds) to establish an average feed rate. The goal during dry feeding was to maintain glass pool level above the electrode holders, provide make-up for melter losses through volatization or draining, and maintain a stable "batch blanket" on top of the molten glass pool. Optimization of batch blanket thickness provided reduced particulate carryover off-gas, reduced melter plenum temperature, and allowed operator observation of melter pool refinement via the CCTV system. 
WHC-SD-WM-VI-031

Revision 0

Table 3-4. VECTRA Glass Feed Composition.

\begin{tabular}{|l|r|r|}
\hline \multirow{2}{*}{ Component } & \multicolumn{2}{c|}{ Glass weight $\%$} \\
\cline { 2 - 3 } & As batched & Oxide bas is \\
\hline Silica & 41.17 & 54.40 \\
\hline Boric Acid & 11.17 & 8.32 \\
\hline Dolomite & 7.52 & 4.91 \\
\hline Magnesium Carbonate & 0.49 & 0.27 \\
\hline Alumina & 12.10 & 10.40 \\
\hline Iron 0xide & 0.72 & 0.92 \\
\hline Soda Ash & 26.87 & 20.78 \\
\hline TOTAL & 100.0 & 100.00 \\
\hline
\end{tabular}

Glass pool "freeboard" level and batch blanket thickness were periodically checked by the operators by removing a $6^{\prime \prime}$ insulated plug from the melter head and inserting a calibrated dip stick into the molten glass pool. During VECTRA Glass feed operation, draining was periodically performed to control glass pool level. When operator inspection revealed sufficient glass pool refinement and adequate freeboard level, glass draining was initiated by opening the remote drain stopper plug. Melter weight was transmitted from load cells and monitored by the operator at the control system. Drain rate was dependent upon glass and drain ring temperature. Draining was stopped by closing the drain stopper plug when the desired weight of glass had been removed or when the desired freeboard level was achieved.

The melter electrodes were operated in automatic power control. Glass temperature and refining rate were controlled by 1 imiting electrode power level. One electrode phase supplied power to a welding machine connected to the drain ring lug. Power to the welding machine was manually adjusted to maintain drain ring temperature less than $1093^{\circ} \mathrm{C}\left(2000^{\circ} \mathrm{F}\right)$ and keep the melter bottom glass molten.

During a three day period over the Easter Hol iday Weekend (4/14/95 through 4/16/95) the melter was operated at idle operations with an average power level of approximately $85 \mathrm{kw}$. During this time period $135 \mathrm{~kg}$ (297 1b) of VECTRA Glass batch feed was added to the melter, and >136 kg (300 1b) of glass product were drained.

Over the eleven (11) day period of VECTRA Glass feed operations, $5401 \mathrm{~kg}$ $(11,909$ 1b) of VECTRA Glass dry feed was added to the melter. A total of $>3886 \mathrm{~kg}$ (8569 1b) of glass product was drained from the melter. This glass was green upon cooling. The average feed throughput was $30.2 \mathrm{~kg} / \mathrm{hr}$ $(66.6 \mathrm{lb} / \mathrm{hr})$ with a peak throughput of $88.5 \mathrm{~kg} / \mathrm{hr}(195.2 \mathrm{lb} / \mathrm{hr})$. Since the Phase 1 VE-SKULL" Melter has a $0.56 \mathrm{~m}^{2}\left(6.1 \mathrm{ft}^{2}\right)$ active glass surface area, the peak feed throughput corresponds to $156.2 \mathrm{~kg} / \mathrm{hr}-\mathrm{m}^{2}\left(32.01 \mathrm{~b} / \mathrm{hr}-\mathrm{ft}^{2}\right)$. The active glass surface area is defined as the glass pool cross sectional area 
(8.1 $\left.\mathrm{ft}^{3}\right)$ adjusted for a 2.5" radial cold zone along the melter wall. Since the VECTRA batch feed was approximately $25 \%$ water and other volatiles (75\% glass by weight), the calculated glass throughput is $117.1 \mathrm{~kg} / \mathrm{hr}-\mathrm{m}^{2}$

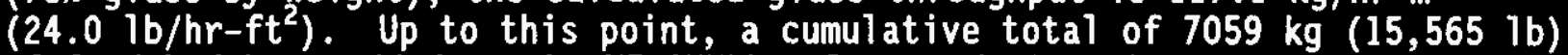
of feed had been added to the VE-SKULL melter and $>3886 \mathrm{~kg}(8,569 \mathrm{lb})$ of glass product had been drained.

\subsubsection{LLRU Slurry Feed Operations}

Operation of the VE-SKULL" melter with LLRW simulant slurry feed was performed from 4/18/95 through 4/21/95. Liquid LLRW simulant, dry glass formers, and dry sucrose reductant were premixed and injected into the melter as a slurry. The dry glass formers were identical to the glass former Mix B utilized for initial RVR dryer testing and are shown in Table 3-5.

Sucrose was added to the LLRW simulant and RVR glass formers (Mix B) for reaction with $\mathrm{NaNO}_{2}$ and $\mathrm{NaNO}_{3}$ to form $\mathrm{Na}_{2} \mathrm{CO}_{3}$ and nitrogen in the off-gas. The various slurry compositions utilized during slurry testing are given in Table 3-6.

The original slurry feed system consisted of a slurry mix tank with internal mixer, diaphragm recirculation pump, $0.71 \mathrm{~m}^{3}\left(25 \mathrm{ft}^{3}\right)$ dry feeder, LLRW simulant drum mixer, and LLRW simulant drum transfer pump. During initial slurry feed operations on $4 / 18 / 95$, the feed 1 ine plugged, and steady feed flow could not be maintained.

The slurry feed system was modified to add a 341 liter (90 gal) batch drum (slurry feed day tank), larger diaphragm recirculation pumps, recirculation lines to the melter stand, and a positive displacement peristaltic tubing pump. The peristaltic pump took a suction on the batch drum and injected slurry through tubing into the melter via the 16" off-gas/ feed plenum. The slurry batch drum was calibrated for level which was periodically monitored. Weight of simulant transferred from the slurry mix tank to the batch drum was monitored on the slurry mix tank scale.

Table 3-5. RVR Glass Formers (Mix B) Composition.

\begin{tabular}{|l|r|c|}
\hline \multirow{2}{*}{ Glass former } & \multicolumn{2}{c|}{ Weight percent } \\
\cline { 2 - 3 } & As batched & Weight oxide \\
\hline Silica Sand & 59.61 & 62.40 \\
\hline Boric Acid & 16.17 & 14.67 \\
\hline Dolomite & 10.87 & 8.65 \\
\hline Magnesium Carbonate & 0.70 & 0.48 \\
\hline Alumina & 11.61 & 12.16 \\
\hline Iron 0xide & 1.04 & 1.64 \\
\hline Total & 100.00 & 100.00 \\
\hline
\end{tabular}


WHC-SD-WM-VI-031

Revision 0

Table 3-6. Slurry Feed Batch Recipes.

\begin{tabular}{|l|c|c|c|c|}
\hline \multirow{2}{*}{ Slurry batch } & \multicolumn{4}{|c|}{ Weight $\%$} \\
\cline { 2 - 5 } & LLRW simulant & $\begin{array}{c}\text { RVR glass } \\
\text { formers }\end{array}$ & $\begin{array}{c}\text { Sucrose } \\
\text { reductant }\end{array}$ & Total \\
\hline Batches 1,2 & 48.04 & 49.96 & 2.00 & 100.0 \\
\hline Batches $3,4,5,6$ & 47.06 & 48.94 & 4.00 & 100.0 \\
\hline
\end{tabular}

An average feed throughput of $36.2 \mathrm{~kg} / \mathrm{hr}(79.8 \mathrm{lb} / \mathrm{hr})$ and peak feed throughput of $77.3 \mathrm{~kg} / \mathrm{hr}(170.5 \mathrm{lb} / \mathrm{hr})$ slurry feed were processed by the melter. This translates to a peak throughput of $136.4 \mathrm{~kg} / \mathrm{hr}-\mathrm{m}^{2}(28.01 \mathrm{~b}$ feed/hr-ft ${ }^{2}$ ) of active glass surface area. Note that evaporation of water adds an additional heat load to the melter electrodes. Since the slurry feed was approximately $57 \%$ glass by weight, an equivalent calculated peak glass throughput of $77.8 \mathrm{~kg} / \mathrm{hr}-\mathrm{m}^{2}$ (15.9 $\mathrm{lb} \mathrm{glass} / \mathrm{hr}-\mathrm{ft}^{2}$ ) was observed.

There were a few variations of the slurry batch. These variations did not change the formulation and the oxide composition of the slurry by more than $\pm 2 \%$ by weight.

During slurry feed operations, a total of $3563 \mathrm{~kg} \mathrm{(7857} \mathrm{lb)} \mathrm{of} \mathrm{LLRW}$ simulant slurry was added, and a total of $>1437 \mathrm{~kg}$ (3169 1b) of glass was drained. This glass product became a darker green with each pour. At this point a cumulative total of $10,622 \mathrm{~kg}(23,422 \mathrm{1b})$ of feed had been added to the melter and a total of $>5323 \mathrm{~kg}(11,738 \mathrm{lb})$ of glass product had been drained.

With the completion of Melter Test Preparations (MT-1) the VE-SKULL" melter had processed dry and slurry feed. Homogeneous glass had been produced. All melter systems had been tested and were operational. Additionally, the melter chemistry had been adjusted to approximate levels expected for the remaining tests. Since a LLRW melter glass volume is approximately $1224 \mathrm{~kg}(2700 \mathrm{lb})$, over one melter glass volume had been processed with slurry feed prior to commencement of the remaining tests (MT-2 through MT-4).

\subsection{MT-2: REACT/VITRIFY GLASS FORMER, LLRW SIMULANT, AND REDUCTANT SLURRY FEED}

For Melter tests MT-2 (S1urry Feed), MT-3 (V-SIM), and MT-4 (Calcined feed), the target glass composition was the same and is given in Table 3-7.

Once the melter was operating at nearly steady state with slurry feed, melter test sequence MT-2 was initiated. Testing with LLRW simulant, glass formers, and sucrose reductant slurry was performed from 4/21/95 to 4/22/95. Three (3) separate batches were mixed during test MT-2. The batch slurry feed makeup fractions are shown in Table 3-8. 
WHC-SD-WM-VI-031

Revision 0

Table 3-7. Target GTass Composition.

\begin{tabular}{|l|c|}
\hline \multicolumn{1}{|c|}{ Component } & Target composition \\
\hline $\mathrm{SiO}_{2}$ & 52.80 \\
\hline $\mathrm{B}_{2} \mathrm{O}_{3}$ & 8.00 \\
\hline $\mathrm{Na}_{2} \mathrm{O}$ & 20.00 \\
\hline $\mathrm{CaO}$ & 2.90 \\
\hline $\mathrm{MgO}$ & 2.10 \\
\hline $\mathrm{CsCl}$ & 0.14 \\
\hline $\mathrm{Al}_{2} \mathrm{O}_{3}$ & 10.00 \\
\hline $\mathrm{Fe}_{2} \mathrm{O}_{3}$ & 1.00 \\
\hline Other & 23.95 \\
\hline Total & 100.00 \\
\hline
\end{tabular}

Table 3-8. MT-2 Slurry Feed Composition.

\begin{tabular}{|l|c|c|c|c|}
\hline \multirow{2}{*}{ Slurry batch } & \multicolumn{4}{|c|}{ Weight \% } \\
\cline { 2 - 5 } & LLRW simulant & $\begin{array}{c}\text { RVR glass } \\
\text { formers }\end{array}$ & $\begin{array}{c}\text { Sucrose } \\
\text { reductant }\end{array}$ & Total \\
\hline Batch la & 49.72 & 46.38 & 3.90 & 100.0 \\
\hline Batches 2a, 3a & 46.38 & 49.72 & 3.90 & 100.0 \\
\hline
\end{tabular}

The slurry feed system was operated identical to slurry feed operations in MT-1. A total of $141 \mathrm{~kg}$ (310 1b) of batch 1 and $724 \mathrm{~kg}(1596 \mathrm{lb})$ of batches $2 a$ and $3 a$ was added during MT-2. A fourteen (14) hour steady state mass balance run was performed. Mass balance data including off-gas samples were taken at the beginning and end of the run. Mass balance off-gas, glass, feed, and liquid sample results are given in Appendix $3 \mathrm{C}$.

During the MT-2 mass balance run, an electrode phase imbalance was observed indicating possible erosion on the Phase B electrode. The Phase B electrode was lowered into the melter and the three electrode phases were again balanced. Post run inspection indicated that a piece of the electrode broke off due to corrosion at the glass/gas interface.

The slurry feed had favorable melting characteristics. Its melt rate was the fastest of the three different feeds. The batch blanket was well formed on top of the glass pool and the tendency to foam was minimal.

During the MT-2 mass balance run, a total of $865 \mathrm{~kg}$ (1907 lb) of slurry feed was added to the melter. A total of $>406 \mathrm{~kg}(896 \mathrm{lb})$ of glass product 
WHC-SD-WM-VI-031

Revision 0

was drained from the melter. The average feed throughput was $59.9 \mathrm{~kg} / \mathrm{hr}$ $(132.0 \mathrm{lb}$ feed $/ \mathrm{hr}$ ). This corresponds to an average feed throughput of $105.6 \mathrm{~kg} / \mathrm{hr}-\mathrm{m}^{2}\left(21.6 \mathrm{lb} \mathrm{feed} / \mathrm{hr}-\mathrm{ft}^{2}\right)$ of active glass surface area. Since the slurry feed was approximately $57 \%$ glass by weight, an equivalent calculated average glass throughput of $60.2 \mathrm{~kg} / \mathrm{hr}-\mathrm{m}^{2}\left(12.3 \mathrm{lb} \mathrm{glass} / \mathrm{hr}-\mathrm{ft}^{2}\right)$ was observed.

\subsection{MT-3: VITRIFY SIMULATED DRY CALCINED PRODUCT}

Following slurry feed testing (MT-2) the slurry feed system was shut down and the melter was prepared for dry feed operations. Pre-batched simulated dry calcined feed (V-SIM) was supplied in pre-weighed bags [approximately $36 \mathrm{~kg}(80 \mathrm{lb})$ each]. The composition of the V-SIM feed is shown in Table 3-9.

MT-3 was performed on 4/22/95. The V-SIM feed was added to the melter dry feeder as was done with the VECTRA Glass batch (section 6.1.2). V-SIM feed was processed for approximately 15 hours. During this time period a three (3) hour steady state mass balance run was performed. Mass balance data and samples were taken at the beginning and end of the run. Mass balance offgas, glass, feed, and liquid sample results are included in Appendix 3B.

During the MT-3 mass balance run, a total of $100 \mathrm{~kg}$ (220 lb) of V-SIM dry feed was added to the melter. A total of $>69 \mathrm{~kg}(151 \mathrm{lb})$ of $\mathrm{glass}$ product was drained from the melter. The glass product was dark green/black. An average feed throughput of $43.2 \mathrm{~kg} / \mathrm{hr}(95.2 \mathrm{lb} / \mathrm{hr})$ was observed. This corresponds to an average feed throughput of $76.2 \mathrm{~kg} / \mathrm{hr}-\mathrm{m}^{2}\left(15.6 \mathrm{lb} \mathrm{feed} / \mathrm{hr}-\mathrm{ft}^{2}\right)$ of act ive glass surface area. Since the V-SIM was approximately $75 \% \mathrm{glass}$ by weight, an equivalent calculated average glass throughput of $57.1 \mathrm{~kg} / \mathrm{hr}-\mathrm{m}^{2}(11.7 \mathrm{lb}$ glass $/ \mathrm{hr}^{-\mathrm{ft}^{2}}$ ) was observed.

The V-SIM batch melt characterization was less desirable than that of the slurry feed. It was more difficult to get a uniform batch blanket on top of the glass pool because of the dry feed. The V-SIM also had a slower melt rate than the slurry feed. It did have less of a tendency to foam and was easier to handle in that respect.

\subsection{IT-4: VITRIFY DRY CALCINED PRODUCT}

Test MT-4 was performed on 4/23/95 to determine the melter performance with actual dry calcined product that was generated during the fluid bed calciner pilot scale testing (See WHC-VIT-03). The dry calcined fluid bed product was provided in 208 liter ( 55 gallon) drums. Since boric acid could not be processed with the glass formers at fluid bed temperatures $\left(500^{\circ} \mathrm{C}\right.$ ) the boric acid was mixed with the dry calcined product just prior to feed to melter. The ratio of dry calcined product to boric acid is shown in Table 3-10.

Calcined feed was processed for approximately eight (8) hours. During this time period a four (4) hour steady state mass balance run was performed. Mass balance data and samples were taken at the beginning and end of the run. Mass balance off-gas, glass, feed, and liquid sample results are attached in Appendix 3B. 
WHC-SD-WM-VI-03I

Revision 0

Table 3-9. V-SIM Feed Composition.

\begin{tabular}{|c|c|c|c|}
\hline \multicolumn{2}{|c|}{ Target Oxide Composition } & \multicolumn{2}{|c|}{ Actual V-SIM Batch } \\
\hline Component & Target Wt\% & Component & $\begin{array}{c}\text { As Batched } \\
\text { (wt\%) }\end{array}$ \\
\hline SiO(2) & 52.80 & Silica & 39.18 \\
\hline$B(2) 0(3)$ & 8.00 & Boric Acid & 10.52 \\
\hline $\mathrm{CaO}$ & 2.90 & Dolomite & 7.27 \\
\hline $\mathrm{MgO}$ & 2.10 & Magnes ium Carbonate & 0.04 \\
\hline $\mathrm{Na}(2) 0$ & 20.00 & $\mathrm{Na}_{2} \mathrm{CO}_{3}$ & 23.95 \\
\hline$A 1(2) 0(3)$ & 10.00 & Alumina & 11.53 \\
\hline $\mathrm{Fe}(2) 0(3)$ & 1.00 & Iron 0xide & 0.68 \\
\hline Moo(3) & 0.15 & $\mathrm{NaMoO}_{4}$ & 0.01 \\
\hline $\operatorname{cr}(2) 0(3)$ & 0.04 & $\mathrm{~K}_{2} \mathrm{Cr}_{2} \mathrm{O}_{7}$ & 0.03 \\
\hline$K(2) 0$ & 1.52 & $\mathrm{~K}_{2} \mathrm{CO}_{3}$ & 0.83 \\
\hline $\mathrm{MnO}(2)$ & 0.0023 & $\mathrm{KMnO}_{4}$ & 0.00 \\
\hline Sro & 0.11 & $\mathrm{SrCO}_{3}$ & 0.12 \\
\hline $\operatorname{cs}(2) 0$ & 0.15 & $\mathrm{CsCl}$ & 0.14 \\
\hline$P(2) 0(5)$ & 0.20 & Tri-Sodium Phosphate & 0.79 \\
\hline $\mathrm{I}$ & 0.07 & $\mathrm{NaIO}_{3}$ & 0.09 \\
\hline $\mathrm{Cl}$ & 0.29 & $\mathrm{NaCl}$ & 0.29 \\
\hline$F$ & 0.28 & $\mathrm{NaF}$ & 0.46 \\
\hline \multirow[t]{6}{*}{ SOx } & 0.22 & $\mathrm{Na}_{2} \mathrm{SO}_{4}$ & 0.21 \\
\hline & & $\mathrm{KOH}$ & 0.67 \\
\hline & & $\mathrm{NaNO}_{3}$ & 0.68 \\
\hline & & $\mathrm{NaNO}_{2}$ & 0.28 \\
\hline & & Carbon & 2.23 \\
\hline & & Total & 100.0 \\
\hline
\end{tabular}


WHC-SD-WM-VI-031

Revision 0

Table 3-10. Boric Acid Addition to Dry

Calcined Feed Product.

\begin{tabular}{|l|c|}
\hline \multicolumn{1}{|c|}{ Component } & Weight \% \\
\hline Dry Calcined Product & 86.36 \\
\hline Boric Acid & 13.64 \\
\hline Total & 100.0 \\
\hline
\end{tabular}

During the MT-4 mass balance run, a total of $162 \mathrm{~kg}$ (357 $1 \mathrm{~b})$ of dry calcined feed was added to the melter. A total of $>136 \mathrm{~kg}(300 \mathrm{lb})$ of $\mathrm{glass}$ product was drained from the melter on two separate drain operations. The glass product was dark black. Only a short run was performed due to the limited quantity of calcine product available.

An average throughput of $25.7 \mathrm{~kg} / \mathrm{hr}(56.6 \mathrm{lb}$ feed $/ \mathrm{hr}$ ) was observed. This corresponds to an average feed throughput of $45.3 \mathrm{~kg} / \mathrm{hr}-\mathrm{m}^{2}(9.3 \mathrm{lb}$ feed $/ \mathrm{hr}-$ $\mathrm{ft}^{2}$ ) of active glass surface area. Since the Calcined feed was approximately 95\% glass by weight, an equivalent calculated average glass throughput of $43.0 \mathrm{~kg} / \mathrm{hr}-\mathrm{m}^{2}\left(8.8 \mathrm{lb} \mathrm{glass} / \mathrm{hr}-\mathrm{ft}^{2}\right)$ was observed.

The dry calcined feed was significantly more difficult to melt due to low $\mathrm{NO}_{3} / \mathrm{NO}_{2}$ levels in the calcined product; however, $\mathrm{NOx}$ levels in the off-gas were negligible. The batch blanket that formed with this product was thick and cold with respect to the other two feed streams. Because of this batch blanket, foaming was non-existent.

\subsection{V-SIM HIGH THROUGHPUT TEST}

A high throughput run was performed using the V-SIM feed from 4/24/95 through $4 / 26 / 95$. Off-gas data was not obtained during this test. The test results are summarized in Table 3-11.

\subsection{SLURRY HIGH THROUGHPUT TEST}

As with the V-SIM, a high throughput run was also performed using slurry feed mixed to the composition of batch la (Table 3-8). The testing was performed on 4/26/95. Off-gas data was not obtained. A summary of test results is presented in Table 3-12.

\subsection{HIGH SODIUN TESTING}

In order to investigate the impact of higher $\mathrm{Na}_{2} \mathrm{O}$ feed levels on melter performance, a run was performed with a separate transition feed batch prepared to the composition summarized in Table 3-13. 
WHC-SD-WM-VI-031

Revision 0

Table 3-11. V-SIM High Throughput Summary.

\begin{tabular}{|c|c|}
\hline Parameter & Value \\
\hline Average Electrode Power (kw) & 119.4 \\
\hline $\begin{array}{l}\text { Average Electrode Power during Peak } \\
\text { Throughput period }(\mathrm{kw})\end{array}$ & 129.2 \\
\hline Total feed, $\mathrm{kg}(\mathrm{lb})$ & $2809(6193)$ \\
\hline Total glass poured, $\mathrm{kg}$ (1b) & 2101 (4632) \\
\hline Duration of high throughput run (hrs) & 58.5 \\
\hline Average throughput, $\mathrm{kg} / \mathrm{hr}(\mathrm{lb} / \mathrm{hr})$ & $47.4(104.6)$ \\
\hline Peak throughput, $\mathrm{kg} / \mathrm{hr}(1 \mathrm{~b} / \mathrm{hr})$ & $71.0(156.5)$ \\
\hline Peak throughput, $\mathrm{kg} / \mathrm{hr}-\mathrm{m}^{2}\left(1 \mathrm{~b} / \mathrm{hr}-\mathrm{ft}^{2}\right)$ & $125.2(25.7)$ \\
\hline $\begin{array}{l}\text { Calculated Peak Glass throughput, } \\
\mathrm{kg} / \mathrm{hr}-\mathrm{m}^{2}\left(1 \mathrm{~b} / \mathrm{hr}-\mathrm{ft}^{2}\right), 75 \% \mathrm{glass}\end{array}$ & $93.9(19.2)$ \\
\hline
\end{tabular}

Table 3-12. LLRW S1urry Feed High Throughput Summary.

\begin{tabular}{|c|c|}
\hline Parameter & Value \\
\hline Average Electrode Power (kw) & 121.3 \\
\hline Total feed, kg. (lb) & $375(826)$ \\
\hline Total glass poured, $\mathrm{kg}(\mathrm{lb})$ & $310(684)$ \\
\hline Duration of high throughput run (hrs) & 5.8 \\
\hline Average throughput, $\mathrm{kg} / \mathrm{hr}(\mathrm{lb} / \mathrm{hr})$ & $67.5(148.9)$ \\
\hline Average throughput, $\mathrm{kg} / \mathrm{hr}-\mathrm{m}^{2}\left(\mathrm{lb} / \mathrm{hr}-\mathrm{ft}^{2}\right)$ & $119.1(24.4)$ \\
\hline $\begin{array}{l}\text { Calculated Average G1ass throughput, } \\
\mathrm{kg} / \mathrm{hr}-\mathrm{m}^{2}\left(1 \mathrm{~b} / \mathrm{hr}-\mathrm{ft}^{2}\right), 57 \% \text { glass }\end{array}$ & $67.9(13.9)$ \\
\hline
\end{tabular}

Table 3-13. High Sodium Run Batch Makeup.

\begin{tabular}{|l|c|}
\hline \multicolumn{1}{|c|}{ Component } & Weight \% \\
\hline V-SIM Pre-batched Dry Feed & 60.00 \\
\hline Soda Ash $\left(\mathrm{Na}_{2} \mathrm{CO}_{3}\right)$ & 40.00 \\
\hline Total & 100.0 \\
\hline
\end{tabular}


The goal of the High Sodium Test was to achieve 26-28 wt\% $\mathrm{Na}_{2} \mathrm{O}$ in glass. Lab analyses of the high sodium glass is attached in Appendix 3A. A summary of high sodium test results is presented in Table 3-14.

\subsection{HELTER DRATHDOW, SHUTDOW, AWD IMSPECTION}

The VE-SKULL" Melter was completely drained into two stainless steel 208 liter (55 gallon) drums on 4/27/95. The 208 liter ( 55 gallon) drums were placed within 341 liter (90 gal) carbon steel salvage drums. The annulus between the outside of the stainless steel drum and the inside of the salvage drum was filled with silica sand. Molten glass was drained directly into the stainless steel drum via a short graphite trough which ran from the melter bottom drain assembly to the stainless steel 208 1iter (55-gallon) drum. A total of approximately $917 \mathrm{~kg}(2022 \mathrm{lb})$ of glass product was drained into the two drums. It took approximately 5 hours to drain the entire melter.

When draindown was complete, electrode power was shut off and the melter allowed to cooldown.

On 4/28/95, following melter cooldown, the melter was disassembled and inspected. The following was observed:

A11 three electrode holders were severely eroded.

All three electrodes were severely eroded. Short sections of electrodes A and B had separated and fallen to the bottom of melter.

Refractory appeared undamaged.

Glass film on refractory was very thin $\left(<1 / 8^{\prime \prime}\right)$.

Table 3-14. High Sodium Run Throughput Summary.

\begin{tabular}{|l|c|}
\hline \multicolumn{1}{|c|}{ Parameter } & Value \\
\hline Average Electrode Power (kw) & 106.4 \\
\hline Total feed, kg (lb) & $408(900)$ \\
\hline Total glass poured, kg (1b) & $239(528)$ \\
\hline Duration of high throughput run (hrs) & 8.2 \\
\hline Average throughput, $\mathrm{kg} / \mathrm{hr}(\mathrm{lb} / \mathrm{hr})$ & $40.0(88.2)$ \\
\hline Average throughput, $\mathrm{kg} / \mathrm{hr}-\mathrm{m}^{2}\left(1 \mathrm{~b} / \mathrm{hr}-\mathrm{ft}^{2}\right)$ & $70.6(14.5)$ \\
\hline $\begin{array}{l}\text { Calculated Average } \mathrm{Glass} \text { throughput, } \\
\mathrm{kg} / \mathrm{hr}-\mathrm{m}^{2} \text { (1b/hr- } \mathrm{ft}^{2} \text { ), 75\% glass }\end{array}$ & $52.9(10.8)$ \\
\hline
\end{tabular}


The two drums of glass, together with outer salvage drum and sand, were allowed to air cool. The partially full [approximately $408 \mathrm{~kg}(900 \mathrm{lb})$ ] drum was a1lowed to air cool to ambient and the full drum [approximately $500 \mathrm{~kg}$ (1100 (b)] was wrapped in $2.5 \mathrm{~cm}\left(1^{\mathrm{N}}\right)$ of kaowool insulation and allowed to air cool to ambient. The uninsulated drum took approximately 36 hrs to cool to less than $71^{\circ} \mathrm{C}\left(160^{\circ} \mathrm{F}\right)$ and the insulated drum took approximately $168 \mathrm{hrs}$ to cool to less than $71^{\circ} \mathrm{C}\left(160^{\circ} \mathrm{F}\right)$ on outer surface of salvage drum. The glass in the center and top surface of the insulated drum was sampled and analyzed. Lab results are attached in Appendix $3 A$.

\subsection{MELTER THROUGHPUT}

VECTRA's VE-SKULL" Melter was operated continuousiy following start-up from $4 / 7 / 95$ to $4 / 27 / 95$. During this time period, a variety of different feed material was processed to accomplish melter test objectives. Generally, emphasis was placed on testing melter performance rather than maximizing melter throughput. Cumulative feed (kg) vs. Time (hrs) was plotted for each Phase 1 test period, and a linear fit of the resulting data scatter was performed. The slope of the resulting trend 1 ine is the average feed throughput $(\mathrm{kg} / \mathrm{hr})$. For some short duration tests (MT-2 slurry, MT-3 V-SIM, and MT-4 Calcined), data from periods before and after the test were utilized in order to generate representative throughput results. For tests with sufficient recorded data (MT-1 VECTRA glass, MT-1 slurry, and V-SIM high throughput), extended high throughput periods ( $>6$ hours) were identified and expanded to reveal the peak steady state feed throughput. Graphs of the Phase 1 feed throughput data are included in Figures 3-1 through 3-12.

The feed throughput results [ $\mathrm{kg} / \mathrm{hr}(1 \mathrm{~b} / \mathrm{hr})]$ were divided by the VE-SKULL" Melter active glass surface area $\left[0.57 \mathrm{~m}^{2}\left(6.1 \mathrm{ft}^{2}\right)\right]$ to reveal standard units of $\mathrm{kg} / \mathrm{hr}-\mathrm{m}^{2}\left(1 \mathrm{~b} / \mathrm{hr}-\mathrm{ft}^{2}\right)$. The active glass surface area is defined as the glass pool cross sectional area $\left(8.1 \mathrm{ft}^{3}\right)$ adjusted for a $2.5^{\prime \prime}$ radial cold zone along the melter wall. Since each Phase 1 feed material contained water and other volatiles, the melter glass throughput was calculated by adjusting the graphical feed throughput results by the corresponding glass weight percent for each type of feed.

A detailed accounting of quantities of melter feed processed and glass produced over the entire Phase 1 melter test period is presented in Appendix 3B, Throughput Log. A throughput summary is presented in Table 3-15.

\subsection{MELter mass balance}

Mass balance calculations were performed on melt tests MT-2 and MT-3. A mass balance calculation was not performed on test MT-4 due to the small amount of feed that was used during this test. The amount of material would not have allowed any amount of change in glass chemistry that could be detected above the normal laboratory error present in the chemical analyses.

The mass balance calculations are presented in Appendix $3 \mathrm{C}$. 


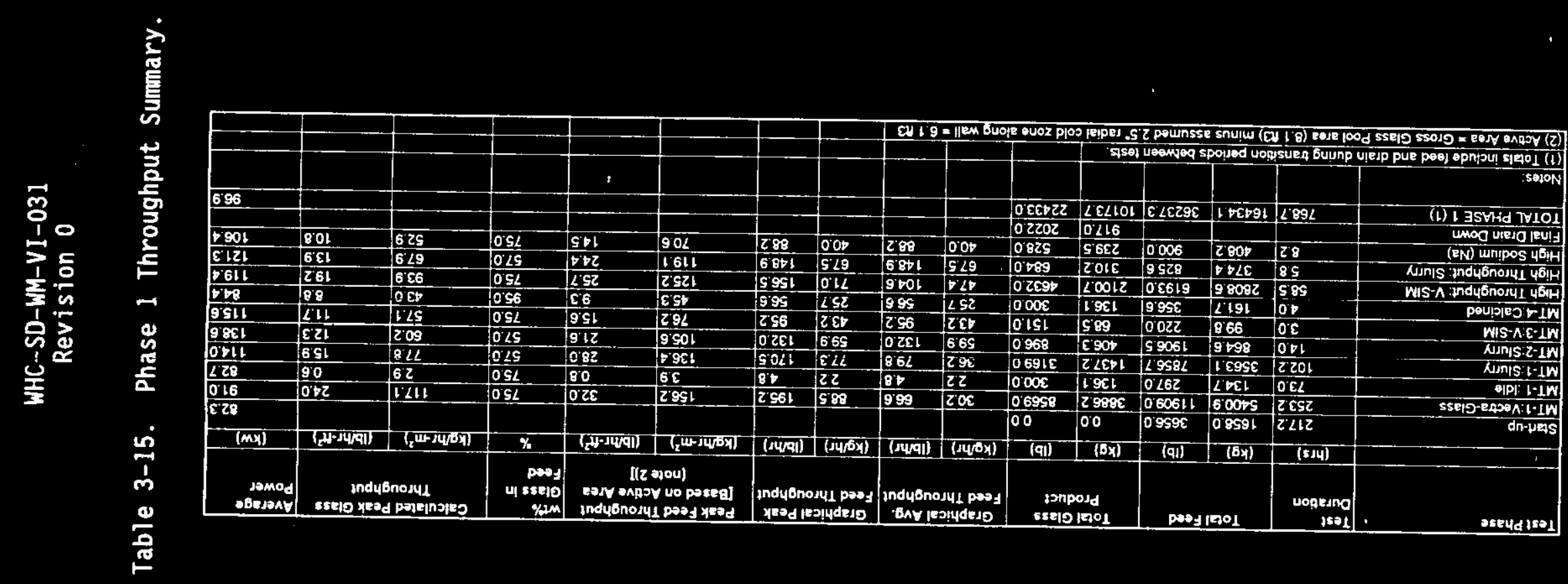


$\downarrow z-\varepsilon$

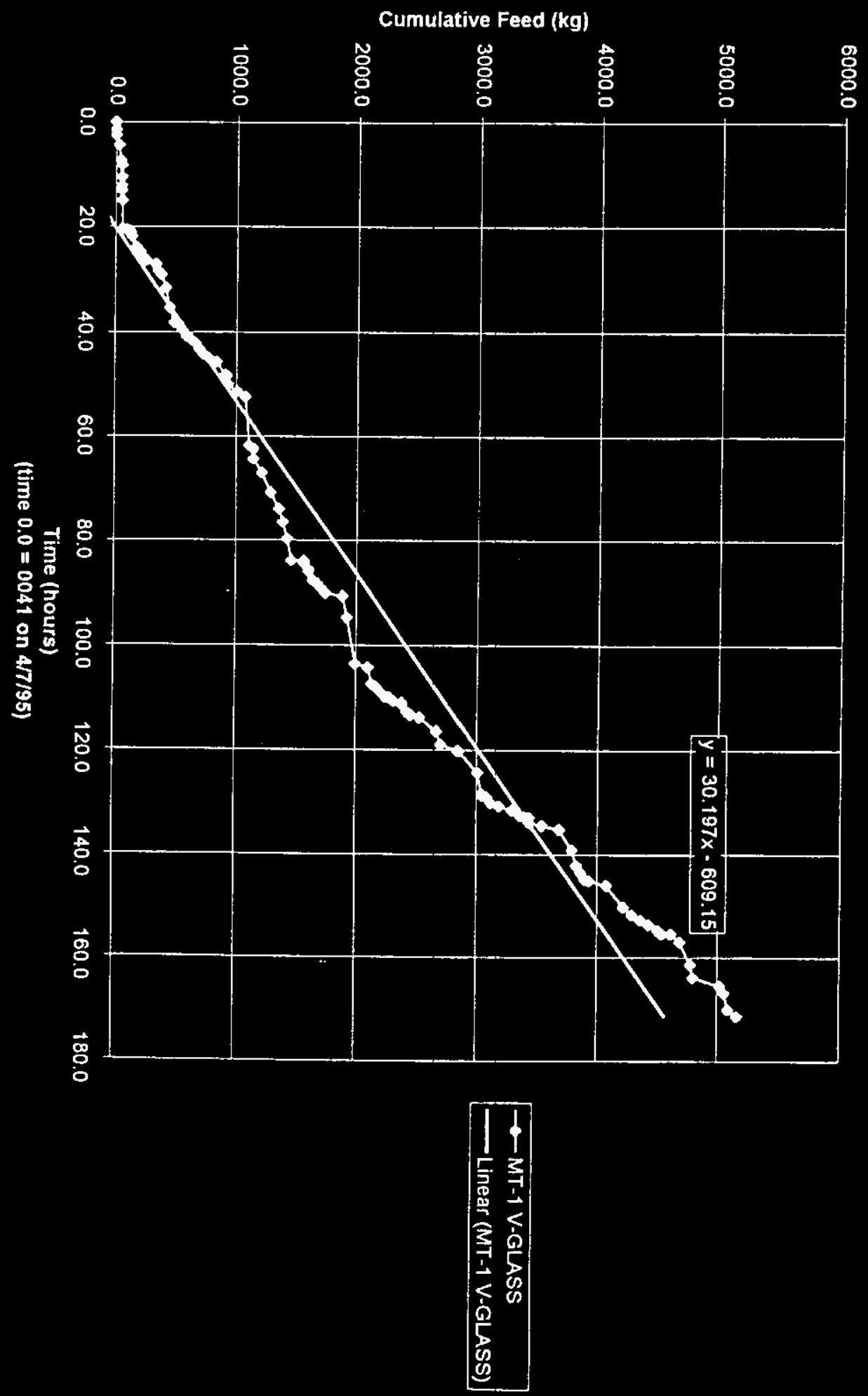

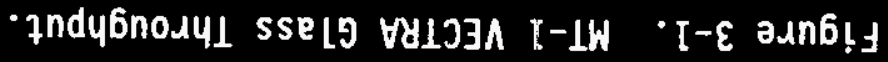

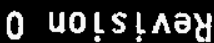

IEO-IA-WM-OS-JHM 
WHC-SD-WM-VI-031

Revision 0

Figure 3-2. MT-1 VECTRA Glass Peak Throughput.

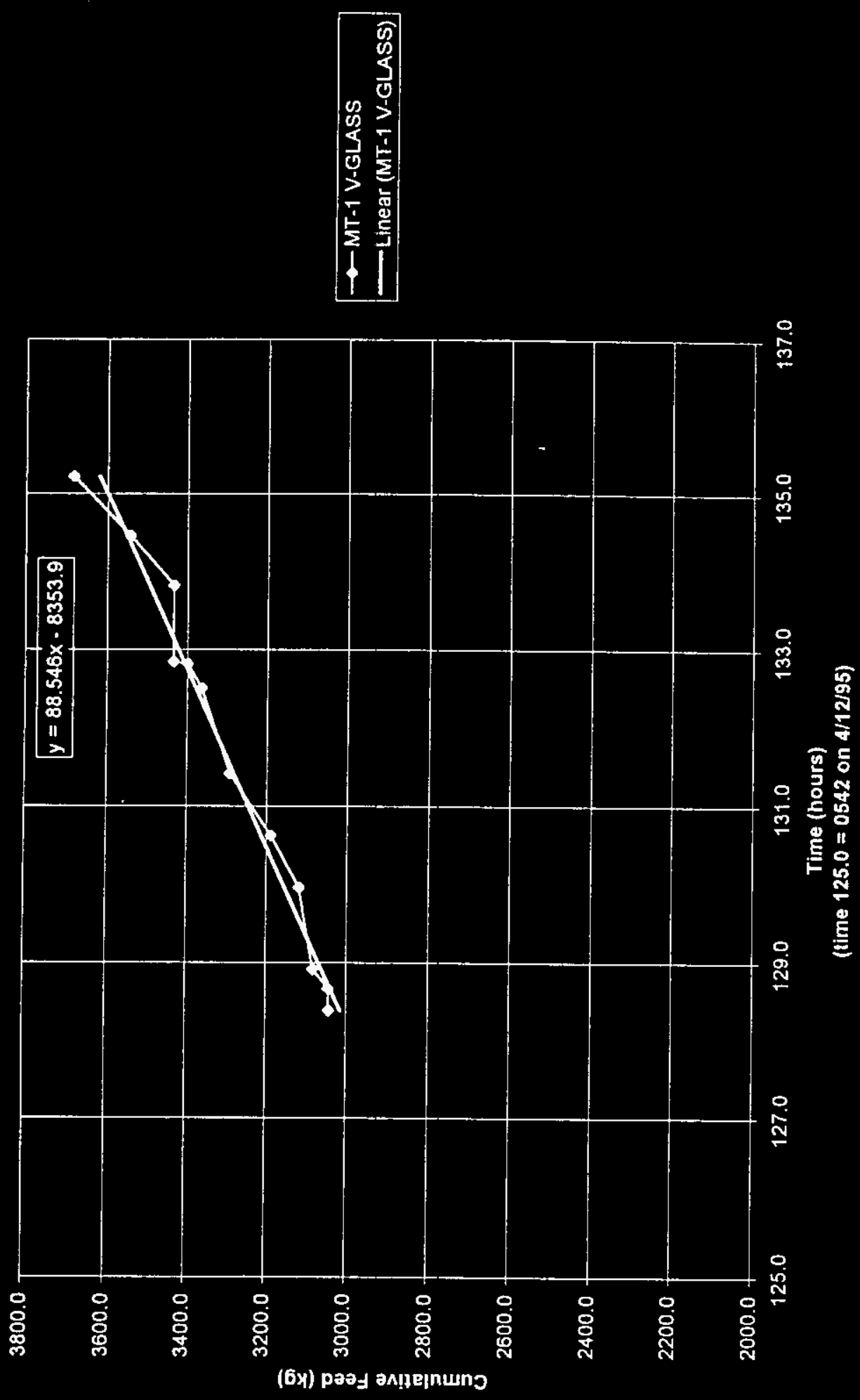


WHC-SD-WM-VI-031

Revision 0

Figure 3-3. VECTRA Glass Idle Throughput.
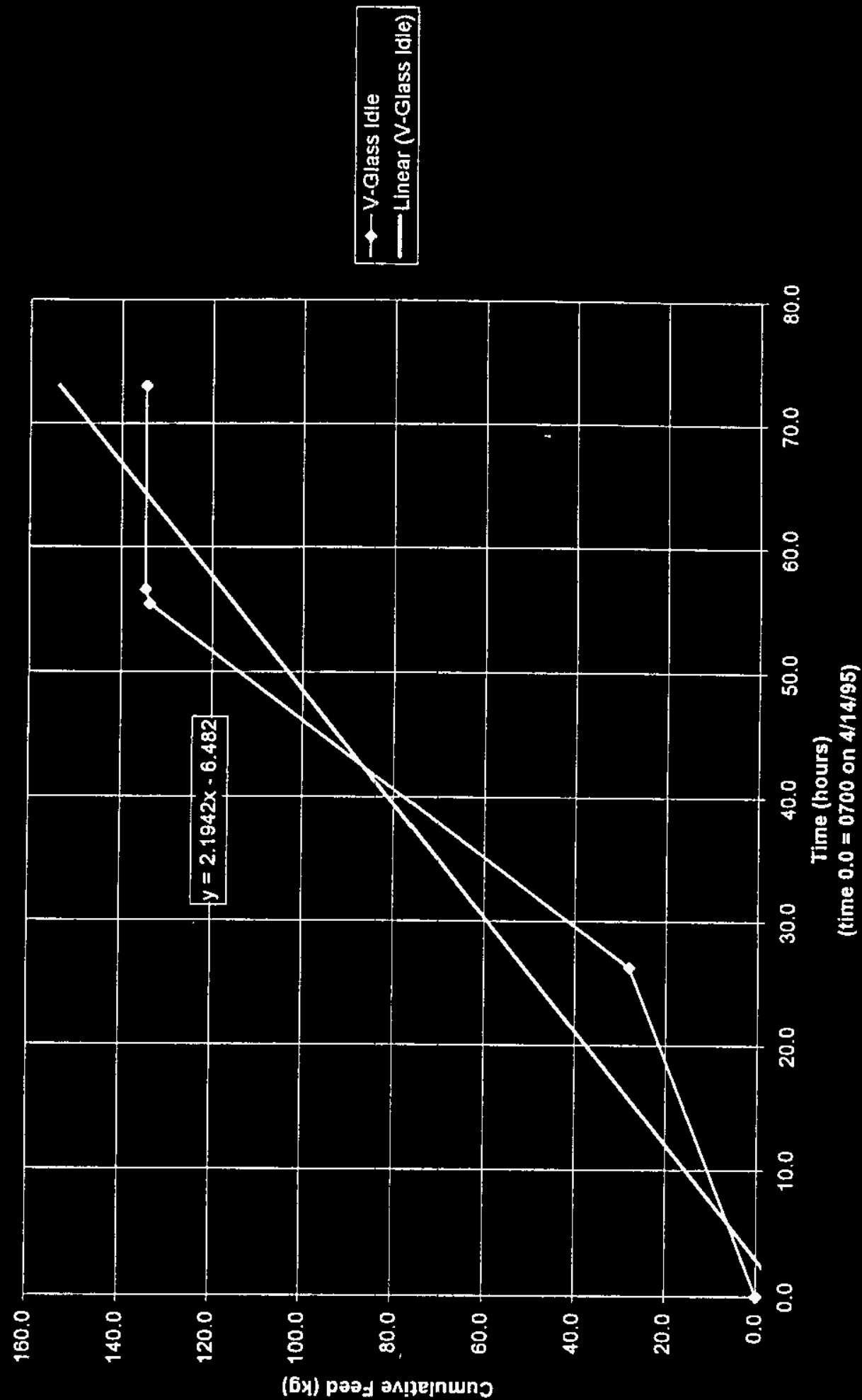
WHC-SD-WM-VI-031

Revision 0

Figure 3-4. MT-1 Slurry Throughput.
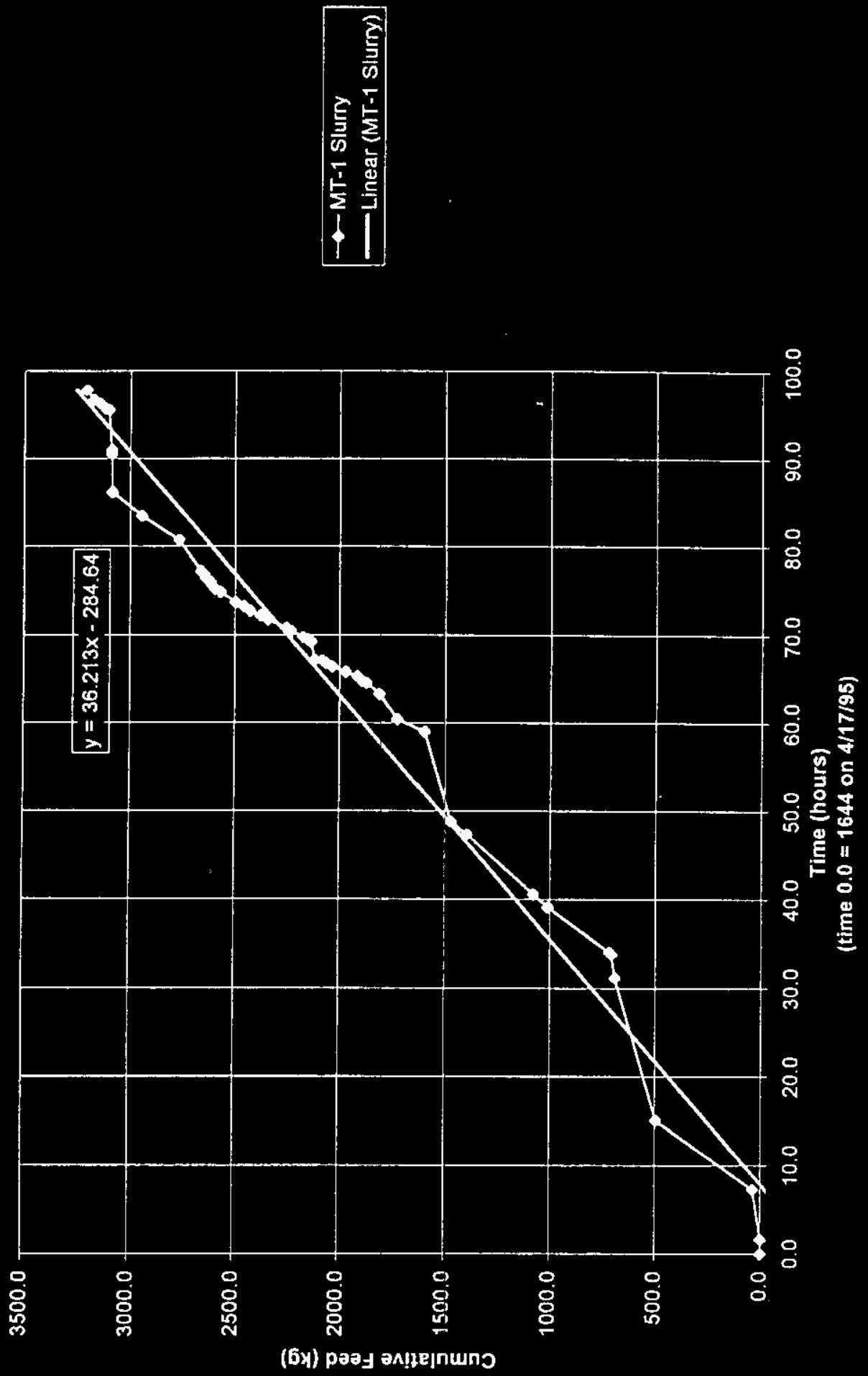
WHC-SD-WM-VI-03I

Revision 0

Figure 3-5. MT-1 Slurry Peak Throughput.
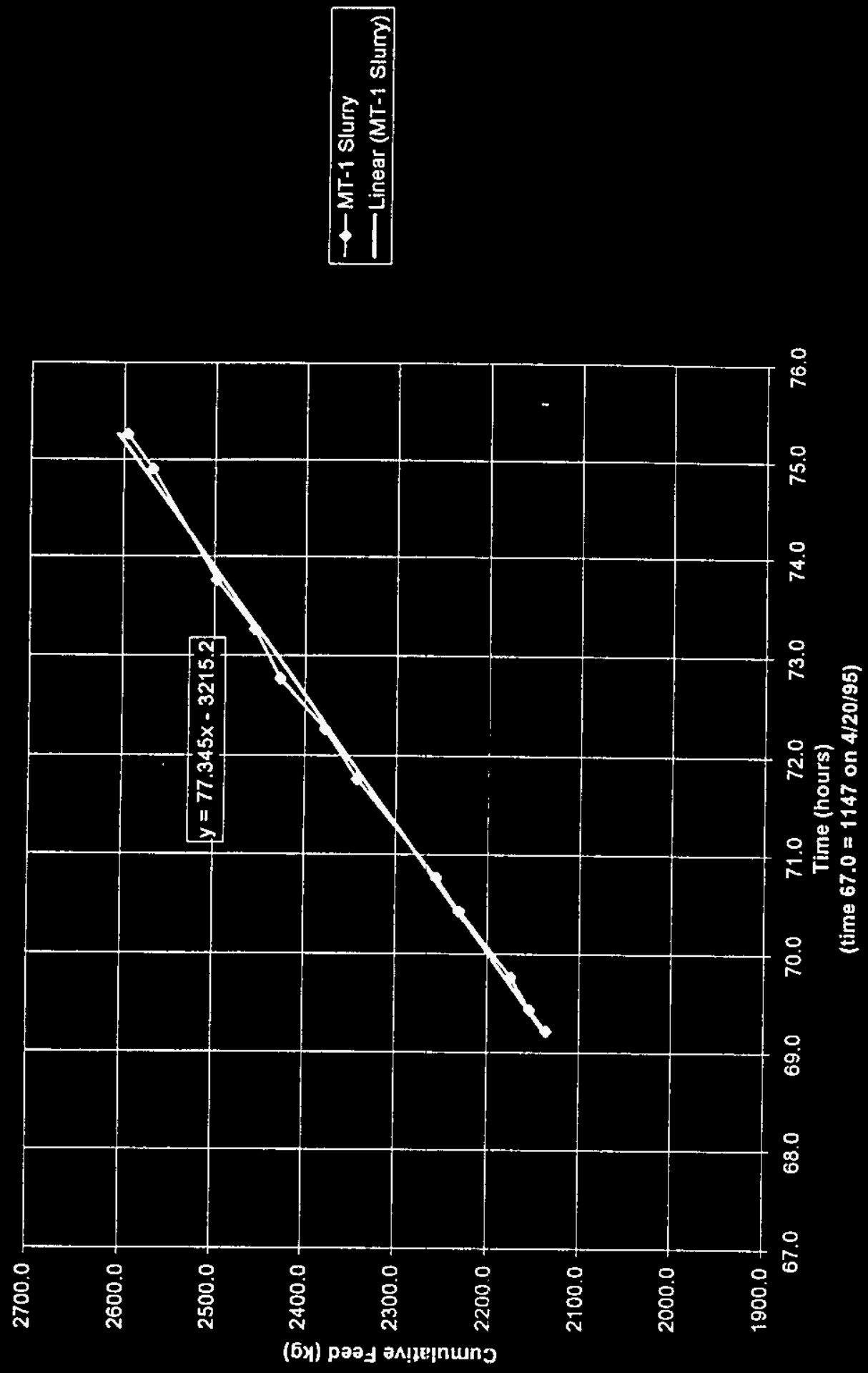
WHC-SD-WM-VI-031

Revision 0

Figure 3-6. MT-2 Slurry Throughput.

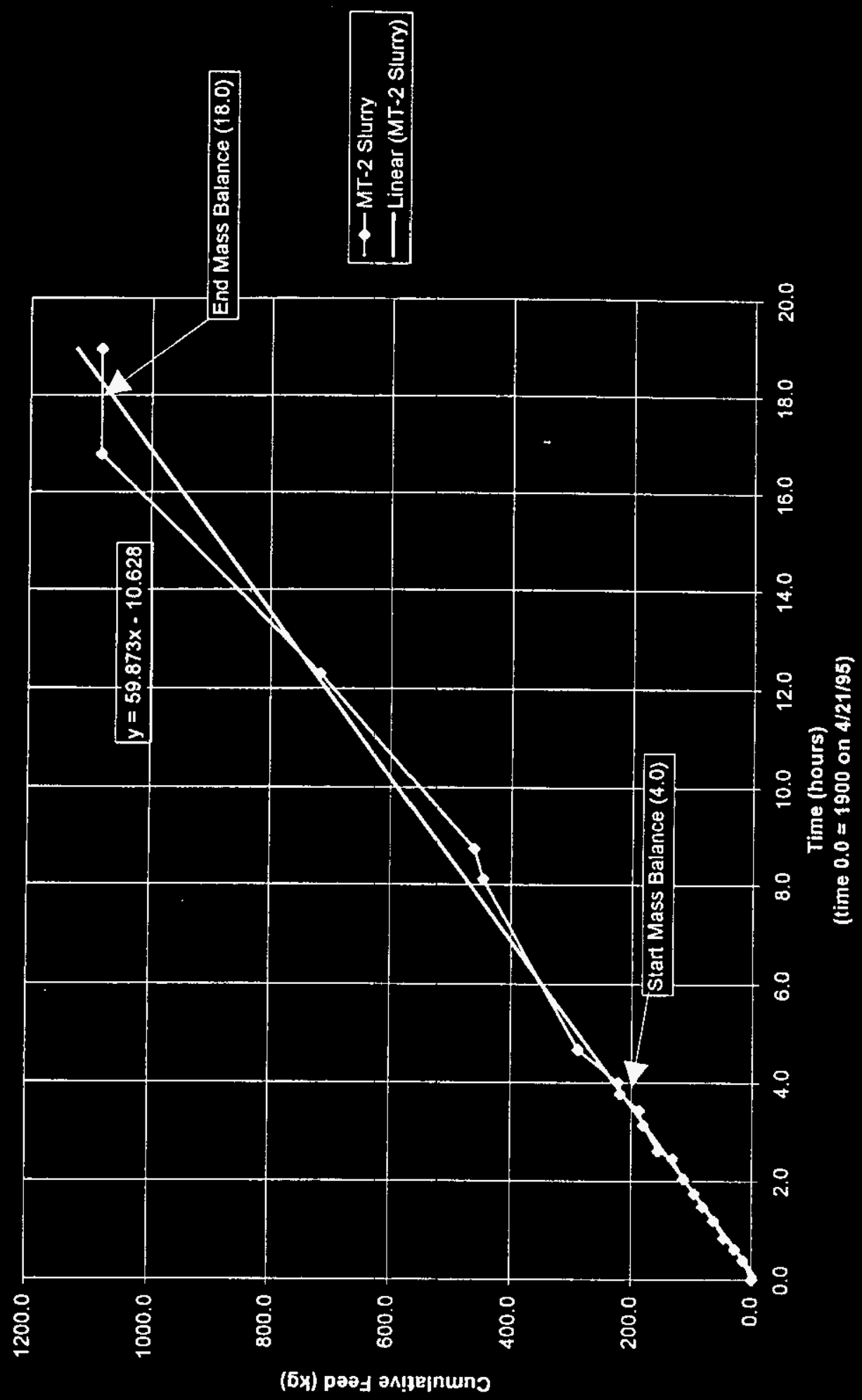


WHC-SD-WM-VI-031

Revision 0

Figure 3-7. MT-3 V-SIM Throughput.
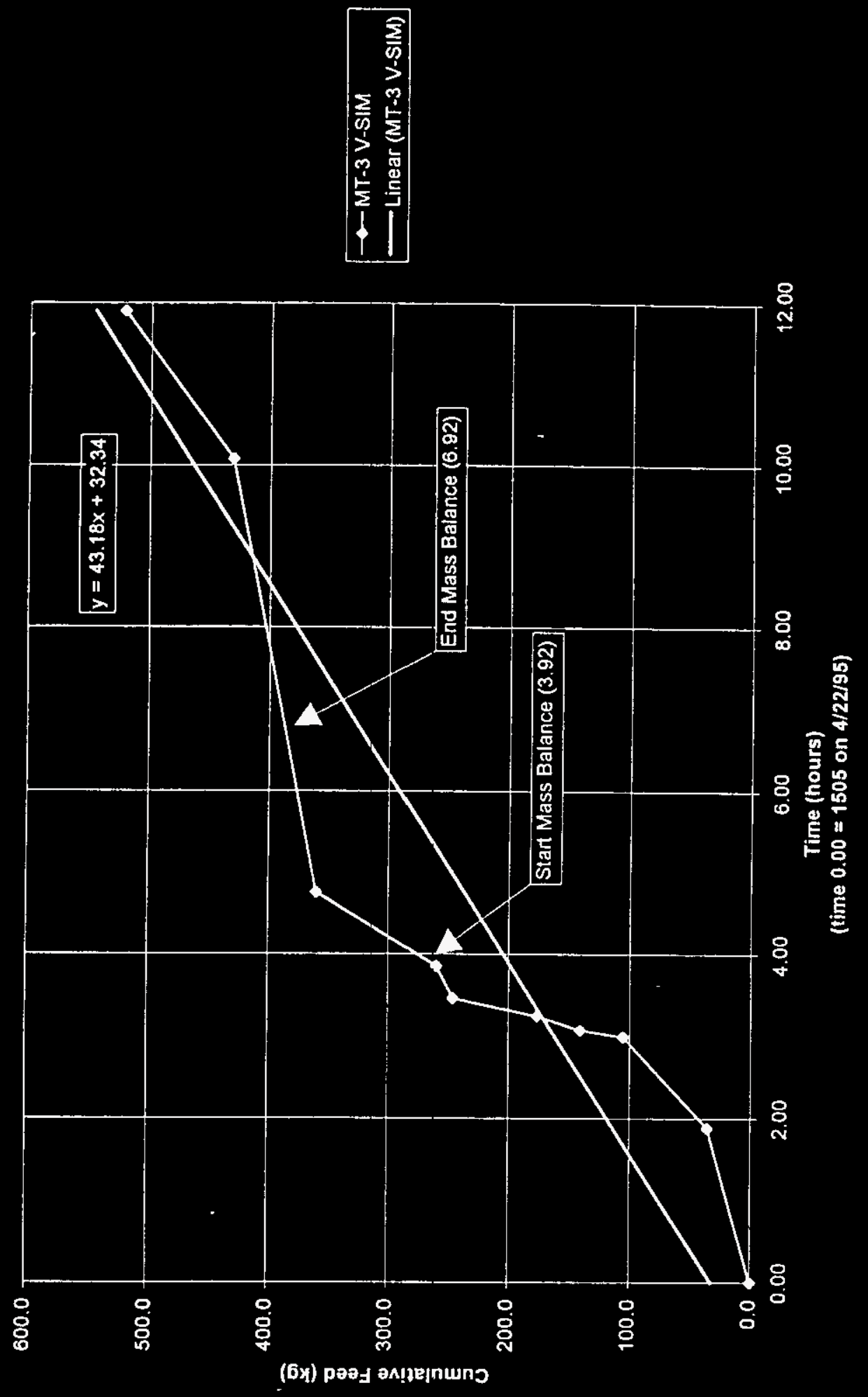


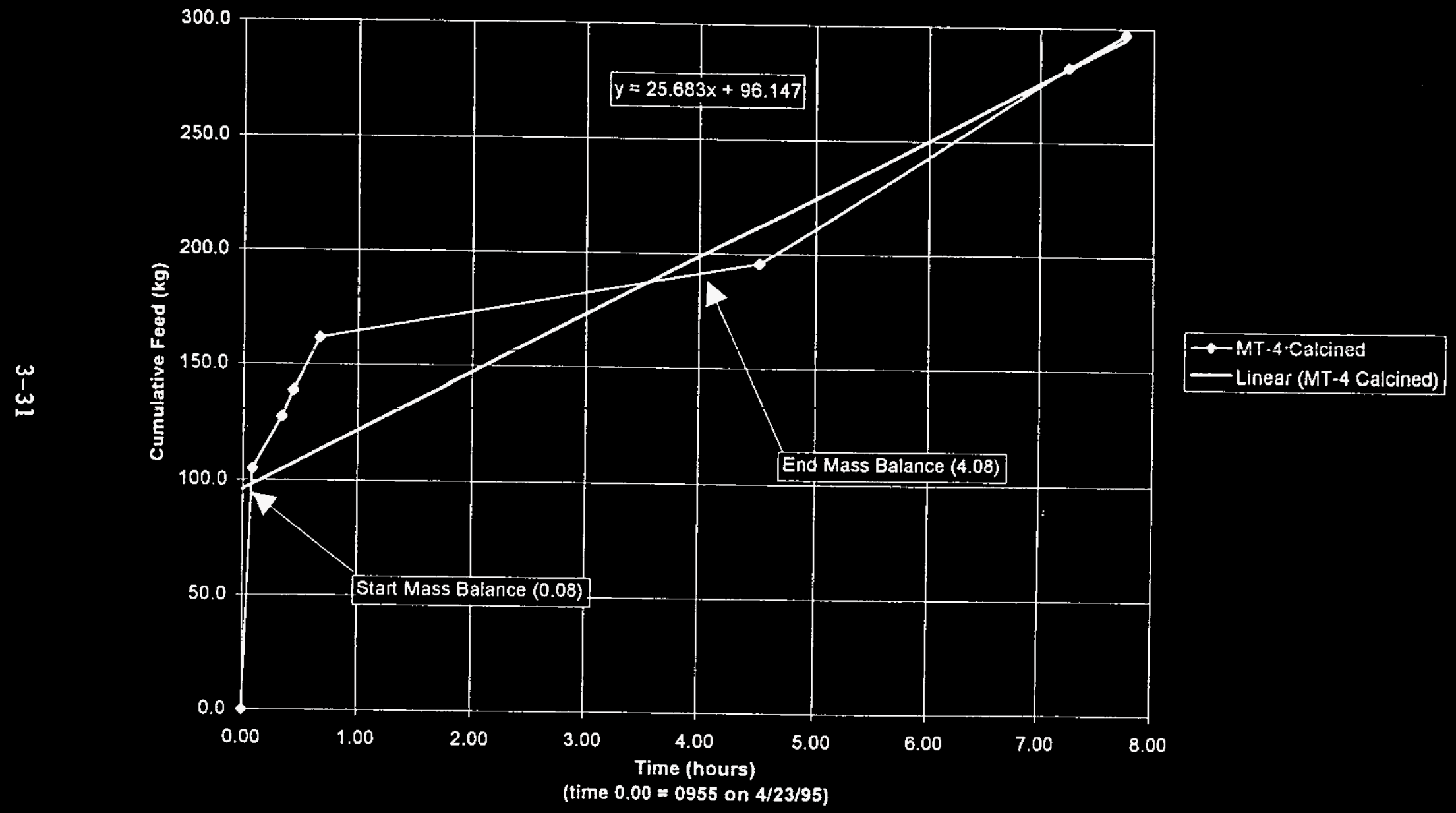

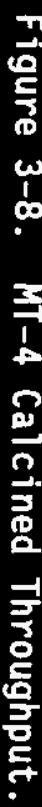


WHC-SD-WM-VI-031

Revision 0

Figure 3-9. V-SIM High Throughput (HT) Run.

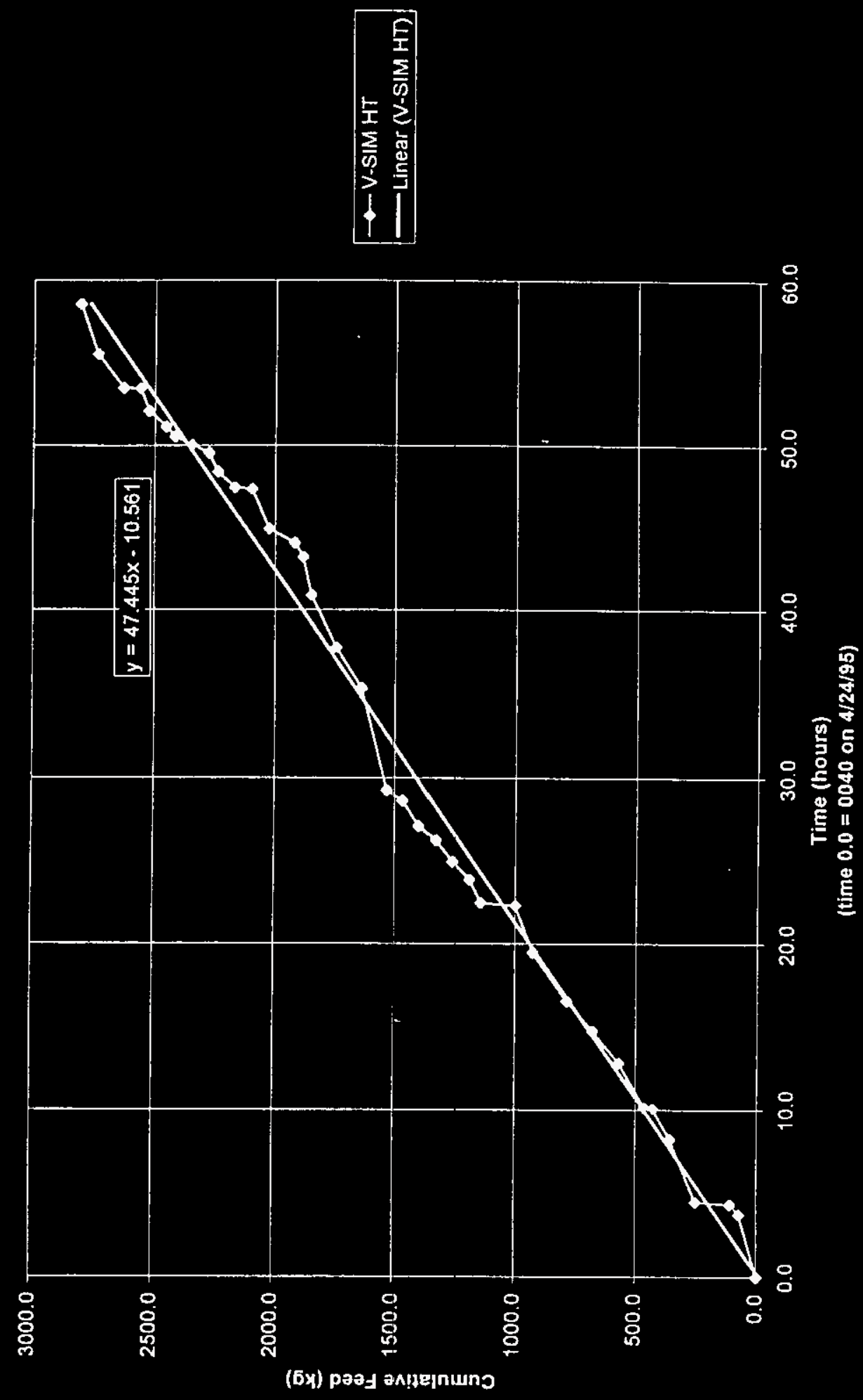


WHC-SD-WM-VI-031

Revision 0

Figure 3-10. V-SIM High Throughput (HT) Run Peak Throughput.
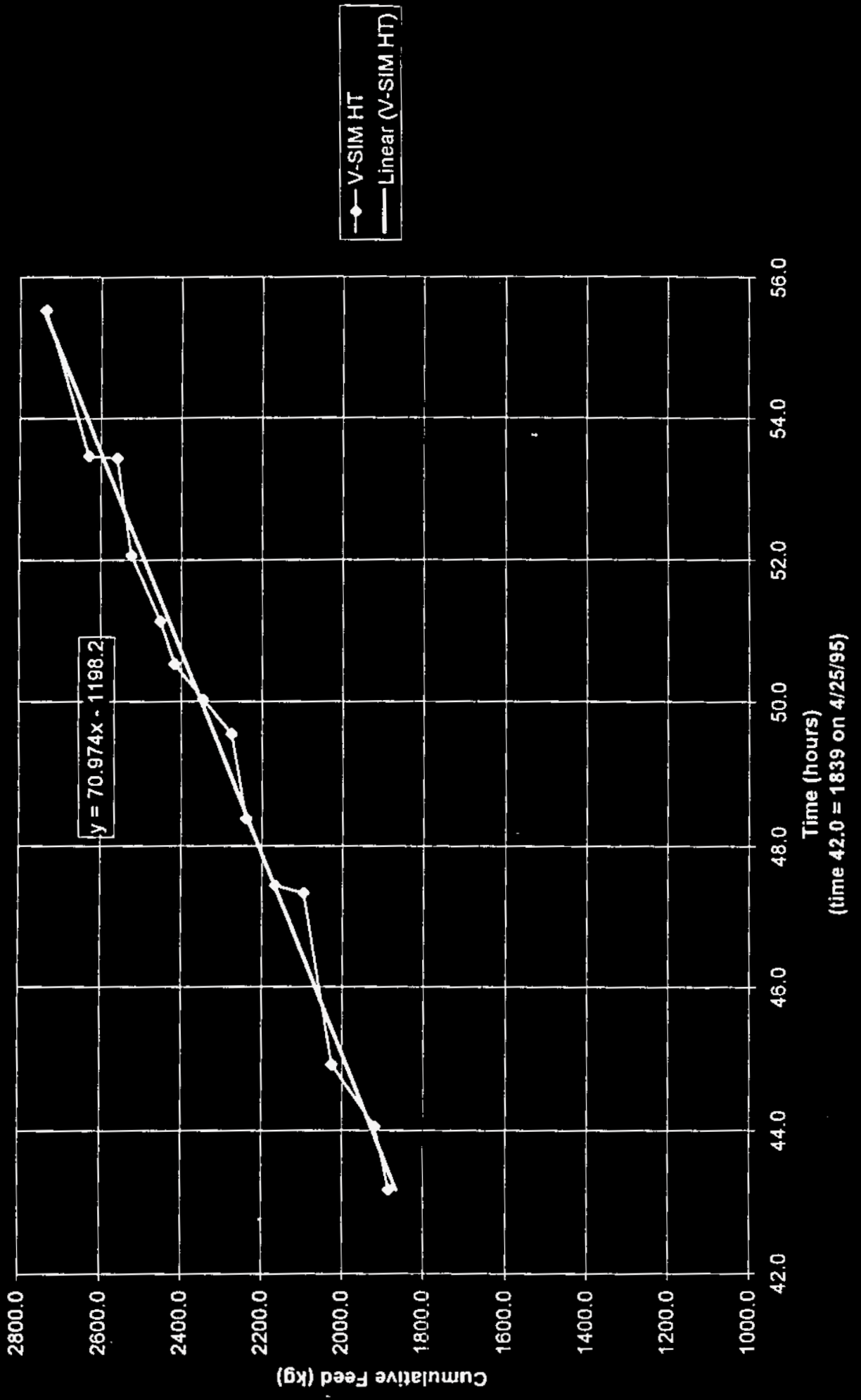
WHC-SD-WM-VI-031

Revision 0

Figure 3-11. Slurry High Throughput (HT) Run.

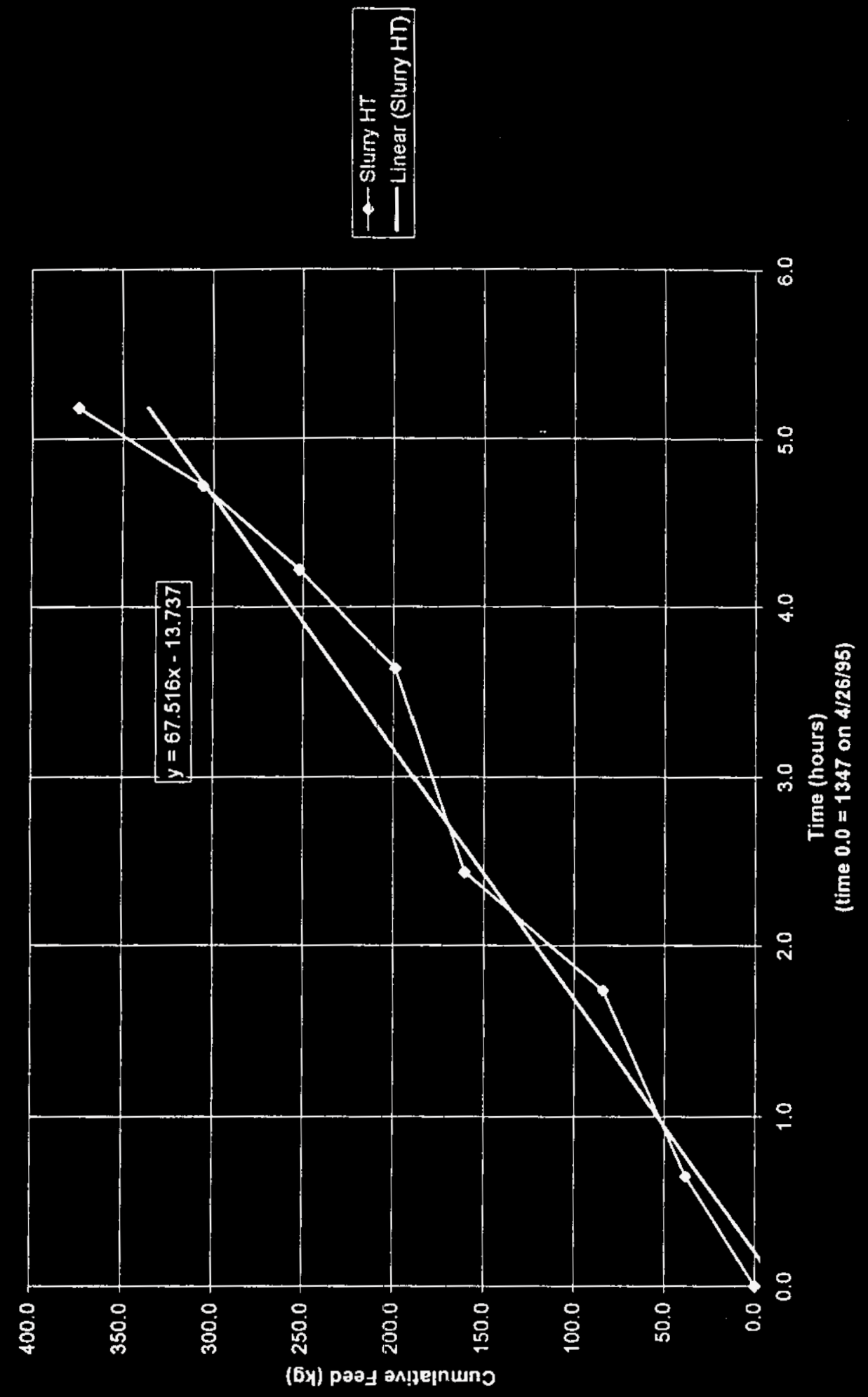


WHC-SD-WM-VI-031

Revision 0

Figure 3-12. High Sodium (Na) Run Throughput.

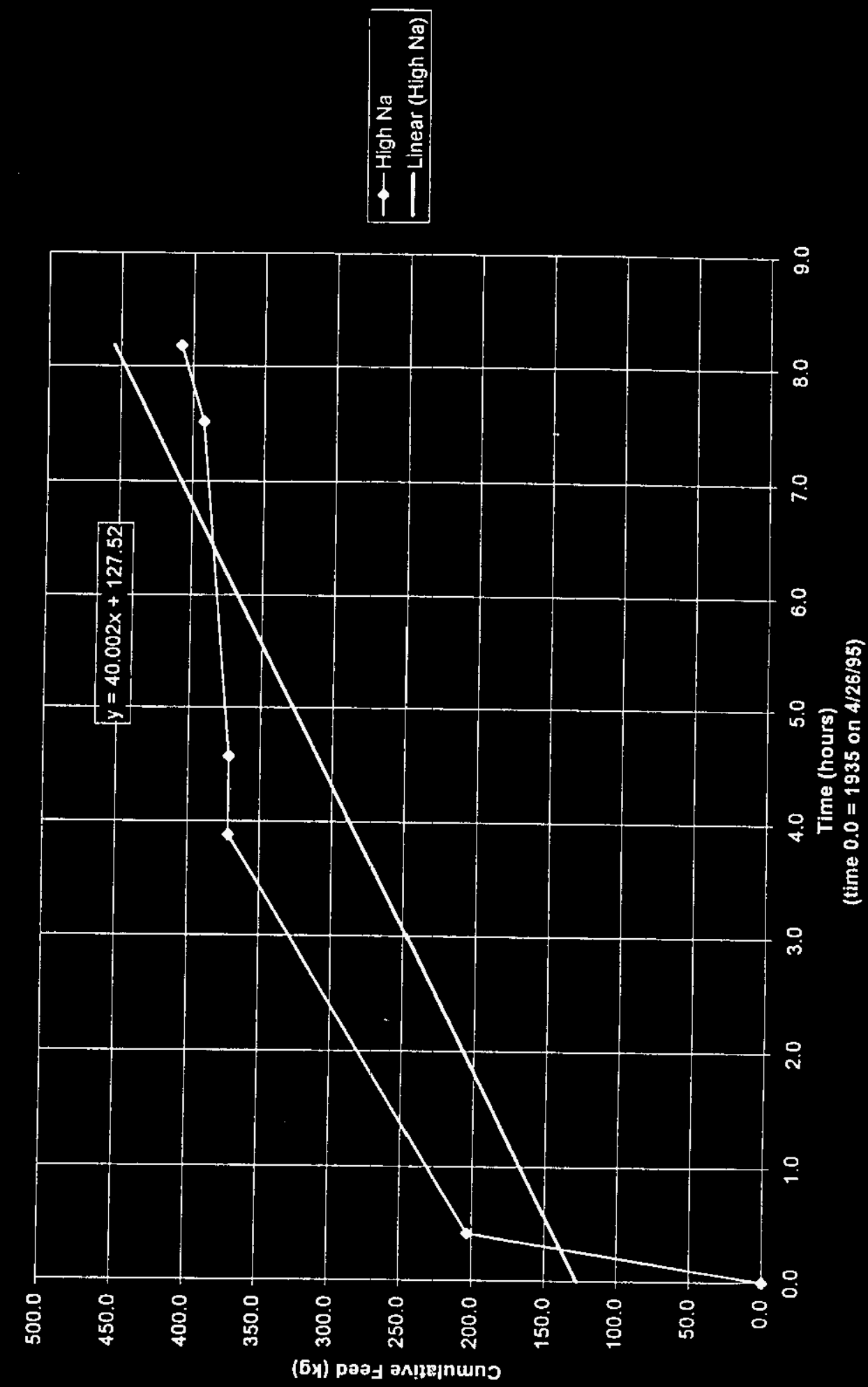


WHC-SD-WM-VI-031

Revision 0

\subsection{MELTER SCALE-UP}

Phase 1 testing provided throughput basis for various types of melter feed. With corresponding increases in number and size of electrodes, electrode power supplies, glass surface area, glass pool depth, and feed components, the melter glass throughput $(\mathrm{kg} / \mathrm{hr})$ per active glass crosssectional area $\left(\mathrm{m}^{2}\right)$ is expected to remain relatively constant as the melter is scaled up. Since improved throughput is expected due to increased glass mixing in larger melters, the Phase 1 throughput basis has been adjusted by a scale-up factor. Melter scale-up will be further verified during Phase 2 testing.

For basis of scale-up, the active glass area equals melter refractory inside diameter cross section area $\left(8.1 \mathrm{ft}^{3}\right.$ ) less a $2.5^{\prime \prime}$ radial cool zone around inside perimeter of melter. For the Phase 1 melter, the active glass area was $0.57 \mathrm{~m}^{2}\left(6.1 \mathrm{ft}^{2}\right)$.

Est imated VE-SKULL TM Melter sizing specifications are presented in Table 3-16.

\subsection{CONCLUSIONS} testing.

The following conclusions are drawn from the Phase 1 VE-SKULL ${ }^{\text {TH }}$ melter

- Single point dry or slurry feed into VE-SKULL"melter results in a uniform batch blanket covering the molten glass pool with no mechanical spreading required. However, it is expected that multiple point or feed spreading will allow use of thicker, more efficient batch blanket with resultant increased throughput.

- VE-SKULL" Melter start-up with high iron content "magnesium" boring bars is not recommended. Start-up with gas burners can be performed in a controlled manner.

- The joule heated bottom pour assembly, air operated drain plug, and water cooled bottom support ring functioned properly to control glass draining. Occasional minor glass flow around drain ring block necessitates future addition of water cooled seal under drain ring.

- Simulated, dry batched calcine feed throughputs were significantly higher than actual dry calcined product throughputs. The presence of very low nitrate levels in calcined product hinders melting rate. Moderate nitrate levels increase melting rates.

- Feed with blended slurry made up of 1 iquid LLRW simulant, dry glass formers, and dry sucrose reductant results in a uniform batch blanket, excellent glass refinement, and significantly higher feed throughputs than dry calcined feed. Slurry feed is recommended feed
method. 
Table 3-16. VE-SKULL" Melter Sizing Specifications.

\begin{tabular}{|c|c|c|c|c|c|c|}
\hline Itom & $\begin{array}{l}\text { Phase } 1 \text { Dry } \\
\text { Foed }\end{array}$ & $\begin{array}{c}\text { Phase } 1 \text { slurry } \\
\text { Feod }\end{array}$ & $\begin{array}{l}10 \text { TPD Dry } \\
\text { Food }\end{array}$ & $\begin{array}{l}50 \text { TPD Dry } \\
\text { Food }\end{array}$ & $\begin{array}{l}60 \text { TPD } \\
\text { slury Foed }\end{array}$ & $\begin{array}{l}100 \text { TFD } \\
\text { Dry Foed }\end{array}$ \\
\hline Outeide Diamoter (ft) & 4.3 & 4.3 & 7.5 & 14.5 & 17.0 & 19.5 \\
\hline Not Inside Diamoter $(\mathrm{ft})$ & 3.2 & 3.2 & 6.5 & 13.4 & 15.8 & 18.3 \\
\hline Metter Overall Haight (ft) & 5.5 & 5.5 & 8.5 & 10.0 & 10.0 & 11.0 \\
\hline Molter Croses Sectional Area & 8.1 & 8.1 & 33.2 & 140 & 195 & 265 \\
\hline \multicolumn{7}{|l|}{ Throughput Basis: } \\
\hline - Dry Foed (lb/hr-ft2) & 30 & 22 & 30 & 30 & 22 & 30 \\
\hline - Melter Size Factor [3] & 3.0 & 1.0 & 1.1 & 1.15 & 1.15 & 1.2 \\
\hline - Design Bacis Glace & 30 & 22 & 33 & 35 & 25 & 36 \\
\hline - Active Glacs Surface & 6.1 & 6.1 & 28 & 130 & 185 & 255 \\
\hline Total Design Throughput & 2 & 2 & 10 & 50 & 60 & 100 \\
\hline Scalo-Up Factor & Bacis & Basis & 5.0 & 5.0 & 5.0 & 10.0 \\
\hline Power Rating, KW [4] & 140 & 160 & 350 & 1050 & 1200 & 1600 \\
\hline No. Electrodes & 3 & 3 & 3 & 6 & 6 & 12 \\
\hline Eloctrode Diameter (in) & 2.5 & 2.5 & 4.0 & 4.0 & 4.0 & 4.0 \\
\hline Electrode Material & moly & moly & moly & moly & moly & moly \\
\hline Glase Depth (in) & $20-30$ & $20-30$ & $42-48$ & $48-60$ & $48-60$ & $48-60$ \\
\hline Glas: Volume (ft3) & 20 & 20 & 150 & 600 & 880 & 1200 \\
\hline Electrode Current Density & 9.1 & 9.1 & 5.3 & 8.0 & 9.1 & 6.0 \\
\hline \multicolumn{7}{|l|}{ Waste Foed Ports } \\
\hline - Number & one & one & one & three & three & four \\
\hline - Size, ID (in) & $16[5]$ & $16[5]$ & $6[6]$ & 6 & 6 & 6 \\
\hline \multicolumn{7}{|l|}{ Off-Gas Ports } \\
\hline - Number & one & one & one & one & one & one \\
\hline - Size, ID (in) & $16[5]$ & $16[5]$ & $18[6]$ & 20 & 20 & 24 \\
\hline \multicolumn{7}{|l|}{ Woight [2] } \\
\hline - Empty thbs & 18,300 & 18,300 & 55,000 & 155,000 & 195,000 & 285,000 \\
\hline - Operating (lb) & 21,500 & 21,500 & 80.000 & 250,000 & 335,000 & 480,000 \\
\hline Glass Drain & one & one & one & one & one & one \\
\hline - Glass Drain Typo & joule heoted & joule heated & Induction & Induction & Induction & Induction \\
\hline - Materials of Conatruction & proprietary & proprietary & proprietary & propriotary & propriotary & propriotary \\
\hline
\end{tabular}


- The VECTRA Glass composition is a suitable feed mixture for idle melter operations. Short term idle melter operation can continue with a minimum amount of feeding or glass draining.

- Lower melter NOX and off-gas emissions resulted with dry, reacted, calcined feed than with VECTRA Glass, simulated calcined feed, or LLRW simulant slurry feed.

- Severe corrosion and failure of stainless steel electrode holders was experienced. New electrode holder design is required to allow angle and height adjustment on electrodes while in service. Holders must be water cooled to el iminate electrode corrosion at glass/gas interface. Glass pool level should be maintained above the holder/ electrode interface.

- Drain ring extension should be placed into melter to allow simple melting of startup cullet in bottom zone of melter to drain ring.

- Partial dissolution of mortar between refractory blocks indicates that refractory blocks seams should be minimized and gaps eliminated to the maximum extent possible.

- The nitrogen purge system should be improved to prevent molybdenum oxidation when draining glass from melter. 
WHC-SD-WM-VI-031

Revision 0

APPENDIX 3A

LAB AKALYSES

3-39 


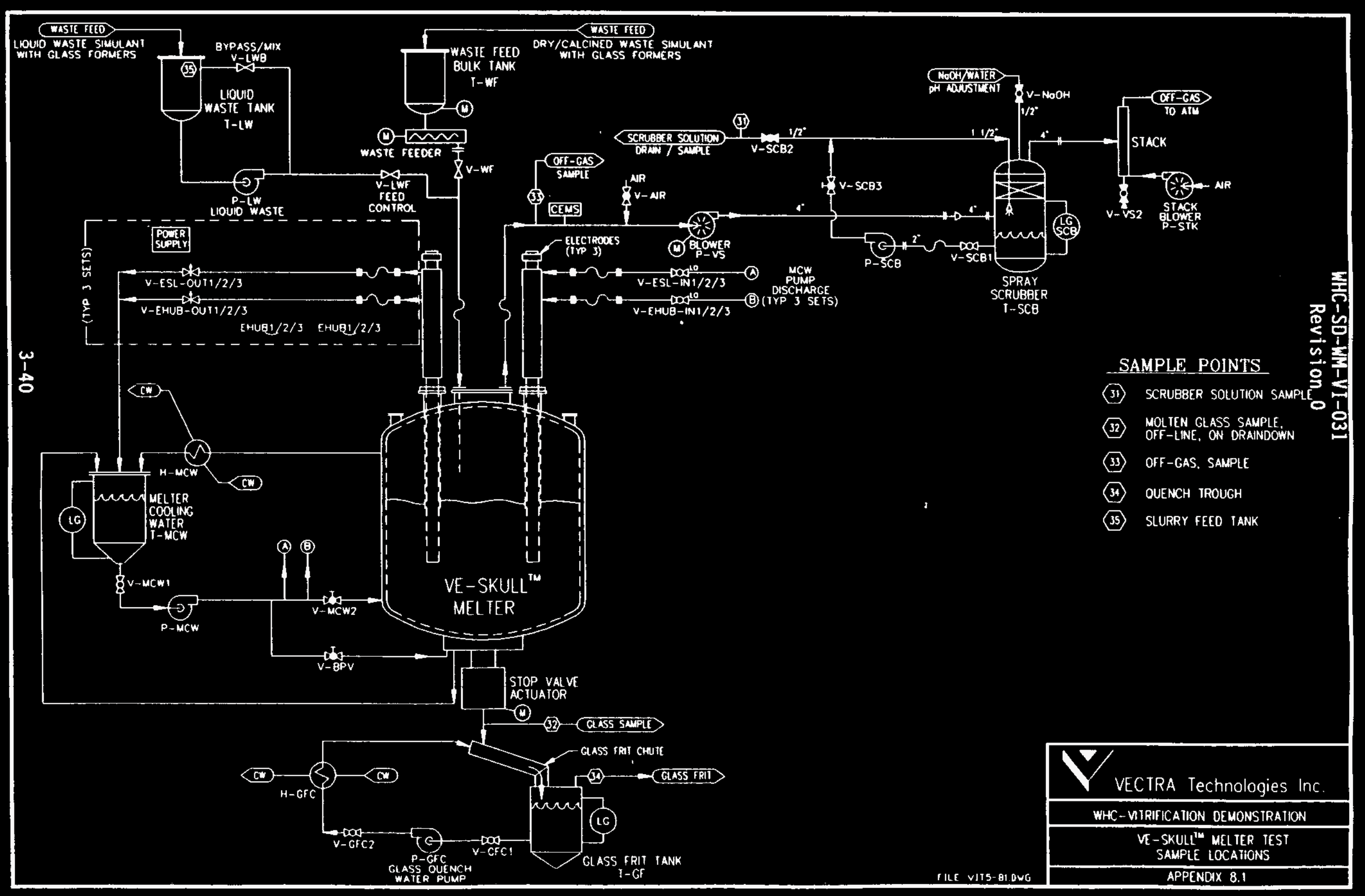




\begin{tabular}{|c|c|c|c|c|c|c|}
\hline & & TNd & $(z \bmod )$ ssefo & StS0 & $t / 22$ & d $610-2 \varepsilon 0 / 2 W 1 \Lambda$ \\
\hline & & әм!़ेग & ( ( ) ) بy sselD & coso & $t / 22$ & $\forall 810^{-}+\varepsilon D / Z W I \Lambda$ \\
\hline & & 8tụuos & 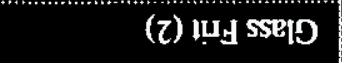 & 0050 & $6 / 22$ & $\partial \angle 10+\varepsilon D / Z W 1 \Lambda$ \\
\hline $096 \mathrm{ESS}-\mathrm{a} 78$ 6S6Е8S - व & $S 6 / t z / t$ & sosn & ( 2$) \eta_{y}$ sseto & 0050 & $6 / 2 z$ & $\cap 910^{-}+\varepsilon D / Z W \mid \Lambda$ \\
\hline \multirow[t]{2}{*}{ LOSI } & $56 / 62 / 6$ & TNd & (z) $\prod_{\mathrm{y}}$ sseip & OOSO & $t / 22$ & $\mathrm{~d} S I O-Z \varepsilon D / Z W \mid \Lambda$ \\
\hline & $\$ 6 / 6 / 5$ & & (1) ssepo & $0 \varepsilon z 2$ & $t / I Z$ & 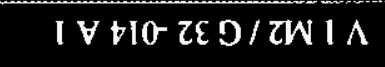 \\
\hline 21 & $56 / 72 / 6$ & 8umuos & ( I mod) sselo & oczz & $t / I Z$ & I J $\varepsilon 10^{-} Z \varepsilon D / Z W 1 \Lambda$ \\
\hline $8 \subseteq 6 £ 8 \subseteq \cdot \square \Rightarrow \angle S 6 £ 8 S \cdot a$ & $56 / t z / t$ & SOSn & (I mod) sselo & oczz & $t / 12$ & $\operatorname{I\cap } 210-2 \varepsilon D / Z W I \Lambda$ \\
\hline \multirow{6}{*}{ IOSI } & $\mathbf{5} 6 / \mathrm{tz} / \mathrm{t}$ & TNd & (I mod) sselo & $0 \varepsilon z 2$ & $t / I Z$ & $\mathrm{I} \mathrm{d} \mathrm{IIO}-\tau \varepsilon D / Z W \mathrm{I} \Lambda$ \\
\hline & & & $\tau$ Peog KumIS & $00 \varepsilon 2$ & $\forall / 12$ & $0 I 0-S E \$ / Z W I \Lambda$ \\
\hline & $\varsigma 6 / \uparrow z / t$ & TNd & 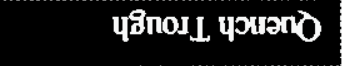 & 0002 & $t / I Z$ & $\operatorname{Id} 600+\varepsilon M / Z W \mid \Lambda$ \\
\hline & & 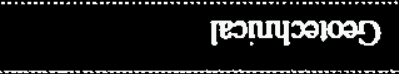 & yônoLl youeno & 0002 & $t / 12$ & IV800 $\downarrow \varepsilon M / Z W$ I $\Lambda$ \\
\hline & & sOSn & yônoul youeno & 0002 & $6 / 12$ & I $\angle 00-\downarrow \varepsilon M / Z W I \Lambda$ \\
\hline & & & 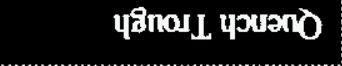 & 0002 & $t / 12$ & I $900 \sqcup E M /$ ZW I $\Lambda$ \\
\hline & & 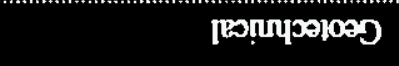 & uonnoOS Ieqquies & 0002 & $t / 12$ & IVSOO IEM/ZWI $\Lambda$ \\
\hline \multirow[t]{4}{*}{ S6-68tI } & $S 6 / \hbar 2 / b$ & TNd & uop!njos reqquiss & $000 z$ & $t / I Z$ & Id toot IEM / ZW I $\Lambda$ \\
\hline & & SOSn & uopmpos reqquLs & $000 z$ & $t / 12$ & $I \cap \varepsilon 00-I \varepsilon M / Z W \mid \Lambda$ \\
\hline & & & uonmpos deqpnuss & 0002 & $t / I Z$ & I ZOO- IE M / ZW I $\Lambda$ \\
\hline & & opdures pox!̣N RunIS & VI peog funis & $00 L I$ & $t / 12$ & IOO-SE $A / Z W I \Lambda$ \\
\hline \# ITdWVS gVT & GLYG INGWdIHS & GVT NOLLVNILSGA & cdxL cTdWVS & TWIL & GLVA & yGgWNN GTdWVS \\
\hline
\end{tabular}




\begin{tabular}{|c|c|c|c|c|c|c|}
\hline SAMPLE NUMBER & DATE & TIME & SAMPLE TYPE & DESTINATION LAB & SHIPMENT DATE & LAB SAMPLE \# \\
\hline $\mathrm{V} 1 \mathrm{M} 2 / \mathrm{G} 32-020 \mathrm{U}$ & $22 / 4$ & 0545 & Glass (2) & USGS & & \\
\hline V $1 \mathrm{M} 2 / \mathrm{G} 32-021 \mathrm{C}$ & $22 / 4$ & 0545 & Glass (2) & Coming & & \\
\hline $\mathrm{V} 1 \mathrm{M} 2 / \mathrm{G} 32-022 \mathrm{~A}$ & $22 / 4$ & 0545 & Glass (2) & Anchive & & \\
\hline $\mathrm{V} 1 \mathrm{M} 2$ / F $35-023$ & $22 / 4$ & 0550 & Slurry Feed & & & \\
\hline $\mathrm{V} 1 \mathrm{M} 2 / \mathrm{G} 32-024 \mathrm{P}$ & $22 / 4$ & 0800 & Glass (3) & PNL & & \\
\hline $\mathrm{V} 1 \mathrm{M} 2$ / G $32-025 \mathrm{U}$ & $22 / 4$ & 0800 & Glass (3) & USGS & $4 / 26 / 95$ & D - 583977 \& D - 583978 \\
\hline $\mathrm{V} 1 \mathrm{M} 2 / \mathrm{G} 32-026 \mathrm{C}$ & $22 / 4$ & 0800 & Glass (3) & Coming & & \\
\hline $\mathrm{V} 1 \mathrm{M} 2 / \mathrm{G} 32-027 \mathrm{~A}$ & $22 / 4$ & 0800 & Glass (3) & Archive & & \\
\hline $\mathrm{V} 1 \mathrm{M} 2 / \mathrm{W} 34-028 \mathrm{P} 2$ & $22 / 4$ & 1100 & Quench Trough & $\mathrm{PNL}$ & $4 / 24 / 95$ & $1495-95$ \\
\hline $\mathrm{V} 1 \mathrm{M} 2 / \mathrm{W} 34-029 \mathrm{U} 2$ & $22 / 4$ & 1100 & Quench Trough & USGS & & \\
\hline $\mathrm{V} 1 \mathrm{M} 2 / \mathrm{W} 34-030 \mathrm{~A} 2$ & $22 / 4$ & 1100 & Quench Trough & Geotechnical & & \\
\hline $\mathrm{V} 1 \mathrm{M} 2 / \mathrm{W} 34-0312$ & $22 / 4$ & 1100 & Quench Trough & & & \\
\hline $\mathrm{V} 1 \mathrm{M} 2 / \mathrm{W} 31-032 \mathrm{P} 2$ & $22 / 4$ & 1145 & Scrubber Solution & PNL & $4 / 24 / 95$ & $1490-95$ \\
\hline $\mathrm{V} 1 \mathrm{M} 2 / \mathrm{W} 31-033 \mathrm{U} 2$ & $22 / 4$ & 1145 & Scrubber Solution & USGS & . & \\
\hline $\mathrm{V} 1 \mathrm{M} 2$ / W $31-034 \mathrm{~A} 2$ & $22 / 4$ & 1145 & Scrubber Solution & Geotechnical & & . \\
\hline $\mathrm{V} 1 \mathrm{M} 2 / \mathrm{W} 31-0352$ & $22 / 4$ & 1145 & Scrubber Solution & & & \\
\hline $\mathrm{V} 1 \mathrm{M} 2 / \mathrm{G} 32-036 \mathrm{P}$ & $22 / 4$ & 1100 & Glass (4) & PNL & & \\
\hline $\mathrm{V} 1 \mathrm{M} 2 / \mathrm{G} 32-037 \mathrm{C}$ & $22 / 4$ & 1100 & Glass (4) & Corning & & \\
\hline $\mathrm{V} 1 \mathrm{M} 2 / \mathrm{G} 32-038 \mathrm{U}$ & $22 / 4$ & 1100 & Glass (4) & USGS & $4 / 26 / 95$ & D - $583975 \& D$ - 583976 \\
\hline $\mathrm{V} 1 \mathrm{M} 2 / \mathrm{G} 32-039 \mathrm{~A}$ & $22 / 4$ & 1100 & Glass (4) & Gootochnical & & \\
\hline $\mathrm{V} 1 \mathrm{M} 2 / \mathrm{G} 32-040 \mathrm{P} 2$ & $22 / 4$ & 1400 & Glass (5) & PNL & $4 / 24 / 95$ & 1502 \\
\hline $\mathrm{V} 1 \mathrm{M} 2$ / G $32-041 \mathrm{C} 2$ & $22 / 4$ & 1400 & Glass (5) & Coming & $4 / 24 / 95$ & 3,4 \\
\hline
\end{tabular}




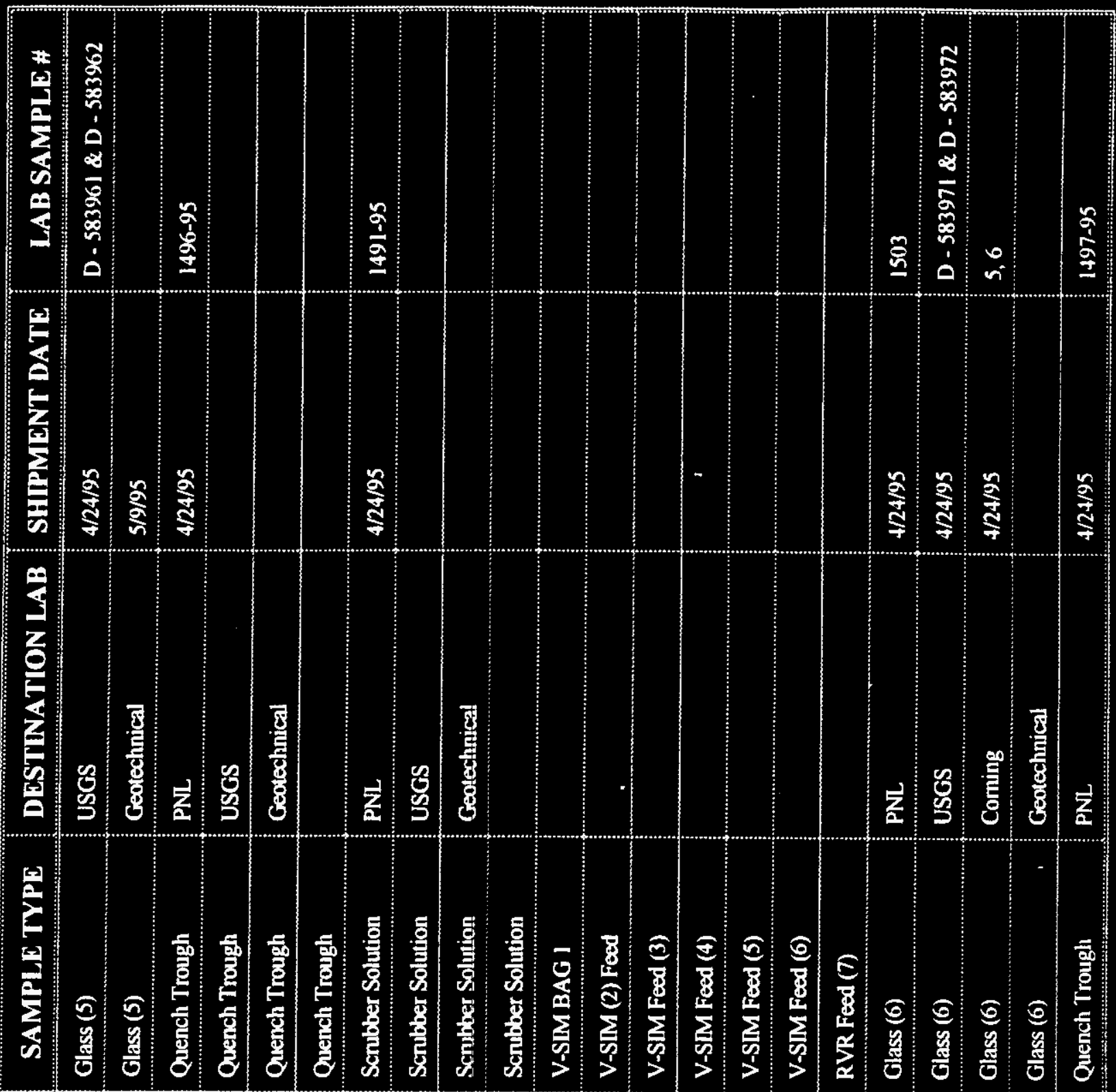

鄫

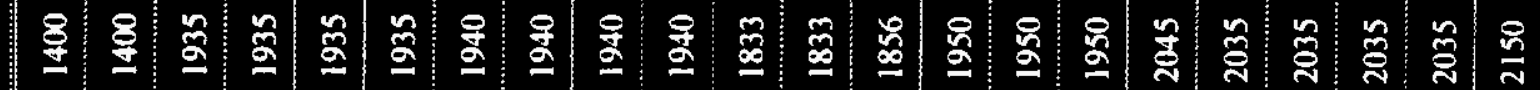

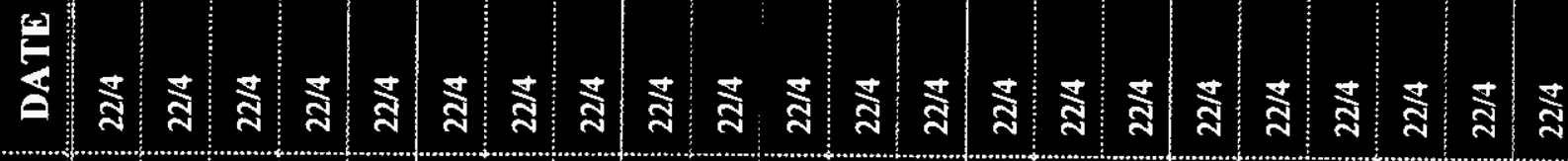
兽

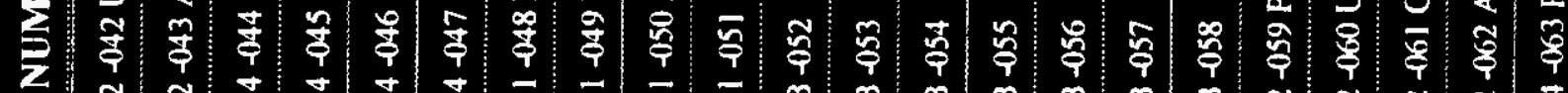
(2) N

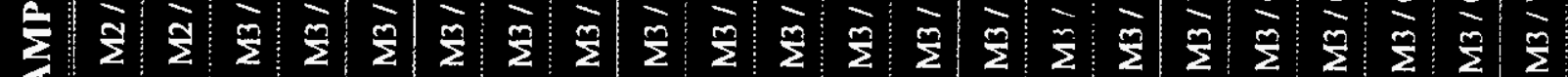

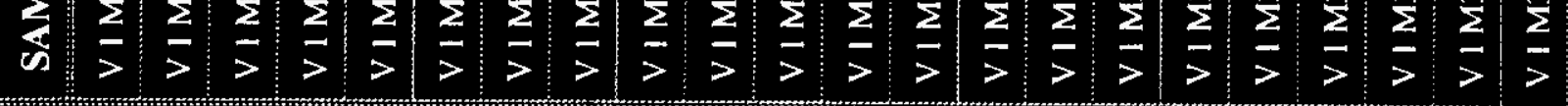




\begin{tabular}{|c|c|c|c|c|c|c|}
\hline SAMPLE NUMBER & DATE & TIME & SAMPLE TYPE & DESTINATION LAB & SHIPMENT DATE & LAB SAMPLE \# \\
\hline $\mathrm{V} / \mathrm{M} 3 / \mathrm{W} 34-064 \cup 2$ & $22 / 4$ & 2150 & Quench Trough & USGS & & \\
\hline $\mathrm{V} 1 \mathrm{M} 3 / \mathrm{W} 34-065 \mathrm{~A} 2$ & $22 / 4$ & 2150 & Quench Trough & Geotechnical & & \\
\hline V I M3 / W $34-0662$ & $22 / 4$ & 2150 & Quench Trough & & & \\
\hline$V_{1} M_{3} / \mathrm{W} 31-067$ P 2 & $22 / 4$ & 2155 & Scrubber Solution & PNL & $4 / 24 / 95$ & $1492-95$ \\
\hline $\mathrm{V} 1 \mathrm{M} 3$ / W 31-068 U 2 & $22 / 4$ & 2155 & Scrubber Solution & USGS & & \\
\hline $\mathrm{V} 1 \mathrm{M} 3 / \mathrm{W} 31-069 \mathrm{~A} 2$ & $22 / 4$ & 2155 & Scnubber Solution & Geotechnical & & \\
\hline $\mathrm{V} 1 \mathrm{M} 3 / \mathrm{W} 31+0702$ & $22 / 4$ & 2155 & Scrubber Solution & & & \\
\hline $\mathrm{V} / \mathrm{M} 3 / \mathrm{G} 34-071 \mathrm{P}$ & $22 / 4$ & 2200 & Glass Frit (6) & PNL & $4 / 24 / 95$ & 1508 \\
\hline$V / M 3 / G 34-72 U$ & $22 / 4$ & 2200 & Glass Frit (6) & USGS & $4 / 24 / 95$ & $D-583963 \& D-583964$ \\
\hline $\mathrm{V} 1 \mathrm{M} 3 / \mathrm{G} 34-073 \mathrm{C}$ & $22 / 4$ & 2200 & Glass Frit (6) & Corning & & \\
\hline V 1 M3 / G $34-074 A$ & $22 / 4$ & 2200 & Glass Frit (6) & Geotechnical & & \\
\hline $\mathrm{V} 1 \mathrm{M} 3$ / G $32-075$ P 2 & $22 / 4$ & 2240 & Glass (7) & PNL & $4 / 24 / 95$ & 1504 \\
\hline $\mathrm{V} 1 \mathrm{M} 3 / \mathrm{G} 32-076$ U 2 & $22 / 4$ & 2240 & Glass (7) & USGS & $4 / 24 / 95$ & D - $583965 \&$ D - 583966 \\
\hline $\mathrm{V} 1 \mathrm{M} 3 / \mathrm{G} 32-077 \mathrm{C} 2$ & $22 / 4$ & 2240 & Glass (7) & Corning & $4 / 24 / 95$ & 7,8 \\
\hline $\mathrm{V} 1 \mathrm{M} 3 / \mathrm{G} 32-078 \mathrm{~A} 2$ & $22 / 4$ & 2240 & 'Glass (7) & Geotechnical & & 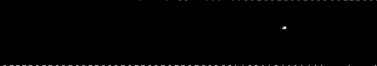 \\
\hline V 1 M4 / F SB -079 & $23 / 4$ & 0312 & Mixed Calcined Feed & & & \\
\hline $\mathrm{V} / \mathrm{M} 3 / \mathrm{G} 32-080 \mathrm{P}$ & $23 / 4$ & 0530 & Glass (8) & PNL & & \\
\hline $\mathrm{V} 1 \mathrm{M} 3 / \mathrm{G} 32-081 \mathrm{U}$ & $23 / 4$ & 0530 & Glass (8) & USGS & & \\
\hline $\mathrm{V} 1 \mathrm{M} 3 / \mathrm{G} 32-082 \mathrm{C}$ & $23 / 4$ & 0530 & Glass (8) & Corning & & \\
\hline V $1 \mathrm{M} 3 / \mathrm{G} 32-083 \mathrm{~A}$ & $23 / 4$ & 0530 & Glass (8) & Geotechnical & & \\
\hline V 1 M4 / W $31-084$ P 1 & $23 / 4$ & 1045 & Scrubber & PNL & $4 / 24 / 95$ & $1493-95$ \\
\hline V $1 \mathrm{M} 4$ / W $31-085$ U 1 & $23 / 4$ & 1045 & Scnubber & USGS & & \\
\hline
\end{tabular}




\begin{tabular}{|c|c|c|c|c|c|c|}
\hline SAMPLE NUMBER & DATE & TIME & SAMPLE TYPE & DESTINATION LAB & SHIPMENT DATE & LAB SAMPLE \# \\
\hline $\mathrm{V} 1 \mathrm{M} 4 / \mathrm{W} 31-086 \mathrm{~A} 1$ & $23 / 4$ & 1045 & Scnubber & Geotechnical & & \\
\hline V I M4 / W $31-0871$ & $23 / 4$ & 1045 & Scrubber & & & \\
\hline $\mathrm{V} 1 \mathrm{M} 4 / \mathrm{G} 32-088 \mathrm{P}]$ & $23 / 4$ & 1015 & Glass (9) & PNL & $4 / 24 / 95$ & 1505 \\
\hline $\mathrm{V} 1 \mathrm{M} 4 / \mathrm{G} 32-089 \mathrm{Cl}$ & $23 / 4$ & 1015 & Glass (9) & Corning & $4 / 24 / 95$ & 9,10 \\
\hline $\mathrm{V} 1 \mathrm{M} 4$ / G $32-090 \mathrm{U} 1$ & $23 / 4$ & 1015 & Glass (9) & USGS & $4 / 24 / 95$ & D - $583967 \&$ D - 583968 \\
\hline $\mathrm{V} I \mathrm{M} 4$ / G $32-091 \mathrm{~A} 1$ & $23 / 4$ & 1015 & Glass (9) & Geotechnical & & \\
\hline $\mathrm{V} 1 \mathrm{M4} / \mathrm{W} 34-092 \mathrm{P} 1$ & $23 / 4$ & 1300 & Quench Trough & PNL & $4 / 24 / 95$ & $1498-95$ \\
\hline V 1 M4 / W $34-093$ U 1 & $23 / 4$ & 1300 & Quench Trough & USGS & & \\
\hline V ! M4 / W $34-094$ A ! & $23 / 4$ & 1300 & Quench Trough & Geotechnical & & \\
\hline V I M4 / W $34-095$ I & $23 / 4$ & 1300 & Quench Trough & & & \\
\hline $\mathrm{V} 1 \mathrm{M} 4 / \mathrm{G} 32-096 \mathrm{P} 2$ & $23 / 4$ & 1315 & Glass (10) & PNL & $4 / 24 / 95$ & 1506 \\
\hline V I M4 / G $32-097$ C 2 & $23 / 4$ & 1315 & Glass (10) & Corning & $4 / 24 / 95$ & 11,12 \\
\hline $\mathrm{V} 1 \mathrm{M} 4 / \mathrm{G} 32-098 \mathrm{U} 2$ & $23 / 4$ & 1315 & Glass (10) & USGS & $4 / 24 / 95$ & D - $583969 \&$ D - 583970 \\
\hline V 1 M4 / G $32-099$ A 2 & $23 / 4$ & 1315 & Glass (I0) & & $\therefore$ & \\
\hline $\mathrm{V} 1 \mathrm{M} 4 / \mathrm{W} 34-100 \mathrm{P} 2$ & $23 / 4$ & 1600 & Quench Trough & PNL & $4 / 24 / 95$ & $1499-95$ \\
\hline V 1 M4 / W 34 -101 U 2 & $23 / 4$ & 1600 & Quench Trough & & & \\
\hline $\mathrm{V} I \mathrm{M} 4 / \mathrm{W} 34-102 \mathrm{~A} 2$ & $23 / 4$ & 1600 & Quench Trough & & & \\
\hline V 1 M4 / W $34-1032$ & $23 / 4$ & 1600 & Quench Trough & & & \\
\hline $\mathrm{V} 1 \mathrm{M} 4 / \mathrm{W} 31-104 \mathrm{P} 2$ & $23 / 4$ & 1600 & Scrubber & PNL & $4 / 24 / 95$ & $1494-95$ \\
\hline $\mathrm{VIM} / \mathrm{W} 31-105 \mathrm{U} 2$ & $23 / 4$ & 1600 & Scrubber & & & \\
\hline $\mathrm{VI} M 4$ /W $31-106 \mathrm{~A} 2$ & $23 / 4$ & 1600 & Scrubber & & & \\
\hline V I M4 / W $31-1072$ & $23 / 4$ & 1600 & Scrubber & & & \\
\hline
\end{tabular}




\begin{tabular}{|c|c|c|c|c|c|c|}
\hline SAMPLE NUMBER & DATE & TIME & SAMPLE TYPE & DESTINATION LAB & SHIPMENT DATE & LAB SAMPLE \# \\
\hline V I M2 / F $35-108 P$ & $22 / 4$ & 0550 & Slurny & PNL & $5 / 1 / 95$ & \\
\hline $\mathrm{V} / \mathrm{M} 2 / \mathrm{F} 35 \cdot 109 \mathrm{U}$ & $22 / 4$ & 0550 & Slurry & & & \\
\hline $\mathrm{V} 1 \mathrm{M} 2 / \mathrm{F} 35-110$ & $22 / 4$ & 0550 & Slurny & & & \\
\hline $\mathrm{V} 1 \mathrm{M} 2 / \mathrm{F} 35-111 \mathrm{~A}$ & $22 / 4$ & 0550 & Slurry & Geotech & & \\
\hline V 1 MS/F $35-112$ & $26 / 4$ & 1300 & Slurry & & & \\
\hline V $1 \mathrm{M} 5 / 033-113$ & $28 / 4$ & 1400 & $\begin{array}{l}\text { Pipe Scale Build Up } \\
\text { (Horizontal Pipe) }\end{array}$ & & & \\
\hline V 1 MS/O $33-114$ & $28 / 4$ & 1400 & $\begin{array}{l}\text { Pipe Scale Build Up } \\
\text { (Plenum Cross) }\end{array}$ & & & \\
\hline V I M5/0 $33-115$ & $28 / 4$ & 1400 & $\begin{array}{l}\text { Pipe Scale Build Up } \\
\text { (Lower Elbow) }\end{array}$ & & & \\
\hline $\mathrm{V} 1 \mathrm{M5} / \mathrm{G} \mathrm{IB}-116 \mathrm{C}$ & $18 / 5$ & 1430 & Inside Barrel & Coming & $5 / 18 / 95$ & Sample 1 \\
\hline V I MS/G OB - $117 \mathrm{C}$ & $18 / 5$ & 1430 & Outside Barrel & Coming & $5 / 18 / 95$ & Sample 2 \\
\hline V 1 M5/G $32-118 C$ & $26 / 4$ & 1035 & Glass from V-Sim & Coming & $5 / 18 / 95$ & Sample 3 \\
\hline
\end{tabular}


WHC-SD-WM-VI-031

Revision 0

\section{SUPPLENENTARY GLASS ANALYSES}

Three glass samples were directly submitted to Corning by Vectra and analyzed under a separate subcontract. Sample description and results of analyses are as follows.

\section{Sample Description}

Sample VIM5/GIB-116C was taken from a drum of glass poured during final melter draining. This drum was wrapped with an insulating fiber mat during cooldown and the sample was taken near the center of the drum. This glass sample therefore was cooled at a relatively slow rate.

Sample VIM5/GOB-117C was taken from the same drum but from near the outside. This glass therefore was cooled at a significantly faster rate than the first sample.

Sample VIM5/G32-118C was taken during a normal glass pour at the end of the V-Sim high-throughput test (see Section 5.5).

Quantitative Chemical Analysis Results

CELS Client No.: 15334-001

Lab I.D. No.: 12120-95

\begin{tabular}{|l|c|c|c|c|}
\hline \multicolumn{4}{|c|}{ Results (wt\%) } & Test method \\
\hline Sample & VIM5/GIB-116C VIM5/GOB-117C VIM5/G32-118C \\
\hline $\mathrm{K}_{2} \mathrm{O}$ & 1.15 & 1.15 & 1.24 & FES \\
\hline $\mathrm{Na}_{2} \mathrm{O}$ & 26.8 & 27.0 & 20.3 & FES \\
\hline $\mathrm{Al}_{2} \mathrm{O}_{3}$ & 8.94 & 8.87 & 9.91 & PLS \\
\hline $\mathrm{B}_{2} \mathrm{O}_{3}$ & 7.45 & 7.46 & 8.08 & PLS \\
\hline $\mathrm{CaO}$ & 2.52 & 2.50 & 2.73 & PLS \\
\hline $\mathrm{Cr}_{2} \mathrm{O}_{3}$ & 0.27 & 0.27 & 0.29 & PLS \\
\hline $\mathrm{Fe}_{2} \mathrm{O}_{3}$ & 0.87 & 0.87 & 0.92 & PLS \\
\hline $\mathrm{MgO}^{4}$ & 1.83 & 1.81 & 1.97 & PLS \\
\hline $\mathrm{MnO}_{2}$ & 0.010 & 0.010 & 0.013 & PLS \\
\hline $\mathrm{P}_{2} \mathrm{O}_{5}$ & 0.18 & 0.15 & 0.17 & PLS \\
\hline $\mathrm{SiO}_{2}$ & 48.0 & 47.6 & 52.5 & PLS \\
\hline $\mathrm{TiO}_{2}$ & 0.012 & 0.012 & 0.012 & PLS \\
\hline $\mathrm{ZrO}_{2}$ & 0.094 & 0.085 & 0.077 & PLS \\
\hline $\mathrm{C}$ & 0.01 & 0.02 & 0.01 & LECO \\
\hline
\end{tabular}


WHC-SD-WM-VI-031

Revision 0

\begin{tabular}{|l|c|c|c|c|}
\hline \multicolumn{4}{|c|}{ Results (wt\%) } & Test method \\
\hline Sample & VIM5/GIB-116C VIM5/GOB-117C VIM5/G32-118C \\
\hline $\mathrm{SO}_{3}$ & 0.11 & 0.12 & 0.12 & LECO \\
\hline $\mathrm{O}_{2}$ & 39.6 & 39.1 & 41.3 & LEC0 \\
\hline $\mathrm{F}^{-}$ & 0.20 & 0.20 & 0.22 & PRYO \\
\hline $\mathrm{FeO}^{-5}$ & 0.69 & 0.67 & 0.65 & COLOR \\
\hline $\mathrm{Cl}^{-}$ & 0.14 & 0.14 & 0.17 & TITR \\
\hline
\end{tabular}

FES = Flame Emission Spectroscopy

PLS = Plasma/Emission Spectroscopy

LECO = LECO Instrumentation

COLOR = Colorimetry

TITR = Titrimetry

Product Consistency Test (PCT) Results

Lab I.D. No.: 12040-95

\begin{tabular}{|c|c|c|c|c|c|}
\hline \multirow[t]{2}{*}{ Sample I.D. } & \multicolumn{5}{|c|}{ Results (ppm) } \\
\hline & Cs & $\mathrm{Na}$ & Al & $\mathrm{Si}$ & $\mathrm{Sr}$ \\
\hline $\begin{array}{l}\text { VIM5/GIB-116C } \\
\text { Dissolution } 1 \\
\text { Dissolution } 2\end{array}$ & $\begin{array}{l}0.55 \\
0.55\end{array}$ & $\begin{array}{l}217 \\
217 \\
\end{array}$ & $\begin{array}{l}31 \\
31 \\
\end{array}$ & $\begin{array}{l}168 \\
168 \\
\end{array}$ & $\begin{array}{l}0.021 \\
0.023\end{array}$ \\
\hline $\begin{array}{l}\text { VIM5/GIB-116C } \\
\text { Dissolution } 1 \\
\text { Dissolution } 2\end{array}$ & $\begin{array}{l}0.56 \\
0.56\end{array}$ & $\begin{array}{l}219 \\
220\end{array}$ & $\begin{array}{l}31 \\
31\end{array}$ & $\begin{array}{l}169 \\
168\end{array}$ & $\begin{array}{l}0.021 \\
0.023\end{array}$ \\
\hline $\begin{array}{l}\text { VIM5/GOB-117C } \\
\text { Dissolution } 1 \\
\text { Dissolution } 2\end{array}$ & $\begin{array}{l}0.53 \\
0.53\end{array}$ & $\begin{array}{l}215 \\
212\end{array}$ & $\begin{array}{l}31 \\
31\end{array}$ & $\begin{array}{l}165 \\
164\end{array}$ & $\begin{array}{l}0.025 \\
0.027\end{array}$ \\
\hline $\begin{array}{l}\text { VIM5/GOB-117C } \\
\text { Dissolution } 1 \\
\text { Dissolution } 2\end{array}$ & $\begin{array}{l}0.57 \\
0.57\end{array}$ & $\begin{array}{l}208 \\
211\end{array}$ & $\begin{array}{l}30 \\
30\end{array}$ & $\begin{array}{l}161 \\
161\end{array}$ & $\begin{array}{l}0.026 \\
0.025\end{array}$ \\
\hline $\begin{array}{l}\text { VIM5/G32-118C } \\
\text { Dissolution } 1 \\
\text { Dissolution } 2\end{array}$ & $\begin{array}{l}0.13 \\
0.13\end{array}$ & $\begin{array}{l}51 \\
50\end{array}$ & $\begin{array}{l}12 \\
12\end{array}$ & $\begin{array}{l}71 \\
71\end{array}$ & $\begin{array}{l}0.012 \\
0.011\end{array}$ \\
\hline $\begin{array}{l}\text { VIM5/G32-118C } \\
\text { Dissolution } 1 \\
\text { Dissolution } 2\end{array}$ & $\begin{array}{l}0.13 \\
0.13\end{array}$ & $\begin{array}{l}50 \\
51\end{array}$ & $\begin{array}{l}12 \\
12\end{array}$ & $\begin{array}{l}71 \\
72\end{array}$ & $\begin{array}{l}0.012 \\
0.013\end{array}$ \\
\hline
\end{tabular}


WHC-SD-WM-VI-03I

Revision 0

\begin{tabular}{|c|c|c|}
\hline Sample I.D. & & Results \\
\hline \multicolumn{3}{|c|}{$\mathrm{pH}$} \\
\hline $\begin{array}{l}\text { VIM5/GIB-1I6C } \\
\text { Dissolution } 1 \\
\text { Dissolution } 2\end{array}$ & \multicolumn{2}{|l|}{$\begin{array}{l}12 \\
12\end{array}$} \\
\hline $\begin{array}{l}\text { VIM5/GOB-117C } \\
\text { Dissolution } 1 \\
\text { Dissolution } 2\end{array}$ & \multicolumn{2}{|l|}{$\begin{array}{l}12.1 \\
12.2\end{array}$} \\
\hline $\begin{array}{l}\text { VIM5/G32-118C } \\
\text { Dissolution } 1 \\
\text { Dissolution } 2\end{array}$ & \multicolumn{2}{|l|}{$\begin{array}{l}11.3 \\
11.3\end{array}$} \\
\hline \multicolumn{3}{|c|}{$\begin{array}{l}\text { Test methods: Cs by } \mathrm{Fl} \text { ame Emission Spectroscopy. } \mathrm{Na}, \mathrm{Al}, \mathrm{Si}, \mathrm{Sr} \text { by } \\
\text { Plasma/Emission Spectroscopy. }\end{array}$} \\
\hline
\end{tabular}




\begin{tabular}{|c|c|c|c|c|c|c|c|c|c|c|c|c|c|c|}
\hline & & & & & & & 80160 & & $00 \angle 0^{\circ} 0$ & & $8600^{\circ} 0$ & & 690 & 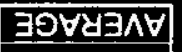 \\
\hline & & & & & & & & & & & & & & \\
\hline & & & & & & & & & & & & & & \\
\hline & & & & & & & 02600 & & & & 06000 & & 890 & \\
\hline & & & & & & & $0022^{\circ} 0$ & & & & $0010^{\prime} 0$ & & 890 & \\
\hline & & & & & & & $0960^{\circ} 0$ & & & & $0600^{\circ} 0$ & & $89^{\circ} 0$ & \\
\hline & & & & & & & $0260^{\circ} 0$ & & & & 66000 & & $\angle 90$ & \\
\hline & & & & & & 910 & $0080^{\circ} 0$ & 100 & & & $0600^{\circ} 0$ & $\angle 90$ & 690 & \\
\hline & & & & & & $9 \mathcal{L}^{\circ} 0$ & $0080^{\circ} 0$ & 100 & & & $6600^{\circ} 0$ & 890 & 690 & \\
\hline & & & & & & Sr.0 & 08600 & 100 & & & 06000 & $\angle 9^{\circ} 0$ & 690 & \\
\hline & & & & & & s. 0 & 00010 & 100 & & & $8600^{\circ} 0$ & $\angle 9^{\prime} 0$ & 690 & \\
\hline & & & & & & sosn! & 1 oupunos & Sosn & 6uinuog & sosn & Eupuos & sosn & 116 6utuso5 & \\
\hline & 14600 & & & & & (s)o & (z)d & $\mathrm{OL}^{4}$ & WW & (2)0 & Duw & & 2) प्र & \\
\hline & 124000 & & 60 & & $1890^{\circ}$ & & 8800 & & OSTOL & & $E t^{\circ} 0$ & & 900 & 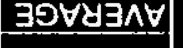 \\
\hline & & & & & & & & & & & & & & \\
\hline & 00800 & & 820 & & 9900 & & $2 \geqslant 00$ & & & & 010 & & & \\
\hline & $0 \rightarrow 200$ & & 820 & & $\angle 900$ & & 2000 & & & & $p 10$ & & & \\
\hline & $\frac{0820^{\circ} 0}{0820^{\circ}}$ & & 820 & & $\angle 900$ & & 1600 & & & & sio 0 & & & \\
\hline & $\frac{08200}{0900^{\circ} 0}$ & LE'O & 820 & & $\angle 90^{\circ} 0$ & & 1600 & & & & sto 0 & & & \\
\hline & $\frac{09000}{0 \angle 000}$ & $\frac{\mid E \cdot O}{\text { LE. }}$ & 620 & 6900 & 8000 & 9600 & $p 800$ & & & Elo & Eto & $\angle 00$ & $\angle 0^{\circ}$ & \\
\hline & $0800^{\circ} 0$ & LEO & $\frac{060}{620}$ & $\frac{6500}{1900}$ & $\begin{array}{l}87000 \\
66000\end{array}$ & $\frac{0000}{980^{\circ} 0}$ & $\frac{b 80_{0}^{\circ}}{t \varepsilon 0^{\circ} 0}$ & $008 \mathrm{Lt}$ & & D10 & E. 0 & 200 & $\frac{\angle 00}{c 00}$ & \\
\hline aN & 09000 & IE'O & 620 & $1 \mathrm{SOO}$ & 6000 & 9800 & 6000 & 0098 & & $\frac{E 10}{160}$ & $\begin{array}{l}\mathrm{Cl} 0 \\
\mathrm{ZLO} 0\end{array}$ & $\frac{60}{50} 0$ & $\frac{50}{90}$ & \\
\hline SOSn & 6upuos & Sอsก & 64 6400 & sosn & Bujuos & sosก & 6ujujos & sosn & Butusos & sosn & Bu!uioj & sosn & 6ug̣uło & \\
\hline (c) & gs & (E)a & (2) 10 & o & is & $\alpha(2)$ & 280 & 1 & & \pm & & 10 & 0 & \\
\hline & LOL & & ES96 & & ezz & & 862 & & L9G & & $F \varepsilon^{2} L$ & & 5989 & 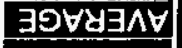 \\
\hline & & & & & & & & & & & & & & \\
\hline & 660 & & g96 & & E\&'2 & & $86 z$ & & ob $\mathrm{gl}$ & & $89 \mathrm{~L}$ & & $08 \angle 9$ & \\
\hline & 001 & & 196 & & 622 & & 662 & & $06 \mathrm{sl}$ & & 29.2 & & $0 \angle 89$ & \\
\hline & $10 \%$ & & ES: 6 & & 622 & & 662 & & $00 \mathrm{st}$ & & $99 \mathrm{~L}$ & & 0989 & \\
\hline & 101 & & 696 & & 622 & & 862 & & opst & & $69 L$ & & $0 \angle 89$ & \\
\hline $60 \%$ & 001 & 296 & 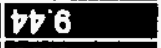 & BzZ & $0 z 2$ & 662 & 982 & $00 \mathrm{Gl}$ & OZ91 & $8 Z L$ & 622 & $0 \angle 8 S$ & $0 Z 8 S$ & \\
\hline 201 & 001 & 896 & 686 & LE? & 122 & $\angle 62$ & $\angle 8 Z$ & 06 b & OZ $9 \mathrm{~L}$ & SEL & $92 \mathrm{~L}$ & $0 \angle 89$ & 0089 & \\
\hline 201 & $00 \%$ & 996 & $p+6$ & 222 & $E 22$ & 682 & 162 & OG'SL & oz 91 & 969 & $2 \varepsilon L$ & 0889 & 0689 & \\
\hline $90 \%$ & $10 \%$ & 596 & $66^{\prime} 6$ & 122 & 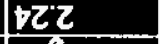 & 682 & 862 & $0 G^{\prime} \mathcal{S}$ & $0 z 91$ & 089 & $92 L$ & 0689 & 0069 & \\
\hline sosn & 6บุแ०ว & s9sn & Bบyบ00 & sosn & 6utuog & sosn & 6uाuा०5 & sosn & 6uiniog & sesn| & 6uluiog & sosn & 6ulujo & \\
\hline (E)o & (2) 3 at & (ع)O & (2) & $\sigma_{0}$ & ow & $0^{e}$ & e. & $0 / 2$ & $\partial E N$ & (घ) & (ट) 8 & (2)o & OIS & \\
\hline
\end{tabular}




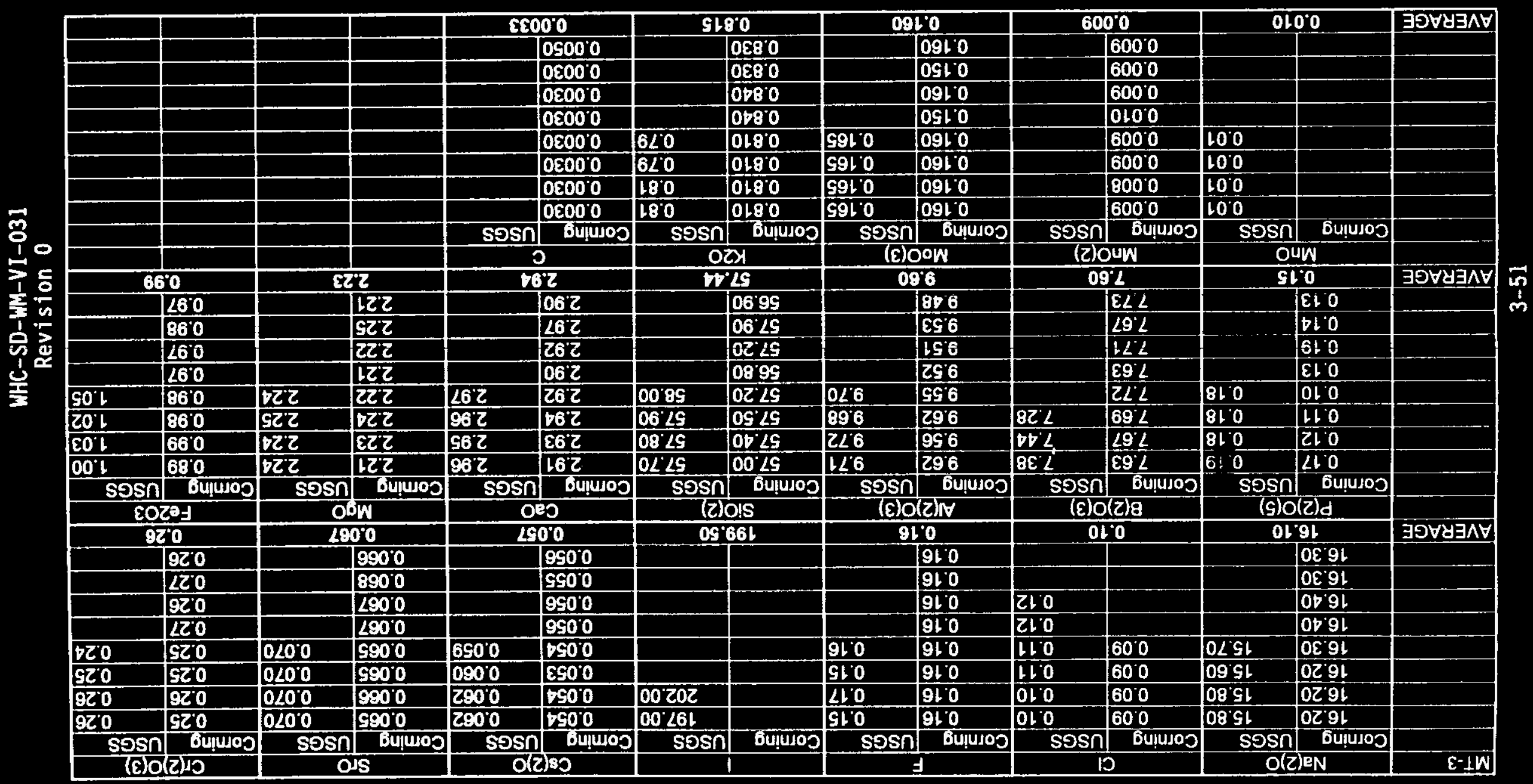


May 16, 1995

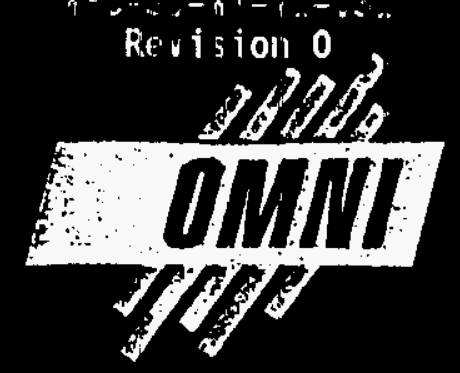

Mike Hill

Vectra Technologies, Inc.

One Harbison Way, Suite 209

Columbia, South Carolina 29212-3408

Dear Mr. Hill:

This letter serves to transmit OMNI Environmental Services, Inc̈. (OMNI) data report for testing services conducted on the vitrification process "melter" at the Vectra Technologies, Inc. Richland, Washington facilities during the period from April 21 to April 23, 1995. OMNI conducted isokinetic sampling and gaseous emissions analysis procedures on the exhaust stack which vents the process melter. The sampled exhaust gases consisted of a mixture of fumes generated by the heating of feedstock materials in the melter and ambient air which is drawn through the melter vent system to carry melter fumes to the emissions control/treatment system before being discharged to the atmosphere. OMNI sampled all emissions at a point in the exhaust-duct system which is more than 8 diameters downstream and 2 diameters upstream from disturbances and located between the melter and the emission control/treatment equipment.

Mr. Mike Hill was the project manager and technical liaison for Vectra Technologies and Mr. Paul Tiegs was the on-site project manager for OMNI.

Six one-hour test runs were performed on the following schedule:

Run Number 1 from 2305, April 21, 1995 to 0005, April 22, 1995

Run Number 2 from 1156, April 22, 1995 to 1256, April 22, 1995

Run Number 3 from 1910, April 22, 1995 to 2010, April 22, 1995

Run Number 4 from 2105, April 22, 1995 to 2205, April 22, 1995

Run Number 5 from 1041, April 23, 1995 to 1141, April 23, 1995

Run Number 6 from 1202, April 23, 1995 to 1302, April 23, 1995

Two runs were conducted for each of three different melter feedstock materials:

Run Numbers 1 and 2 were conducted while slurry \#1 was used, Run Numbers 3 and 4 were conducted while slurry $\$ 2$ was used, and Run Numbers 5 and 6 were conducted while a dry calcined feedstock was used.

Isokinetically (U.S. EPA Method 5 Type) sampled particulate material was analyzed for total mass and for the elemental and cation/anion constituents presented in Table 1. Except for methane and nitrous oxide $\left(\mathrm{N}_{2} \mathrm{O}\right)$, the exhaust-gas gaseous constituents were measured on-site with

OMNI Environmental Services, Inc.

Consulting + Enggineering + Testing 


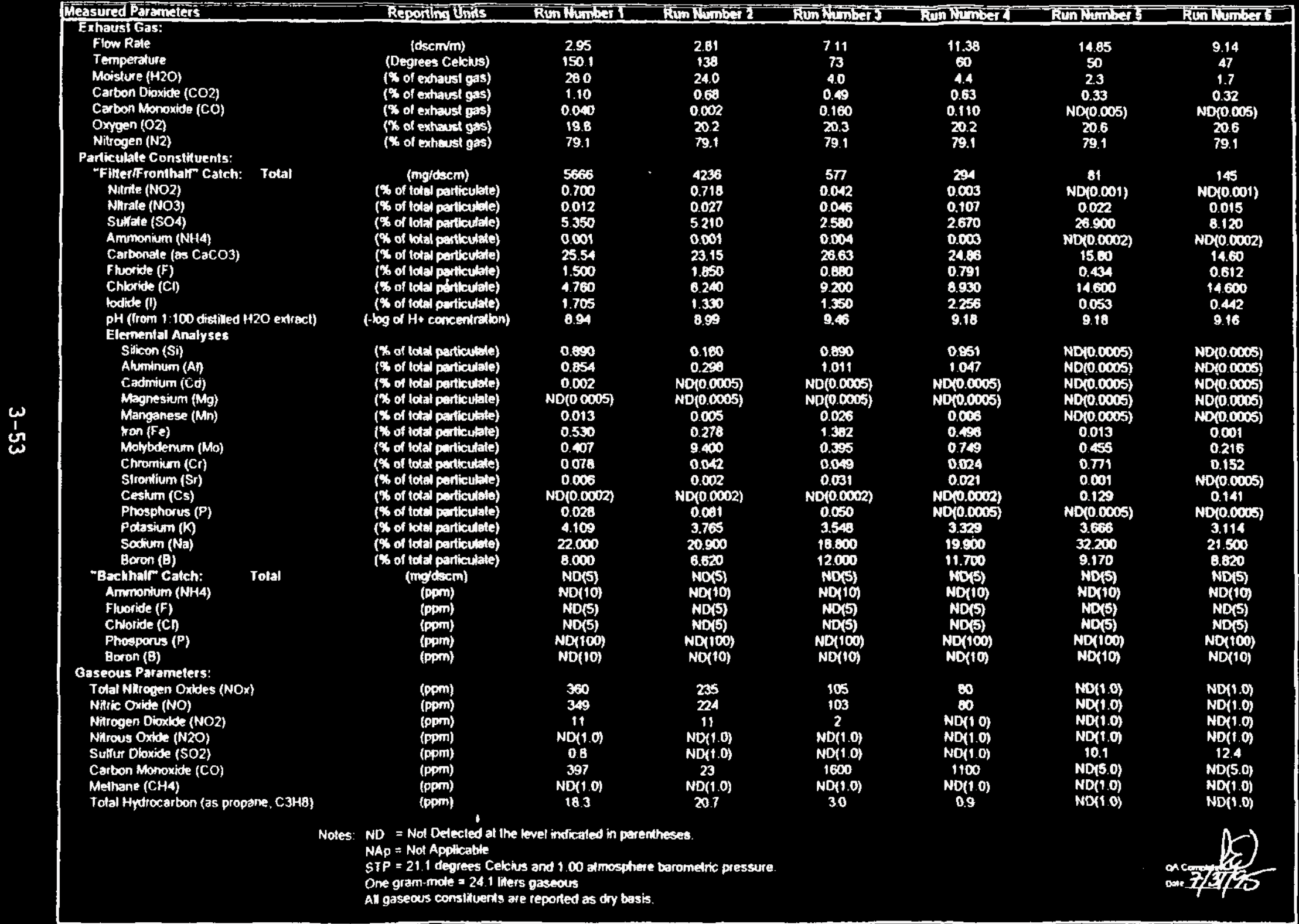


WHC-SD-WM-VI-031

Revision 0

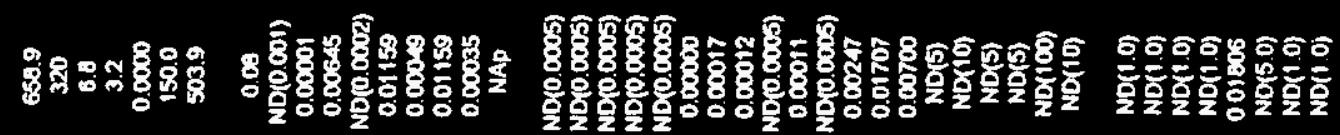
$2 \frac{12}{2} 20$

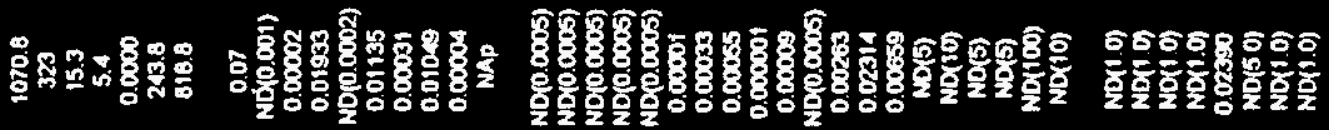

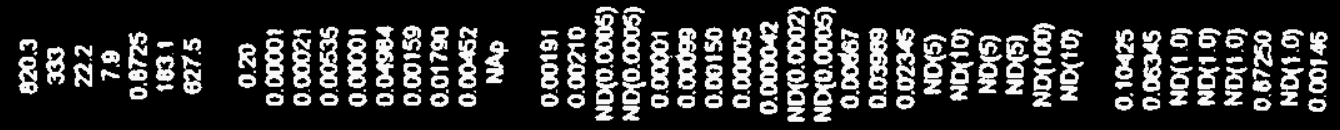

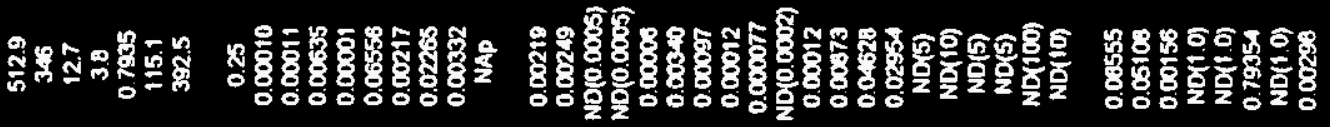

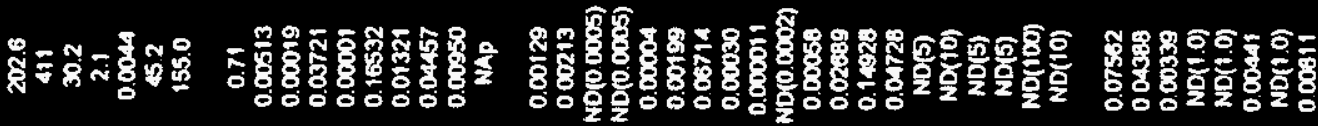

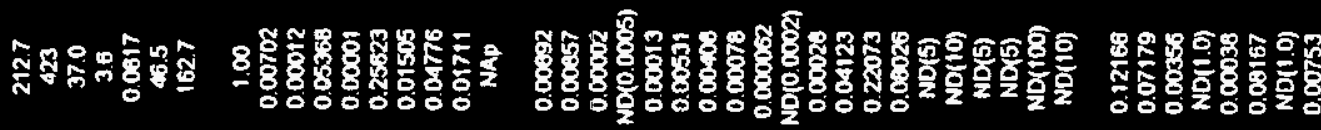

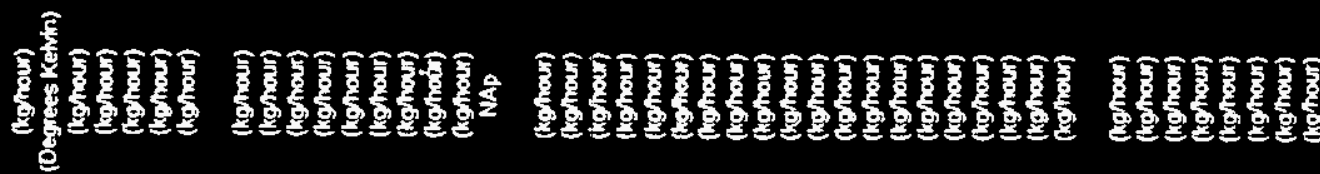

变

腹

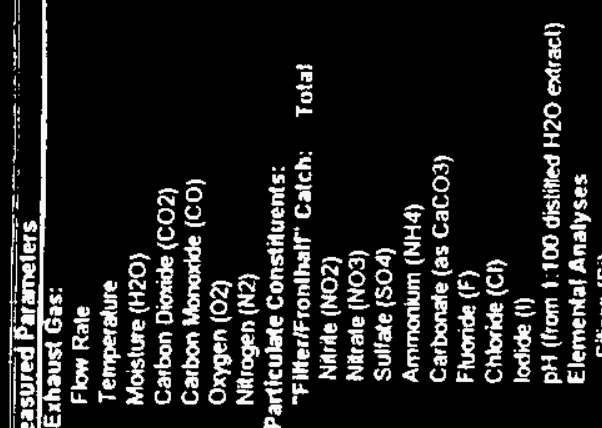

产 
WHC-SD-WM-VI-031

Revision 0

APPENDIX 3B

THROUEHPUT LOG 


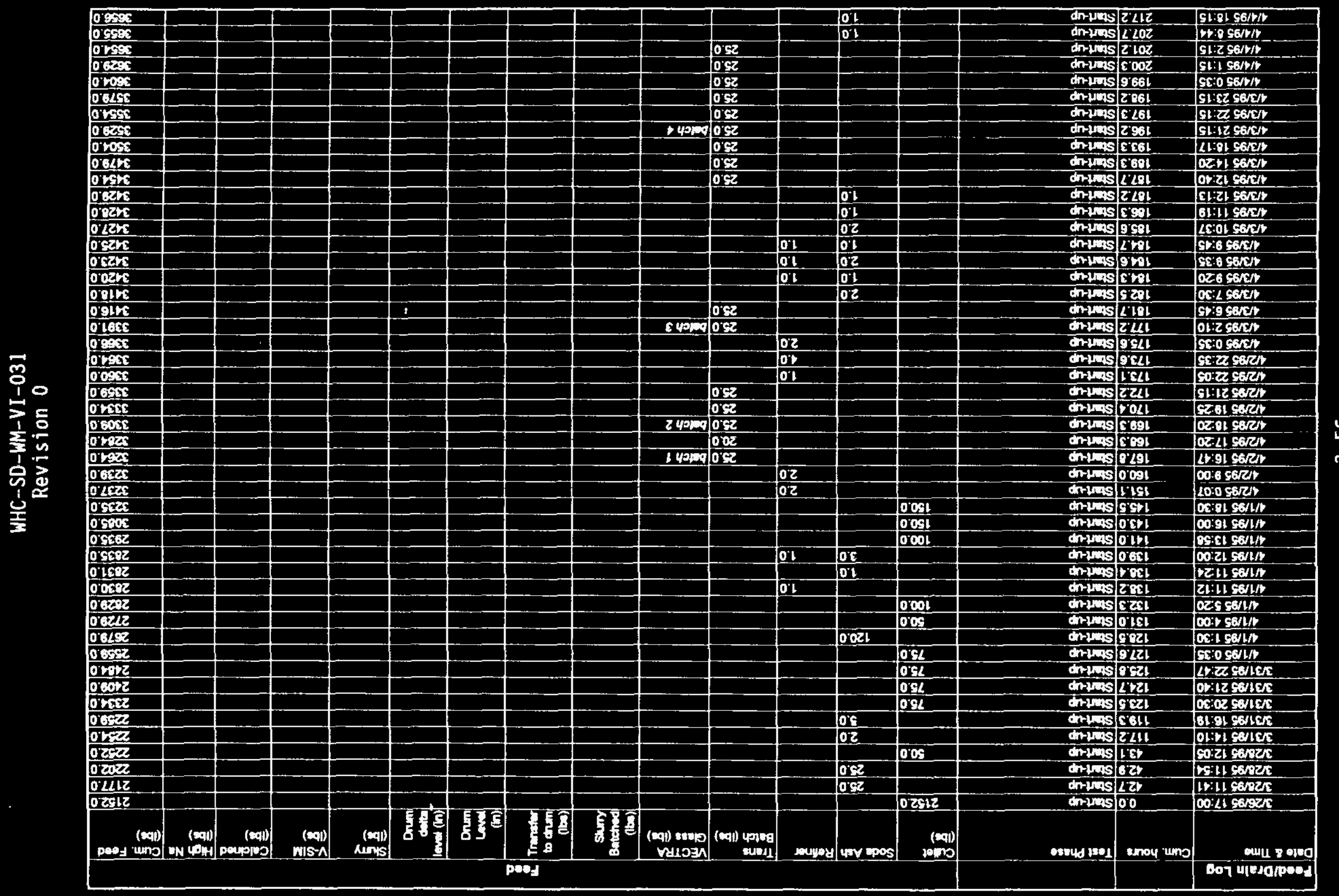




\begin{tabular}{|c|c|c|c|c|c|c|c|c|c|c|c|c|c|c|c|c|}
\hline \multirow{2}{*}{ Foedprain Log } & \multirow[b]{2}{*}{ Cum hours } & \multirow[b]{2}{*}{ Toat Phase } & \multicolumn{14}{|c|}{ Foed } \\
\hline & & & $\begin{array}{l}\text { Cullot } \\
\text { (libs) }\end{array}$ & Sodit Ach & Roliner & $\begin{array}{l}\text { Trans } \\
\text { Batch (lbos) }\end{array}$ & $\begin{array}{l}\text { VECTRA } \\
\text { Clases (bos) }\end{array}$ & 要 & $8 \overline{8}$ & & $\overline{\frac{5}{4}}$ & Sinty & $\begin{array}{l}\text { V-sin } \\
\text { (lba) }\end{array}$ & $\mid \begin{array}{l}\text { Calchned } \\
(\mathrm{lba})\end{array}$ & $\begin{array}{l}\mathrm{Hilah} \mathrm{Na} \\
\text { (lba) }\end{array}$ & $\begin{array}{l}\text { Cum. Feod } \\
\text { (lbas) }\end{array}$ \\
\hline $4 / 750: 41$ & 271.7 & MTT-1, Volaes START & & & & & 70.0 & & & & & & & & & $37 \overline{26.0}$ \\
\hline $4 / 1952: 07$ & 273.1 & MT-1, V-ones & & 2.0 & & & & & & & & & & & & 3720.0 \\
\hline $4 \pi / 952: 13$ & 273.2 & $\overline{M T}-1, V_{-0}$ & & & & & & & & & & & & & & 37200 \\
\hline $4 \pi 1953: 10$ & $274 . \overline{2}$ & WT-1, Votanes & & 2.0 & & & & & & & & & & & & 3730.0 \\
\hline $471505: 00$ & 276.1 & DT-1, V-tans & & & & & $\mathbf{5 0 . 0}$ & & & & & & & & & 3700.0 \\
\hline $4 i 55800$ & 278.0 & MT-1, V-twen & & & & & 25.0 & & & & & & & & & 3605.0 \\
\hline 47159000 & 200.0 & $\overline{m T-1}, \mathrm{~V}-\overline{1080}$ & & & & & 25.0 & & & & & & & & & 3050.0 \\
\hline $4 / F 511: 00$ & 202.1 & DT-1, V-cous & & 2.0 & & & & & & & & & & & & 36.00 \\
\hline WTSO 12:48 & 203.8 & DT-1. Voters & & 2.0 & & & & & & & & & & & & 36340 \\
\hline $\operatorname{tros} 13: 47$ & 2040 & XT-1, Vtans & & 2.0 & & & & & & & & & & & & 3053.0 \\
\hline $4 / \sqrt{65} 15: 12$ & 20.7 & DT-1. V-omes & & 4.0 & & & & & & & & & & & & 30.40 .0 \\
\hline $475321: 04$ & $2 \div 2.1$ & DT-1, V-Doses & & & & & & & & & & & & & & 3.40 .0 \\
\hline $4 \pi 521: 15$ & 2322 & DT-1, V.tenes & & & & & 80.0 & & & & & & & & & 3920.0 \\
\hline $415021: 40$ & $2 \div 2.7$ & XT-1. V-tuses & & & & & 77.0 & & & & & & & & & 3897.0 \\
\hline $4 \pi 032: 30$ & $20,3.5$ & WT-1, V-cases & & & & & 250 & & & & & & & & & 4020 \\
\hline $20050: 30$ & 205 & Tा-1, V-atas & & & & & 77.0 & & & & & & & & & 4090.0 \\
\hline Novis 1:30 & 203 & Dr-1, volas & & & & & 78.0 & & & & & & & & & 41770 \\
\hline $40,32: 13$ & 207.2 & DT-1, V.Jas & & & & & 25.0 & & & & & & & & & 4202.0 \\
\hline $\cos 3.00$ & 200 & DTT-1, Voluss & & & & & 80.0 & & & & & & & & & 4202.0 \\
\hline $40.33: 50$ & 200 & mr-1, volases & & & & & 1760 & & & & & & & & & 4400 \\
\hline 2005500 & 300.0 & MT-1, Vetases & & & & & 40.0 & & & & & & & & & 4500.0 \\
\hline woros 5.45 & 3000 & DT-1, V-ing & & & & & 60.0 & & & & & & & & & 4560.0 \\
\hline $40508: 15$ & 303.2 & TIT-1, vols & & & & & 80.0 & & & & & & & & & $\$ 40.0$ \\
\hline $40 \% 50$ & 307.0 & DT-1, volas & & & & & 75.0 & & & & & & & & & 4715.0 \\
\hline $405514: 52$ & 300.0 & TT-1, Votass & & & & & 75.0 & & & & & & & & & 4750.0 \\
\hline 1/205 14:53 & 3000 & DT-1.Vedase & & & & & & & & & & & & & & 4750.0 \\
\hline WOSS 15:04 & 310.1 & DT-1. V-ans & & & & & 75.0 & & & & & & & & & 4055.0 \\
\hline 40501620 & 311.3 & CDT-1, Vocoses & & & & & 0.0 & & & & & & & & & 4945.0 \\
\hline $4 / 65$ & 312.5 & 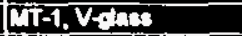 & & & & & 77.0 & & & & & & & & & 502.0 \\
\hline 4005 10.00 & 313.0 & (MT-1, V-ones & & & & & 77.0 & & & & & & & & & 5690.0 \\
\hline 4/655 10.47 & 313.0 & מেT-1, V-thes & & & & & 80.0 & & & & & & & & & 5170.0 \\
\hline 40052000 & 315.0 & TT-1, Volases & & & & & 770 & & & & & & & & & 5256.0 \\
\hline $402521: \infty$ & 316.0 & CTT-1, V-dases & & & & & 76.0 & & & & & & & & & 5332.0 \\
\hline WOOS 21:34 & 316.6 & CrT-1, V-ciase & & & & & 70.0 & & & & & & & & & S410.0 \\
\hline $16 \times 527$ & 317.4 & 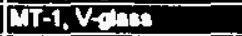 & & & & & 163.0 & & & & & & & & & 5583.0 \\
\hline Whos 1:00 & $3 \geq 0.0$ & DT-1, V-toes & & & & & 153.0 & & & & & & & & & 5724.0 \\
\hline क⿻川口s $1: 10$ & 320.2 & DT-1, V. & & & & & 30.0 & & & & & & & & & 5754.0 \\
\hline T205 2:40 & 321.7 & DTT-1, Vy,aus & & & & & 30.0 & & & & & & & & & 57.0 \\
\hline 4015 4:10 & 523.2 & (mT-1, V-uases & & & & & 157.0 & & & & & & & & & 5941.0 \\
\hline thes 5:10 & 324.2 & DT-1, V-thess & & & & & 156.0 & & & & & & & & & 609.0 \\
\hline $49 / 5514.20$ & 333.3 & CTT-1, Votass & & & & & & & & & & & & & & 60.97 .0 \\
\hline $400514: 22$ & 333.4 & ITT-1, Voclass & & & & & 70.0 & & & & & & & & & 6167.0 \\
\hline $4969515: 07$ & 334.1 & MT-1, V-dats & & & & & 70.0 & & & & & & & & & 52450 \\
\hline $4 / 96517: 00$ & 336.0 & MT-1, V-tats & & 2.0 & & & & & & & & & & & & 62470 \\
\hline $4 / 9255$ 19:40 & 336.7 & MT-1, V-tats & & & & & 153.0 & & & & & & & & & 6405.0 \\
\hline $4 / 9 / 9523: 30$ & 342.5 & MT-1, V-dacs & & & & & 155.0 & & & & & & & & & 60600 \\
\hline $4 / 10 / 052: 35$ & 345.6 & MT-1, V-tass & & & & & 155.0 & & & & & & & & & 6715.0 \\
\hline $4 / 10 / 955: 10$ & $348 . \overline{2}$ & MT-1, V-Jass & & & & & 75.0 & & & & & & & & & 6790.0 \\
\hline $4 / 10 / 958.21$ & 351.3 & MT-1, V-dane & & & & & 81.0 & & & & & & & & & 6.71 .0 \\
\hline
\end{tabular}




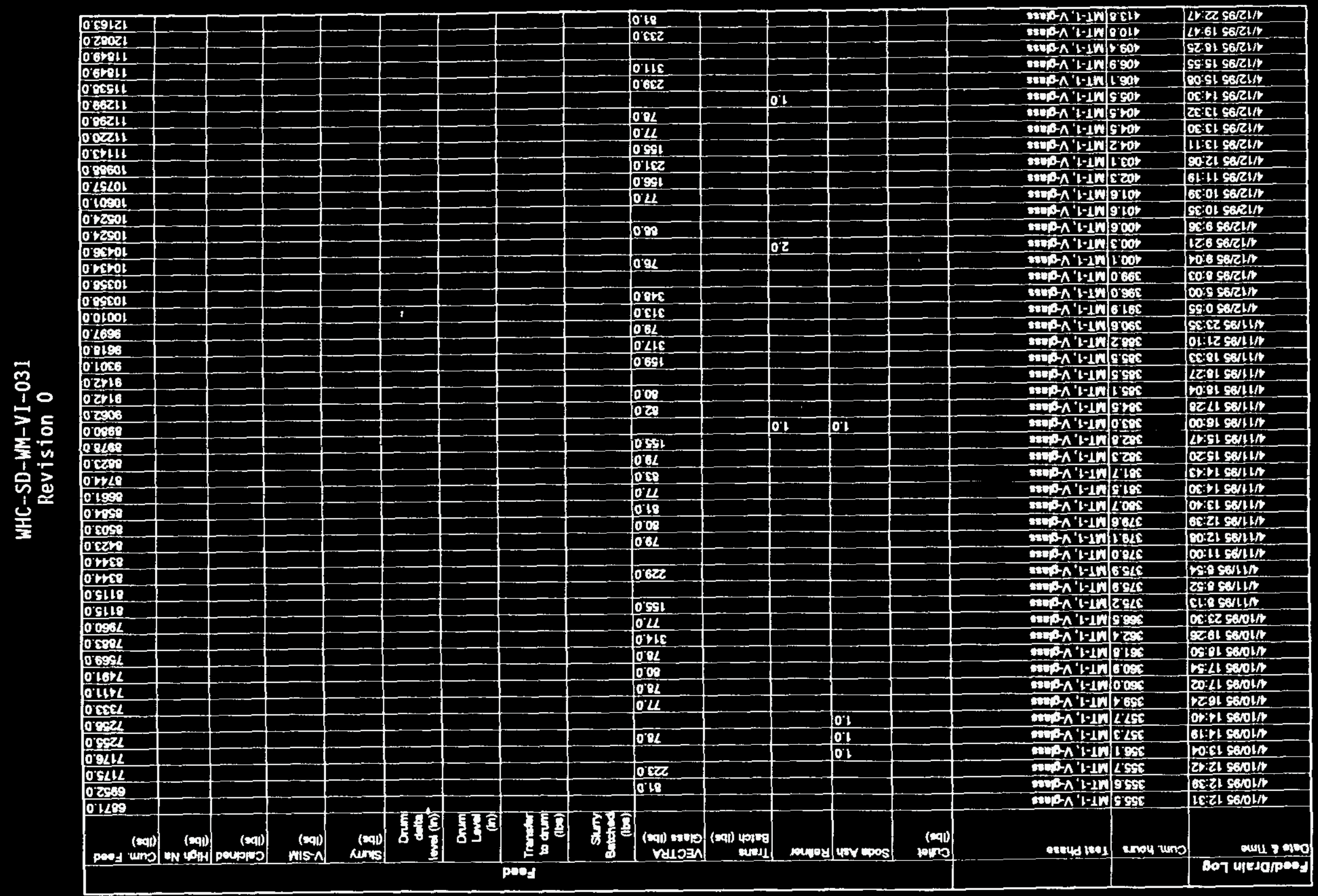




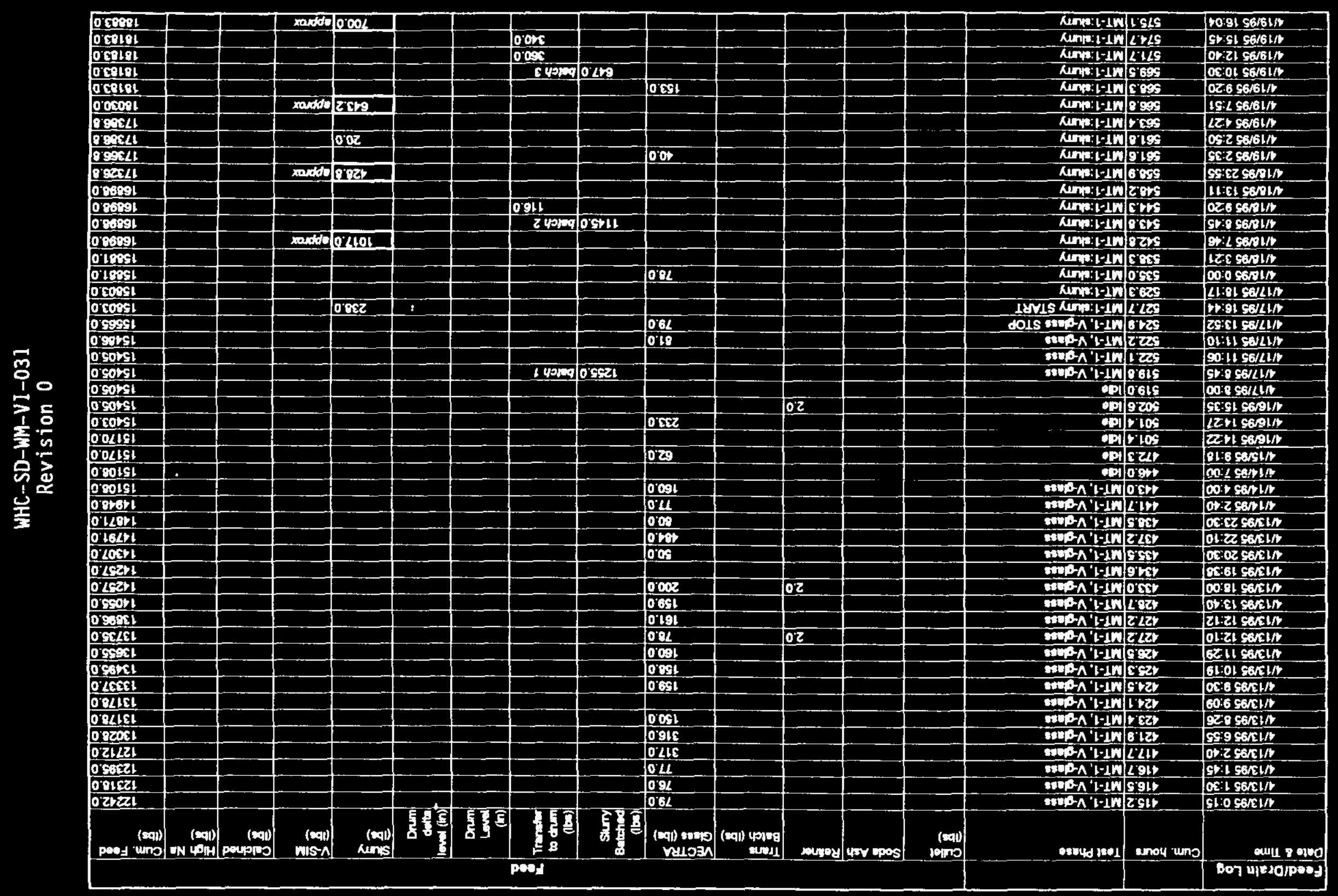




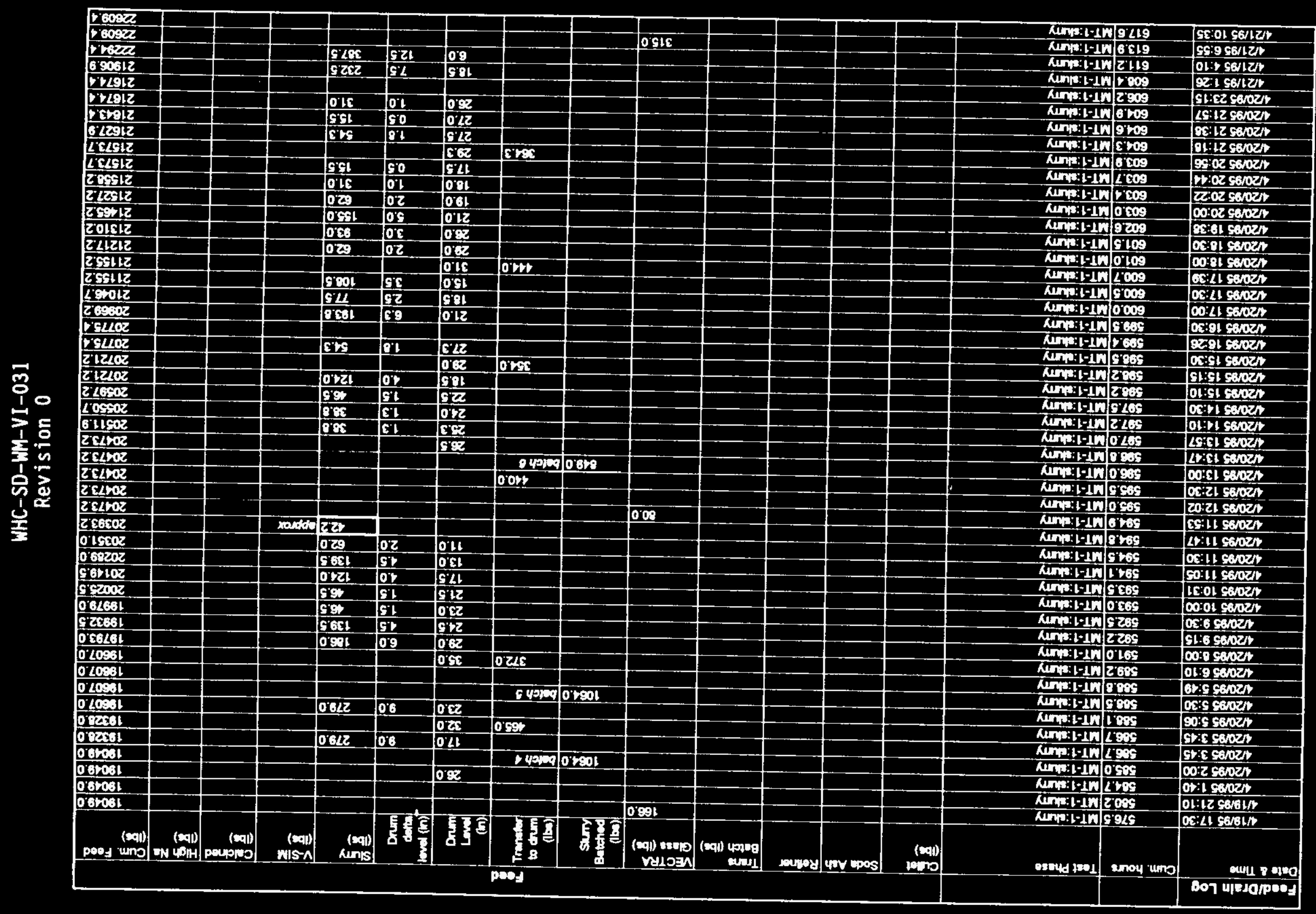




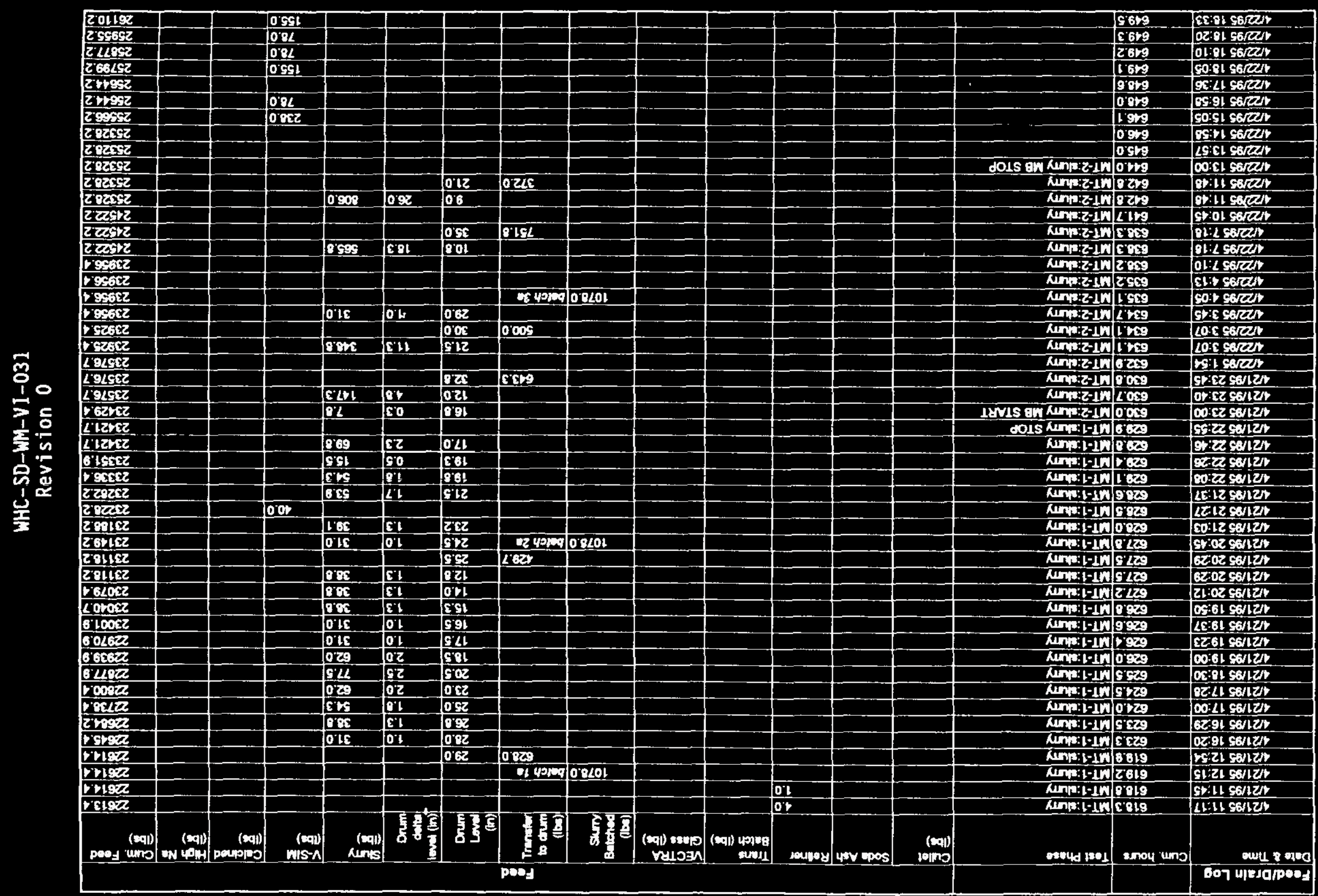




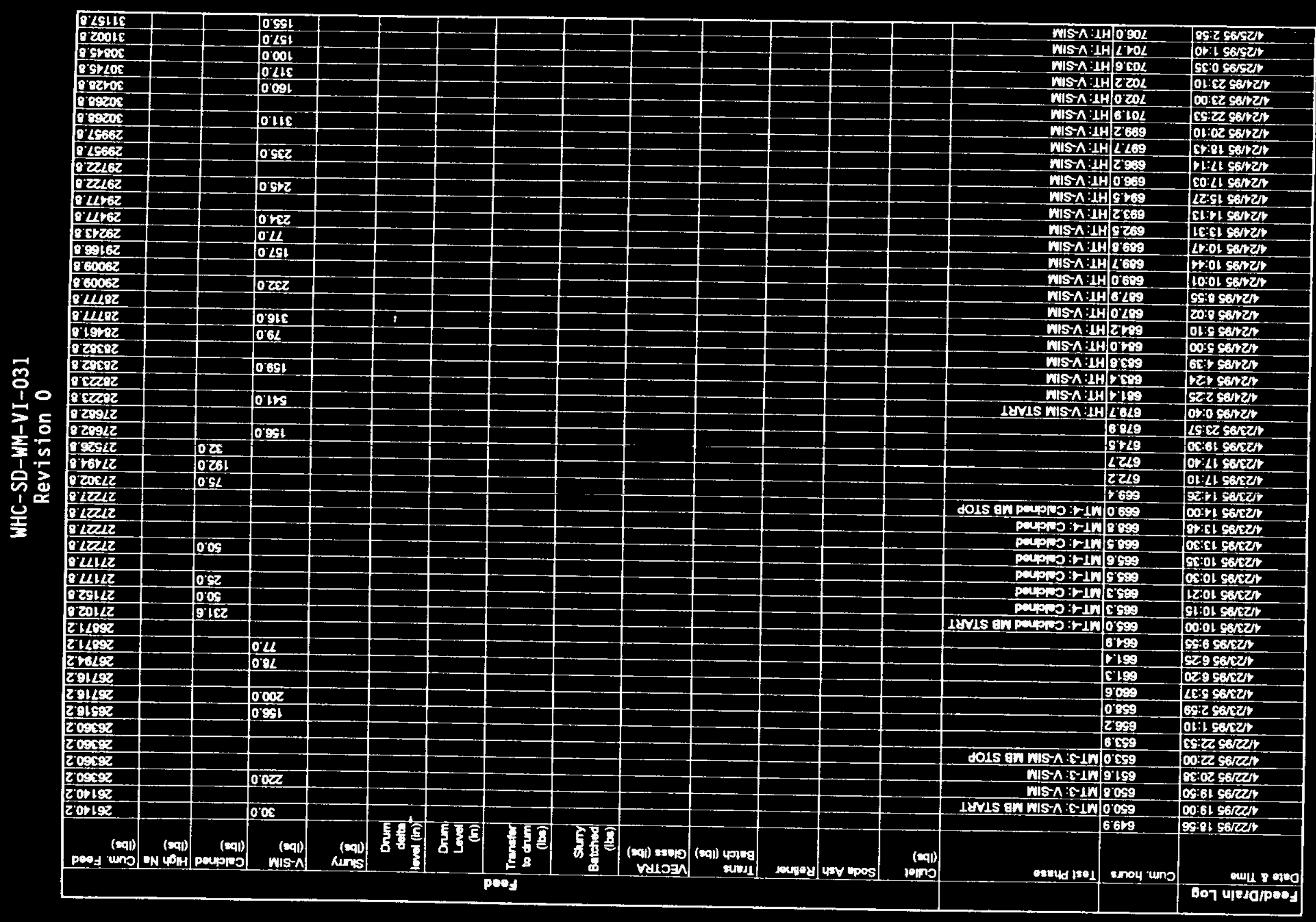




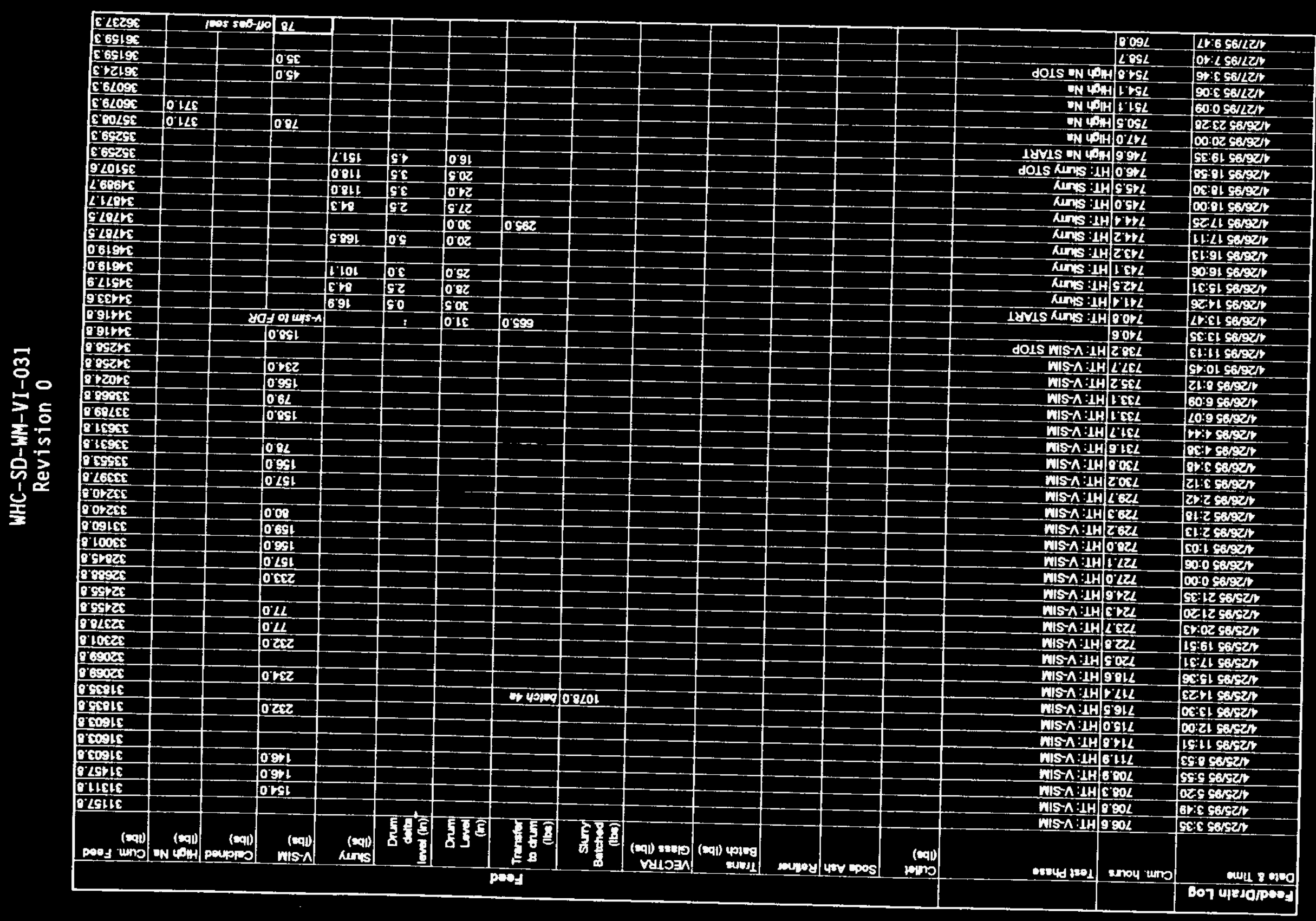


THROUOKPUT LOO

\begin{tabular}{|c|c|c|c|c|c|c|c|c|c|c|c|c|c|c|c|c|}
\hline \multirow{2}{*}{\begin{tabular}{|l} 
Foed/Draln Loo \\
Dote of Time
\end{tabular}} & \multirow[b]{2}{*}{ Cum. hows } & \multirow[b]{2}{*}{ Tout Phase } & \multicolumn{14}{|c|}{ Food } \\
\hline & & & $\begin{array}{l}\text { Cullat } \\
\text { (bios) }\end{array}$ & Sode Ash & Potiner & Batch (bas) & GECTRA & 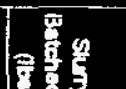 & 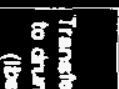 & $=5$ & 重。 & Situry & T.sim & $\begin{array}{l}\text { Calcinod } \\
\text { (ibs) }\end{array}$ & $(\operatorname{liligh} N a$ & $\operatorname{l}_{(b s)}^{\text {Cum Feed }}$ \\
\hline [ $42719517: 43$ & 76007 & & & . & & & 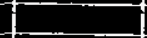 & & & & & & & & & 36237.3 \\
\hline Torals & 768.7 & & 3032.0 & $\mathbf{2 1 3 . 0}$ & 31.0 & 309.0 & 12711.0 & 10338.0 & 7530.0 & & & 9728.7 & .8700 .0 & 855.0 & 7420 & 36237.3 \\
\hline
\end{tabular}

Paga 9

WHCVIT05, XLS 
THROUOHPUT LOO

\begin{tabular}{|c|c|c|c|c|c|c|c|c|c|c|}
\hline \multirow{2}{*}{$\begin{array}{l}\text { Feed/Drain LoO } \\
\text { Dato \& Time }\end{array}$} & \multirow[b]{2}{*}{ Cum. hours } & \multirow[b]{2}{*}{ Teat Phase } & \multicolumn{2}{|c|}{ Oraphleal Data } & \multicolumn{4}{|c|}{ Drain } & \multicolumn{2}{|c|}{ Throughput } \\
\hline & & & $\begin{array}{l}\text { Tost Cum } \\
\text { Howrs }\end{array}$ & $\begin{array}{l}\text { Tont Cum } \\
\text { Feed }(k g)\end{array}$ & Drain & Drah (163) & $\begin{array}{l}\text { Avo orah } \\
\text { Rete } \\
\text { (beanin) }\end{array}$ & 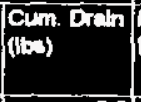 & Foed This & $\begin{array}{l}\text { Clones this } \\
\text { Perlod (lbstr) }\end{array}$ \\
\hline $3 / 26 / 9517: 00$ & 0.0 & Stantep & & & - & & & 0.0 & & - \\
\hline $3 / 20 / 9511: 41$ & 42.7 & Statep & & & & & & 0.0 & 0.6 & \\
\hline$\frac{3 / 20 / 95}{3 / 2: 05}$ & -129 & Sentep & & & & & & 0.0 & 115.4 & \\
\hline$\frac{3 / 2019512: 05}{3 / 31: 5614: 10}$ & 43.1 & Statup & & & & & & 0.0 & 272.7 & \\
\hline$\frac{3 / 31 / 95}{3 / 310: 10}$ & 117.2 & Starte & & & & & & 0.0 & 0.0 & \\
\hline$\frac{3 / 31 / 5016: 19}{3 / 31 / 5020: 30}$ & 118.3 & Stotep & & & & & & 0.0 & 2.3 & \\
\hline$\frac{3 / 316520: 30}{3 / 31 / 5521: 40}$ & 123.3 & Stitep & & & & & & 0.0 & 17.9 & \\
\hline$\frac{3 / 31 / 3521: 40}{3 / 31 / 452247}$ & 124.7 & strtep & & & & & & 0.0 & 64.3 & \\
\hline$\frac{3 / 31 / 9522: 47}{4 / 1950: 35}$ & 125.0 & Stantep & & & & & & 0.0 & 67.2 & \\
\hline $4 / 1950: 35$ & 127.6 & Start-10 & & & & & & 0.0 & 41.7 & \\
\hline $4 / 1 / 151: 30$ & 120.5 & Stetep & & & & & & 0.0 & 1309 & \\
\hline 41195400 & 131.0 & Statep & & & & & & 0.0 & 20.0 & \\
\hline $41 / 953: 20$ & 1323 & Sintup & & & & & & 0.0 & 75.0 & \\
\hline $410511: 12$ & 133.2 & $\sin -4 p$ & & & 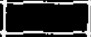 & & & 0.0 & 0.2 & \\
\hline 411051124 & 130.4 & Statep & & & & & & 0.0 & 5.0 & \\
\hline 11\%5 12:00 & 139.0 & Stantup & & & 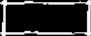 & & & 0.0 & 6.7 & \\
\hline $4 / 1 / 5513: 50$ & 141.0 & sintelp & & & & & & 0.0 & 50.8 & \\
\hline $41 / 051600$ & 143.0 & sintep & & & + & & & 0.0 & 73.8 & \\
\hline $410510: 30$ & 145.3 & sentep & & & & & & 0.0 & 60.0 & \\
\hline $421900: 07$ & 151.1 & Sintep & & & 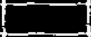 & & & 8.0 & $\overline{0.4}$ & \\
\hline 123538000 & 160.0 & stintep & & & & & & 0.0 & $0 . \overline{2}$ & \\
\hline $4 / 25316: 47$ & 167.6 & Sting & & & & & & 0.0 & 3.2 & \\
\hline 42851720 & 160.3 & Etatup & & & 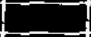 & & & 0.0 & 36.4 & \\
\hline 625518.20 & 169.3 & Statep & & & . & - & & 0.0 & 25.0 & \\
\hline $4 / 2051925$ & 170.4 & Sartep & & & & & & 0.0 & 23.1 & \\
\hline $12 \operatorname{ses} 21: 15$ & 172.2 & Stortep & & & & 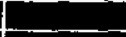 & & 0.0 & 13.6 & \\
\hline 1229522005 & 173.1 & Sortep & & & & & & 0.0 & 1.2 & \\
\hline $424322: 35$ & 173.6 & Sintep & & & & & & 0.0 & 8.0 & \\
\hline $431550: 35$ & 175.6 & Stritep & & & & & & 0.0 & 1.0 & \\
\hline Nass 2:10 & 177.2 & Stritup & & & & & & 0.0 & 15.0 & \\
\hline 43056.45 & 101.7 & stite? & & & & & & 0.0 & 5.5 & $i$ \\
\hline 4355730 & 102.5 & Strite & & & . & & & 0.0 & 2.7 & \\
\hline $4 / 3190920$ & 10.3 & Stitep & & & & & & 0.0 & 1.1 & \\
\hline$\sqrt{3} / 50 \times 35$ & 16.6.6 & stitep & & & & & & 0.0 & 12.0 & \\
\hline $\operatorname{coses} 9.45$ & 10.7.7 & Stap & & & & & & 0.0 & 12.0 & \\
\hline 1310510.37 & 105.6 & Sten & & & & & & 0.0 & 2.3 & \\
\hline $4 / 3 T=511: 19$ & 10.3 & Sotep & & & - & & & 0.0 & 1.4 & \\
\hline $4 / 25512: 13$ & 107.2 & Stentip & & & & & & 0.0 & 1.1 & \\
\hline $1 / 39512: 00$ & 167.7 & Stantep & & & 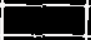 & & & 0.0 & $35 . \overline{6}$ & \\
\hline $43 \times 31420$ & 169.3 & $\sin t-4$ & & & $\because$ & & & 0.0 & 15.0 & \\
\hline 4/305 10:17 & 193.3 & $\sin -\bar{p}$ & & & 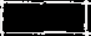 & & & 0.0 & 6.3 & \\
\hline $4 / 310021: 15$ & 193.2 & Sant th & & & & & & 0.0 & 8.4 & \\
\hline $4 / 3 / 952210$ & 197.3 & Surteup & & & 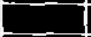 & & & 0.0 & 25.0 & \\
\hline $4315523: 15$ & 198.2 & Sint-up & & & & & & 0.0 & 25.0 & \\
\hline 4/495 0.35 & 199.6 & statipe & & & & & & 0.0 & 18.8 & \\
\hline 4/495 1:15 & 200.3 & Starteup & & & & & & 0.0 & 37.5 & \\
\hline $4 / 4952: 15$ & 201.2 & $\operatorname{sentup}$ & & & & & & 0.0 & 25.0 & \\
\hline $4 / 495844$ & 207.7 & Start-up & & & & & & 0.0 & 0.2 & \\
\hline A/4 9510.15 & 217.2 & Stantep & & & & & & 0.0 & 0.1 & \\
\hline
\end{tabular}




\begin{tabular}{|c|c|c|c|c|c|c|c|c|c|c|}
\hline & $\frac{\sqrt{692}}{0.62}$ & $\frac{0.0 \cos 2}{0.0028}$ & & & & Eszer & 262 & 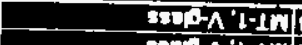 & E.tSE & LZ: S S6/OL \\
\hline & Eos & $\frac{0.002}{0.0025}$ & & & & $\frac{96951}{95081}$ & $\frac{592}{68 L}$ & 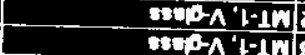 & zok & Ol:S s601/4 \\
\hline & $\frac{1205}{E 6 g}$ & $\frac{0.0092}{10002 z}$ & & & & $\cos 2$ & 002 & $\operatorname{sen} 2-1+16$ & 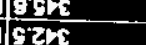 & 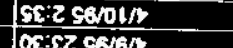 \\
\hline & 12 & 00 & & & & 0 oflzl & 029 & $=\sin 3-1-10$ & 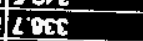 & $\frac{12.25}{06.6161612}$ \\
\hline & $0+06$ & 0000 & & & - & Eegh & $\mathrm{Crg}$ & $\operatorname{sen}-\Lambda 1+100$ & 0 & $00: 45616 \pi$ \\
\hline & 92 & 00022 & & & & $\frac{5211}{0.011}$ & 5 & $n+a-1+10$ & LEE & Lo:Fi sas/s \\
\hline bit & & 00002 & 89 & 0.200 & 6 & & 2.19 & $200-\Lambda^{2}-14$ & rese & Cetrl serot \\
\hline & $0 \% 9$ & Defle & & & & Equot & sisa & $\cos 2 \Lambda^{\prime} 1-10$ & ecese & $0 z+p 1$ sest \\
\hline & LNOL & 0 octe & & & & 5rool & S18 & 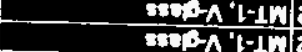 & 202 & ToL:S SESt \\
\hline & 000 & 0 oxt2 & & & 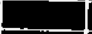 & Ecso & 000 & 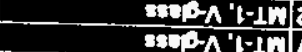 & zece & of: $\cos 6$ \\
\hline & 0002 & 0 opriz & & & & $\angle O 16$ & Gat & 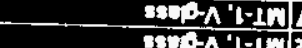 & $\angle 122$ & 101:2 s6/ $6 / \%$ \\
\hline & 029 & 0.8212 & & & & [leos & cot & $m-n^{\prime} 1-10$ & 2028 & ol:1 cast \\
\hline & g9o/ & oepl2 & & & & Simen & & & & \\
\hline & $g<\varepsilon 1$ & 0012 & & & & Legs & & & & \\
\hline & $0 \%$ & 0 atz & & & & E.02 & & & & \\
\hline & Eहg & $08+12$ & & & & & Evt & $\operatorname{sen} 2 \Lambda^{\prime}-1 \omega$ & Dots & $00: 12 \operatorname{sen} \theta$ \\
\hline 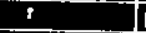 & 1201 & $0 \mathrm{cplz}$ & & & & & EE & $200-\Lambda^{\prime} l-1 \omega b$ & OGIE & Do:0z sarol \\
\hline & otsi & gentz & & & & $\frac{0659}{1208}$ & Liz & $\operatorname{sen} \sin ^{\prime} l-10$ & QRE & 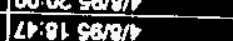 \\
\hline & 09 & gatz & & & & $\frac{203}{0.03}$ & EL & $\min -n^{\prime} l-10$ & Qcis & 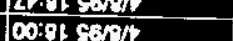 \\
\hline & 289 & Detro & & & & $\alpha<s$ & oos & $5019-\Lambda 1-10$ & gzzis & 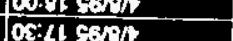 \\
\hline & oqces & ocetiz & & & & $a^{2} \cos ^{2}$ & $\angle \theta$ & $\operatorname{sen}-\Lambda \cdot 10$ & E.LE & 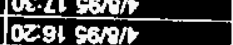 \\
\hline 6iv & & 0 ostz & 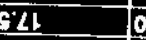 & 0.200 & $E^{2}+2$ & gois & pos & $0.5-1,1-1$ & LOLE & thosisest \\
\hline & 28 & orotst & & & & & & $\operatorname{mon} 1-1 n$ & 6002 & $\sin \operatorname{sen} x$ \\
\hline & 0002 & oorst & & & & 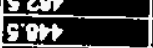 & $\frac{20 x}{\varepsilon c s}$ & 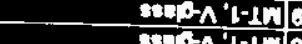 & Geos & Cs: $\lambda \mathrm{sen} /$ \\
\hline & are & ockst & & & & Sout & $\frac{2}{918}$ & $\left.-2-n^{\prime} l-1\right]$ & $0.20 x$ & oo:zl $\sec n$ \\
\hline & 000 & 00 at & & & & & & $\operatorname{man}-1-102$ & 280 & Siloger \\
\hline & Ex & onat & & & & & & & & \\
\hline & gelz & $006 \mathrm{k}$ & & & & & EQ?z & $=n^{0}-1=0$ & 000 & $\frac{1000}{1005} 560 \%$ \\
\hline & $\angle 801$ & oonel & & & & $62 x$ & 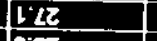 & $\operatorname{sen} \Lambda^{\circ}-1 n$ & 000 & $\frac{0.550 \%}{0.56 / t}$ \\
\hline & EEE & oons & & & & 239 & $2 g$ & 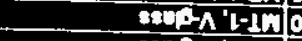 & 0002 & 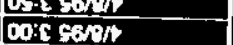 \\
\hline & $0 \%$ & 00 ari & & & & $\frac{6 \cos 2}{\cos 2}$ & $90 z$ & $\cos \theta-1-102$ & Z2LZ & $\frac{10 x \cosh }{\sin 2 \cosh }$ \\
\hline & GKE & orokel & & $-1+2>$ & & $\frac{5 x+2}{7 x y}$ & arz & $\sin n^{\prime}+1-10$ & 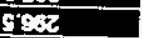 & 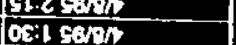 \\
\hline E & 00 & $000 \mathrm{~s}$ & & & & 2609 & 02 & $\operatorname{sen} 3-\Lambda^{\prime} 1-10$ & Gesz & $\frac{\cos 10 \sin s}{\cos 0 \cos \theta}$ \\
\hline & ani & 00061 & - & & & inis & 812 & $=2 \cdot \Lambda^{\circ}-10$ & 6862 & 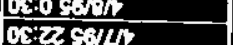 \\
\hline & $n$ & $000=1$ & & & & $\frac{6 z a_{1}}{10}$ & $a x z$ & $\left.=2-\lambda^{\prime} l-1\right) L$ & $\angle 262$ & 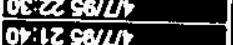 \\
\hline Ger & & oovel & 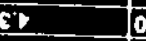 & 0.200 & $z$ & $\frac{000}{20}$ & got & $\operatorname{sen}+1 \cdot 1=2$ & zzzaz & 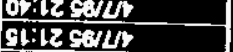 \\
\hline & 12 & Ocis & & & & $\frac{2.15}{2.15}$ & 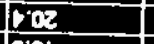 & 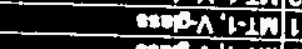 & 1262 & Lo:12seg/L \\
\hline & 12 & 0 . & & & & $\frac{615}{66 t}$ & $\frac{0.91}{1.81}$ & $\operatorname{mon}(-102$ & $\operatorname{Lg} 2$ & 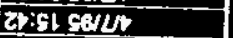 \\
\hline & 21 & oges & & & & 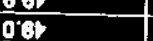 & & and 1010 & onz & 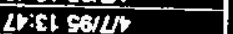 \\
\hline & 80 & Oels & & & & tot & $\frac{1.21}{601}$ & $n^{\prime} 1-100$ & $\theta \operatorname{sez}$ & $65: 215824$ \\
\hline & $0 \underline{G}$ & $0 \mathrm{els}$ & & & & 业 & SOLL & $20-n^{\prime}-10$ & 1202 & co:ll captu \\
\hline & 60 & OEIS & & & & 24 & 80 & $\cos ^{\prime} 1-100$ & 0002 & $00.6 \mathrm{ge} / \mathrm{ht}$ \\
\hline & $\mathrm{FEz}$ & tocks & & & & $8 x$ & 82 & $2 x-1-1-100$ & 0622 & $00: 86,21$ \\
\hline & 6i & o. & & & & $\frac{5 z}{2 z}$ & it & $m-n^{\prime}-I D L$ & 1922 & co:s cestst \\
\hline 61 & & OEL & 92 & 08 & & $\theta$ & $q z$ & $\operatorname{sen} 1-1-2$ & 202 & 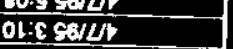 \\
\hline & Bt & 00 & & & & 60 & & Solo-A1-1nz & $Z C L Z$ & El:z $5 B / L t$ \\
\hline A & E't & 0.0 & & & & 00 & $\frac{11}{00}$ & $=\operatorname{mon} 1-10$ & IELZ & $\angle 0: 256 / 24$ \\
\hline & & & (4) & & & & & 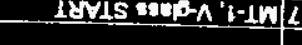 & $\leq 122$ & $1+0$ s6/Lt \\
\hline 4 & 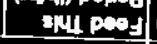 & 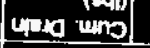 & 40000y & 9a) 400 & und & $\begin{array}{l}\text { (ox) poos: } \\
\text { who youl }\end{array}$ & ung reor & ognud jese & sno yum & \\
\hline indyet & & & 마렁 & & & ADO 1001 & गमdन्डo & & & 607 uleuaposes \\
\hline
\end{tabular}




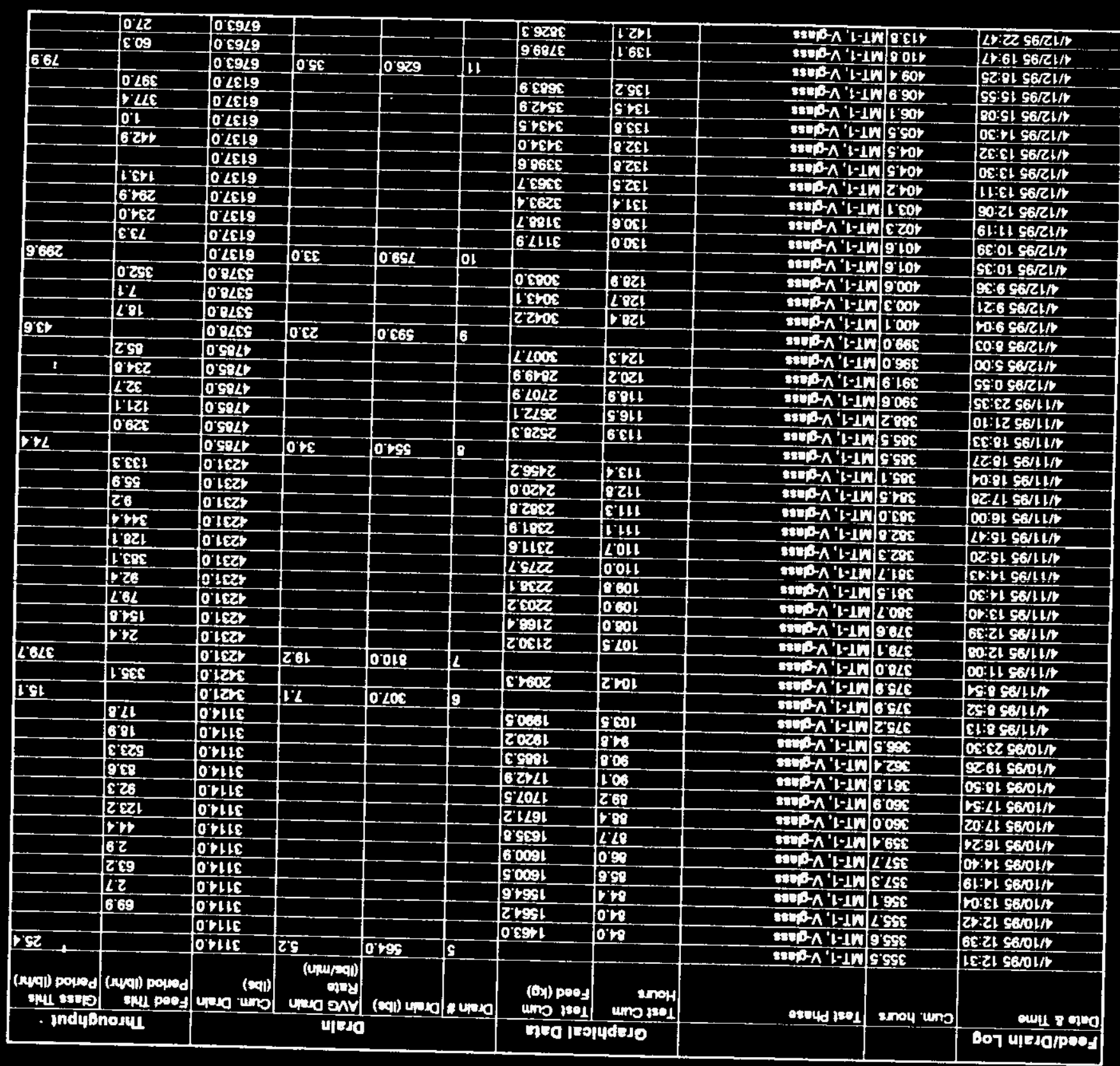




\begin{tabular}{|c|c|c|c|c|c|c|c|c|c|c|}
\hline & 0.01 & 0 gile6 & & & & 89651 & 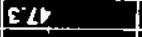 & $\operatorname{lan}_{1-1}$ & $15<s$ & $1009196 / 61 / \mathrm{t}$ \\
\hline & & 0 & & & & & & Aump:-1-1n & 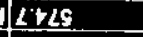 & stisl $96 / 6 \mathrm{~L} / \mathrm{t}$ \\
\hline & & $\frac{0.9168}{0.968}$ & & & & & & $\cos 1-10 \mid$ & $\angle 1 \angle 9$ & $0 \mathrm{t}: 21 \mathrm{56/61/t}$ \\
\hline & EOL & $\frac{10963}{0.9163}$ & & & & & & Anp: $1-\operatorname{In} \mid \mathrm{s}$ & 969 & os:ol se/6t/1 \\
\hline & $\frac{10.16}{2: 021}$ & & & & & 26201 & gop & Alm:-1WE & 509 & $02: 856 / 61 /$ \\
\hline$\frac{65 t}{6501}$ & 2021 & $\frac{0.968}{0.6618}$ & 28 & $0 \% 91$ & 12. & 000102 & Les & RIn: & 092 & 19:L S618L/L \\
\hline geol & & 06918 & 00 & $0 . \angle 61$ & $0 z$ & & & Aense: $1-1$ end & 689 & LZt' G6/6t/t \\
\hline & $\frac{0.09}{0.91}$ & $\frac{0 \cos s}{0.060}$ & & & & coll & IKE & Aunp:-1-100 & 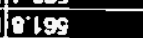 & $00.256 / 61 / 2$ \\
\hline$\theta \bar{z}$ & $\frac{0 \mathrm{gl}}{90 ?}$ & $0 \operatorname{zecs} 3$ & 0 & $0 \leq 28$ & 61. & $260 L$ & CIEE & kens:1-1ws & 918 & 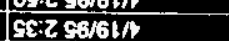 \\
\hline & $9 \%$ & $0<2086$ & & & & 1.169 & Z1E & Aun:L-Iw 6 & $6 \mathrm{cos}$ & Ss:ez s6\%L/t \\
\hline 201 & & $0<\angle 828$ & $\angle \varepsilon$ & avol & $\mathfrak{a l}$ & & & Ans:l-1w2 & 20 & HLEL SGML \\
\hline & & $\frac{0.8916}{0.6918}$ & & & & & & Rump:1-10: & ens & $0 z 6 \mathrm{sen} / \mathrm{h}$ \\
\hline & & $\frac{08916}{0.0916}$ & & & & & & Amp:1-1:1] & egrs & st:0 gonth \\
\hline & 6008 & 008916 & & & & 9005. & osi. & 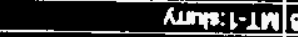 & azrs & 9t: $1 \mathrm{~s} 6 \mathrm{NL} / \mathrm{h}$ \\
\hline 95 & & 00926 & bs & 0009 & $\underline{L}$ & & & 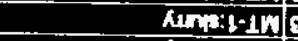 & enss & $12.8 \mathrm{sghelh}$ \\
\hline द्या & 201 & $\frac{0.659}{0.6590}$ & 0.22 & 000 & & 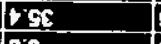 & $E 2$ & 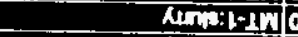 & 0.589 & $00: 05600$ it \\
\hline & 009 & $\frac{1}{0.6959}$ & & 0008 & 92 & 00 & 51 & Aun:1-10 & E.62s & LI:Ol SGLLL \\
\hline & 662 & 060959 & & & & 00 & 00 & 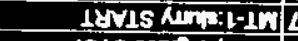 & LLES & Erigl se/Llth \\
\hline & ir & 0609 & & & & & & 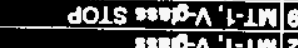 & GBSG & $250156124 / 1$ \\
\hline 62 & & 0605 & $\overline{O E}$ & $0 \mathrm{zOL}$ & 童 & & & 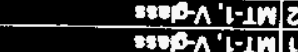 & Ezas & $01: 1 / 56 / 21 / 2$ \\
\hline & & 0.20 & & & & & & 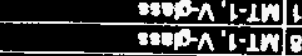 & toss & $50: 1 / 56 / 24 / 4$. \\
\hline & & Q 290 & & & & Lket & $O E L$ & 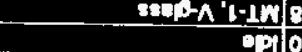 & Bels & 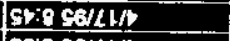 \\
\hline & 01 & 020 & & & & LtEL & 89 & openc & 0618 & 000 SGLLL \\
\hline & 00 & 0 LSO & & & & Beql & 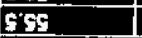 & 90 & gzos & 58:G1 56/91/ \\
\hline Et & & 020 & I & 000 & b & & & optic & ilog & $12+1,9601 / 4$ \\
\hline & $1+2$ & $0<2910$ & & & & [og & $E \%$ & opite & $\frac{1004}{2 z 2 t}$ & $2 \mathrm{ar}$ ! cagih \\
\hline & & 0.2918 & & & & 00 & 00 & opio & $\frac{1201}{0.04 t}$ & 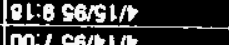 \\
\hline & Dozi & 02010 & & & & 6.019 & E.KL & 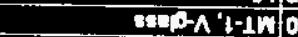 & $00 \mathrm{ct}$ & 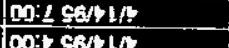 \\
\hline & 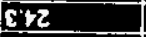 & 02960 & & & & 66009 & 0.024 & $\sec 2-n \cdot t-10$ & Lith & 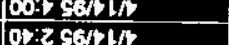 \\
\hline & 0.09 & $0<240$ & & & & reas & 0991 & $9=0-\lambda^{\prime} l-10$ & gost & 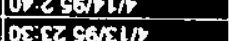 \\
\hline & 6062 & OLOLO & & & & 1005 & $\mathrm{gcsl}$ & $20-\lambda^{\prime} l-1 \omega$ & $2 \angle E t$ & 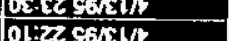 \\
\hline & 002 & 0.2918 & & & & $900<2$ & $0 \mathrm{cos}$ & $50-n^{\prime} l-1$ & 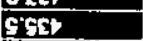 & ol.ec soluth \\
\hline Eas & & 0 2018 & ooㅏ & dots & Et & & & 8x-1-n & grtst & ootoc sonis \\
\hline & 995 & 0.0192 & & & & oqut & E. & 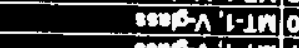 & 0 ost & $\cos \theta t \sec 1 / t$ \\
\hline & ragl & 06192 & & & & nest & 049 & $\sin \wedge \mathrm{L}-10 \mathrm{~L}$ & 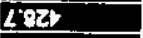 & Oorcl sckint \\
\hline & C:95: & 0619 & & & & Ects & 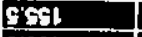 & $\operatorname{mon} 1-10]$ & ELZZ & ZLIZt SGSL $/ 1$ \\
\hline & & 0062 & & & & 26054 & S요 & $\operatorname{sen}-n^{\prime} 1-102$ & 2128 & OL:zl serth \\
\hline & BLE & 0619 & & & & 6zost & pris & $\operatorname{sen} 1-10 \mathrm{~s}$ & 6:37 & EZII sactis \\
\hline & Sig6! & 00.19 & & & & bosent & 8891 & $\operatorname{sen} 11-10$ & cs33 & $61: 01$ sedit \\
\hline & L61 & $0019 L$ & & & & 2005 & 8잔. & 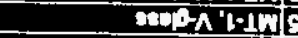 & Ginat & $00: 8 \sec 14$ \\
\hline 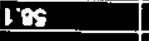 & & 06192 & 89 & 0009 & 21 & & & 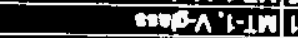 & $1+2 t$ & $60 \times 6 \mathrm{cosin}$ \\
\hline & 680 & 0.8929 & & & & genzp & pisi & $m\left[n^{\prime} 1-1 w\right.$ & tezt & $800951 / 2$ \\
\hline & $B 2$ & 0.8929 & & & & 90126 & 20,1 & 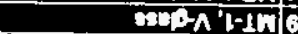 & 6.125 & sogestots \\
\hline & pgre & 0.0049 & & & & colot & $0 \%$ & $\theta+\Lambda 1-1 \mathrm{~N} L$ & LLL & ot:2 $26 \mathrm{sen}$ \\
\hline & 0000 & 0.8019 & & & & GLEOE & is & $m \Lambda^{\prime} n-1, L$ & LGL & st:1 casint \\
\hline & $\frac{809}{689}$ & $\frac{0.829}{0.0919}$ & & & & 9965 & $\frac{0+1}{001}$ & $\operatorname{sen} 2-1+10$ & S9tr & OQ: 1 SGKLW \\
\hline & & |lox $x \leq 469$ & (LTwes) & & & tises & $98 \mathrm{~g}$ & 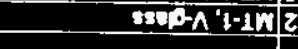 & Esth & Gl:o seselot \\
\hline 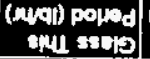 & {$\left[\begin{array}{c}\text { (n/qul) poped } \\
\text { sul peos }\end{array}\right.$} & unso (ans) & upany & (cail) uppol: & areal & (Dxp peos & sinot| & 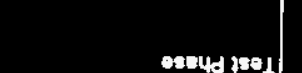 & - mu-um & \\
\hline ind & ก०गया & & पIFId & & & 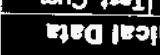 & 44d010 & 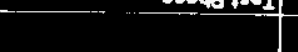 & 20040 & 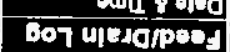 \\
\hline
\end{tabular}




\begin{tabular}{|c|c|c|c|c|c|c|c|c|c|c|}
\hline Q & & 0 gigh & ss & Dege & $L Z$ & & & Anp:l-1w & $19<19$ & SE:01 SG/LZt \\
\hline & $\frac{5011}{8+21}$ & $\frac{0 z z 211}{0.2 z z 11}$ & & & & ospos & 209 & 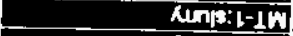 & 6019 & sg:9 s6/L2/ \\
\hline & $\frac{\log 25}{2 \cdot 3}$ & $\frac{0.28211}{0.28211}$ & & & & $0+6 z$ & $r \varepsilon$ & Alons: $1-1 w$ & 2119 & 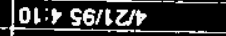 \\
\hline & $\angle \mathrm{Qg}$ & $\frac{0.25211}{0.20211}$ & & & & 20922 & $\angle 00$ & Auns: $1-10$ & 10009 & 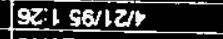 \\
\hline 6.28 & 1626 & $\frac{0.25216}{0.10011}$ & Q.5 & $0.18 z$ & 92 & & & Run: $1-1$ N & 2009 & S1:82 56/021- \\
\hline & sos & $\frac{0.10041}{0.10011}$ & & & & $0 \cos 2$ & 24 & Anto:10 & 6700 & LS:12 96021 \\
\hline & & $\frac{0.10011}{0.10011}$ & & & & $\angle 8+2$ & 692 & Alnp:L-10 & 900 & $0 \times 1256021$ \\
\hline & & $\frac{0.1001 !}{0.10015}$ & & & & $\angle 1+2$ & 892 & Antiviln & eros & ol:12 s6rozit \\
\hline & & 0.10014 & & & & & & Mns: $1-10$ & 6809 & SS:02 5602020 \\
\hline & & 0.10014 & & & & FLIS & $0 \%$ & Amp: 1 -10 & $\angle 809$ & to: 5602 \\
\hline & $\frac{570}{1691}$ & 0.10021 & & & & 0018 & 902 & Auns:t-10 & $\sqrt{6809}$ & 20202561020 \\
\hline & $\frac{1691}{8991}$ & 0.10011 & & & & 089022 & sol & Aint: $1-10$ & Jogos & $00: 025602010$ \\
\hline & $\log 1$ & o'sons & & & & 62.002 & 62 & Ann:L-10 & 9200 & 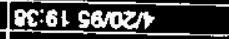 \\
\hline & 0001 & 0.10011 & & & & $9<62 z$ & $0 \Omega 2$ & 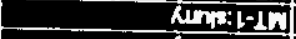 & 5.109 & 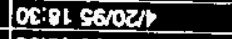 \\
\hline & 0.2द1 & 0.2001 & & & & peser & $\varepsilon \varepsilon 2$ & Anra: $1-10$ & 0.109 & $0001 \mathrm{sen} 0 \mathrm{~s}$ \\
\hline & & olooll & & & & & & Antip:-10 & $\angle 000$ & $68: 456024$ \\
\hline & $0<2$ & 0.10021 & & & & E.Lar2 & 021 & Anpa:l-10 & 5000 & $100: \angle 1,56021$ \\
\hline & 0.091 & 0.10015 & & & & $10 \angle 82$ & C.2L & Anip:i-1) & 10000 & $00: 42 \operatorname{sen} 2 \pi$ \\
\hline$\div$ & p861 & 0.10011 & & & & berez & 0.12 & Ant:1-10 & 5669 & 06:9l s6/02 b \\
\hline En & & 0.10041 & 601 & $0,6 \varepsilon 2$ & $g z$ & & & Anns:l-10 & 1869 & 1291 s6024 \\
\hline & $\leq 2$ & ozayos & & & & $1.50 x$ & 002 & Alas:Lin & 5865 & 08: 이 560211 \\
\hline & & ozzerol & & & & & & Ann:L-10 & 2005 & St is se/021 \\
\hline & 0001 & araxol & & & & Fose & DOL & Antil-10 & 2005 & los:si sepert \\
\hline & 5681 & $072 \%$ & & & & $2 \times<12$ & 089 & Anp:L-1w & E2Ls & $05+16602 \pi$ \\
\hline & $0 \% 2<1$ & 020201 & & & & Logle & t69 & AnploL & 2465 & ol:bl sanzes \\
\hline & Let & 020201 & & & & 95012 & 269 & An:Lin & 0205 & $\angle G \Omega 1$ csond \\
\hline & & 0.29201 & & & & & & An:2:10 & Deas & LPCI senz" \\
\hline & & $02 z<01$ & & & & & & Alris:-10 & 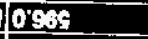 & $00: 81$ sarat \\
\hline & & o'zelol & & & & & & $\left.x_{1+1}: 1-1\right)$ & 6ogs & os: 21 sanzest \\
\hline act & & 102901 & sit & Qto & $\underline{z}$ & & & Anti-1) & 0005 & $20: 21$ s61020 \\
\hline & 00000 & otosol & & & & poukz & 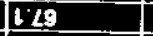 & Auns:l-10 & ares & $00: 11 \cos 020$ \\
\hline & osent & o.toest & & & & $\angle \log 2$ & $0<9$ & Antivitin & oros & Lt:Al senzot \\
\hline & post & 0 losol & & & & 9202 & ges & Ans:1-1 & S.6s & loxil s6/020 \\
\hline & $2 \operatorname{sos} 2$ & 0.0001 & & & & Shooz & $\mathrm{cos}$ & Anlp:L-10 & $1+6 s$ & E0:11 S6,021 \\
\hline & 0002 & 0 logat & & & & $2<<6$ & 698 & $\operatorname{Ren}: 1-1)$ & 7869 & 15:ol sesozth \\
\hline & 008 & oloen & & & & 0 cstol & cos & Aunsilin & 0869 & Do:0l sejors \\
\hline & 0.81 & otrent & & & & 6899 & pos & Mns:L & 0268 & $00: 696021$ \\
\hline & s.t1 & ologol & & & & $0<2<1$ & Sto & Ann:L-1n & 2265 & S1:6 canozts \\
\hline & tik & o.toges & & & & seopl & 509 & Antil-1M & 0,165 & $00: 0 \cos 210$ \\
\hline & & 0 tonol & & & & & & Axp: & $26 \%$ & $001: 9$ seopes \\
\hline $85 s$ & & OLloeol & $0 \pi$ & 0200 & $\underline{z Z}$ & & & Ann: & $\theta \cos$ & Gr:s s602 \\
\hline & & $06<101$ & & & & & & Anp:l-1w & $\operatorname{sen}$ & $00: 5 \sin 2 \pi$ \\
\hline & $\angle 902$ & 064101 & & & & zsal & 609 & Ans: 1.10 & 100 & $00: 556 \sqrt{211}$ \\
\hline & & 062101 & & & & & & Anp:-1-1 & $\angle \operatorname{ses}$ & 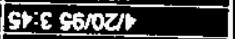 \\
\hline & 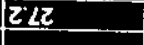 & 064101 & & & & 9664 & 069 & $\operatorname{Min}=1-1 \omega$ & $2 \operatorname{ses}$ & Stic seopest \\
\hline & & $06<101$ & & & & & & Anrip:1-10 & 0.505 & 002560021 \\
\hline & & $06<<101$ & & & & & & Antip:1-10 & Lhes & 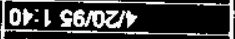 \\
\hline D.61 & & $06<101$. & 0.21 & 0 rez & $\bar{c}$ & & & Auns:-1:14 & $2 \cos 5$ & OL: $1256 / 610$ \\
\hline & osil & ocises & & & & $t z<1$ & 8 & Anp: $1-10$ & sols & $08: \angle 1$ SG/6th \\
\hline 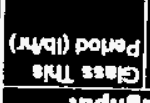 & 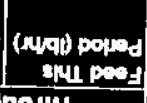 & 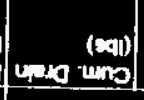 & 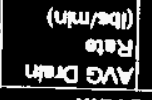 & (ai1) 느므미 & | & 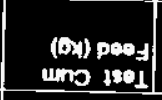 & unp jeos & $0504 \mathrm{t} 100 \mathrm{~s}$ & enoy uno & - \\
\hline \multicolumn{2}{|c|}{ indyonoulu } & \multicolumn{3}{|c|}{ upead } & & \multicolumn{2}{|c|}{ Gra leajudeso } & & & 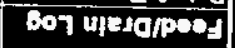 \\
\hline
\end{tabular}


THROUOHPUT LOO

\begin{tabular}{|c|c|c|c|c|c|c|c|c|c|c|}
\hline \multirow{2}{*}{$\begin{array}{l}\text { Feod/Drain Loo } \\
\text { Date \& Tme }\end{array}$} & \multirow[b]{2}{*}{ Cum. hours } & \multirow[b]{2}{*}{ Tost Phase } & \multicolumn{2}{|c|}{ Oraphlcal Data } & \multicolumn{4}{|c|}{ Dratn } & \multicolumn{2}{|c|}{ Throughput } \\
\hline & & & $\begin{array}{l}\text { Toat Cum } \\
\text { Hours }\end{array}$ & $\begin{array}{l}\text { Test Cum } \\
\text { Foed }(\mathrm{kg})\end{array}$ & Drain 1 & Drain (bes) & $\begin{array}{l}\text { AvG Draln } \\
\text { Rato } \\
\text { (lba/min) }\end{array}$ & Cum. Orain & $\begin{array}{l}\text { Foed Thls } \\
\text { Pentod (lohr) }\end{array}$ & $\begin{array}{l}\text { Canss This } \\
\text { Pertiod (Ib/a) }\end{array}$ \\
\hline 4/21/9511:17 & 616.3 & WT-1:aluy & 50.5 & 3008.6 & & & & 116150 & 0.8 & \\
\hline $421 / 9511: 45$ & 610.8 & Wit-1:uny & 91.0 & 3009.1 & & & & 11615.0 & 2.1 & \\
\hline $4 / 21 / 6512: 15$ & 619.2 & MT-1:nity & & & & & & 11615.0 & & \\
\hline $4 / 21 / 9512: 54$ & 618.9 & MT-1:hry & & & & & & 11615.0 & & \\
\hline $4 / 21 / 951620$ & 623.3 & MT-1any & 95.6 & 3103.1 & & & & 11615.0 & 6.8 & \\
\hline $4 / 21 / 9516: 29$ & 623.5 & pitoling & 05.7 & 3120.7 & & & & 11615.0 & 230.3 & \\
\hline $421 / 1031700$ & 621.0 & {$[\pi-1: d y$} & 83.3 & 3145.3 & & & & 11615.0 & 105.0 & \\
\hline$\sqrt{2121031720}$ & 624.5 & mitcony & 8.7 & 3173.4 & & & & 11615.0 & 132.9 & \\
\hline 4/2163 18:30 & 625.3 & Wit-1:ming & 97.6 & 320.6 & & & & 11616.0 & 75.0 & \\
\hline 4/2100 10:00 & 623.0 & Fitolay & 0.0 & 0.0 & & & & 11616.0 & 1240 & \\
\hline 4/21/50 10:23 & 626.4 & Mitiny & 0.4 & 14.1 & & & & 11615.0 & 0.0 & \\
\hline 421/53 18:37 & 62.6 & (IT-1) dny & 0.6 & 20.1 & & & & 11815.0 & 132.8 & \\
\hline $4 / 21 / 5019: 50$ & 620 & $\bar{m}-1 \cdot \cos y$ & 8.0 & 45.7 & & & & 11615.0 & 178.8 & \\
\hline $4 / 21 / 5320: 12$ & 627.2 & MT-1:Lry & 1.2 & 63.3 & & & & 11615.0 & 165.7 & \\
\hline 421352020 & 627.51 & Tite-1:nuy & 1.5 & 80.0 & & & & 11815.0 & 133.8 & \\
\hline 421552020 & 627.5 & 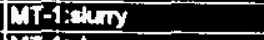 & & & & & & 11615.0 & & \\
\hline $4215320: 45$ & 627.0 & Dit-1:nty & 1.0 & 89.9 & & & & 11615.0 & 116.3 & \\
\hline 4/2150 21:03 & 620.0 & DTr-1:duty & 2.1 & 112.6 & & & & 11615.0 & 130.2 & \\
\hline 4/21/5021:27 & 628.5 & DT-1:Dry & 2.5 & 130.8 & & & & 11615.0 & 100.0 & \\
\hline $4 / 215521: 37$ & 6206 & MT-10uty & 2.6 & 153.2 & & & & 11615.0 & 323.6 & \\
\hline 4/210522:00 & 629.1 & WT-1:ary & 3.1 & 178.8 & & & & 11615.0 & 16.0 & \\
\hline 0210122.23 & 623.4 & DT-4:DTy & 3.4 & 106.0 & & & & 11615.0 & 31.7 & \\
\hline $4 / 21 / 252246$ & 620 & Th+1:1/ny & 3.8 & 216.3 & & & & 11615.0 & 200.2 & \\
\hline $4210522: 55$ & 629.8 & TT-1:DIY STOP & & & 28. & 123.0 & 6.2 & 11730.0 & & 10.0 \\
\hline 4/21:95 23:00 & 630.0 & DT-2:AUY ME START & 4.0 & 2020 & & & & 11736.0 & 33.2 & \\
\hline 4/21/9523:40 & 630.7 & DT-2: & 4.7 & 20.8 & & & & 11735.0 & 20.9 & \\
\hline $4 / 2119523: 43$ & 630.8 & DT-2:Dy & & & & & & 11735.0 & & \\
\hline $420501: 54$ & 632.8 & प्रT-2:any & & & 29 & 197.0 & 49 & 11935.0 & & 68.0 \\
\hline $420503: 07$ & 634.1] & MT2:anty & 0.1 & 446.9 & & & & 11935.0 & 101.1 & \\
\hline $420253: 07$ & 634.1 & 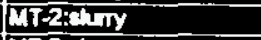 & & & & & & 11935.0 & & \\
\hline $4222053: 45$ & 634.7 & m-2:5ny & 8.0 & 461.0 & & & & 11936.0 & 48.8 & $t$ \\
\hline 4202505 & 635.1 & MT-2:num & & & & & & 11935.0 & & \\
\hline $4 \sqrt[2005]{4: 13}$ & 635.2 & Tit-2:any & & & 30 & 193.0 & 6.4 & 12120.0 & & 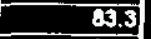 \\
\hline $4 / 221957: 10$ & 633.2 & DT-2:any & & & 31 & 189.0 & 5.4) & 12317.0 & & 64.1 \\
\hline 4/22195 7:10 & 630.31 & Tit-2:ainy & 12.3 & 717.6 & & & & 12317.0 & 159.4 & \\
\hline $4 / 22057: 10$ & 630.3[ & WT-2:ouny & & & & & & 12317.0 & & \\
\hline 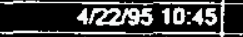 & 641.7. & CिT-2:dury & & & 32. & 317,0 & 5.3 & 12634.0 & & 8.5 \\
\hline $4 / 2219511: 40$ & 642.0[ & DT-2:any & 16.0 & 1003.1 & & & & 12639.0 & 179.1 & \\
\hline $4 \sqrt[2205]{11: 40}$ & 642.0 & MT-2:Diny & & & & & & rasuo & & \\
\hline $4 \sqrt{2095} 13000$ & 644.0[ & WT2:CUTy MB STOP & & & & & & 12334.0 & & \\
\hline$4 \longdiv { 2 2 1 9 5 } 1 3: 57$ & 645.0 & & 19.0 & 1003.1 & 33 & 74.0 & 7.4 & 12700.0 & & 23.1 \\
\hline $472219514: 50$ & 646.0 & & & & 39- & 150.0 & 17.0 & 12050.0 & & 147.5 \\
\hline $12229515: 05$ & 646.1 & & 0.00 & 0.0 & & & & 12550.0 & 72.5 & \\
\hline $4220516: 50$ & 64.0 & & 1.00 & 35.4 & & & & 12050.0 & 41.4 & \\
\hline 12arss 17:36 & 640.6 & & & & 35 & 2040 & 20.4 & 13062.0 & & 77.5 \\
\hline $4 / 220518: 05$ & 649.1 & & 3.00 & 105.7 & & & & 130.02 .0 & & \\
\hline $122 / 9518: 10$ & 649.2 & & 3.00 & 141.0 & & & & 130.2 .0 & 194.2 & \\
\hline 40219510.20 & 649.3 & & 3.25 & 176.4 & & & & 13062.0 & 465.0 & \\
\hline $1 / 229510: 33$ & 649.5 & & 3.47 & 246.7 & & & & 130.02 & 715.4 & \\
\hline
\end{tabular}


THROUOHPUT LOO

\begin{tabular}{|c|c|c|c|c|c|c|c|c|c|c|}
\hline \multirow{2}{*}{$\begin{array}{l}\text { Feed/Draln Log } \\
\text { Doto \& Tims }\end{array}$} & \multirow[b]{2}{*}{ Cum. hours } & \multirow[b]{2}{*}{ Test Phase } & \multicolumn{2}{|c|}{ Oraphical Data } & \multicolumn{4}{|c|}{ Drain } & \multicolumn{2}{|c|}{ Throushput } \\
\hline & & & \begin{tabular}{|l|l} 
Tost Cum & T \\
Hours
\end{tabular} & $\begin{array}{l}\text { Tost Cum } \\
\text { Foed }(k-1)\end{array}$ & Drant 7 & Drain (ibs) & $\begin{array}{l}\text { Ave Draln } \\
\text { Rato } \\
\text { (lbow/min) }\end{array}$ & Cum. Drain & Foed This & $\begin{array}{l}\text { Chass This } \\
\text { Perlod (Ib/x) }\end{array}$ \\
\hline $\begin{array}{l}4209510: 36 \\
4 / 2219519: 00\end{array}$ & 649.9 & & 3.05 & 250.3 & & & & 15052.0 & 76.3 & \\
\hline$\frac{1 / 22951900}{42005} 10: 00$ & $\frac{650.0}{650.0}$ & MT-3:VSIM MB START & & & & & & 13052.0 & & \\
\hline $\begin{array}{l}4 / 225510: 50 \\
12250520: 30\end{array}$ & $\frac{650.8}{651.6}$ & MT-3:V-SIM & 4.75 & 360.1 & & & & 130.02 & 244.4 & \\
\hline$\sqrt{2205} 22: 00$ & ES3.0 & |ut-3: V-sin & & & 3.5 & 151.0 & 21.6 & 13213.0 & & $\overline{49.8}$ \\
\hline$\sqrt{202052: 53}$ & 653.9 & & & & & & & 13213.0 & & \\
\hline $1235031: 10$ & 65.2 & & & & 37 & 162.0 & 5.2 & 13375.0 & & 72.0 \\
\hline $4 / 23152.59$ & 650.0 & & 10.00 & 430.0 & & & & 13357.0 & 29.3 & \\
\hline 1/23:55:37 & 600.6 & & 11.00 & 321.5 & & & & 13375.0 & 110.1 & \\
\hline 42355620 & 631.3 & & & & 3.]. & 300.0 & 11.4 & 13603.0 & & 45.7 \\
\hline 423505205 & 601.4 & & & & & & & 153.3.0 & & \\
\hline $4 / 2355060$ & 86.0 & & & & & & & 1332.0 & 45.1 & \\
\hline 1235s 10:00 & 665.0 & WTA: Celend W'B STRT & 0.00 & 0.0 & 30. & 104.0 & 8.6 & 13\%.07.0 & & 42.0 \\
\hline $4 \sqrt{23505} 10: 15$ & 635.3 & DTt-4: colded & 0.00 & 105.0 & & & & 136.67 .0 & 6.6. & \\
\hline $4 \sqrt{2353} 1021$ & 635.31 & WTat: cosined & 0.35 & 127.7 & & & & 136.67 .0 & 200.0 & \\
\hline $4 / 2315510: 30$ & 63.5 & 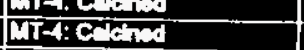 & 0.43 & 139.0 & & & & 136.37 .0 & 20.0 & \\
\hline$\sqrt{23535} 10.35$ & 635.61 & 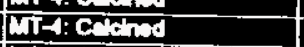 & & & & & & 13637.0 & & \\
\hline $4 \sqrt{2355} 13: 30$ & 63.5] & RT-4: Colched & 0.67 & 161.7 & & & & 130.67 .0 & 214.3 & \\
\hline $4 / 2355313: 40$ & 630.01 & DTt-4: Cenchod & & & & & & 13.37 .0 & & \\
\hline 4235051400 & 669.01 & 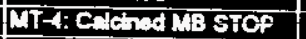 & & & (4) & 300.0 & 7.8 & 14167.0 & & $\overline{7.3}$ \\
\hline $1 / 23531426$ & 669.4 & & & & & & & 14167.0 & & \\
\hline 1/235S 17:10 & 672.2 & & $\begin{array}{ll}4.52 \\
7.203\end{array}$ & 150.7 & & & & 14167.0 & 19.5 & \\
\hline $4 \sqrt{2365} 17: 00$ & 672.7 & & 7.25 & 2.2 .8 & & & & 14167.0 & 70.2 & \\
\hline $42359519: 30$ & 674.5 & & 7.75 & 297.3 & & & & 14167.0 & 6.0 & \\
\hline $4 \sqrt{23} / 9523: 57$ & 670.8 & & & & & & & 14167.0 & 85.1 & \\
\hline $424 / 950: 40$ & 679.7 & HT: V-SID START & 0.0 & & 41) & 220.0 & 13.0 & 143.7 .0 & & 21.7 \\
\hline $4 / 24002225$ & 601.4 & Hir: V-sin & & 0.0 & & & & 143.7 .0 & 104.7 & \\
\hline $4 \sqrt{2405} 4.24$ & 6.3.4 & HT:V-SIM & 3.7 & & 42 & 197.0 & 7.8 & 1453.0 & & 78.9 \\
\hline /24/95 4:39 & 6.3 .6[ & HT: VSIM & 3.71 & 72.1 & & & & 145040 & 42.6 & \\
\hline $4 / 24 / 955: 00$ & 604.01 & HT:VSIM & & & 43 & 34.0 & 24.5 & 14820.0 & & 159.0 \\
\hline$\sqrt{24 / 55} 5: 10$ & 63.2. & MT: VSIM & $\begin{array}{l}4.3 \\
4.5\end{array}$ & 107.9 & & & & 14920.0 & & \\
\hline $4 / 24958.02$ & 6.7 .0 & HT: V-SIM & 4.5 & 251.2 & & & & 14920.0 & 515.2 & 8 \\
\hline $4 / 24906: 53$ & 637.0 & HI:V-SIM & & & 44 & 186.0 & 18.0 & 150060 & & 49.7 \\
\hline $4 / 24 \% 510: 01$ & 60.0 & HI: V-Sin & 8.3 & 356.5 & & & & 150060 & 61.9 & \\
\hline $424 / 8510: 44$ & 609.7 & HT: VSIM & & & 45 & 254.0 & 10.0 & 15350.0 & & 120.1 \\
\hline $4 / 24 / 5510: 47$ & 609.01 & HT:V-SiM & 10.1 & 427.7 & & & & 15350.0 & & \\
\hline $4 / 24 / 5513: 31$ & 692.5 & HT: V SIN & 10.1 & 452.6 & & & & 15350.0 & 125.4 & \\
\hline $424 / 9514: 13$ & 693.2 & AT: Visim & 12.8 & 560.7 & & & & 15350.0 & 85.6 & \\
\hline $4 / 24 / 9515.27$ & 69.5 & HT:VSIM & & & 45 & 234.0 & 10.0 & 15504.0 & & 55.7 \\
\hline $4 / 24 / 95$ 17:03 & 603.01 & HT:V SIM & 14.0 & 679.8 & & & & 15504.0 & 126.7 & \\
\hline $4 / 24 / 9517: 14$ & $696.2 \mathrm{f}$ & HT: VSIM & & & 47 & 312.0 & 24.0 & 15096.0 & & T10.1 \\
\hline $4 \sqrt{24 / 95} 78: 43$ & 697.7 & HT: V-silu & 16.6 & 766.4 & & & & 15053.0 & 131.8 & \\
\hline $4 \sqrt{24 / 95} 20: 10$ & 699.21 & HT: V-sin & & & 48 & 79.0 & 13.0 & 15975.0 & & 47.4 \\
\hline$4 \longdiv { 2 4 / 9 5 } \overline { 2 2 : 5 3 }$ & $701.9 \mathrm{~F}$ & HT: V-SIM & 19.5 & 927.4 & & & & 15975.0 & 106.0 & \\
\hline $4 / 24 / 9523: 00$ & $702.0 \mathrm{t}$ & HT: VSIM & & & 49 & 254.0 & 5.6 & 16229.0 & & 61.0 \\
\hline $4 / 24 / 9523: 10$ & 702.2 & HT: V-SIM & $\frac{22.3}{223}$ & 1000.0 & & & & 16220.0 & & \\
\hline $1 / 25 / 950: 35$ & $703.6 \mathrm{~F}$ & HT: V-Sin & 22.3 & 1143.0 & & & & 1622.0 & 159.0 & \\
\hline 4/25/95 $1: 40$ & $704.7 \mathrm{~F}$ & HT: V-SIM & 23.9 & 1189.1 & & & & 16229.0 & 70.6 & \\
\hline $4 / 25 / 952: 50$ & $705.0 \mathrm{t}$ & HT: VSIM & 25.0 & 1260.3 & & & & 16229.0 & 144.9 & \\
\hline & & & 26.3 & 1330.6 & & & & 16020.0 & 119.2 & \\
\hline
\end{tabular}


THROUOHPUT LOO

\begin{tabular}{|c|c|c|c|c|c|c|c|c|c|c|}
\hline \multirow{2}{*}{$\begin{array}{l}\text { Fesd/Draln Lod } \\
\text { Doto \& Timo }\end{array}$} & \multirow[b]{2}{*}{ Cum hows } & \multirow[b]{2}{*}{ Teat Phaso- } & \multicolumn{2}{|c|}{ Oraphleal Data } & \multicolumn{4}{|c|}{ Drain } & \multicolumn{2}{|c|}{ Throughput } \\
\hline & & & $\begin{array}{l}\text { Tost Cum } \\
\text { Hours }\end{array}$ & $\begin{array}{l}\text { Toat Cun } \\
\text { Foed (ko) }\end{array}$ & Drain ar & Drain (be) & $\begin{array}{l}\text { Avo Draln } \\
\text { Roto } \\
\text { (lbaimin) }\end{array}$ & Cum. Dran & $\begin{array}{l}\text { Foed This } \\
\text { Perlod (lbar) }\end{array}$ & $\begin{array}{l}\text { Glass This } \\
\text { Period (lb/hr) }\end{array}$ \\
\hline$4 \longdiv { 2 5 9 5 } 3: 35$ & 703.6 & HT: VSIM & & & 50 & 409.0 & 5.3 & 16710.0 & & 10.0 \\
\hline $425 / 553: 48$ & 706.8 & HI: V.SIM & 27.1 & 1400.5 & & & & 16710.0 & 101.2 & \\
\hline 12055 5:20 & 700.3 & HT: V-SIM & 20.7 & 160.7 & & & & 16710.0 & 6.3 & \\
\hline $1 / 2505603$ & 700.0 & HiT: v-sim & 20.3 & 1532.0 & & & & 16710.0 & 250.3 & \\
\hline $425 / 008: 53$ & 711.8 & AT: V SIM & & & 51 & 176.0 & 7.0 & 16090 & & 33.2 \\
\hline $425 \times 0011: 51$ & 714.8 & HT: VSII & & & 52 & $1 \% 0$ & 5.8 & 17000 & & 64.0 \\
\hline$\sqrt{25}$ & 715.0 & HiT: VSIM & 35.3 & 1633.1 & & & & 1704.0 & 30.1 & \\
\hline $4 / 25513: 30$ & 716.5 & HT: V-SIn & & & & & & 1700.0 & & \\
\hline 425631423 & 717.4 & HI:VSII & 37.7 & 174.2 & & & & 17004.0 & 83.2 & \\
\hline W25SS 15:39. & 718.6 & मT:V-SID & & & 53 & 20.0 & 7.0 & 1720.0 & & 5.4. \\
\hline $425 \% 3517: 31$ & 720.5 & HI:V-SID & 40.0 & 100.4 & & & & 17200.0 & 74.0 & \\
\hline 4/25SS 19:51 & Fad & HT:V-SII & 43.2 & 1004.4 & & & & 17250.0 & 33.0 & \\
\hline $4 / 23 / 9520443$ & 723.7 & HT:VSIM & $44 . \overline{0}$ & 1010.3 & & & & 17200.0 & 0.0 & \\
\hline$\sqrt{235 O 5120}$ & 724.3 & HT: V-SIM & & & 59 & 533.0 & 17.3 & 176360 & & 83.0 \\
\hline $4 \sqrt{25} 5521: 35$ & 724.6 & HI:VsIM & 44.8 & 20.24 .9 & & & & 17036.0 & 265.8 & \\
\hline$\sqrt{26 / 95} 0: 00$ & 727.0 & HI:VSIM & 47.3 & 2006.1 & & & & 17620.0 & & \\
\hline $4 / 26 / 50,00$ & 727.1 & HI:VSII & 47.4 & 2163.9 & & & & 176260 & 124.4 & \\
\hline$\sqrt{22185} 1: 03$ & 720.0 & HT: V-STM & 40.4 & 22390.0 & & & & 1760.0 & 167.4 & \\
\hline प20\%5 2:13 & 729.2 & HI:V-SID & 40.5 & 2275.3 & & & & 17626.0 & 6.6.6 & \\
\hline $4 / 2552210$ & 720.3 & HT:V-sin & & & 35 & 455.0 & 21.7 & 10201.0 & & 91.8 \\
\hline 426552.42 & 729.7 & AT:V-SIM & 50.0 & 2343.5 & & & & 10201.0 & 324.8 & \\
\hline $4 / 25 \% 53: 12$ & 730.2 & HT:VSIM & 50.5 & 2417.2 & & & & 18201.0 & 312.0 & \\
\hline$\sqrt{26 \% 53} 340$ & 730.8 & सिT:V-SIM & 51.1. & 265.6 & & & & 16301.0 & 130.0 & \\
\hline $4 / 2 \mathrm{BSS} 4: 30$ & 731.6 & HTr:V-Sin & & & 53 & 501.0 & 11.1 & 16702.0 & & 214.7 \\
\hline$\sqrt{26395} 4: 44$ & \begin{tabular}{|l|}
731.7 \\
\end{tabular} & HT:V-SIM & 52.1 & 2524.3 & & & & 107020 & 169.3 & \\
\hline 1/20\%56 6:07 & 733.1 & AT:V-sin & 53.4 & 2560.1 & & & & 10702.0 & & \\
\hline $4 / 26 / 356: 09$ & 733.1 & HT: V-STM & 53.5 & 2630.6 & & & & 10702.0 & 165.9 & \\
\hline 4/25/95 6:12 & 735.2 & HAT:V-SIM & 55.5 & 2737.0 & & & & 107.02 .0 & 114.1 & \\
\hline $4 \sqrt{26 / 55} 10: 45$ & 737.7 & HT:V-SIM & & & 57 & 237.0 & 4.5 & 19010.0 & & 33.7 \\
\hline $4 / 25 / 9511: 13$ & 733.2 & AिT: VSIM STOP & 3.5 & 2000.6 & & & & 15019.0 & 52.4 & \\
\hline $42665513: 35$ & 740.6 & & & & & & & 15019.0 & & $I$ \\
\hline 4/2:69513:47 & 740.8 & HT: SUIY START & 0.0 & 0.0 & & & & 15019.0 & 6.6 & \\
\hline 4/26/95 14:26 & 741.4 & AT: SiUTy & 0.7 & 3..2 & & & & 19019.0 & 129.6 & \\
\hline $4 \sqrt{26} / 95$ 15:31 & 742.5 & HT: Sinty & 1.7 & 4.1. & & & & 19019.0 & 83.5 & \\
\hline 4/26/95 16:06 & 743.1 & HT: SUTY & & & 50 & 403.0 & 5.0. & 19sin.0 & & 80.3 \\
\hline 42:655 16:13 & 743.2 & HT: Sinty & 2.4 & 160.5 & & & & 18502.0 & 240.7 & \\
\hline 4/26/95 17:11 & 744.2 & HT: STIY & & & & & & 195020 & & \\
\hline 4/26/95 $17: 25$ & 744.4 & HT: Sinty & 3.6 & 190.7 & & & & 16502.0 & 70.2 & \\
\hline $4260510: 00$ & 745.0 & HT: Siñy & 4.2 & 252.2 & & & & 18502.0 & 202.2 & \\
\hline $4 / 26 / 9510: 30$ & 745.5 & AT: SIITy & 4.7 & 305.7 & & & & 1850.0 & 235.9 & \\
\hline $4 / 26510530$ & 763.00 & HT: STIY STOP & 5.2 & 374.4 & & & & 15000.0 & 325.0 & \\
\hline 4/2595 19:35 & 746.6 & FWA NA START & 0.0 & 0.0 & 59 & 201.0 & 5.7 & 10703.0 & & 57.7 \\
\hline $4 / 26 / 9520: 00$ & 747.0 & Fing & 0.4 & 203.6 & & & & 19700.0 & 434.5 & \\
\hline $426 \% 523: 20$ & 750.50 & Finno & 3.9 & 371.0 & & & & 19703.0 & 107.0 & \\
\hline $4 / 27 / 950: 09$ & $731.1 \mathrm{t}$ & High $\mathrm{No}$ & 4.6 & 371.9 & 60 & 327.0 & 2.3 & 20050.0 & & 71.6. \\
\hline 4/27/953:06 & 754.1 & $\operatorname{Aon} \mathrm{Na}$ & 7.5 & 392.3 & & & & 20030.0 & 12.4 & \\
\hline $427 / 953: 46$ & 754.0 & FII Na STOP & 8.2 & 400.2 & & & & 20030.0 & 52.5 & \\
\hline 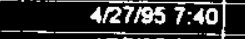 & 753.7 & & & & 61 & 351.0 & 8.7 & 20411.0 & & 50.7 \\
\hline 1/27/959:47 & 760.8 & & & & & & & 2011.0 & & \\
\hline
\end{tabular}


THROUOHPUT LOO

\begin{tabular}{|c|c|c|c|c|c|c|c|c|c|c|}
\hline \multirow{2}{*}{$\begin{array}{l}\text { Foed/Draln Loo } \\
\text { Date \& Timo }\end{array}$} & \multirow[b]{2}{*}{ Cum hours } & \multirow[b]{2}{*}{ Test Phise } & \multicolumn{2}{|c|}{ Oraphical Data } & \multicolumn{4}{|c|}{ Draln } & \multicolumn{2}{|c|}{ Throughput } \\
\hline & & & $\begin{array}{l}\text { Teat Cum } \\
\text { Hows }\end{array}$ & $\begin{array}{l}\text { Teat Cun } \\
\text { Food (kg) }\end{array}$ & Drain 4 & Drain (lbs) & $\begin{array}{l}\text { Ave Drein } \\
\text { Rato } \\
(\text { abe/min) }\end{array}$ & $\begin{array}{l}\text { Cum Drain } \\
\text { (bib) }\end{array}$ & $\begin{array}{l}\text { Fond This } \\
\text { Peotod (botr) }\end{array}$ & $\begin{array}{l}\text { Class Th/s } \\
\text { Pectod (b/om) }\end{array}$ \\
\hline $4 / 27 / 9517: 43$ & 760.7 & & & & 五 & 2022.0 & 6.6 & 22433.0 & & 201.2 \\
\hline TOTALS & 768.7 & & & & 620 & 22433.0 & 11.7 & 22433.0 & 100.4 & 09. \\
\hline
\end{tabular}

Page 18 
WHC-SD-WM-VI-031

Revision 0

This page intentionally left blank. 
WHC-SD-WM-VI-031

Revision 0

APPENDIX 3C

masS BaLANCE CALCULATIONS 
Revision 0

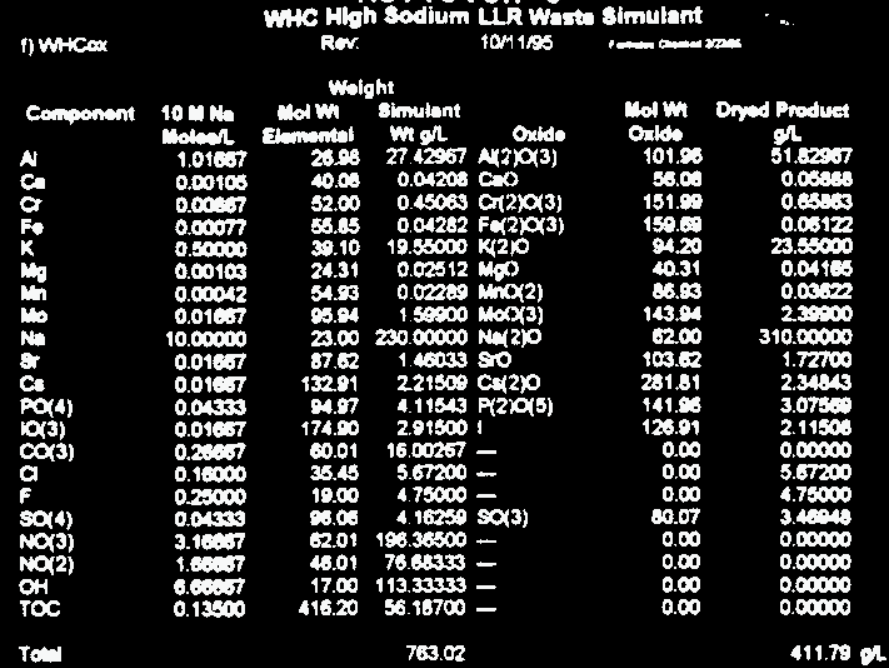
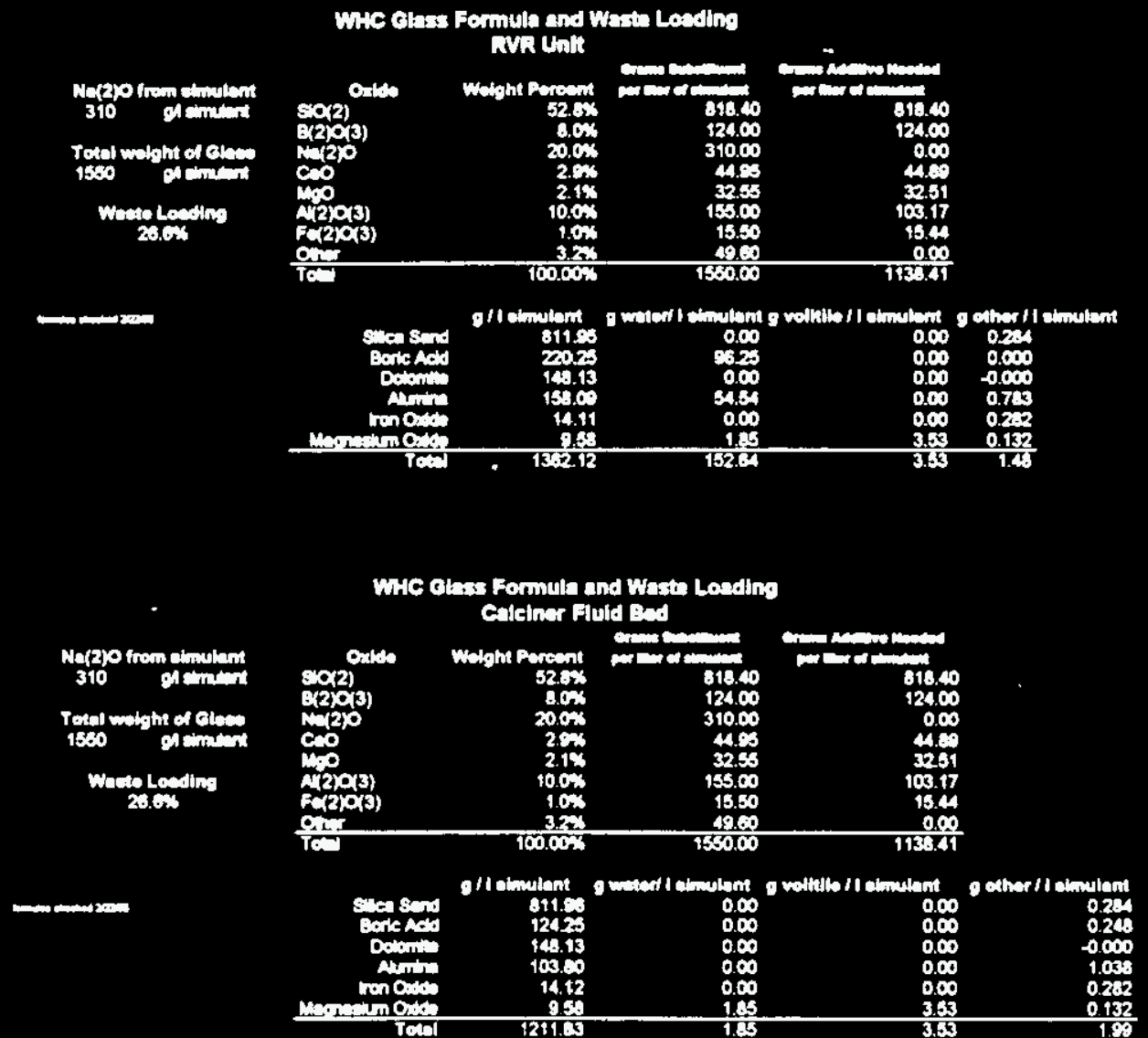

RVR Glass Formors

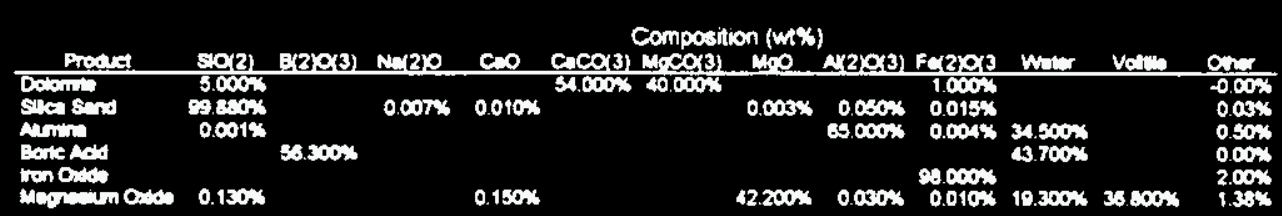

Calciner Glase Formers

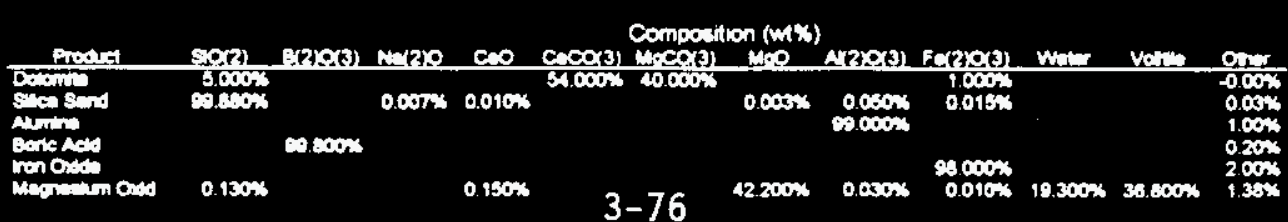




\section{Revision 0}

Expected Na(2)O Levels and Glass Production for Slurry Feed Glass

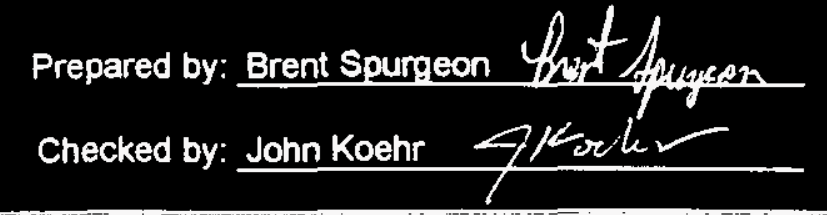

$\begin{array}{llcl} & & \text { Na(2)O level } \\ \text { Assume: } & \text { Density of simulant }= & 1.49 \mathrm{~g} / \mathrm{ml} & 17.71 \% \text { per and of ealc } \\ & \text { Molarity of Simulant }= & 10 \mathrm{Molar} & \end{array}$

Feed Ratio, Ib of RVR mix/b Simulant =

1.07

For Batch 2A \& $3 A$

Total Batch Weight
Date: $\quad 08 / 17 / 95$

Date: 08/17/95

Total Oxides from RVR Mix:

$3 \mathrm{lb}$ or $1362 \mathrm{~g}$ of RVR Mix Yields...

$\left.\begin{array}{lr}\mathrm{SiO}(2) & 818.40 \mathrm{~g} \\ \mathrm{~B}(2) \mathrm{O}(3) & 124.00 \mathrm{~g} \\ \mathrm{Na}(2) \mathrm{O} & 0.00 \mathrm{~g} \\ \mathrm{CaO} & 44.89 \mathrm{~g} \\ \mathrm{MgO} & 32.51 \mathrm{~g} \\ \mathrm{Al}(2) \mathrm{O}(3) & 103.17 \mathrm{~g} \\ \mathrm{Fe}(2) \mathrm{O}(3) & 15.44 \mathrm{~g} \\ \text { Other } & 1.48 \mathrm{~g} \\ \hline \text { Total } & 1138.41 \mathrm{~g}\end{array}\right\} \quad$ per f)WHCox.w61

RVR mix lb
Above yields for

Total Oxides From RVR Mix

\begin{tabular}{lr}
$\mathrm{SiO}(2)$ & $146.22 \mathrm{~kg}$ \\
$\mathrm{~B}(2) \mathrm{O}(3)$ & $22.15 \mathrm{~kg}$ \\
$\mathrm{Na}(2) \mathrm{O}$ & $0.00 \mathrm{~kg}$ \\
$\mathrm{CaO}$ & $8.02 \mathrm{~kg}$ \\
$\mathrm{MgO}$ & $5.81 \mathrm{~kg}$ \\
$\mathrm{Al}(2) \mathrm{O}(3)$ & $18.43 \mathrm{~kg}$ \\
$\mathrm{Fe}(2) \mathrm{O}(3)$ & $2.76 \mathrm{~kg}$ \\
Other & $0.26 \mathrm{~kg}$ \\
\hline Total & $203.66 \mathrm{~kg}$
\end{tabular}




\section{Revision 0}

Oxides from Simulant

$\begin{array}{lcr}\text { Density of Simulant }= & 1.49 \mathrm{~g} / \mathrm{ml}= & 3.28 \mathrm{lb} \boldsymbol{} \\ \text { Total liters of simulant }= & 152.21 \mathrm{I} \text { simulant }\end{array}$

Oxides from Simulant

\begin{tabular}{|c|c|c|}
\hline $\begin{array}{c}\text { Oxide } \\
\text { Al(2)O(3) }\end{array}$ & $\begin{array}{r}g / \text { simulant } \\
51.83\end{array}$ & $\begin{array}{r}\mathrm{kg} \text { total } \\
7.89\end{array}$ \\
\hline $\mathrm{CaO}$ & 0.06 & 0.01 \\
\hline $\mathrm{Fe}(2) \mathrm{O}(3)$ & 0.06 & 0.01 \\
\hline $\mathrm{MgO}$ & 0.04 & 0.01 \\
\hline $\mathrm{Na}(2) \mathrm{O}$ & 310.00 & 47.19 \\
\hline$\overline{\text { Total }}$ & 361.99 & 55.10 \\
\hline
\end{tabular}

\section{Total Oxides}

\begin{tabular}{lrr}
$\quad$ Oxide & \multicolumn{1}{c}{$\mathbf{k g}$} & \\
$\mathrm{SiO}(2)$ & 146.22 & $54.89 \%$ \\
$\mathrm{~B}(2) \mathrm{O}(3)$ & 22.15 & $8.32 \%$ \\
$\mathrm{Na}(2) \mathrm{O}$ & 47.19 & $17.71 \%$ \\
$\mathrm{CaO}$ & 8.03 & $3.01 \%$ \\
$\mathrm{MgO}$ & 5.85 & $2.20 \%$ \\
$\mathrm{Al}(2) \mathrm{O}(3)$ & 26.32 & $9.88 \%$ \\
$\mathrm{Fe}(2) \mathrm{O}(3)$ & 2.77 & $1.04 \%$ \\
Other & 7.85 & $2.95 \%$ \\
\hline Total & 266.38 & $100.00 \%$
\end{tabular}

Others

\begin{tabular}{|c|c|c|}
\hline Oxide & gl simulant & $\mathrm{kg}$ total \\
\hline $\mathrm{Cr}(2) \mathrm{O}(3)$ & 0.65863 & 0.10 \\
\hline$K(2) 0$ & 23.55000 & 3.58 \\
\hline $\mathrm{MnO}(2)$ & 0.03622 & 0.01 \\
\hline $\mathrm{MoO}(3)$ & 2.39900 & 0.37 \\
\hline Sro & 1.72700 & 0.26 \\
\hline $\mathrm{Cs}(2) \mathrm{O}$ & 2.34843 & 0.36 \\
\hline$P(2) O(5)$ & 3.07569 & 0.47 \\
\hline 1 & 2.11508 & 0.32 \\
\hline $\mathrm{Cl}$ & 5.67200 & 0.86 \\
\hline$F$ & 4.75000 & 0.72 \\
\hline $\mathrm{SO}(3)$ & 3.46948 & 0.53 \\
\hline
\end{tabular}

Glass to Slumy Ratio:

$56.69 \%$ 


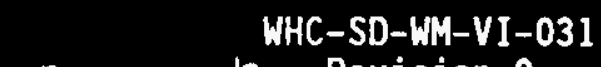

$\frac{7}{2}$

$\frac{8}{2}$

$\frac{8}{8}$

ก กับ

है

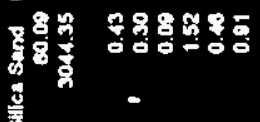

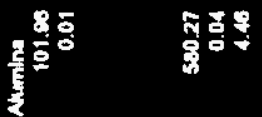

$\stackrel{8}{\circ}$

WHC-SD-WH-VI-031



$8898 \%$

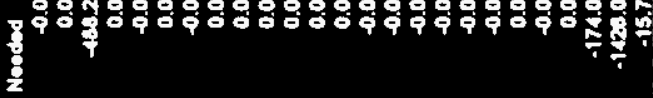

के

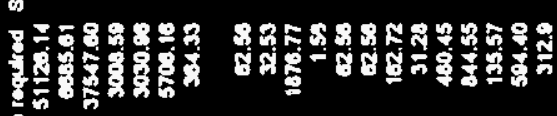

हू

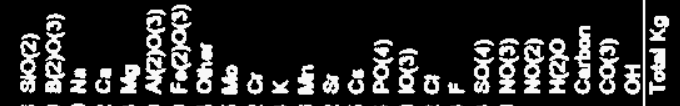

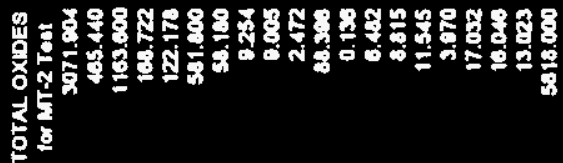

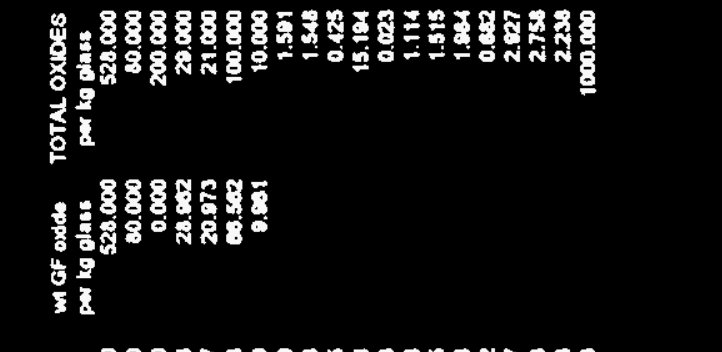

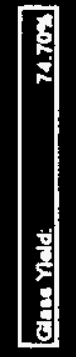

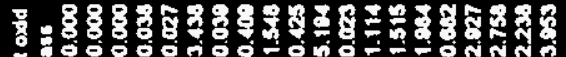

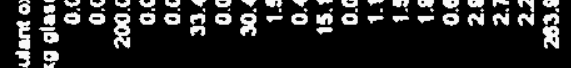

寒

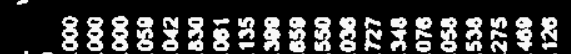

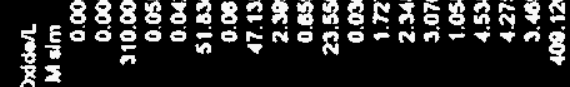
홍

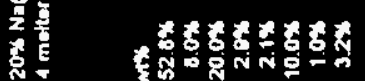

Fon

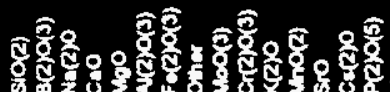

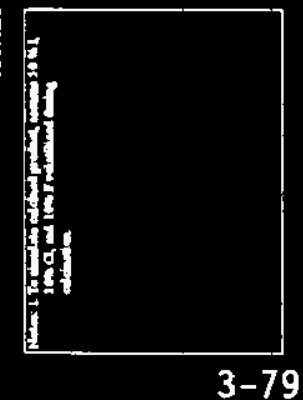




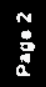

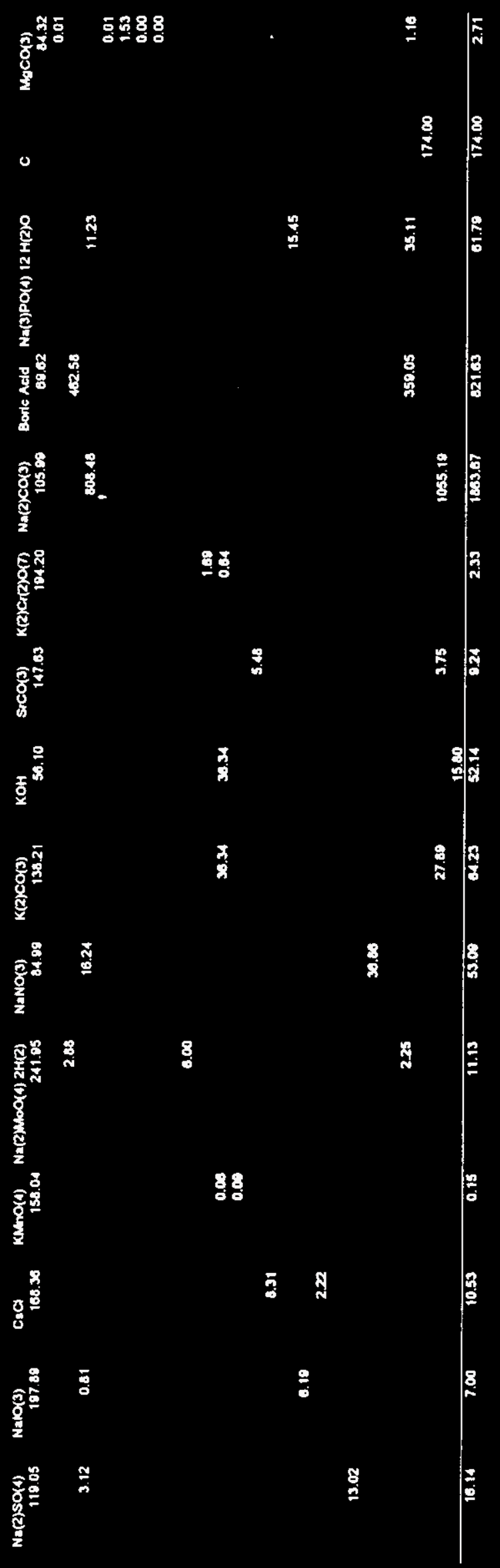


Mass Balance for MT-2, slurry feed

Prepared by: Brent Spurgeon $1 / 0$

Date: $08 / 17 / 95$

Checked by: John Koehr

Q⿻

Date: $08 / 17 / 95$

\section{General Information}

whevitos.xts $=$ Throughpur Summary

Test Duration:

$14.0 \mathrm{hr}$

per wherito5 $x$ ds

Total Slumy Feed:

$864.60 \mathrm{~kg}$

Batch Ratio: $w$. of RVR mix / $\mathrm{m}$. Simulant

1.07

$\mathrm{m}$. of Sugar $/ \mathrm{m}$. simulant

0.084

Density of Simulant:

$1.49 \mathrm{kghiter}$

pem WHC inform

Molarity of Simulant:

$10.00 \mathrm{M}$

per wheoxub1

Total Simulant:

$401.39 \mathrm{~kg}$

269.39 liters

Total RVR Mix:

$429.49 \mathrm{~kg}$

Total Sugar:

$33.72 \mathrm{~kg}$

Total Glass Product:

$406.42 \mathrm{~kg}=896.0 \mathrm{lb}$

per whowes dis:

Average Off-Gas flow rate:

Total Off-Gas:

$204.70 \mathrm{~kg} / \mathrm{hr}$

$2865.80 \mathrm{~kg}$

Average Particulate in Off-Gas:

$0.855 \mathrm{~kg} / \mathrm{hr}=$ $11.97 \mathrm{~kg}$

Total Particulate in Off-Gas:

Glass Composition:

\begin{tabular}{lrrrr} 
Component & Target Composition & Actual Average Composition & & \\
SiO(2) & $52.8 \%$ & $58.65 \%$ & $11.08 \%$ & high \\
$\mathrm{B}(2) \mathrm{O}(3)$ & $8.0 \%$ & $7.34 \%$ & $8.25 \%$ & low \\
$\mathrm{Na}(2) \mathrm{O}$ & $20.0 \%$ & $15.61 \%$ & $21.95 \%$ & low \\
$\mathrm{CaO}$ & $2.9 \%$ & $2.93 \%$ & $1.15 \%$ & high \\
$\mathrm{MgO}$ & $2.1 \%$ & $2.26 \%$ & $7.62 \%$ & high \\
$\mathrm{Al}(2) \mathrm{O}(3)$ & $10.0 \%$ & $9.53 \%$ & $4.70 \%$ & low \\
$\mathrm{Fe}(2) \mathrm{O}(3)$ & $1.0 \%$ & $1.01 \%$ & $1.00 \%$ & high \\
Other & $3.2 \%$ & $2.67 \%$ & $16.56 \%$ & low \\
\hline Total & $100.00 \%$ & $100.00 \%$ & &
\end{tabular}


RVR Mix Composition:

Silica Sand

Boric Acid

Dolomite

Alumina

Iron Oxide

Mag. Carb.
$59.61 \%$

$16.17 \%$

$10.87 \%$

$11.61 \%$

$1.04 \%$

$0.70 \%$ 


\section{Sodium Balance}

Sodium In:

Sodium In Simulant:

230.00 g/iter

269.39 liter

Total Simulant In:

$61.96 \mathrm{~kg} \mathrm{Na}$

per wheox,wbt

Total Sodium from Simulant:

Sodium Out:

Sodium in Glass:

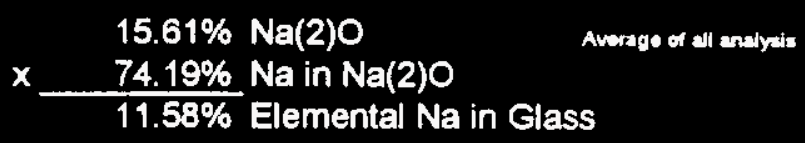

$47.07 \mathrm{~kg} \mathrm{Na}$

Sodium in Off-Gas particulate: Total Particulate during MT-2:

$21.45 \% \mathrm{Na}$ in Off-gas

$11.97 \mathrm{~kg}$

Total Na in the Particulate:

$2.57 \mathrm{~kg} \mathrm{Na}$

Percent loss to Off-gas on an Oxide Basis:

Oxide equivalant in Slumy:

$83.52 \mathrm{~kg} \mathrm{Na(2)O}$

Oxide equivalant in Off-Gas:

Percent Na(2)O to Off-Gas:

$4.14 \%$ of Feed

$\begin{array}{ll}\text { Error Analysis: } & \text { Total Sodium In: } \\ & \text { Total Sodium Out: } \\ & \text { Difference }\end{array}$

Percent Error:
$61.96 \mathrm{~kg}$

$49.64 \mathrm{~kg}$

$12.32 \mathrm{~kg}$

$19.89 \%$ not accounted for 


\section{Chloride Balance}

Chloride In:

Chloride in Simulant:

Total Simulant In:

$5.672 \mathrm{~g} \Lambda$

269.39 liter

per WHCaxwb:

Total Chloride from Simulant

\section{Chloride Out:}

$\% \mathrm{Cl}$ in Off-Gas Particulate: $\quad \mathbf{5 . 5 0} \%$

Total Particulate Out: $\quad 11.97 \mathrm{~kg}$

Total Chloride Out in Off-Gas: $\quad 0.66 \mathrm{~kg}$

Percent Chloride lost to Off-Ga $\quad \mathbf{4 3 . 0 9} \%$

- Chloride in Class:

Total Glass Out:

$0.06 \%$

Total Chloride in glass:

$406.42 \mathrm{~kg}$

$0.248 \mathrm{~kg}$

Total Cl Out:

$0.906 \mathrm{~kg}$

$\% \mathrm{Cl}$ not accounted for: $\quad 40.69 \%$

\section{Fluoride Balance}

Fluoride In:

Fluoride in Simulant:

Total Simulant In:
$4.75 \mathrm{~g} /$ 269.39 liter
Total Fluoride from Simulant:
per wHCoxinbt
$1.28 \mathrm{~kg}$

Fluoride Out:

$\% \mathrm{FI}$ in Off-Gas Particulate:

$1.68 \%$

Total Particulate Out:

$11.97 \mathrm{~kg}$

Total Fluoride Out in Off-Cas:

$0.20 \mathrm{~kg}$

Percent Fluoride lost to Off-Ga

$15.67 \%$

Floride in Glass:

$0.13 \%$

Total Glass Out:

$406.42 \mathrm{~kg}$

Total Floride in glass:

$0.539 \mathrm{~kg}$

Total F Out:

$0.739 \mathrm{~kg}$

$\% \mathrm{~F}$ not accounted for:

$42.25 \%$ 


\section{lodide Balance}

lodide in:

lodide in Simulant:

$2.115 \mathrm{~g} \Lambda$ 269.39 liter

per WHCox.mb1

Total Simulant In:

\section{Total lodide from Simulant:}

$0.57 \mathrm{~kg}$

\section{lodide Out:}

$\%$ lodide in Off-Gas Particulate

Total Particulate Out:

$1.52 \%$

$11.97 \mathrm{~kg}$

Total lodide Out in Off-Gas:

$0.18 \mathrm{~kg}$

Percent lodide lost to Off-Gas:

$31.88 \%$

lodide in Glass:

Total Glass Out:

Total lodide in glass:

$203.00 \mathrm{ppm}$

$406.42 \mathrm{~kg}$

$0.083 \mathrm{~kg}$

\section{Total I Out:}

$0.264 \mathrm{~kg}$

$\%$ I not accounted for:

$53.64 \%$ 


\section{Cesium Balance}

Cesium In:

Cesium in Simulant:

Total Simulant in:

\section{Cesium Out:}

Cesium in Off-Gas:

Cesium in Glass:

Total Glass Out:

Total Cesium in glass:

$\%$ Cs not accounted for:
$2.22 \mathrm{~g} A$

269.39 liter

Total Cesium from Simulant: per WMCox.wb1

$0.60 \mathrm{~kg}$

\author{
Non Detectable \\ $386.00 \mathrm{ppm}$ \\ $406.42 \mathrm{~kg}$ \\ $0.149 \mathrm{~kg}$
}

Total Cs Out:

$0.149 \mathrm{~kg}$

$75.07 \%$

\section{Strontium Balance}

Strontium In:

Strontium in Simulant:

Total Simulant in:

1.46 gniter

269.39 liter

Total Strontium In:

$393.40 \mathrm{~g}$

\section{Strontium Out:}

Strontium in Off-Gas:

Total Particulate Out:

$0.0040 \%$

$11.97 \mathrm{~kg}$

Total Strontium in Off-Gas:

$0.4788 \mathrm{~g}$

Percent Strontium lost to Off-Gas:

$0.12 \%$

Strontium in Glass:

Total Glass Out:

$505.00 \mathrm{ppm}$

$406.42 \mathrm{~kg}$

Strontium out in glass:

$205.24 \mathrm{~g}$

Total Strontium Out:

$205.72 \mathrm{~g}$

$\%$ Strontium not accounted for.

$47.71 \%$ 


\section{Chromium Balance}

Chromium in:

$\mathrm{Cr}(2) \mathrm{O}(3)$ in Simulant:

Total Simulant in:

total $\operatorname{Cr}(2) \mathrm{O}(3)$ :

Chromium out:

$\mathrm{Cr}(2) \mathrm{O}(3)$ in glass:

Total Glass out:

$\mathrm{Cr}(2) \mathrm{O}(3)$ out in glass:

Cr out with particulate: total Particulate Out:

- Total Cr Out:

$\% \mathrm{Cr}$ in $\mathrm{Cr}(2) \mathrm{O}(3)$ :

$\mathrm{Cr}(2) \mathrm{O}(3)$ equivalent:

Total $\mathrm{Cr}(2) \mathrm{O}(3)$ out:

\% Excess:
0.659 g/iter

269.39 liter

$0.177 \mathrm{~kg}$
$2060.00 \mathrm{ppm}$

$406.42 \mathrm{~kg}$

$0.84 \mathrm{~kg}$

$0.06 \%$

$11.97 \mathrm{~kg}$

$0.0072 \mathrm{~kg}$

$68.42 \%$

$0.0105 \mathrm{~kg}$

$0.85 \mathrm{~kg}$

$477.78 \%$

\section{Potassium Balance}

Potassium in:

$K(2) O$ in Simulant:

Total Simulant In:

23.55 gaiter

Total K(2)O from Simulant:

Potassium Out:

$\mathrm{K}(2) \mathrm{O}$ in glass:

$0.63 \%$

Total Class Out:

$406.42 \mathrm{~kg}$

Total K(2)O in Glass:

$2.56 \mathrm{~kg}$

Potassium in Particulate:

$3.94 \%$

Total Particulate Out:

$11.97 \mathrm{~kg}$

Total Potassium in Particulate:

$\% \mathrm{~K}$ in $\mathrm{K}(2) \mathrm{O}$ :

$K(2) O$ equivalent:

$0.471 \mathrm{~kg}$

$83.02 \%$

$0.568 \mathrm{~kg}$

total $K(2) O$ out:

$3.13 \mathrm{~kg}$

$\% \mathrm{~K}(2) \mathrm{O}$ not accounted for:

$50.69 \%$ 


\section{Manganese Balance}

Manganese in:
$M n O(2)$ in Simulant:
0.0362 ghiter
Simulant In: 269.39 liter
Total $\mathrm{MnO}(2)$ in from Simulant:
$0.0098 \mathrm{~kg}$
Manganese Out:
$\mathrm{MnO}(2)$ in Glass:
Total Glass Out:
$0.0096 \%$
$406.42 \mathrm{~kg}$
$0.0388 \mathrm{~kg}$
Total $\mathrm{MnO}(2)$ in Glass:
Mn in Particulate:
$0.0090 \%$
total Particulate Out:
$11.97 \mathrm{~kg}$
Total Mn in Particulate:
$\%$ Mn if $M n O(2):$
$0.0011 \mathrm{~kg}$
$63.22 \%$
$\mathrm{MnO}(2)$ equivilent:
$0.0017 \mathrm{~kg}$
total $\mathrm{MnO}(2)$ out:
$0.0405 \mathrm{~kg}$
$\%$ excess $\operatorname{MnO}(2)$ :
$415.23 \%$

\section{Phosphorus Balance}

Phosphorus in:

$P(2) O(5)$ equivilant in Simulant:

Total amount of Simulant in:

Total $P(2) O(5)$ equivilant in:

\author{
3.08 galiter \\ 269.39 liter \\ $0.829 \mathrm{~kg}$
}

\section{Phosphorus Out:}

Phosphorus in Off-Gas Particulate:

Total Particulate Out:

Total Phosphorus out:

$$
\begin{gathered}
0.055 \% \\
11.97 \mathrm{~kg} \\
0.006524 \mathrm{~kg}
\end{gathered}
$$

$P(2) O(5)$ equivilant out:

percent $P(2) O(5)$ in Off-Gas:

$0.014949 \mathrm{~kg}$

$1.80 \%$

$P(2) O(5)$ in Class:

$0.11 \%$

$0.466 \mathrm{~kg}$

Total $P(2) O(5)$ Out:

$0.481 \mathrm{~kg}$

$\% \mathrm{P}(2) \mathrm{O}(5)$ not accounted for:

$41.91 \%$ 


\section{Silicon Balance}

Silicon In:

\%Silica Sand in RVR MIX:

SiO(2) in Silica Sand:

total SiO(2) in RVR mix:

\section{Silicon Out:}

SiO(2) in product:

Total Product Out:

SiO(2) out:

$\%$ SiO(2) not accounted for:
$5961 \%$

$9988 \%$

$255.71 \mathrm{~kg}$

$58.65 \%$

406.42

$238.36 \mathrm{~kg}$

$6.78 \%$

\section{Boron Balance}

Boron In:

Boric Acid in RVR Mix:

Total RVR Mix:

Total Boric Acid In:

Boric Acid ->

Total Boron on Oxide Basis:

Boron Out:

Boron in Off-Gas Particulate: Total Particulate:

Total Boron in Particulate:

$B$ in $B(2) O(3)$ :

Total Boron on Oxide Basis:

Percent Lost to Off-Gas:

$B(2) O(3)$ in glass:

Total $B(2) O(5)$ out:

$\%$ not accounted for
$16.17 \%$ $429.49 \mathrm{~kg}$ $69.45 \mathrm{~kg}$

$56.30 \% \mathrm{~B}(2) \mathrm{O}(3)$

$$
\begin{array}{r}
7.31 \% \\
11.97 \mathrm{~kg} \\
0.88 \mathrm{~kg}
\end{array}
$$

$31.03 \%$

$2.82 \mathrm{~kg} \mathrm{~B}(2) \mathrm{O}(3)$

$7.21 \%$

$7.45 \%$

$30.28 \mathrm{~kg}$

$33.10 \mathrm{~kg}$

$15.35 \%$ par Whacosubt

per WHCox,wit

per Ond Ropent 


\section{Calcium Balance}

$\mathrm{CaCO}(3)$ in RVR Mix:

\begin{tabular}{lr} 
Silica Sand & $0.03 \mathrm{~kg}$ \\
Boric Acid & $0.00 \mathrm{~kg}$ \\
Dolomite & $14.12 \mathrm{~kg}$ \\
Alumina & $0.00 \mathrm{~kg}$ \\
Iron Oxide & $0.00 \mathrm{~kg}$ \\
Magnesium Carbonate & $0.005 \mathrm{~kg}$ \\
CaO in Simulant: & $0.016 \mathrm{~kg}$ \\
\hline Total CaO In: & $14.16 \mathrm{~kg}$ \\
& \\
\hline & \\
CaO in Glass: & $2.93 \%$ \\
& $11.92 \mathrm{~kg}$ \\
\hline & $15.83 \%$
\end{tabular}

\section{Magnesium Balance}

MgO in RVR Mix:

\begin{tabular}{lc} 
Silica Sand & $0.008 \mathrm{~kg}$ \\
Boric Acid & $0.000 \mathrm{~kg}$ \\
Dolomite & $8.929 \mathrm{~kg}$ \\
Alumina & $0.000 \mathrm{~kg}$ \\
Iron Oxide & $0.000 \mathrm{~kg}$ \\
Magnesium Carbonate & $1.269 \mathrm{~kg}$ \\
MgO in Simulant: & $0.011 \mathrm{~kg}$ \\
\hline Total MgO In: & $10.216 \mathrm{~kg}$ \\
& \\
MgO in Glass: & $2.26 \%$ \\
& $9.19 \mathrm{~kg}$ \\
& \\
\% Missing: & $10.09 \%$
\end{tabular}




\section{Alumina Balance}

$\mathrm{Al}(2) \mathrm{O}(3)$ in RVR Mix:

$\begin{array}{lr}\text { Silica Sand } & 0.121 \mathrm{~kg} \\ \text { Boric Acid } & 0.000 \mathrm{~kg} \\ \text { Dolomite } & 0.000 \mathrm{~kg} \\ \text { Alumina } & 32.411 \mathrm{~kg} \\ \text { Iron Oxide } & 0.000 \mathrm{~kg} \\ \text { Magnesium Carbonate } & 0.001 \mathrm{~kg}\end{array}$

$\mathrm{Al}(2) \mathrm{O}(3)$ in Simulant: $\quad 13.962 \mathrm{~kg}$

$\overline{T o t a l ~ A(2) O(3) ~ i n: ~} \quad 46.503 \mathrm{~kg}$

- $\mathrm{Al}(2) \mathrm{O}(3)$ in Glass: $\quad 9.53 \%$

$38.73 \mathrm{~kg}$

Iron Balance

$\%$ Missing:

$16.71 \%$

$\mathrm{Fe}(2) \mathrm{O}(3)$ in RVR Mix:

\begin{tabular}{lr} 
Silica Sand & $0.038 \mathrm{~kg}$ \\
Boric Acid & $0.000 \mathrm{~kg}$ \\
Dolomite & $0.467 \mathrm{~kg}$ \\
Alumina & $0.002 \mathrm{~kg}$ \\
Iron Oxide & $4.377 \mathrm{~kg}$ \\
Magnesium Carbonate & $0.000 \mathrm{~kg}$ \\
Fe(2)O(3) in Simulant: & $0.016 \mathrm{~kg}$ \\
& \\
\hline Total Fe(2)O(3) In: & $4.901 \mathrm{~kg}$ \\
& \\
Fe(2)O(3) in Glass: & $1.01 \%$ \\
& $4.10 \mathrm{~kg}$
\end{tabular}

\% Missing: $\quad 16.25 \%$ 


\section{Nitrogen Balance}

Nitrogen In Simulant:

NO(3) in Simulant:

Total NO(3):

$\% \mathrm{~N}$ in $\mathrm{NO}(3)$ :

$\mathrm{N}$ from $\mathrm{NO}(3)$ :

$\mathrm{NO}(2)$ in Simulant:

Total NO(2):

$\% N$ in $N O(2)$ :

$\mathrm{N}$ from $\mathrm{NO}(2)$ :

$\mathrm{Na(4)EDTA}$ in Simulant:

Total $\mathrm{Na}(4) E D T A$ :

$\% \mathrm{~N}$ in $\mathrm{Na}(4)$ EDTA:

$\mathrm{N}$ from $\mathrm{Na}(4) E D T A$ :

Total Nitrogen from Simulant:

$$
\begin{gathered}
196.365 \mathrm{~g} / \mathrm{iter} \\
52.90 \mathrm{~kg} \\
22.58 \% \\
11.94 \mathrm{~kg}
\end{gathered}
$$

$76.683 \mathrm{~g} / \mathrm{iter}$

$20.66 \mathrm{~kg}$

$30.44 \%$

$6.29 \mathrm{~kg}$

56.187 g/iter

$15.14 \mathrm{~kg}$

$6.73 \%$

$1.02 \mathrm{~kg}$ per WHCox,wh1

per WHCox.mbt

$19.25 \mathrm{~kg}$

\section{Nitrogen compound out of melter:}

Total Particulate Out:

$11.97 \mathrm{~kg}$

per OANI

$N O(3)$ in particulate:

$0.020 \%$

$0.0023 \mathrm{~kg}$

per oman

$\mathrm{N}$ from $\mathrm{NO}(3)$ :

$0.0005 \mathrm{~kg}$

$\mathrm{NO}(2)$ in particulate:

$0.709 \%$

$0.0849 \mathrm{~kg}$

$\mathrm{N}$ from $\mathrm{NO}(2)$ :

$0.0258 \mathrm{~kg}$

$\mathrm{NH}(3)$ in Particulate:

$0.001 \%$

$0.00012 \mathrm{~kg}$

$\mathrm{N}$ in $\mathbf{N H}(3):$

$82.35 \%$

$\mathrm{N}$ from $\mathrm{NH}(3)$ :

$9.9 E-05 \mathrm{~kg}$

NOx in Off-Gas:

$0.09864 \mathrm{~kg} / \mathrm{hr}$

Total NOx in Off-Gas:

$1.38096 \mathrm{~kg}$

$30.44 \%$

$N$ in NOx:

$0.420295 \mathrm{~kg}$

per ount

per onsi

Total N from NOx:

$0.12 \%$

\% NO(2)NO(3) lost of particulate:

$97.55 \%$ 


\section{Sulfur Balance}

Sulfur in:

$\mathrm{SO}(4)$ in Simulant:

Total Simulant In:

Total SO(4) in:

$\% S$ in SO(4):

4.16 g/iter

269.39 iter

$1.12 \mathrm{~kg}$

$33.33 \%$

Total $\mathrm{S}$ in:

Sulfur out:

SO(3) in Glass:

Total Glass Out:

Total SO(3) out:

$\% \mathrm{~S}$ in SO(3):

Total $S$ in Glass:

$S O(2)$ in Off-Gas:

SO(4) in Off-Gas Particulate:

Total SO(4) out:

$\%$ S in SO(4):

Total S in Particulate:

Total S out:

$\%$ missing:
0.239

$0.017 \%$

$406.42 \mathrm{~kg}$

$0.070 \mathrm{~kg}$

$40.00 \%$

$0.028 \mathrm{~kg}$

ND

\author{
$5.28 \%$ \\ $0.632 \mathrm{~kg}$ \\ $33.33 \%$ \\ $0.211 \mathrm{~kg}$
}

$36.19 \%$ 
Mass Balance for MT-3, V-SIM

Prepared by: Brent Spurgeon

Date: $\quad 08 / 17 / 95$

Checked by: John Koehr $4 k$

Date: $\quad 08 / 17 / 95$

\section{General Information}

V-SIM composition:

\begin{tabular}{lr} 
Component & \multicolumn{1}{c}{ W\% } \\
\hline Alumina & $11.500 \%$ \\
Silica Sand & $39.100 \%$ \\
Dolomite & $7.250 \%$ \\
Iron Oxide & $0.683 \%$ \\
NaNO(2) & $0.277 \%$ \\
NaCl & $0.286 \%$ \\
NaF & $0.456 \%$ \\
Na(2)SO(4) & $0.207 \%$ \\
NalO(3) & $0.090 \%$ \\
CsCl & $0.135 \%$ \\
KMnO(4) & $0.002 \%$ \\
NaMoO(4) & $0.014 \%$ \\
NaNO(3) & $0.681 \%$ \\
K(2)CO(3) & $0.825 \%$ \\
KOH & $0.669 \%$ \\
SrCO(3) & $0.119 \%$ \\
K(2)Cr(2)O(7) & $0.030 \%$ \\
Na(2)CO(3) & $23.900 \%$ \\
Boric Acid & $10.500 \%$ \\
Na(3)PO(4) ${ }^{-12 H(2) O ~}$ & $0.793 \%$ \\
Carbon & $2.230 \%$ \\
Mag Carb. & $0.035 \%$ \\
& $100 \%$
\end{tabular}

whovitos.xs = Throughpux Summary

Test Duration:

$3 \mathrm{hr}$

Total Feed Durring Test:

$$
220.0 \mathrm{lb}=
$$

$99.79 \mathrm{~kg}$

Average Off-Gas flow rate:

Total Off-Gas:

$657.05 \mathrm{~kg} / \mathrm{hr}$ $1971.15 \mathrm{~kg}$

Average Particulate in Off-Gas: $\quad 0.225 \mathrm{~kg} / \mathrm{hr}$

Total Particulate in Off-Gas:

$$
0.675 \mathrm{~kg}
$$

Total Glass Out:

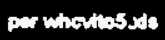

$151.0 \mathrm{lb}=$

$68.49 \mathrm{~kg}$
Per OMni Runs

324 


\section{Sodium Balance}

Sodium In:

Sodium in V-Sim

Components with Sodium

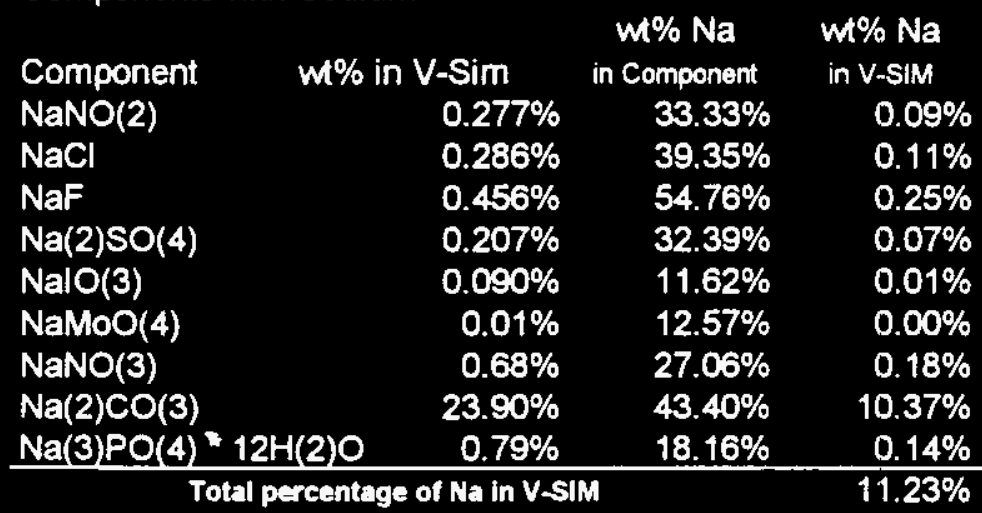

Total V-Sim Feed:

$99.79 \mathrm{~kg}$

Total Sodium Feed:

Equivalent $\mathrm{Na}(2) \mathrm{O}$ :

$15.11 \mathrm{~kg}$

Sodium Out:

Sodium in Off-Gas Particulate:

$19.35 \%$

Total Particulate in Off-Gas:

$0.675 \mathrm{~kg}$

Total Sodium Out:

$0.131 \mathrm{~kg}$

$\mathrm{Na}(2) \mathrm{O}$ equivalent:

$0.176 \mathrm{~kg}$

Percent Na lost to Off-Gas:

$1.17 \%$

$\mathrm{Na}(2) \mathrm{O}$ in Glass:

$16.10 \%$

Total glass Out:

$68.49 \mathrm{~kg}$

Total $\mathrm{Na}(2) \mathrm{O}$ out of glass:

$11.03 \mathrm{~kg}$

Total Na(2)O Out:

11.20

$\%$ not accounted for

$25.85 \%$ 


\section{Chloride Balance}

Chloride In:

Chloride in V-Sim:

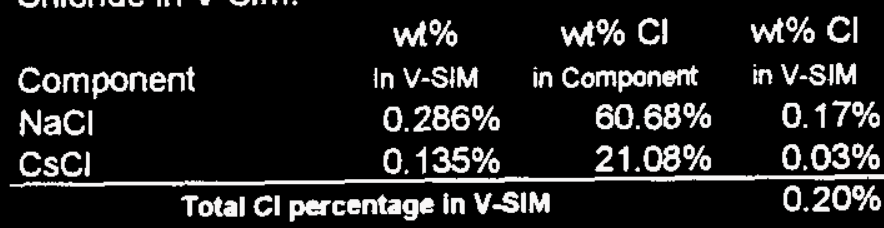

Total V-Sim Feed:

$99.79 \mathrm{~kg}$

Total Chloride Feed:

$0.20 \mathrm{~kg}$

Chloride Out:

Chloride in Particulate:

$9.07 \%$

Total Particulate in Off-Gas:

$0.675 \mathrm{~kg}$

Total Chloride in Particulate:

$0.06 \mathrm{~kg}$

Percent loss to Off-Gas:

$30.35 \%$

Chloride in Glass:

$0.10 \%$

Total Glass Out:

$68.49 \mathrm{~kg}$

Total Chloride in Class:

$0.068 \mathrm{~kg}$

$\%$ not accounted for:

$66.02 \%$ 


\section{Fluoride Balance}

Fluoride In:

Fluoride in V-Sim:

Component

$w \% \quad w \% F \quad w \%$

NaF

In V-SIM in Component in V-SIM

$0.00456 \quad 45.24 \% \quad 0.21 \%$

Total V-Sim Feed:

Total Fluoride Feed:

$99.79 \mathrm{~kg}$

$0.21 \mathrm{~kg}$

Fluoride Out:

Fluoride in Particulate:

$0.84 \%$

Total Particulate in Off-Gas:

Total Fluoride in Particulate:

$0.675 \mathrm{~kg}$

$0.01 \mathrm{~kg}$

Percent loss to Off-Gas:

$2.74 \%$

Fluoride in Glass:

Total Glass Out:

Total F in Class:

$0.16 \%$

$68.49 \mathrm{~kg}$

$0.109 \mathrm{~kg}$

Total F out:

$0.115 \mathrm{~kg}$

$\%$ not accounted for:

$44.30 \%$ 


\section{lodide Balance}

lodide In:

lodide in V-Sim:

Component

$\begin{array}{ccc}\text { w\% } & \begin{array}{c}\text { w } \% \text { I } \\ \text { in V-SIM }\end{array} & \begin{array}{c}\text { in Component } \\ \text { in V-SIM }\end{array} \\ 0.090 \% & 64.14 \% & 0.06 \%\end{array}$

Total V-Sim Feed:

Total lodide Feed:

$99.79 \mathrm{~kg}$
$0.06 \mathrm{~kg}$

lodide Out:

lodide in Particulate:

$1.80 \%$

Total Particulate in Off-Gas:

Total lodide in Particulate:

$0.675 \mathrm{~kg}$

$0.01 \mathrm{~kg}$

Percent loss to Off-Gas:

$21.13 \%$

I in Glass:

Total glass Out:

199.5 PPM

$68.49 \mathrm{~kg}$

$0.014 \mathrm{~kg}$

Total 1 out:

$0.026 \mathrm{~kg}$

$\%$ not accounted for:

$55.15 \%$ 


\section{Cesium Balance}

Cesium In:

lodide in V-Sim:

$\begin{array}{lccc}\text { Component } & w \% & w \% \text { Cs } & w \% \text { Cs } \\ \text { CsCl } & \text { in V-siM } & \text { in Component } & \text { in V-SiM } \\ \text { (n) } & 0.135 \% & 78.93 \% & 0.11 \%\end{array}$

Total V-Sim Feed:

$99.79 \mathrm{~kg}$

Total Cesium in Feed:

$0.11 \mathrm{~kg}$

\section{Cesium Out:}

Cesium in Off-Gas:

Cs(2)O in Glass

Total Glass out:

Total Cs(2)O in Glass:

Total Cs in glass:

$\%$ not accounted for

\section{Non Detectable}

$$
\begin{aligned}
& 0.057 \% \\
& 68.49 \mathrm{~kg} \\
& 0.039 \mathrm{~kg} \\
& 0.037 \mathrm{~kg}
\end{aligned}
$$

$65.38 \%$ 


\section{Strontium Balance}

Strontium In:

Strontium in V-Sim:

$\begin{array}{lcrc} & w \% & w \% \text { Sr } & w \% \text { Sr } \\ \text { Component } & \text { In V-SIM } & \text { in Component } & \text { in V-SIM } \\ \text { SrCO(3) } & 0.119 \% & 58.96 \% & 0.07 \%\end{array}$

Total V-Sim Feed:

Total Strontium Feed:

$99.79 \mathrm{~kg}$

$0.07 \mathrm{~kg}$

Sro equivalent out:

$0.083 \mathrm{~kg}$

\section{Strontium Out:}

Strontium in Particulate:

$0.03 \%$

Total Particulate in Off-Gas:

Total Strontium in Particulate:

$0.675 \mathrm{~kg}$

Sro Equivalent:

\section{$0.00018 \mathrm{~kg}$}

$0.00021 \mathrm{~kg}$

Percent loss to Off-Gas:

$0.25 \%$

Sro in Glass:

$0.067 \%$

Total Glass Out:

68.49

Total SrO in Glass:

$0.046 \mathrm{~kg}$

Total Sro Out:

0.046

$\%$ not accounted for:

$44.33 \%$ 


\section{Phosphorus Balance}

Phosphorus in:

Phosphorus in V-Sim:

\begin{tabular}{|c|c|c|c|c|}
\hline & & $\begin{array}{c}w \% \\
\text { in V-sIM }\end{array}$ & $\begin{array}{c}\text { W\% P } \\
\text { in Component }\end{array}$ & $\begin{array}{l}W \% P \\
\text { in V-SIM }\end{array}$ \\
\hline 12 & $12 \mathrm{H}(2) \mathrm{O}$ & $0.793 \%$ & $8.16 \%$ & $0.06 \%$ \\
\hline
\end{tabular}

Total V-Sim Feed:

$99.79 \mathrm{~kg}$

Total Phosphorus Feed:

$P(2) O(5)$ equivalent:

$0.148 \mathrm{~kg}$

Phosphorus Out:

Phosphorus in Particulate:

Total Particulate in Off-Gas:

Total Phosphonus in Particulate:

$P(2) O(5)$ equivalent:

$0.03 \%$

$0.675 \mathrm{~kg}$

$0.0002 \mathrm{~kg}$

$0.0004 \mathrm{~kg}$

Percent loss to Off-Gas:

$0.26 \%$

$P(2) O(5)$ in Class:

$0.15 \%$

Total Glass Out:

$68.49 \mathrm{~kg}$

Total $P(2) O(5)$ in Class:

$0.103 \mathrm{~kg}$

Total $P(2) O(5)$ Out:

0.103

$\%$ not accounted for:

$30.25 \%$ 


\section{Boron Balance}

Boron In:

Boron in V-Sim:

Component

$w \% \quad w \% B \quad w \% B$

Boric Acid

In V-SIM in Component in V-SIM

$10.500 \% \quad 17.47 \% \quad 1.83 \%$

Total V-Sim Feed:

Total Boron Feed:

$99.79 \mathrm{~kg}$

$1.83 \mathrm{~kg}$

$B(2) O(3)$ equivalent:

$5.90 \mathrm{~kg}$

Boron Out:

Boron in Particulate:

Total Particulate in Off-Gas:

Total Boron in Particulate:

$B(2) O(3)$ equivalent:

Percent loss to Off-Gas:

$4.37 \%$

$B(2) O(3)$ in Glass:

$7.60 \%$

Total Glass Out:

$68.49 \mathrm{~kg}$

Total $\mathrm{B}(2) \mathrm{O}(3)$ in glass:

$5.21 \mathrm{~kg}$

Total $B(2) O(5)$ OUT:

$5.46 \mathrm{~kg}$

$\%$ not accounted for:

$7.94 \%$ 


\section{Alumina Balance}

Alumina In:

\begin{tabular}{lcrr} 
Component & $\begin{array}{c}\text { WH\% } \\
\text { in V-Sim }\end{array}$ & $\begin{array}{c}\text { Wt\% Al(2)O(3) } \\
\text { in Alumina }\end{array}$ & $\begin{array}{c}\text { WH Al(2)O(3) } \\
\text { Total }\end{array}$ \\
\hline Alumina & $11.500 \%$ & $65.00 \%$ & $7.48 \%$
\end{tabular}

Total V-Sim Feed:

$99.79 \mathrm{~kg}$

Total Al(2)O(3) in:

Alumina Out:

$\begin{array}{lc}\text { Al in Particulate out: } & 1.029 \% \\ \text { Total Particulate Out: } & 0.675 \\ \text { Total Al Out: } & 0.0069 \mathrm{~kg} \\ \text { Al(2)O(3) equivalent: } & 0.0131 \mathrm{~kg} \\ & \\ \text { Al(2)O(3) in Glass: } & 9.60 \% \\ \text { Total Glass Out: } & 68.49 \mathrm{~kg} \\ \text { Total Al(2)O(3): } & 6.58 \mathrm{~kg} \\ \text { Total Al(2)O(3) out: } & 6.59 \\ \text { \% not accounted for: } & 11.68 \%\end{array}$

\section{Silica Balance}

Silica In:

\begin{tabular}{|c|c|c|c|}
\hline Component & $\begin{array}{c}W \% \\
\text { in V-sim }\end{array}$ & $\begin{array}{l}\text { Wh SiO(2) } \\
\text { in Componert }\end{array}$ & $\begin{array}{c}\text { Wo SiO(2) } \\
\text { Total }\end{array}$ \\
\hline Silica Sand & $39.100 \%$ & $99.80 \%$ & $39.02 \%$ \\
\hline Dolo & $7.250 \%$ & $5.00 \%$ & $\frac{0.36 \%}{39.38 \%}$ \\
\hline
\end{tabular}

Total V-Sim Feed:

$99.79 \mathrm{~kg}$

Total SiO(2) in:

Silica Out:

Si in Particulate:

Total Particulate:

Total Si in Particulate:

$0.675 \mathrm{~kg}$

SiO(2) equivalent:

$0.0062 \mathrm{~kg}$

$0.0133 \mathrm{~kg}$

SiO(2) in Glass:

Total Glass Out:

$57.44 \%$

Total SiO(2) in Glass:

$68.49 \mathrm{~kg}$

$39.34 \mathrm{~kg}$

Total SiO(2) Out:

$39.36 \mathrm{~kg}$

$\%$ excess:

$0.14 \%$ 


\section{Calcium Balance}

Calcium In:

\begin{tabular}{lrrr} 
Component & $\begin{array}{c}\text { Wt\% } \\
\text { in V-Sim }\end{array}$ & $\begin{array}{c}\text { W\% CaO } \\
\text { in Component }\end{array}$ & $\begin{array}{c}\text { Ww CaO } \\
\text { Total }\end{array}$ \\
\hline Doiomite & $7.250 \%$ & $30.24 \%$ & $2.19 \%$
\end{tabular}

Total V-Sim Feed:

$99.79 \mathrm{~kg}$

Total CaO in:

Calcium Out:

CaO in Glass:

$2.94 \%$

Total Glass Out:

$68.49 \mathrm{~kg}$

Total CaO in Glass:

$\%$ not accounted for:

$8.09 \%$

\section{Magnesium Balance}

Mgo in:

\begin{tabular}{lrrr} 
Component & $\begin{array}{c}\text { Wt\% } \\
\text { in V-Sim }\end{array}$ & $\begin{array}{c}\text { W\% Mgo } \\
\text { in Component }\end{array}$ & $\begin{array}{c}\text { W\% Mgo } \\
\text { Total }\end{array}$ \\
\hline Dolomite & $7.250 \%$ & $19.13 \%$ & $1.39 \%$ \\
Mag Carb. & $0.035 \%$ & $42.20 \%$ & $0.01 \%$ \\
\hline & & & $1.40 \%$
\end{tabular}

Total V-Sim Feed:

$99.79 \mathrm{~kg}$

Total MgO:

Mgo Out:

MgO in Glass:

$2.23 \%$

Total Glass Out:

Total MgO in Glass:

\% excess:

$9.22 \%$ 


\section{Iron Balance}

Iron In:

\begin{tabular}{lrrr} 
Component & $\begin{array}{c}W \% \\
\text { in V-Sim }\end{array}$ & $\begin{array}{r}W \% \mathrm{Fe}(2) \mathrm{C}(3) \\
\text { in Componemt }\end{array}$ & $\begin{array}{r}\text { Wt\% Fe(2)o } \\
\text { Total }\end{array}$ \\
\hline Iron Oxide & $0.683 \%$ & $98 . C 0 \%$ & $0.67 \%$
\end{tabular}

Total V-Sim Feed:

$99.79 \mathrm{~kg}$

Total $\mathrm{Fe}(2) \mathrm{O}(3)$ in:

$0.67 \mathrm{~kg}$

Iron Out:

$\mathrm{Fe}(2) \mathrm{O}(3)$ in Glass:

$0.99 \%$

Total Glass Out:

Total $\mathrm{Fe}(2) \mathrm{O}(3)$ in Glass:

$\%$ excess:

$1.86 \%$

\section{Chromium Balance}

Chromium In:

\begin{tabular}{|c|c|c|c|}
\hline & $\begin{array}{c}\text { Wt\% } \\
\text { in V-Sim }\end{array}$ & $\begin{array}{l}\text { Wh\% } \mathrm{Cr}(2) \mathrm{O}(3) \\
\text { in } \mathrm{Component}\end{array}$ & $\begin{array}{c}\text { wr\% Cr(2)o } \\
\text { Total }\end{array}$ \\
\hline ( & 0 & $\bar{T}$ & 800 \\
\hline
\end{tabular}

Total V-Sim Feed:

$99.79 \mathrm{~kg}$

Total $\mathrm{Cr}(2) \mathrm{O}(3)$ in:

$0.0155 \mathrm{~kg}$

Chromium Out:

Chromium in Particulate:

Total Particulate Out:

Total Chromium in Particulate:

$\mathrm{Cr}(2) \mathrm{O}(3)$ equivalent:

$0.037 \%$

$0.675 \mathrm{~kg}$

$0.00025 \mathrm{~kg}$

$0.00036 \mathrm{~kg}$

$\mathrm{Cr}(2) \mathrm{O}(3)$ in Glass:

Total Glass Out:

$0.26 \%$

Total $\mathrm{Cr}(2) \mathrm{O}(3)$ in Class:

$68.49 \mathrm{~kg}$

$0.1781 \mathrm{~kg}$

Total $\operatorname{Cr}(2) \mathrm{O}(3)$ Out:

$0.1784 \mathrm{~kg}$

\% excess:

$1052.89 \%$ 
Manganese Balance

Manganese In:

\begin{tabular}{lrrr} 
Component & $\begin{array}{c}\text { Wt\% } \\
\text { in V-Sim }\end{array}$ & $\begin{array}{r}\text { Wt\% MnO(2) } \\
\text { in Component }\end{array}$ & $\begin{array}{c}\text { W\% MnO(2 } \\
\text { Total }\end{array}$ \\
\hline $\mathrm{KMnO}(4)$ & $0.002 \%$ & $55.03 \%$ & $0.0011 \%$
\end{tabular}

Total V-Sim Feed:

Total $\mathrm{MnO}(2)$ in:

$99.79 \mathrm{~kg}$

$0.0011 \mathrm{~kg}$

Manganese Out:

$\mathrm{MnO}(2)$ in Glass:

Total Glass Out:

$0.009 \%$

Total MnO(2) in Glass:

$68.49 \mathrm{~kg}$

$0.006 \mathrm{~kg}$

\% Excess:

$479.99 \%$

\section{Molybdenum Balance}

Molybdenum In:

\begin{tabular}{lrrr} 
Component & $\begin{array}{c}\text { Wt\% } \\
\text { in V-Sim }\end{array}$ & $\begin{array}{c}\text { W\% MoO(3) } \\
\text { in Component }\end{array}$ & $\begin{array}{c}\text { Wh\% MoO(3 } \\
\text { Total }\end{array}$ \\
\hline NaMoO(4) & $0.014 \%$ & $78.63 \%$ & $0.0110 \%$
\end{tabular}

Total V-Sim Feed:

Total MoO(3) in:

$99.79 \mathrm{~kg}$

$0.0110 \mathrm{~kg}$

Molybdenum Out:

$\mathrm{MoO}(3)$ in Glass:

$0.16 \%$

Total Class Out:

$68.49 \mathrm{~kg}$

Total MoO(3) in Glass:

$0.11 \mathrm{~kg}$

\% Excess:

$885.13 \%$ 


\section{Potassium Balance}

Potassium In:

\begin{tabular}{|c|c|c|c|}
\hline Component & $\begin{array}{c}\text { Wt\% } \\
\text { in V-Sim }\end{array}$ & $\begin{array}{l}\text { W\% K(2)O } \\
\text { in Component }\end{array}$ & $\begin{array}{c}\text { wt\% K(2)o } \\
\text { Total }\end{array}$ \\
\hline $\mathrm{KMnO}(4)$ & $0.002 \%$ & $59.46 \%$ & $0.0012 \%$ \\
\hline $\mathrm{K}(2) \mathrm{CO}(3)$ & $0.825 \%$ & $68.12 \%$ & $0.5620 \%$ \\
\hline $\mathrm{KOH}$ & $0.669 \%$ & $167.86 \%$ & $1.1230 \%$ \\
\hline $\mathrm{K}(2) \operatorname{Cr}(2) \mathrm{O}(7)$ & $0.030 \%$ & $31.97 \%$ & $\begin{array}{r}0.0096 \% \\
1.70 \%\end{array}$ \\
\hline
\end{tabular}

Total V-Sim In:

$99.79 \mathrm{~kg}$

Total K(2)O in V-Sim:

Potassium Out:

Potassium in Particulate:

\section{$3.44 \%$}

Total Particulate out:

$0.675 \mathrm{~kg}$

$0.023 \mathrm{~kg}$

Total Potassium in Particulate:

$K(2) O$ equivalent:

$K(2) O$ in Class:

$0.82 \%$

Total Glass Out:

$68.49 \mathrm{~kg}$

Total K(2)O in Glass:

$0.558 \mathrm{~kg}$

Total K(2) O Out:

$0.586 \mathrm{~kg}$

$\%$ not accounted for:

$65.36 \%$ 


\section{Carbon Balance}

Carbon in:

\begin{tabular}{lrrr} 
Component & $\begin{array}{c}\text { Wi\% } \\
\text { in V-Sim }\end{array}$ & $\begin{array}{c}\text { wi\% C } \\
\text { in Component }\end{array}$ & $\begin{array}{l}\text { W\% C } \\
\text { Total }\end{array}$ \\
\hline Dolomite & $7.250 \%$ & $12.17 \%$ & $0.8825 \%$ \\
$\mathrm{~K}(2) \mathrm{CO}(3)$ & $0.825 \%$ & $8.68 \%$ & $0.0716 \%$ \\
$\mathrm{SrCO}(3)$ & $0.119 \%$ & $8.13 \%$ & $0.0097 \%$ \\
$\mathrm{Na}(2) \mathrm{CO}(3)$ & $23.900 \%$ & $11.32 \%$ & $2.7057 \%$ \\
Carbon & $2.230 \%$ & $100.00 \%$ & $2.2300 \%$ \\
Mag Carb. & $0.035 \%$ & $10.09 \%$ & $0.0035 \%$ \\
\cline { 2 - 4 } & & & $5.9031 \%$
\end{tabular}

Total V-Sim In:

total C in V-Sim:

$99.79 \mathrm{~kg}$

$5.89 \mathrm{~kg}$

\section{Carbon Out:}

C in Off-Gass:

Carbon Dioxide Out:

$5.75 \mathrm{~kg} / \mathrm{hr}$

$17.25 \mathrm{~kg}$

Carbon Equivalent:

$4.71 \mathrm{~kg}$

Carbon Monoxide Out:

$0.82 \mathrm{~kg} / \mathrm{hr}$

Carbon equivalent:

$2.46 \mathrm{~kg}$

$1.06 \mathrm{~kg}$

Total C in Off-Gas:

$5.76 \mathrm{~kg}$

C in Glass:

Total Carbon in Class

$0.0033 \%$

Total Glass Out:

$68.49 \mathrm{~kg}$

Total C in Glass:

$0.0023 \mathrm{~kg}$

Total C Out:

$5.77 \mathrm{~kg}$

$\%$ not accounted for.

$2.10 \%$ 


\section{Nitrate/Nitrite Conversion}

Nitrate/Nitrite in:

\begin{tabular}{|c|c|c|c|}
\hline Component & $\begin{array}{c}\text { Wt\% } \\
\text { in V-Sim }\end{array}$ & $\begin{array}{c}\text { Wt\% } \\
\text { in Compenent }\end{array}$ & $\begin{array}{l}\text { W\% } \\
\text { Total }\end{array}$ \\
\hline \multirow{4}{*}{$\begin{array}{l}\text { Nitrate: } \\
\text { NaNO(3) } \\
\text { Nitrite: } \\
\text { NaNO(2) }\end{array}$} & $0.681 \%$ & $54.19 \%$ & $0.37 \%$ \\
\hline & & & \\
\hline & $0.277 \%$ & $66.67 \%$ & $0.18 \%$ \\
\hline & & Total & $0.55 \%$ \\
\hline \multicolumn{2}{|l|}{$\begin{array}{l}\text { Total V-Sim in: } \\
\text { Total Nitrate/Nitrite: }\end{array}$} & \multicolumn{2}{|c|}{$\begin{array}{r}99.79 \mathrm{~kg} \\
0.55 \mathrm{~kg}\end{array}$} \\
\hline \multicolumn{4}{|l|}{ NOx Out: } \\
\hline \multicolumn{2}{|l|}{ NOx in Off-Gas, (as NO(2)) } & \multicolumn{2}{|c|}{$\begin{array}{l}0.0949 \mathrm{~kg} / \mathrm{hr} \\
0.2847 \mathrm{~kg}\end{array}$} \\
\hline \multicolumn{2}{|l|}{$\begin{array}{l}\text { Nitrate/Nitrite in Particulate: } \\
\text { Total Particulate Out: } \\
\text { Total Out: }\end{array}$} & \multicolumn{2}{|c|}{$\begin{array}{r}\text { Negligible } \\
0.675 \mathrm{~kg} \\
0.0000 \mathrm{~kg}\end{array}$} \\
\hline Conversion to $N(2)$ : & & $48.47 \%$ & \\
\hline
\end{tabular}


WHC-SD-WM-VI-031

Revision 0

This page intentionally left blank. 


\section{DISTRIBUTION SHEET}

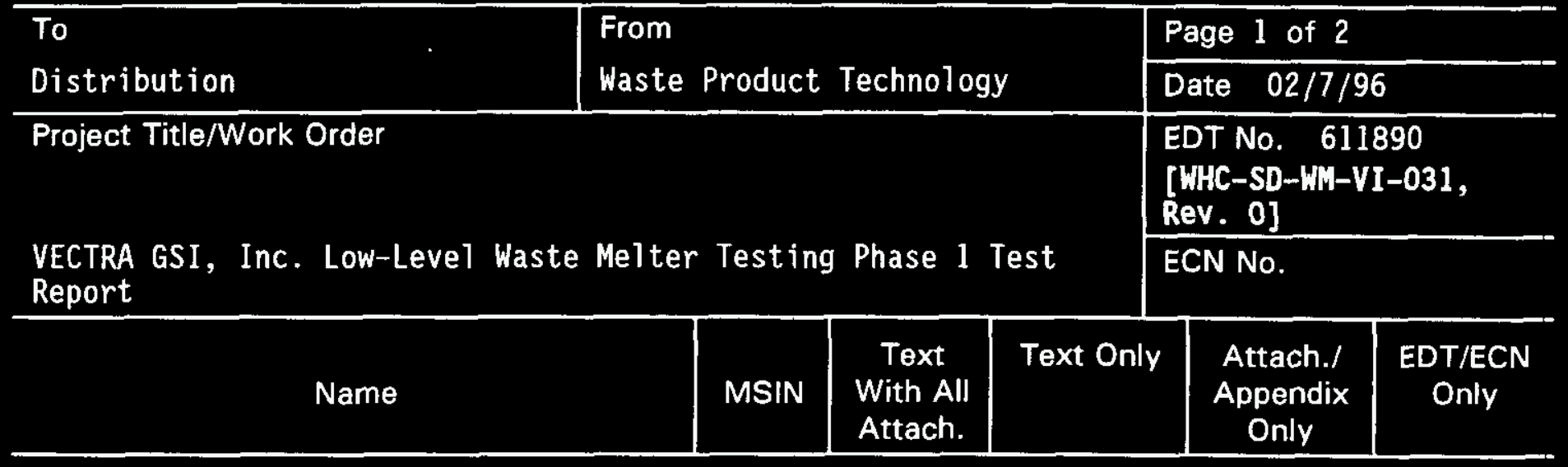

\section{OFFSITE}

VECTRA GSI, Inc.

500 Exectutive Parkway, Suite 500

San Ramon, CA 94583

G. Klein (2)

VECTRA Technologies

2939 Richardson Road

Richland, WA 99352

M. Hall (2)

B. Spurgeon

VECTRA Technologies

107 Carpenter Drive, Suite 110

Sterling, VA 20164

K. Allen (2)

$x$

VECTRA Waste Services

One Harbison Way, Suite 209

Columbia, SC 29212-3408

P. Denault (2)

$x$

Roger B. EK \& Associates, Inc

2711226 th Ave SE

Issaquah, WA 98027

R. B. Ek

$x$

Washington State Department

of Ecology

Nuclear Waste Program

P.0. Box 47600

01ympia, WA 98504-7600

J. Granthum (2) 


\section{DISTRIBUTION SHEET}

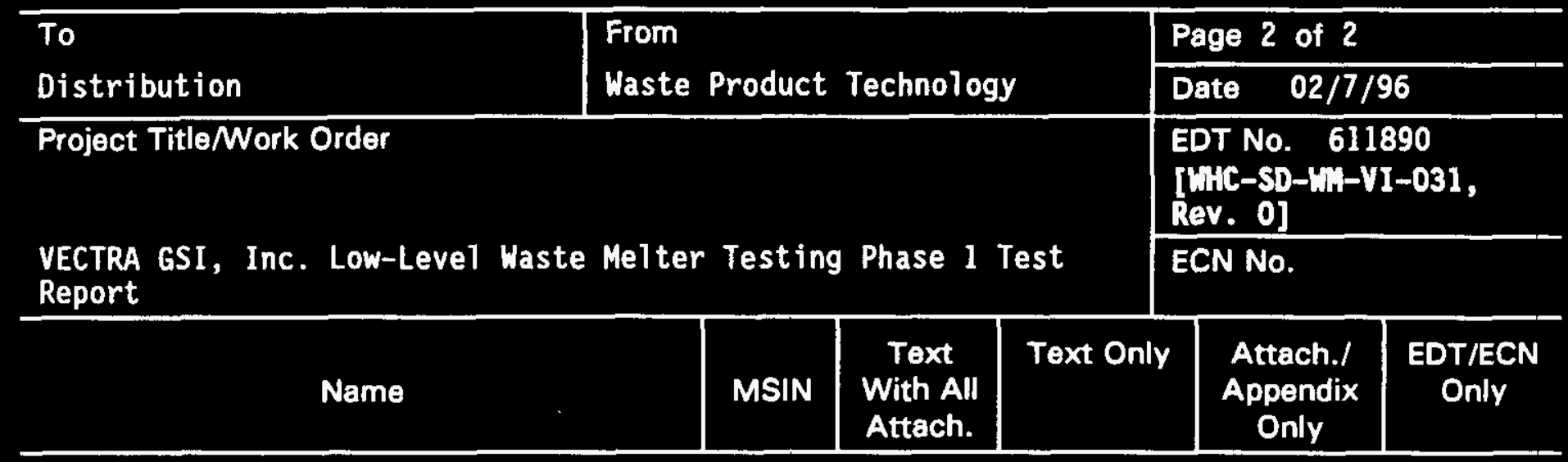

ONSITE

U.S. Department of Energy. Richland Operations office

N. R. Brown

L. A. Huffman

J. C. Peschong

G. H. Sanders

C. L. Sohn

Pacific Northwest National Laboratory

C. J. Freeman

D. Vela

J. H. Westsik

G. A. Whyatt

\section{Westinghouse Hanford Company}

M. J. Bowman

K. C. Burgard

R. P. Colburn

D. W. Duncan

W. C. Eaton

P. Felise

J. S. Garfield

R. L. Gibby

D. W. Hendrickson

B. A. Higley

S. L. Lambert

E. S. Mast

R. J. Murkowski

S. R. Nelson

R. M. Orme

C. A. Petersen

E. H. Randklev

J. W. Shade

G. E. Stegen

D. J. Washenfelder

C. N. Wilson (5)

Central Files (orig. +1)
$\begin{array}{ll}K 6-51 & X \\ K 6-51 & X\end{array}$

K6-51

S7-53

K6-51

P7-41

K6-51

K9-80

P7-19

$x$

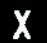

G1-25 $X$

H5-03 $X$

H5-27 $X$

B4-55

H5-27

B4-55

H5-49

H5-27

L5-31

H5-27

H5-27

S3-90

H5-03

B4-55

H5-27

H5-27

H5-27

H5-27

H5-27

H5-61

H5-27

A3-88

$X$
$X$

$\mathbf{X}$$$
x
$$

x

$x$
$x$
$x$
$x$

x 